Value, Competition and Exploitation 
To Katie. J.C.

A te. R.V. 


\section{Value, Competition and Exploitation \\ Marx's Legacy Revisited}

Jonathan F. Cogliano

Dickinson College, USA

Peter Flaschel

Bielefeld University, Germany

Reiner Franke

University of Kiel, Germany

Nils Fröhlich

University of Cooperative Education Glauchau, Germany

Roberto Veneziani

Queen Mary University of London, UK

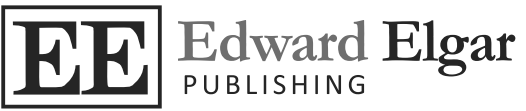

Cheltenham, UK • Northampton, MA, USA 
(c) Jonathan F. Cogliano, Peter Flaschel, Reiner Franke, Nils Fröhlich, Roberto Veneziani 2018

Cover image: CC - BY - 2.0 - (Flickr user: Russian_Constructivism). "Beat the Whites with the Red Wedge" - El Lissitzky

All rights reserved. No part of this publication may be reproduced, stored in a retrieval system or transmitted in any form or by any means, electronic, mechanical or photocopying, recording, or otherwise without the prior permission of the publisher.

Published by

Edward Elgar Publishing Limited

The Lypiatts

15 Lansdown Road

Cheltenham

Glos GL50 2JA

UK

Edward Elgar Publishing, Inc.

William Pratt House

9 Dewey Court

Northampton

Massachusetts 01060

USA

A catalogue record for this book

is available from the British Library

Library of Congress Control Number: 2018931754

This book is available electronically in the Elgaronline

Economics subject collection

DOI 10.4337/9781786430649

ISBN 9781786430632 (cased)

ISBN 9781786430649 (eBook) 


\section{Contents}

List of Figures $\quad \mathrm{x}$

List of Tables xiii

Preface $\quad \mathrm{xv}$

List of Abbreviations xviii

Notation xix

General Introduction 1

Part I Classical Competition: Theory, Evidence, and Criticism

1 François Quesnay: The Circular Flow of Income and InputOutput Analysis

1.1 Introduction 23

1.2 An early example of global economic reasoning 24

1.3 Input-output analysis: Surplus, multipliers and connectedness

1.4 Contemporary empirical input-output tables 41

1.5 Conclusions 49

1.6 Appendix: Further properties of matrices 50

2 Adam Smith: The "Invisible Hand" and Accumulation 53

2.1 Ruthless competition: The invisible hand in the early and rude state of society 53

2.2 The structure of the economy 57

2.3 Classical competition 60

2.4 CCE: Equilibrium and efficiency 63

2.5 Real wages and Malthusian population dynamics: Efficient pauperization 
2.6 Conclusions

2.7 Appendix: The neoclassical theory of the firm and capital accumulation

3 Adam Smith II: The "Invisible Hand" and "Natural Prices" 83

3.1 Restless competition II: Values and natural prices 83

3.2 Values and prices in the early and rude state 84

3.3 The multi-sector economy. Economic propositions and mathematical theorems

3.4 Empirical examples of wage-profit curves 98

3.5 Conclusions

4 David Ricardo: Long-Period Prices, Accumulation, and the Invariable Measure of Value

4.1 Introduction

109

4.2 Production data and labor values

110

4.3 Price equations and the wage-profit curve

114

4.4 Quantity equations and the consumption-growth curve

4.5 Income distribution, savings and the Classical closure

123

4.6 Differentiated saving habits and multiple equilibria

4.7 Proportions in production and relative price movements

4.8 The choice of numéraire: The foredoomed search for an invariable measure of value

4.9 Actual income shares in the open economy

4.10 Substitution and the choice of technique

154

4.11 Conclusions

5 The von Neumann-Sraffa Model 165

5.1 The von Neumann growth model 166

5.2 The gravitation of market prices in a square von Neumann-Sraffa system

5.3 Process and product extinction in the classical gravitation process

5.4 Conclusions 
Part II Value and Exploitation: Marx's Legacy

6 Labor Values: An Axiomatic Approach 189

$\begin{array}{lll}6.1 & \text { Introduction } & 189\end{array}$

6.2 The labor content of commodities: The real side of social interdependence 191

6.3 Basic principles when generalizing labor values 193

6.4 Simple quantitative features of the Labor Theory of Value

6.5 Pragmatic uses and applications of the notion of labor values

6.6 Conclusions 204

7 Labor Productivity and the Law of Decreasing Labor Content

7.1 Introduction 209

7.2 The labor content of commodities and the measurement of labor productivity

7.3 Technical change and the law of decreasing labor content

7.4 Conclusions

8 The Sources of Aggregate Profitability: Marx's Theory of Surplus Value Revisited

8.1 Introduction

8.2 The SNA approach to Marxian labor values

8.3 Average value and price gross rates of profit

242

8.4 Labor values and the LDLC: Empirical results 246

8.5 Three main sources of aggregate profitability 248

8.6 Conclusions 252

8.7 Appendix 253

9 Actual Labor Values with Multiple Activities 261

9.1 Introduction 261

9.2 Average and individual labor values in single product systems 263

9.3 Individual and average labor values 268

9.4 The measurement of labor contents 271 
9.5 Prices, profits, and rate(s) of exploitation 273

9.6 Conclusions 276

10 Joint Production in a (Marxian) System of National Accounts 279

10.1 Introduction 279

10.2 Joint production: The litmus test 282

10.3 Input-output methods: Which one to choose? 283

10.4 The 'Commodity Technology' and 'Industry Technology' Hypotheses 293

10.5 The determination of labor contents via U.N. IO tables 297

10.6 A 'physical' approach to the determination of the labor contents of commodities 300

10.7 Conclusions 303

11 Actual Labor Values with Joint Production 305

11.1 Introduction 305

11.2 An alternative definition of labor values 308

11.3 Properties of value and price relationships 316

11.4 Joint production and the main products of industries 321

11.5 Employment multipliers and labor values in joint production systems: Steedman after Stone 323

11.6 Labor values 326

11.7 Conclusions 332

11.8 Appendix 333

12 Production Prices and Imperfect Competition, Part I 343

12.1 Introduction 343

12.2 Differentiated sectoral wage and profit rates 344

12.3 Production prices with persistent wage and profit rate differentials

12.4 Production prices assuming real wage rates to be given 349

12.5 Distributive conflict and the wage-profit frontier $\quad 354$

12.6 Conclusions 355

13 Production Prices and Imperfect Competition, Part II 357

13.1 Introduction 357

13.2 Neo-Ricardian theory and empirical analysis $\quad 360$ 
13.3 Theoretical aspects of wage-profit curve measurements

364

13.4 Wage-profit curve measurement: An example

369

13.5 Conclusions

13.6 Appendix 1: Linear wage-profit curves and capital intensities

13.7 Appendix 2: Capital consumed and capital advanced

14 Conclusions 


\section{List of Figures}

2.1 The optimal allocation of labor in corn production 68

2.2 Income distribution in a classical corn economy 70

2.3 Adjustment path towards the steady state after an increase in agricultural productivity or when agricultural taxation is reduced

2.4 Income distribution in the neoclassical manufacturer world

3.1 The wage-profit curve and the conflict over income distribution with manufactured means of production

3.2 Empirical wage-profit curves under different consumption baskets

4.1 The wage-profit curve for $\kappa \equiv k_{2} / k_{1}=1$ (measured in terms of good 2)

$4.2 w(r)$ and $p_{1}(r)$ curves for $\kappa \neq 1$

4.3 The consumption-growth trade-off for $\kappa \neq 1$

4.4 The determination of average capital intensity $p_{1} k \quad 125$

4.5 The closure of the classical model by subsistence wages $w=$ $\bar{w}$ and the savings hypothesis $g=s_{k} r$; for $\kappa>1\left(p_{2}=1\right)$

4.6 The growth-profit curve for $k_{k}=0$, i.e. $g p_{1} k=s_{w}(w+$ $\left.r p_{1} k\right)$

4.7 The $g-r$ savings relationship for non-uniform capital intensities

4.8 The $g$ - $r$ curve for $k_{1}=k_{2}$

4.9 Pasinetti $\left(\bar{w}_{2}\right)$ and Anti-Pasinetti $\left(\bar{w}_{1}\right)$ outcomes under the classical closure

4.10 Sectoral labor intensities for $p y=1$ as function of the rate of profit $r$

4.11 Sectoral labor intensities for $p y=1$ over selected ranges of the rate of profit $r$

4.12 The prices of sectors $2-4$ for $p y=1$ as a function of the profit rate $r$ 
4.13 The prices of sectors 5-7 for $p y=1$ as a function of the profit rate $r$

4.14 The sectoral labor intensities for $p q^{n}=1$ as function of the rate of profit $r$

4.15 The prices of sectors $2-4$ for $p q^{n}=1$ as function of the rate of profit $r$

4.16 The prices of sectors 5-7 for $p q^{n}=1$ as function of the rate of profit $r$

4.17 Sectoral capital intensities $k_{j}$ as ratios of $\bar{k}$ as a function of the profit rate

4.19 National income and the wage-profit curve $\left(l(I-\delta A)^{-1} b=\right.$ $1=p b)$

4.20 Normalizing by net national product $(p y=1=l(I-$ $\delta A)^{-1} y$ ) for varying $r$

4.21 The illusion of a linear subdivision of national income

4.22 A simplification of the theory of income distribution? $[0<$ $\left.s_{w}<s_{k} \leq 1\right]$

4.23 Representing income distribution using the workers' consumption basket as the numéraire $(p c=p b=1, p y, w(r)$ from top to bottom)

4.24 Representing income distribution by way of Sraffa's Standard Commodity $\left(p q^{n}=1, p y, w(r)\right.$ from top to bottom)

4.25 The representation of income shares in net national product $\left(p q^{n}, p y=1, w(r)\right.$ from top to bottom)

4.26 Wage (lower line) and wage-plus-profit (upper line) shares in national product proper: $y=x-A^{d} x$

4.27 The wage-profit frontier of three alternative techniques in sector 1 and a single technique in sector $2\left(p_{2}=1\right.$ in all three cases, $\left.k=a_{1} / l\right)$

4.28 The wage-profit frontier for equation (4.24) and $p_{1}=1$

4.29 Smooth factor substitution in the one-good case $(\delta=0, k=$ $\left.a_{1} / l\right)$

4.30 Each single technique is in operation at exactly one point of the enveloping strictly convex wage-profit frontier $\left(w^{\max }=\right.$ $\left.1 / l, r^{\max }=1 / a_{1}\right)$

4.31 The classical closure and the choice of technique

4.32 The classical closure of the two degrees of freedom in the case of substitution for $\kappa>1, \kappa=1, \kappa<1$ 
7.1 An increase in net production possibilities and a decrease in the conventional measure of aggregate labor productivity $\left(p(0)=e^{\prime}\right)$

8.1 Comparing conventional and Marxian labor productivity indices: $\pi_{j}^{c}, 1 / v_{j}$

12.1 Differentiated sectoral rates of profit under exogenous real wage rates

13.1 Four wage-profit curves and their envelope 


\section{List of Tables}

1.1 An IO representation of the Tableau Économique for a closed economy

1.2 An aggregation example as starting point for a macroeconomic analysis of the circuit of income

1.3 The physical background of the Tableau Économique 27

1.4 The unit-output representation of the linear technology behind Quesnay's Tableau

1.5 The basic structure of a nominal IO table $\left(\hat{p}\left[X_{i j}\right]\right)$

1.6 The nominal unit coefficients of a linear technology behind Table 1.5: $A^{\text {nom }}=\hat{p} X \hat{x}^{-1} \hat{p}^{-1}$

1.7 The physical coefficients of a linear technology assumed behind the IO Table 1.5: $A=\hat{p}^{-1} A^{\text {nom }} \hat{p}=X \hat{x}^{-1}, x=$ $\left[x_{1}, \ldots, x_{n}\right]^{\prime}, p=\left[p_{1}, \ldots, p_{n}\right]$

1.8 A simple two-sector IO matrix

1.9 The two-sectoral standard aggregation 45

1.10 The 7-sector standard aggregation 47

1.11 Technological coefficients of the 7-sector aggregation (Germany, 2000)

1.12 Import shares (in percent) for the 7-sector aggregation 48

1.13 The Leontief-inverse $(I-A)^{-1}$ for the 7 -sector aggregation (Germany, 2000)

3.1 Commodity valuations in Smith's "early and rude state"

3.2 Labor coefficients $l$, capital intensities $k$, and consumption structure $c$ (Germany, 1995)

3.3 Eigenvalues of the technological matrix $A=A_{T}$ from Table 1.11

3.4 Right-hand (RH) and left-hand (LH) eigenvectors of $A=$ $A_{T}$

3.5 Production prices from equation (3.11)

103

3.6 Production prices in terms of labor commanded $(w=1) \quad 106$

7.1 The standard form of an IO table 
7.2 Elementary IO table in matrix notation

7.3 A two-sector economy with profitable capital-using and labor-saving technical change (at constant prices $p(0)=$ $\left.e^{\prime}, w=1\right)$

8.1 Technological coefficients of the 7-sectoral aggregation IO matrix (Germany, 1995, sectors 1-7 as columns, per million Euro of output value)

8.2 Depreciation matrix $A^{\delta}$ (Germany, 1995) 240

8.3 Capital stock matrix $K$ (Germany, 1995) 242

8.4 Consumption vs. final demand (million Euro) 243

8.5 Labor values or total labor costs (persons per million Euro of output value) 247

8.6 Main results 250

8.7 Employment, labor value of capital stock, price value of capital stock, and relative value of capital stock (measured in million persons)

8.8 Data underlying the construction of matrices $K$ and $A^{\delta} \quad 255$

8.9 CVAR resulting from different values of $\delta^{p l t}$ and $\delta^{e q t} \quad 256$

8.10 Empirical sectoral rates of profit (Germany, 1995) 257

8.11 German sectoral profit rates (profits per unit of capital goods advanced) over the 1990s

9.1 A version of Steedman's two-sector corn economy 264

10.1 A joint production example economy 285

10.2 The monetary equivalent of Table $10.1 \quad 286$

10.3 An arbitrary homogeneity assumption 286

10.4 An arbitrary sales transfer assumption 287

10.5 Negative coefficients in single IO tables 291

10.6 A method that rules out negative coefficients in single IO tables

10.7 The theoretical IO framework of the SNA 297

11.1 The two-sector corn economy again 336

11.2 The two-sector corn economy with rising efficiency 338

11.3 The two-sector corn economy with Sraffa's vintage prices 340

12.1 Sectoral price components (Germany, 1995) 346

12.2 Empirical two-sectoral data for equation (12.7) (Germany, 1995) 


\section{Preface}

This book arises out of our dissatisfaction with the current state of economic theory, especially after the recent financial crisis. The crisis of 2008 represented an opportunity to overturn and rethink much of the stale or misguided parts of economic theory to build a theoretically rich and empirically meaningful social science. However, this kind of reinvention has not taken place, and the response of economic theory - broadly speaking - has not done a great deal to move the discipline forward. The standard approach to economics remains largely unchanged, the only changes that appear to be taking place are some minor reconsiderations around the edges while maintaining the same core body of ideas as before the crisis. In our view, this state of affairs will not suffice to better understand the deep structural forces that drive everyday market behavior and contribute to the development of crises. Something different is needed.

This book offers an alternative to the standard, or neoclassical, approach to understanding notions of value, prices, and competitionconcepts that serve as the foundation for a theoretically and empirically robust economic theory - by reconsidering the classicalMarxian tradition using the modern tools of economic analysis. The broad literature in classical-Marxian political economy has made great strides in elucidating the causes and consequences of the growth and development of capitalist economies, while forcefully showing the central importance of class conflict. However, there remain vigorous debates around key tenets of Marxian value theory. Chief among these is the longstanding debate over the saliency of Marx's labor theory of value (LTV) - started right after the posthumous publication of volume three of Capital in 1894.

The primary arguments of this book turn specifically on these issues and where they stand today. The main objectives are to provide a formally rigorous and empirically informed approach to the classical theories of value and price and to demonstrate that Marx's LTV remains a valuable tool to understand the structure and dynamics of capitalist economies when viewed as part of Richard Stone's (1968) 
System of National Accounts (SNA). The connection between Stone's SNA and Marx's LTV is likely unintentional, but the implications are of interest to those active in Marxian political economy, Keynesian macroeconomics (broadly defined) and the neo-Ricardian tradition. An added benefit of the approach detailed in this book is that Marxian value theory can be shown to provide an arguably deeper analytical framework than current mainstream theory.

The central concepts of mainstream, neoclassical economics are largely familiar. They are frequently used in the media and have entered popular discussions. Further, they are usually considered to capture directly measurable magnitudes and events of immediate empirical import. The central categories of classical and Marxian economics are less familiar nowadays and they are also more difficult to grasp, because they aim to capture deep processes occurring under the surface of observable economic events and they refer to magnitudes that often are not directly measurable.

The aim of this book is to provide a comprehensive and rigorous, but accessible, analysis of classical and Marxian price and value theory. The exposition will proceed chronologically focusing first on the classical authors and then on Marx. This will allow us to introduce the reader to some interesting and important-but often neglected-issues in the history of economic thought and provide the necessary historical context for our discussion. However, our interest is not primarily historical or exegetical, nor do we aim to prove that Marx and the classics 'were right', and their analyses can be straightforwardly applied to the economies of the 21st century. Rather, the book aims to show that the broad conceptual framework and methodology of the classical authors and Marx provides interesting and relevant insights on the basic structure and evolution of modern capitalist economies. It is not a matter of 'going back to the classics', and reading their texts in order to find replies to the urgent contemporary questions. But it is possible to reconsider the core of their approach in the light of recent developments in economics, and also in mathematical tools, and to gain a different, arguably deeper perspective into today's problems than is offered by mainstream approaches. Our hope is to convince the reader that this exercise is indeed fruitful.

Much of what is contained within this book stems from the research program of Peter Flaschel. Peter's efforts serve as the driving force behind bringing this body of work together, and this book builds on and extends Peter's seminal contributions on value theory and the classical-Marxian tradition. The interpretation of Marxian theory developed in this book is part of a larger project of reconstruction of 
a broad, integrated Marx-Keynes-Schumpeter model of capitalism that encompasses both microeconomics (the main topic of this book) and macroeconomic dynamics. ${ }^{*}$

This book is the product of a long-term analysis of values and prices in capitalist economies and builds on it. Thus, some material in the book is drawn from already published papers. Specifically, we reuse the following articles, with changes to notation and exposition where appropriate:

- Chapter 7 repurposes Flaschel, Franke, and Veneziani (2013), "Labor productivity and the Law of Decreasing Labor Content," Cambridge Journal of Economics 37, pp. 379-402;

- Chapter 8 adapts Flaschel, Fröhlich, and Veneziani (2013a), "The sources of aggregate profitability: Marx's theory of surplus value revisited," Intervention - European Journal of Economics and Economic Policies 10, pp. 299-312;

- Chapter 13 is drawn from Flaschel, Franke, and Veneziani (2012a), "The measurement of prices of production: An alternative approach," Review of Political Economy 24, pp. 417-435.

We are grateful to the publishers for permission to reuse these works.

We are indebted to several people for detailed and constructive comments on earlier versions of the manuscript. We would like to thank Clopper Almon, Donald Katzner, Heinz Kurz, Fred Lee, Theodore Mariolis, Gary Mongiovi, Simon Mohun, Neri Salvadori, Anwar Shaikh, Ian Steedman, and Naoki Yoshihara for their comments and suggestions on various parts of the manuscript. Roberto Veneziani has worked on this project while visiting the University of Massachusetts, Amherst: their hospitality and support are gratefully acknowledged. The feedback we have received has significantly helped us in clarifying the arguments of this book but the responsibility of any remaining errors is ours. Lastly, we are also grateful for support provided by the Research and Development Committee of Dickinson College.

\footnotetext{
* For an overview of Peter Flaschel's intellectual journey, see Flaschel (2013).
} 


\section{List of Abbreviations}

CS-LS Capital-Saving Labor-Saving Technical Change

CS-LU Capital-Saving Labor-Using Technical Change

CTH Commodity Technology Hypothesis

CU-LS Capital-Using Labor-Saving Technical Change

CVAR Capital/Value Added Ratio

FMT Fundamental Marxian Theorem

IO Input-Output

ITH Industry Technology Hypothesis

KU-LS Fixed Capital-Using Labor-Saving Technical Change

LDLC Law of Decreasing Labor Content

LTV Labor Theory of Value

MAT Marxian Aggregation Theorem

MPT Marxian Proportionality Theorem

SNA System of National Acounts 


\section{Notation}

For any two vectors $x=\left(x_{1}, \ldots, x_{n}\right)$ and $y=\left(y_{1}, \ldots, y_{n}\right)$ in the $n$ dimensional Euclidean space, the notation for vector inequalities is as follows: $x>y$ if and only if $x_{i}>y_{i}$ for all $i=1, \ldots, n ; x \geqq y$ if and only if $x_{i} \geq y_{i}$ for all $i=1, \ldots, n$; and $x \geq y$ if and only if $x_{i}>y_{i}$ for some $i$ and $x \neq y . x=y$ denotes two vectors for which all elements are equal, i.e. $x_{i}=y_{i}$ for all $i=1, \ldots, n$, whereas for some scalar $\phi>0$, $x=\phi y$ denotes proportionality between the vectors $x$ and $y . x, y \geqq 0$ denotes nonnegative vectors $x$ and $y, x, y \geq 0$ denotes semi-positive vectors $x$ and $y$, and $x, y>0$ denotes strictly positive vectors $x$ and $y$. The same notation applies for matrices of any dimension.

Steady state values are indicated by a superscript ' $o$ '. When no confusion arises, letters $F, G, H$ may also define certain functional expressions in a specific context.

$\begin{array}{ll}p & \left.\text { production price vector (a row: } p=\left(p_{1}, \ldots, p_{n}\right)\right) \\ p^{w} & \text { production price vector in labor commanded } \\ w & \text { wage rate } \\ \omega & \text { the real wage } \\ \bar{\omega} & \text { natural wage in classical theory } \\ Y & \text { output } \\ K & \text { capital stock or matrix of capital coefficients } \\ s & \text { savings rate } \\ L & \text { total hours worked by employed workers } \\ h & \text { number hours worked per worker per day } \\ r & \text { profit rate } \\ R=1+r & \\ \rho & \text { rent on land } \\ g & \text { growth rate } \\ \epsilon & \text { rate of exploitation } \\ i & \text { interest rate } \\ \delta & \text { depreciation rate } \\ \pi & \text { labor productivity }\end{array}$


$v \quad$ vector of labor values (a row: $\left.v=\left(v_{1}, \ldots, v_{n}\right)\right)$

$\Omega \quad$ organic composition of capital

$\kappa=k_{i} / k_{j} \quad$ ratio of capital intensities

$k$

c

$x$

$y$

$\nu$

b

$A=\left[a_{i j}\right]$

$A^{k}$

$A^{\prime}=\left[a_{j i}\right]$

$l$

$A_{\star j}$

$A_{i \star}$

$A B$

vector of capital-labor ratios

consumption per unit of labor

subsistence or consumption vector

into industry $j$

$k$-th power of $A$

the transposed of the matrix $A$

vector of labor inputs $\left(\right.$ a row $\left.l=\left(l_{1}, \ldots, l_{n}\right)\right)$

the $j$-th column of the matrix A

the $i$-th row of the matrix A
$A^{b}=A+b l$

vector of activity levels (a row: $\left.x^{\prime}=\left(x_{1}, \ldots, x_{n}\right)\right)$

vector of final demands (a row $\left.y^{\prime}=\left(y_{1}, \ldots, y_{n}\right)\right)$

vector of values added, or net output per worker

matrix of physical intermediate inputs of industry $i$

product of matrices $A$ and $B\left(A B_{i k}=\left[A_{i *} B_{* k}\right]\right)$

workers' subsistence basket

$A^{c}=A+c l$ matrix of physical inputs augmented by

workers' consumption basket

$B \quad$ matrix of physical outputs

$\hat{x} \quad$ matrix with elements of vector $x$ on the diagonal

$\mathbb{R} \quad$ the set of real numbers

$e_{j} \quad$ the $j$-th vector $(0, \ldots, 0,1,0, \ldots, 0)$ of the

canonical basis of $\mathbb{R}$

$\mathbb{R}^{n} \quad$ vector space of dimension $n$

$\mathbb{R}^{n \times m} \quad$ vector space of $n \times m$ vectors or matrices

$\mathbb{R}_{+}^{n}, \mathbb{R}_{++}^{n} \quad$ the nonnegative respectively positive orthant of $\mathbb{R}^{n}$

e

$\mathcal{S}$

$I$

$M=\left[m_{i j}\right]$

$M^{-1}$

$\|\cdot\|$

$\frac{d}{d x}, \frac{d^{2}}{d x^{2}}, \ldots$ the vector $(1, \ldots, 1))$

summation matrix

identity matrix $(I=\hat{e})$

difference matrices $(M=I-A$ etc. $)$

inverse matrix of $M$

Euclidean norm of scalars, vectors and matrices

differential operators (first, second derivative)

eigenvalues 


\section{General Introduction}

This book is placed within a long tradition of formal, mathematical analysis of Marxian economics, and indeed aims to revive it. Two related streams of literature are directly relevant to our project. The first stream concerns Marxian value theory, specifically the relationship between values and prices and the labor theory of value. For Marx values are the amount of labor time socially necessary to produceembodied in - a commodity and serve as underlying regulators of the structure and dynamics of market prices. The labor theory of value purports that there is a direct correspondence of prices to values, but this idea has run aground on a series of mathematical and theoretical issues: the so-called "transformation problem". The transformation problem has generated a vast literature with contributions from those trying to salvage Marx's theory as it is, those trying to show its unescapable defects, and those attempting to provide coherent reinterpretations in the spirit of Marx's original work.

The transformation problem and the associated issues are not new, with the debates around it beginning shortly after Marx's death. Discussions of the transformation problem make cyclical appearances in the literature, with long periods of relative inactivity punctuated by spurts of heated debate. One of the pioneers of a rigorous approach to value theory, and a central figure in the first wave of debates, is Ladislaus von Bortkiewicz (1949). His analysis of the relation between prices of production and labor values can be considered as the starting point of the rigorous treatment of the transformation problem. This is not the place to provide a comprehensive discussion of von Bortkiewicz's contribution and of the subsequent literature. For our purposes, the key point is that von Bortkiewicz showed that, even in the simplest linear economies, labor values - as traditionally definedcannot be thought of as determining (relative, equilibrium) prices in any meaningful sense. Thus, the (standard interpretation of the) LTV is flawed.

It is important to stress that von Bortkiewicz's results are mathematically true, and in a sense, von Bortkiewicz set the parameters 
of the discussion, becoming the point of reference-positive or negative - for most of the subsequent contributions. Some authors have denied the relevance of von Bortkiewicz's analysis arguing that he focused on the wrong price or value magnitudes, and that appropriately defined values do determine appropriately defined prices. More persuasively, other authors have interpreted von Bortkiewicz's results as the starting point for a reconsideration of the role of value theory within Marxian economics. The LTV may be inadequate to explain relative prices, ${ }^{1}$ but this does not mean that labor accounts are irrelevant to explain some fundamental characteristics of capitalist economies that are relevant from both a positive and a normative viewpoint.

The key point to note is that there is no single, natural interpretation of Marxian value theory. The received view is that the main aim of the LTV is predictive: labor values are meant to explain (relative, equilibrium) prices. Yet even within a predictive interpretation, labor magnitudes may be relevant to explain other phenomena of capitalist economies. For example, one may argue that the LTV establishes a relation between profits and exploitative relations, thus allowing one to explain investment and growth. More generally, however, it is not clear that Marxian value theory can only be interpreted as a predictive exercise. For "there are at least three distinct non-metaphysical interpretations of the labour theory of value, viz. (i) descriptive, (ii) predictive and (iii) normative" (Sen 1978, 175).

One descriptive interpretation of the LTV is that of capturing the process of formation of equilibrium prices in capitalist economies, as in the standard view. But this is certainly not the only possibility. One may argue that in the LTV "it is the activity of production that is being described... [with a focus] on 'personal participation'" (Sen 1978, 177). Or it may be relevant to measure and understand some important characteristics of capitalist economies - such as the dynamics of productivity and profitability, or the implications of technical progress - that are not immediately visible by focusing on monetary, market magnitudes.

\footnotetext{
${ }^{1}$ It is worth noting, however, that the second important, albeit often neglected, contribution of von Bortkiewicz's seminal analysis is the early solution to the problem of the 'transformation' of labor magnitudes into price magnitudes based on the proof of the existence of a clear relation between the sphere of labor accounts and the sphere of monetary accounts. For a comprehensive discussion and review of the literature see Desai (1988). For more general, formal analyses see Morishima $(1973,1974)$ and Roemer (1981).
} 
But the LTV can also be interpreted primarily as providing the foundations for a normative, evaluative exercise and an indictment of capitalist relations of production. For example, one may argue that it explains the origin of profits as accruing from the exploitation of workers and therefore shows the illegitimacy of capitalist income, and the source of significant inequalities of well-being. Or it may be taken as providing the foundations of a distributive approach based on contribution and effort.

None of these interpretations are metaphysical, and none of them are affected by the standard criticisms leveled against Marxian value theory based on the transformation problem. ${ }^{2}$

The second stream of literature focuses on the Marxian theory of exploitation and classes. In the early stages of the Marxian economics literature, the solution of the 'transformation problem', and the proof that relative prices are determined by labor values was considered to be central to the Marxian project of proving that capitalism is fundamentally exploitative. One of the major contributions of Nobuo Okishio is the proof that - in linear, two-class economies with homogeneous labor - aggregate profits are positive if and only if the aggregate rate of exploitation is positive (Okishio 1963). In other words, even setting aside the issue of whether labor values determined relative prices, it is possible to prove that capitalism is inherently linked with the exploitation of workers. ${ }^{3}$ Indeed, the result has often-somewhat misleadingly - been interpreted as proving the Marxian insight that exploitation is the only, or main, source of profits. The significance of this result is such that it has been dubbed the Fundamental Marxian Theorem (FMT), and it has sparked a substantial literature. ${ }^{4}$

The contributions by von Bortkiewicz and Okishio, and the subsequent literature, allowed the establishment of some key insights of Marxian value theory - albeit properly qualified - in simple, twoclass linear economies. Yet they left open the fundamental question of

${ }^{2}$ For a detailed analysis of the variety of interpretations of Marxian value theory and a novel axiomatic interpretation of the LTV see Mohun and Veneziani (2017).

3 The result has been successfully extended to Leontief economies with heterogeneous labor by Bowles and Gintis (1977), Krause (1981), Fujimori (1982), and Veneziani and Yoshihara (2011).

4 The literature is too vast for a comprehensive list of references. In addition to the classic contributions cited in the text, it is worth mentioning the more recent discussions by Fleurbaey (1996), Mohun (2003), Flaschel (2010), Veneziani and Yoshihara (2012), and Veneziani and Yoshihara (2015a). For a survey see Yoshihara (2017). 
the validity of their results in more general settings. The key claim of Steedman's (1977) famous analysis is precisely that, outside of the simplest Leontief economies, not only are labor values irrelevant to determine prices of production: labor accounts are irrelevant to understand capitalist economies, labor values are logically flawed and the notion of exploitation is at best useless. Steedman (1977) analyzed von Neumann economies characterized by joint production and showed, by means of examples, that in this more general setting labor values could turn out to be negative and that the FMT does not hold: it is possible to have positive profits with negative surplus value and thus a negative exploitation rate.

These conclusions sparked a new wave of debates. Given the rather heated controversy, and the sometimes unfair criticisms (motivated by the perceived political relevance of the conclusions that seemed to question the core of Marxian economics), it is worth stressing that Steedman's results are plain mathematical truths. Within the formal and conceptual framework adopted by Steedman, there is no way of escaping his conclusions, and any criticisms denying this are a plain red herring. Rather, Steedman's seminal contribution can be interpreted as clarifying the terms of the analysis. Certain simpleminded generalizations of concepts that hold in Leontief economies are inappropriate in more general settings and yield paradoxical conclusions. Consider the notion of labor values first. In simple Leontief economies, the labor values of commodities are the standard Leontief employment multipliers. Steedman's key assumption is that this definition of labor values holds also in more general settings. This is by no means trivial and many authors have criticized this approach on formal, methodological and even exegetical grounds. Morishima (1974) and Morishima and Catephores (1978), for example, rejected Steedman's definition because it allowed for technically inefficient processes to determine labor values. ${ }^{5}$ More radically, since the early 1980s, the 'New Interpretation' originally developed by Duménil (1980) and Foley $(1982)^{6}$ has questioned the traditional dualist interpretation of Marxian value theory that has dominated the debate from the publication of Capital Volume III up until the 1970s-including both

\footnotetext{
${ }^{5}$ Morishima (1974) and Morishima and Catephores (1978) define the labor embodied in a bundle of goods as the minimum amount of labor necessary to produce the bundle as net output among all existing production techniques.

${ }^{6}$ See also Foley (1986b), Mohun (1993, 2004, 2009), Foley and Duménil (2008), Duménil, Foley, and Lévy (2009), Foley and Mohun (2016), and Cogliano (2013).
} 
Steedman (1977) and Morishima (1973). In the dualist approach, money plays no role and labor values and monetary magnitudes are assumed to form two conceptually separate systems. There is an underlying (intrinsic, invisible, essential) system of labor values and associated exploitation, and a phenomenal (extrinsic, visible, superficial) system of prices and profit rate. Marxian value theory is then interpreted as a predictive tool that bridges the gap between the two systems: relative labor values are meant to explain equilibrium relative prices. According to the New Interpretation, instead, money and labor accounts are expression of the same set of underlying mechanisms and form a single system within the circuit of capital. Thus, it is possible to define a variable, called the monetary expression of labor time (the ratio of net national income over total labor spent in production), which acts as a conversion rate between value and price magnitudes, allowing one to move from monetary accounts to labor accounts and vice versa.

Both Morishima's approach and the New Interpretation have raised inevitable controversy, but it is fair to say that their definitions of labor values in general economies are arguably closer to the spiritif not the letter - of Marx's own notion of labor values than the employment multipliers used by Steedman (1977). Further, by severing the link between the notion of labor values and that of employment multipliers, they avoid the paradoxical results derived by Steedman: negative labor values do not appear in either approach. ${ }^{7}$ Similar, and theoretically related, doubts have been raised on Steedman's definition of exploitation as the difference between the amount of labor expended by workers and the value of labor power, where the latter is defined as the amount of labor embodied in the workers' consumption bundle, computed using employment multipliers. According to Morishima

\footnotetext{
${ }^{7}$ Nor do they emerge in other approaches proposed in the literature sparked by Steedman's (1977) book. The discussion here does not aim to provide a comprehensive review of the debate. Rather, it focuses on those contributions that are conceptually and/or methodologically closer to the approach laid out in this book. Other interpretations of value theory that reject Steedman's conceptual and/or formal framework include Fine and Harris (1979); the 'Single-System Interpretation' by Wolff, Roberts, and Callari (1982); the 'Temporal Single-System Interpretation' (see for example, Freeman and Carchedi (1996), Kliman and McGlone (1999), for a thorough critical discussion see Veneziani (2004, 2005) and Mohun and Veneziani (2007, 2009)); the 'Macro-Monetary' interpretation by Moseley (2000, 2016); and the approach by Shaikh (1998, 2016). For a comprehensive survey, see Mohun and Veneziani (2017).
} 
(1974), for example, employment multipliers should not be used to compute the labor embodied in the workers' consumption bundle. If his definition of labor values is used, instead, then the FMT can be shown to hold in the balanced growth equilibria of von Neumann economies. ${ }^{8}$ According to the New Interpretation, instead, the value of labor power should be defined as the monetary wage divided by the monetary expression of labor time and not - as in standard dualistic approaches as the labor embodied in the bundle consumed by workers. Using this notion of the value of labor power, it is not difficult to show that the perverse examples provided by Steedman (1977) do not arise and a robust relation between profits and the exploitation of the working class can be shown to hold in general economies (Veneziani and Yoshihara 2012, 2015a, 2017b).

In summary, Steedman's contribution is important and it has significantly helped to clear the ground from erroneous interpretations. Yet it is not the last word on Marxian economics. The debate around and after Marx after Sraffa has helped to rigorously pose, and address, some fundamental questions concerning the role, scope and fundamental tenets of Marxian value theory. This book aims to revive and contribute to this tradition.

To be specific, we explore and develop an interpretation of Marxian value theory originally proposed by Flaschel (1983a). Conceptually, this approach is analogous to other recent interpretations of Marxian economics, especially the New Interpretation, in its emphasis on the monetary dimension of modern capitalist economies and on the relevance of an empirically grounded notion of values. Marxian economics, and especially Marxian value theory, is not relegated to the sphere of pure theory. It is a practical tool to understand the deep dynamic forces underlying capitalist development.

The solution to the so-called 'transformation problem' offered in this book is, conceptually, very simple. Marx's labor values are an inputoutput (IO) based accounting construct and should be defined within a

\footnotetext{
${ }^{8}$ Yet Roemer (1981) showed that if Morishima's (1974) definition is adopted, the FMT does not hold, in general, in economies with a convex cone technology, if a different notion of equilibrium is adopted, namely that of a reproducible solution (Roemer 1980a). Later, Roemer (1982) proposed an alternative definition of exploitation, which according to him would generalize the FMT (and other Marxian propositions) to convex cone economies at reproducible solutions. Veneziani and Yoshihara (2012, 2015a) have shown that neither Morishima's nor Roemer's definition preserve the FMT in economies with a convex technology and homogeneous labor.
} 
given accounting system. In particular, as in Richard Stone's "System of National Accounts" (SNA), labor values are simply the total labor costs defined in the SNA. Thus, as soon as joint production is allowed for, there can be no purely technological definition of labor values. Labor values constructed in this way do not determine relative prices. They are measures of real costs of production focusing on human labor, in order to describe and understand the deep structural characteristics of production in capitalist economies beneath the surface of market phenomena. They are an integral part of a SNA, whose aim is not to explain prices, but to define real growth and inflation rates at the aggregate level, the dynamics of real labor productivity at the sectoral level, and the fundamental determinants of profitability.

From this perspective, the contributions of Sraffa (1960) and the neo-Ricardians (including Steedman), while important, are all but the last word on Marxian economics, and the so-called 'transformation problem' can be considered an empty expression. Rather than thinking in terms of Marx after Sraffa, at least as far as Marx's LTV is concerned, we would say that Marx after Stone may be the proper slogan for future research.

\section{Themes of the project}

For the Classicals, observable magnitudes and events are more often than not mere epiphenomena of deeper structures, and a focus on market outcomes would yield at best a preliminary, superficial understanding of economic processes. This is not to deny their relevance in everyday economic life. The market prices of commodities (including labor), for example, are observable and important magnitudes in determining agents' decisions, the survival of firms, government policies, and so on. However, according to the Classicals, market prices are influenced by a large number of unpredictable and short-lived influences and thus are not very informative of the deep underlying structures of capitalist economies. Thus, although we will discuss market prices, this book will introduce and analyze the classical notions of values and exploitation, and of 'natural' prices.

In the classical perspective, observable market prices are the effect of largely unpredictable and impermanent events, but their fluctuations usually occur around some stable magnitudes, whose determinants can be investigated and whose values can be predicted. Such centers of gravitation of the dynamic processes in the economy are the 'natural' prices of commodities and the proper object of economic analysis. Different authors have provided different definitions of natural prices. 
In the Sraffa-von Neumann approach, advocated by neo-Ricardians, for example, natural (or production) prices require both the existence of perfect second-hand markets for fixed capital goods (conceived of as joint products of production activities) and the equalization of profit rates across sectors on circulating capital. This approach is theoretically rigorous and formally elegant but several of its assumptions have been questioned. First, the treatment of fixed capital as a joint product does not properly reflect the actual behavior of firms. As argued by Bródy (1970) in his stock-flow analysis, fixed capital is arguably not a jointly marketable product. Further, and related, the notion of the sectoral rates of profit implied by the Sraffian approach neglects replacement investment and, perhaps more importantly, it relates profits to the flow of material inputs rather than to the sectors' capital stock that ties up the money invested.

In this book, we adopt a different concept of natural, or production, prices that builds on the seminal IO approach developed by Andras Bródy and Wassily Leontief. We shall focus, in particular, on Leontief's (1953) almost forgotten notion of a capital stock matrix, which emphasizes the notions of capital advanced, capital consumed, and the related turnover times as well as the importance of stock-flow accounting in general. The relevant profit rate, we shall argue, should be computed on the capital tied up into production processes. Further, we shall consider a deviation from the standard assumption of equalized profit and wage rates across production sectors. This assumption is motivated by the idea that competitive forces tend to predominate, at least in the long-run. Yet, the structure of modern capitalist economies is far from the competitive benchmark, and the empirical evidence on the existence of a tendency towards equalization is rather mixed. In fact, a hierarchy of profit and wage rates tends to emerge, and persist over time, across sectors. The question then arises whether the key insights of the classical-Marxian theory of production prices - such as the existence of a fundamental distributive conflict between workers and capitalists - continue to hold when the assumption of a uniform profit rate is relaxed. Therefore we explore a generalization of the standard model which incorporates fixed capital and a constant hierarchy of profitability across sectors.

The other key concept that will be introduced is that of (labor) values. This notion was prominent in classical economics, as it was thought to identify some deep structural aspects of the production process, independent of the vagaries of market forces. The subjectivist approach to value that has come to predominate in mainstream economics has relegated the analysis of value to the sideline of academic 
economics. The received view (both in mainstream and, alas, in much of heterodox economics), in fact, is that the notion of labor value is at best redundant (Samuelson's famous blackboard theorem) and likely logically incoherent, especially outside of extremely simple economies.

In this book, we propose a notion of labor values as the total labor costs of produced commodities, that is different-in generalfrom employment multipliers; is logically coherent and general; avoids paradoxical results; and is based on the actual accounting practices of capitalist firms. Then we show that the notion is relevant to understand some deep - if invisible dynamics of modern economies, including the trends in (aggregate and sectoral) productivity and aggregate profitability. Far from being a metaphysical construct, labor values and labor accounts in general can be used in empirical studies of actual economies.

Based on this notion of labor values, we shall advance a general definition of exploitation that is also logically coherent (in general production economies) and relevant to understand the deep trends of actual capitalist economies. At the aggregate level, the rate of exploitation will be defined as the difference between the amount of labor performed by workers and the value of their labor power. Again, not only is this concept of exploitation based on well defined, empirically measurable magnitudes; it can also be shown to be one of the key determinants of the general rate of profit - arguably the most relevant variable in capitalist economies, as it is the main determinant of capitalist production and investment decisions.

In this book, we will not analyze the exploitation status of individual agents, nor will we discuss the normative relevance of the concept of exploitation. ${ }^{9}$ These are important questions which must be addressed in order to provide a general theory of exploitation. Nonetheless, our analysis should show that these questions are worth asking and, contrary to the received wisdom, a general, logically coherent and empirically relevant definition of exploitation can be provided which helps to understand modern capitalist economies. ${ }^{10}$

\footnotetext{
${ }^{9}$ Further, we focus only on the distributive aspects of the notion of exploitation in a static context. We examine neither the relevance of power and coercion in exploitative relations, nor the dynamics of exploitation. For a discussion of power and the persistence of exploitation, see Veneziani (2007, 2009a,c, 2013), Veneziani and Yoshihara (2015b), and Cogliano, Veneziani, and Yoshihara (2016). For a critique of merely distributive approaches, see Veneziani (2008, 2009b, 2012).

${ }^{10}$ According to some critics, the notion of exploitation does not add much to our understanding of capitalist economies because it is just a complicated
} 


\section{Structures: One-, two-, and $n$-sectoral linear production models}

The nature of our investigation is primarily theoretical: we aim to rigorously define some important concepts that allow one to analyze modern capitalist economies, and investigate their properties. For this purpose, we will set up and analyze abstract models of production economies. To be specific, we will focus on linear production models (IO matrices with constant returns to scale in each sector).

There are two main reasons for this choice. First, the vast majority of the literature in the classical tradition has indeed focused on linear models. Although our concepts and results hold more generally, it is useful to focus on linear economies in order to compare our work with the rest of the literature. Second, and perhaps more important, linear production models are at the core of the IO literature and, as we will argue in the rest of the book, there are many deep theoretical connections between IO theory and the classical-Marxian approach. In this context, linear models do not necessarily reflect assumptions on technology and substitutability between factors of production. Rather, IO matrices provide a theoretically informed ex post portrayal of the production side of the economy at the sectoral level.

For expositional reasons, we shall sometimes consider economies with only one, or two, produced commodities. This will allow us to introduce the reader to the most complex concepts of the book in the clearest possible way, by abstracting from all technical complexities and also to clarify the theoretical issues that arise by moving from one-good models to more general economies with multiple commodities. The transformation problem, for example, can be conceptualized precisely as an aggregation problem that arises when one considers more than one produced commodity.

It should be stressed, however, that our analysis of IO structures is not purely theoretical. In fact, the book contains numerous empirical illustrations of the main concepts and results, focusing in particular on the German economy at the beginning of the 21st century. More deeply, as we shall argue repeatedly in the course of the book, unlike in much of the mainstream and heterodox literature, we adopt what

way of capturing the productivity of the economy. This objection is misguided and in the modern approaches to value theory, the existence of profits is not synonymous with the existence of a surplus denominated in any arbitrary commodity (as the Generalized Commodity Exploitation Theorem implies, see for example Roemer (1981)). For a discussion see Veneziani and Yoshihara (2010, 2013a). 
might be called an empirically-informed theoretical approach, whereby empirical reality and stylized facts - e.g. concerning firm's actual profit maximizing practices - act as constraints on theoretical constructs, and guide research efforts. Thus, for example, our definition of labor values is based on the actual accounting and production practices of capitalist firms. We conceive of fixed capital as capital tied up in production, rather than presuming the existence of perfect second-hand markets for used machines. We explore the implications of persistent barriers to competition for production prices by explicitly considering intersectoral wage and profit rate differentials. And so on. From this perspective, the classical-Marxian approach developed in this book has natural applications to the analysis of empirical questions.

In Part I of the book, we analyze simple models with no joint production, uniform turnover time, and circulating capital only. This partly reflects the chronological order of our exposition: the simpler linear models are more appropriate to capture the theoretical framework of early classical authors. In Part II, we will relax some of these assumptions and deal with the complex issues raised by joint production, fixed capital (or, more precisely, capital tied up in production) and different turnover times in production.

Most of our analysis is conducted at the microeconomic level, and focuses on the intersectoral relations in general $n$-commodity economies, as we aim to show that a logically coherent and empirically relevant interpretation of Marxian value theory can be provided which meets the standards of analytical clarity and rigor of modern economic theory. A number of results are proved for the disaggregated $n$ good economy that provide interesting insights on the structure and laws of capitalist economies. We see our analysis as a first step in the development of a Marxian value-theoretic understanding of modern capitalism, and in the construction of a bridge between the microeconomic foundations of Marxian theory and the analysis of the macro-dynamics of capitalist economies. Indeed, this book can be seen as part of the construction of a broader theory inspired by the work of Marx, Keynes and Schumpeter.

\section{Readership}

The book aims to reach a wide readership. The topics analyzed should be of immediate relevance for scholars working in the classical and Marxian traditions, which this book explicitly aims to revive. However, some of the fundamental concepts (such as, among the others, the classical notion of competition and prices, the treatment of fixed 
capital, the classical concept of equilibrium) and of the issues raised (for example, the efficiency of capitalist economies) should be of interest to all economic theorists. Indeed, our analysis is conducted using the standards of rigor of contemporary economic analysis and we adopt the formal, methodological approach common to much economic theory. General equilibrium theorists, mathematical economists and IO theorists, for example, should find many of the topics discussed in the book familiar.

It should be stressed, however, that our approach is not purely theoretical, and readers with more empirical inclinations should find some food for thought too. In fact, as noted earlier, the book contains numerous empirical illustrations of the main concepts and results, and the classical-Marxian approach developed in this book has natural applications to the analysis of empirical questions.

Although our aim is not exegetical, the themes developed in the book should appeal also to readers interested in the history of economic thought (and, more broadly, the history of economic ideas). To be sure, we shall not provide a detailed analysis of classical texts, nor do we aim to provide the correct interpretation of classical and Marxian economics. The main purpose of this book is to develop a conceptual framework inspired by classical authors, and especially Marx, that allows us to derive important insights on modern capitalist economies. We will not try to show that classical authors, or indeed Marx, "are right": theories developed almost two centuries ago at the dawn of the capitalist era can hardly be expected to be valid today. Yet we hope that the analysis of classical concepts and theories with the modern tools of mathematical economics will not only provide a different perspective on these authors, perhaps shedding new light on their works, but it will also convince readers that there is much still to be learned in the classical and, especially, Marxian approaches.

In particular, we hope that students $(\mathrm{PhD}$ candidates, MSc and advanced undergraduate students) will find the book stimulating and useful. For this purpose, we have tried to make the book as accessible as possible. In particular, no previous knowledge of the theories and approaches developed in the book is required. The structure of the book allows every reader to follow the argument by building up in complexity and sophistication from chapter to chapter. Further, we have tried to keep the formal analysis at the simplest possible level compatible with the fundamental standard of clarity and generality of modern economic theory. For most of the models and results presented in the book, a basic knowledge of linear algebra is all that is required to understand the logic and implications of our arguments. In most cases, especially in 
Part I of the book, we introduce relevant definitions and concepts and derive basic results first in the simpler one- or two-commodity settings and then show that they can be generalized in more general economies by means of matrix algebra.

But even when the concepts, models and propositions may seem forbidding, we hope that the readers, and especially the younger ones, will not be discouraged. In an era where mainstream economics, with its toolbox of representative agent models, efficient markets hypothesis, perfect competition assumptions, and so on, is in disrepute, our hope is that a new look at the Classicals and Marx can bring a breath of fresh air, and encouragement to search outside the box.

\section{Plan of the book}

Part I introduces the main classical authors. These chapters describe the historical roots and provide the context of our analysis. Sadly, modern mainstream economics behaves as a discipline without a history, often giving birth to old ideas. Contrary to this widespread attitude, Part I of the book explicitly shows the evolution of the fundamental ideas of our approach in history and places our analysis clearly within the history of economic thought. The chronological order of the chapters, however, also allows us to introduce the reader to increasingly complex and sophisticated concepts and tools of analysis.

Chapter 1 focuses on François Quesnay and his Tableau Économique, which represents the first attempt at constructing a complete formal model of the circular flow of income and of the relations between different sectors in the economy. In this sense, the Tableau Économique can be seen as the forerunner of modern IO analysis, and we shall indeed analyze it using the tools and concepts of IO theory. We characterize production structures which are productive and profitable, and define the concepts of productiveness and profitability for general $n$-sectoral IO tables interpreted as linear production technologies. We show the equivalence of these two concepts and provide some further characterizations focusing on Leontief-inverses and their use in multiplier analysis, which together demonstrate the dual structure (concerning quantities and prices) of IO analysis.

Having introduced the building blocks of the IO representation of economic systems in Chapter 1, Chapters 2 and 3 analyze Adam Smith (2000) and his notions of competition and prices. We focus on two central themes in Smith's work, namely the increasing division of labor and its implications for commodity exchange and prices, and the (unintended) economic consequences of individual (economic) actions. 
We start from Smith's "early and rude state of society," a pure labor and corn economy. In this context, the relationships between the total labor costs embodied in the various commodities, the natural prices that guarantee a uniform rate of profit on the basis of a uniform wage rate, and labor commanded prices, representing purchasing power in terms of labor, can be clearly seen. We then use again Quesnay's simple structure of production in order to see how normal prices, labor costs and labor commanded prices can diverge once produced means of production enter the picture. Then we prove that the strict pursuit of self-interest does not lead to economic and social chaos, but insteadas Smith claimed - to the maximum corn production that is possible with given labor resources and given land of decreasing fertility (as assumed by the classical authors). In other words, the classical notion of ruthless competition provides a theory of long-period prices as the center of gravity for market prices, and it allows us to show that, under certain conditions, the ruthless pursuit of self-interest by individual agents may lead to maximum production.

In Chapter 4, we turn to David Ricardo (1951) and his theory of natural or long-period prices of production (and the underlying wageprofit curve), balanced growth paths (and the underlying consumptiongrowth curve), and technical change in a manufacturing environment. We will investigate questions of changing income distribution, their implications for price formation and the choice of technique and balanced economic reproduction, from a long-period perspective, in order to understand some fundamental relationships that characterize the process of capital accumulation and price formation in a capitalist economy.

We provide tools for an economic analysis of price-quantity relationships that question (i) standard results of the neoclassical theory of economic growth and distribution, (ii) a narrow and onesided understanding of labor values as the main force that drives price formation, and (iii) the Sraffian interpretation of the Standard Commodity as a means to exploring the causes of changing relative prices of production due to changes in income distribution or even technical change. The attempts to construct 'real' magnitudes (be it capital, embodied labor, or invariable measures of value) behind the surface of nominal price-quantity expressions therefore demand closer examination.

Chapter 5 extends the analysis of Chapter 4 to explore the von Neumann model with joint production and multiple activities, and to examine the dynamics of prices and quantities. We analyze a model of the gravitation of market prices around classical prices of production 
and balanced growth. The dynamics need not be convergent and it can be formulated in a very general way, in a rectangular system with joint production that allows for process as well as product extinction in general $n$-good economies. We show that Sraffa/von Neumann production prices are centers of gravity for market prices, but convergence may take a rather long time. Chapter 5 concludes the analysis of the classical authors.

Having introduced the basic definitions and concepts, and the main theoretical tenets of classical economics in Part I of the book, Part II engages with Karl Marx's contribution and, in particular, with his LTV. Each chapter deals with different aspects of Marx's theory considering the challenges that multiple activities, joint products, fixed capital, and so on, pose to the LTV, while at the same time showing some limitations of the standard classical approach to prices and values. The starting point of Part II is two well-known results in mathematical Marxian economics. First, as shown in Part I of the book, apart from very special cases, production prices are not proportional to labor values. Therefore, contrary to the standard reading of the LTV, equilibrium prices are not determined by labor values. Second, and perhaps more worryingly, Steedman (1977) famously showed that, outside of the simplest Leontief economies, labor values - defined in the conventional fashion as the standard employment multipliers - and surplus value can be negative. In the light of these results, two related questions immediately arise: if they are not good predictors of equilibrium prices, what are labor values good for? And, in any case, does the concept of labor values only make sense in the simplest linear economies?

Rather than presenting our definition of labor values straight away, in Chapter 6, we start from first principles and discuss the role of labor values ('what labor values are good for') and, consequently, the properties that a proper, general definition of labor values should have. We argue, among other things, that the appropriate definition should be such that labor values are always nonnegative, and the labor value of any commodity is zero if and only if its price is zero. Further, different types of labor should be homogenized using wage rates are the relevant conversion rates. We also state some results that hold in simple Leontief economies, including a relation between the existence of exploitation and positive profits at the aggregate level (the FMT), the proportionality between prices and values when the rate of profit is zero, and the aggregate equality between total direct labor spent in production activities and the labor value of the net product. We argue that these relations represent the core of Marxian value theory and any 
appropriate definition of labor values should preserve them. ${ }^{11}$ It may be objected that these relations should be, and indeed usually are, proved as results in a given economic environment, under certain conditions. Yet the central relevance of each such relation in value theory is such that "its epistemological status in our understanding is as a postulate" (Roemer 1982, 152).

In Chapter 7, we examine the role of labor values and labor accounts in economic theorizing. We provide a comprehensive analysis of technical change and the notion of labor productivity both at the sectoral and at the aggregate level. We analyze the conventional indices of labor productivity used in systems of national accounts (SNA) based on notions of sectoral real value-added per unit of labor and the IO productivity measure, namely the reciprocal of the IO employment multipliers that is, the labor values of Marxian economic theory. We show that the latter provide the theoretically sound measures of sectoral and economy-wide labor productivity, with purely technological foundations insofar as IO coefficients can be interpreted as pure quantity magnitudes.

First, a unified theoretical framework for the analysis of productivity measures is provided, which is based on a novel axiomatic method. Rather than comparing different measures in terms of their implications in various scenarios, we start from first principles and formalize some theoretically desirable properties that any measure of labor productivity should satisfy. The main axiom focuses on changes in productivity and states that labor productivity at time $t$ in the production of good $i$ has increased relative to the base period, if a unit increase in the net product of good $i$ demands less labor than in the base period. This is a weak restriction which incorporates the key intuitions behind the main productivity measures in the literature. Yet it characterizes the IO measures, whereas the conventional SNA indices do not satisfy it in general owing to their inherent dependence on relative prices and final demand. The second major contribution of this chapter is a rigorous analysis of the conditions under which profitable innovations lower labor values, thereby raising productivity and increasing consumption and investment opportunities. In a model with fixed capital and possibly differentiated sectoral profit and wage rates, we derive a theoretical foundation for the Law of Decreasing Labor Content (LDLC). The inherent functioning of the capitalist

${ }^{11}$ For a discussion of the role of formal models in Marxian economics, see Veneziani and Yoshihara (2017b) and in economic theory in general, see Mohun and Veneziani (2012). 
system, in particular the forces driving technical change, including class struggle, leads to a tendential decrease in the amount of labor necessary to produce (or, indeed, embodied in) commodities. To be specific, profitable fixed-capital-using labor-saving innovations lead to productivity increases. Given that capital-using labor-saving technical change has characterized most of the phases in the evolution of capitalism, this result provides theoretical foundations for the conclusion that labor values tend to fall, and labor productivity tends to rise, over time in capitalist economies.

In Chapter 8, we extend our analysis and show that labor valuesor total labor costs, in our interpretation - provide important insights on one of the most important issues in Marxian economics, namely the determinants of the general, or economy-wide, rate of profit, and the LTV explains the key variables determining profitability. More generally, far from being metaphysical constructs, labor values can be used to investigate empirically some of the fundamental dynamic laws and tendencies of capitalist economies. Thus, we introduce the notions of capital consumed and capital advanced in production and discuss Leontief's notion of a capital stock matrix, and how to compute it based on the available IO data. This is important because profitability should be measured in relation to the stock of capital advanced, and tied up in production both at the sectoral and at the aggregate level.

We identify three main determinants of the value rate of profit: the creation of absolute and relative surplus value, and technical change and accumulation (via their effects on the value of the total capital stock). We show that differences between the value rate of profit and the price rate of profit depend on the differences between the wage share and the value of labor power, and between the price and value measures of the total capital stock. Then, empirically, we show that the LDLC holds and it affects the evolution of aggregate profitability in the German economy 1991-2000. We also show that, although prices and labor values of individual commodities may deviate, at the aggregate level any such differences are irrelevant and the key insights of the Marxian theory of exploitation and of the profitability of a capitalist economy are valid.

In Chapters 7 and 8, we assume that each sector produces a single commodity by using one method of production, and so labor values can be defined as the standard IO employment multipliers and have all of the usual, desirable properties. Chapter 9 extends our analysis to production economies in which each sector produces a single good but multiple activities are used to produce the various goods. The key step for the generalization of the concept of labor 
values as measuring the real (labor) costs of producing goods is to note that the existence of alternative methods of production requires, following Marx, distinguishing (average) labor values from individual values. The distinction between individual and average requirements is standard in IO methodology, which derives a conventional square IO table by aggregating the activities of the given sectors using the activity levels that characterize the single activities into some suitably defined 'average' technology $A^{\prime}, l^{\prime}$, which is derived from the original IO structure $A, l$. Labor values are then defined as the solution of $v A^{\prime}+l^{\prime}=v$, and represent the average total labor costs of producing the various commodities with respect to the multiple activities that are operated in each sector. We show that this extension of the concept of labor values has all the desired properties and it allows us to generalize the standard propositions of Marx's economics.

The presence of multiple activities requires the introduction of the distinction between individual and (average) labor values, but Marxian value theory can still be analyzed based on merely physical, production-based quantities. If joint production is considered, a purely technological definition of labor values is not necessarily appropriate theoretically and it is quite distant from the actual accounting practices of profit maximizing firms. In Chapter 10, alternative methods of determining the total labor costs, or requirements, of commodities both in money value and in physical terms are discussed and different extensions of the definition of labor values are considered based on the actual accounting practices of capitalist firms. We argue that it is conceptually impossible to separate price and quantity magnitudes. We argue that, the appropriate way to disentangle the joint outputs of each sector or activity is by using the so-called "industry technology hypothesis" which splits up all inputs in proportion to the relative value of output in the output basket of the joint production activity. In the full-cost accounting techniques of business administration, this is called the "sales value method", since the relative proceeds of the items in a joint bundle determine the amount of joint inputs these single items have to bear.

In Chapter 11, we show that once the impossible quest for pure physical values is abandoned, it is possible to provide definitions of labor values - actual labor values - that are theoretically robust and empirically meaningful, and that preserve the main propositions of Marxian value theory. To be specific, we show that if jointly produced outputs and their inputs are disentangled by means of economic imputations which reflect the benefit received from each unit of costing - i.e. using the "sales value method"-then individual 
and average labor values can be defined as in the case with multiple activities based on the square input matrix (and its corresponding labor input vector) obtained from IO methodology in the case of the industry technology hypothesis.

We show that actual labor values are well defined, positive and unique, and preserve the main properties of Marxian value theory in linear economies with joint production, including the key propositions on price-value relationships, such as the FMT. In fact, actual labor values display none of the paradoxical features shown in Steedman's (1977) famous examples. We argue that such counterintuitive results derive entirely from Steedman's definition of labor values as the standard employment multipliers - a definition which is conceptually inappropriate in the case of joint production or multiple activities.

The last two chapters deal with two further extensions of price and value theory. In the classical long-period approach, production prices entail the equalization of profit rates and wage rates across sectors. Conceptually, this is meant to reflect the idea that prices of production are centers of gravity of market prices and emerge due to competitive pressures that tend to remove any sectoral differences in wage and profit rates. The starting point of Chapter 12 is the acknowledgement that, empirically, there is remarkably little evidence of a strong tendency towards profit rate or wage rate equalization. Actually, a number of empirical studies, and our own analysis of the German economy 19912000 suggest that intersectoral differences are persistent and indeed show a remarkable stability over time. Therefore we extend the classical production price equations to incorporate the existence of persistent, and stable, wage and profit rate differentials. We show that, under the usual assumptions on technology, the generalized production prices are unique, well-defined and strictly positive. Moreover, all of the standard results of production price theory - including the existence of a negative relation between wages and profits - continue to hold.

In Chapter 12, we drop the standard assumption of uniform wage and profit rates, but the model is otherwise standard. In particular, fixed capital is ignored and the profit rate is computed as the rate of return only on so-called circulating capital. Chapter 13 extends the analysis by considering the implications of imperfect competition for the classical analysis of technical change, choice of technique, and distribution, from both a theoretical and an empirical perspective in economies with fixed capital. We reconsider the classical notion of prices of production when both of the standard Sraffian assumptions are dropped, namely profit rate and wage rate equalization and the presence of perfect second hand markets for capital used in production 
(and treated as a joint product): both assumptions are at odds with the actual features of modern capitalist economies. In contrast, we define classical prices of production assuming the existence of persistent wage and profit rate differentials across different sectors and incorporate the notion of fixed capital as capital tied up in production, using Leontief's notion of capital matrices. We derive the so-called wage-profit curves in this more general framework and prove that the main classical-Marxian insights concerning technical innovations and distribution remain valid in this more general setting. Then, we analyze data from the German economy at the beginning of the 21 st century to show that our general theoretical framework is more useful for empirical analyses than the standard neo-Ricardian approach. Interestingly, our analysis shows that the empirical wage-profit curves of the German economy (2000-2010) are fairly close to straight lines, independently of the choice of scaling, which confirms a host of similar results in the literature.

The analysis of imperfect competition and fixed capital takes us at the cutting-edge of the modern approach to Marxian price and value theory. We point out some directions for further research in the spirit of this book in the concluding chapter. 


\section{PART I}

\section{Classical Competition: Theory, Evidence, and Criticism}





\section{François Quesnay: The Circular Flow of Income and Input-Output Analysis}

\subsection{Introduction}

Economic theorizing dates back to the time of Aristotle, but it was François Quesnay (1694-1774) who first formulated a model describing a whole economy, with empirical relevance and clear-cut, radical policy implications for the French economy and society. In this chapter we use his model as an introduction to input-output (IO) tables and IO analysis, focusing on a classic translation of Quesnay's (1759) Tableau Économique into IO language by Barna (1975). From this perspective, Quesnay's model provides an IO matrix with two commodities, corn and manufactured goods, where the corn input into the production of corn (agriculture) and manufacturing (including trade) also includes the subsistence consumption of workers as a representation of their direct labor input (as if they were cattle).

In this relatively simple two-commodity framework, we characterize productive and profitable production structures, and provide the general definition of the concepts of productiveness and profitability for general $n$-sectoral IO tables interpreted as linear production technologiesthat is, IO matrices with constant returns to scale in each sector. We show the equivalence of these two concepts and consider some related notions, such as Leontief-inverses and the multiplier analysis that is based on them, which together illustrate the dual structure (concerning quantities and prices) of IO analysis.

We then consider issues of decomposability for such IO models, leading to the Sraffian distinction between basic and nonbasic commodities and the analysis of non-cyclical technology structures. In the two-sectoral case considered by Quesnay we provide a complete taxonomy for these concepts, starting from Adam Smith's "early and rude state of society" (no physical inputs) and ending with a strictly 
positive IO matrix (such as the standard highly aggregated empirical IO tables).

We close the chapter with an application of the IO approach to the actual data of the German economy (1991-2000). We also briefly consider the United Nations' System of National Accounts (SNA) which will be important as a modeling framework in the following chapters. Consistent with the general approach adopted in this book, the main purpose of this chapter is not a textual exegesis of the work of François Quesnay, but rather an introduction to IO analysis that focuses on Quesnay's early approach to the circular flows of income and on the monetary circuit from which these flows derive. Blaug (1985) and Kurz and Salvadori (1995) provide further introductions to this important early example of macroeconomic theorizing.

\subsection{An early example of global economic reasoning}

The economy-wide model of an economic circuit which we will describe and discuss in this section, has been developed by François Quesnay, the personal physician of Madame de Pompadour at the court of King Louis XV in France. Quesnay was the leader of the Physiocrat school which stressed the role of nature as a source of wealth. In 1758, he presented his Tableau Économique to the king who showed no understanding of it or interest in it. This Tableau Économique applied the theory of the circulatory system in animals to an economy as a whole. Using this theoretical background, Quesnay wanted to contrast the desolate state of the French economy of his time with an ideal type of an economy with a healthy circulation of income.

In the Tableau Quesnay distinguishes two sectors of production: agriculture and manufacturing; and three social classes: property owners, entrepreneurs, and workers. For Quesnay, entrepreneurs and workers in the agriculture sector are part of the 'productive class', while those in the manufacturing sector constitute the 'sterile class'. Property owners consist of landlords, the king (including the court), and the church, where the latter two receive tax income and tithe payments out of the rent paid to landlords. In this scheme of circulation, landlords receive ground rent from agriculture, from which they transfer a certain amount to the king and church in the form of taxes. In broad strokes, the Tableau outlines how income circulates between the three classes in society, while also allowing an examination of the interaction of two sectors of production. Quesnay's Tableau also contains normative elements: it was meant in part as a proposal of economic reform addressed to the French ruling class. The 'state of 
bliss' presented in the Tableau represents the French economy operating at its optimal state of economic activity, in contrast to its actual state in the middle of the 18th century. The zig-zagging patterns depicting the interconnections between sectors and actors in the Tableau has been replaced by modern diagrams of the circular flow of goods and income in introductory economics texts, but the Tableau remains an informative starting point for introducing the logic of IO analysis.

Quesnay's model and physiocratic theory in general attemptedincluding 30 maxims or comparative static exercises - to provide the description of an optimal steady-state for an economy at the macro level. He provided an original, albeit crude image of a whole economy, by structuring it appropriately in its interdependencies in order to determine its stationary equilibrium in which the economy reproduces itself. However, the presentation of Quesnay's Tableau can be improved and made more general and flexible, if the monetary circuit of the economy in a particular year is represented by means of an IO accounting table. ${ }^{1}$

The first two columns of the IO Table 1.1 show the two sectors of Quesnay's Tableau: agriculture (total output 2625) and manufacturing (total output 1313). The middle three columns show the three actors of the Tableau, or types of households, while the next two columns show the government and church as the constituent parts of the state. Note that according to Quesnay only landlords have to pay taxes and tithe: 300 and 150, respectively. This represents an income transfer between households that is shown in the lower right-hand side of Table 1.1. The axiom of a closed circuit implies that column totals must equal row totals. Finally, social product net of intermediate inputs is $2625+1313-525-525=2888 .^{2}$

Table 1.1 shows that an IO representation of the whole economy (whether actual or idealized) is of great help for analyzing the transactions between the sectors in a given period. ${ }^{3}$ Along these lines, Table 1.1 serves as a starting point for both macro- and microeconomic analysis. As a foundation of macroeconomic analysis we can for example derive from Table 1.1 the following aggregated table for the French economy seen in Table 1.2.

The magnitudes in Table 1.1 are nominal and in order to move to

\footnotetext{
${ }^{1}$ As suggested by Barna (1975) in a pioneering contribution.

${ }^{2}$ Looking at individual sectors, it is important to note that in modern terminology, 'manufacturing' exhibits a positive value added, i.e., it is not unproductive or sterile as Quesnay claimed it to be.

${ }^{3}$ Of course, IO tables are nowadays structured differently, see United Nations (1993).
} 


\begin{tabular}{|c|c|c|c|c|c|c|c|c|}
\hline & \multicolumn{2}{|c|}{ Firms } & \multicolumn{3}{|c|}{ Households } & \multicolumn{2}{|c|}{ State } & \multirow[b]{2}{*}{ Total } \\
\hline & $\begin{array}{l}\text { Agric- } \\
\text { ulture }\end{array}$ & $\begin{array}{c}\text { Manu- } \\
\text { factur- } \\
\text { ing }\end{array}$ & $\begin{array}{l}\text { Far- } \\
\text { mer }\end{array}$ & \begin{tabular}{|c} 
Manu- \\
factu- \\
rer
\end{tabular} & $\begin{array}{c}\text { Land- } \\
\text { lord }\end{array}$ & $\begin{array}{l}\text { Gover- } \\
\text { nment }\end{array}$ & $\begin{array}{c}\text { Chu- } \\
\text { rch }\end{array}$ & \\
\hline $\begin{array}{l}\text { Agric. } \\
\text { prod. }\end{array}$ & 525 & 525 & 525 & 525 & 300 & 150 & 75 & 2625 \\
\hline $\begin{array}{l}\text { Manf. } \\
\text { prod. }\end{array}$ & - & - & 525 & 263 & 300 & 150 & 75 & 1313 \\
\hline $\begin{array}{l}\text { Farm } \\
\text { inco- } \\
\text { me }\end{array}$ & 1050 & - & & & & & & 1050 \\
\hline $\begin{array}{l}\text { Manf. } \\
\text { inco- } \\
\text { me }\end{array}$ & - & 788 & & & & & & 788 \\
\hline Rent & 1050 & - & & & & & & 1050 \\
\hline Taxes & - & - & - & - & 300 & - & - & 300 \\
\hline Tithe & - & - & - & - & 150 & - & - & 150 \\
\hline Total & 2625 & 1313 & 1050 & 788 & 1050 & 300 & 150 & \\
\hline
\end{tabular}

Table 1.1: An IO representation of the Tableau Économique for a closed economy

\begin{tabular}{|c||c|c|c||c|}
\hline & Firms & Households & Government & Total \\
\hline \hline Consumption & 1050 & 2438 & 450 & 3938 \\
Gross Income & 2888 & - & - & 2888 \\
Taxes & - & 450 & - & 450 \\
(Government \& Church) & & & & \\
\hline \hline Total & 3938 & 2888 & 450 & \\
\hline
\end{tabular}

Table 1.2: An aggregation example as starting point for a macroeconomic analysis of the circuit of income

real magnitudes we assume that the prices of the two commodities in 
Quesnay's model are both equal to 1, i.e. we assume that the table has been deflated in a commodity specific way (using double-deflation rather than single-deflation techniques, see Chapter 7 below). Thus by assumption quantity and value are of the same size for all entries, except for values added, because there is no real magnitude behind them. In a two commodity world the physical table reads as in Table $1.3 .^{4}$

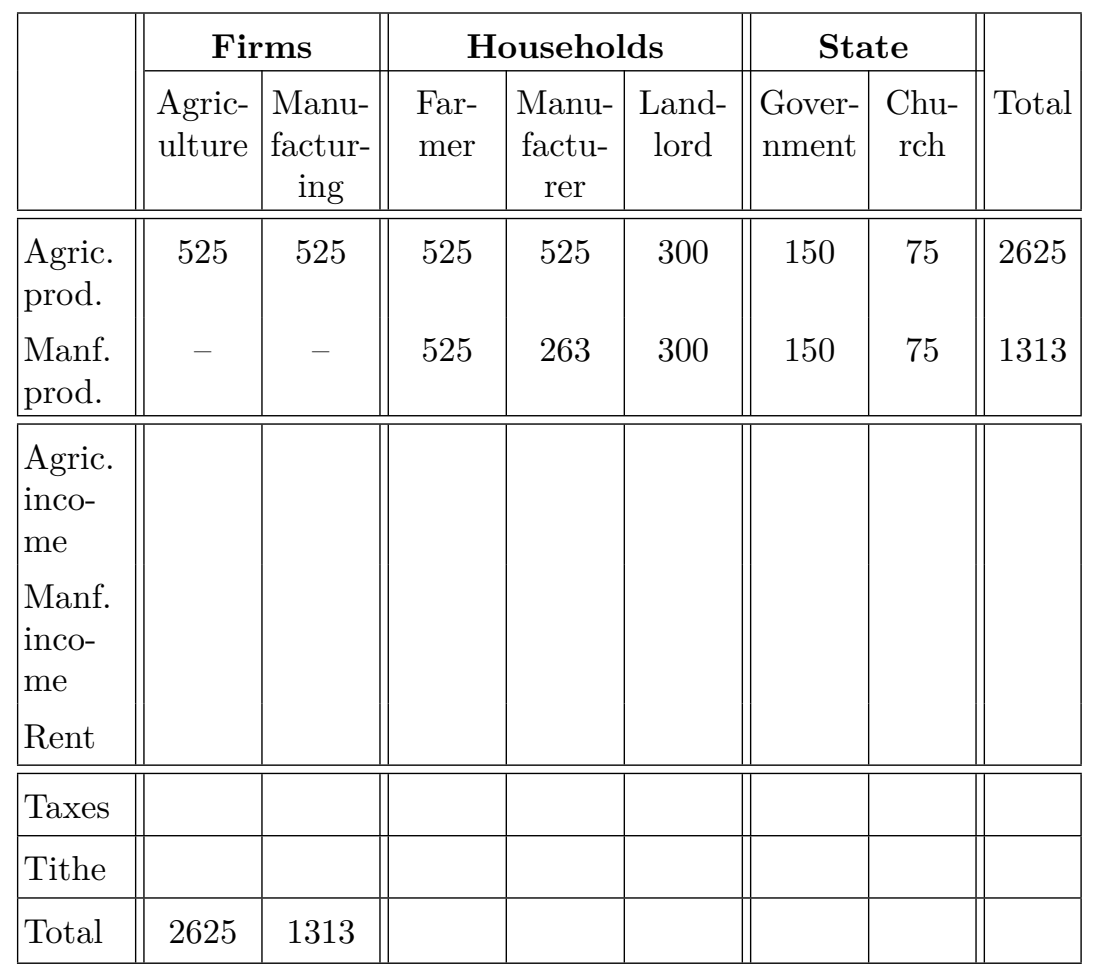

Table 1.3: The physical background of the Tableau Économique

This physical representation of the stationary circuit of a physiocrat world does not provide much microeconomic information. It shows neither how commodity prices are formed, nor how the allocation of quantities has been established, nor how the process of income distribution factors into the determination of price and quantity expressions. However, if we also assume the nominal rent payment of Quesnay's scheme (see Table 1.1), we can derive the actual profit

\footnotetext{
${ }^{4}$ This table departs in some aspects from today's IO scheme as explained in Section 1.4 below.
} 
rates $r_{a}$ and $r_{m}$ in the two production sectors, with subscripts $a$ and $m$ respectively denoting agriculture and manufacturing. ${ }^{5}$ These two profit rates read:

$r_{a}=\frac{2625-525-1050}{525}=\frac{1050}{525}=2, \quad r_{m}=\frac{1313-525}{525}=\frac{788}{525}=1.5$.

The number 525 in the numerator and the denominator indicates that the agricultural product (and only this) has been used in both sectors as an intermediate input, therefore denoting the capital advanced and the reference against which profit rates are calculated. Both sectors are profitable with profit rates of 200 and 150 percent, respectively. Profits before rent payments in agriculture are higher than in manufacturing. This fits with Quesnay's theory that agriculture is responsible for generating all of the transfer payments in this economy, thus this sector must be highly productive. In the profit rate calculations it is assumed that workers' wages are contained in the intermediate inputs of firms - if wages are considered at all, they only guarantee subsistence (in terms of corn). The surpluses obtained in this framework are consistent with the Marxian notion of surplus, since they represent the excess product, or value created by workers.

In general, IO theory considers average IO coefficients and assumes constant returns to scale. This allows for the determination of actual physical inputs per unit of output. On this basis, we arrive at the following IO matrix, or technology $A=\left[a_{i j}\right]$ of physical intermediate inputs: ${ }^{6}$

\begin{tabular}{|l|c|c|}
\hline & agriculture & manufacturing \\
\hline agric. products & $a_{11}=\frac{525}{2525}=0.2$ & $a_{12}=\frac{525}{1313}=0.4$ \\
\hline manuf. products & $a_{21}=0$ & $a_{22}=0$ \\
\hline output & 1 & 1 \\
\hline
\end{tabular}

Table 1.4: The unit-output representation of the linear technology behind Quesnay's Tableau

So far we have assumed that both commodity prices are equal to 1 .

${ }^{5}$ Uniform profit rates resulting from ruthless competition will be analyzed in the next chapter.

${ }^{6}$ It is worth noting that the IO matrix $A$ and its elements $a_{i j}$ should include some notion of time $t$, i.e. $A_{t}$ or $a_{i j ; t}$. However, for simplicity, time subscripts will be dropped in the following presentation. 
These prices can be understood as administered prices since Quesnay wanted to support agriculture compared to 'trade', the 'sterile' sector of his model. As we have seen, under this assumption the profit rates in the two sectors are 200 and 150 percent. In order to show that a sector of the economy is truly sterile, the profit rate in trade would need to be zero. This occurs, if, rather than $p_{a}=p_{m}=1$, we have $p_{a}=1$ and $p_{m}=0.4$, thus $p_{m} \cdot 1313-p_{a} \cdot 525=0$. In this scenario manufacturing does not create a surplus and the physical IO scheme would have to be changed since the monetary magnitudes now imply different final demands.

Alternatively, in place of administered prices intended to support agriculture, one can also follow Quesnay and assume that competition is freed from regulations and assume that profit rates are equalized by capital flows across the two sectors. In the case of a linear technology and with rent payment fixed in nominal terms, the resulting profitand price-structure is: $p_{a}=1$ (and so $r_{a}=\left(1-a_{11}-2 / 5\right) / a_{11}=2$ ), $p_{m}=\left(1+r_{a}\right) a_{12}=1.2 .{ }^{7}$

\subsection{Input-output analysis: Surplus, multipliers and connectedness}

The tables of Section 1.2 show that Quesnay's Tableau can be viewed as a precursor to modern IO economics. We now consider the standard IO framework that relies on a single table to represent the circuit between production, income, and demand. This type analysis was initiated by Wassily Leontief in the 1930s and grew to become the standard treatment for analyzing linear economies as IO systems, an approach which lays the foundation for the analysis conducted throughout the rest of the book.

Consider a closed economy with $n$ industries producing $n$ goods that are also used as intermediate inputs. Let $X_{i j}$ denote the physical amount of the commodity produced by sector $i$ transferred to sector $j$ and let $p_{i}$ denote the price of commodity $i$. Sector $i$ also sells its product as final demand (a final good) $y_{i}$, thus its total output is $x_{i}=\sum_{1}^{n} X_{i j}+$ $y_{i}$. In contrast to Quesnay's Tableau, for the sake of simplicity, we consider the annual production of $n$ industries, the distribution between them, and final demands in a single aggregated column in Table 1.5. The diagonal matrix formed from a price vector $p$ is denoted as $\hat{p}$. The value added by each sector $i$ is denoted by $\nu_{i}$, with $\nu$ denoting the vector of sectoral value added across the $n$ sectors.

\footnotetext{
${ }^{7}$ Competitive prices will, however, change demand again thereby changing output, an issue beyond our current consideration.
} 
The connections between industries in commodity production are represented in Table 1.5 in terms of quantities $X_{i j}$ as well as values $p_{i} X_{i j}$. Magnitudes without explicit reference to prices $p_{j}$ represent pure value magnitudes that cannot be meaningfully split into a single price and a single quantity component ('value added' being the difference between homogeneous sales and heterogenous costs). Note that in such a consistent ex post representation of the activities of a whole economy total value added $\sum_{i} \nu_{i}$ of the $n$ branches must be identical to the value of total final demand $\sum_{i} p_{i} y_{i}$, i.e. $\sum_{i} p_{i} y_{i} \equiv \sum_{i} \nu_{i}$. However, such an identity is not true for sectoral values added and their final demands.

\begin{tabular}{|l||c|c|c|c||c|}
\hline $\begin{array}{l}\text { Delivery from } \downarrow \\
\text { to } \rightarrow\end{array}$ & Sector 1 & $\ldots$ & Sector $n$ & $\begin{array}{c}\text { Final } \\
\text { demand }\end{array}$ & $\begin{array}{c}\text { Row sum } \\
\text { (Total } \\
\text { production) }\end{array}$ \\
\hline \hline Sector 1 & $p_{1} X_{11}$ & $\ldots$ & $p_{1} X_{1 n}$ & $p_{1} y_{1}$ & $p_{1} x_{1}$ \\
\hline Sector 2 & $p_{2} X_{21}$ & $\ldots$ & $p_{2} X_{2 n}$ & $p_{2} y_{2}$ & $p_{2} x_{2}$ \\
\hline$\ldots$ & $\ldots$ & $\ldots$ & $\ldots$ & $\ldots$ & $\ldots$ \\
\hline Sector $n$ & $p_{n} X_{n 1}$ & $\ldots$ & $p_{n} X_{n n}$ & $p_{n} y_{n}$ & $p_{n} x_{n}$ \\
\hline Value added & $\nu_{1}$ & $\ldots$ & $\nu_{n}$ & & \\
\hline $\begin{array}{l}\text { Column sum } \\
\text { (Total } \\
\text { production) }\end{array}$ & $p_{1} x_{1}$ & $\ldots$ & $p_{n} x_{n}$ & & $\sum_{i} p_{i} x_{i}$ \\
\hline
\end{tabular}

Table 1.5: The basic structure of a nominal IO table $\left(\hat{p}\left[X_{i j}\right]\right)$

If one divides the first $n$ columns of Table 1.5 by the total value of the corresponding output $p_{j} x_{j}$ one obtains the matrix, shown in Table 1.6, whose elements are all dimensionless. Letting the superscript nom represent nominal quantities, the matrix $A^{\text {nom }}$ represents the intermediate inputs of the different sectors per value of one unit of output and can be written as follows:

$$
A^{\text {nom }}=\left[a_{i j}^{n o m}\right]=\left[p_{i} X_{i j} / p_{j} x_{j}\right]=\left[p_{i} a_{i j} / p_{j}\right], \quad \text { with } \quad a_{i j}=X_{i j} / x_{j} .
$$

Table 1.6 does not provide a pure quantity representation of the economy, since quantities are still multiplied by the appropriate relative prices. If one removes relative prices from the IO table one finally obtains the physical data of Table 1.7 as a starting point for the IO analysis. The theoretical IO coefficients $a_{i j}=X_{i j} / x_{j}$ represent the 


\begin{tabular}{|l|c|l||c|}
\hline $\begin{array}{l}\text { Delivery from } \downarrow \\
\text { to } \rightarrow\end{array}$ & Sector 1 & $\ldots$ & Sector $n$ \\
\hline Sector 1 & $a_{11}^{\text {nom }}$ & $\ldots$ & $a_{1 n}^{\text {nom }}$ \\
\hline$\ldots$ & $\ldots$ & $\ldots$ & $\ldots$ \\
\hline Sector $n$ & $a_{n 1}^{\text {nom }}$ & $\ldots$ & $a_{n n}^{\text {nom }}$ \\
\hline Value added & $\nu_{1} /\left(p_{1} x_{1}\right)$ & $\ldots$ & $\nu_{n} /\left(p_{n} x_{n}\right)$ \\
\hline $\begin{array}{l}\text { Unit output } \\
\text { (value) }\end{array}$ & 1 & $\ldots$ & 1 \\
\hline
\end{tabular}

Table 1.6: The nominal unit coefficients of a linear technology behind Table 1.5: $A^{\text {nom }}=\hat{p} X \hat{x}^{-1} \hat{p}^{-1}$

amount of product $i$ per unit of product $j$ and therefore can no longer be added to each other, in contrast to the previous table (unlike in Table 1.6) where both row and column summation are possible. The average IO coefficients $a_{i j}$ (the average amount of good $i$ that is used up in the production of good $j$ ) are generally interpreted as representing a linear technology (fixed proportions in production and constant returns to scale) - this interpretation is retained throughout the book.

\begin{tabular}{|l|c|l||c|}
\hline $\begin{array}{l}\text { Delivery from } \downarrow \\
\text { to } \rightarrow\end{array}$ & Sector 1 & $\ldots$ & Sector $n$ \\
\hline \hline Sector 1 & $a_{11}$ & $\ldots$ & $a_{1 n}$ \\
\hline$\ldots$ & $\ldots$ & $\ldots$ & $\ldots$ \\
\hline Sector $n$ & $a_{n 1}$ & $\ldots$ & $a_{n n}$ \\
\hline $\begin{array}{l}\text { Unit output } \\
\text { (quantity) }\end{array}$ & 1 & $\ldots$ & 1 \\
\hline
\end{tabular}

Table 1.7: The physical coefficients of a linear technology assumed behind the IO Table 1.5: $A=\hat{p}^{-1} A^{n o m} \hat{p}=X \hat{x}^{-1}, x=\left[x_{1}, \ldots, x_{n}\right]^{\prime}$, $p=\left[p_{1}, \ldots, p_{n}\right]$

\subsubsection{Productive and profitable input-output matrices}

The linear production structure given by the nonnegative $n \times n$ matrix $A \geq 0$ allows us to define the notions of 'productiveness' and 'profitability'. Unlike in Quesnay's model, we consider labor inputs $l \in \mathbb{R}^{n}$ (a row vector) explicitly rather than in the intermediate inputs 
via a given consumption basket $c \in \mathbb{R}^{n}$ (a column). Considering $l$ separately incorporates labor costs as part of the value added of each sector, therefore, a positive value added does not necessarily imply positive profits.

\section{Definition 1.1. ('Productive' and 'Profitable').}

(1) The matrix of intermediate inputs $A \in \mathbb{R}^{n \times n}$ is called productive, if there is a vector of activity levels $x \geq 0$ in $\mathbb{R}_{+}^{n}$ for the $n$ industries, such that $A x<x$ holds, i.e., for all rows $A_{i \star}$ of $A$, $A_{i \star} x=\sum_{j} a_{i j} x_{j}<x_{i}$ holds.

(2) The matrix of intermediate inputs $A \in \mathbb{R}^{n \times n}$ is called profitable, if there is a price vector $p \geq 0$ in $\mathbb{R}_{+}^{n}$ for the $n$ commodities, such that $p A<p$ holds, i.e., for all columns $A_{\star j}$ of $A, p A_{\star j}=\sum_{i} p_{i} a_{i j}<p_{j}$ holds.

Productive matrices are therefore characterized by the existence of a nonnegative vector of activity levels $x^{\prime}=\left(x_{1}, \ldots, x_{n}\right)$ of the $n$ industries of our model economy, such that the output $x_{i}$ of each industry $i$ (rows of $A$ ) is larger than the total consumption $A_{i \star} x$ of good $i$ by the $n$ industries. All sectors of the economy produce a positive surplus in this case. Similarly, an IO matrix is profitable, if there is a nonnegative vector of prices $p$, such that unit production costs (excluding labor costs) in each industry $j$ (columns of $A$ ) $p A_{\star j}$ are always lower than the price of the commodity $p_{j}$. The second criterion considers each sector in isolation (but with a common price structure for all sectors), while the first criterion compares the consumption and output for each commodity over the whole set of industries. We show below that a matrix $A$ is productive if and only if it is profitable - in a sense, both comparisons lead to the same set of nonnegative matrices which provide the basis for all later price-quantity considerations - the central subject of this book.

Let $M=I-A$, where $I$ is the $n$-dimensional identity matrix. Definition 1.1 still applies to $A$ via $M$ by $M x=y$, where $y$ represents the vector of final demands $\left(y^{\prime}=\left(y_{1}, \ldots, y_{n}\right)\right)$ and $p M=\nu>0$. Stated differently, these equation systems can be solved for at least one semipositive vector of final demands $y>0$ and at least one semi-positive vector of values added $\nu>0$ by means of gross output levels $x \geq 0$ or commodity prices $p \geq 0$. This is more formally stated by Proposition 1.1, which summarizes results of Nikaido (1968).

\section{Proposition 1.1.}

(1) The matrix of intermediate inputs $A$ is productive if and only if for all nonnegative vectors of final demands $y \in \mathbb{R}_{+}^{n}$ there is a 
nonnegative vector of activity levels $x \in \mathbb{R}_{+}^{n}$, which provides the surplus $y$, i.e., such that $M x=y$.

(2) The solution $x$ of the linear equation system $M x=y$ is given by:

$$
\begin{aligned}
x & =M^{-1} y=(I-A)^{-1} y=\left(I+A+A^{2}+A^{3}+\ldots\right) y \\
& =y+A y+A^{2} y+A^{3} y+\ldots,
\end{aligned}
$$

if and only if $A$ is productive. Because the matrix $I-A$ is then invertible $(I-A)^{-1}$, the so-called "Leontief-inverse", is nonnegative and representable by means of a standard multiplier formula (or geometric series that holds for all dimensions $n$ ).

(3) The matrix of intermediate inputs $A$ is profitable if and only if for all nonnegative vectors of value added $\nu \in \mathbb{R}_{+}^{n}$ there is a nonnegative vector of prices $p \in \mathbb{R}_{+}^{n}$ which generates value added $\nu=\left(\nu_{1}, \ldots, \nu_{n}\right)$ per unit of output of the $n$ industries, i.e., such that $p M=\nu$.

(4) The solution $p$ of this equation is then (and only then) given by the following expressions:

$$
\begin{aligned}
p & =\nu M^{-1}=\nu(I-A)^{-1}=\nu\left(I+A+A^{2}+A^{3}+\ldots\right) \\
& =\nu+\nu A+\nu A^{2}+\nu A^{3}+\ldots
\end{aligned}
$$

The matrix $M=I-A$ is then invertible and nonnegative.

In other words the possibility of surplus production in each branch of the economy implies that every semi-positive demand for the $n$ commodities of the $n$ sectors can be satisfied by choosing an appropriate uniquely determined vector of activity levels. Similarly, the economy can generate any semi-positive value added vector $\nu=\left(\nu_{1}, \ldots, \nu_{n}\right)$ by choosing an appropriate vector of commodity prices. Moreover, such activity and price levels needed are determined by applying the relevant Leontief multipliers. Finally, as shown in Proposition 1.2, any one of these conditions implies the validity of all others, for example one only needs to assume a productive IO structure in order to fulfill all other assertions on the flexibility of such an IO system, with completely rigid proportions in production.

Proposition 1.2. (Hawkins-Simon Conditions). Consider the IO matrix $A \geq 0$, and the corresponding difference matrix $M=I-A$. The following conditions are equivalent:

(1) The matrix of intermediate inputs $A$ is productive.

(2) The matrix A is profitable. 
(3) The upper left-hand principal minors of $M$ are all positive. $\operatorname{det}\left(m_{i j}\right)>0, i=1, \ldots, k, j=1, \ldots, k,(k=1, \ldots, n)$.

(4) All principal minors of $M$ are positive: $\operatorname{det}\left(m_{i i}\right)>0, \quad i \in N, N \subset$ $\{1, \ldots, n\}$.

For low dimensional economies condition (3) of Proposition 1.2 provides an easily applicable routine, to check the productiveness and profitability of nonnegative IO matrices. For example, in the case $n=2$, it is sufficient to check that $m_{11}$ and $\operatorname{det} M$ are both positive and in the case $n=3$ we have to check in addition that $m_{11} m_{22}-m_{12} m_{21}>0$ holds true.

\subsubsection{1 'Productiveness' and 'profitability' in Quesnay's economy}

The notions of productiveness and profitability can be illustrated by means of a simple two-commodity example based on the IO matrix from Table 1.8, which is a more general version of the IO matrix in Table 1.4 - effectively our earlier example of Quesnay's Tableau.

\begin{tabular}{|l|c|c|}
\hline & Agriculture & $\begin{array}{c}\text { Manu- } \\
\text { facturing }\end{array}$ \\
\hline \hline Agricultural products & $a_{11}$ & $a_{12}$ \\
\hline Manufacturing products & 0 & 0 \\
\hline \hline Total output & 1 & 1 \\
\hline
\end{tabular}

Table 1.8: A simple two-sector IO matrix

The IO matrix $A$, the difference matrix $M$, and the corresponding vectors of activity levels $x$ and prices $p$ of Table 1.8 can be written as

$$
\begin{gathered}
A=\left[\begin{array}{cc}
a_{11} & a_{12} \\
0 & 0
\end{array}\right], \quad M=\left[\begin{array}{cc}
1-a_{11} & -a_{12} \\
0 & 1
\end{array}\right], \\
x=\left[\begin{array}{l}
x_{1} \\
x_{2}
\end{array}\right], \quad p^{\prime}=\left[\begin{array}{l}
p_{1} \\
p_{2}
\end{array}\right] .
\end{gathered}
$$

The $A$ matrix is immediately shown to be productive as well as profitable, for example by means of the vectors $x^{\prime}=(1,1)$ and $p=(1,1)$, provided $a_{11}<1$, which ensures that the principal minors of $M$ are positive. As a result, the geometric series in Proposition 1.1 are well-defined and converge towards the Leontief-inverse. Thus, it is 
possible to calculate the vectors of activity levels and prices implied by given vectors of final demand or monetary surpluses. In detail, the matrix powers read as follows:

$$
\begin{gathered}
A^{2}=\left[\begin{array}{cc}
a_{11}^{2} & a_{12} a_{11} \\
0 & 0
\end{array}\right], \quad A^{3}=\left[\begin{array}{cc}
a_{11}^{3} & a_{12} a_{11}^{2} \\
0 & 0
\end{array}\right], \\
A^{4}=\left[\begin{array}{cc}
a_{11}^{4} & a_{12} a_{11}^{3} \\
0 & 0
\end{array}\right], \quad \text { etc. }
\end{gathered}
$$

Carrying the procedure further and summing these matrices with the two-dimensional identity matrix $I$ :

$$
I+A+A^{2}+A^{3}+A^{4}+\ldots=\left[\begin{array}{cc}
1 /\left(1-a_{11}\right) & a_{12} /\left(1-a_{11}\right) \\
0 & 1
\end{array}\right]
$$

This result is an analog to macroeconomic multiplier analysis. Typically, in macroeconomics, multipliers are formulated in a onecommodity world and the expenditure multiplier is derived with reference to households' marginal propensity to consume, for given levels of investment and government expenditures in place of intermediate products. Similarly, the above result can be viewed as the sum of the indirect effects of autonomous changes in demand, thereby capturing the essence of the well-known multiplier effect in macroeconomics.

Testing the consistency of the above calculation, the vector of activity levels $x=\left(x_{1}, x_{2}\right)^{\prime}$ corresponding to the vector of final demands $y=\left(y_{1}, y_{2}\right)^{\prime}$ reads:

$$
x=\left[\begin{array}{cc}
1 /\left(1-a_{11}\right) & a_{12} /\left(1-a_{11}\right) \\
0 & 1
\end{array}\right] y=\frac{1}{1-a_{11}}\left[\begin{array}{c}
y_{1}+a_{12} y_{2} \\
\left(1-a_{11}\right) y_{2}
\end{array}\right]
$$

and thus

$$
A x=\frac{1}{1-a_{11}}\left[\begin{array}{c}
a_{11}\left(y_{1}+a_{12} y_{2}\right)+a_{12}\left(1-a_{11}\right) y_{2} \\
0
\end{array}\right]
$$

and, moving back to the vector of final demands: 


$$
\begin{aligned}
y & =x-A x \\
& =\frac{1}{1-a_{11}}\left[\begin{array}{c}
-a_{11} y_{1}+a_{12} a_{11} y_{2}-a_{12} y_{2}-a_{12} a_{11} y_{2}+y_{1}+a_{12} y_{2} \\
\left(1-a_{11}\right) y_{2}
\end{array}\right] \\
& =\frac{1}{1-a_{11}}\left[\begin{array}{c}
\left(1-a_{11}\right) y_{1} \\
\left(1-a_{11}\right) y_{2}
\end{array}\right]=\left[\begin{array}{l}
y_{1} \\
y_{2}
\end{array}\right]
\end{aligned}
$$

consistently with the calculation of the vector $x$. A similar exercise can be performed exploring the relationship between profits and prices.

In summary, we have added to Quesnay's Tableau Économique a set of structural propositions concerning questions of reproducibility and profitability at different scales of production and for different prices. (Although, of course, not all sectoral price and activity levels lead to profitable or productive situations.) Leontief's IO theory thus leads us from an ex post determination of IO tables for entire economies to interesting, though still basic, propositions of IO analysis as a foundation for questions of income distribution (via cost and price determination) and growth (as determined by investment and quantity formation).

\subsubsection{Connectedness and decomposability of input-output structures}

Before turning to questions of distribution and growth, some helpful definitions and propositions are introduced below. The aim is to clarify the Sraffian concept of basic and nonbasic commodities, and some useful concepts of sectoral interdependencies. In an $n$-good economy a commodity is basic if it enters directly or indirectly the production of all $n$ commodities, as intermediate good, or as intermediate good for intermediate good, and so on. In the matrices above, for example in Table 1.4, there is one basic (good 1) and one nonbasic commodity (good 2). Following Nikaido (1968), the concept of a basic commodity and the related notions of decomposable and primitive matrices can be formally defined: ${ }^{8}$

Definition 1.2. Let $A=\left(a_{i j}\right)$ be the IO matrix of intermediate inputs per unit of output of a closed economy.

(1) We define the $i$-th sector of $A$ (as a supplier) to be directly or indirectly connected to the $j$-th sector and denote this by $i \rightarrow j$, if there exists a chain of intermediate sectors $k_{1}, \ldots, k_{m}$ with $\left(k_{0}=i, k_{m+1}=j\right)$ such that all neighboring sectors of the chain

\footnotetext{
${ }^{8}$ For a detailed analysis of primitive matrices see also Seneta (1973).
} 
exhibit a direct delivery relationship, in the sense that $a_{k_{s}, k_{s+1}}>0$ for all $s \in\{0, \ldots, m\}$.

(2) A commodity $i$ is called a basic commodity, if $i \rightarrow j$ for all $j \in$ $\{1, \ldots, n\}$.

(3) The matrix $A$ is called decomposable, if there exists a proper nonempty subset $J$ of $\{1, \ldots, n\}$ such that

$$
a_{i j}=0, \quad i \notin J, j \in J .
$$

(4) The matrix $A$ is called indecomposable, if it is not decomposable.

(5) The indecomposable matrix $A$ is called primitive, if there exists a positive integer $k$ such that $A^{k}>0$ (in the opposite case the matrix is called imprimitive).

Definition 1.2(1) implies that increases in the final demand for good $j$ must eventually lead to increases in the demand for good $i$, and if the IO matrix $A$ is decomposable, then the $n$ produced commodities can be renumbered in such a way that the resulting matrix is of the form

$$
\left[\begin{array}{cc}
A_{11} & A_{12} \\
0 & A_{22}
\end{array}\right]
$$

where the matrix $A_{11}$ represents the interrelationships between the commodities of the set $J, A_{22}$ represents those of the entries $\{1, \ldots, n\}-$ $J$, and $A_{12}$ represents the intermediate inputs from the $J$ sectors to $\{1, \ldots, n\}-J$ subsectors, while $A_{21}=0$, i.e. the $\{1, \ldots, n\}-J$ subsectors do not deliver anything to the $J$ sectors. The latter are therefore independent from the former; however, the commodities produced by sectors $J$ are not necessarily basic commodities while those of subsector $\{1, \ldots, n\}-J$ are nonbasic commodities. If $A_{12}=0$ then both subsystems $J$ and $\{1, \ldots, n\}-J$ are independent of each other and there are no basic commodities. Finally, if the subsystem $A_{11}$ is indecomposable, we have reached the lowest level of decomposability, a situation which we will call a Sraffa matrix. ${ }^{9}$

Before presenting Proposition 1.3 it is helpful to define a cyclical subdivision for an IO matrix. A cyclical subdivision in $r$ subsectors

$$
s_{i} \subset\{1, \ldots, n\}, \quad \bigcup_{i=1}^{i=r} s_{i}=\{1, \ldots, n\}, \quad s_{i} \cap s_{j}=\emptyset, \quad i \neq j, \quad s_{i} \neq \emptyset,
$$

\footnotetext{
${ }^{9}$ See Nikaido (1968, II.7-8) and Seneta (1973, Ch.1) for an expanded discussion of the principles underlying Proposition 1.3.
} 
for some IO matrices $A_{s_{i}, s_{i+1}} \geq 0$ implies a matrix with the following structure:

$$
\left[\begin{array}{ccccc}
0 & A_{s_{1}, s_{2}} & 0 & \ldots & 0 \\
0 & 0 & A_{s_{2}, s_{3}} & \ldots & 0 \\
\ldots & \ldots & \ldots & \ldots & \ldots \\
0 & 0 & 0 \ldots & \ldots & A_{s_{r-1}, s_{r}} \\
A_{s_{r}, s_{1}} & 0 & 0 & \ldots & 0
\end{array}\right] .
$$

\section{Proposition 1.3.}

(1) A productive IO matrix $A$ is indecomposable if and only if its Leontief-inverse

$$
(I-A)^{-1}=I+A+A^{2}+A^{3}+\ldots
$$

is strictly positive. This holds if and only if all commodities are basic.

(2) An indecomposable IO matrix $A$ is primitive if and only if there exists no proper and disjoint cyclical subdivision in $r$ subsectors

$$
s_{i} \subset\{1, \ldots, n\}, \quad \bigcup_{i=1}^{i=r} s_{i}=\{1, \ldots, n\}, \quad s_{i} \cap s_{j}=\emptyset, \quad i \neq j, \quad s_{i} \neq \emptyset,
$$

with corresponding IO matrices $A_{s_{i}, s_{i+1}} \geq 0$.

(3) Let $A$ be a productive matrix, $i$ is basic if the $i$-th row of the Leontiefinverse is strictly positive.

(4) Let $A$ be a decomposable matrix of type (1.1). The set of commodities $J$ corresponds to the set of basic commodities if there is at least one basic commodity in the considered subsystem and the matrix $A_{11}$ is indecomposable.

Proposition 1.3 indicates that it may be of great interest to order the given set of commodities to uncover the hierarchies hidden in the coefficients of their IO relationships. First the basic commodities, then the basic commodities of order two (concerning only the remaining sectors), and so on, given their cyclical structure (see Proposition 1.3(2)), imprimitive matrices should play no role in such a restructuring of an IO matrix from the empirical point of view, due to the implausible decoupling of the last level of production to the very first one (from where production starts again in a strictly hierarchical manner). The importance of Proposition 1.3 can be made more clear by providing 
a sense of its proof in Lemma 1.1. The next Lemma provides some methods to check these properties.

Lemma 1.1. Let $A$ be an IO matrix and let $a_{i j}^{(k)}$ represent the entry $A_{i j}^{k}$ of $A$. Then:

(1) $a_{i j}^{(k+l)}=\sum_{\eta=1}^{n} a_{i, \eta}^{(k)} a_{\eta, j}^{(l)}$

(2) There exists a positive integer $k$ such that $a_{i j}^{(k)}>0$ if and only if $i \rightarrow j$.

(3) The matrix $A$ is indecomposable for all sectors represented by $i$ and $j$ if and only if there exists a positive integer $k$ such that $a_{i j}^{(k)}>0$ holds.

(4) A commodity $i$ is basic if and only if for all sectors $j$ there exists a positive integer $k$ such that $a_{i j}^{(k)}>0$ holds.

Observe that in general the choice of $k$ in part (4) depends on the choice of $j$ except for primitive IO matrices where $k$ can be chosen independently of $j$.

The simplest example of an indecomposable matrix $A$ which is imprimitive is given by

$$
A=\left[\begin{array}{cc}
0 & 0.5 \\
0.5 & 0
\end{array}\right]=0.5\left[\begin{array}{ll}
0 & 1 \\
1 & 0
\end{array}\right] .
$$

For this matrix there holds

$$
\begin{gathered}
A^{2}=\left[\begin{array}{cc}
0 & 0.5 \\
0.5 & 0
\end{array}\right]\left[\begin{array}{cc}
0 & 0.5 \\
0.5 & 0
\end{array}\right]=0.25\left[\begin{array}{ll}
1 & 0 \\
0 & 1
\end{array}\right], \\
A^{3}=\left[\begin{array}{cc}
0 & 0.5 \\
0.5 & 0
\end{array}\right]\left[\begin{array}{cc}
0 & 0.5 \\
0.5 & 0
\end{array}\right]\left[\begin{array}{cc}
0 & 0.5 \\
0.5 & 0
\end{array}\right]=0.125\left[\begin{array}{ll}
0 & 1 \\
1 & 0
\end{array}\right], \quad \text { etc. }
\end{gathered}
$$

In this example there is a permanent switch between a main-diagonal and an off-diagonal situation, which prevents any matrix $A^{k}$ from becoming strictly positive. The same also holds for matrices of the form:

$$
0.5\left[\begin{array}{lll}
0 & 1 & 0 \\
0 & 0 & 1 \\
1 & 0 & 0
\end{array}\right],
$$

and so on. From the economic point of view these matrices imply that commodity $i$ with $i=1, \ldots, n-1$ is only used by sector $i+1$, for example 
we have a clear hierarchical structure between the considered sectors. However, the last commodity in this hierarchy, commodity $n$, is used by sector 1 and only there, a situation we may find in biological food chains or at the level of firms, but not at the level of entire economies. ${ }^{10}$

In the following, we assume that by suitable renumbering of commodities the matrix structure in equation (1.1) can be obtained where the $s \times s$ matrix $A_{11}$ is primitive; the first $s$ are basic commodities (and thus the $s \times(n-s)$ matrix $A_{12}$ is semi-positive in a specific way); and the $(n-s) \times(n-s)$ matrix $A_{22}$ is just semi-positive. This allows us to focus on the production structure $A_{11}$, since nonbasic commodities are largely irrelevant for the analysis of price and quantity formation, as shown in the next chapter.

Before considering empirical IO tables, it is helpful to quickly sketch out some examples of two-commodity matrices.

1. No means of production: Smith's "early and rude state" of society:

$$
A=\left[\begin{array}{ll}
0 & 0 \\
0 & 0
\end{array}\right] \text {. }
$$

2. Good 1 used for producing good 2, but not vice versa, i.e. Quesnay to Sraffa matrices:

$$
A=\left[\begin{array}{cc}
0 & a_{12} \\
0 & 0
\end{array}\right], \quad A=\left[\begin{array}{cc}
a_{11} & a_{12} \\
0 & 0
\end{array}\right], \quad A=\left[\begin{array}{cc}
a_{11} & a_{12} \\
0 & a_{22}
\end{array}\right]
$$

3. Indecomposable and primitive (not cyclical, Leontief matrices):

$$
A=\left[\begin{array}{ll}
a_{11} & a_{12} \\
a_{21} & a_{22}
\end{array}\right] .
$$

4. Indecomposable, but not primitive (circular production structures):

$$
A=\left[\begin{array}{cc}
0 & a_{12} \\
a_{21} & 0
\end{array}\right] .
$$

5. Completely decomposable (basic two country situation):

\footnotetext{
${ }^{10}$ Further discussion on helpful rearrangements of rows and columns of matrices can be found in the Appendix to this chapter.
} 


$$
A=\left[\begin{array}{cc}
a_{11} & 0 \\
0 & a_{22}
\end{array}\right] .
$$

Even in the case of only two commodities, we find a number of qualitatively different production structures which can be classified focusing on good $1 .{ }^{11}$ The seven matrices can be called, respectively, Smith-matrices, Austrian-matrices, Quesnay-matrices, Sraffa-matrices, von-Neumann-matrices, (biological) hypercycle-matrices, and twocountry-matrices.

\subsection{Contemporary empirical input-output tables}

\subsubsection{Input-output tables in open economies}

In the rest of the book, we will assume that the IO matrices $A \in \mathbb{R}_{+}^{n \times n}$ are productive. Then, the basic input-output equation for an economy,

$$
x=A x+y,
$$

is well-defined for any given vector of final demands $y \in \mathbb{R}_{+}^{n}$.

In Section 1.2 the matrix coefficients $a_{i j}$ were defined to represent the quantity of good $i$ used up in the production of one unit of good $j$. This characterization involves an implicit assumption. Since the vector $x$ on the left-hand side of equation (1.2) refers to production in a given country, say, the home country, a sector $j$ obtains the quantity $a_{i j} x_{j}$ on the right-hand side of equation (1.2) exclusively from the domestic sector $i$. If $a_{i j}$ is the quantity of good $i$ that is technologically required per unit of output $j$, sector $j$ buys all it needs on the domestic market, and the same is true for all sectors. Therefore, equation (1.2) refers to a closed economy, or at least to an economy that imports no intermediate goods.

The assumption of a closed economy may have been reasonable at the time Quesnay designed his Tableau Économique, but it is certainly obsolete today. Hence we must distinguish between what is technologically needed, and what is bought in the home country and abroad, respectively. To this effect, let $A_{T}, H$, and $A$ be $n \times n$ matrices with respective elements $a_{T ; i j}, h_{i j}$, and $a_{i j}$. The coefficient $a_{T ; i j}$ represents the quantity of good $i$ that is technologically required to produce one unit of output $j, h_{i j}$ is the share of input good $i$ that sector $j$ buys in the home country (the home share), and $a_{i j}$ is the

$\overline{11}$ There are corresponding further cases if one looks at these structures focusing on good 2 . 
quantity of input $i$ per unit of output that sector $j$ buys on the domestic market. The coefficients specified above are related through equation (1.3):

$$
\underset{\text { domestic coefficient }}{a_{i j}}=\underset{\text { home share }}{h_{i j}} \quad \cdot \quad a_{T, i j} .
$$

With the above interpretation of the matrix $A$, equation (1.2) continues to apply as the basic IO equation for open economies.

Monetary relations must also be modified accordingly. Let $\nu_{j}$ be the value added per unit of output $j$, which for the time being may be thought to be exogenously given. For the sake of notational simplicity suppose that there are no price differentials at home and abroad. The price equation for sector $j$ would read $p_{j}=\sum_{i} a_{T ; i j} p_{i}+\nu_{j}$ or, in vector form:

$$
p=p A_{T}+\nu
$$

In closed economies, where $A=A_{T}$, equations (1.2) and (1.4) constitute a nice dual representation of the economy, but the direct correspondence is lost in open economies.

\subsubsection{Aggregation of input-output tables}

In theory, we can conceive of an economy producing $n$ goods and refer to the quantities of all these goods. Yet there are thousands of goods on the market. In the real world even the most detailed statistics cannot record each and every good, and even at the most elementary level several goods are combined into aggregate goods. Thus in applied work the expression "quantity of good $i$ " must not be taken literally. Rather, it means something like: "One unit of good $i$ is a bundle of (physically different) goods that are assigned to a category $i$. Valued at prices of a given base year, it is worth one million Euros." 12

It is important to note that the prices in the base year $p^{b}$ are all unity: $p_{i}^{b}=1$ for all $i$. Hence, we may add up the column coefficients of a real IO table: $\sum_{i} a_{i j}$ should then be interpreted as $\sum_{i} p_{i}^{b} a_{i j}$, which reflects the value of the intermediate inputs in terms of the base year prices per unit of output $j$. However, with respect to some other price vector $p \neq p^{b}$ a term like $p_{i} x_{i}$ would mean the value of a bundle of goods assigned to category $i$, which if valued at the base year prices, would be worth $x_{i}$ million Euros. Under this proviso, we can refer to $p_{i} x_{i}$ as the value of (the quantity of) $x_{i}$ units of good $i$.

$\overline{12}$ One million Euros in prices of the year 1995 is the monetary unit underlying the real (as opposed to the nominal) IO tables for Germany over the decade 1991-2000. It does not matter whether it is a million Euros or another sum of money, this is just a matter of scale. 
This brief sketch of how to interpret the "quantities" of goods is the basis of all aggregation procedures for IO tables. In preparing IO tables for national economies, statistical offices necessarily perform a great deal of aggregation. For example, the IO tables of Germany distinguish 71 categories of goods, produced in 71 sectors. This is a relatively high degree of differentiation that is useful for a variety of applications. In order to study the sectoral interrelationships and their implications, however, $71 \times 71$ tables are often quite unwieldy and it may be appropriate to aggregate these data further.

The basic tool in aggregating IO matrices is a so-called binary "summation matrix" $\mathcal{S}$. In order to illustrate the general procedure, consider first a simple three-good economy where the first two goods are to be aggregated into one composite good. Consider again the matrix $X$ of intersectoral flows

$$
X=\left[\begin{array}{lll}
a & b & c \\
d & e & f \\
g & h & i
\end{array}\right] .
$$

Let $X^{\star}$ be the aggregated version of $X$, where $x_{11}^{\star}$ is the sum of all flows between the two original sectors 1 and $2 ; x_{21}^{\star}$ sums up the flows of the original sectors 1 and 2 to the original sector 3 , which is now counted as sector 2 ; etc. Thus we get

$$
X^{\star}=\left[\begin{array}{cc}
a+b+d+e & c+f \\
g+h & i
\end{array}\right] .
$$

The summation matrix $\mathcal{S}$ describes the transition from $X$ to $X^{\star}$ in a compact way. It is a matrix of order $2 \times 3$, specified as

$$
\mathcal{S}=\left[\begin{array}{lll}
1 & 1 & 0 \\
0 & 0 & 1
\end{array}\right] \text {. }
$$

It is then easily checked that

$$
X^{\star}=S X \mathcal{S}^{\prime} .
$$

Equation (1.5) remains valid even if, where there are $1, \ldots, n$ original sectors that are aggregated to $1, \ldots, M$, macro sectors. To be specific, let $J_{m}$ be the index set of the original sectors that are assigned to macro sector $m\left(m=1, \ldots, M\right.$; so, $J_{1}=\{1,2\}$ and $J_{2}=\{3\}$ in our example). 
In this case, $\mathcal{S}$ is of order $M \times n$, and its entries are given by

$$
s_{m j}= \begin{cases}1 & \text { if the original sector } j \in J_{m} \\ 0 & \text { otherwise. }\end{cases}
$$

Besides (1.5), matrix $\mathcal{S}$ is also used to describe how to move from an original activity vector $x \in \mathbb{R}_{+}^{n}$ to the aggregated vector $x^{\star} \in \mathbb{R}_{+}^{M}$ with components $x_{m}^{\star}=\sum_{j \in J_{m}} x_{j}$ :

$$
x^{\star}=\mathcal{S} x,
$$

and likewise for the final demand vectors $y$ and $y^{\star}$, or any other quantity vectors.

Things are a bit more involved when we turn from the total flows in $X$ and $X^{\star}$ to the coefficient matrix $A \in \mathbb{R}_{+}^{n \times n}$ and its aggregated counterpart $A^{\star} \in \mathbb{R}_{+}^{M \times M}$. To explore these complications, let $\widehat{x}$ denote the $n \times n$ diagonal matrix where entries $x_{j}$ on the main diagonal are the elements of the production vector $x \in \mathbb{R}_{+}^{n}$; likewise for $\widehat{x}^{\star} \in \mathbb{R}_{+}^{M}$. The transition from $A$ to $A^{\star}$ is summarized by Proposition 1.4.

Proposition 1.4. Let $A \in \mathbb{R}_{+}^{n \times n}$ be an IO matrix and $x \in \mathbb{R}_{++}^{n}$ a strictly positive activity vector that is associated with it. Let the aggregation of the $n$-sectoral economy into an $M$-sectoral economy be described by the matrix $\mathcal{S}$ in equation (1.6). Let $x^{\star}=S x$, then the aggregated IO matrix $A^{\star} \in \mathbb{R}_{+}^{M \times M}$ can be obtained from $A$ and $x$ as follows

$$
A^{\star}=S A \widehat{x} \mathcal{S}^{\prime}\left(\widehat{x}^{\star}\right)^{-1} .
$$

Proof. By definition, for all $i, j, a_{i j}=X_{i j} / x_{j}$. Therefore, in matrix form $A \widehat{x}=X$. By the same token, $A^{\star} \widehat{x}^{\star}=X^{\star}$. Thus, $A^{\star} \widehat{x}^{\star}=X^{\star}=$ $\mathcal{S} X \mathcal{S}^{\prime}=\mathcal{S} A \widehat{x} \mathcal{S}^{\prime}$, where the second equality follows from (1.5). The result follows by post-multiplying the outer terms in this chain by the inverse of $\widehat{x}^{\star}$.

The relationship applies whether $A$ is the matrix of domestic coefficients or (with $A=A_{T}$ ) of technological coefficients. It should be noted that this aggregation procedure is based on a given output vector containing the empirical sectoral outputs.

\subsubsection{Examples of aggregated input-output tables}

Going back to Quesnay, Table 1.4 provides an early example of a $2 \times 2 \mathrm{IO}$ table. It has, however, two unrealistic features: the coefficients appear 
too small (as reflected in the high associated profit rates), and the matrix is not only decomposable, but one of the two goods does not enter production at all. With a basic understanding of the notion of aggregation, we can now set up a more relevant numerical example. It is constructed from the German IO tables (in real terms), where the original 71 production sectors are aggregated up to two (macro) sectors: the industry sector and the services sector. This constitutes our twosectoral standard aggregation. The relative size of the two sectors is illustrated in Table 1.9, which displays the sectoral output shares for Germany in the year 2000. This will serve as our empirical reference point throughout the book. ${ }^{13}$

1: The industry sector $42.5 \%$

2: The services sector $57.5 \%$

Table 1.9: The two-sectoral standard aggregation

For the same country and year, the matrices $A_{T}$ and $A$ in equation (1.8) present the technological and the domestic IO coefficients computed from the original $71 \times 71$ tables, using the procedure outlined in Proposition 1.4. It is evident that the distinction in section 1.4.1 between the technological and domestic coefficients is relevant, especially for the intrasectoral flows in the industry sector. About a third of this composite input good is imported from abroad $(0.432-0.286) / 0.432=0.340$. Similarly, the services sector buys $7.8 \%$ of the services necessary for production on foreign markets.

$$
A_{T}=\left[\begin{array}{ll}
0.432 & 0.073 \\
0.199 & 0.312
\end{array}\right], \quad A=\left[\begin{array}{ll}
0.286 & 0.060 \\
0.191 & 0.287
\end{array}\right]
$$

As should be clear from section 1.4.1, the notion of the Leontief-inverse involves the matrix $A$ of the domestic coefficients. Given equation (1.8), the Leontief-inverse is

13 The "industry sector" comprises agriculture, manufacturing, and construction. The percentage numbers are the sectoral output shares in gross output of Germany in 2000. 


$$
\begin{aligned}
(I-A)^{-1} & =\frac{1}{\left(1-a_{11}\right)\left(1-a_{22}\right)-a_{12} a_{21}}\left[\begin{array}{cc}
1-a_{22} & a_{12} \\
a_{21} & 1-a_{11}
\end{array}\right] \\
& =\left[\begin{array}{ll}
1.432 & 0.121 \\
0.383 & 1.436
\end{array}\right] .
\end{aligned}
$$

According to equation (1.9), an increase in the final demand for the industrial good by one million Euros in 1995 prices raises the output of this sector by 1.432 million Euros. The resulting increase in services to provide the production processes with the necessary inputs is 0.383 million Euro. Thus a one million increase in final demand raises total economic activity by 1.815 million Euros. Foreign countries profit too, but in this basic framework we are ignoring this possible effect and any possible feedback effects on the home country.

The impact of an increase in the final demand for services on total economic activity is less pronounced. Here, the "multiplier" is only 1.556 (the difference from $0.121+1.436=1.557$ is due to rounding errors).

A two-sector aggregation may be convenient to illustrate some basic features - in other respects it is, of course, very crude. For a more detailed picture of the economy, we occasionally work with a 7 -sector aggregation. The industry sector is split up into agriculture, manufacturing, and construction. Within manufacturing itself, we separate out another subsector which for an export-oriented country like Germany should be of particular importance. It comprises the four single production sectors (among the 71 original sectors) with the highest exports: chemicals, pharmaceuticals, machinery, and motor vehicles. We call this macro sector the export core. ${ }^{14}$

The services sector produces very different output 'goods'. We distinguish between three of them: business-related services, consumer services, and social services. The term business-related services needs further clarification. For one of the 71 original sectors of the German IO tables is labeled thus, and has grown considerably over the 1990s with an output share that in 2000 rose to almost seven percent (it is thus larger than the construction sector). This single sector may be viewed as business-related services in a narrow sense. For our aggregation, however, we understand this term in a broader sense and include the

\footnotetext{
${ }^{14}$ The specification of this export core has proved fruitful in the investigation of the impact that the structural change in the industrial sector has on the other sectors; see Kalmbach, Franke, Knottenbauer, and Krämer (2005).
} 
following sectors: wholesale trade, communications, finance, leasing, computer and related services, research and development services. In contrast consumer services comprise: retail trade, repair, transport, insurance, real estate services, and personal services. ${ }^{15}$

Table 1.10 summarizes the seven (macro) sectors obtained. For a better assessment of their relative importance, it also indicates the sectoral output shares (in the year 2000). According to this criterion, agriculture could have well been included in what we have called other manufacturing (which would be sensible given the industrial character of today's agriculture and fisheries). We consider it separately for conventional reasons. The technological coefficients of the 7 -sectoral aggregation are reported in Table 1.11.

\begin{tabular}{|lr|}
\hline (1): Agriculture (Agrc.) & 1.33 \\
(2): Manufacturing, the export & 12.37 \\
& core (Manf.) \\
(3): Other manufacturing & 22.55 \\
$\quad$ (Oth.Mf.) & \\
(4): Construction (Cstrt.) & 6.29 \\
(5): Business-related services & 21.36 \\
& (Bus.Svcs.) \\
(6): Consumer services & 23.35 \\
& (Cns.Svcs.) \\
(7): Social services (Soc.Svcs.) & 12.75 \\
\hline
\end{tabular}

Table 1.10: The 7-sector standard aggregation

Note: The numbers in the last column are the sectoral output shares (in percent) for Germany in 2000.

${ }^{15}$ Business-related services do not exclusively work for enterprises and consumer services do not exclusively serve the consumer. Our distinction between the two is justified by the relatively high share of $68.9 \%$ of the output of business-related services that is purchased as intermediate inputs by the other sectors (and themselves, in 2000). This is almost 30 percentage points above the average share of $40.2 \%$ of total output in the economy. By contrast, the consumer services' share of output used as intermediate inputs is as low as $32.3 \%$. The shares of real estate services and insurance (without social insurance), which are assigned to the consumer services, are actually not much higher: they amount to $35.2 \%$ and $33.5 \%$, respectively. 


\begin{tabular}{lccccccc}
\hline & $(1)$ & $(2)$ & $(3)$ & $(4)$ & $(5)$ & $(6)$ & $(7)$ \\
& Agrc. Manf. & Oth.Mf. Cstrt. & Bus.Svcs. & Cns.Svcs. Soc.Svcs. \\
\hline Agrc. & 0.028 & 0.000 & 0.045 & 0.000 & 0.000 & 0.002 & 0.002 \\
Manf. & 0.090 & 0.282 & 0.050 & 0.022 & 0.003 & 0.008 & 0.011 \\
Oth.Mf. & 0.142 & 0.232 & 0.324 & 0.287 & 0.030 & 0.055 & 0.065 \\
Cstrt. & 0.007 & 0.003 & 0.006 & 0.017 & 0.006 & 0.028 & 0.016 \\
Bus.Svcs. & 0.142 & 0.121 & 0.140 & 0.107 & 0.332 & 0.134 & 0.096 \\
Cns.Svcs. & 0.036 & 0.053 & 0.051 & 0.108 & 0.072 & 0.152 & 0.049 \\
Soc.Svcs. & 0.031 & 0.006 & 0.011 & 0.007 & 0.007 & 0.013 & 0.024 \\
\hline
\end{tabular}

Table 1.11: Technological coefficients of the 7-sector aggregation (Germany, 2000)

Regarding the domestic coefficients for the 7 -sector aggregation, it is more insightful to directly report the import shares for the different intermediate goods (referring to (1.3), these shares are given by $1-h_{i j}$ ). This is done in Table 1.12.

\begin{tabular}{|c|c|c|c|c|c|c|c|}
\hline & (1) & $(2)$ & (3) & (4) & $(5)$ & (6) & $(7)$ \\
\hline & Agrc. & Manf. & Oth.Mf. & Cstrt. & Bus.Sves. & . Cns.Sves. & Soc.Svcs. \\
\hline Agrc. & 12.2 & 57.4 & 26.1 & 0.0 & 0.0 & 22.2 & 21.3 \\
\hline Manf. & 56.4 & 38.4 & 46.1 & 28.1 & 69.0 & 21.7 & 47.7 \\
\hline Oth.Mf. & 16.4 & 30.2 & 37.8 & 16.1 & 19.2 & 21.1 & 24.4 \\
\hline Cstrt. & 0.3 & 0.4 & 8.8 & 41.6 & 0.3 & 0.4 & 0.2 \\
\hline Bus.Sves. & 0.1 & 5.3 & 1.9 & 0.5 & 9.6 & 2.9 & 4.4 \\
\hline Cns.Sves & 7.5 & 8.6 & 7.4 & 6.3 & 7.8 & 9.9 & 4.9 \\
\hline Soc.Sves. & 0.0 & 11.6 & 2.0 & 0.0 & 12.2 & 0.0 & 17.2 \\
\hline
\end{tabular}

Table 1.12: Import shares (in percent) for the 7-sector aggregation

Tables 1.11 and 1.12 provide all the information needed to compute the Leontief-inverse for the 7-sector economy; first compute matrix $A$ of the domestic coefficients, and subsequently invert matrix $I-A$. The Leontief-inverse is shown in Table 1.13. The last row of the table 
computes the column sums. Just as in the two-sector example discussed above, they give the increase in total economic activity induced by a unit increase of final demand for the respective good, and they may be interpreted as economy-wide multipliers.

Regarding the impact on the output of the different sectors, we point out that if we discount for the direct effects (i.e. if unity is subtracted from the diagonal elements), then it is the business-related services that profit the most from increases in final demand (except for an increase in demand for construction). ${ }^{16}$ In short, the fifth row dominates the other rows. This could not be easily predicted from Table 1.11, where the entries of the fifth row are not clearly dominant. ${ }^{17}$ The dominant output gains of the business-related services are one example of intersectoral IO relationships leading to results that are not a priori obvious.
(1) (2)
(3)
(4)
(5)
(6)
(7)

Agrc. Manf. Oth.Mf. Cstrt. Bus.Svcs. Cns.Svcs. Soc.Svcs.

\begin{tabular}{llllllll}
\hline Agrc. & 1.032 & 0.010 & 0.044 & 0.012 & 0.002 & 0.005 & 0.005 \\
Manf. & 0.056 & 1.220 & 0.046 & 0.033 & 0.005 & 0.014 & 0.011 \\
Oth.Mf. & 0.184 & 0.268 & 1.287 & 0.332 & 0.057 & 0.088 & 0.081 \\
Cstrt. & 0.013 & 0.011 & 0.012 & 1.019 & 0.013 & 0.036 & 0.020 \\
Bus.Srcs. & 0.276 & 0.278 & 0.292 & 0.260 & 1.468 & 0.249 & 0.170 \\
Cns.Svcs. & 0.077 & 0.107 & 0.100 & 0.160 & 0.118 & 1.188 & 0.076 \\
Soc.Srvcs. & 0.038 & 0.014 & 0.019 & 0.015 & 0.012 & 0.019 & 1.024 \\
\hline \multirow{2}{*}{$:$} & 1.676 & 1.907 & 1.800 & 1.830 & 1.674 & 1.599 & 1.387 \\
\hline
\end{tabular}

Table 1.13: The Leontief-inverse $(I-A)^{-1}$ for the 7-sector aggregation (Germany, 2000)

\subsection{Conclusions}

This chapter has analyzed Quesnay's Tableau Économique in order to introduce some fundamental concepts of IO theory. We have started from simple, small-scale examples similar to those used by

${ }^{16}$ Observe that the numbers in the entries $i, j$ of Table 1.13 can be directly compared, the common unit being "bundles of goods of category $i$ worth one million Euros in prices of 1995".

17 This characterization also holds for the matrix $A$. 
Quesnay himself and quickly advanced to full-scale examples of modern economies with extensive division of labor. The properties of IO matrices that are of primary concern reflect, on the one hand, their potential to generate physical and economic surpluses across sectors, and on the other hand, the degree of connectedness of an $n$-sector economy.

The IO tools introduced in this chapter allow us to provide a complete description of the flows of goods and services in the economy, capturing the physical interconnections between sectors, and a snapshot of the technological knowledge and productive structure of the economy. However, they also provide the foundations for the analysis of the classical notions of production prices and balanced growth pathsintroduced by classical authors like Smith, Ricardo, and Marx - that will be considered in the rest of the book. Certainly, the structure of advanced capitalist economies is far more complicated than the matrices of intermediate inputs considered in this chapter (which, for example, exclude the existence of joint products and fixed capital). We shall consider these complications in the rest of the book by gradually relaxing some of the simplifying assumptions of the basic IO model.

As a first step, while this chapter examined IO structures in which labor is considered part of the means of production (by implicitly assuming a given subsistence wage), the next chapter examines concepts directly relevant to labor - such as labor values, the rate of exploitation, and changes in real wages - more explicitly.

\subsection{Appendix: Further properties of matrices}

1.6.1 Dominant diagonal matrices

A matrix $M=I-A$ has a dominant diagonal if

$$
\left|m_{i i}\right|>\sum_{i \neq j}\left|m_{i j}\right| \text { for all } i=1, \ldots, n .
$$

It has a dominant diagonal in the extended sense if and only if there exist real numbers $d_{j}>0 \quad(j=1, \ldots, n)$ such that:

$$
d_{i}\left|m_{i i}\right|>\sum_{i \neq j} d_{j}\left|m_{i j}\right| \quad \text { for all } \quad i=1, \ldots, n .
$$

Finally the matrix $M$ has a positive dominant diagonal if and only if there exist $d_{i}>0, i=1, \ldots, n$, such that 


$$
d_{i}\left|m_{i i}\right|>\sum_{i \neq j} d_{j}\left|m_{i j}\right|, \quad \text { and } \quad m_{i i}>0 \text { for } i=1, \ldots, n \text {. }
$$

The latter two criteria are sufficient to imply the Hawkins-Simon conditions and thus all four statements in Proposition 1.2. In terms of the difference matrix $M$ this means that each sector produces more of its own product than it consumes and (as the first condition) that this surplus dominates the coefficients $\left|m_{i j}\right|=-a_{i j}$ that describe the consumption of good $i$ by the other sectors of the economy (when combined with appropriate weights).

If applied to the matrix $M=I-A$, where $A$ is nonnegative, a dominant diagonal is equivalent to the productivity of $A$. In fact, if $A$ is productive there exists a vector $\breve{x} \geq 0$ with $\breve{x}>A \breve{x}$. If $\breve{x}$ contains some zero components, a slight perturbation of $\breve{x}$ yields a strictly positive vector $x$ with this property. Thus, we have $M x>0$ or

$$
m_{i i} x_{i}+\sum_{i \neq j} m_{i j} x_{j}>0 \text { for all } i
$$

Since $m_{i j}<0$ for $j \neq i$, the latter inequality can be rewritten as

$$
\left|m_{i i}\right| x_{i}>\sum_{i \neq j}\left|m_{i j}\right| x_{j} \quad \text { for all } i .
$$

So it only remains to replace $x_{j}$ by $d_{j}$. The converse statement follows from similar considerations.

If instead of row sums, the definition of dominant diagonal were in terms of column sums, then dominant diagonal applied to $M=I-A$ would be equivalent to the profitability of the matrix $A$.

1.6.2 Useful manipulations of rows and columns of matrices The rows and columns of the matrix

$$
A=\left[\begin{array}{llll}
2 & 0 & 1 & 1 \\
0 & 6 & 0 & 5 \\
3 & 1 & 4 & 0 \\
0 & 7 & 0 & 8
\end{array}\right]
$$

can be rearranged so as to get the following input structure 


$$
A=\left[\begin{array}{llll}
2 & 1 & 0 & 1 \\
3 & 4 & 1 & 0 \\
0 & 0 & 6 & 5 \\
0 & 0 & 7 & 8
\end{array}\right] .
$$

Therefore this IO matrix exhibits two basic and two nonbasic commodities or industries.

Strictly hierarchically structured production technologies are given by matrices that can be triangularized as follows:

$$
\left[\begin{array}{ccccc}
a_{11} & a_{12} & a_{13} & \ldots & a_{1 n} \\
0 & a_{22} & a_{23} & \ldots & a_{2 n} \\
0 & 0 & a_{33} & \ldots & a_{3 n} \\
\ldots & \ldots & \ldots & \ldots & \ldots \\
0 & 0 & 0 \ldots & \ldots & a_{n-1, n} \\
0 & 0 & 0 \ldots & \ldots & a_{n n}
\end{array}\right], \quad\left[\begin{array}{ccccc}
0 & a_{12} & a_{13} & \ldots & a_{1 n} \\
0 & 0 & a_{23} & \ldots & a_{2 n} \\
\ldots & \ldots & \ldots & \ldots & \ldots \\
0 & 0 & 0 \ldots & \ldots & a_{n-1, n} \\
0 & 0 & 0 \ldots & \ldots & 0
\end{array}\right],
$$

possibly with further decomposable structures within the triangular part. Clearly, the first matrix is indecomposable and primitive, while the second is decomposable and with no basic commodity. 


\section{Adam Smith: The "Invisible Hand" and Accumulation}

\subsection{Ruthless competition: The invisible hand in the early and rude state of society}

In the Wealth of Nations, Adam Smith considered a wide range of topics, such as the industrial revolution, the division of labor, the measure and cause of value, the cost-of-production theory of prices, supply-determined prices, wages, profits, rent, a social unit of accounting, the trend in prices, banking, productive and unproductive labor, optimum investment patterns, taxation and public debt, and so on. In this chapter, and in the next, we concentrate on two central themes in Smith's work: the increasing division of labor, and its implications for commodity exchange and prices.

In this chapter we analyze Smith's views on the unintended economic consequences of individual actions - often summarized in his idea of the "invisible hand"1_in a simple economy consisting of labor and corn, which Smith defined the the "early and rude state of society". To be specific, this chapter analyzes income distribution and efficiency in a general equilibrium model that captures the key aspects of the competitive process as conceptualized by Smith and the Classics. It has long been acknowledged that, despite relevant differences between

\footnotetext{
1 "Every individual is continually exerting himself to find out the most advantageous employment of whatever capital he can command. It is his own advantage, indeed, and not that of the society, which he has in view. But the study of his own advantage naturally, or rather necessarily leads him to prefer that employment which is most advantageous to society" (Smith 2000, 482)... [B]y directing that industry in such a manner as its produce may be of the greatest value, he intends only his own gain, and he is in this, as in many other cases, led by an invisible hand to promote an end which was no part of his intention. Nor is it always the worse for the society that it was not part of it. By pursuing his own interest he frequently promotes that of society more effectually than when he really intends to promote it" (Smith 2000, 485).
} 
the various authors, it is possible to define a classical approach to competition, distribution, and growth that is an alternative to the neoclassical paradigm (Kurz and Salvadori (1992, 1995, 2002) and Flaschel (2010)). Classical political economy starts from given economic data and adopts a method-long-period analysis - which are different from those of neoclassical economics. ${ }^{2}$ Setting aside the complexities and differences of the various theories put forward by classical authors, the key classical views on the macroeconomic features of capitalist economies have been formalized by Kaldor (1955), Pasinetti (1960), Samuelson (1978), Morishima (1989), among others, in what has been defined "the canonical Classical model". This macroeconomic model captures the essential features of Ricardo's corn economy, with three classes (capitalists, workers, and landowners), scarce land, and one basic good used for workers' consumption. It provides a clear and rigorous framework to analyze the distribution of income between the three classes, and the macro-dynamics of the economy, from a classical perspective.

However, the microeconomic foundations of the model and the decentralized, competitive mechanism underlying the macroeconomic outcomes are less clearly specified. This is somewhat unsatisfactory as "ruthless competition" (Samuelson 1978) between agents plays an important role in classical theories, as the driving force of wage rate and profit rate equalization, but also in the determination of rents over different qualities of land. ${ }^{3}$ This chapter explicitly analyzes the microeconomic process of ruthless competition over wages, profits, and rents underlying the canonical classical model. Following the Classicals, the economy is described only in terms of observable magnitudes and with no reference to individual or societal preferences (Kurz and Salvadori 2002). There are three sets of agents: workers who own labor which they supply in fixed quantity, capitalists who own capital which is used to buy labor for production, and landowners who own pieces of land of different quality. As in Ricardo's corn model, and in the subsequent literature on the canonical classical model, we assume that

\footnotetext{
${ }^{2}$ According to Kurz and Salvadori (2002), in long-period analysis the givens of the classical authors are: (i) the set of available techniques; (ii) the size and composition of the social product; (iii) the ruling real wage rate for common labor; and (iv) the quantities of the different qualities of land available and the known stocks of depletable resources. In contrast, neoclassical givens are preferences, technology, and endowments.

${ }^{3}$ An interesting exception is the recent contribution by Salvadori (2004), which analyzes the Ricardian theory of rent in a game-theoretic perspective.
} 
only one good, namely corn, is produced by means of land and labor; corn is the only wage good; and capital in agriculture consists entirely of the wage bill.

One of the core features of the model is that, unlike in neoclassical approaches, capital is not a physical good tied up in the production of final commodities. Capital is conceptualized as a magnitude of purchasing power that can be freely allocated to different land/labor combinations (vis-à-vis technologies). Consistently with this interpretation, the key behavioral assumption is that as long as the expected profit rate is positive, capitalists put all their capital to productive use and, in particular, they try to allocate capital to the technologies that yield the highest rate of profit.

In Section 2.2, we analyze the functioning of decentralized, ruthless competition between agents in the short-run, where total labor supply and total capital are fixed. We define a Classical competitive equilibrium $(\mathrm{CCE})$ as an allocation in which: a unique market clearing real wage rate prevails; land/labor combinations are chosen by capitalists so as to maximize the rate of profit; rents adjust so that a unique profit rate emerges on all lands in operation; and all capital is put to productive use. The CCE, in classical terminology, is thus defined as a type of market equilibrium, or a moving equilibrium (Pasinetti 1960), in that the economy has not reached its natural, or stationary state.

In the CCE, ruthless competition between agents leads to a welldefined distribution of income between the three classes, and in particular to a precise division of the surplus between total profits and total rents. Further, in equilibrium the types of lands in operation, and the rents paid to their owners are uniquely determined in a model where both extensive and intensive diminishing marginal returns (and therefore rents) are accounted for. Only lands yielding a nonnegative profit rate are operated.

Section 2.3 proves the existence and uniqueness of the CCE for a large class of economies. It also provides a formal proof of the classical claim that ruthless competition leads to a social optimum. The optimal solution of a social planner's problem can be decentralized, provided capitalist farmers choose maximum-profit-rate techniques and competition between agents eliminates profit rate and wage rate differentials. Supply side dynamics are such that no reallocation of workers over the existing lands can yield a higher corn output than is generated by ruthless competition. The optimal amount of corn is distributed to labor, capitalist farmers, and landlords in a uniquely determined way. Further, an aggregate production function with the usual properties can be derived that describes production possibilities 
in this economy. This result provides the foundations for the use of aggregate production functions in the literature on the canonical Classical model. ${ }^{4}$ In this setting, the strict pursuit of self-interest leads - as Smith claimed - to the maximum possible corn production, given existing constraints.

In Section 2.4, the long-run dynamics of the classical model are analyzed. Two laws of motion describing the movement of the main state variables - namely, population and capital - are formalized. The first incorporates a Malthusian mechanism driving changes in labor supply. The other incorporates standard classical consumption and saving habits, and a version of Say's law: workers and landlords consume all of their income, and capitalist farmers invest the entirety of their income. The evolution of the wage fund over time depends on the profitability of the system. It is shown that a stationary state exists, which coincides with the Ricardian natural, long-run equilibrium, whereby wages are at the subsistence level, the profit rate is zero, and population and capital are stationary. The stationary state is stable and so we conclude that the decentralized economy settles on a path of moving market equilibria which asymptotically approach the natural equilibrium.

In the Appendix, we briefly review the canonical neoclassical model and the standard interpretation of Smithian competition adopted throughout much of the economics discipline and compare them to the classical concept of competition.

Two observations are worth making at this point. First, consistently with the canonical classical model, we assume that labor and land are the only inputs in the production of corn. This is only a simplifying assumption and it can be relaxed to include capital by focusing on the so-called "dose of labor-cum-capital" (Samuelson 1978) as a production input, although at the cost of an increase in technicalities. Further, some recent contributions focusing on the macro-dynamics of distribution and growth have generalized the classical framework to allow for human capital, too (see, for example, Dutt and Veneziani (2010, 2011)). Second, we analyze the classical model from a contemporary perspective and with modern tools. The aim is not to provide a careful textual exegesis of classical works, but rather to analyze some key classical insights in a simple and theoretically rigorous way.

\footnotetext{
${ }^{4}$ Kurz and Salvadori (1992) prove a similar result, albeit in a rather different
} model. 


\subsection{The structure of the economy}

Consider an economy with a set $\mathcal{K}=\{1, \ldots, n\}$ of lands with distinct fertility available for farming (corn production). Farming requires only labor as an input, and the period of production is uniform and normalized to one on each piece of land. Each piece of land $i \in \mathcal{K}$ is characterized by a production function $Y_{i}=f_{i}\left(L_{i}\right)$, which gives the amount of agricultural output that can be produced with a given labor input. For the sake of simplicity, in what follows, we assume that $f_{i}($.$) is$ twice differentiable, with positive but decreasing marginal productivity, and that no output can be obtained without labor. Formally, for all $i \in \mathcal{K}, f_{i}(0)=0, f_{i}^{\prime}()>$.0 and $f_{i}^{\prime \prime}()<$.0 , for all $L_{i} \in \mathbb{R}_{+}$where $f_{i}^{\prime}($. and $f_{i}^{\prime \prime}($.$) denote, respectively, the first and the second order derivative$ of $f_{i}($.$) . The use of differentiable production functions on every piece$ of land allows us to analyze both extensive and intensive rent (see Pasinetti (1960) and the discussion in Kurz and Salvadori (1992)).

Without loss of generality, we assume that if land $i \in \mathcal{K}$ has a higher harvest than land $j \in \mathcal{K}$ for some labor input $L>0$, it also has a higher harvest for every other positive labor input, so that lands can be unambiguously ordered in terms of their fertility. ${ }^{5}$ Formally:

\section{Assumption 2.1.}

$$
f_{1}(L)>f_{2}(L)>\ldots>f_{n}(L) \quad \text { for all labor inputs } L>0 \text {. }
$$

There are three classes, or sets of agents: workers, capitalist farmers, and landowners. At the beginning of each period, landowners own land, possibly of different type, and maximize rent. Each worker is endowed with a certain amount of (homogeneous) labor, which is normalized to one, and her labor supply is perfectly inelastic. Aggregate labor supply is therefore given and it is denoted by $\bar{L}$. Workers compete in order to obtain the highest possible (real) wage. Each capitalist farmer, denoted as $c$, is endowed with a certain amount of wage-funds $\Omega^{c}$, which can be used to pay workers in advance in order to activate (either alone, or as part of a consortium) a certain type of land (production technique). Capital is only advanced by capitalists to buy labor and it is not used as an intermediate good in production. Each capitalist can decide to pool her wage fund with other capitalists and form one, or more production coalitions. Since wages are paid in advance, gross profits also include

\footnotetext{
${ }^{5}$ We note in passing that an ordering of lands in terms of fertility is more problematic in models where intermediate goods are also used in production (see Kurz and Salvadori $(1992,232)$ ).
} 
the return on the wage fund to capitalist farmers. Rents are paid out of the net product.

In line with the existing literature, we assume extreme classical saving habits by postulating that there is no saving out of wages or rents, whereas all profits are saved. Thus the wage-fund for the next production period is equal to current aggregate gross profits made by capitalist farmers.

The key behavioral assumption of this section is that capitalist farmers compete on two markets as follows. Each period is divided into two stages. In the first stage, capitalists compete in order to hire workers: insofar as the expected rate of return on productive investments in agriculture is positive, they use all of their wage-funds in order to hire as many workers as possible to be used in agricultural production. In the second stage - the production stage - they compete in order to get those lands which yield the highest profit rate, taking as given the rent paid to landowners and the real wage rate. ${ }^{6}$

Formally, let $C$ be the set of capitalists in the economy. Let $\omega$ be the real wage rate paid in advance to workers and let $\rho_{i}$ denote the rent paid on land of type $i$, with $\omega$ and $\rho_{i}$ taken as given. ${ }^{7}$ For all $i \in \mathcal{K}$, let $r_{i}=\frac{f_{i}\left(L_{i}\right)-\rho_{i}-\omega L_{i}}{\omega L_{i}}$ be the profit rate from agricultural production on land of type $i$, if $L_{i}$ workers are employed on it. Let $L^{c}$ denote the labor demand of capitalist $c$. Then, our behavioral assumption implies that for all $c \in C, L^{c}=\Omega^{c} / \omega$, whenever there is some $i$ such that $r_{i} \geq 0$, for some $L_{i}>0$, supposing that each capitalist owns a sufficiently small proportion of the total capital stock.

Let $C_{i} \subseteq C$ be a subset of capitalists forming a productive coalition, and let $\Omega^{C_{i}}=\sum_{c \in C_{i}} \Omega^{c}$ be the total amount of wage funds of coalition $C_{i}$. We assume that there are no costs and no barriers in the formation of productive coalitions, and that each capitalist may belong to more than one coalition. The maximum profit rate that a coalition of farmers $C_{i}$ (which could be a singleton) can obtain on land $i \in \mathcal{K}$ is given by

$$
\begin{gathered}
\max _{L_{i}} r_{i}=\frac{f_{i}\left(L_{i}\right)-\rho_{i}-\omega L_{i}}{\omega L_{i}}=\frac{f_{i}\left(L_{i}\right)-\rho_{i}}{\omega L_{i}}-1, \\
\text { subject to } 0 \leq L_{i} \leq \frac{\Omega^{C_{i}}}{\omega} .
\end{gathered}
$$

For a given real wage $\omega$ (paid ex-ante) and rent $\rho_{i}$ for land $i$ (paid

\footnotetext{
${ }^{6}$ In their analysis of Malthus's theory, Costabile and Rowthorn (1985) also divide each period in two stages, a production stage and a circulation stage.

${ }^{7} \mathrm{~A}$ unique real wage is paid to all workers because of labor mobility.
} 
ex-post, as part of the surplus), $M P_{1}$ gives the maximum rate of profit that capitalists can earn on $i$, provided they can hire the optimal amount of workers $L_{i}$. In other words, we depart here from the standard neoclassical emphasis on profit maximization by focusing more explicitly on profit rates. This is in line with the classical view that the rate of profit is the key variable driving competition between capitalists. In the economy, capital can freely move between given technologies (lands), and thus appropriate coalitions of farmers can simply compare the maximum rates of profit that can be earned on the various plots of land by the optimal choice of investment and employment. Thus, maximizing the rate of profit implies maximizing the sum of profits on capital advanced, since each farmer is endowed with part of the wage fund and is free to choose the most profitable 'technology', that is, the type of land that she will rent. She will therefore attempt to rent that piece of land (or part of it, through appropriate coalition formation) that maximizes the rate of return on the capital that she can advance.

At this point it is important to stress that the classical notion of competition adopted in this section is different from the neoclassical concept of perfect competition. For example, we do not assume that all agents have zero market power and we allow for some concentration of ownership of resources. In the Ricardian theory of rent, "some concentration of landed property is [compatible] with free competition. And free competition is... perfectly compatible with the existence of rent" (Gehrke and Kurz 2001, 474). The notion of ruthless competition adopted here requires only that competitive forces are strong enough to enforce the law of one price, thus establishing a unique price for labor, a unique rent for each type of land, and a unique rate of profit across the economy. ${ }^{8}$

Furthermore, the assumption that capitalist farmers choose land/labor combinations that maximize the profit rate implies that they obtain the highest total profits. Yet our behavioral assumption is different from assuming that they maximize total profits $f_{i}\left(L_{i}\right)-$ $\rho_{i}-\omega L_{i}$. Formally, the solution to the latter problem would imply $f_{i}^{\prime}\left(L_{i}^{*}\right)=\omega^{*}$ and would therefore not give rise to the classical theory of rent. Theoretically, profit maximization essentially assumes that it is technologies (lands) that are distributed in a fixed way to capitalist

\footnotetext{
8 Yet a significant concentration of land ownership may require an explicit analysis of strategic interdependence between landowners. Salvadori (2004) develops an interesting analysis of Ricardian extensive rent theory in a game-theoretic framework.
} 
farmers, instead of wage-fund endowments (that can be perfectly adjusted to investment needs through appropriate coalition formation).

\subsection{Classical competition}

In this section, we analyze the notion of classical competition and the implications of the classical mode of competition between agents. To be precise, we provide a formal definition of a Classical competitive equilibrium (CCE). This equilibrium notion is conceptually related to Pasinetti's (1960) "market solutions", whereby equalization of profit rates is achieved and capital and labor endowments (rather than the real wage) are given. This type of equilibrium does not represent a stationary state for the economy and there may be forces that lead the equilibrium to move over time. The $\mathrm{CCE}$ is thus a moving equilibrium. The steady state and the basic dynamic laws of motion of the economy are analyzed later in the chapter.

Let a classical economy be defined by a set $\mathcal{K}=\{1, \ldots, n\}$ of lands; a set $\mathcal{F}=\left\{f_{1}(),. \ldots, f_{n}().\right\}$ of production functions satisfying $f_{i}^{\prime}()>$. and $f_{i}^{\prime \prime}()<$.0 , all $i \in \mathcal{K}$; a set $C$ of capitalists; a vector of wage funds $\left(\Omega^{c}\right)_{c \in C}$; and an aggregate labor endowment $\bar{L}>0$. Let $r_{i}^{*}$ be the maximum rate of profit that can be obtained on land of type $i$. Let $\bar{\Omega}=\sum_{c \in C} \Omega^{c}$ denote the aggregate amount of wage-funds in the economy. The CCE can be defined as follows:

Definition 2.1. (Classical competitive equilibrium (CCE)). The classical competitive equilibrium of a classical economy is a nonnegative tuple $\left(\omega_{o},\left\{r_{i}^{*}\right\}_{i=1, \ldots, n},\left\{\rho_{i}^{*}\right\}_{i=1, \ldots, n}\right)$ and the associated actions $\left\{L_{i}^{*}\right\}_{i=1, \ldots, n}$ such that

(i) $L_{i}^{*}$ solves $M P_{1}$ for all $C_{i} \subseteq C$ (profit rate maximization);

(ii) $r_{i}^{*}=r^{*} \geq 0$, for all $i \in \mathcal{K}$ such that $L_{i}^{*}>0$ (profit rate equalization);

(iii) $\omega^{*}=\bar{\Omega} / \bar{L}$ (labor market equilibrium);

(iv) $\sum_{i=1}^{n} L_{i}^{*}=\bar{\Omega} / \omega^{*}$ (capital market equilibrium).

In other words, in equilibrium (i) capitalist farmers choose land/labor combinations that maximize the rate of profit on each type of land. Furthermore, (ii) (ruthless) competitive behavior ensures the equalization of the profit rate earned on every type of land in operation. Condition (iii) incorporates the idea that in the short-run the real wage rate is determined by market forces that equalize labor demand and labor supply. As Kurz and Salvadori $(2002,372)$ note: "In Ricardo's view, demand and supply regulate the 'market' prices of commodities, 
whereas the normal or 'natural' prices are the prices that obtain in a cost-minimizing system of production". ${ }^{9}$

In line with classical assumptions, labor supply is completely inelastic and need not be at its stationary level. As for labor demand, as noted in the previous section, at the beginning of the period, there is a given wage-fund $\bar{\Omega}$, i.e. corn in the possession of capitalist farmers: provided the expected rate of profit is nonnegative, all of this fund is put on the market to buy labor at the given real wage rate. Competition among workers ensures a uniform real wage rate given by an amount of corn $\omega$. For each such $\omega$ the aggregate labor demand of capitalist farmers is $L^{C}=\bar{\Omega} / \omega$. Setting $\bar{L}=L^{C}$ yields condition (iii): the implicit dynamics of the labor market thus leads to a uniform market clearing real wage rate which is independent of rents and profits. The equilibrium level of the real wage is assumed as given for the employment decisions of capitalist farmers.

Finally, condition (iv) guarantees that all of the capitalists' funds advanced for the purchase of labor are actually used. Together with condition (ii), this implies that in equilibrium employment decisions taken in the first stage are also rational ex post. ${ }^{10}$

We are interested in the distribution of income between the three classes determined by the ruthless competition between agents, and in the efficiency of the CCE. In order to explore both issues, we first analyze the key characteristics of the decentralized allocation of resources in the classical economy.

First, Definition 2.1(iii) determines the equilibrium real wage rate. Given the real wage rate, for any given vector of rents the necessary and sufficient conditions for an interior solution of $M P_{1}$ with $L_{i}^{*}>0$ on land $i$ are:

\footnotetext{
${ }^{9}$ In the classical approach, the wage rate is usually taken as given, and equal to some sort of subsistence level. This is going to be the case in the long-run analysis in Sections 2.4 and 2.7. However, even in the short-run, although the wage rate is not given, but changes in order to equilibrate demand and supply, there is an asymmetry in the model and the wage is determined prior to and independently of profits and rents, but it enters the determination of all of them.

${ }^{10}$ Note that condition (iv) implies that at any CCE at least one type of land must be operated, and therefore it must be $\bar{\Omega} / \bar{L}<f_{i}^{\prime}(0)$ for at least one $i \in \mathcal{K}$. Note also that coalition formation is not a problem in equilibrium because farmers are indifferent between various coalitions at a CCE. For by condition (ii) every type of land yields the same rate of profit and by conditions (iii)-(iv) all capital is used.
} 


$$
\begin{aligned}
\rho_{i} & =f_{i}\left(L_{i}^{*}\right)-f_{i}^{\prime}\left(L_{i}^{*}\right) L_{i}^{*} \equiv H_{i}\left(L_{i}^{*}\right), \\
f_{i}^{\prime}\left(L_{i}^{*}\right) L_{i}^{*} & =\left(1+r_{i}^{*}\right) \omega^{*} L_{i}^{*}, \\
H_{i}^{\prime}\left(L_{i}^{*}\right) & =f_{i}^{\prime}\left(L_{i}^{*}\right)-f_{i}^{\prime}\left(L_{i}^{*}\right)-f_{i}^{\prime \prime}\left(L_{i}^{*}\right) L_{i}^{*}=-f_{i}^{\prime \prime}\left(L_{i}^{*}\right) L_{i}^{*}>0 .
\end{aligned}
$$

Therefore, the maximal profit rate on land $i, r_{i}^{*}$, is positive whenever $f_{i}^{\prime}(0)>\omega^{*}$, and $L_{i}^{*}>0$ at the optimum. Instead, if $r_{i}^{*}<0$, then $L_{i}^{*}=0$ and the land is not used. Formally, for all $i \in \mathcal{K}$,

$$
\begin{aligned}
& L_{i}^{*}=H_{i}^{-1}\left(\rho_{i}\right)>0, \quad \text { for } \quad f_{i}^{\prime}(0)>\omega^{*} ; \\
& L_{i}^{*}=0 \text { otherwise. }
\end{aligned}
$$

These conditions actually identify a global maximum, since there is a uniquely determined solution to the first order conditions. ${ }^{11}$

It is important to note that the optimal amount of labor allocated to every piece of land, and therefore the maximum profit rate that can be obtained on such land, depends on the rent $\rho_{i}$. Therefore, for an arbitrary vector of rents, a vector of different optimal rates of profit would emerge from the maximizing behavior of capitalist farmers.

Differential profit rates are a disequilibrium phenomenon: ruthless competition among farmers about land driven by profit rate differentials raises or lowers rents, and it leads in equilibrium to uniform rates of profit. Conditions (i)-(iii) in Definition 2.1 imply: ${ }^{12}$

$$
\begin{aligned}
& r_{i}^{*}=r^{*}=\frac{f_{i}^{\prime}\left(L_{i}^{*}\right) L_{i}^{*}-\omega^{*} L_{i}^{*}}{\omega^{*} L_{i}^{*}} \\
&=\frac{f_{i}^{\prime}\left(L_{i}^{*}\right)}{\omega^{*}}-1 \geq 0, \quad i=1, \ldots, k \leq n, \\
& f_{i}^{\prime}(0) \leq \omega^{*}, \quad k+1 \leq i \leq n ;
\end{aligned}
$$

and

$$
\begin{aligned}
& \rho_{i}^{*}=f_{i}\left(L_{i}^{*}\right)-f^{\prime}\left(L_{i}^{*}\right) L_{i}^{*}, \quad i=1, \ldots, k \leq n, \\
& \rho_{i}^{*}=0, \quad k+1 \leq i \leq n
\end{aligned}
$$

where the number $k$ of utilized lands is a cut off in the fertility hierarchy. If more productive land of type $j \leq k$ were idle, and less fertile soils $l>$

$\overline{11}$ The first condition holds if, for example, all $i \in \mathcal{K}$ production possibilities can be described by a Cobb-Douglas production function $f_{i}\left(L_{i}\right)=\left(L_{i}\right)^{\beta_{i}}$, for some $\beta_{i}>0$.

${ }^{12}$ If for all $i \in \mathcal{K}$ technology is given by $\beta_{i} f\left(L_{i}\right)$, for some $\beta_{i}>0$, equilibrium rents are larger the more productive the land is, but this is not true in general. 
$k$ were used, there would be an offer for land $j$ that would increase rent payments and profits as compared to $l$. Utilized lands thus represent a connected sequence with respect to the fertility ordering. There must therefore be a unique $k \in \mathcal{K}$-which depends on the size $\bar{L}$ of the workforce - which separates utilized from non-utilized land.

To summarize: different profit rates would induce extra rent offers from farmers with low profit rates to those landlords where high profit rates are achieved, thus lowering the latter. This process continues until all profit rates are equalized and rents are endogenously adjusted, such that marginal products are all equal to $\left(1+r^{*}\right) \omega^{*}$, and thus equal to the difference of total corn production minus marginal products times employment on each land that is used. In CCE we thus have an endogenous explanation of wages, rents, the general rate of profit and the number $k \leq n$ of lands that are utilized:

$$
\begin{aligned}
\rho_{i}^{*}, L_{i}^{*} & >0, \quad i=1, \ldots, k, \\
\rho_{i}^{*}, L_{i}^{*} & =0, \quad i=k+1, \ldots, n, \\
r^{*} & =r_{1}^{*}=\ldots=r_{k}^{*} \geq 0, \\
\omega^{*} & =\bar{\Omega} / \bar{L},
\end{aligned}
$$

such that the marginal product of labor is equalized on all land in operation (and irrelevant elsewhere):

$$
\frac{f_{i}^{\prime}\left(L_{i}^{*}\right)-\omega^{*}}{\omega^{*}}=r^{*} \geq 0 \quad \text { i.e. } \quad f_{i}^{\prime}\left(L_{i}^{*}\right)=\left(1+r^{*}\right) \omega^{*} \geq \omega^{*} .
$$

Two points should be made about the classical theory of rent. First, in our model, rent on the marginal land in use is in general positive. The classical view that the marginal land in use pays no rent would require a continuum of types of land and associated production technologies, such as $a \cdot f(L), a \in[0, \bar{a}]$, for some $\bar{a}>0$. Second, the set of idle lands may be empty, depending on technology. It is empty, for example, if $f_{i}^{\prime}(0)=\infty$, for all $i \in \mathcal{K}$. Alternative assumptions on technology produce the result shown in Figure 2.1 below.

\subsection{CCE: Equilibrium and efficiency}

In order to investigate the existence and efficiency of the CCE consider the problem of a social planner that maximizes corn-production from a given amount of labor $\bar{L}$ to be allocated to the different types of land. Formally: 


$$
\begin{gathered}
\max _{L_{1}, \ldots, L_{n}} f_{1}\left(L_{1}\right)+f_{2}\left(L_{2}\right)+\ldots+f_{n}\left(L_{n}\right), \\
\text { subject to } L_{1}+L_{2}+\ldots+L_{n} \leq \bar{L}, \\
L_{i} \geq 0, \quad i=1, \ldots, n .
\end{gathered}
$$

The optimization program $M P_{2}$ is well defined, as it entails the maximization of a continuous (actually, twice differentiable) function on a compact set. By standard economic reasoning, we expect that the maximization of output requires the equalization of marginal products in each type of land operated. Further, if only $k<n$ types of land are operated, then these should be the $k$ most productive ones, as defined in Assumption 2.1. Proposition 2.1 proves that these intuitions are correct, and there is a unique optimal allocation of labor.

Proposition 2.1. Under Assumption 2.1, the social planner's problem $M P$ has a unique optimal solution $\left(L_{1}^{*}, L_{2}^{*}, \ldots, L_{n}^{*}\right)$ such that all labor is used, $\sum_{i} L_{i}^{*}=\bar{L}$, and there is a number $k \leq n$ such that $L_{i}^{*}>0$ for $i \leq k$ and $L_{i}^{*}=0$ for $k+1 \leq i \leq n$. Furthermore, all lands in operation have the same marginal product,

$$
f_{1}^{\prime}\left(L_{1}^{*}\right)=f_{2}^{\prime}\left(L_{2}^{*}\right)=\ldots=f_{k}^{\prime}\left(L_{k}^{*}\right) .
$$

Proof. In order to apply the standard Kuhn-Tucker theorem, we transform $M P_{2}$ into a minimization problem:

$$
\begin{aligned}
\min _{\mathbf{L}=\left(L_{1}, \ldots, L_{n}\right)} \varphi(\mathbf{L}) & \equiv-\sum_{i} f_{i}\left(L_{i}\right), \\
\text { such that } g(\mathbf{L}) & \equiv \sum_{i} L_{i}-\bar{L} \leq 0, \\
L_{i} & \geq 0, \quad i=1, \ldots, n .
\end{aligned}
$$

Existence follows from the fact that the assumptions of the KuhnTucker theorem are satisfied: the feasible set $\mathcal{S} \equiv\left\{\mathbf{L} \in \mathbb{R}_{+}^{n}: g(\mathbf{L}) \leq\right.$ $0\}$ has a non-empty interior; and the functions $g$ and $\varphi$ are convex and continuously differentiable on $\mathcal{S}$. Uniqueness follows from the strict convexity of $\varphi$.

The Lagrange function of this problem $\mathcal{L}: S \times \mathbb{R}_{+} \rightarrow \mathbb{R}$ is

$$
\mathcal{L}(\mathbf{L}, \lambda) \equiv \varphi(\mathbf{L})+\lambda g(\mathbf{L}) .
$$

The Kuhn-Tucker theorem ensures that a nonnegative vector $\mathbf{L}^{*}$ is an optimal solution if and only if there exists $\lambda^{*} \geq 0$ such that the following conditions are fulfilled: 


$$
\begin{aligned}
\frac{\partial \mathcal{L}\left(\mathbf{L}^{*}, \lambda^{*}\right)}{\partial L_{i}} & =-f_{i}^{\prime}\left(L_{i}^{*}\right)+\lambda^{*} \geq 0, \quad \text { for all } i, \\
\frac{\partial \mathcal{L}\left(\mathbf{L}^{*}, \lambda^{*}\right)}{\partial \lambda} & =\sum_{i} L_{i}-\bar{L} \leq 0, \\
\sum_{i} L_{i}^{*} \frac{\partial \mathcal{L}\left(\mathbf{L}^{*}, \lambda^{*}\right)}{\partial L_{i}} & =L_{i}^{*}\left[-f_{i}^{\prime}\left(L_{i}^{*}\right)+\lambda^{*}\right]=0, \\
\lambda^{*} \frac{\partial \mathcal{L}\left(\mathbf{L}^{*}, \lambda^{*}\right)}{\partial \lambda} & =\lambda^{*}\left[\sum_{i} L_{i}-\bar{L}\right]=0 .
\end{aligned}
$$

Since $f_{i}^{\prime}>0$, all $i \in \mathcal{K}$, we have $\lambda^{*}>0$ in condition (2.3) and thus $\sum_{i} L_{i}-\bar{L}=0$ from condition (2.6). Condition (2.5) entails that $-f_{i}^{\prime}\left(L_{i}^{*}\right)+\lambda^{*}=0$ in condition (2.3) if $L_{i}^{*}>0$. Hence all lands on which $L_{i}^{*}>0$ have the same marginal product. It is immediate to show that the constraint qualification holds.

To show that an allocation whereby $\tilde{L}_{i}=0$ and $\tilde{L}_{i+1}>0$ cannot be optimal observe that by Assumption 2.1, the reallocation $L_{i}=\tilde{L}_{i+1}$ and $L_{i+1}=0$ would yield a higher harvest, without violating the constraints.

Lands with higher fertility do not necessarily employ more labor, because the fertility ordering in Assumption 2.1 refers to output levels, not to marginal products. If, however, the marginal products satisfy the same unambiguous ordering for all labor inputs $L>0,{ }^{13}$ then, at the optimal solution to $M P_{2}$ :

$$
L_{1}^{*}>L_{2}^{*}>\ldots>L_{k-1}^{*}>L_{k}^{*} .
$$

Given Proposition 2.1, we can derive our main results on classical general competitive equilibrium. Theorem 2.1 proves the existence of a unique CCE for a large class of economies.

Theorem 2.1. Under Assumption 2.1, for any $(\mathcal{K} ; \mathcal{F} ; C ; \bar{L})$, there is a $\Omega^{*}>0$ such that a unique CCE exists for any $\left(\Omega^{c}\right)_{c \in C}$ such that $\sum_{c \in C} \Omega^{c}=\bar{\Omega} \in\left(0, \Omega^{*}\right)$.

Proof. 1. By Proposition 2.1, for any $(\mathcal{F} ; \bar{L})$, the social planner's problem $M P_{2}$ has a unique optimal solution $\left(L_{1}^{*}, L_{2}^{*}, \ldots, L_{n}^{*}\right)$ such that all labor is used, $\sum_{i} L_{i}^{*}=\bar{L}$, and there is a number $k \leq n$ such that $L_{i}^{*}>0$ for $i \leq k$ and $L_{i}^{*}=0$ for $i \geq k+1$. Furthermore,

\footnotetext{
${ }^{13}$ This is the case, for example, with Cobb-Douglas production functions: $f_{i}\left(L_{i}\right)=L_{i}^{\beta_{i}}$ and $\beta_{1}>\beta_{2}>\ldots>\beta_{n}>0$. A similar assumption on marginal products underlies Figure 2.1 below.
} 
$f_{1}^{\prime}\left(L_{1}^{*}\right)=f_{2}^{\prime}\left(L_{2}^{*}\right)=\ldots=f_{k}^{\prime}\left(L_{k}^{*}\right)$, for all $i \leq k$. We need to prove that there is a $\Omega^{*}>0$ such that for any $\bar{\Omega} \in\left(0, \Omega^{*}\right)$, the social planner's optimum can be decentralized.

2. At the solution to $M P_{2}$, there is a unique well defined $\lambda^{*}>0$. Therefore, for any $\bar{L}>0$, let $\Omega^{*}>0$ be such that $\lambda^{*} \bar{L}=\Omega^{*}$. For any $\bar{\Omega} \in\left(0, \Omega^{*}\right)$, it will be $\lambda^{*} \bar{L}>\bar{\Omega}$. Hence, for any $\bar{\Omega} \in\left(0, \Omega^{*}\right)$, let $\omega=\bar{\Omega} / \bar{L}$ and let $r$ solve $\lambda^{*}=(1+r) \omega$. Note that for any $\bar{\Omega} \in\left(0, \Omega^{*}\right)$, $r>0$. Further, for any $i \in \mathcal{K}$, let $\rho_{i}=f_{i}\left(L_{i}^{*}\right)-f^{\prime}\left(L_{i}^{*}\right) L_{i}^{*}, i=1, \ldots, k \leq$ $n$, and $\rho_{i}=0, k+1 \leq i \leq n$. By the strict concavity of $f_{i}($.$) for all$ $i \in \mathcal{K}$, it follows that $\rho_{i} \geq 0$ for all $i \in \mathcal{K}$. We show that the nonnegative tuple $\left(\omega,\left\{r_{i}\right\}_{i=1, \ldots, n},\left\{\rho_{i}\right\}_{i=1, \ldots, n}\right)$, where $r_{i}=r$, all $i \leq k$, and $r_{i}=0$, all $i \geq k+1$ is a CCE.

3. First, note that since there are no costs and no strategic motives in the formation of coalitions, we need not worry about the initial distribution of wage funds. Second, since $\omega=\bar{\Omega} / \bar{L}$, then Definition 2.1(iii) is satisfied. Third, given $\left(\omega,\left\{\rho_{i}\right\}_{i=1, \ldots, n}\right)$, it is immediate to show that $L_{i}=L_{i}^{*}$, all $i \in \mathcal{K}$, solves $M P_{1}$ for all $C_{i} \subseteq C$ (Definition 2.1(i)) and $r_{i}=r \geq 0$, for all $i \in \mathcal{K}$ such that $L_{i}>0$ (Definition 2.1(ii)). Finally, by construction $\sum_{i=1}^{n} L_{i}=\bar{\Omega} / \omega$, so that Definition 2.1(iv) is also satisfied, which concludes the proof.

Theorem 2.1 proves the existence of a unique equilibrium for economies with any concave technology and any initial labor endowment, provided capital is not abundant, a result in line with classical intuitions. The result is obtained by starting from the social planner's problem and showing that the optimal solution (which always exists and is unique) can be decentralized. Theorem 2.2 establishes the converse result: if a $\mathrm{CCE}$ exists, it is efficient.

Theorem 2.2. If a CCE exists, then it solves $M P_{2}$.

Proof. 1. At any CCE, it must be $\sum_{i=1}^{n} L_{i}=\bar{L}$, by Definition 2.1(iii)(iv). Similarly, by Proposition 2.1, at the solution of $M P$, all labor will be used.

2. Let $k^{C} \leq n$ and $k^{M P} \leq n$ denote the last land in the fertility ordering that is operated, respectively, at a $\mathrm{CCE}$ and at the solution of $M P_{2}$. By Proposition 2.1, we need to prove that $k^{C}=k^{M P}$ and $f_{i}^{\prime}\left(L_{i}^{*}\right)=\lambda^{*}$, all $i \leq k^{C}$.

3. At a CCE, equation (2.1) must hold and therefore it follows that $f_{i}^{\prime}\left(L_{i}^{*}\right)=\left(1+r^{*}\right) \omega^{*}=f_{j}^{\prime}\left(L_{j}^{*}\right)$, for all $i, j \in\left\{1, \ldots, k^{C}\right\}$. Suppose, by way of contradiction, that $\left(1+r^{*}\right) \omega^{*} \neq \lambda^{*}$. If $\left(1+r^{*}\right) \omega^{*}<\lambda^{*}$, then by the strict concavity of $f_{i}($.$) for all i \in \mathcal{K}$, and step 1 of the proof, this implies that $k^{C}<k^{M P}$, and it is possible to reallocate labor from some land 
$i \in\left\{1, \ldots, k^{C}\right\}$ to another land $j \in\left\{k^{C}+1, \ldots, k^{M P}\right\}$, so as to obtain a higher profit rate, which contradicts profit rate maximization.

If $\left(1+r^{*}\right) \omega^{*}>\lambda^{*}$, then by the strict concavity of $f_{i}($.$) for all$ $i \in \mathcal{K}$, and step 1 of the proof, this implies that $k^{C}>k^{M P_{2}}$. However, by Proposition 2.1, $f_{j}^{\prime}(0) \leq \lambda^{*}$, for all $j \in\left\{k^{M P}+1, \ldots, k^{C}\right\}$, a contradiction.

4. By step 2 of the proof, we have $f_{i}^{\prime}\left(L_{i}^{*}\right)=\left(1+r^{*}\right) \omega^{*}=\lambda^{*}$, for all $i \in\left\{1, \ldots, k^{C}\right\}$. Then it is immediate to show that $\sum_{i=1}^{n} L_{i}=\bar{L}$ and $f_{i}^{\prime}(0) \leq \lambda^{*}$ for all $i>k^{M P}$ implies $k^{C}=k^{M P}$, which completes the proof.

By Theorem 2.1, decentralized ruthless competition leads to the optimum corn production that can be obtained from a given amount of labor. This result is interesting per se, but also because a well defined relation can be identified between aggregate labor, on the one hand, and total output and income distribution in the classical economy, on the other hand. Consider again the social planner problem. Note that if $\bar{L}$ changes, so does the solution vector $\mathbf{L}^{*}=\left(L_{1}^{*}, \ldots, L_{n}^{*}\right)$ of $M P_{2}$. In order to make the dependence of $\mathbf{L}^{*}$ on $\bar{L}$ explicit, we write the optimal labor inputs as functions $\phi_{i}$ of $\bar{L}$, i.e. $L_{i}^{*}=\phi_{i}(\bar{L}), i \in \mathcal{K}$. Similarly, we denote total output by $F(\bar{L})$,

$$
Y^{p}=F(\bar{L}) \equiv \sum_{i=1}^{n} f_{i}\left[\phi_{i}(\bar{L})\right]
$$

By Proposition 2.1, $F(\bar{L})$ is well-defined and it summarizes the optimal solution of the social planner's problem. Proposition 2.2 demonstrates that it also has the properties of a standard aggregate production function.

Proposition 2.2. The function $\bar{L} \mapsto F(\bar{L})$ is twice differentiable with

$$
F^{\prime}(\bar{L})>0, \quad F^{\prime \prime}(\bar{L})<0, \quad \text { for all } \bar{L}>0 .
$$

Proof. Differentiability follows from the fact that the production functions $f_{i}$, all $i \in \mathcal{K}$, are strictly concave and twice differentiable, which implies that the functions $\phi_{i}, i \in \mathcal{K}$, are also differentiable. Furthermore, using standard comparative statics results, it is immediate to prove that $\phi_{i}^{\prime} \geq 0, i \in \mathcal{K}$, with strict inequality for $i=1, \ldots, k$. Hence, noting that at the optimal solution $f_{i}^{\prime}(\cdot)=f_{j}^{\prime}(\cdot)$ for all $i, j$ and $\sum_{i=1}^{n} \phi_{i}(\bar{L})=\bar{L}$, we can differentiate $Y^{p}=F(\bar{L})=$ $\sum_{i=1}^{n} f_{i}\left(\phi_{i}(\bar{L})\right)$ with respect to $\bar{L}$ to obtain: 


$$
F^{\prime}(\bar{L})=\sum_{i=1}^{n} f_{i}^{\prime}(\cdot) \phi_{i}^{\prime}(\bar{L})=f_{i}^{\prime}(\cdot) \sum_{i=1}^{n} \phi_{i}^{\prime}(\bar{L})=f_{i}^{\prime}(\cdot), \quad i=1, \ldots, k,
$$

for all $L_{1}, \ldots, L_{n} \geq 0$, and $\sum_{i=1}^{n} L_{i}=\bar{L}$. Differentiating the latter expression with respect to $\bar{L}$ completes the proof.

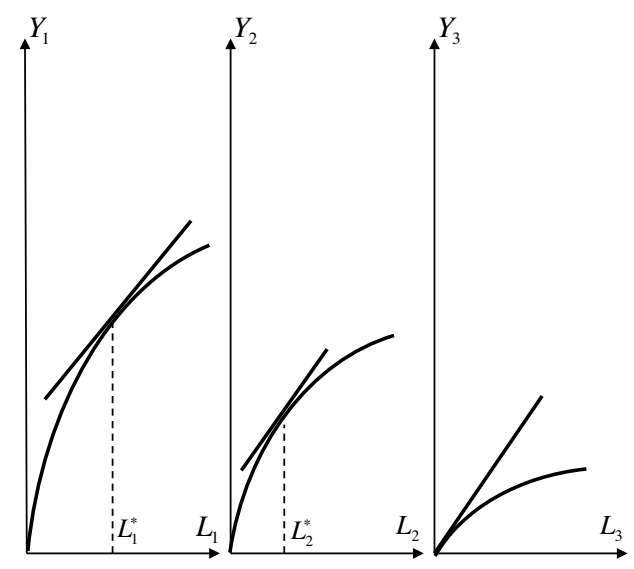

Figure 2.1: The optimal allocation of labor in corn production

Propositions 2.1 and 2.2 are illustrated in Figure 2.1 under the assumption that $f_{i}^{\prime}(0)<\infty$ for some lands $i \in \mathcal{K}$. Starting from $\bar{L}=0$, as aggregate labor increases, it is allocated to the most fertile land first, until marginal fertility falls below $f_{2}^{\prime}(0)$. At this point, the two most fertile lands are utilized on the basis of uniform marginal productivity, until their marginal products fall below $f_{3}^{\prime}(0)$. At this point, the third type of land enters the production of corn, and so on. Figure 2.1 shows the point in which land three comes into operation. All operated lands have equal marginal products and an increase in aggregate labor supply yields a movement along the economy-wide production function. In the economy described in Figure 2.1, an increase in total labor-if allocated according to the marginal productivity rule - would lead both to a more intensive use of land in the first two farms and to a more extensive usage of land, since the third land would also be taken into operation. It is actually a general property of the economy that both the intensive and the extensive use of land can vary with labor supply.

Theorem 2.2 and Proposition 2.2 imply that, contrary to 
Morishima's $(1989,103)$ claim, an aggregate production function exists for the decentralized economy which coincides with that of the social planning problem $M P_{2}$ : given an aggregate supply of labor $\bar{L}$, the total amount of corn produced under ruthless competition can be written as $Y^{p}=F(\bar{L}) \cdot{ }^{14}$

Perhaps more interestingly, by Theorem 2.2 and Proposition 2.2 it is possible to use the aggregate production function to provide an alternative representation of the income distribution between the three classes - workers, capitalist farmers and landlords - determined by decentralized ruthless classical competition. Given aggregate labor supply $\bar{L}$, aggregate output at a CCE is: $Y^{p}=F(\bar{L})$, wages are: $\omega^{*}=\bar{\Omega} / \bar{L}$, and gross profits of farmers are: $\left(1+r^{*}\right) \omega^{*} \bar{L}=F^{\prime}(\bar{L}) \bar{L}$, or in terms of the main state variables

$$
r^{*}=\left(F^{\prime}(\bar{L})-\bar{\Omega} / \bar{L}\right) /(\bar{\Omega} / \bar{L}) .
$$

Therefore the aggregate amount of income accruing to landlords is:

$$
\begin{aligned}
\rho^{*}= & \sum_{i=1}^{n} \rho_{i}^{*}=F(\bar{L})-F^{\prime}(\bar{L}) \bar{L}, \\
& \text { due to } \quad \int_{0}^{\bar{L}} F^{\prime}(L)=F(\bar{L})-F(0)=F(\bar{L}) .
\end{aligned}
$$

The income distribution between workers, capitalist farmers, and landowners at a CCE, is shown in Figure 2.2. An increase in $\bar{L}$ decreases $\omega^{*}$, the equilibrium real wage, while it increases the aggregate rents $\rho^{*}$ paid to landlords out of current production. The effect on the rate of profit is less clear-cut and depends on the elasticity of the aggregate marginal productivity of labor. If the aggregate marginal product does not react much to a change in aggregate labor, then the rate of profit increases. In contrast, the accumulation of wage funds by capitalist farmers leads to an increase in the real wage rate and a decrease in the equilibrium profit rate, while leaving rents unchanged. Interestingly, Figure 2.2 also shows that Quesnay's proposal of a single (proportional) tax on rent would not disturb the equilibrium allocation of the economy.

Although our analysis fundamentally differs from the literature on the canonical classical model - in that it focuses on the decentralized competitive behavior of economic agents - the results on the aggregate distribution of income are in line with the rest of the literature. The

${ }^{14}$ Kurz and Salvadori (1992) also prove the existence of an aggregate production function by assuming a linear technology. 
equation $\rho^{*}=\sum_{i=1}^{n} \rho_{i}^{*}=F(\bar{L})-F^{\prime}(\bar{L}) \bar{L}$ also holds in Pasinetti (1960, 83, fn. 1) and in Samuelson (1978, 1420), for example. In Pasinetti (1960), however, it is a definitional relation and is not explicitly derived from profit (rate) maximization. Samuelson (1978) derives it from the assumption that labor (together with capital) gets its marginal product and total rent is a residual after the payment of labor (and capital). For, given full employment of labor and capital, marginal productivity determines the wage rate (and similarly for the profit rate), whereas in our model the causality is the other way round, consistently with the classical approach. ${ }^{15}$

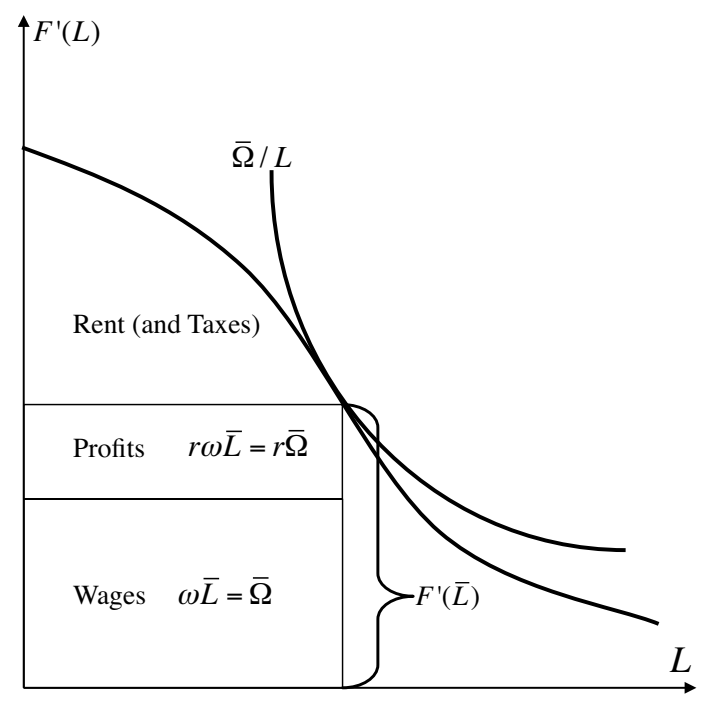

Figure 2.2: Income distribution in a classical corn economy

\subsection{Real wages and Malthusian population dynamics: Efficient pauperization}

These then are the laws by which wages are regulated, and by which the happiness of far the greatest part of every community is governed. Like all other contracts, wages should be left to the fair and free competition of the market, and should never be controlled by the interference of the legislature. The clear and direct tendency of the poor laws is in direct opposition to these obvious principles: it is not,

15 The aggregate incomes accruing to workers and farmers in Pasinetti (1960, 84) are also similar to our model. See also Kurz and Salvadori $(1992,234)$. 
as the legislature benevolently intended, to amend the condition of the poor, but to deteriorate the conditions of both poor and rich... (Ricardo 1951, Ch. V).

The previous analysis focused on temporary, or moving equilibria. In this section we analyze the laws of motion of these equilibria and their convergence in the long-run to a stationary state. From a classical perspective, the key state variables in the economy are the wage-fund, $\Omega$, and labor supply, $L .{ }^{16}$ Despite some relevant differences among the Classicals, we would argue that the widely shared views were that capitalist economies are characterized by an inherent drive to accumulate, and that short-run labor market conditions interacted with long-run population dynamics. In discrete time, the classical assumptions can be formalized as follows: ${ }^{17}$

$$
\begin{aligned}
\Omega_{t+1} & =F^{\prime}\left(L_{t}\right) L_{t} \\
L_{t+1} & =L_{t}+g\left(\frac{\Omega_{t}}{\bar{\omega} L_{t}}-1\right) L_{t}, \quad g(0)=0, g^{\prime}(\cdot)>0
\end{aligned}
$$

where $\Omega_{t} / L_{t}=\omega_{t}$ and $F(\cdot)$ is the aggregate production function derived in Section 2.4. Equation (2.7) describes capital accumulation: farmers' proceeds $F^{\prime}\left(L_{t}\right) L_{t}$ (corn output minus rent payments) at $t$ determine their wage fund at $t+1$. Equation (2.8) describes the dynamics of the labor force, based on Malthus's population law: the growth rate of labor supply is strictly increasing in the real wage rate, and it is zero if and only if the wage rate is at the subsistence level $\bar{\omega}>0$. If the real wage is above (below) subsistence, then labor supply expands (shrinks) due to population increases (decreases). Although the size of the workforce is given in the short-run, in the long-run "the required size of the common workforce is essentially generated alongside the accumulation process" (Kurz and Salvadori 2002, 375).

With a constant wage fund, equation (2.8) implies that the real wage rate cannot be above subsistence forever, since the workforce would become infinitely large and eventually exercise downward pressure on

${ }^{16}$ For the sake of notational simplicity, in this section we denote aggregate wage-funds and the aggregate labor supply simply as $\Omega$ and $L$, respectively.

17 Similar laws of motion can be found in Pasinetti (1960). Samuelson (1978) has a similar law of motion for the workforce but he assumes that changes in the capital stock are driven by the profit rate. Costabile and Rowthorn (1985) adopt a version of Malthus's population law that focuses on earnings, rather than on the real wage. This distinction is less relevant here given full employment. 
real wages and thus on the growth rate of labor supply via the shrinking marginal product of labor. Equation (2.7) strengthens this tendency towards convergence: as shown below, the interaction of the movement in the wage fund with population dynamics leads to a stationary state with the real wage approaching the subsistence level and the size of the workforce settling at a level such that the marginal product of labor is equal to the subsistence wage, and profits are zero. ${ }^{18}$ In other words, the invisible hand allocates total labor supply efficiently to different types of land at each $t$, but it also leads the economy to a stationary state where worker households consume what they produce. ${ }^{19}$

In order to show the monotonic convergence to the stationary state, we slightly reformulate the above dynamics as follows:

$$
\begin{aligned}
\Omega_{t+1}-\Omega_{t} & =F^{\prime}\left(L_{t}\right) L_{t}-\Omega_{t}, \\
L_{t+1}-L_{t} & =g\left(\frac{\Omega_{t}}{\bar{\omega} L_{t}}-1\right) L_{t} .
\end{aligned}
$$

The eigenvalues of the right-hand side Jacobian $J$ of this system at the steady state are equal to those of equations (2.7)-(2.8) minus one. Therefore, if we show that they lie in the interval $(-2,0)$, this implies that the eigenvalues of the original system are real and in the interval $(-1,1)$, yielding the desired result. The point of rest of equations $(2.9)-$ (2.10) — and of equations (2.7)-(2.8) - is:

$$
\omega^{o}=\bar{\omega}, \quad F^{\prime}\left(L^{o}\right)=\bar{\omega}, \quad \Omega^{o}=\bar{\omega} L^{o} .
$$

Hence, the stationary position of the system is entirely determined by the level of subsistence wage $\bar{\omega}$. Further, $r^{o}=\left(F^{\prime}\left(L^{o}\right)-\omega^{o}\right) / \omega^{o}=0,{ }^{20}$ and rents are maximized (as compared to lower levels of employment). The stationary state is unique provided the nonlinear equation $F^{\prime}\left(L^{o}\right)=\bar{\omega}$ has a unique solution, which is true if $F^{\prime}(0)>\bar{\omega}$ and $\bar{\omega}>F^{\prime}(\infty)$, as in Pasinetti (1960), for example.

The right-hand side Jacobian $J$ of the reformulated system is given by:

${ }^{18}$ We are implicitly assuming that capitalist farmers also receive the real wage in their additional role as workers.

19 According to classical authors, however, such a state may be disturbed by a commercial crisis, when the economy comes close to it.

${ }^{20}$ Unlike in Samuelson (1978) where the long-run rate of profit is positive. 


$$
\begin{aligned}
J & =\left[\begin{array}{cc}
-1 & F^{\prime \prime}\left(L^{o}\right) L^{o}+F^{\prime}\left(L^{o}\right) \\
\left(g^{\prime}(\cdot) /\left(\bar{\omega} L^{o}\right)\right) L^{o} & -\left(g^{\prime}(\cdot) \Omega^{o} /\left(\bar{\omega} L^{o-2}\right)\right) L^{o}
\end{array}\right] \\
& =\left[\begin{array}{cc}
-1 & \bar{\omega}+F^{\prime \prime}\left(L^{o}\right) L^{o} \\
g^{\prime}(\cdot) / \bar{\omega} & -g^{\prime}(\cdot)
\end{array}\right] .
\end{aligned}
$$

Since $g^{\prime}(\cdot)>0$, the trace of $J$ is negative. The $\operatorname{determinant,~} \operatorname{det} J$, is:

$$
\begin{aligned}
\operatorname{det} J & =g^{\prime}(\cdot)-g^{\prime}(\cdot)\left(1+F^{\prime \prime}\left(L^{o}\right) L^{o} / \bar{\omega}\right) \\
& \left.=g^{\prime}(\cdot)\left(-F^{\prime \prime}\left(L^{o}\right) L^{o} / \bar{\omega}\right)\right)=g^{\prime}(\cdot)\left(-\epsilon_{F^{\prime}, L}\left(L^{o}\right),\right.
\end{aligned}
$$

where $-\epsilon_{F^{\prime}, L}\left(L^{o}\right) \approx\left(\Delta F^{\prime}\left(L^{o}\right) / F^{\prime}\left(L^{o}\right)\right) /\left(\Delta L^{o} / L^{o}\right)$ is the absolute value of the elasticity of $F^{\prime}(L)$ with respect to $L$. Therefore $\operatorname{det} J>0$, so that the eigenvalues of $J$ at the steady state are either both real and negative, or conjugate complex with negative real parts.

Because population dynamics are in general fairly sluggish, the slope of the function $g$ is fairly close to zero. Setting it equal to zero gives $\operatorname{det} J=0$, implying that the eigenvalues of $J$ are equal to -1 and 0 . Therefore, by continuity, it follows that a sufficiently small increase in the slope of $g$ does not move the eigenvalues out of the interval $[-2,0]$, since they remain real (due to the smaller eigenvalue) and negative (since stability is given). We thus have monotonic convergence to the steady state under normal conditions and for all relevant sizes in the adjustment speed of the workforce population. ${ }^{21}$

The conclusion that profits vanish in the stationary state is unsurprising in this economy, and it was a common view among classical economists, in the absence of technical progress. The next question of interest, therefore, concerns the impact of technological change. Suppose that the economy is initially in the steady state. We assume that innovations increase the marginal products of the different types of land uniformly at all levels of employment. Hence, the economy-wide marginal product of the old stationary level of employment is increased, which raises the wage fund of next year, $\Omega_{1}$ and thus the real wage. This in turn increases next year's population $L_{2}$. If we assume that $-\epsilon_{F^{\prime}, L}<1$, then $F^{\prime}(L) L$ is increasing in $L$ and this increases the wage fund further, and so on, leading to a 'staircase' path towards the new long-run equilibrium with higher population and wage-fund. In other words, this type of technical progress makes it possible for a larger

${ }^{21}$ It is worth noting that the case with fluctuations in the wage fund and the labor supply represents a Classical theory of the cycle which was heavily criticized by Marx. See Flaschel (2009, Ch. 5) for a thorough discussion. 
population to subsist in the long-run. This movement is depicted in Figure 2.3.
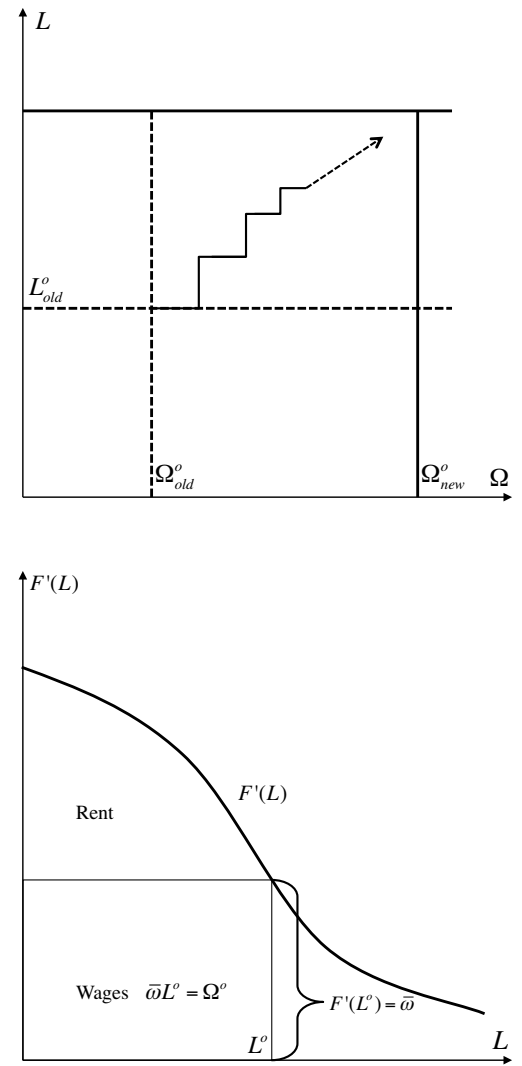

Figure 2.3: Adjustment path towards the steady state after an increase in agricultural productivity or when agricultural taxation is reduced

Interestingly, a similar adjustment process occurs after a change in taxation. Suppose that initially there is a tax on profits at the constant rate $\tau$, giving rise to the following modified dynamical system.

$$
\begin{aligned}
\Omega_{t+1} & =(1-\tau) F^{\prime}\left(L_{t}\right) L_{t} \\
L_{t+1} & =L_{t}+g\left(\frac{\Omega_{t}}{\bar{\omega} L_{t}}-1\right) L_{t} .
\end{aligned}
$$

The steady state population must satisfy $F^{\prime}\left(L^{o}\right)=\bar{\omega} /(1-\tau)$ where 
$L^{o}$ is strictly decreasing in $\tau$, and it is immediate to show that, unlike a tax on rents, taxing profits has an negative impact on the economy in the long-run. Therefore suppose - as originally proposed in François Quesnay's Tableau Économique - that the tax on profits was replaced by a single tax on rents: in our model this is equivalent to deducting $\tau F^{\prime}(L)$ from $\rho$. It is easy to see that this change in taxation policy would have a similar effect as a productivity increase: it would improve the situation in agriculture as shown above, leading to an allocation of labor on lands as in the model without taxation, since a tax on rents (however it is raised) is non-distorting, as long as rents remain positive.

\subsection{Conclusions}

This chapter examines the concept of classical competitive equilibrium (CCE) in order to analyze income distribution and efficiency in a general equilibrium model that captures the process of competition envisaged by the Classicals. In this framework, capital is not conceived of as a physical good tied up in the production process, as in the standard approach. Rather, it is a magnitude of purchasing power that can be freely allocated to different uses and agents allocate capital to the uses that generate the highest rate of profit. In a large class of economies, the CCE exists and is unique, and it determines a unique distribution of income between profits, rents, and wages. The notion of CCE provides a rigorous formal and conceptual framework to analyze growth and distribution in a classical perspective and will be the basis of the following discussion. Further, the planner's optimum allocation is also a social optimum, and an aggregate production function can be derived with the usual properties.

We have also extended our analysis to illustrate classical views on the long-run behavior of the economy. To be specific, we have proved that there exists a unique, stable steady state to which the sequence of temporary CCEs converges. At the steady state, output and rents reach their maximum values while the profit rate falls to zero, and wages fetch the marginal product of labor on lands in operation. Whether this is a desirable outcome of the invisible hand or not depends on the class perspective adopted and on the value of a pristine environment vis-à-vis the maximum sustainable production.

The CCE thus provides a general economic framework that captures the classical views on competitive behavior while encompassing both short-term market equilibrium and long-period dynamics. Thus the CCE represents an ideal starting point for the analysis of relevant economic and policy issues in a classical vein, as well as serving as 
a reference point for more in-depth examinations of pressing issues of growth and distribution in subsequent chapters.

The next chapter continues to develop the classical framework beyond the one-commodity world to broach the longstanding issues posed by the two- or $n$-commodity world.

\subsection{Appendix: The neoclassical theory of the firm and capital accumulation}

This appendix presents a typical neoclassical growth model to highlight the differences between the classical approach detailed above and the neoclassical approach. For this presentation we drop the classical axioms of diminishing returns (in agriculture) and of a Malthusian population dynamics, replacing them with constant returns (in a capital-using manufacturing world) and constant labor force growth, as in the celebrated Solow (1956) model. We show the working of the invisible hand in a one-commodity world where output can be used for consumption and investment purposes. We consider a given distribution of the capital stock $K=K_{1}+\ldots+K_{n}$ over $n$ firms characterized by identical production functions (so that only the distribution of the capital stock - the result of past investment decisions - differentiates firms from one another). The situation shown in Figure 2.1 thus applies here as well with respect to the variable production factor $L_{i}$, with the shape of the short-run production functions $F\left(L_{i}\right)$ being determined by $K_{i}$. Land is no longer considered a factor of production and wages are assumed to be paid ex post. The objective of capitalists is profit rather than profit rate maximization, since each firm is associated with a single technology $K_{i}$.

Following Sargent's (1987, 7-10) presentation of the theory of the firm in a one-good economy under perfect competition, the $i$ firms are assumed to be price-takers and endowed with a given capital stock of size $K_{i}$. If there are no rents and firms maximize profits, the result is analogous to that obtained in Section 2.4. In this scenario, land is no longer involved in production and the residual role of rent is taken up by profits, which are residually determined by the marginal products of labor equalized real wages $\omega=w / p$ in each firm. ${ }^{22}$

Formally, consider an economy with $n$ perfectly competitive firms ( $n$ being large) which produce the same good and utilize the same technology (production function) $F: \mathbb{R}_{+}^{2} \rightarrow \mathbb{R}_{+}$. The output $Y_{i}$ of the $i$-th firm at any moment is

${ }^{22}$ The rate of profit $r$ is now given by $\partial F(\cdot) / \partial K$ in place of $r=(\partial F(\cdot) / \partial L-$ $\omega L) /(\omega L)$. 


$$
Y_{i}=F\left(K_{i}, L_{i}\right), \quad i=1, \ldots, n .
$$

At a given point in time, $K_{i}$ is given because firms cannot change their technology instantaneously, unlike capitalist farmers in the classical model. The production function is characterized by positive though diminishing marginal products of capital and labor, and by a direct dependence of the marginal product of capital on employment - with the converse holding as well:

$$
\frac{\partial F(\cdot)}{\partial K}, \frac{\partial F(\cdot)}{\partial L}>0, \quad \frac{\partial^{2} F(\cdot)}{\partial K^{2}}, \frac{\partial^{2} F(\cdot)}{\partial L^{2}}<0, \quad \frac{\partial F(\cdot)}{\partial K \partial L}=\frac{\partial F(\cdot)}{\partial L \partial K}>0 .
$$

The production function $F$ is assumed to be linearly homogeneous in $K_{i}$ and $L_{i}$, so that by virtue of Euler's theorem we have

$$
Y_{i}=\frac{\partial F\left(K_{i}, L_{i}\right)}{\partial K_{i}} K_{i}+\frac{\partial F\left(K_{i}, L_{i}\right)}{\partial L_{i}} L_{i} .
$$

Additionally, by virtue of the linear homogeneity of $F$ we have for all $\lambda>0$

$$
\frac{\partial F\left(K_{i}, L_{i}\right)}{\partial K_{i}}=\frac{\partial F\left(\lambda K_{i}, \lambda L_{i}\right)}{\partial K_{i}} \quad ; \quad \frac{\partial F\left(K_{i}, L_{i}\right)}{\partial L_{i}}=\frac{\partial F\left(\lambda K_{i}, \lambda L_{i}\right)}{\partial L_{i}},
$$

and setting $\lambda=1 / L_{i}$, we have

$$
\frac{\partial F\left(K_{i}, L_{i}\right)}{\partial K_{i}}=\frac{\partial F\left(K_{i} / L_{i}, 1\right)}{\partial K_{i}} \quad ; \quad \frac{\partial F\left(K_{i}, L_{i}\right)}{\partial L_{i}}=\frac{\partial F\left(K_{i} / L_{i}, 1\right)}{\partial L_{i}},
$$

so that the marginal products depend only on the ratio of capital to labor.

In this one-good economy capital represents the accumulated stock of output. At any moment the capital stock is fixed both for the economy and for each individual firm, consistent with the nature of capital as a state variable. Assuming that capital is fixed for each firm at a given point in time amounts to ruling out the existence of a perfect capital market in which individual firms can trade capital. The absence of such a market may be rationalized by positing that, once in place, capital becomes completely specialized to each firm and therefore useless for other firms.

While firms cannot trade capital, they are able to vary employment instantaneously. Firms operate in a competitive labor market in which at any moment they can hire all the labor they want at the going money wage $w$. The output market is also perfectly competitive, and each firm 
can sell any quantity of output at the given market price, $p$. The $i$-th firm's profits $\Pi_{i}$ are

$$
\Pi_{i}=p F\left(K_{i}, L_{i}\right)-w L_{i}-\delta p K_{i},
$$

where $\delta$ is the rate of physical depreciation of capital. Each firm chooses labor to maximize its profits, taking its capital stock as fixed. The firm's employment is then described by the familiar first-order condition

$$
F_{L_{i}}\left(K_{i}, L_{i}\right)=w / p
$$

which states that the firm equates the marginal product of labor to the real wage. Equation (2.15) describes the firm's demand function for labor which, given $K_{i}$, relates the firm's demand for employment inversely to the real wage. For each firm, equation (2.15) determines the capital-labor ratio, which is identical for all firms since all face a common real wage. Because at any moment the $n$ firms have different amounts of capital $K_{i}, i=1, \ldots, n$, employment varies proportionally with $K_{i}$ across firms.

Our assumption about a uniform production function and firms' profit-maximizing behavior in perfectly competitive markets for output and labor imply that there exists a well defined aggregate production function. Aggregate output $Y$, is given by

$$
Y=\sum_{i=1}^{n} Y_{i}=\sum_{i=1}^{n} F\left(K_{i}, L_{i}\right)
$$

By Euler's theorem we have

$$
Y=\sum_{i=1}^{n} \frac{\partial F\left(K_{i}, L_{i}\right)}{\partial K_{i}} K_{i}+\sum_{i=1}^{n} \frac{\partial F\left(K_{i}, L_{i}\right)}{\partial L_{i}} L_{i} .
$$

But since the marginal products of capital and labor depend only on the capital-labor ratio and since that ratio is the same for all firms, the marginal products of capital and of labor are the same for all firms. Thus, we can write

$$
Y=\frac{\partial F\left(K_{i} / L_{i}, 1\right)}{\partial K} \sum_{i=1}^{n} K_{i}+\frac{\partial F\left(K_{i} / L_{i}, 1\right)}{\partial L} \sum_{i=1}^{n} L_{i} .
$$

Let $K=\sum_{i=1}^{n} K_{i}$ and $L=\sum_{i=1}^{n} L_{i}$. Because the ratios $K_{i} / L_{i}$ are the same for all $n$ firms, they must be equal to the aggregate ratio $K / L$. 
Consequently, we have

$$
Y=\frac{\partial F(K / L, 1)}{\partial K} K+\frac{\partial F(K / L, 1)}{\partial L} L .
$$

By applying Euler's theorem to $F$ equation (2.16) can be written as

$$
Y=F(K, L): \quad y=f(k)=F(K / L, 1), \quad y=Y / L, k=K / L .
$$

Moreover, because $\partial F(\cdot) / \partial L$ and $\partial F(\cdot) / \partial K=f^{\prime}(k)$ equal, respectively, the marginal product of labor and capital for each firm it is legitimate to carry out our analysis in terms of the aggregate production function, equation (2.16), and focus on the equality between the real wage $\omega$ and the partial derivative $\partial Y / \partial L$ :

$$
w / p=\omega=\frac{\partial F(K, L)}{\partial L} K \quad\left[=f(k)-\frac{\partial f(k)}{\partial k} k\right] .
$$

This analysis provides another example of Smith's invisible hand for a modern capitalist economy. For equation (2.16) is a valid description of the aggregate productive relationship between $Y, L$, and $K$ only if the $L_{i}$ are distributed optimally across firms according to equation (2.15), given the fixed distribution of $K$ across firms. And it is possible to derive equation $(2.17)$ by maximizing profits with respect to employment across the economy. ${ }^{23}$

Although the previous analysis holds at a given moment in time, it can be extended to consider the dynamics of the economy. Consistent with the standard neoclassical model, we ignore Malthusian population dynamics and assume an exogenous growth rate of population. Indeed, without loss of generality, we abstract from population growth and, for the sake of comparison with the classical model, technical progress. We also assume classical saving habits, although we allow for $0=s_{w}<$ $s_{c} \leqq 1$.

Given the aggregate production function $Y=F(K, L)$ we investigate the dynamics of capital intensity $k=K / L$, which, noting that $L$ is constant, can be written as:

${ }^{23}$ The concept of an aggregate production function-derived in this section on the basis of a one-good economy - has been heavily criticized from the viewpoint of multisectoral economies and the classical theory of production prices, see in particular Chapter 4. See also Harcourt (1972), Shaikh (1974), Felipe and Fisher (2003), and Rada and Taylor (2006) for further criticisms of aggregate production functions. 


$$
\dot{k}=\dot{K} / L=S / L=s_{c}(Y-\delta K-\omega L) / L=s_{c}(f(k)-\delta k-\omega),
$$

where $S$ denotes aggregate savings and $f(k)=F(K / L, 1)$ is strictly concave and increasing in $k$. In equilibrium, the real wage $\omega$ satisfies equation (2.17). Therefore the law of motion of capital intensity $k$ is:

$$
\begin{aligned}
\dot{k}= & s_{c}\left(f(k)-\delta k-\left(f(k)-f^{\prime}(k) k\right)\right)=s_{c}\left(f^{\prime}(k) k-\delta k\right) \\
& \quad \text { or } \quad \dot{k} / k=s_{c}\left(f^{\prime}(k)-\delta\right) .
\end{aligned}
$$

Because $f^{\prime}(k)>0, f^{\prime \prime}(k)<0$, and assuming $f$ to satisfy the Inada conditions - so that $f^{\prime}(k)$ maps $\mathbb{R}$ onto $\mathbb{R}$ - there is a unique stationary point $k^{o}$ where $f^{\prime}\left(k^{o}\right)=\delta$ that is globally asymptotically stable in $\mathbb{R}_{++}$, since $f^{\prime}(k)-\delta$ is positive to its left and negative to its right. The working of the invisible hand in the short-run leads the economy again to a stationary state in the long-run.

If constant population growth $\left(\dot{L} / L=n_{L}\right)$ is added, the law of motion of $k$ becomes:

$$
\dot{k} / k=s_{c}\left(f^{\prime}(k)-\delta\right)-n_{L}, \quad \text { with } \quad f^{\prime}\left(k^{o}\right)=\delta+n_{L} / s_{c} .
$$

The steady state of these extended dynamics depends on the savings rate out of profits and the question can be posed: which savings rate leads to the highest per capita consumption by workers? Because $f^{\prime}\left(k^{o}\right)$ is a strictly decreasing function of $k^{o}$, for given $n_{L}, \delta$ the highest value of $k^{o}$ occurs when $s_{c}=1$. Furthermore:

$$
\omega^{o}\left(k^{o}\right)=f\left(k^{o}\right)-f^{\prime}\left(k^{o}\right) k^{o}, \quad \text { and } \quad \omega^{o \prime}\left(k^{o}\right)=-f^{\prime \prime}\left(k^{o}\right) k^{o}>0 .
$$

Therefore $s_{c}=1$ also yields the highest level of consumption per worker. "Accumulate, accumulate," as Marx says, is thus in the workers' interest and the socially beneficial role of capitalist firms emerges from the model.

Compare this result with the standard Keynesian assumption of a constant, uniform saving rate $s=s_{c}=s_{w}>0$ out of net income. In this case, the law of motion of $k$ is:

$$
\dot{k}=s(f(k)-\delta k)-n_{L} k
$$

Therefore, again, there exists a unique, globally asymptotically stable steady state, $f\left(k^{o}\right) / k^{o}=\delta+n_{L} / s$, on $\mathbb{R}_{+}$, since the function $f\left(k^{o}\right) / k^{o}$ is strictly decreasing:

$$
h^{\prime}\left(k^{o}\right)=f^{\prime}\left(k^{o}\right) / k^{o}-f\left(k^{o}\right) /\left(k^{o}\right)^{2}=\left(f^{\prime}\left(k^{o}\right)-f\left(k^{o}\right) /\left(k^{o}\right)\right) / k^{o}<0 .
$$


The value of the average rate of savings $s$ that maximizes consumption per person is

$$
c^{o}=f\left(k^{o}\right)-\delta k^{o}-n_{L} k^{o} \rightarrow \max , \quad \text { i.e. } \quad f^{\prime}\left(k^{o}\right)=\delta+n_{L} .
$$

The latter expression implies

$$
\frac{\partial F(K, L)}{\partial K} K / L=f^{\prime}\left(k^{o}\right) k^{o}=\delta+n_{L}=S / L, \quad \text { i.e. } \quad \frac{\partial F(K, L)}{\partial K} K=S .
$$

In this optimal steady state, consumption per head is at its maximum if and only if aggregate savings equals aggregate profits. This "golden rule of accumulation" in the neoclassical theory of growth mirrors the implications of classical savings habits (optimal consumption per head if profits equal savings), even though the assumptions are quite different. The problem for the invisible hand here is to identify the optimal savings rate $s$ that allows for such a result, and how it can be achieved by individual behavior or the government.

Finally, Figure 2.4 illustrates the neoclassical theory of functional income distribution $Y=\omega L+r K$, or $y=\omega+r k$ between workers and capitalists. This figure is based on the equality of the rate of profit and the marginal product of capital $\partial F(\cdot) / \partial K$, i.e. $r=f^{\prime}(k)$, and holds both for temporary equilibrium positions and at the steady state.

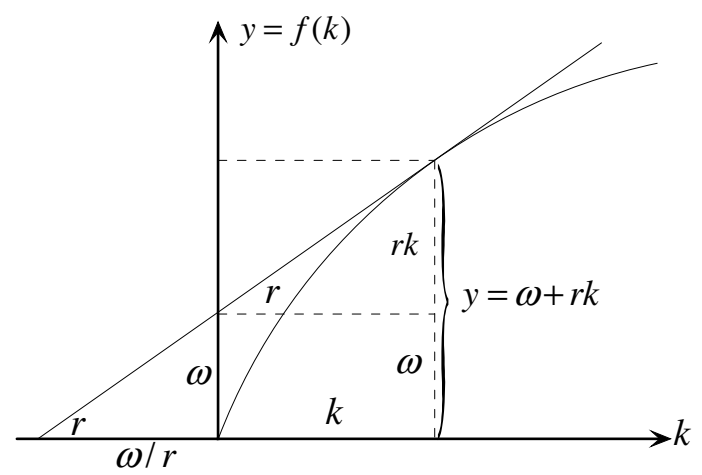

Figure 2.4: Income distribution in the neoclassical manufacturer world

Figure 2.4 shows a negative relationship between capital intensity $k$ and the rate of profit $r$, and a positive relationship between $k$ and the factor price ratio $\omega / r$, both in the steady state and at each moment in time. In the next chapter we show that this result of the neoclassical theory of distribution is not robust: even in a two-commodity case, with one investment good and one pure consumption good, such 
relationships do not hold in general. Therefore, they are more akin to a fable, rather than a good approximation of the relationship between labor and capital, even if only income distribution is considered, and not yet the class conflict between labor and capital in the sphere of production. 


\section{Adam Smith II: The "Invisible Hand" and "Natural Prices"}

\subsection{Restless competition II: Values and natural prices}

In the previous chapter, we analyzed one of the two main themes in Smith's work that are central to our analysis: the increasing division of labor brought about by capitalist relations of production and the effects of decentralized decision-making in competitive markets. In this chapter, we focus on the other main theme of Smith's work, namely the implications of the division of labor, and competition, for commodity exchange and prices.

To be precise, in this chapter we examine the fundamental concepts of values and natural prices, starting from Smith's thought experiment of the "early and rude state of society" in which labor is the only means of production. We rigorously define the notions of total labor costs embodied in commodities, natural prices that guarantee a uniform rate of profit, and labor commanded prices and prove that in this context the "commodity law of exchange" (Foley 2011; Foley and Mohun 2016) holds and there exists a well-defined relationship between the three magnitudes, such that one can conclude that labor values determine relative (natural or labor commanded) prices.

We then extend our analysis to Quesnay's structure of production, as seen in Chapter 1, in which both labor and capital are used to produce commodities. We illustrate a well-known result in price and value theory: in a capitalist economy with produced means of production, in general the commodity law of exchange does not hold and natural prices, labor costs, and labor commanded prices diverge. It is superseded by the "capitalist law of exchange" (Foley 2011; Foley and Mohun 2016), specified as the determination of prices that support an equalized rate of profit. This creates problems to the standard, predictive interpretation of the labor theory of value as a theory of the determination of relative prices, but it does not necessarily mean that the classical-Marxian theory of prices and values is irremediably flawed. 
We deal with value theory in Part II of the book. In this chapter, we show that the classical-Marxian notion of natural, or production prices is theoretically and formally well-defined and can be generalized to an $n$-sector Leontief economy by means of the Perron-Frobenius theory of nonnegative matrices. Therefore, as far as the valuation of different commodities is concerned, the classical theory of restless competition analyzed in the previous chapter provides a theory of long-period natural prices as centers of gravity for market prices. Unlike in the neoclassical approach, it also provides the foundations for an analysis of income distribution as the product of class conflict, rather than merely technological factors. For, technology only determines the parameters within which class conflict takes place, and the set of feasible income distributions - the wage-profit curve. The actual income distribution is the product of social, cultural, institutional as well as broadly economic forces. Indeed, the concepts of natural prices and wage-profit curves are not purely theoretical constructs: in this chapter, we empirically analyze the feasible income distributions in the German economy in 1995 by deriving the relevant wage-profit curves.

\subsection{Values and prices in the early and rude state}

In this chapter, we consider again the two-sector economy of Chapter 1 and investigate the output prices and commodity values with uniform wage and profit rates. Before returning to the Quesnay-type economy (with corn as the only intermediate input in production), we first consider an economy with two produced commodities, goods 1 and 2, which are produced solely by means of labor - as in Smith's early and rude state of society.

Formally, suppose that the result of one production period can be represented ex post (with frozen production conditions as in a stationary state) by

$$
L_{i} \mapsto x_{i},
$$

where $L_{i}, i=1,2$ is the amount of labor spent in the production of the two goods. Good 1 can be interpreted as beaver hunting in Smith's example and good 2 can be interpreted as deer hunting. The average IO structure is $l=\left(l_{1}, l_{2}\right)=\left(L_{1} / x_{1}, L_{2} / x_{2}\right), A=0$, which is again augmented by the assumption of constant returns to scale. At all levels of production, we need labor $l_{1}$ (respectively $l_{2}$ ) to produce one extra unit of good 1 (respectively good 2).

Unlike in Chapter 1, we do not replace the nominal wage $w$ with a given bundle of commodities $p_{1} c$ (and thus do not obtain an augmented IO matrix similar to those in Chapter 1), because labor effort must 
be explicitly considered in order to define labor values. Despite the possible connections to the Quesnay-type economy, we do not consider given subsistence wage baskets, and specify an arbitrary numéraire.

Representation of the technology, the classical concepts of labor embodied, natural prices (or prices of production in Marx's terminology), and labor commanded prices can be defined in Table 3.1 based on a given uniform rate of profit $r$ and a uniform nominal wage rate $w$ :

$$
\begin{array}{llrl}
v_{1} & =l_{1}, & v_{2}=l_{2} & \text { (labor embodied) } \\
p_{1}=(1+r) w l_{1}, & p_{2}=(1+r) w l_{2} & \text { (nominal natural prices) } \\
p_{1 w}=(1+r) l_{1}, & p_{2 w}=(1+r) l_{2} & \text { (labor commanded) }
\end{array}
$$

Table 3.1: Commodity valuations in Smith's "early and rude state"

Observe that Table 3.1 displays the labor commanded natural prices, namely natural prices normalized in terms of the nominal wage. Labor commanded prices may also be measured by dividing actual nominal market prices $p_{i}^{n}$ by the wage rate $w$, which may correspond to differentiated rates of profit.

Valuations in terms of labor embodied, or labor values, are defined by the total amount of labor spent directly in the production of one unit of output. Later on, such total labor costs will also include the labor time spent in the production of the means of production, of their means of production, and so on. In principle, the definition of labor values attempts to be as general as possible and to be applicable to actual economies.

Natural prices, in the early and rude state of society, are based on labor costs $\left(l_{1}, l_{2}\right)$, but they are expressed in terms of wage costs $w l_{i}$, and multiplied by $(1+r)$ to reflect normal profits at the rate $r$. At first sight, it would seem that increases in $w$ or $r$ simply lead to increases in $p_{1}$ and $p_{2}$. However, a robust understanding of classical competition and the relation between prices and distribution is obtained via Ricardo's analysis of the wage-profit relationship below.

Labor commanded prices, finally, are natural prices normalized by the wage rate. They represent the amount of labor that can be bought by one unit of good 1 or 2, respectively. Stated simply, 'a household has to work $p_{w_{1}}$ time units in order to get one unit of commodity 1 
for consumption purposes', which therefore 'commands' this amount of labor. $^{1}$

In the early and rude state of society, these value and price concepts all imply the same relative magnitudes, or relative prices

$$
\frac{v_{1}}{v_{2}}=\frac{p_{1}}{p_{2}}=\frac{p_{1 w}}{p_{2 w}} .
$$

Labor embodied, natural prices, and labor commanded prices thus appear to be just three different ways of looking at one and the same thing. ${ }^{2}$ However, this result does not hold outside of the early and rude state of society, as shall be shown below.

\subsubsection{Conflict in the early and rude state}

At first sight, natural prices as formulated above do not seem to imply anything that looks like a conflict between profit-oriented capitalist households and consumption-oriented worker households. Yet, consider a real consumption basket for workers $c \in \mathbb{R}_{+}$, measured per workhour, and suppose only for simplicity that $c$ is an amount of commodity 1 that fulfills $w=p_{1} c$, i.e. $c=w / p_{1}$, then the equations for natural prices become:

$$
\begin{aligned}
1 & =(1+r) c l_{1}, \\
p_{2} & =(1+r) c l_{2},
\end{aligned}
$$

yielding

$$
r=\frac{1-c l_{1}}{c l_{1}}=\frac{1}{c l_{1}}-1 .
$$

Therefore, there is a strictly negative relationship between the real wage rate and the profit rate earned by capitalists $r$. Ricardo, to whom the formulation of this relationship is often attributed, was later accused, by

\footnotetext{
${ }^{1}$ Prices measured in terms of the wage unit (actual, natural, or competitive prices) are used by Keynes (1936) when he discusses the choice of units for his macroeconomic theory, and in particular, the measurement of output as a whole.

${ }^{2}$ Recent work by Cogliano (2013), Cogliano and Jiang (2016), and Wright (2008) employs an agent-based computational approach to show that the correspondence of relative prices and relative labor values can be achieved in settings of dispersed commodity production and decentralized exchange with many agents. In these approaches, particularly Cogliano (2013) and Cogliano and Jiang (2016), the correspondence between relative prices and relative values holds in a statistical sense, where relative labor values act as the center of gravity for oscillations in relative prices.
} 
economists of his time, of having formulated a theory of class conflict. Today, the relationship between $c$ and $r$ is taken to represent the tradeoffs in the distribution of domestic product, while it is still possible to see the inverse relationship between $c$ and $r$ as the root of Marx's theory of the conflict between labor and capital over the distribution of income. ${ }^{3}$

3.2.2 Values and prices reconsidered

Let us now extend the analysis to the production technology with labor and one intermediate good of Chapter 1 . Consider an average (or linear) IO structure (with exogenous, or stationary output levels $x^{\prime}=\left(x_{1}, x_{2}\right)$ ):

$$
l=\left(l_{1}, l_{2}\right)>0, \quad A=\left[\begin{array}{cc}
a_{11} & a_{12} \\
0 & 0
\end{array}\right] \geq 0, \quad \text { with } \quad y=x-A x \geq 0 .
$$

In other words, instead of a corn economy we are now considering an economy producing corn, and, say iron, where corn enters the production of iron as well as its own production. In this case labor embodied, labor values, or total labor costs, can be written as

$$
\begin{aligned}
& v_{1}=l_{1}+v_{1} a_{11}, \\
& v_{2}=l_{2}+v_{1} a_{12} .
\end{aligned}
$$

In matrix notation for the case of $n$ commodities, labor values are given by $v=l+v A$ and fulfill the aggregate relationship

$$
v y=v(I-A) x=l x=L,
$$

where $L$ is the amount of labor used up in the production of the gross output vector $x$, and it is equal to the total labor cost embodied in the production of net output $y$.

In the case of a productive economy $\left(a_{11}<1\right)$, the solution to equations (3.1)-(3.2) reads:

$$
\left(v_{1}, v_{2}\right)=\left(l_{1}, l_{2}\right)(I-A)^{-1}=\sum_{k=0}^{\infty}\left(l_{1}, l_{2}\right) A^{k}, \quad A^{0}=I
$$

As proved in Chapter 1, the solution vector is uniquely determined and strictly positive if the matrix $A$ is productive $\left(a_{11}<1\right)$ and if $\left(l_{1}, l_{2}\right)>0$ holds. In the current two-commodity case, however, the solution $\left(v_{1}, v_{2}\right)$ can be obtained recursively by solving equation (3.1) with respect to

\footnotetext{
${ }^{3}$ Marx's innovations on the Classical tradition are detailed in Part II.
} 
$v_{1}=l_{1} /\left(1-a_{11}\right)$ and by inserting this result into equation (3.2). Labor values $v_{i}, i=1,2$ must be positive for the concept of embodied labor to be economically meaningful. They can be interpreted as the accumulated sum of labor efforts - at all simultaneously considered stages of production - needed to produce one unit of good 1 or 2, and they do not depend on the distribution of income, unlike the price vectors.

Assuming a uniform nominal wage rate $w$ and a uniform rate of profit $r$, the equations for natural prices in this two-good economy are: ${ }^{4}$

$$
\begin{aligned}
& p_{1}=(1+r)\left(w l_{1}+p_{1} a_{11}\right) . \\
& p_{2}=(1+r)\left(w l_{2}+p_{1} a_{12}\right) .
\end{aligned}
$$

Thus both labor and intermediate inputs are paid for at the beginning of the production period, and represent capital advanced and the basis for the calculation of profit rates:

$$
r=\frac{p_{1}-\left(w l_{1}+p_{1} a_{11}\right)}{w l_{1}+p_{1} a_{11}}=\frac{p_{2}-\left(w l_{2}+p_{1} a_{12}\right)}{w l_{2}+p_{1} a_{12}} .
$$

The solution of equations (3.3)-(3.4) is easy to obtain if one observes that only two of the variables $p_{1}, p_{2}, w, r$ can be determined. We take $w$ as exogenously given and normalize $p_{1}$ by setting it equal to ' 1 ', i.e. we consider the real wage $\omega=w / p_{1}$, then:

$$
\begin{aligned}
1+r & =\frac{1}{\omega l_{1}+a_{11}} \text { or } r=\frac{1-\left(\omega l_{1}+a_{11}\right)}{\omega l_{1}+a_{11}}=\frac{1}{\omega l_{1}+a_{11}}-1, \\
p_{2} & =(1+r(\omega))\left(\omega l_{2}+a_{12}\right) .
\end{aligned}
$$

Note that commodity 1 is basic, commodity 2 is nonbasic, and the profit rate only depends on basic commodities, because $a_{22}=0$ holds in the nonbasic sector.

Because $A$ is productive $r$ and $p_{2}$ are positive for wages rates $\omega$ close to zero. There is, however, a maximum $\omega$ where $r$ is no longer positive, which is given by: $\omega^{\max }=\left(1-a_{11}\right) / l_{1}$. Equation (3.5) therefore defines what is called a wage-profit curve $r(\omega)$ in the literature. It is easy to show that

\footnotetext{
${ }^{4}$ None of our conclusions depend on wages being paid ex ante. Ex post payment of wages is considered in Chapter 4.
} 


$$
r^{\prime}(\omega)=-\frac{l_{1}}{\left(\omega l_{1}+a_{11}\right)^{2}}<0, \quad r^{\prime \prime}(\omega)=\frac{2 l_{1}^{2}}{\left(\omega l_{1}+a_{11}\right)^{3}}>0
$$

and that the wage-profit curve is always decreasing and strictly convex with $r^{\max }=\frac{1-a_{11}}{a_{11}}$ and $\omega^{\max }=\frac{1-a_{11}}{l_{1}}$ as depicted in Figure 3.1.

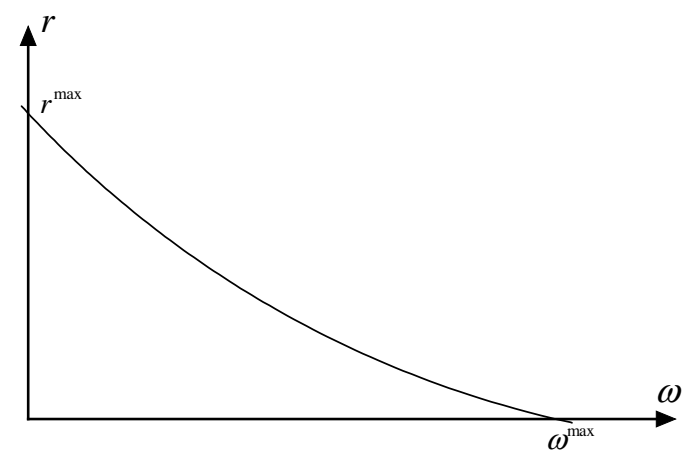

Figure 3.1: The wage-profit curve and the conflict over income distribution with manufactured means of production

Ricardo's theory of conflict over income distribution thus holds: real wages can only be increased through a reduction in the profit rate earned by capitalists. Since good 1 is the physical input in the production of goods 1 and 2, and the consumption good (corn) of the workforce (and thus good 2 is a luxury good not consumed by workers), our normalization $p_{1}=1$ implies that $w$ is the real wage or 'corn' wage since $\omega=w / p_{1}$. If $w=\omega^{\max }$, the surplus $1-a_{11}$ obtained in the production of corn is fully paid out as wages, while it is paid out entirely as profit at the rate $r^{\max }$ where $w=0$ holds. Then there is a strictly convex, inverse relationship between corn wages and the rate of profit. ${ }^{5}$ Therefore, in real terms there is no basis for Smith's proposed 'adding up' theory of prices, but rather a subdivision of the surplus $1-a_{11}$ between capital and labor that does not vary linearly with changes in the wage rate $\omega=w / p_{1}$.

The price of the luxury good is $p_{2}(\omega)=(1+r(\omega))\left(\omega l_{2}+a_{12}\right)$ and SO:

${ }^{5}$ The wage-profit curve need not be strictly convex in more general economies. 


$$
\begin{aligned}
p_{2}^{\prime}(\omega) & =-\frac{l_{1}}{\left(\omega l_{1}+a_{11}\right)^{2}}\left(\omega l_{2}+a_{12}\right)+\frac{1}{\omega l_{1}+a_{11}} l_{2} \\
& =\frac{a_{11} l_{2}-a_{12} l_{1}}{\left(\omega l_{1}+a_{11}\right)^{2}} \\
& =\frac{a_{11} / l_{1}-a_{12} / l_{2}}{l_{1} l_{2}\left(\omega l_{1}+a_{11}\right)^{2}} \\
& =\frac{k_{1}-k_{2}}{l_{1} l_{2}\left(\omega l_{1}+a_{11}\right)^{2}},
\end{aligned}
$$

where $k_{1}=a_{11} / l_{1}$ and $k_{2}=a_{12} / l_{2}$ represent the capital intensities of the two processes $\left(k_{i}=K_{i} / L_{i}=a_{1 i} \bar{x}_{i} /\left(l_{i} \bar{x}_{i}\right)\right)$. Therefore $p_{2}$ will increase (decrease) with the wage rate $\omega$ if and only if $k_{1}>k_{2}\left(k_{1}<k_{2}\right)$, and will not depend on $\omega$ if and only if $k_{1}=k_{2} \cdot{ }^{6}$ Further, differentiating $p_{2}(\omega)$ twice it is easy to see that $p_{2}(\omega)$ is strictly concave (convex) if and only if $k_{1}>k_{2}\left(k_{1}<k_{2}\right)$. Labor values thus cannot provide a theory of long-period prices (except when either $r=0$ or $k_{1}=k_{2}$ holds) and this indeed should not be their role in national accounting and economic theorizing, as we argue throughout the book.

Let us now consider the normalization of natural prices in terms of labor commanded or prices measured in terms of the wage. Letting $w=1$ :

$$
\begin{aligned}
& p_{1, w}=(1+r)\left(l_{1}+p_{1, w} a_{11}\right), \\
& p_{2, w}=(1+r)\left(l_{2}+p_{1, w} a_{12}\right),
\end{aligned}
$$

or, in matrix notation,

$$
p_{w}\left[\frac{1}{1+r} I-A\right]=l=\left(l_{1}, l_{2}\right), \quad p_{w}=\left(p_{1, w}, p_{2, w}\right)
$$

The solution to this matrix equation is:

$$
p_{w}=\phi\left[\begin{array}{c}
1 / w \\
p_{2}(\omega) / w
\end{array}\right],
$$

where $p_{2}(\omega)=(1+r(\omega))\left(\omega l_{2}+a_{12}\right)$ is given by equation (3.6) and $\phi$ is a constant scalar. Labor commanded prices are economically meaningful for all $w \in\left(0, w^{\max }\right]$, but in order to solve the system with respect to natural prices we take $r$ as exogenous (and $w=1$ ) to obtain

\footnotetext{
${ }^{6}$ It is possible for these monotonicity results to hold in higher dimensional IO tables. See the discussion of Table 3.5 below.
} 


$$
\begin{aligned}
p_{w} & =l\left[\frac{1}{1+r} I-A\right]^{-1}=l\left[\frac{1}{1+r}(I-(1+r) A)\right]^{-1} \\
& =(1+r) l[I-(1+r) A]^{-1} \\
& =(1+r) l\left(I+(1+r) A+(1+r)^{2} A^{2}+(1+r)^{3} A^{3} \ldots\right) \\
& =(1+r) l \sum_{k=0}^{\infty}((1+r) A)^{k} \\
& =(1+r) l\left[\begin{array}{cc}
\frac{1}{1-(1+r) a_{11}} & \frac{(1+r) a_{12}}{1-(1+r) a_{11}} \\
0 & 0
\end{array}\right]>0 .
\end{aligned}
$$

This follows from Chapter 1 , as long as $r<r^{\max }$, because $I-$ $(1+r) A$ is profitable and productive, and therefore nonnegatively invertible, i.e. the Leontief-inverse exists and is representable by means of the above geometric matrix series. These results also show that the following equation systems are equivalent

$$
p_{w}(I-(1+r) A)=(1+r) l ; \quad p_{w}\left[\frac{I}{1+r}-A\right]=l .
$$

Proposition 3.1. Assume that the matrix $A$ is productive. Then

(1) If $r=0$ and $\omega=\omega^{\max },\left(p_{1, w}, p_{2, w}\right)=\left(v_{1}, v_{2}\right)$.

(2) If $r>0$ then $p_{w}>v>0$, i.e., labor commanded prices are strictly larger than labor values.

(3) $p_{2, w} / p_{1, w}$ is strictly increasing, decreasing, or constant if and only if $k_{1}>k_{2}, k_{1}<k_{2}$, or $k_{1}=k_{2}$, respectively.

Proof. Assertion (1) is immediate. Assertion (2) follows from:

$p_{w}=(1+r) l \sum_{k=0}^{\infty}((1+r) A)^{k}>l \sum_{k=0}^{\infty} A^{k}=l\left[\begin{array}{cc}\frac{1}{1-a_{11}} & \frac{a_{12}}{1-a_{11}} \\ 0 & 0\end{array}\right]=v>0$

since $l>0$. Assertion (3) follows from the previous analysis, noting that $p_{2, w} / p_{1, w}=p_{2} / p_{1}=p_{2}(\omega)$ for $p_{1}=1$.

Proposition 3.1 has various implications. First, natural prices in terms of labor commanded can command more labor per unit of commodity than is embodied in this commodity if the profit rate is positive. Second, the normalized prices $p_{1, w}, p_{2, w}$ strictly increase with the profit rate, the faster increase taking place in the sector $i$ with the higher composition $k_{i}=a_{1 i} / l_{i}$. Third, relative natural prices $p_{2} / p_{1}$ are 
identical to relative values $v_{2} / v_{1}$-independent of their normalizationif and only if the capital-labor ratio $k_{i}=a_{1 i} / l_{i}$ is the same in both sectors $i=1,2$. This follows from the fact that labor values are equal to natural prices in the case of zero profits $(r=0)$ and relative prices do not change in the case of a uniform capital intensity across processes.

In summary, embodied labor values and natural prices are not proportional and thus the former are not centers of gravity around which market prices oscillate. However, labor embodied is always smaller than labor commanded for a given commodity (if profits are positive), suggesting that labor values provide a lower bound for prices. Adam Smith did not clearly distinguish natural prices (in terms of wages or any other numéraire) from labor values or the labor time embodied in commodities. As argued in the next chapter, the same holds in David Ricardo's analysis, though to a lesser extent. While Ricardo clearly distinguished values and prices, he could not solve the problem of the choice of a suitable numéraire (labor in the case of labor values) that would not vary over time, i.e. an 'invariable standard' of value. Such an invariable standard would allow one to understand the causes behind changes in all nominal prices throughout an economy. As Sraffa (1970) notes in the introduction to Ricardo's Principles, the search for an invariable standard of value occupied a great deal of Ricardo's time, yet was never completed.

In the next section we generalize labor values, natural prices, and their renormalization in terms of labor commanded using powerful mathematical theorems on the dominant eigenvalue of nonnegative and square IO matrices of dimension $n$.

\subsection{The multi-sector economy. Economic propositions and mathematical theorems}

In this section, we consider a nonnegative, square and productive $n \times n$ intermediate-input-output matrix $A=\left(a_{i j}\right) \geq 0$. We later add a strictly positive vector of labor inputs $l=\left(l_{1}, \ldots, l_{n}\right)$, which together with the matrix $A$ characterizes the (average) inputs of the $n$ activities per unit of output. Whenever convenient we will augment the matrix $A$ by the $n \times n$ matrix $c l=\left(c_{i} l_{j}\right)$ which then adds the reference basket $c^{\prime}=$ $\left(c_{1}, \ldots, c_{n}\right)$ of workers weighted by the direct labor input $l_{j}$ to the intermediate inputs of each activity $j$. We denote the resulting $n \times n$ matrix by $A^{c}$. If $\omega=w / p c$ denotes the real wage in terms of the basket $c$ we denote the resulting augmented matrix by $A^{c}(\omega)=A+\omega c l$ which then varies linearly with the real wage $\omega$. All quantity expressions are considered as given magnitudes. 
From the mathematical point of view, but motivated by economic analysis long before the theorems of this section became available, we investigate the eigenvalue problems

$p A=\lambda(A) p, \quad \lambda(A) \geqq 0, \quad p \geq 0, \quad A x=\lambda(A) x, \quad \lambda(A) \geqq 0, \quad x \geq 0$.

From the economic point of view we examine the balanced situations

$$
\begin{aligned}
& (1+r) p A=p, \quad(1+r) \geqq 0, \quad p \geq 0, \\
& (1+g) A x=x, \quad(1+g) \geqq 0, \quad x \geq 0 .
\end{aligned}
$$

In other words, we are looking for nonnegative (non-zero) price systems and activity vectors such that the vector of production cost $p A$ and the vector of intermediate input consumption $A x$ are proportional to the price system $p$ and the activity vector $x$, respectively. This is again the question of uniform profitability with the rate of profit formula $1+r=1 / \lambda(A)$, and of balanced expansion with the rate of growth formula $1+g=1 / \lambda(A)$, assuming $\lambda(A)>0$. Note that both $r$ and $g$ can be negative, but are assumed to be larger than -1 . We focus on the price equation, but observe that all results can be extended to the activity equation, provided a linear technology is assumed. The analysis of (average) price systems is independent of this extra assumption. Although we focus on $A$, similar results can be obtained for the eigenvalues of $A^{c}, A^{c}(\omega)$, and other IO matrices.

The eigenvalues of $A$ are obtained from the so-called characteristic equation $\operatorname{det}(\lambda I-A)=0$, with solutions in the complex plane in general. In the case of a nonnegative matrix economic intuition suggests that solutions of the above problems should exist. At the beginning of the last century, mathematicians established that this intuition is correct and that there exists a uniquely determined eigenvalue $\lambda(A)$, which dominates any other eigenvalue $\lambda$ in modulus $\lambda(A) \geqq\|\lambda\|$ (but may be a multiple root of the above characteristic equation). This is called the dominant root. Let $\Phi(A)$ be the set of $r$ such that the matrix $I-(1+r) A$ is nonnegatively invertible, Proposition 3.2 then relates our statements on the Leontief-inverse in the previous section to dominant roots. $^{7}$

\section{Proposition 3.2.}

(1) The set $\Phi(A)$ is given by $\left(-1, r^{\max }\right)$ with $r^{\max }>-1$.

(2) For the above $r^{\max }$ there is some $p \geq 0$ such that $\left(1+r^{\max }\right) p A=p$.

\footnotetext{
7 The presentation and the proofs of all the theorems considered in this section can be found in Nikaido (1968).
} 
Formally, the definition of $r^{\max }$ amounts to the consideration of eigenvalues $\lambda=1 /\left(1+r^{\max }\right)$. Economically, we are, however, asking whether there is a maximum uniform profit rate for the economy if intermediate inputs are the only costs. This profit rate indeed exists and is well defined. Because the IO matrix $A$ satisfies the Hawkins-Simon conditions stated in Proposition 1.2 (i.e. it is productive and profitable) it has a nonnegative Leontief-inverse. Therefore, by Proposition 3.2, there exists a maximum positive profit rate $r^{\max }=1 / \lambda(A)-1$, i.e. the matrix $M=I-(1+r) A$ is nonnegatively invertible for all rates of profit $r \in\left[0, r^{\max }\right)$.

We now add the vector $l=\left(l_{1}, \ldots, l_{n}\right)>0$ of labor inputs used in the $n$ sectors.

\section{Proposition 3.3.}

(1) There is a unique, strictly positive vector $v=\left(v_{1}, \ldots, v_{n}\right) \in \mathbb{R}^{n}$ which fulfills

$$
v=l+v A,
$$

called the vector of embodied labor, or briefly, the vector of labor values.

(2) For profit rates $r<r^{\max }$ there is a unique, strictly positive vector $p=\left(p_{1}, \ldots, p_{n}\right) \in \mathbb{R}^{n}$ which fulfills

$$
p=(1+r)[\omega l+p A]=(1+r) p[\omega c l+p A],
$$

for given positive $\omega \in \mathbb{R}$ measured in terms of the consumption basket $c(p c=1, \omega=w / p c)$. This is the vector of natural prices.

(3) There is a unique, strictly positive vector $p_{w}=\left(\overline{p_{1, w}, \ldots, p_{n, w}}\right) \in$ $\mathbb{R}^{n}$ which fulfills

$$
p_{w}=(1+r)\left[l+p_{w} A\right]
$$

this is the vector of labor commanded prices, i.e. measured in terms of the wage unit.

(4) If $r>0$, then there always holds: $p_{w}>v$, i.e., $p_{i, w}>v_{i}$ for all $i=1, \ldots, n$.

All of the assertions of Proposition 3.3 follow from the fact that for all $r<r^{\max }$ the sequence of matrix multipliers

$(I-(1+r) A)^{-1}=I+(1+r) A+(1+r)^{2} A^{2}+(1+r)^{3} A^{3} \cdots \geq(I-A)^{-1}$

is well defined and converges, and noting that $(1+r) l$ and $v=l(I-A)^{-1}$ are strictly larger than $l$. Observe that labor values are equal to labor 
commanded prices when $r=0$ and that they are proportional to labor values for all admissible $r>0$ if $v A$ is proportional to $l$, i.e. if the ratio of labor indirectly embodied in the means of production to direct labor is the same in all $n$ sectors. In fact, in this case $\phi v$ for some scalar $\phi>0$ solves equation (3.9) since $\phi v$ for positive $p h i$ is then a solution of the type in Proposition 3.2.

By Proposition 3.3, the labor $v_{i}$ embodied in commodities $i=1, \ldots, n$ (or the total labor costs needed for producing these commodities), is uniquely determined and always positive. Due to the multiplier formula it is the direct labor $l_{i}$ used in the production of $i$ plus the direct labor used in the production of the intermediate inputs $l A_{\star i}$ plus the direct labor used in the production of the intermediate inputs used to produce the intermediate inputs $l A\left(A_{\star i}\right)=l A^{2} e_{i}$ and so on. The concept of labor embodied or the labor values of commodities is therefore well defined and economically meaningful.

Proposition 3.3 further suggests that the concept of labor values yields interesting insights. For it states that, due to the inclusion of profits in the definition of labor commanded, one obtains more labor in exchange for a commodity than is embodied in it. This insight should hold even more generally; dividing the actual price, not only the natural price, by the wage rate $w$ should lead to a magnitude that is larger than the labor embodied in the commodity or its total cost of production in terms of labor (provided profits are positive in each sector). A first empirically important assertion is that we can easily get an upper estimate for labor values by dividing actual prices by the money wage rate.

Next, we state the Frobenius-Perron theorem for nonnegative square matrices that identifies many intuitively plausible properties of the dominant eigenvalue of such matrices. According to the fundamental theorem of algebra, the roots of the polynomial $\operatorname{det}(\lambda I-A)$ of degree $n$ in the variable $\lambda$ are always $n$ when solved in the complex plane. The generally complex-valued eigenvectors corresponding to these eigenvalues may not reach the same dimensionality if the characteristic polynomial has multiple roots. There are thus a number of difficulties when arbitrary matrices $A$ and all of their eigenvalues and eigenvectors are considered. In the case of square semi-positive matrices however we can - in close correspondence to the classical considerations of natural prices and balanced growth paths - focus on the dominant roots and formulate the following set of propositions which are quite intuitive once the dominant roots are transformed into the uniform rate of profit they imply. 
Theorem 3.1. Let $A$ be nonnegative and square. Then:

(1) A has a nonnegative eigenvalue. A nonnegative eigenvector is associated with the largest nonnegative eigenvalue $\lambda$ denoted by $\lambda(A)$.

(2) $I-(1+r) A$ is nonnegatively invertible if and only if $r<1 / \lambda(A)-1$.

(3) If $A x \geqq \lambda x$ for a real number $\lambda$ and a semi-positive vector $x \geq 0$, then $\lambda(A) \geqq \lambda$.

(4) $\lambda(A) \geqq\|\lambda\|=\sqrt{a^{2}+b^{2}}$ for any eigenvalue $\lambda=a+$ bi of $A$.

There are further useful properties of the dominant root $\lambda(A)$.

Theorem 3.2. Let $A$ be nonnegative. Then:

(1) $\lambda(A)=\lambda\left(A^{\prime}\right)$.

(2) $\lambda(\phi A)=\phi \lambda(A)$ for $\phi \geqq 0$.

(3) $\lambda\left(A^{k}\right)=(\lambda(A))^{k}$ for any positive integer $k$.

(4) $\lambda(A) \geqq \lambda(B)$ if $A \geqq B \geq 0$.

(5) $\lambda(A) \geqq \lambda(B)$ for any principal minor matrix $B$ of $A$.

(6) $\lambda(A)=0$ if and only if $A^{k}=0$ for some positive integer $k$.

These results allow us to extend some properties of the wage-profit frontier in Figure 3.1 to a multi-sector economy. To show this consider the matrix $A^{c}(\omega)=A+\omega c l$ : using the normalization rule $p c=1$ for natural prices $p$ we have the equation system:

$p=(1+r) p A^{c}(\omega)=(1+r) p[A+\omega c l]=(1+r)[p A+\omega p c l]=(1+r)[p A+\omega l]$,

where $\omega$ is the real wage in terms of the wage basket $c$ and determines the scale of consumption of this basket that workers can realize.

We know that $r=r^{\max }$ whenever $\omega=0$, and expect that the normalization $p c=1$ defines a wage-profit curve that is decreasing in $r$. This is shown with the help of Theorem 3.2 as follows. Recall that $\omega c l=\omega\left(c l_{1}, \ldots, c l_{n}\right)$ is a nonnegative $n \times n$ matrix describing the consumption of workers of the wage basket $c$ for each production sector $j,\left(c l_{j}\right)$. Since the augmented matrix $A^{c}(\omega)=A+\omega c l$ is increasing in $\omega$, then by Theorem 3.2(4), $\lambda\left(A^{c}(\omega)\right)$ is increasing with $\omega$ and $r=$ $1 / \lambda(A(\omega))-1$ therefore decreases as $\omega$ increases.

The following result strengthens the insights of Theorems 3.1 and 3.2 by focusing on indecomposable matrices.

Theorem 3.3. Let $A$ be nonnegative and indecomposable. Then:

(1) Any nonnegative eigenvector associated with $\lambda(A)$ is positive. Moreover $\lambda(A)>0$.

(2) The eigenvector of $A$ associated with $\lambda(A)$ is unique up to scalar multiplication. 
(3) $\lambda(A)$ is a simple root of its characteristic equation.

(4) If $A \geq B \geq 0$, and one of $A$ or $B$ is indecomposable, then $\lambda(A)>$ $\lambda(B)$.

In other words, if $A$ is indecomposable, we have positive profit factors $1+r$ and positive and unique relative prices $p$. Furthermore, considering $A^{c}(\omega)$, the wage-profit frontier would be strictly decreasing in $\omega$.

In two-good economies the eigenvalues of the matrix $A$ are:

$$
\begin{aligned}
\lambda_{1,2} & =\operatorname{tr} A / 2 \pm \sqrt{(\operatorname{tr} A)^{2} / 4-\operatorname{det} A} \\
& =\frac{a_{11}+a_{22}}{2} \pm \sqrt{\left(\frac{a_{11}-a_{22}}{2}\right)^{2}+a_{12} a_{21}} .
\end{aligned}
$$

Only real solutions obtain in this case, since at least one root must be real. In the case of only one basic commodity $\left(a_{21}=0\right)$ this in particular gives

$\lambda_{1,2}=\frac{a_{11}+a_{22}}{2} \pm \sqrt{\left(\frac{a_{11}+a_{22}}{2}\right)^{2}-a_{11} a_{22}}=\frac{a_{11}+a_{22}}{2} \pm \frac{\left\|a_{11}-a_{22}\right\|}{2}$.

If $a_{11}>a_{22}$ then $\lambda_{1}=a_{11}>a_{22}$, if $a_{11}<a_{22}$ then $\lambda_{2}=a_{11}<a_{22}$ with right and left hand eigenvectors $x^{\prime}=(1,0), p=\left(1, a_{12} /\left(a_{11}-a_{22}\right)\right)$ and $x^{\prime}=\left(1, a_{11} /\left(a_{22}-a_{11}\right)\right), p=(0,1)$, respectively. In the even simpler case where $a_{22}=0$ also, we get $\lambda(A)=a_{11}$.

It is not implausible to assume that the matrix $A^{c}(\omega)=A+\omega c l$ is indecomposable, since this amounts to assuming that each good is used directly or indirectly for the production of intermediate goods or consumption goods of workers (so that not too many goods are excluded from consideration). For indecomposable $A^{c}(\omega)=A+\omega c l$ Theorem 3.3 implies $\lambda\left(A^{c}(\omega)\right)$ is strictly increasing with $\omega$-and thus $r=1 / \lambda\left(A^{c}(\omega)\right)-1$ strictly decreasing - in $\omega$. Furthermore, there is a unique value $\omega^{\max }$ where

$$
\lambda\left(A^{c}\left(\omega^{\max }\right)\right)=1, \quad \text { i.e., } \quad r\left(\omega^{\max }\right)=0 .
$$

Thus, in the multi-sector economy there is a wage-profit curve as shown in Figure 2.1 (there measured in terms of commodity 1 in place of the basket $c$ ), but one that need not be convex (or concave, see the next chapter) and that is defined by:

$$
p=(1+r)[\omega l+p A], \quad p c=1, \quad \omega \in\left[0, \omega^{\max }\right],
$$

for some maximum real wage $\omega^{\max } \in \mathbb{R}$. 
Finally, we note that in the case of decomposable matrices we assume that at least one basic commodity exists and that an IO matrix can be reduced to its canonical form in terms of basic and nonbasic commodities (represented by the matrices $A_{11}, A_{22}$ ):

$$
\left[\begin{array}{cc}
A_{11} & A_{12} \\
0 & A_{22}
\end{array}\right]: \quad \lambda(A)=\lambda\left(A_{11}\right) \geqq \lambda\left(A_{22}\right),
$$

where $A_{11}$ is a primitive matrix (no cyclical hierarchies in the ordering of industries). ${ }^{8}$ Note that the matrix $A_{22}$ itself can be structured as we have structured the matrix $A$, and so on. Note further that the assumption of the existence of basic commodities implies that all columns in the matrix $A_{12}$ must be semi-positive, but that $A_{22}$ may consist of zeros throughout. Matrices with $\lambda\left(A_{11}\right)<\lambda\left(A_{22}\right)$ i.e. matrices where nonbasic commodities dominate the basic ones, since their maximum profit rate restricts the profit rate of the basic sector, have no real economic meaning, as the Frobenius-Perron theorems suggest (the prices of the basic commodities would be zero in general), and are therefore ignored in the following analysis.

Therefore, the maximum rate of profit supported by the basic sector is at most equal to the one of the sector of nonbasics ${ }^{9}$ implying that the Leontief-inverse for nonbasics is always well-defined if the same holds for the sector of basic commodities. In this case the Leontief-inverse of the matrix $(1+r) A$ is:

$$
\begin{aligned}
& (I-(1+r) A)^{-1}= \\
& {\left[\begin{array}{cc}
\left(I-(1+r) A_{11}\right)^{-1} & \left(I-(1+r) A_{11}\right)^{-1} A_{12}\left(I-(1+r) A_{22}\right)^{-1} \\
0 & \left(I-(1+r) A_{22}\right)^{-1}
\end{array}\right] .}
\end{aligned}
$$

In the next section we provide some examples of empirical wageprofit curves that illustrate the properties discussed above.

\subsection{Empirical examples of wage-profit curves}

Let $A$ be a productive matrix and let $l>0$. Then consider the natural price equations with wages paid either ex ante or ex post:

\footnotetext{
${ }^{8}$ Recall that a matrix with these properties is called a Sraffa matrix as defined in Chapter 1.

9 This is quite a natural assumption, since the matrix $A_{22}$ neglects all inputs of the basic sector into the sector of the nonbasic commodities.
} 


$$
\begin{aligned}
& p=(1+r)(p A+w l), \\
& p=(1+r) p A+w l .
\end{aligned}
$$

Setting $w=1$, the prices $p=p_{w}(r)$ in terms of labor commanded are:

$$
\begin{aligned}
& p_{w}(r)=(1+r) l(I-(1+r) A)^{-1}, \\
& p_{w}(r)=l(I-(1+r) A)^{-1} .
\end{aligned}
$$

Generalizing the logic of Proposition 3.2, we know that over the halfopen interval $\left[0, r^{\max }\right)$ each price $p_{i, w}(r)$ is a strictly increasing function of $r$.

The real wage rate is measured in a given wage, or consumption, basket $c \in \mathbb{R}_{+}^{n}$ (a column vector). Formally, the ratio

$$
\omega=\omega(r)=\frac{1}{p_{w}(r) c} \quad \text { or } \quad p=(1+r)(p A+\omega l), \quad p c=1,
$$

indicates how many baskets $c$ the nominal wage rate $w=1$ can buy. The geometric locus of the pairs $r$ and $\omega(r)$ is the corresponding wageprofit curve for $0 \leq r<r^{\max }$.

To derive empirical examples of wage-profit curves, we can refer to the 1995 technological matrix $A=A_{T}$, of the German economy in Table 1.11. In addition, we have to provide data for the labor coefficients $l$ and a consumption basket $c$. Coefficient $l_{i}$ for a (macro) sector $i$ is obtained by dividing employment in this sector by its output. The German IO tables document, sector by sector, the annual number of persons, but unfortunately they do not distinguish between persons employed and independent business men, while the reported total salaries refer to employed persons only, and have no information on something like "entrepreneurial wages". ${ }^{10}$ Given the lack of better data, we simply divide the number of persons in 1995 by the sectoral output and treat the resulting ratio as our labor coefficients.

For the seven sectors of our standard aggregation (agriculture, manufacturing, other manufacturing, construction, business-related services, consumer services, and social services), labor coefficients are displayed in the first row of Table 3.2. The units are number of persons per 1 mill. Euro of output in 1995 prices. Given this type of scaling the coefficients are directly comparable. As expected, the industrial sectors require the smallest number of employees, the most

\footnotetext{
${ }^{10}$ Any error resulting from limited data availability is likely to be greatest in sectors 1 (agriculture) and 6 (consumption services). On the whole, independent businesses make up a ratio of roughly ten percent.
} 
"productive" sectors, the export core (sector 2), being ahead of other manufacturing (sector 3). ${ }^{11}$ On the other hand, social services and agriculture are the least "productive" sectors. Regarding the wage basket $c$, the most natural choice is to select the economy's actual consumption vector in $1995 .{ }^{12}$ Its structure is reported in the third row of Table 3.2. Note that here a non-negligible part of the business-related (sector 5) services is also delivered to final consumers.
(1)
(2)
(3)
(4)
(5)
(6)
(7)

Agrc. Manf. Oth.Mf. Cstrt. Bus.Svcs. Cns.Svcs. Soc.Svcs.

\begin{tabular}{|c|c|c|c|c|c|c|c|}
\hline$l:$ & 20.23 & 5.29 & 7.01 & 12.50 & 9.65 & 12.53 & 20.03 \\
\hline$k^{\prime}$ & : 18.95 & 55.07 & 73.87 & 39.33 & 31.48 & 29.36 & 12.29 \\
\hline$c^{\prime}$ & 1.61 & 6.30 & 24.64 & 0.40 & 7.78 & 52.79 & 6.48 \\
\hline
\end{tabular}

Table 3.2: Labor coefficients l, capital intensities $k$, and consumption structure c (Germany, 1995)

In studying the wage-profit relationship, let us begin with the 1995 matrix $A=A_{T}$ from Table 1.11 and an illustration of Theorem 3.3, according to which the eigenvalue $\lambda^{\star}$ of $A$ with maximum modulus is real and its associated (real) eigenvector is strictly positive. Table 3.3 lists the real and imaginary parts of all seven eigenvalues and their modulus. It shows that $A$ has only one pair of complex eigenvalues, which is dominated by several real eigenvalues. The largest of them, $\lambda^{\star}=\lambda_{1}$, gives rise to a maximum rate of profit of

$$
r^{\max }=\frac{1-\lambda^{\star}}{\lambda^{\star}}=\frac{1-0.49768}{0.49768}=100.93 \% .
$$

We note in passing that the maximum rate of profit in the two-sector aggregation comes out a little different, which is only natural since dominant eigenvalue $\lambda^{\star}$ is not invariant to the level of aggregation of a given IO structure. In detail, we compute for $A_{T}$ in Chapter 1.4.3, equation (1.8), the value $\lambda^{\star}=0.492$ (rounded), so that here the

\footnotetext{
11 These coefficients have also shown the strongest fall over the 1990s. To be exact, "productivity" is here used as a common language expression and not as an economic term, where the labor inputs are related to value added rather than gross output.

${ }^{12}$ For completeness it should be mentioned that $c$ includes imports.
} 
maximum rate of profit amounts to $r^{\max }=103.08 \%$. The imprecision seems nevertheless tolerable.

The right-hand (column) and left-hand (row) eigenvectors associated with $\lambda^{\star}$, and the dominant eigenvalue $\lambda^{\star}=0.498$, are reported in Table 3.4. As predicted by Theorem 3.1, both of them are strictly positive. In the table they are scaled such that the components sum up to 100 percent (apart from rounding errors). Let the Standard Commodity be defined as the dominant eigenvector on the quantity side of the IO system $A x=\lambda(A) x$ (the concept of the Standard Commodity is investigated further in Chapter 4). Observe that the consumption vector $c$ in Table 3.2, is quite distinct from the proportions of the Standard Commodity (mainly in the components 5 and 6 ). The wageprofit curve expressed in the consumption basket $c$ will therefore deviate from a straight line.

\begin{tabular}{rrrc}
\hline & $\operatorname{Re} \lambda$ & $\operatorname{Im} \lambda$ & modulus \\
\hline$\lambda_{1}:$ & 0.498 & 0.000 & 0.498 \\
$\lambda_{2}:$ & 0.281 & 0.000 & 0.281 \\
$\lambda_{3}:$ & 0.157 & 0.000 & 0.157 \\
$\lambda_{4}:$ & 0.119 & 0.000 & 0.119 \\
$\lambda_{5}:$ & 0.018 & 0.021 & 0.028 \\
$\lambda_{6}:$ & 0.018 & -0.021 & 0.028 \\
$\lambda_{7}:$ & -0.007 & 0.000 & 0.007 \\
\hline
\end{tabular}

Table 3.3: Eigenvalues of the technological matrix $A=A_{T}$ from Table 1.11

Before we turn to the computation of the price systems, it is important to note that the numerical values of the profit rates that we obtain can be quite misleading, as they are calculated referring only to circulating capital, an assumption that is at odds with capitalist production processes.

1. The capital advanced in production is not just the intermediate inputs but, much more important, the money that has been invested in plant and equipment. The sectoral profits should therefore be related to the sector's fixed capital.

2. While fixed capital remains in existence for longer than our 


\begin{tabular}{|c|c|c|c|c|c|c|c|}
\hline & (1) & $(2)$ & (3) & (4) & (5) & (6) & $(7)$ \\
\hline & Agrc. & Manf. & Oth.Mf & Cstrt. & Bus.Svcs. & Cns.Svcs. & Soc.Svcs. \\
\hline RH EV & 3.38 & 8.66 & 32.90 & 2.39 & 36.55 & 13.69 & 2.43 \\
\hline LH EV & 15.03 & 24.00 & 21.56 & 17.17 & 7.26 & 8.49 & 6.48 \\
\hline
\end{tabular}

Table 3.4: Right-hand (RH) and left-hand ( $L H)$ eigenvectors of $A=A_{T}$

Note: The table displays the eigenvectors associated with the dominant eigenvalue $\lambda^{\star}$ normalized so that their components sum up to 100 percent.

accounting period of one year, it is nevertheless subject to depreciation. Hence, the notion of profits should include deductions for the corresponding replacement investment.

3. If, for the moment, we accept the intermediate inputs in the denominator of the profit rates as a (very imperfect) proxy for fixed capital, we trade this simplification for the problem of different sectoral turnover times. With "just-in-time" production in the industrial sectors, we may expect that the average time elapsing between buying the intermediate inputs and selling the output is substantially shorter than in the services sectors. As a result, the uniformity of the profit rates in equations (3.10) and (3.11) would be superficial and purely formal: earning, say, $10 \%$ on an investment in three months is more profitable than earning it in six months.

We will try to address these points below, where we improve our modeling as well as the empirical examples. For the moment, however, we analyze the basic production price in equations (3.10) and (3.11). ${ }^{13}$

Consider first equation (3.11), where wages are paid ex-post. The vector of production prices is shown in Table 3.5 as the uniform profit rate increases from 0 to $r^{\max }$. The data in Table 3.5 are based on $A=A_{T}$ from Table 1.11 and $l$ from Table 3.2, with the components summing to 100 percent. Individual prices are expressed as a share of their total sum, i.e. prices are normalized by setting $p e=100, e^{\prime}=$ $(1, \ldots, 1)$, facilitating comparison between the last price vector in Table 3.5 with the left-hand eigenvector in Table 3.4. See Proposition 3.1 for

${ }^{13}$ Acknowledging the presence of fixed capital, it would be more appropriate to call $r$ the profit margin, rather than the profit rate, however we stick to the conventional terminology. 
a motivation of the statement (there in the two commodities case) that prices have to rise in those sectors where capital intensity is above the average and have to fall in the other ones (if prices are normalized in the here considered way).

\begin{tabular}{lccccccc}
\hline$r$, in\% & $(1)$ & $(2)$ & $(3)$ & $(4)$ & $(5)$ & $(6)$ & $(7)$ \\
& Agrc. & Manf. & Oth.Mf. & Cstrt. & Bus.Svcs. & Cns.Svcs. Soc.Svcs. \\
\hline $0.00:$ & 21.38 & 11.74 & 13.47 & 14.58 & 11.34 & 12.23 & 15.27 \\
20.00: & 20.24 & 13.36 & 14.75 & 14.93 & 11.07 & 11.83 & 13.82 \\
40.00: & 19.03 & 15.30 & 16.16 & 15.36 & 10.62 & 11.30 & 12.23 \\
$60.00:$ & 17.75 & 17.63 & 17.74 & 15.86 & 9.92 & 10.60 & 10.50 \\
$80.00:$ & 16.43 & 20.43 & 19.50 & 16.45 & 8.87 & 9.71 & 8.62 \\
$100.00:$ & 15.09 & 23.83 & 21.46 & 17.14 & 7.34 & 8.56 & 6.58 \\
$100.90:$ & 15.03 & 23.99 & 21.55 & 17.17 & 7.26 & 8.50 & 6.49 \\
\hline
\end{tabular}

Table 3.5: Production prices from equation (3.11)

Apart from the general feature that the relative prices change considerably over the full range of profit rates, five points are worth mentioning:

(a) The price vector resulting from $r=0$ is proportional to labor values as defined in Proposition 3.3.

(b) As $r \rightarrow r^{\max }$, the price vector $p=p(r)$ converges to the left-hand eigenvector of $A$ associated with the dominant eigenvalue $\lambda^{\star}$, which is also the price vector brought about by the maximum rate of profit (presupposing a uniform normalization; cf. the second row in Table 3.4).

(c) Price variations seem monotonic, with prices either strictly increasing or decreasing. Yet, this result may be an artifact of the rather large step increases in $r$ chosen, and in any case, it cannot be generalized to other IO structures (presumably matrices with relatively larger off-diagonal entries).

(d) Given the choice of quantity units, actual market prices in 1995 are equal to the unit vector. Evidently, this is very different from all of the production prices in the table.

(e) The second row of Table 3.2 shows the sectoral capital intensities $k_{i}$. The (weighted) average capital intensity $\bar{k}$ over the whole economy (with sectors weighted based on their share in total output) is given 
by $\bar{k}=38.72$ which implies that sectors 2,3 , and 4 exhibit a capital intensity above the average and the remaining sectors a capital intensity below the average. ${ }^{14}$ This result should be compared with Table 3.5 to see that natural prices increase (decrease) with the profit rate in sectors with an above (below) average capital intensity. This is intuitive, since wages are assumed there to be paid ex post so that the rate of profit is only applied to intermediate goods implying that increasing rates of profit put more pressure on prices where capital intensity is high. The normalization rule $p e=100$ then implies that prices rise when capital intensities are above the average and fall otherwise. This appears to be a neat empirical result for price-profit rate relationships. However, this result has been brought into question in the theoretical literature (Sraffa 1960, Ch. III).

Consider now the empirical wage-profit curve. We are interested in two points: the shape of the wage-profit curves for different wage baskets $c$, and the order of magnitude of real wages. We show four cases in Figure 3.2. In the first three the wage basket consists of a single (composite) good, namely: the good produced by other manufacturing, $c_{3}=1$; social services, $c_{7}=1$; and consumer services, $c_{6}=1$. In addition, we consider the empirical consumption vector $c$ in Table 3.2, normalized by setting $\sum_{i} c_{i}=1$. In all four cases, the goods represented by $c$ are worth 1 mill. Euro at 1995 prices. For convenience, we multiply the wages from $(3.14), \omega=1 / p_{w}(r) c$, by 1000 . The real wage rate thus obtained is the number of consumption bundles each of which would be worth 1000 Euro at 1995 prices. The outcome of these computations is shown in Figure 3.2; in all four panels the outer curve results from ex-post wage payments, equation (3.11), the inner curve from wages paid in advance, equation (3.10).

Focusing on (3.11), we see that the same matrix can give rise either to a convex $\left(c_{3}=1\right)$ or to a concave wage-profit curve $\left(c_{6}=1\right.$ or, even more pronounced, $\left.c_{7}=1\right)$. Using the actual empirical composition of consumption, a slightly concave relationship emerges. Regarding exante wage payments, the upper-right panel in Figure 3.2 shows a curve that is convex for lower and concave for higher rates of profit, though only slightly so.

Figure 3.2 also shows the scope for real wages in the presence of

${ }^{14}$ In Table 3.2, the labor coefficients $l_{i}$ are the number of persons per 1 mill. Euro of output $i$ in 1995 prices. The capital intensities $k_{i}$ are measured in 1000 Euro per head. The components $c_{i}$ of total consumption are given in percentages. 


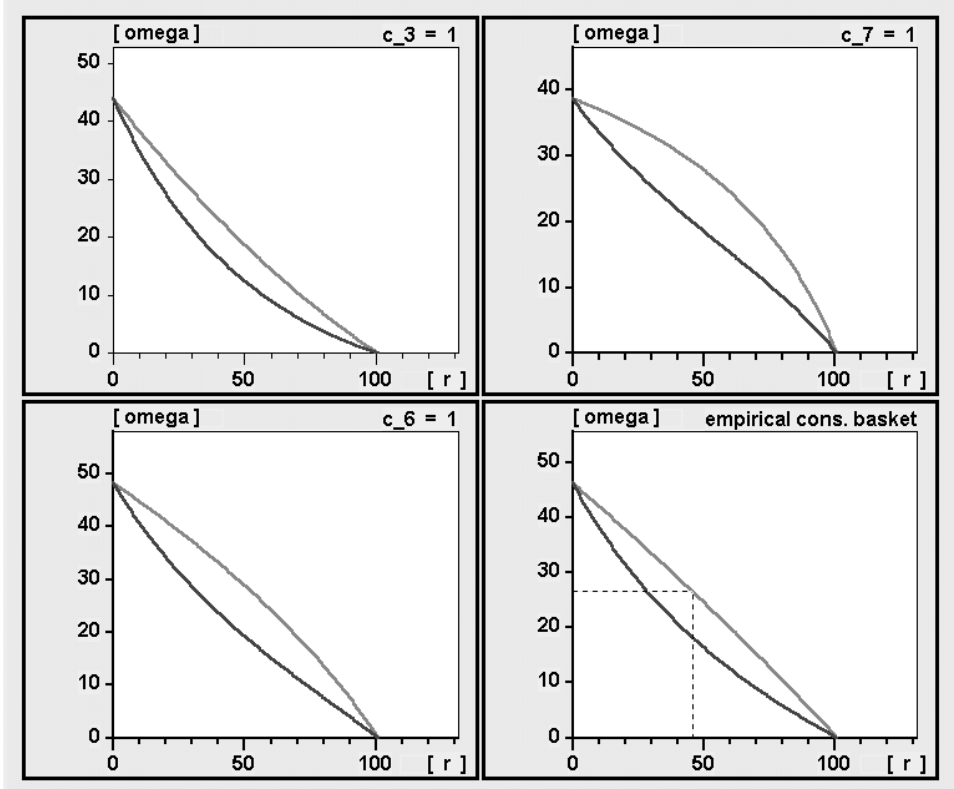

Figure 3.2: Empirical wage-profit curves under different consumption baskets

Note: Wages are paid ex-post on the upper curve and ex-ante on the lower curve. Underlying is the 7-sector matrix $A=A_{T}$ from Table 1.11 and the labor coefficients $l$ from Table 3.2. See text for the four different consumption baskets. The real wage rate $\omega$ is the number of these baskets, where scaling is such that one basket is worth 1000 Euro in prices of 1995.

zero profits (and no allowances for depreciation). Depending on the composition of the wage basket, the real wage rate would then range between an equivalent of 38000 and 48000 Euro per year.

We may ask which point(s) on the wage-profit curves correspond to the 1995 data - setting aside all the conceptual shortcomings pointed out above. In order to identify them we need to know the wage rate in 1995. The German IO tables report a total of 37.382 mill. persons working and (gross) wage payments summing up to 996900 mill. Euro. Therefore, subject to the provisos made when introducing the labor coefficients in Table 3.2, we obtain a wage rate of 26668 Euro per year. Taking the empirical 1995 consumption vector, a real wage rate 
$\omega=0.001 \cdot 26.7$ in (3.11) is associated with a profit rate $r=45.5 \% .{ }^{15}$ This combination is shown by the dotted lines in the lower right panel of Figure 3.2.

\begin{tabular}{lccccccc}
\hline$r$, in\% & $(1)$ & $(2)$ & $(3)$ & $(4)$ & $(5)$ & $(6)$ & $(7)$ \\
& Agrc. & Manf. & Oth.Mf. & Cstrt. & Bus.Svcs. & Cns.Svcs. Soc.Svcs. \\
\hline 0.00: & 0.36 & 0.20 & 0.23 & 0.25 & 0.19 & 0.20 & 0.26 \\
20.00: & 0.42 & 0.27 & 0.30 & 0.31 & 0.23 & 0.24 & 0.28 \\
40.00: & 0.51 & 0.41 & 0.43 & 0.41 & 0.28 & 0.30 & 0.33 \\
60.00: & 0.69 & 0.68 & 0.69 & 0.61 & 0.38 & 0.41 & 0.41 \\
80.00: & 1.22 & 1.52 & 1.45 & 1.23 & 0.86 & 0.72 & 0.64 \\
100.00: & 24.99 & 39.45 & 35.53 & 28.38 & 12.16 & 14.16 & 10.90 \\
$100.90:$ & 721.15 & 1150.91 & 1033.89 & 823.67 & 348.36 & 407.56 & 311.22 \\
\hline
\end{tabular}

Table 3.6: Production prices in terms of labor commanded $(w=1)$

Note: Based on $A=A_{T}$ from Table 1.11 and $l$ from Table 3.2. We have rescaled production prices here in terms of 100 Euro per unit of good, since they become very large when the maximum rate of profit is approached. Note that $r=0$ now gives the vector of labor values.

Inspection of the price vectors shown in Table 3.6 shows that prices in terms of the wage unit are strictly increasing in the rate of profitstarting from labor values at $r=0$. Profits become the dominant part of these prices, broadly speaking, from rates of profits above 40 percent, and completely outweigh the total labor costs embodied in commodities as $r$ approaches $r^{\max }$. Sectors 2, 3, and 4 have capital intensities above the average (see Table 3.2) and dominate changes in relative prices when the rate of profit increases.

\subsection{Conclusions}

We have expanded the analysis of Chapters 1 and 2 to define and explore the concepts of labor values, natural prices, and labor commanded prices. We have shown that the standard interpretation of

\footnotetext{
${ }^{15}$ Once again, recalling the aforementioned shortcomings 1-3 discussed at the beginning of this section, this level of the profit rate should not be taken literally.
} 
Marx's LTV is untenable: in general, outside of Smith's "early and rude state of society" labor values are different from natural prices - the socalled 'transformation problem'. This creates problems to the standard, predictive interpretation of the LTV as a theory of the determination of relative prices, but it does not necessarily mean that the classicalMarxian theory of prices and values is irremediably flawed.

Indeed, we have shown that both labor values and classical natural, or production prices are theoretically well defined and empirically relevant in $n$-good linear Leontief economies. In the next two chapters we further explore the usefulness of Adam Smith's concepts of labor embodied prices, labor commanded prices, and natural prices in terms of a given numéraire commodity. We analyze price-quantity interactions in a balanced growth equilibrium and extend the classical theory to economies with (pure) joint production. In Part II, we argue that Marxian labor values are also well-defined, and although they do not explain prices, they are theoretically important to understand the laws and dynamics of capitalist economies, and in this role they are an integral part of the United Nations' SNA. We shall offer no solution to the transformation problem because, in our view, no transformation from value to price magnitudes is either meaningful or necessary, and therefore no problem arises. 



\section{David Ricardo: Long-Period Prices, Accumulation, and the Invariable Measure of Value}

\subsection{Introduction}

In this chapter, we turn to Ricardo's theory of prices, growth and technical change in a manufacturing economy. The concept of price is that of natural or long-period prices, and from our long-period perspective we investigate questions of changing income distribution and its implications for price formation and the choice of technique. We quickly move from Ricardo's own presentation of his theoretical framework to a modern approach allowing us to analyze wage-profit curves, balanced growth paths, the capital controversies of the 1960s, and more generally the fundamental relationships that characterize the process of capital accumulation and price formation in capitalist economies.

To be sure, in his Principles, Ricardo studied many other important topics, including, for example, the implications of ruthless competition in agriculture and the formation of rents considered in Chapter 2. In this chapter, we abstract from the complications related to the presence of an agricultural sector (such as the presence of diminishing returns) and focus on economic systems characterized by long-period prices as well as balanced growth paths, making appropriate assumptions on the savings of capitalists and workers and on natural growth. Regarding the latter, we replace Malthus's population law with the assumption of constant natural growth in the labor supply in a manufacturing economy producing one pure investment and one pure consumption good.

We demonstrate that even in a two-sector linear economy there exist no simple monotonic functional relationships between capital intensity $k$ and, on the one hand, the rate of profit $r$ and, on the other hand, the so-called "factor price ratio" $\omega / r$. In fact, at the aggregate level, capital 
intensity is determined by a combination of price and quantity effects and therefore cannot be considered independently from, and prior to, questions concerning income distribution and balanced growth.

The results derived in this chapter have several implications. In particular, they cast serious doubts on the standard results associated with the neoclassical theory of growth and distribution, but also on the narrow, one-sided conception of labor values as the primary determinants of natural prices - what Marx later calls prices of production. Finally, this chapter calls into question the Sraffian interpretation of the so-called Standard Commodity, as a tool to identify changes in relative prices as being due to variations in distribution or technical change. Thus the attempts to conceive of something 'real' behind the 'nominal' sphere - be it 'capital', embodied labor, or invariable measures of value - must be examined more closely, or indeed wholly reconsidered.

\subsection{Production data and labor values}

We consider an economy producing one pure investment good (commodity 1) and one pure consumption good (commodity 2) by means of capital (commodity 1) and labor. For most of this chapter, we assume as the 'natural' time unit, or period of production, the time span characterizing the wage payments - 1 month for example. These payments are supposed to be made at the end of the period, i.e. wages are paid ex post.

Good 1 is a fixed capital good. In order to avoid the complications arising from different vintages of capital goods, we assume that a constant fraction $\delta$ of good 1 is consumed within each period. This includes the case $\delta=1$ of so-called circulating capital, which is completely used up during one production period, considered thus far. It will later be argued that the apparently technological distinction between fixed and circulating capital goods is not entirely convincing from the economic point of view and should give way to the concepts of 'capital advanced' versus 'capital consumed'. However, for the time being we accept the former 'technological view' as our point of departure, since the capital-theoretic literature is generally based on it. ${ }^{1}$

The economy produces with a linear technology. In each sector $i$ $(i=1,2), a_{1 i}$ units of the capital good have to be installed at the beginning of the period to produce one unit of good $i$. If we think of capital as machines, it can be said that they are run at a constant

\footnotetext{
${ }^{1}$ The presentation of the technology is based on Jaeger (1979).
} 
efficiency level, so that their number can be identified with the hours that they are in use. This is the capital to be advanced. Assuming the same rate of depreciation $\delta$ in both sectors, $\delta a_{1 i}$ units of the capital good are consumed over the period per unit of output $i$. As in the previous chapters, only productive technologies are considered and so we assume $0 \leq \delta a_{11}<1$. The labor input per unit of output $i$ is given by the quantity $l_{i}$.

On the basis of the analysis in Chapter 3 , the labor values $v_{1}, v_{2}$ of the two goods are:

$$
\begin{aligned}
& v_{1}=l_{1}+v_{1} \delta a_{11}, \\
& v_{2}=l_{2}+v_{1} \delta a_{12} .
\end{aligned}
$$

The equations state that the labor values of the goods are determined by the sum of the direct labor expended in their production plus the labor time that has gone into the production of the consumed means of production. Hence equations (4.1)-(4.2) measure the direct and indirect labor that, on the whole, is needed in the production of one unit of each good. By their very definition, labor values represent theoretically motivated accounting concepts - whose usefulness remains to be established.

As the first equation is decoupled from the second, the labor value of the investment good is easily calculated as

$$
v_{1}=\frac{l_{1}}{1-\delta a_{11}}=l_{1}+\delta a_{11} l_{1}+\left(\delta a_{11}\right)^{2} l_{1}+\cdots
$$

Accordingly, the amount of labor embodied in one unit of the investment good is given by the direct labor per unit of the corresponding net product $\left(1-\delta a_{11}\right)$. Alternatively, if reference is made to the geometric series, the labor value is equal to the direct labor expended to produce one unit of good 1, plus the direct labor expended in the production of the amount of capital $\delta a_{11}$, and so on. The labor value $v_{1}$ is thus the sum of direct labor expended in the infinite production sequence that leads to one unit of output of the capital good.

At this level, we have a concept of labor values that is, on the one hand, technological in nature and, on the other hand, a matter of purely theoretical (labor) cost accounting. And by equation (4.2), the same applies to the labor value of the consumption good, which is given by the direct labor used to produce this good plus the labor embodied 
in the means of production $\delta a_{12}$ that are used up in the production of one unit of good 2.

By definition, labor values represent real magnitudes rather than nominal market - or natural - prices. In a given economy, they might therefore allow us to detect the real processes behind the statics or the dynamics of nominal magnitudes. As a first step in such an analysis, let us suppose that the production levels $x_{1}$ and $x_{2}$ of the two goods are exogenously given. The vector of the corresponding net product $y=\left(y_{1}, y_{2}\right)^{\prime}$ is

$$
y_{1}=x_{1}-\left(\delta a_{11} x_{1}+\delta a_{12} x_{2}\right), \quad y_{2}=x_{2} .
$$

Its labor value is easily shown to be identical to the volume of direct labor employed in the production of the gross output $x=\left(x_{1}, x_{2}\right)^{\prime}$,

$$
v_{1} y_{1}+v_{2} y_{2}=l_{1} x_{1}+l_{2} x_{2} \text {. }
$$

In fact, in the more convenient vector and matrix notation,

$$
A=\left(\begin{array}{cc}
a_{11} & a_{12} \\
0 & 0
\end{array}\right), \quad l=\left(l_{1}, l_{2}\right), \quad v=\left(v_{1}, v_{2}\right)
$$

the system given by (4.1) and (4.2) can be rewritten and then solved as

$$
\begin{aligned}
& v=l+v \delta A=l(I-\delta A)^{-1} \\
& \text { where } \quad(I-\delta A)^{-1}=\frac{1}{1-\delta a_{11}}\left(\begin{array}{cc}
1 & \delta a_{12} \\
0 & 1-\delta a_{11}
\end{array}\right) .
\end{aligned}
$$

Since

$$
x=y+\delta A x=(I-\delta A)^{-1} y,
$$

postmultiplying the labor values by the net product vector finally yields:

$$
v y=l(I-\delta A)^{-1} y=l x .
$$

Therefore the labor time embodied in the net product $y$ is equal to the direct labor time spent in producing the gross product $x$. Hence, in terms of labor values, the definition of the gross national product at the macro level is given by the labor time directly and indirectly spent in its production.

Moreover, an increase in the net product of commodity 1 by one 
unit implies

$$
y \mapsto y+\left(\begin{array}{c}
1 \\
0
\end{array}\right), \quad \text { i.e. } \quad x \mapsto x+(I-\delta A)^{-1}\left(\begin{array}{l}
1 \\
0
\end{array}\right) .
$$

Since

$$
v_{1}=v\left(\begin{array}{l}
1 \\
0
\end{array}\right)=l(I-\delta A)^{-1}\left(\begin{array}{l}
1 \\
0
\end{array}\right),
$$

the labor value $v_{1}$ also represents the amount of direct labor needed to increase net production $y$ by one unit of good 1. Total labor costs to produce this extra unit are thus identical here to the so-called "employment multipliers" that measure the extra employment directly needed for this extra net product:

$$
\begin{aligned}
v_{1} & =v\left(\begin{array}{l}
1 \\
0
\end{array}\right)=l \frac{1}{1-\delta a_{11}}\left(\begin{array}{cc}
1 & \delta a_{12} \\
0 & 1-\delta a_{11}
\end{array}\right)\left(\begin{array}{l}
1 \\
0
\end{array}\right) \\
& =\frac{1}{1-\delta a_{11}} l_{1}=l_{1}+\left(\delta a_{11}\right) l_{1}+\left(\delta a_{11}\right)^{2} l_{1}+\cdots
\end{aligned}
$$

It follows that in this model there is an identity between total labor costs for the production of one unit of good 1 and the direct employment effects that such an increase in net production would have if all indirect employment multiplier effects were taken into account. The same is true for good 2, the consumption good, where net and gross production are identical. The total labor costs (or the employment multipliers) when commodities enter the production of all commodities will be considered in more detail in Chapter 9.

Because, as in Chapter 3, we assume that wages are paid post factum. Prices in terms of labor commanded are given by:

$$
\begin{aligned}
p_{w} & =l+(r+\delta) p_{w} A, \quad \text { or } \\
p_{w}(r) & =l(I-(\delta+r) A)^{-1}=l \sum_{k=0}^{\infty}[(\delta+r) A]^{k} .
\end{aligned}
$$

If $r>0$, this expression provides an upper bound for the labor contents of the various commodities - as shown in the previous chapter-with $\delta=1$. Further, if we assume a uniform composition of capital in both sectors,

$$
v_{1} a_{11} / l_{1}=v_{1} a_{12} / l_{2}, \quad \text { i.e. here } \quad a_{11} / l_{1}=a_{12} / l_{2} \quad \text { or } \quad A_{1 *} \sim l \text {, }
$$


then, as in Chapter 3 , it follows that the vector $p_{w}$ must be proportional to (and strictly larger than) the vector of labor values $v$, which in turn are proportional to (and strictly larger than) the vector $l$ of labor input coefficients. Relative production prices are equal to relative labor values. If, in addition, production prices $p_{w}=p_{w}(r)$ are re-normalized by the condition $p_{w}(r) y=l x$, then from the above equality $v y=l x$ it follows that $p_{w}(0)=v$, if the rate of profit is zero.

Finally, it is worth noting that exchanging products according to the total labor costs embodied in them could be regarded as the exchange of "equivalents", or fair exchange, since agents would then exchange commodities according to the contributions of their labor efforts (including indirect labor efforts of agents at the earlier stages of production). From the viewpoint of uniform labor efforts (and skills) this could be considered as an egalitarian commodity exchange principle, at least in the case of simple commodity production. Agents would exchange their labor efforts on the market and national income would be quite naturally represented by the total labor time spent in the yearly commodity production. The next section shows, however, that the laws of commodity exchange in a capitalist market economy make equal exchange impossible. Nonetheless, in Capital Marx tried to use labor values for real social accounting (and other purposes), while he conceived of the prices of production as the centers of gravity of the market prices in actual capitalist economies. We return to these topics in Part II.

\subsection{Price equations and the wage-profit curve}

In the classical perspective, natural prices are prices which yield a uniform rate of profit in all sectors of production. We have discussed this concept in the previous chapter under the assumption of ex ante wage payments. We now analyze it assuming that wages are paid $e x$ post, which given our assumptions on capital advanced and depreciation yields the following equations:

$$
\begin{aligned}
& p_{1}=w l_{1}+p_{1} \delta a_{11}+r p_{1} a_{11}=w l_{1}+(\delta+r) p_{1} a_{11}, \\
& p_{2}=w l_{2}+p_{1} \delta a_{12}+r p_{1} a_{12}=w l_{2}+(\delta+r) p_{1} a_{12} .
\end{aligned}
$$

This means that profits, given by the vector $\left(p_{1}-w l_{1}-p_{1} \delta a_{11}\right.$, $\left.p_{2}-w l_{2}-p_{1} \delta a_{12}\right)$, are assumed to be uniformly related to capital advanced $\left(p_{1} a_{11}, p_{1} a_{12}\right)$ via the rate $r$ :

$$
r=\frac{p_{1}-w l_{1}-p_{1} \delta a_{11}}{p_{1} a_{11}}=\frac{p_{2}-w l_{2}-p_{2} \delta a_{12}}{p_{1} a_{12}} .
$$


It is convenient to write, and solve, the two price equations in matrix notation,

$$
p=w l+(r+\delta) p A, \quad \text { or } \quad p=w l[I-(r+\delta) A]^{-1} .
$$

Since there are two (nonlinear) equations and four unknowns: prices $p_{1}, p_{2}$, wages $w$ and the uniform rate of profit $r$, two of them must be treated as exogenously given. We choose the profit rate $r$ and the price of the consumption good $p_{2}$ for this purpose. The latter is set equal to one and thus serves as a numéraire. Accordingly, the capital price $p_{1}$ and the wage rate $w$ are endogenously determined, both measured in units of the consumption good. The wage rate $w$ is therefore the real wage. The matrix representation (4.6) becomes

$$
\left(p_{1}, 1\right)=w\left(l_{1}, l_{2}\right) \frac{1}{1-(r+\delta) a_{11}}\left(\begin{array}{cc}
1 & (r+\delta) a_{12} \\
0 & 1-(r+\delta) a_{11}
\end{array}\right) .
$$

The second component reads,

$$
1=\left[w l_{1}(r+\delta) a_{12}+w l_{2}\left(1-(r+\delta) a_{11}\right] /\left[1-(r+\delta) a_{11}\right],\right.
$$

which can be solved for the real wage as follows:

$$
\begin{gathered}
w=\frac{1-(r+\delta) a_{11}}{l_{1}(r+\delta) a_{12}+l_{2}\left(1-(r+\delta) a_{11}\right)} \\
=\frac{1-(r+\delta) a_{11}}{l_{2}+(r+\delta)\left(l_{1} a_{12}-l_{2} a_{11}\right)} \\
=\frac{1-(r+\delta) a_{11}}{l_{2}+(r+\delta) l_{2} a_{11}\left(k_{2} / k_{1}-1\right)}, \quad \text { i.e. } \\
w=\frac{1-(r+\delta) a_{11}}{l_{2}+(r+\delta) l_{2} a_{11}(\kappa-1)}, \quad \kappa=k_{2} / k_{1}, \quad k_{j}=a_{1 j} / l_{j} .
\end{gathered}
$$

Plugging this expression into the first component of (4.7) for the relative price $p_{1}$ yields:

$$
\begin{aligned}
p_{1} & =\frac{1-(r+\delta) a_{11}}{l_{2}+(r+\delta) l_{2} a_{11}\left(k_{2} / k_{1}-1\right)} \cdot \frac{l_{1}}{1-(r+\delta) a_{11}}, \\
& =\frac{l_{1}}{l_{2}+(r+\delta) l_{2} a_{11}(\kappa-1)} .
\end{aligned}
$$


The fractions $k_{2}$ and $k_{1}$ are the capital intensities of the two sectors, so that $\kappa \equiv k_{2} / k_{1}>1(<1)$ means that sector 2 is more (less) capital intensive than sector 1 . The special case of a uniform capital intensity in the economy, $\kappa=1$, is identical to the uniform composition of capital considered in the previous section. The real wage $w$ and the relative prices are then given by the simplified expressions

$$
w=\frac{1-(r+\delta) a_{11}}{l_{2}}, \quad p_{1}=\frac{l_{1}}{l_{2}},
$$

and, concerning labor values, we obtain

$$
\begin{array}{ll}
v_{1} / l_{1}=1+v_{1} \delta k_{1}, & k_{1}=k_{2}(\kappa=1), \\
v_{2} / l_{2}=1+v_{1} \delta k_{2}, & k_{1}=k_{2}(\kappa=1),
\end{array}
$$

i.e.

$$
\left(v_{1} / l_{1}\right) /\left(v_{2} / l_{2}\right)=1, \quad \text { or } \quad v_{1} / v_{2}=l_{1} / l_{2}=p_{1} / p_{2}=p_{1} .
$$

A uniform capital intensity therefore implies that labor values are proportional to the prices of production (for all choices of the numéraire), which in turn are proportional to the direct labor inputs; the structure of labor values and classical prices is identical and quite straightforward. Furthermore, the wage-profit curve is linear as shown in Figure 4.1 (with the hybrid ratio $a_{11} / l_{2}$ providing the value of overall capital intensity $p_{1} k$ ).

In the general case with $k_{1} \neq k_{2}$, by equation (4.1) the slope of the wage-profit curve is:

$$
\begin{aligned}
w^{\prime}(r) & =\frac{-a_{11}\left(l_{2}+(r+\delta) l_{2} a_{11}\left(\frac{k_{2}}{k_{1}}-1\right)\right)-\left(1-(r+\delta) a_{11}\right) l_{2} a_{11}\left(\frac{k_{2}}{k_{1}}-1\right)}{\left(l_{2}+(r+\delta) l_{2} a_{11}\left(\frac{k_{2}}{k_{1}}-1\right)\right)^{2}} \\
& =\frac{-l_{2} a_{11} k_{2} / k_{1}}{\left(l_{2}+(r+\delta) l_{2} a_{11}\left(k_{2} / k_{1}-1\right)\right)^{2}}=\frac{-l_{2} a_{11} \kappa}{D^{2}}<0,
\end{aligned}
$$

where $D=\left(l_{2}+(r+\delta) l_{2} a_{11}(\kappa-1)\right)^{2}$, so that the curvature of the $w-r$ relationship depends on the relative capital intensity $\kappa=k_{2} / k_{1}$ as follows:

$$
\begin{aligned}
w^{\prime \prime}(r) & =\frac{l_{2} a_{11} \kappa l_{2} a_{11}(\kappa-1) 2 D}{D^{4}} \\
& =\frac{2 l_{2}^{2} a_{11}^{2} \kappa(\kappa-1)}{D^{3}} \underset{<}{\geq} 0 \text { if and only if } \quad \kappa \underset{<}{\gtrless} .
\end{aligned}
$$

Similarly, the slope and curvature of the $p_{1}(r)$-curve are given by: 


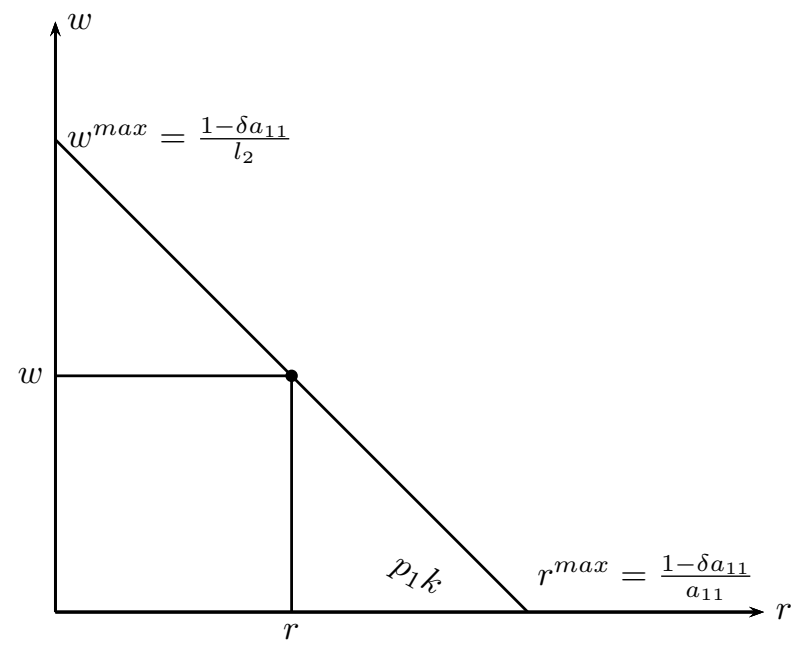

Figure 4.1: The wage-profit curve for $\kappa \equiv k_{2} / k_{1}=1$ (measured in terms of $\operatorname{good} 2)$

$$
\begin{aligned}
p_{1}^{\prime}(r) & =\frac{-l_{1} l_{2} a_{11}\left(k_{2} / k_{1}-1\right)}{\left(l_{2}+(r+\delta) l_{2} a_{11}\left(k_{2} / k_{1}-1\right)\right)^{2}} \\
& =\frac{-l_{1} l_{2} a_{11}(\kappa-1)}{D^{2}} \underset{<}{\gtrless} \quad \text { if and only if } \quad \kappa \stackrel{>}{<}, \\
p_{1}^{\prime \prime}(r) & =\frac{l_{1} l_{2} a_{11}(\kappa-1) l_{2} a_{11}(\kappa-1) 2 D}{D^{4}} \\
& =\frac{l_{1} l_{2}^{2} a_{11}^{2}(\kappa-1)^{2} 2}{D^{3}}>0 \quad \text { if and only if } \quad \kappa \neq 1 .
\end{aligned}
$$

This gives rise to the graphical representations of the $w(r)$ and the $p_{1}(r)$ curves shown in Figures $4.2(\mathrm{a})$ and (b). They demonstrate that classical prices are not at all a simple concept for long-run price determination, not even in the case where capital is a homogeneous good and there is only one consumption good. Furthermore, although there is an unambiguous trade-off between the rate of profit and real wages (measured in terms of the consumption good), this trade-off is generally nonlinear. It is thus not equivalent to the simple subdivision of a given 'cake' between capital and labor. As $\kappa$ approaches 1 , however, the two wage-profit curves in Figure 4.2(a) approach each other without 
a change in their intersection points, and become the straight line shown in Figure 4.1 when $\kappa=1$.

Lastly, recall the labor commanded prices from equation (4.3), assuming the matrix $\delta A$ is productive, the maximum rate of profit $r^{\max }$ is determined by the following eigenvalue equation:

$$
p A=\frac{1}{\delta+r^{\max }} p \quad \text { with } \quad \frac{1}{\delta+r^{\max }}=\lambda(A)=a_{11},
$$

so that the formula for $r^{\max }$ reads,

$$
r^{\max }=\frac{1}{\lambda(A)}-\delta=\frac{1}{a_{11}}-\delta=\frac{1-\delta a_{11}}{a_{11}} .
$$

\subsection{Quantity equations and the consumption-growth curve}

Natural prices may be viewed as the long-run outcome of capitalist competition, but this does not necessarily imply any natural tendency towards balanced growth of the economy. Indeed, it is not unreasonable to conjecture that balanced growth represents an unstable position. Nonetheless, balanced growth can serve as a helpful reference point for understanding the features of disequilibrium growth. Thus, we introduce the notion of balanced growth into the two-sector model. The basic assumptions underlying the following analysis are:

1. capital is always fully utilized;

2. employment is fully adjusted to the capital stock; and

3. there are no restrictions originating from insufficient goods demand, that is, Say's law holds.

Let $g$ be the uniform growth rate of capital across the two sectors, $C$ the aggregate consumption level, and $K$ the aggregate capital available in the economy, and let $x_{1}$ and $x_{2}$ be gross output in the two sectors. The output of sector 1 is used for aggregate gross investment, $x_{1}=$ $(g+\delta) K$, and the output of sector 2 for aggregate consumption, $x_{2}=C$. The demand for capital in the two sectors is given by $a_{11} x_{1}$ and $a_{12} x_{2}$, respectively. The equilibrium condition in the capital market therefore reads,

$$
K=a_{11} x_{1}+a_{12} x_{2}=a_{11}(\delta+g) K+a_{12} C,
$$

and the corresponding volume of employment is

$$
L=l_{1} x_{1}+l_{2} x_{2} .
$$

Normalizing the two equations by employment $L$ gives two nonlinear 


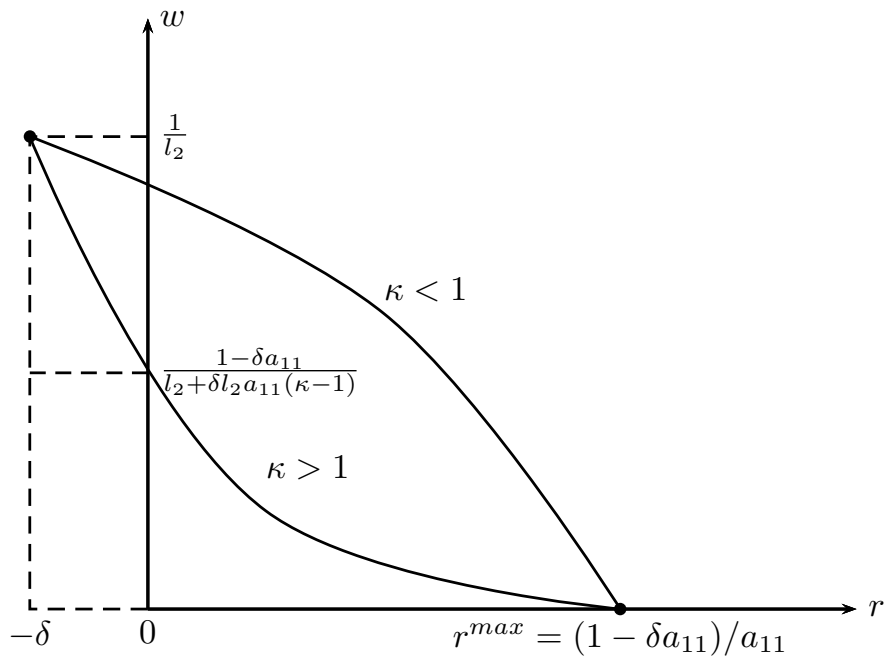

(a) The wage-profit curve for $\kappa \neq 1$

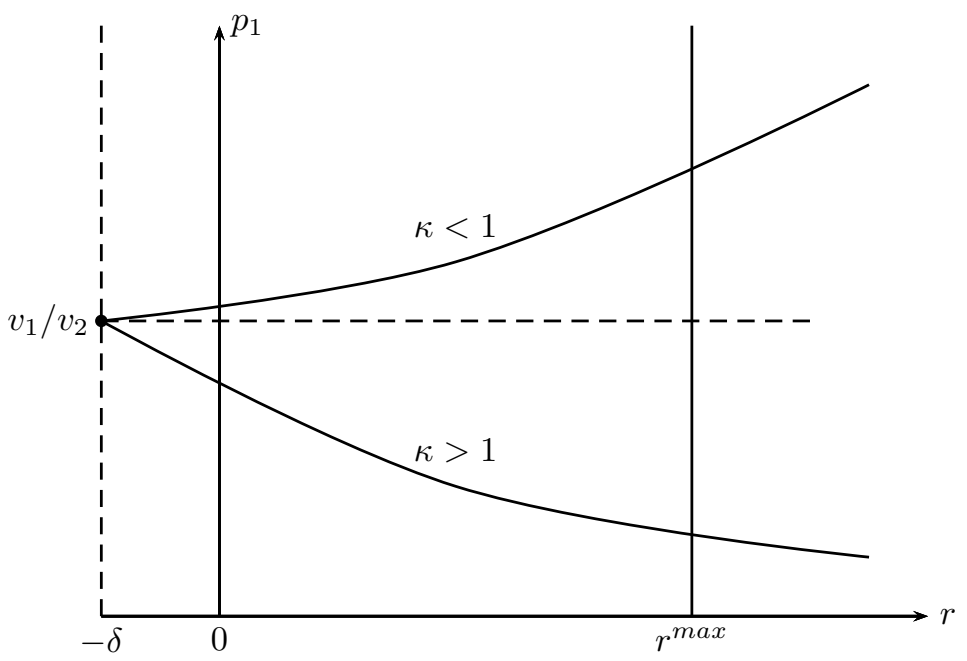

(b) Relative classical prices $p_{1}$ for $\kappa \neq 1\left(p_{2}=1\right)$

Figure 4.2: $w(r)$ and $p_{1}(r)$ curves for $\kappa \neq 1$ 
equations in the two unknowns $g$ and $c$,

$$
\begin{aligned}
k & =a_{11}(g+\delta) k+a_{12} c, \quad k=K / L, c=C / L, \\
1 & =l_{1}(g+\delta) k+l_{2} c .
\end{aligned}
$$

Solving (4.11) for $c$,

$$
c=\frac{1-a_{11}(g+\delta)}{a_{12}} k,
$$

and inserting this into (4.12) yields

$$
1=l_{1}(g+\delta) k+l_{2} \frac{1-a_{11}(g+\delta)}{a_{12}} k,
$$

from which the average physical capital intensity is obtained as

$$
\begin{aligned}
k & =k(g)=\frac{a_{12}}{l_{1}(g+\delta) a_{12}+l_{2}\left(1-a_{11}(g+\delta)\right)} \\
& =\frac{a_{12}}{l_{2}+(g+\delta) l_{2} a_{11}\left(k_{2} / k_{1}-1\right)}=\frac{k_{2}}{D(g)},
\end{aligned}
$$

where $D(g)=1+(g+\delta) a_{11}(\kappa-1)$ and $\kappa=k_{2} / k_{1}$. We note that

$$
k^{\prime}(g)=\frac{-k_{2}(\kappa-1) a_{11}}{D^{2}(g)} \underset{=}{\gtrless} 0 \text { if and only if } 1 \underset{<}{<} \kappa, \text { and } \quad k^{\prime \prime}(g) \leq 0 .
$$

Plugging $k(g)$ into the expression for $c$ from above, we get

$$
c=\frac{k_{2}\left(1-a_{11}(g+\delta)\right) / l_{2}}{1+(g+\delta)(\kappa-1) a_{11}}=\frac{1-(g+\delta) a_{11}}{l_{2}+l_{2} a_{11}(g+\delta)(\kappa-1)} .
$$

This functional relationship has the same shape as the $w$-r relationship in the previous section (mathematically, it is even the same function). Thus,

$$
c^{\prime}(g)<0, \quad c^{\prime \prime}(g) \stackrel{\geq}{<} 0 \text { if and only if } \kappa \stackrel{\geq}{<} 1,
$$

as illustrated in Figure 4.3 for $\kappa \neq 1 .^{2}$

Since the capital stock grows at the rate $g$ by assumption, we have that

$$
(1+g) K=(1+g)\left[a_{11}(\delta+g) K+a_{12} C\right]=(1+g)\left(a_{11} x_{1}+a_{12} x_{2}\right) .
$$

${ }^{2}$ If $\kappa=1$, then the $c(g)$ curve is linear, analogously to Figure 4.1. 


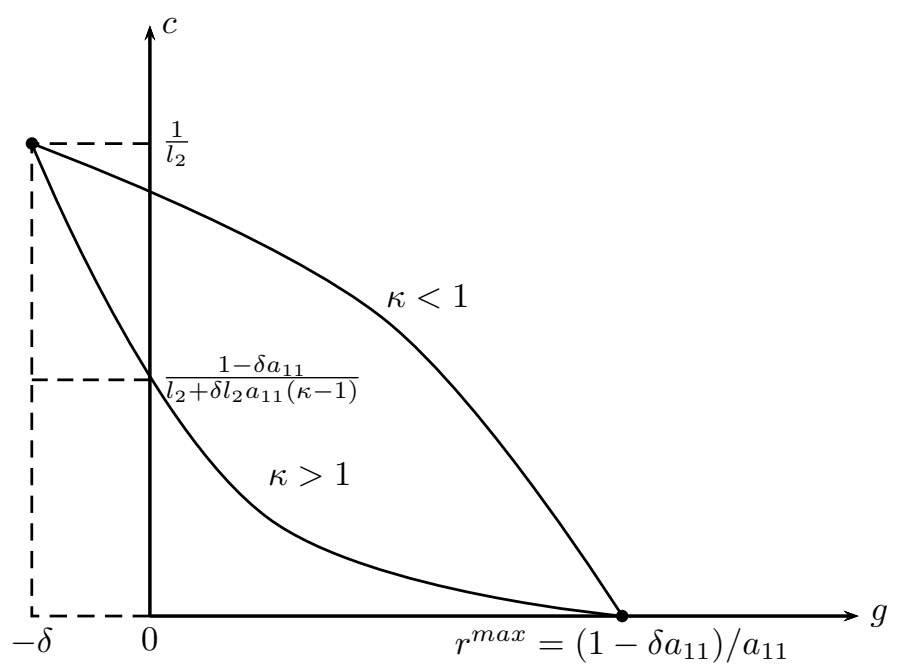

Figure 4.3: The consumption-growth trade-off for $\kappa \neq 1$

Hence both sectors can indeed grow at the same rate $g$, as does employment $L$. Observe that we do not assume full employment, nor do we yet consider the rate of growth of labor supply. Whether the growth of $L$ is sufficient for the maintenance of a given employment rate is therefore left as an open question for the moment.

The calculation of the function $k(g)$ shows the dependence of the average capital intensity on the rate of growth $g$. This function is similar to the function $p(r)$ (see Figure $4.2(\mathrm{~b})$ ), so that we have the dual pair of relationships

$$
w(r), p_{1}(r) \text { and } c(g), k(g)
$$

for income distribution and relative prices on the one hand, and for consumption per head and average capital intensity on the other hand, where referring to Figures $4.2(\mathrm{a})$ and $(\mathrm{b})-r^{\max }$ corresponds to $g^{\max }$ and $w^{\max }$ corresponds to $c^{\max }$.

4.4.1 The $n$-dimensional case with a general choice of numéraire

The duality between the price and quantity sides of the classical economy is not an artifact of the two-commodity assumption. The price equations and the conditions for balanced growth are easily generalized 
to the $n$-dimensional case as follows: ${ }^{3}$

$$
p=(\delta+r) p A+w l, \quad p b=1, \quad x=(\delta+g) A x+c b, \quad l x=1 .
$$

Here the vector $b=\left(b_{1}, \ldots, b_{n}\right)^{\prime}$ represents an arbitrary consumption structure and the scalar $c$ denotes the level of consumption of the goods in this basket. The sectoral activity levels $x=\left(x_{1}, \ldots, x_{n}\right)^{\prime}$ are measured in terms of a given level of employment $l x=L=1$, which need not necessarily be the full employment level and has been normalized to unity.

Assuming the matrix $\delta A$ to be productive, these equations can be explicitly solved by means of the Leontief-inverse,

$p=w l(I-(\delta+r) A)^{-1}, \quad p b=1, \quad x=(I-(\delta+g) A)^{-1}(c b), \quad l x=1$.

The vectors $p$ and $x$ are well-defined and nonnegative if (and only if) the rates of profit or growth, respectively, are less than $1 / \lambda(A)-\delta$. Observe that the same consumption basket $b$ is used in the wage-profit and the consumption-growth curve, where it serves to normalize the prices $p$, on the one hand, and to measure the level of consumption per head by a scalar $c$, on the other hand.

The following equations therefore define identical, downwardsloping wage-profit curves $w(r)$ and consumption-growth curves $c(g)$, as in the two-sector case studied above:

$$
\begin{aligned}
& 1=p b=w l(I-(\delta+r) A)^{-1} b, \quad \text { i.e. } \quad w=\frac{1}{l(I-(\delta+r) A)^{-1} b}, \\
& 1=l x=l(I-(\delta+g) A)^{-1}(c b), \quad \text { i.e. } \quad c=\frac{1}{l(I-(\delta+g) A)^{-1} b} .
\end{aligned}
$$

Because there no longer is a single capital good, average capital intensity $k(g)$ can no longer be defined purely in physical terms, but is instead determined by

$$
\begin{aligned}
k(r, g)= & \frac{p(r) A x(g)}{l x(g)}=w l C(r, g)(c b)=w(r) c(g) l C(r, g) b, \\
& \text { where } C(r, g) \equiv[I-(\delta+r) A]^{-1} A[I-(\delta+g) A]^{-1} .
\end{aligned}
$$

As in the model with one capital good, the capital intensity in value terms depends both on $g$ and on $r$, but it now changes in complicated

\footnotetext{
${ }^{3}$ For notational simplicity, we continue to assume a uniform rate of capital stock depreciation $\delta$. Differentiated rates of depreciation could be easily incorporated by introducing a suitable diagonal matrix.
} 
ways if either variable changes and, with it, the price structure and/or the structure of the activity levels of the $n$ industries. Note that the wage-profit and the consumption-growth curves in the model with one capital good and one consumption good are obtained by specifying the basket $b$ as the unit vector $(0,1)^{\prime}$. The physical capital intensity $k(g)$ in this case can be obtained from the general formula for the average capital intensity $k(r, g)$.

Finally, it is possible to derive the general form of the price and quantity equations

$$
\begin{array}{ll}
p(r)=\frac{l(I-(\delta+r) A)^{-1}}{l(I-(\delta+r) A)^{-1} b}, & p(r) b=1, \\
x(g)=\frac{(I-(\delta+g) A)^{-1} b}{l(I-(\delta+g) A)^{-1} b}, & l x(g)=1 .
\end{array}
$$

This presentation provides a general way of approaching the determination of long-period prices and the corresponding wage-profit and consumption-growth curves for an $n$-dimensional economy.

\subsection{Income distribution, savings and the Classical closure}

We now return to the canonical two-good model and combine the $w(r)$ and the $c(g)$ - curves in order to develop a complete and integrated model of balanced growth and natural prices ('complete' as far as the real sector of a capitalist economy is concerned). To this end, we shall derive the basic national income identity relating income generation and income spending. First, in a balanced growth path, the gross and net output vectors are given by

$$
x=\left(\begin{array}{c}
(\delta+g) K \\
C
\end{array}\right) \text { and } y=x-\delta A x=\left(\begin{array}{c}
g K \\
C
\end{array}\right),
$$

Pre-multiplying the gross output vector by the normalized price vector $\left(p_{1}, 1\right)$ we obtain

$$
p x=\left(p_{1}, 1\right)\left(\begin{array}{c}
(\delta+g) K \\
C
\end{array}\right)=(\delta+g) p_{1} K+C .
$$

Next, multiplying equations (4.4) and (4.5) by $x_{1}$ and $x_{2}$, respectively, and adding the resulting expression yields - noting that good 2 is the numéraire $\left(p_{2}=1\right)$ : 


$$
\begin{aligned}
p x=p_{1} x_{1}+x_{2} & =w\left(l_{1} x_{1}+l_{2} x_{2}\right)+p_{1}(\delta+r)\left(a_{11} x_{1}+a_{12} x_{2}\right) \\
& =w L+(\delta+r) p_{1} K .
\end{aligned}
$$

Finally, dividing net output by the total volume of employment $L$ yields the following series of identities expressing the net national product per unit of labor, $\nu:^{4}$

$$
\begin{aligned}
\nu & =p(x-\delta A x) / L=(w l x+r p A x) / L=w(r)+r p_{1}(r) k(g) \\
& =\left(c a_{o} x+g p A x\right) / L=c+g p_{1} k=c(g)+g p_{1}(r) k(g) .
\end{aligned}
$$

The two distributional functions $w=w(r)$ and $c=c(g)$ explicitly appear in the expression of net national product per unit of labor. Thus, the following question arises: how can the (so far exogenously given) magnitudes $r$ and $g$ be determined such that a unique distribution of income, with respect to sources as well as uses, is generated?

Having established the relationships in equation (4.14), they can be used to derive the following expression for the value of the capital intensity $p_{1} k$ :

$$
p_{1} k=\frac{c-w}{r-g}>0 \text { if } r \neq g, \quad p_{1} k=-w^{\prime}(r)=-c^{\prime}(g) \text { if } r=g .
$$

Since the curves $c=c(g)$ and $w=w(r)$ have the same shape, the situation shown in Figure 4.4 obtains. Hence the capital intensity $p_{1} k=$ $p_{1}(r) k(g)$ is related to the slope and the curvature of the wage-profit curve, and of the consumption-growth curve. The latter curve is strictly convex (as shown) if $\kappa=k_{2} / k_{1}>1$, and strictly concave if $\kappa<1$. In the borderline case $\kappa=1$, we have $c^{\prime}(g)=w^{\prime}(r)=p_{1} k=$ constant and, as already observed, relative prices are always equal to relative labor values.

In interpreting Figure 4.4, it should be emphasized that there are two degrees of freedom, namely $r, g$. To close the model, and know which points on the two curves $w=w(r)$ and $c=c(g)$ are actually realized, two additional equations have to be introduced. With respect to wages, we follow the Classicals and assume an exogenously given real wage $w=\bar{w}$ (maintaining the normalization $p_{2}=1$ ). It may be thought of as a subsistence wage as in Ricardo's theory, or, following

\footnotetext{
${ }^{4}$ The equations describing the sources and the uses of net national income can also be obtained from the matrix equations $p(I-\delta A)=w l+r p A$ and $(I-\delta A) x=c(0,1)^{\prime}+g A x$ by post-multiplying the former by $x$, and by pre-multiplying the latter by $p$.
} 


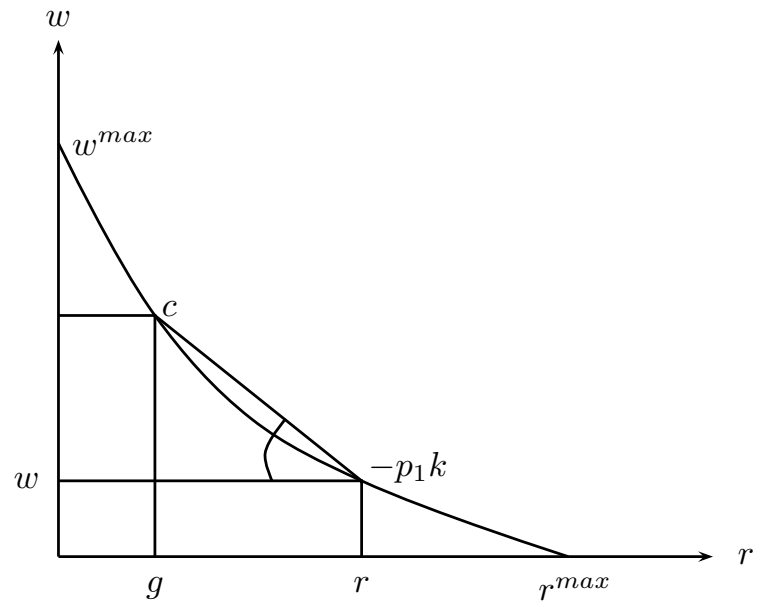

Figure 4.4: The determination of average capital intensity $p_{1} k$

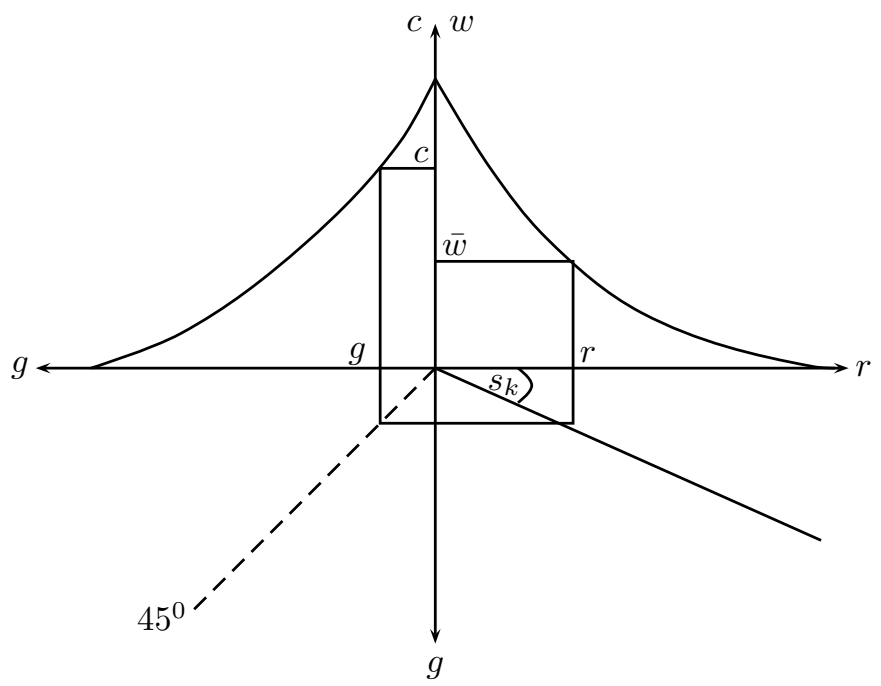

Figure 4.5: The closure of the classical model by subsistence wages $w=\bar{w}$ and the savings hypothesis $g=s_{k} r$ for $\kappa>1\left(p_{2}=1\right)$ 
Marx's approach, as being governed by the conflict between workers and capitalists over income distribution.

The second condition is a relationship between the rate of profit and the rate of growth. This corresponds to the classical notion that workers (as such) do not save and capitalists save a fraction $s_{k}$ of their profits (and consume the rest). Hence,

$$
g=s_{k} r \quad\left(0<s_{k} \leq 1\right) .
$$

With these two assumptions and supposing $\kappa>1$, the situation in Figure 4.5 is obtained. The given real wage $\bar{w}$ determines the rate of profit, which in turn, via the savings propensity $s_{k}$, determines the rate of growth $g$, which then determines consumption per head, $c$. Apart from the curvature, things are qualitatively the same if $\kappa<1$. If $\kappa=1$, the curves in the upper part of the diagram would be linear.

A comparison of different steady state positions is straightforward. For example, a rise in the real wage lowers both the profit and the growth rate, but increases workers' consumption as well as (despite the reduction of capitalist consumption) consumption per head. Therefore, an increase in workers' bargaining power allows them to increase their current welfare, but at the cost of lower growth, which reduces their future employment prospects. In contrast, a rising savings propensity of capitalists lowers consumption per head. Not only does workers' consumption remain unaffected in this case, but they also profit from this structural change as the increase in the rate of growth raises future employment.

\subsubsection{Two degrees of freedom and the Classical closure}

In this section, we have constructed a model which explains the rate of profit, the rate of growth, and consumption per head simultaneously as long period positions. Recall the national accounting identity derived above: $\nu=w(r)+r p_{1}(r) k(g)=c(g)+g p_{1}(r) k(g)$ for all values of $r, g$. If the real wage $w$ increases then aggregate capital intensity $p_{1} k$, falls, but this is due to a fall in prices, $p_{1}(r)$, without any change in technology. If we also assume a given natural rate of growth $\bar{n}=\dot{L} / L=$ constant, we can distinguish two situations of balanced growth in the medium run. We can either have a 'golden' age, where $\bar{n}<g$ and labor would become scarce (unless firms start to recruit foreign labor, a scenario not considered here). Or we can be in a 'leaden' age, where $\bar{n}>g$, which can occur if the employment of the labor force is just a matter of secondary importance.

We close this section noting that, Figure 4.5 can be reduced to linear wage-profit and consumption-growth curves, if $q^{\prime}=(1,0)$ is used 
as the numéraire for prices $p\left(p_{1}=1\right)$ and as a unit of measurement for consumption per head $c$ (which implies - as a matter of consistencythat good 1 is also used as consumption good). In this case, we get

$$
w=\frac{1-(\delta+r) a_{11}}{l_{1}}, \quad c=\frac{1-(\delta+g) a_{11}}{l_{1}},
$$

and

$$
p_{2}=\frac{l_{2}}{l_{1}}+l_{2} k_{1}(\delta+r)(\kappa-1),
$$

so that relative prices $p_{2}$ are also a linear function of $r$ and are strictly increasing (decreasing) if $\kappa>1(\kappa<1) .{ }^{5}$ Below, we generalize this result to $n$-good economies by choosing Sraffa's Standard Commodity (which here corresponds to $q=(1,0)^{\prime}$ as numéraire.

\subsection{Differentiated saving habits and multiple equilibria}

In the previous section, we considered classical saving habits and the classical closure with given wage-profit and consumption-growth curves. In this section, instead, we consider differentiated savings of both workers $\left(s_{w}>0\right)$ and capitalists $\left(s_{k} \in(0,1]\right)$ and assume that $s_{w}<s_{k}$, consistent with the literature and the available empirical evidence. We show that different saving habits give rise to so-called "Pasinetti" and "Anti-Pasinetti" cases as far as the implied shape of the growth-profit curve is concerned (Pasinetti 1962). ${ }^{6}$ However, there may be more than one steady state equilibrium for a given rate of profit.

In our real approach to balanced growth, if workers save, they do so in terms of physical capital goods, i.e. total capital per head $k$ must be owned by workers (or their saving institutions), given $s_{w}>0$. Let $k_{w}$ denote the amount of capital per head owned by workers and let $g_{w}$ denote its growth rate; and let a similar notation hold for capitalists. Unlike pure capitalists, workers have two sources of income, wages per head $w$ and profits $r p_{1} k_{w}$ per head. Then workers' capital holdings (in value) grow according to the following formula

$$
g_{w} p_{1} k_{w}=s_{w}\left(w+r p_{1} k_{w}\right), \quad p_{2}=1 .
$$

Therefore

$$
g_{w}=\frac{s_{w}\left(w+r p_{1} k_{w}\right)}{p_{1} k_{w}}
$$

\footnotetext{
${ }^{5}$ Note that the macroeconomic identity becomes $w+r k=\nu=c+g k, k=$ $a_{11} / l_{1}$.

${ }^{6}$ The growth-profit curve is in the lower-right quadrant of Figure 4.5.
} 
while the corresponding rate for pure capitalists is:

$$
g_{k}=\frac{s_{k} r p_{1} k_{k}}{p_{1} k_{k}}=s_{k} r
$$

Note that $k_{k}$ may be zero (if workers own the whole capital stock) and that $k_{w}+k_{k}=k, 0<k_{w} \leq 1$ must always hold.

Let us first investigate the case with $k_{w}=k$. In this case ${ }^{7}$

$$
g=g_{w}=\frac{s_{w}\left(w+r p_{1} k\right)}{p_{1} k}
$$

with

$$
p_{1}=\frac{l_{1}}{l_{2}+(r+\delta) l_{2} a_{11}(\kappa-1)}, \quad k=\frac{a_{12}}{l_{2}+(g+\delta) l_{2} a_{11}(\kappa-1)},
$$

and

$$
w=\frac{1-(r+\delta) a_{11}}{l_{2}+(r+\delta) l_{2} a_{11}(\kappa-1)}, \quad \frac{w}{p_{1}}=\frac{1-(r+\delta) a_{11}}{l_{1}} .
$$

From the above we get:

$$
\begin{aligned}
\frac{g}{s_{w}} & =r+\frac{1-(r+\delta) a_{11}}{l_{1}} \cdot \frac{l_{2}+(g+\delta) l_{2} a_{11}(\kappa-1)}{a_{12}} \\
& =r+\frac{1-\delta a_{11}}{l_{1}} \cdot \frac{l_{2}}{a_{12}}-r \cdot \frac{k_{1}}{k_{2}}+\left(1-(r+\delta) a_{11}\right) \frac{k_{1}}{k_{2}}(g+\delta)(\kappa-1) \\
& =r \cdot \frac{\kappa-1}{\kappa}+\frac{r^{m a x}}{k_{2}}+\left(1-(r+\delta) a_{11}\right) \frac{(g+\delta)(\kappa-1)}{\kappa}
\end{aligned}
$$

This gives

$$
\frac{\kappa g}{s_{w}(\kappa-1)}=\phi+r+\left(1-(r+\delta) a_{11}\right)(g+\delta),
$$

where $\phi$ is a given constant. Differentiating this equation with respect to the implicitly defined function $g(r)$ gives

$$
\frac{\kappa g^{\prime}(r)}{s_{w}(\kappa-1)}=1-a_{11}(g+\delta)+\left(1-(r+\delta) a_{11}\right) g^{\prime}(r),
$$

or

${ }^{7}$ This equation, and the $g-r$ relationship implied by it, is independent of the choice of numéraire $\left(p_{2}=1\right)$. 


$$
\frac{\kappa-s_{w}(\kappa-1)\left(1-(r+\delta) a_{11}\right)}{s_{w}(\kappa-1)} g^{\prime}(r)=1-a_{11}(g+\delta) .
$$

We have $1-(r+\delta) a_{11}=\left(w / p_{1}\right) l_{1} \in(0,1)$ for all $r<r^{\max }=$ $\left(1-\delta a_{11}\right) / a_{11}$. The numerator on the left-hand side is therefore larger than $\kappa\left(1-s_{w}\right)+s_{w}$, and thus positive, as is the expression on the right-hand side if $g<g^{\max }$. Hence,

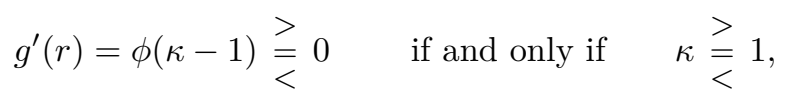

since $\phi$ is a positive real number. Furthermore:

$$
g\left(r^{\max }\right)=s_{w} r^{\max } \quad \text { and } \quad g(0)=\frac{\left[1+\delta a_{11}(\kappa-1)\right] s_{w} r^{\max }}{\kappa+(1-\kappa) a_{11} s_{w} r^{\max }}
$$

Therefore $g(0)=s_{w} r^{\max }$ for $\kappa=1$ and $g(0)>s_{w} r^{\max }, g(0) \in$ $\left(0, s_{w} r^{\max }\right)$ for $\kappa<1\left[g^{\prime}(r)<0, g\left(r^{\max }\right)=s_{w} r^{\max }\right]$, and for $\kappa>1$ : $\left[1-s_{w} a_{11} r^{\max }=1-s_{w}\left(1-\delta a_{11}\right)>0\right]$, respectively. We have thus arrived at the situation shown in Figure $4.6 .^{8}$

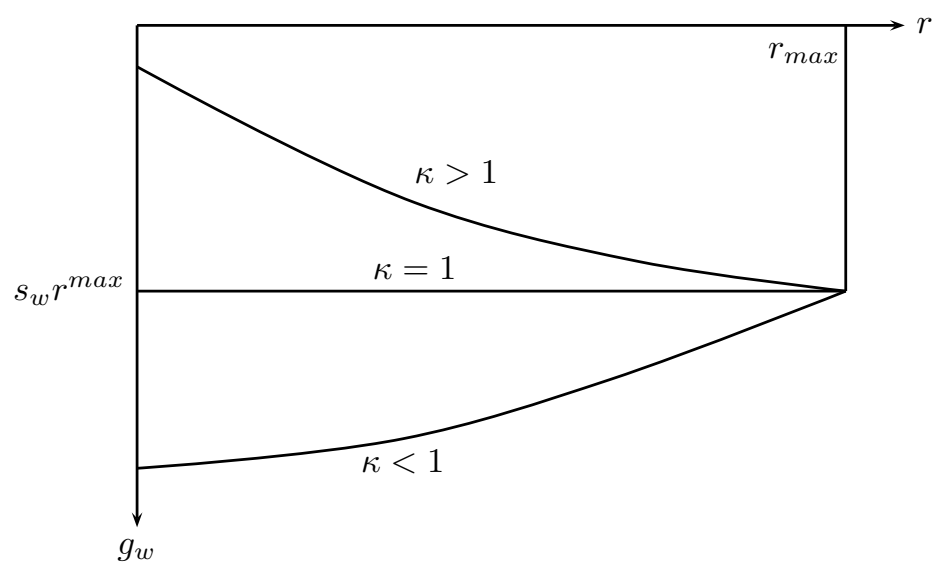

Figure 4.6: The growth-profit curve for $k_{k}=0$, i.e. $g p_{1} k=s_{w}\left(w+r p_{1} k\right)$

Let us now consider the case where $k_{k}>0$ holds in the long-period

${ }^{8}$ Note that the vertical axis $g_{w}$ in Figure 4.6 is inverted for ease of comparison with later figures, i.e. Figure 4.9. It can also be proved that $g^{\prime \prime}(r) \geq 0$ holds in all three cases shown in Figure 4.6 (see Spaventa $(1970))$. 
equilibrium. Then

$$
g p_{1} k=s_{w}\left(w+r p_{1}\left(k-k_{k}\right)\right)+s_{k} r p_{1} k_{k},
$$

i.e.

$$
\begin{aligned}
k_{k} & =\frac{g p_{1} k-s_{w}\left(w+r p_{1} k\right)}{\left(s_{k}-s_{w}\right) r p_{1}} \\
& =\frac{\left(g-g_{w}\right) p_{1} k}{\left(s_{k}-s_{w}\right) r p_{1}},
\end{aligned}
$$

where $g_{w}$ is the growth rate of the economy if $k_{k}=0$. Hence, we have $k_{k}<0$ above the $g_{w}$ curve - which is infeasible - and $k_{k}>0$ below it. Therefore, in the economy with different savings propensities by workers and capitalists, the $g-r$ curves can be of three types, as depicted in Figures 4.7 and 4.8, depending on the value of the relative capital intensity ratio.

Starting from low rates of profit $r$, at first only equilibria where $k_{k}=0, k_{w}=k$, and thus $g=s_{w}\left(w+r p_{1} k\right)$ exist. These are the so-called Anti-Pasinetti solutions, which depict a sort of people's capitalism. For sufficiently high rates of profit (above $\bar{r}$ ) there exist Pasinetti-type solutions where the growth rate of the economy is $g=s_{k} r$. However, in such cases there still exist equilibria with a lower rate of growth in which all capital is owned by workers.

In Pasinetti-type equilibria, we have the so-called "Pasinetti paradox" which states that (within certain limits) changes in the savings rate of workers, $s_{w}$, may have no impact on the equilibrium. If $k_{k}>0$ holds in equilibrium, workers' savings propensity is therefore (up to a certain point) completely irrelevant for the growth rate of the economy and their savings do not matter at all in the long-run. If $k_{k}>0$, we have:

$$
g p_{1} k=s_{w}\left(w+r p_{1} k_{w}\right)+s_{k} r p_{1} k_{k},
$$

and so the steady state $g$-r curve is simply $g=s_{k} r$ so that $s_{k}$ alone determines the speed of economic growth. We summarize these findings in Figure 4.9 for the case $\kappa>1$.

If $\bar{w}=\bar{w}_{2}$, we have multiple equilibria. In addition to the solution:

$$
\bar{w}_{2}, r_{2}, g_{2}, c_{2} \quad \text { with } \quad p_{1}\left(r_{2}\right), k\left(g_{2}\right), k_{k}>0
$$

shown in Figure 4.9, there is another equilibrium associated with $r_{2}$ and $k_{k}=0$ and a lower growth rate: $g=g_{w}\left(r_{2}\right)$ compared to $s_{k} r_{2}$, 

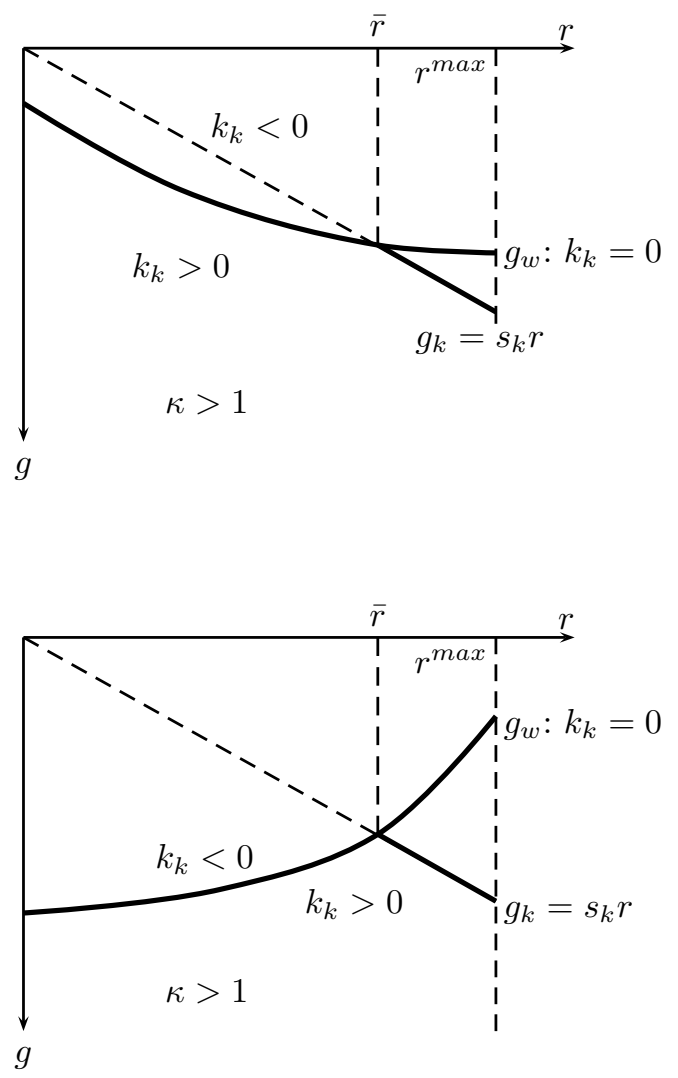

Figure 4.7: The g-r savings relationship for non-uniform capital intensities

but higher per capita consumption. Equilibrium selection can only be adjudicated by means of stability analysis, a topic that we will not explore here. ${ }^{9}$ Further, a sufficiently high increase in $\bar{w}_{2}\left(\rightarrow \bar{w}_{1}\right)$ will always drive out the Pasinetti case and lead to dominant workers' savings, further reduced growth and even higher consumption per head (not solely of workers). Finally, in the case $\kappa<1$, growth can even decline if the profit rate increases (as long as $k_{k}=0$ holds).

The main findings of this section therefore are that:

\footnotetext{
${ }^{9}$ One may however guess that there is a tendency towards the Pasinetti equilibrium, since $k_{k}$ grows at a faster rate than $k_{w}$.
} 


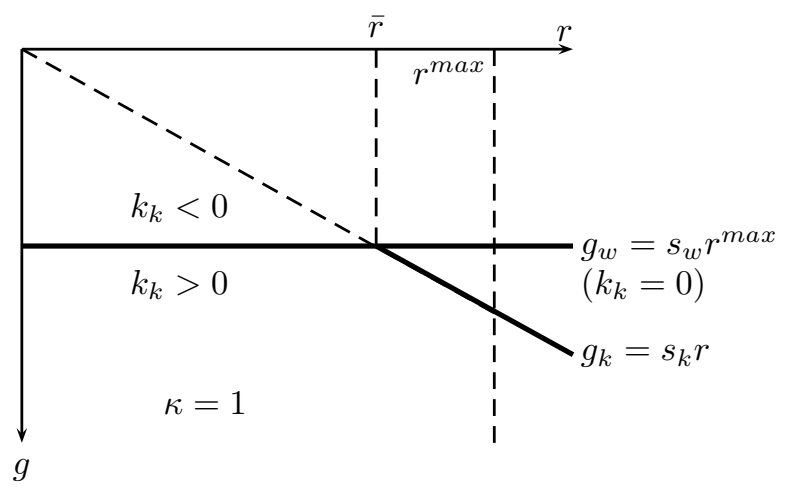

Figure 4.8: The g-r curve for $k_{1}=k_{2}$

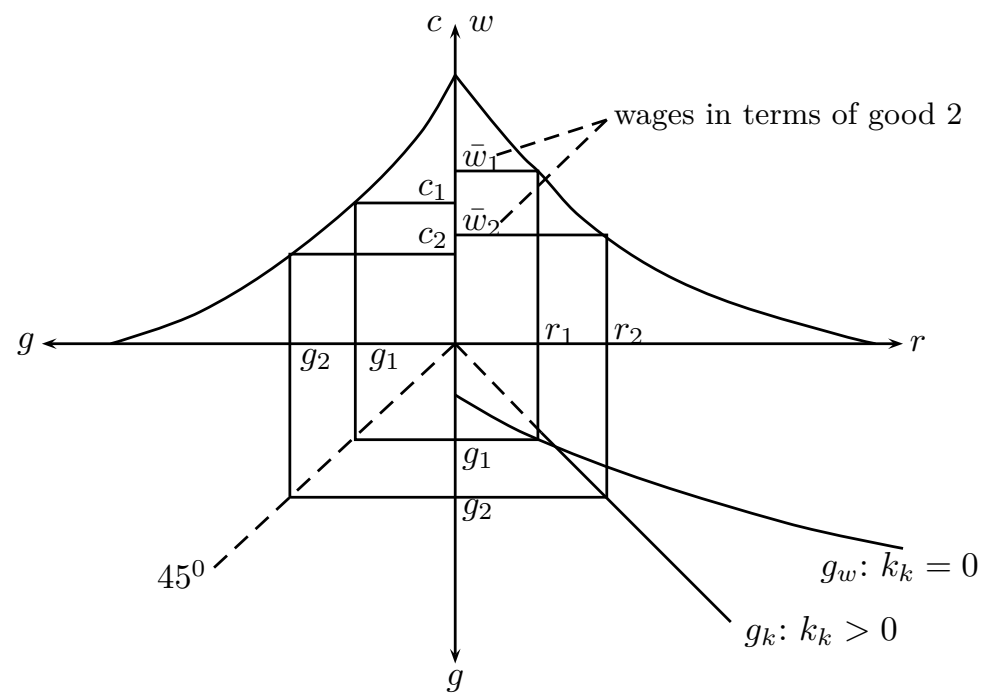

Figure 4.9: Pasinetti $\left(\bar{w}_{2}\right)$ and Anti-Pasinetti $\left(\bar{w}_{1}\right)$ outcomes under the classical closure

1. workers do not influence the equilibrium growth rate if $k_{k}>0$;

2. profitability and growth may move in opposite directions if $k_{k}=0$ 
and the investment good sector is more capital-intensive than the consumption good sector.

\subsection{Proportions in production and relative price movements}

In this section, we investigate price movements deriving from changes in real wages and thus in the general rate profit. Let the net product $y=x-A x$ be the numéraire. Production prices are:

$$
p=\frac{l(I-(\delta+r) A)^{-1}}{l(I-(\delta+r) A)^{-1} y} .
$$

Thus, for example, the relative price of sector 1 is

$$
p_{1}=\frac{l(I-(\delta+r) A)^{-1}(1,0)^{\prime}}{l(I-(\delta+r) A)^{-1} y}=\sum_{k=0}^{\infty} l A^{k}(1,0)^{\prime}(r+\delta)^{k} .
$$

The movement of this relative price, and of all other prices, may therefore be quite complicated depending on changes in the IO matrix $A$ - a point carefully illustrated by Sraffa (1960, Ch. 3). Let the wage $w$-which also represents the wage share take on values from 1 to 0 . The effects of changes in $w$ on the profit rate $r$ and on relative prices $p$ derived by Sraffa (1960) are summarized below: ${ }^{10}$

1. If $w=1$, then $r=0$ and the whole of output goes to workers and there is a correspondence between $p$ and $v$ as stated in the classical LTV.

2. If in this situation wages fell and $r$ rose, relative prices would change in a pattern determined by the proportions of $A_{j}$ and $l_{j}$ utilized in sector $j$, provided the proportions of $A_{j}$ and $l_{j}$ are not uniform across sectors.

3. If there is inequality of "proportions" then relative prices change as $w$ and $r$ vary. For example, when $w$ falls industries with low (high) $l_{j}$ relative to $A_{j}$ will have a deficit (surplus) "on their payments for wages and profits" (Sraffa 1960, Ch. 3, §16).

4. There can be a critical proportion of $l_{j}$ to $A_{j}$ that marks the "watershed" between deficit and surplus industries. An industry at this critical proportion would exhibit a balance, "the proceeds of the wage-reduction would provide exactly what was required for the payment of profits at the general rate" $r$ (Sraffa 1960, Ch. 3, $\S 17)$.

$\overline{10}$ The original passages in Sraffa (1960, Ch. 3) can be found in $\S(13)-(21)$. 
5. When $w$ falls, a change in relative prices is necessary to balance deficit and surplus industries: a rise in price brings balance in the former and vice versa for the latter.

6. However, these price changes may not occur if means of production in deficit (surplus) industries $i$ are produced by industries $j$ that are in relative deficit (surplus) to $i$.

7. Price movements thus depend on the "proportions" in any given industry, as well as the "proportions" of the industries producing their means of production.

8. It is then possible to construct a critical proportion for all industries, for which the industries producing its means of production also exhibit this critical proportion.

In order to illustrate empirically such complex price movements we use our IO table $A=A_{T}$ for Germany (1991-2000) and consider the movement of sectoral labor intensities $\frac{l_{j}}{p A_{* j}}$ as long-period prices vary with decreasing wages. The results are shown in Figure 4.10.

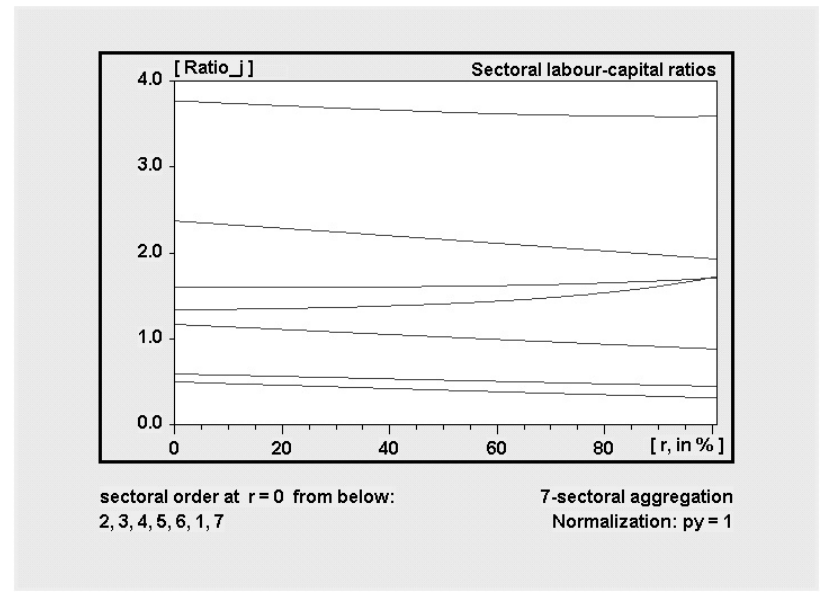

Figure 4.10: Sectoral labor intensities for $p y=1$ as function of the rate of profit $r$

We ignore here international trade for the sake of simplicity and consider the actual net output vector $y=x-A x$, where $x$ is the actual vector of activity levels with respect to the IO table $A=A_{T}: l x=$ 1. Stated differently, we consider the actual net product of the open economy treating imported intermediate inputs as though they were produced domestically. The price normalization rule is thus $p y=1$ and wages (and profits) are measured as shares in this (technologically- 
determined) net product. Following Sraffa we consider decreases in this wage share, from 1 to 0 , and thus increases in the rate of profit from zero to $r^{\max }$.

Sectors 2, 3, and 4 are Sraffa's deficit industries for which labor intensities $\frac{l_{j}}{p A_{* j}}$ are below the average and wage reductions do not increase profits as much as in the surplus sectors 1, 5, 6, and 7. These industries remain deficit sectors over the whole range of profit rates with labor intensities actually falling as $r$ increases. Surplus industries may have either falling or rising labor intensities, as $r$ increases, even if technology does not change. A closer analysis of Figure 4.11 reveals that there can be a reversal in the direction into which labor intensities are changing (in sector 6) and even a reordering of labor intensities (concerning sectors 5 and 6 ). Such revaluation effects are not very strong and seem more or less exceptional. By and large, Figure 4.11 indicates a fairly stable empirical characterization of capital intensity hierarchies, rather than the extreme reversals described by Sraffa (1960, Ch. 3).

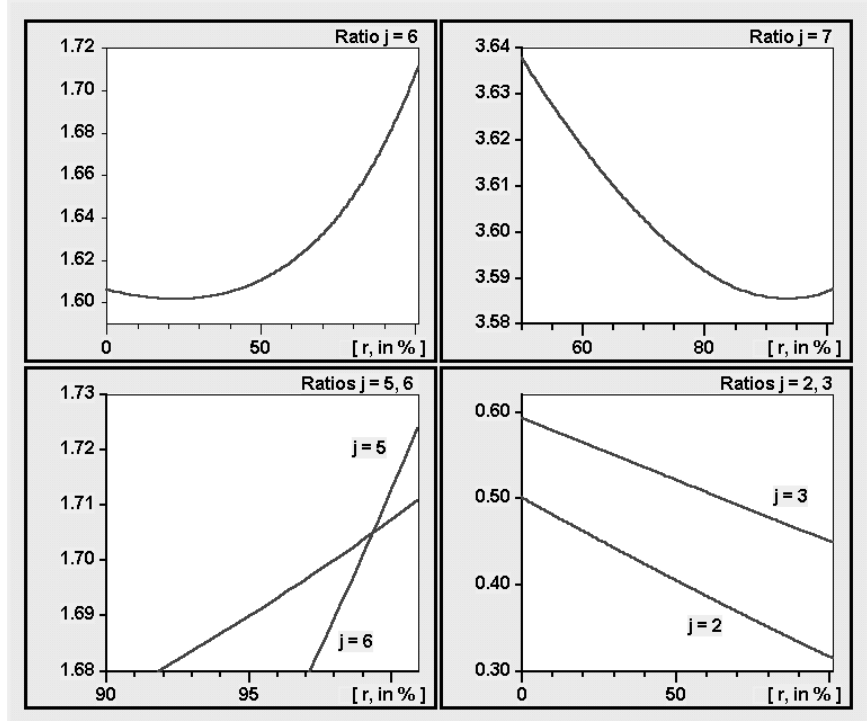

Figure 4.11: Sectoral labor intensities for $p y=1$ over selected ranges of the rate of profit $r$

Deficit industries are depicted in Figure 4.12, which shows that prices rise faster the lower the labor intensity. Empirical measurement thus indicates that changes in labor intensities and in prices of 


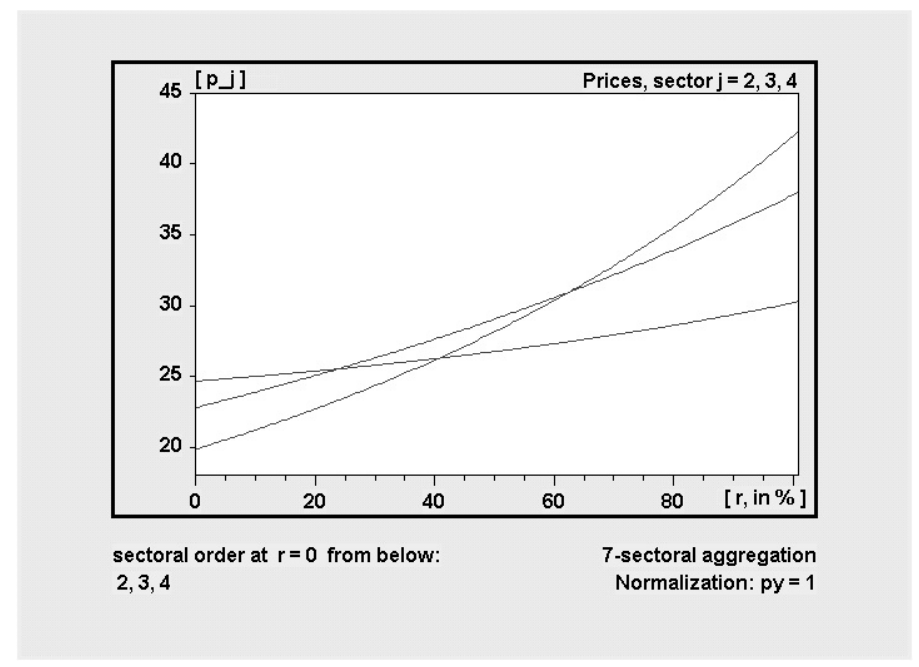

Figure 4.12: The prices of sectors 2-4 for $p y=1$ as a function of the profit rate $r$

production are still very simple - over the whole range of admissible profit rates - at least at a high level of sectoral aggregation with a given technology.

Figure 4.13 shows that a similar conclusion holds in surplus industries where prices - after readjusting a uniform rate of profitappear to fall faster the higher the labor intensity, except possibly for sectors 5 and 6 where the order of labor intensities actually changes at very high rates of profit. Nonetheless, the overall impression is that empirically the movement of prices of production induced by falling real wages (and thus increasing rate of profit) seems considerably less irregular than is suggested by purely theoretical considerations.

\subsubsection{Sraffa's Standard Commodity: Construction and basic implications}

The previous analysis derives some relations between changes in the distributive variables and variations in production prices, based on sectoral capital/labor ratios. Our empirical results display a remarkable degree of regularity. However, Sraffa (1960) famously argued that a proper understanding of changes in relative prices required the construction of an "invariable measure of value"-leading to the construction of the so-called "Standard Commodity". In particular, in chapter 3 Sraffa (1960) de-emphasizes the importance of "critical" 


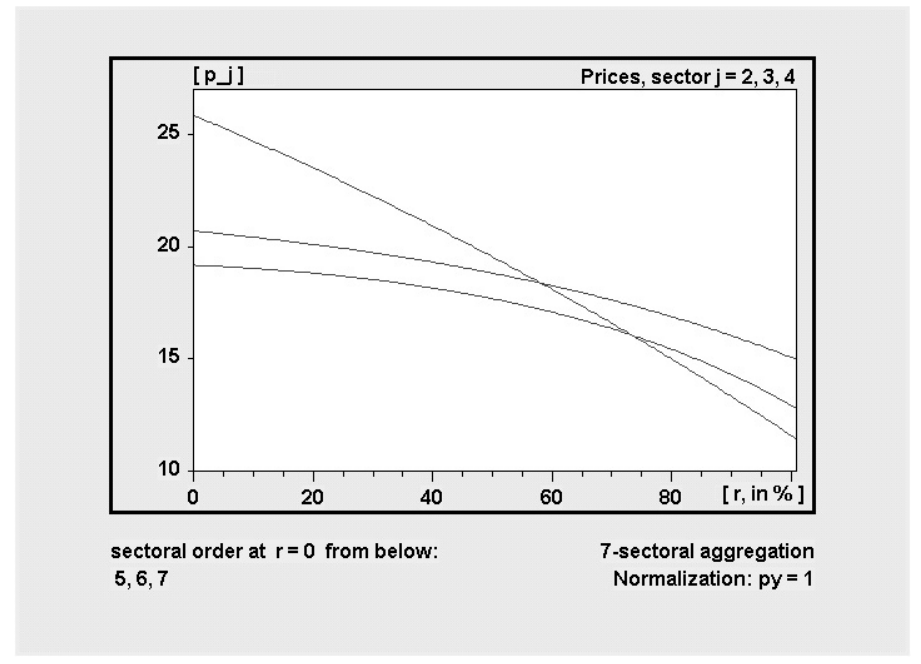

Figure 4.13: The prices of sectors 5-7 for $p y=1$ as a function of the profit rate $r$

proportions of "labour to means of production" in finding a suitable numéraire to provide a clear understanding of changes in relative prices. Rather than focusing on such hybrid ratios of magnitudes measured in money terms and in labor hours, he advocates the use of a homogeneous ratio that no longer refers only to the internal structure of inputs. It is worth quoting him at length:

In trying to identify the 'balancing' proportion it is convenient to replace the hybrid 'proportion' of the quantity of labour to the value of the means of production which we have been using up to this point, with one of the corresponding 'pure' ratios between homogeneous quantities. There are two such corresponding ratios, namely the quantity-ratio of direct to indirect labour employed, and the value-ratio of net product to means of production. We shall adopt the latter here.

While the rate of profits is uniform in all industries, and depends only on the wage, the value-ratio of the net product to the means of production is in general different for each industry and mainly depends on its particular circumstances of production.

There is however an exception to this. When we make the wage equal to zero and the whole of the net product goes to profits, in each industry the value-ratio of net product to means of production 
necessarily comes to coincide with the general rate of profits. However different from one another they may have been at other wage-levels, at this level the 'value-ratios' of all industries are equal.

It follows that the only 'value-ratio' which can be invariant to changes in the wage, and therefore is capable of being 'recurrent' in the sense defined in $\S 21$, is the one that is equal to the rate of profits which corresponds to zero wage. And that is the 'balancing' ratio.

We shall call Maximum rate of profits the rate of profits as it would be if the whole of the national income went to profits. And we shall denote by a single letter, $R$, the two coincident ratios, namely the Maximum rate of profits and the 'balancing' ratio of net product to means of production (Sraffa 1960, Ch. 3).

Sraffa's Standard Commodity is simply defined by the dominant eigenvector - and, of course, dominant eigenvalue of the technology matrix $A=A_{T}$. Formally, setting $\delta=1$ :

$$
A q=\lambda(A) q, l q=1 \quad q^{n}=q-A q=(1-\lambda(A)) q, \quad \lambda(A)=\frac{1}{1+r^{\max }} .
$$

In economies with only basic commodities, these equations have a unique and strictly positive solution. In the more general case of a Sraffa matrix this holds for the sector of basics while all components of $q$ that correspond to nonbasics will be zero. Since Sraffa's discussion of critical proportions in production was meant to simplify the analysis of price movements, in this subsection we analyze whether this new type of numéraire can indeed provide new insights on prices movements.

First, we consider the movement of labor intensities when the Standard Commodity is adopted as numéraire:

$$
p q^{n}=1
$$

Figure 4.14 shows that there is not much new to be observed when the Standard net product is used instead of actual net product as the numéraire. The ordering of labor intensities over the whole range of admissible profit rates is basically the same and even the quantitative changes in these ratios are of comparable size. At this level of analysis, there is nothing to be noted in favor or against the use of the Standard net product instead of the actual one. This conclusion hardly changes if we turn to the investigation of price movements as the uniform profit rate varies from zero to its maximum value, as is obvious from a visual inspection of Figures 4.15 and 4.16 . 


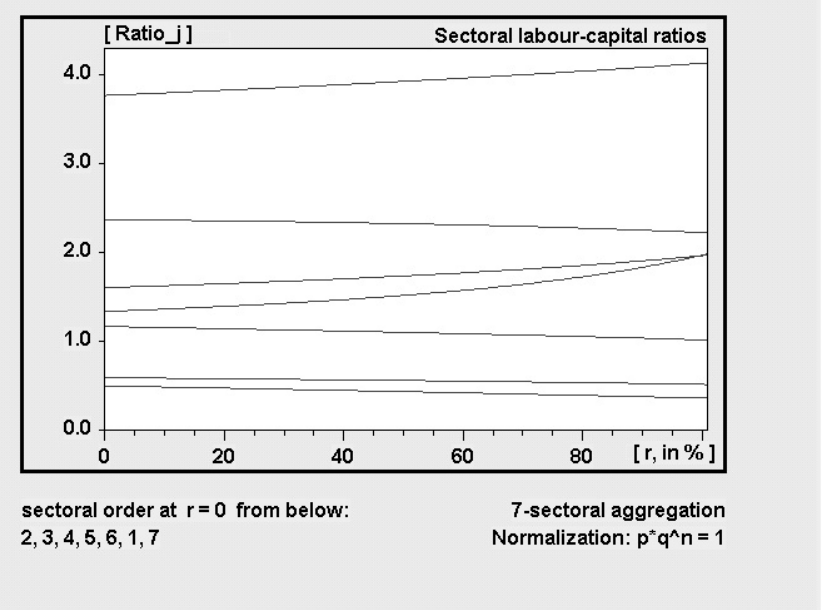

Figure 4.14: The sectoral labor intensities for $p q^{n}=1$ as function of the rate of profit $r$

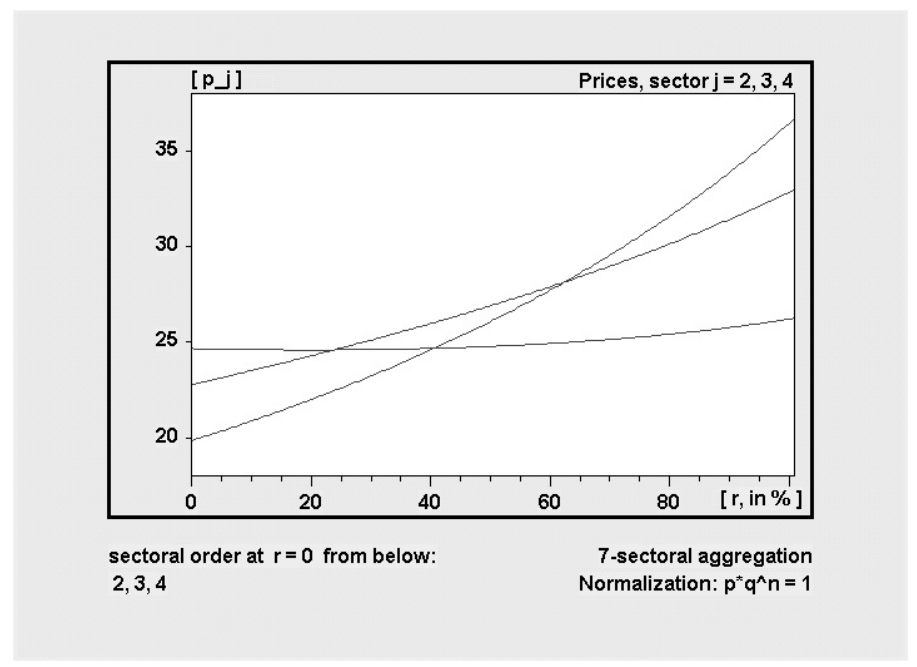

Figure 4.15: The prices of sectors 2-4 for $p q^{n}=1$ as function of the rate of profit $r$

Again, in order to reestablish a uniform rate of profit, prices in deficit industries rise and prices in surplus sectors fall. Nothing else 
is required after a change in the real wage at all admissible levels of $w$. From this perspective, choosing the Standard Commodity as the numéraire makes the analysis of price movements resulting from changes in $w$ neither simpler, nor more complicated.

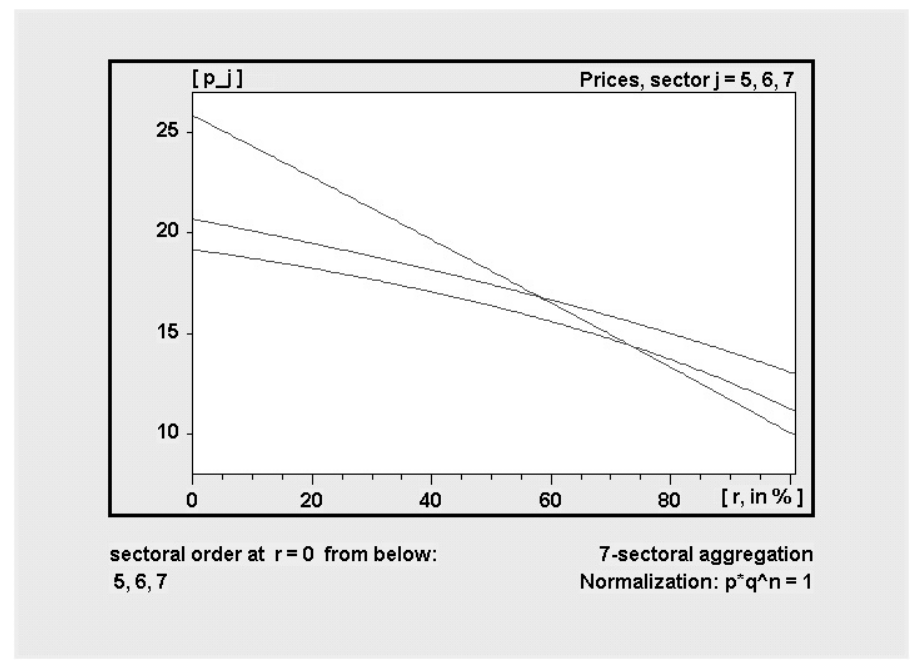

Figure 4.16: The prices of sectors 5-7 for $p q^{n}=1$ as function of the rate of profit $r$

We conclude that the movement in prices of production associated with empirically observed IO tables is far less dramatic than suggested by purely theoretical considerations and it not easier to understand if the Standard net product is used instead of the actual net product as the numéraire. ${ }^{11}$

4.7.2 Relative labor intensities: Numéraire-free investigation of changes in income distribution

In our framework, the "capital intensity" of sector $j$ is the ratio of the value of intermediate inputs to the direct labor input. Formally, given a price vector $p$, it is ${ }^{12}$

$$
k_{j} \equiv(p A)_{j} / l_{j}
$$

${ }^{11}$ For a detailed discussion, see Flaschel (1986). For a counterpoint, see Schefold (1986).

${ }^{12}$ In this subsection, in order to simplify the empirical calculations we only consider intermediate or circulating capital goods by setting $\delta=1$. 
In order to interpret these ratios, we need the concept of an average capital intensity $\bar{k}$, which can be computed from the sectoral intensities weighted by their sectoral output (identified for simplicity with the empirical outputs $x^{95}$ of our reference year 1995). Hence,

$$
\bar{k} \equiv p A x^{95} / l x^{95} \text {. }
$$

Besides allowing us to classify the $k_{j}$ as being relatively high or low, $\bar{k}$ is also useful in order to compare individual values of $k_{j}$ arising from different price vectors. For the intensities $k_{j}$ depend on the normalization of prices, and therefore cannot be compared directly. If, however, they are divided by $\bar{k}$, it is possible to detect any substantial changes arising from variations in the price vector.

As a first step, the capital intensities arising from the empirical prices $p=p^{95}=(1,1, \ldots, 1)$ may be compared with those brought about by the production prices $p=p(\tilde{r})$, when we plug the empirical average rate of profit $\tilde{r}=45.50 \%$ into the production price equations. Though the (relative) components of the two price vectors differ considerably, their impact on the sectoral capital intensities is quite limited. In particular, the sectoral ordering of the $k_{j}$ remains unaffected. Numerically, the ratios $k_{j} / \bar{k}$ are:

$$
\begin{array}{lllllllll}
p=p^{95} & : & 0.49 & 2.46 & 1.91 & 1.01 & 0.97 & 0.76 & 0.32 \\
p=p(\tilde{r}) & : & 0.51 & 2.68 & 2.10 & 1.07 & 0.79 & 0.69 & 0.30 .
\end{array}
$$

The ratios $k_{j} / \bar{k}$ do not change significantly either, when we vary production prices over the whole range of the uniform rate of profit. Figure 4.17 depicts all seven sectoral ratios as a function of $r$. The curves do not intersect, except in one case which is almost negligible. As shown by the third and fourth line from the bottom, the ordering of sectors 5 and 6 is reversed as $r$ approaches its maximum profit rate $r^{\max }=100.93 \%$. For any $r$ between 0 and $99.30, k_{5}$ always exceeds $k_{6}$. From then on $k_{6}$ is slightly above $k_{5}$, but the difference is so small that it is hardly visible in the diagram. For example, for $r=100.92 \%$ : $k_{5} / \bar{k}=0.585<0.590=k_{6} / \bar{k}$. As Figure 4.17 shows, by and large sectors 7, 1, 6, and 5 remain deficit sectors in Sraffa's words (capital intensities remain below 1 ) whereas sectors 4,3 , and 2 are surplus sectors (capital intensities stay above 1) as the profit rate changes.

Finally, as in Section 3.4, we consider the price changes following from changes in the general rate of profit. Figure 4.18(a) shows that the prices of all deficit sectors $(7,1,6,5)$ rise as a percentage of the total 


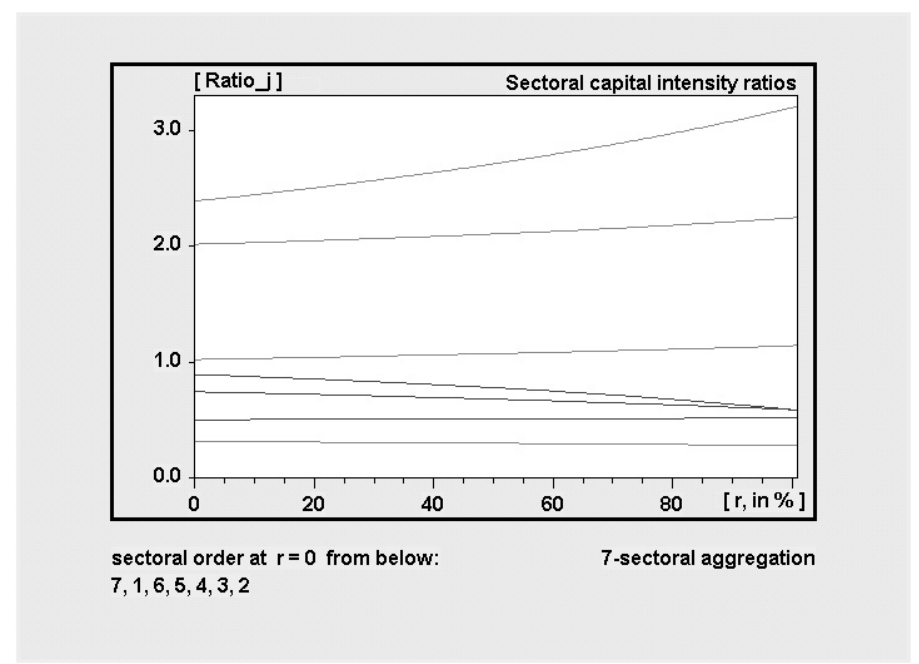

Figure 4.17: Sectoral capital intensities $k_{j}$ as ratios of $\bar{k}$ as a function of the profit rate

price sum (=100), suggesting that Sraffa's chapter 3 consideration of a possible reversal in price movements is not supported by this empirical example. Due to their higher labor intensity, deficit sectors lose in terms of relative profitability when wages increase and thus must raise their output prices in order to obtain a rate of profit equal to the surplus sectors. Figure 4.18 (b) shows that the opposite unambiguously holds for the surplus sectors 2,3 , and 4 with low labor costs relative to capital costs and thus smaller reductions in profitability as compared to the deficit sectors. The relative weight in the price sum therefore declines.

\subsection{The choice of numéraire: The foredoomed search for an invariable measure of value}

In his Introduction to Ricardo's Principles, Sraffa argues that

The search for what has been called 'the chimera of an invariable standard of value' preoccupied Ricardo to the end of his life. However, the problem which mainly interested him was not that of finding an actual commodity which would accurately measure the value of corn or silver at different times and places; but rather that of finding the conditions which a commodity would have to satisfy in order to be invariable in value (Sraffa 1970, xli).

Flaschel (1986) has demonstrated that Sraffa (1960, Ch. 3) has 


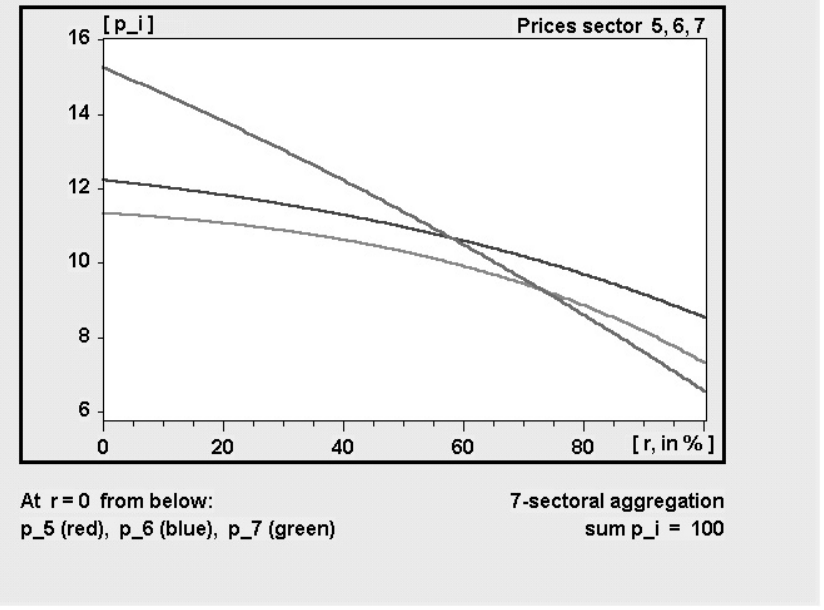

(a) Prices $p_{5}, p_{6}, p_{7}$ from Table 3.5

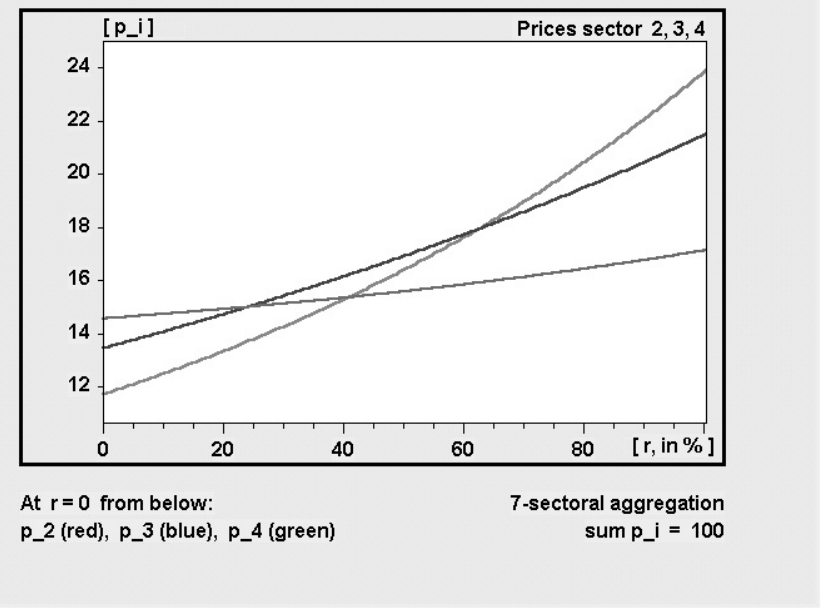

(b) Prices $p_{2}, p_{3}, p_{4}$ from Table 3.5

Figure 4.18: Prices from Table 3.5 
identified "the conditions which a commodity would have to satisfy in order to be invariable in value." But the specific commodity identified in Sraffa (1960, Ch. 4) - the Standard Commodity - does not satisfy this very list of conditions. This discrepancy was not noticed by Sraffa and his followers and, as a consequence, the properties of an "invariable measure of value" were ascribed to a "commodity" which in fact is not "more invariable' in value with respect to changes in income distribution than any other commodity. Ricardo's dream of an "invariable measure" of value (Pasinetti 1977, 120) is not fulfilled by Sraffa's Standard Commodity.

In this section, we analyze this topic from a more pragmatic point of view than in Flaschel (1986). We shall simply compare three ways of normalizing prices of production with respect to their properties for analyzing changes in income distribution with a given technology. As in the previous section, we find that Sraffa's Standard Commodity does not make the situation more transparent compared to other choices of numéraire, such as a normalized consumption basket representing workers' consumption per unit of employment, or the net output vector of the economy per unit of employment.

\subsubsection{The choice of numéraire: Theoretical issues}

At the end of Section 3.3, we briefly considered a general $n$-good economy and the price of production equation $p=(\delta+r) p A+w l$, $p b=1$, normalized by means of a nonnegative consumption basket $b^{\prime}=\left(b_{1}, \ldots, b_{n}\right) \geq 0$. The real wage rate $w$ therefore represents the level at which this consumption basket may be consumed. In this case, we have: ${ }^{13}$

$$
p=w l(I-(\delta+r) A)^{-1}, \quad w=\frac{1}{l(I-(\delta+r) A)^{-1} b} .
$$

Then, for a given (actual) net output vector $y=(I-A) x$, with $l x=1$, the value of (per capita) national product (or income) is:

$$
p y=\frac{l(I-(\delta+r) A)^{-1} y}{l(I-(\delta+r) A)^{-1} b}=l(I-(\delta+r) A)^{-1} y .
$$

Like the wage-profit curve $w(r), p y$ also exhibits a significant nonlinear dependence on the rate of profit $r$, depending on the extent to which the net output basket $y$ differs in structure from the consumption basket $b$. In this case, the situation shown in Figure 4.19 may emerge. Note that

${ }^{13}$ In this section, we consider again the general case with $\delta$ possibly different from one. 
$w(0)$ and $p(0) y$ must both be equal to one at the rate of profit $r=0$, due to the normalization of the gross activity vector. ${ }^{14}$

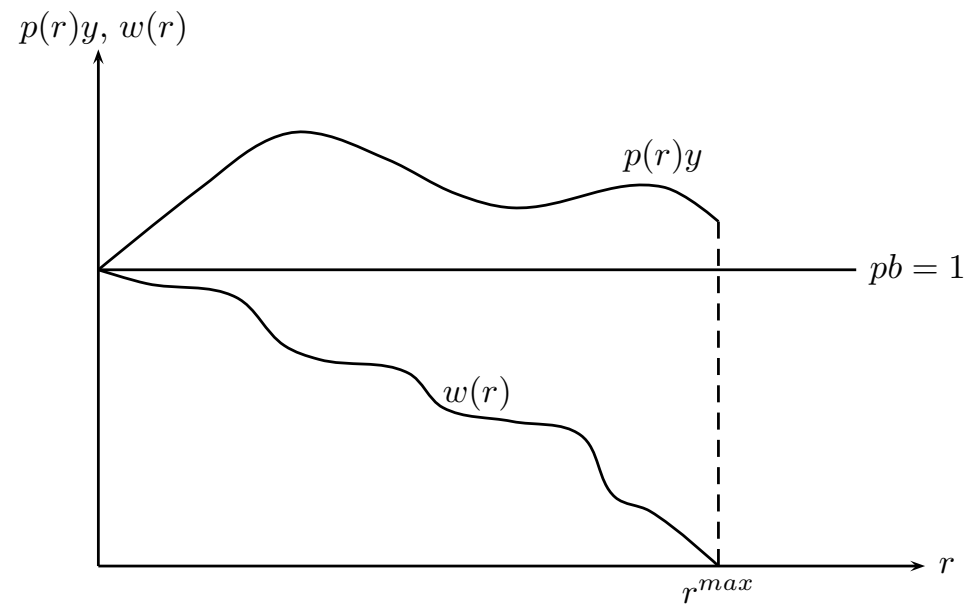

Figure 4.19: National income and the wage-profit curve $\left(l(I-\delta A)^{-1} b=1=p b\right)$

In terms of the basket $b$ everything is well defined from a mathematical perspective. However, neither the wage share nor the profit share can be easily identified by visually inspecting Figure 4.19; only the rate of profit has a clear empirical meaning as a standard variable in national accounting systems. Assuming $b$ to be the normalized consumption basket of workers however provides us with the number of such baskets $w(r)$ that can be consumed by the workforce. We call this the "uses approach" to the question of income distribution as far as the interpretation of the real wage $w$ is concerned.

This scenario changes significantly if we take actual net output $y=$ $x-A x, l x=1$ as the numéraire commodity as shown in Figure 4.20. Note that $w(0)$ must be equal to one at $r=0$, due to the normalization of the gross activity vector.

Figure 4.20 depicts the wage share $w$ and shows that if capital intensities are not uniform then income distribution changes in a nonlinear, but strictly monotone, way with the rate of profit $r$ by the definition of $x$. We call this the "sources approach" to the analysis of income distribution by means of wage-profit curves. The value of the Standard Commodity $q^{n}$ and its change are of no economic importance

$\overline{14}$ This is due to $p(0)=w(0) l(I-\delta A)^{-1},(I-\delta A)^{-1} y=x$. 


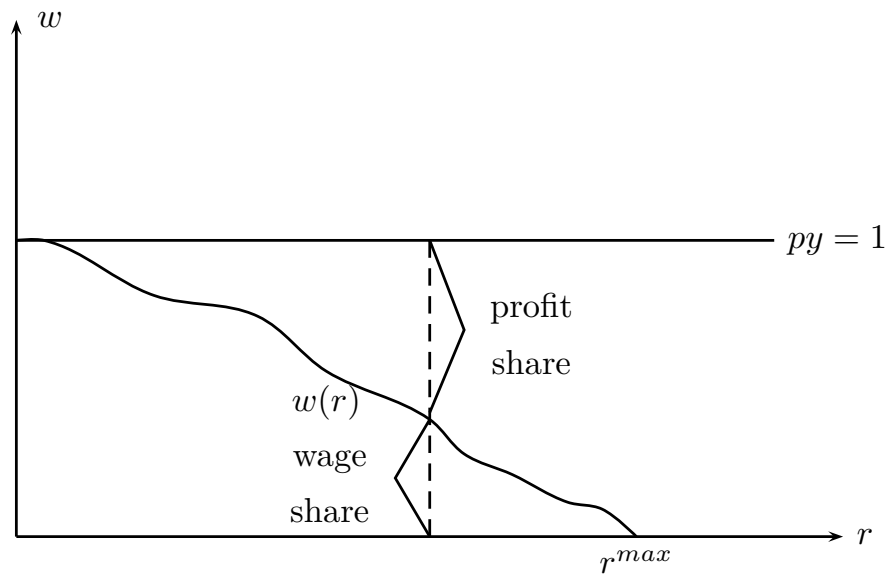

Figure 4.20: Normalizing by net national product $\left(p y=1=l(I-\delta A)^{-1} y\right)$ for varying $r$

in this case $\left(p(r) q^{n}=w(r) r^{\max } /\left(r^{\max }-r\right)\right)$. We note that labor commanded prices $(w=1)$ are not very helpful here, since $w=1$ would then be accompanied by strictly increasing $p(r)$ - values which would not show income distribution changes as clearly as in Figure 4.20 .

Finally, let the Standard Commodity $q$ be defined by $A q=\lambda(A) q$ and normalized by $l q=1$. Let the net Standard Commodity be defined as $q^{n}=(I-\delta A) q$ and suppose that $q^{n}$ is used as the numéraire, so that $p(r)(I-\delta A) q=1$. Then:

$$
\begin{aligned}
& 1=p(I-\delta A) q=r \lambda(A) p q+w \\
& \text { with } \quad p A q=\lambda(A) p q=\frac{\lambda(A)}{1-\delta \lambda(A)}=\frac{1}{r^{\max }}
\end{aligned}
$$

and thus

$$
w=1-(\delta+r) \lambda(A)=1-\frac{\delta+r}{\delta+r^{\max }}=\frac{1}{\delta+r^{\max }}\left(r^{\max }-r\right) .
$$

Hence, the wage-profit curve is a straight line: the change in the wage rate in terms of the Standard Commodity resulting from a given change in the profit rate is the same at all levels of $r$. The severe disadvantage of this renormalization, however, is that it does not tell us much about the 
state of income distribution. The wage rate is measured in terms of the right-hand dominant eigenvector of $A$, which is not very informative. The resulting full picture and income distribution are in Figure 4.21. Note that $w(0)=p(0) y=1$, due to the normalization of the gross activity vector $q$.

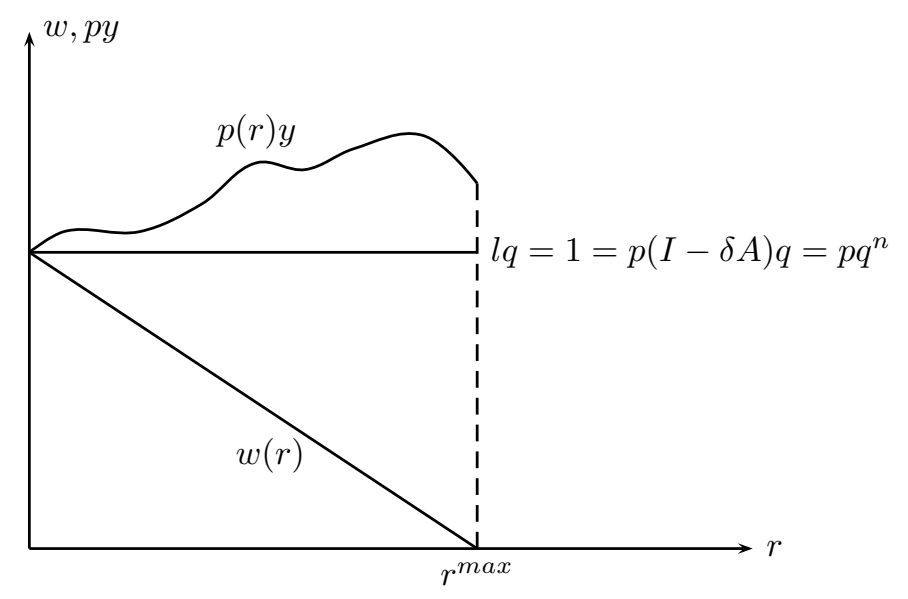

Figure 4.21: The illusion of a linear subdivision of national income

The nonlinearity of the wage-profit curve in the case $p y=1$ has only been shifted onto the $p y$-curve, while $p q^{n}=1$, a fact that is rarely mentioned. The nonlinearity in income distribution is thus by no means removed. Again, Figure 4.21 contains only hidden information on wage and profit shares (as they move with $r$ ) and is thus not at all an improvement as compared to Figure 4.20. The deeper reason for this result - in brief - lies in the fact that Sraffa's Standard Commodity fulfills only some necessary, but not sufficient conditions for an invariable measure of value, or a watershed industry, (see Sraffa (1960, Ch. 3) and Flaschel (1986)). ${ }^{15}$

Figure 4.22 summarizes the situation obtained from using the Standard Commodity $q$ as numéraire for prices $p(r)$. It provides an instructive picture of balanced growth, but it also forcefully shows that the Standard Commodity is not of much help in the pursuit of a simple representation of income distribution, savings and balanced growth. Indeed the search of an invariable measure of value with respect to

${ }^{15}$ See Flaschel (1984) for a more detailed discussion of the choice of a numéraire. 
given production data and changing income distribution may be a purely academic exercise with no actual relevance, and no solution. This conclusion is significantly strengthened if choice of techniques is incorporated, since in this case there is no well-defined, unique Standard Commodity which guarantees the linearity of the alternative wageprofit curves.

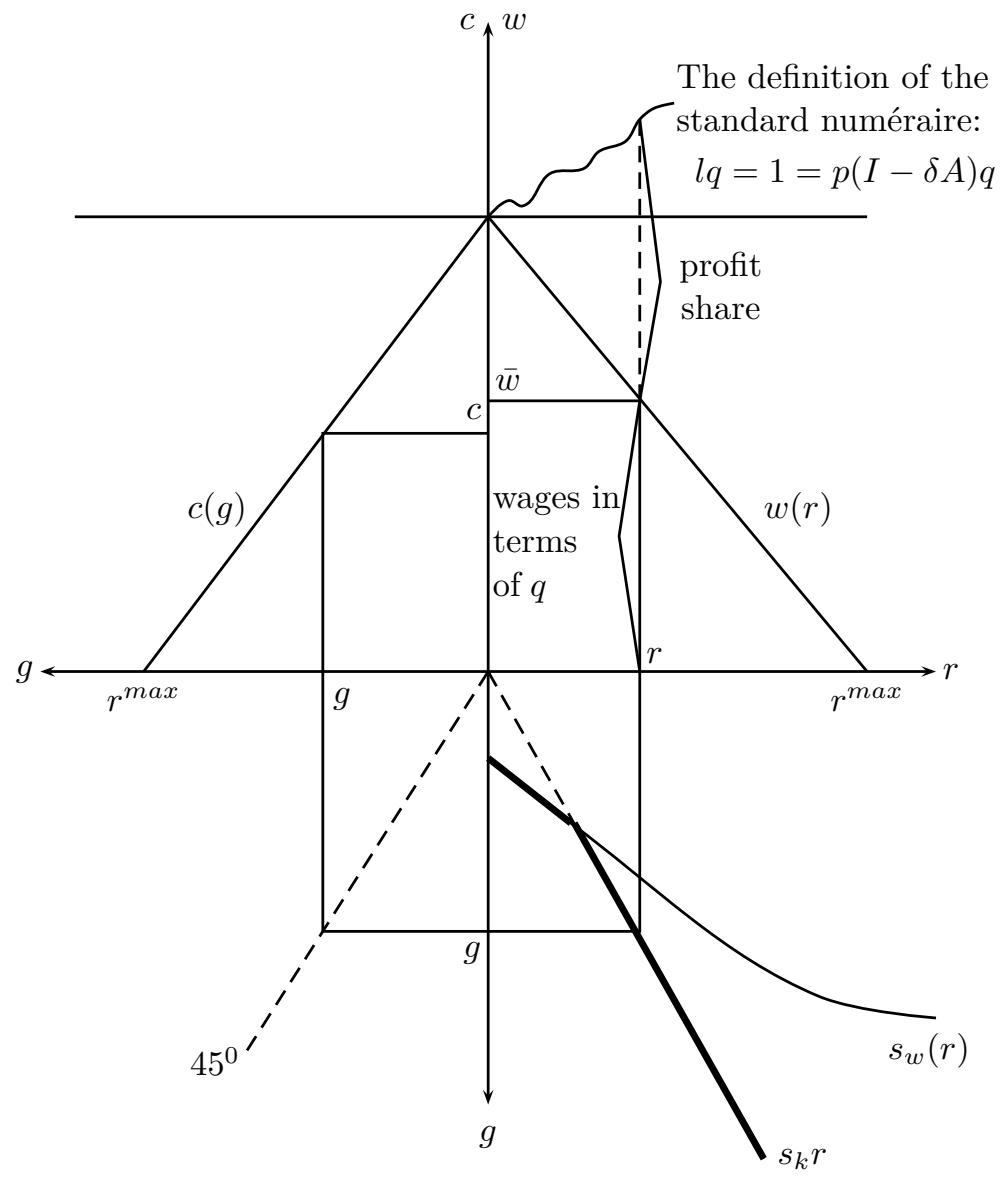

Figure 4.22: A simplification of the theory of income distribution? $\left[0<s_{w}<s_{k} \leq 1\right]$ 
4.8.2 The choice of numéraire: Empirical findings

In this subsection we present the empirical analogs of the alternative theoretical wage-profit curve representations of the previous subsection. We show that wage-profit curves tend to be close to linearity even without using the Standard numéraire and that the normalization of prices by means of py provides the most informative graphical representation, since income shares and their change with the profit rate can immediately be identified. Consider first, however, the schedule of national income and the wage-profit curve for the numéraire $p b=1=$ $l(I-\delta A)^{-1} b$, where $b$ represents the structure of the actual consumption basket of workers (normalized by total labor time expended). The fraction on the vertical axis shows how much of the maximum amount $b$ is indeed consumed by workers on the basis of their wage income (which is entirely spent on consumption goods). Since workers' consumption is the dominant part of the vector of final demands the numéraires $b, y$ provide very similar results in this graphical representation of the income distribution frontier in a given technological environment.

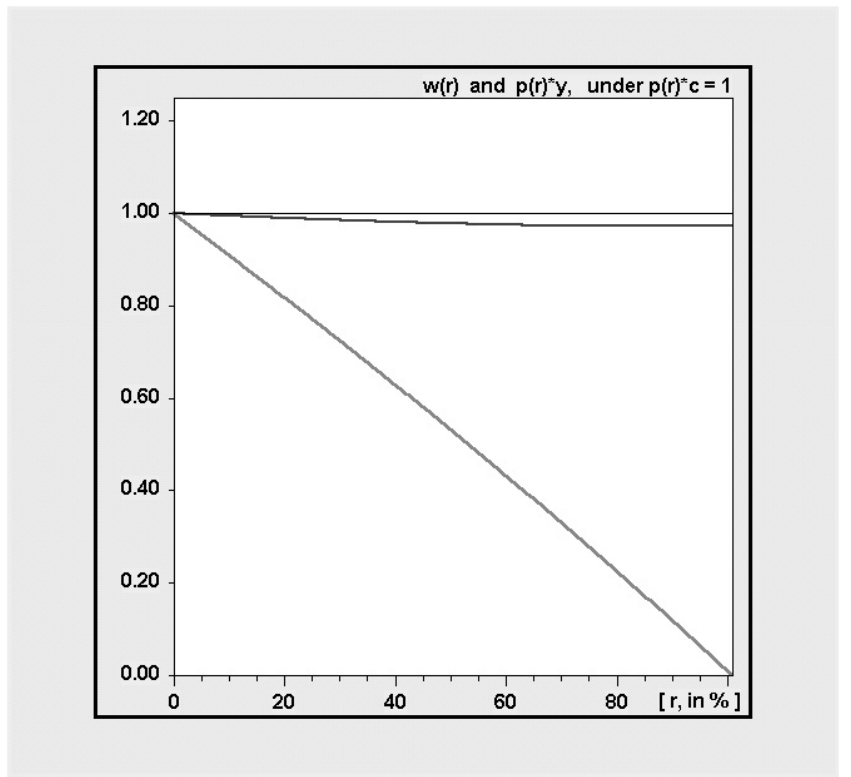

Figure 4.23: Representing income distribution using the workers' consumption basket as the numéraire $(p c=p b=1, p y, w(r)$ from top to bottom)

Next, consider the distributional frontier resulting from Sraffa's 
Standard Commodity. In terms of the Standard net product, both the wage-profit and the national income curves are strictly decreasing, implying that real wages (measured in terms of the Standard Commodity) underestimate the share of wages in national income. However, the wage-profit curve is exactly linear and therefore the change in the profit rate resulting from a given change in real wages is a constant, at all levels of $w$. But since real wages are measured in terms of a commodity reflecting the structure of final demand, if everything is plowed back into production then no real information can actually be obtained.

Finally, in Figure 4.25, we use the net national product as the numéraire commodity. It shows how the value of the standard net product changes, but, again, no useful information is obtained from the observation of its dependence on $r$. By contrast, the $w(r)$ curve- which is slightly nonlinear - shows the shares of wages and profits in national income, after imports have been deducted. In the next subsection, we consider the full physical net production of the economy, without deducting imports, and therefore get an import share in addition to the wage and profit shares. In both situations we have an empirically relevant interpretation of the downward sloping wage curve (and wage plus profit curve), and consideration of the Standard Commodity yields no additional insight.

In summary the Standard Commodity is not of much use in the investigation of price movements and changes in income distribution. This conclusion would have been different, had one established the proposition that Sraffa's analysis of wage changes in the setting $p y=1$ implies invariance for the Standard proportions $l q /(p(r) A q)=$ $1 /(p(r) A q)=\left(r^{\max }+\delta\right) / p(r) q$ between labor and means of production. Yet as we have shown, these proportions do vary with the rate of profit and thus they are not of the critical type discussed earlier.

\subsection{Actual income shares in the open economy}

In an open economy, the technological IO matrix $A_{T}$ is different from the matrix $A^{d}$ of domestic IO coefficients, the net output vector $y$ is

$$
\begin{aligned}
y & =\left(I-A^{d}\right) x, \\
l x & =1=v y, \quad v=l\left(I-A^{d}\right)^{-1}<v_{T}=l\left(I-A_{T}\right)^{-1} .
\end{aligned}
$$

Normalizing total employment at unity in the second equation is not essential but will prove useful below. The matrix $A^{d}$ is not a full 


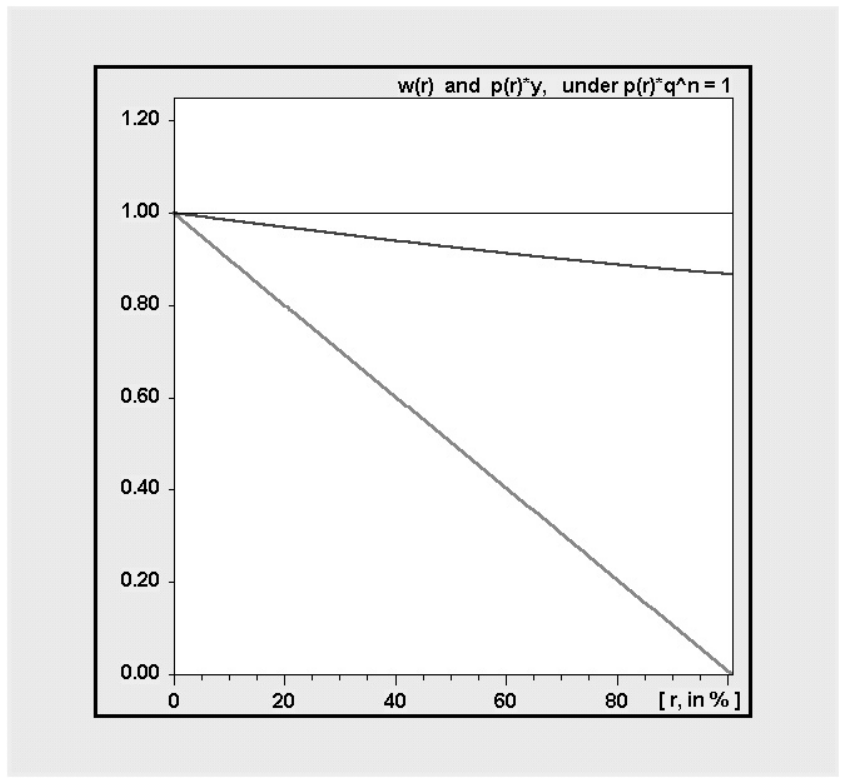

Figure 4.24: Representing income distribution by way of Sraffa's Standard Commodity ( $p q^{n}=1, p y, w(r)$ from top to bottom)

description of the employed technology, but can be used to determine the domestic employment multipliers of aggregate demand increases.

Instead, the production price equations are always based on the matrix $A_{T}$. Here it is convenient to normalize prices such that the national product is unity:

$$
p=(1+r) p A_{T}+w l, \quad p y=1 .
$$

Measuring the real wage rate, too, in terms of net output, we can refer to prices in terms of labor commanded, $p_{w}=p_{w}(r)$, to obtain

$$
w=w(r)=1 / p_{w}(r) y \text {. }
$$

To see this, recall that $p_{w}=(1+r) p_{w} A_{T}+l$. The solution $p$ of equation (4.20) and $p_{w}$ are related by the equation $p=p_{w} / p_{w} y$, if we set $w=$ $1 / p_{w} y$. Since the latter magnitude indicates how many baskets $y$ the nominal wage rate $w$ can buy, the nominal wage rate $w$ and the real wage rate $\omega$ coincide. Formally, $w / p y=w / 1=\omega$. Further, the real 


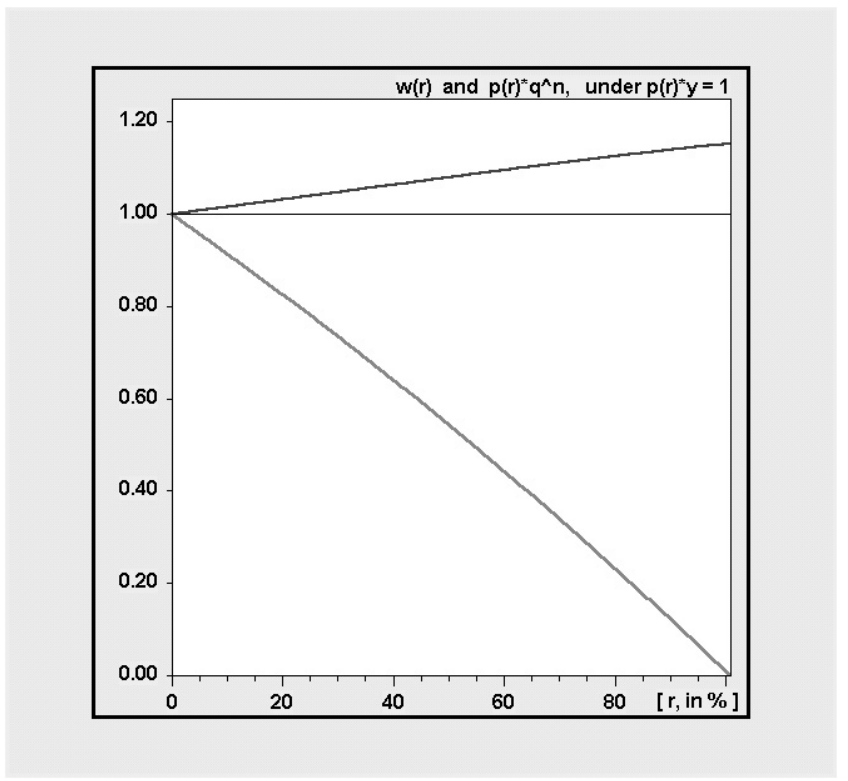

Figure 4.25: The representation of income shares in net national product $\left(p q^{n}, p y=1, w(r)\right.$ from top to bottom)

wage $\omega$ is identical to the wage share $w l x / p y$ in national product if the normalization rule (4.19) for the level of gross output applies.

A consequence of the imports of intermediate inputs and of the above specification of the national product is that the latter is not only distributed among (domestic) workers and capitalists, but part of it must also pay imported inputs. To formalize this, postmultiply equation (4.20) by the vector $x$ and rearrange the resulting equation as $p\left(I-A^{d}\right) x=p\left(A_{T}-A^{d}\right) x+r p A_{T} x+w l x$. Using (4.18), we get

$$
1=\frac{p\left(A_{T}-A^{d}\right) x}{p y}+\frac{r p A_{T} x}{p y}+\frac{w l x}{p y} .
$$

It might be tempting to interpret the first term on the right-hand side of (4.22) as an import share. This expression, however, presupposes that the imported material inputs $\left(A_{T}-A^{d}\right) x$ are valued at the current domestic prices, which would be inappropriate if the prices $p$ were subject to variations. It is therefore clearer to introduce the vector 
$\bar{p}^{i m}$ denoting the prices at which the material imports were actually purchased. ${ }^{16}$

Let $A^{i m}$ be the import matrix, $A^{i m} \equiv A_{T}-A^{d}$. The term $p A^{i m} x=$ $p\left(A_{T}-A^{d}\right) x$ can be decomposed into the actual historical value of the intermediate inputs and gains or losses resulting from the change in the (good-specific) terms of trade, due to the deviations of production prices $p$ from the given import prices $\bar{p}^{i m}$. That is, $p A^{i m} x=\bar{p}^{i m} A^{i m} x+$ $\left(p-\bar{p}^{i m}\right) A^{i m} x$. Therefore:

$$
1=\frac{\bar{p}^{i m} A^{i m} x}{p y}+\frac{\left(p-\bar{p}^{i m}\right) A^{i m} x}{p y}+\frac{r p A_{T} x}{p y}+\frac{w l x}{p y},
$$

where the four ratios on the right-hand side are, respectively, the historical value of imported material inputs in percent of current net product (term 1), ${ }^{17}$ the changes in the terms of trade in percent of the net product (term 2), the profit share (term 3 ), and the wage share (term 4).

The lower line in Figure 4.26 represents the fall of the wage share as the profit rate rises from 0 to $r^{\max }=100.93 \%$. The computation of this curve does not require much extra effort: since labor commanded prices are already available, we only need to compute $y$ in equations (4.18) and (4.19) and subsequently $\omega$ in equation (4.21). Recall that by virtue of the normalization $l x=1$ and $p y=1, \omega=w l x / p y$ coincides with the wage share. The gross output vector employed here is the empirical output vector in 1995.

The upper line in Figure 4.26 is obtained by adding $r p A_{T} x$ to $\omega$ : it represents the sum of the wage and profit shares, which do not sum up to unity. The residual is the sum of terms 1 and 2: for an open economy like Germany it is clearly non-negligible. For example, suppose $r=45.5 \%$. The wage share is $50.85 \%$, and the profit share is $29.28 \%$. Hence, the sum of terms 1 and 2 amounts to $19.87 \%$. It is also apparent that the gap widens as $r$ rises. At $r=0$ it is $11.68 \%$, but at $r=r^{\max }$ it is as high as $34.11 \%$. Since term 1 is a constant, this is due to the change in the structure of the good-specific terms of trade in term 2: the prices

${ }^{16}$ The bar is to point out that $\bar{p}^{i m}$ is treated as a given constant. Because in the IO tables all real magnitudes are based on the 1995 -prices $(1,1, \ldots, 1)$, $\bar{p}^{i m}$ is proportional to the summation vector. The proportionality factor itself depends on the normalization of $p$. Concretely, we can take it to be equal to $W / W^{e m p}$, where $W=w l x$ is obtained from the production price equations and $W^{\text {emp }}$ are empirical total wages.

17 The expression "current net product" is a shorthand for "national product valued at production prices". 


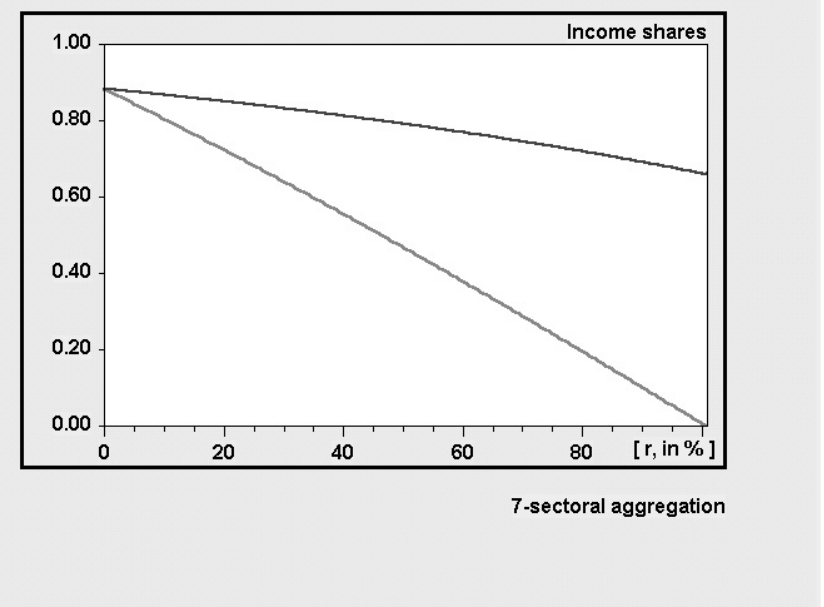

Figure 4.26: Wage (lower line) and wage-plus-profit (upper line) shares in national product proper: $y=x-A^{d} x$

$p_{i}$ of goods $i$ with a relatively high component $\left(A^{i m} x\right)_{i}$ tend to rise, whereas those of goods with a relatively low component $\left(A^{i m} x\right)_{i}$ tend to fall. ${ }^{18}$

\subsection{Substitution and the choice of technique}

\subsubsection{Two-physical-inputs approaches}

In this section we allow for more than one technique in the production of the investment and the consumption good ( 1 and 2 respectively). We assume a given set of "blueprints" for both production activities, represented by

$$
\begin{aligned}
& {\left[\begin{array}{ccccccc}
a_{11}^{1}, & a_{11}^{2}, & a_{11}^{3}, \ldots ; & a_{12}^{1}, & a_{12}^{2}, & a_{12}^{3}, \ldots \\
0, & 0, & 0, & \ldots ; & 0, & 0, & 0,
\end{array}\right]} \\
& \left(l_{1}^{1}, l_{1}^{2}, l_{1}^{3}, \ldots ; l_{2}^{1}, l_{2}^{2}, l_{2}^{3}, \ldots\right),
\end{aligned}
$$

${ }^{18}$ Recall that because of $p y=1$, not all prices can change in the same direction. 
where $a_{i j}^{\zeta}$ is the amount of good $i$ necessary to produce one unit of good $j$ using blueprint $\zeta{ }^{19}$ In principle, every activity in sector 1 can be combined with any activity available for sector 2 , and the vector of activity levels can be written as $x=\left(x_{1}^{1}, x_{1}^{2}, \ldots, x_{2}^{1}, x_{2}^{2}, \ldots\right)$ where $x_{j}^{\zeta}$ is the output of good $j$ produced using technique $\zeta$. Yet some combinations of such activities will not be profit maximizing. To substantiate this claim we proceed as follows.

Suppose choice of technique is possible only in sector 1 (the basic sector of machine production). Following Pasinetti (1977, Ch. 7) we assume cost-minimization as the criterion of choice which is equivalent to profit rate maximization (for a given numéraire and a given wage rate) as well as wage rate maximization (for a given numéraire and a given profit rate). According to Pasinetti $(1977,158)$, the resulting wage-profit frontier - the outer envelope of all wage-profit curves obtained from the alternatives of producing commodity 1- has some properties that can be illustrated in Figure 4.27 in the case of three techniques.

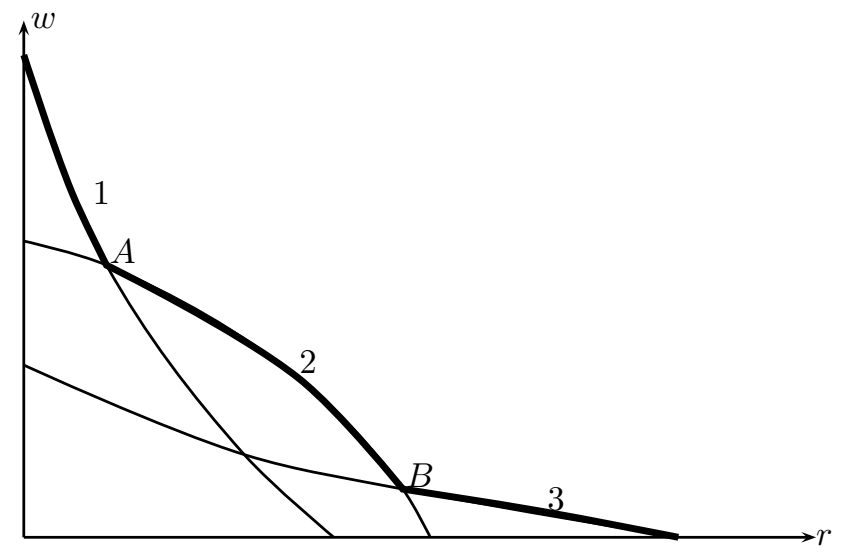

Figure 4.2\%: The wage-profit frontier of three alternative techniques in sector 1 and a single technique in sector $2\left(p_{2}=1\right.$ in all three cases, $k=a_{1} / l$ )

${ }^{19}$ We assume a uniform rate of depreciation across all activities. 


$$
\begin{aligned}
A^{1} & =\left[\begin{array}{cc}
a_{11}^{1} & a_{12} \\
0 & 0
\end{array}\right], A^{2}=\left[\begin{array}{cc}
a_{11}^{2} & a_{12} \\
0 & 0
\end{array}\right], A^{3}=\left[\begin{array}{cc}
a_{11}^{3} & a_{12} \\
0 & 0
\end{array}\right], \\
l^{1} & =\left[\begin{array}{ll}
l_{1}^{1} & l_{2}
\end{array}\right], l^{2}=\left[\begin{array}{ll}
l_{1}^{2} & l_{2}
\end{array}\right], l^{3}=\left[\begin{array}{ll}
l_{1}^{3} & l_{2}
\end{array}\right]
\end{aligned}
$$

Figure 4.27 shows that $\left(A^{1}, l^{1}\right)$ is the most profitable technique at high wage rates $w$ (or low profit rates $r$ ). If the wage rate decreases sufficiently the economy switches to $\left(A^{2}, l^{2}\right)$, and so on. The long-run positions of income distribution are described by the outer envelope of all wage-profit curves.

Concerning such technological frontiers of income distribution possibilities Pasinetti (1977, 158-159) claims: ${ }^{20}$

1. At the switch points A or B in Figure 4.27, each commodity has the same price independently of whether it is produced by the technique to its left or to its right in the set of available techniques (which includes linear combinations of these activities).

2. If one of two techniques from the given technology is more profitable than the other (for given numéraire and real wage), it will yield prices in terms of labor commanded, that are strictly lower than those yielded by the other technique: $r^{i}>r^{j} \Rightarrow p_{w}^{i}>p_{w}^{j}$.

3 . Comparisons in terms of the $w(r)$-curve are independent of the numéraire used for the price system, i.e. changing the numéraire will change the slopes of the wage-price curves, but not the order in which the various techniques appear on the wage-profit frontier. In particular, the resulting switch points remain of the same type.

4. The wage-profit frontier is strictly decreasing, just as the wageprofit curves (which may be infinite in number) of which it is composed.

Further, setting $p_{1}=1$, changes of technique in the nonbasic sector (good 2) only modify the price of this commodity, but not the wageprofit curve as long as our general assumption $\lambda\left(A_{11}\right)>\lambda\left(A_{22}\right)$ holds, which in our model is equivalent to $a_{11}>a_{22}=0$. If, in such a case, $p_{2}=1$ is assumed, the wage-profit curves do change through the way the real wage is measured, but they intersect at the same points and the succession of techniques on their outer envelope - forming the wageprofit frontier - is unchanged.

If technological change only occurs in the sector of basics (good 1$)$, we can operate with linear wage-profit curves if we adopt $p_{1}=1$ as the

${ }^{20}$ The proofs of these assertions are simple and can be found in Pasinetti (1977). 
normalization rule:

$$
w=\frac{1+(\delta+r) a_{11}}{l_{1}} .
$$

The given techniques can therefore appear only once as part of the wage-profit frontier as Figure 4.28 shows.

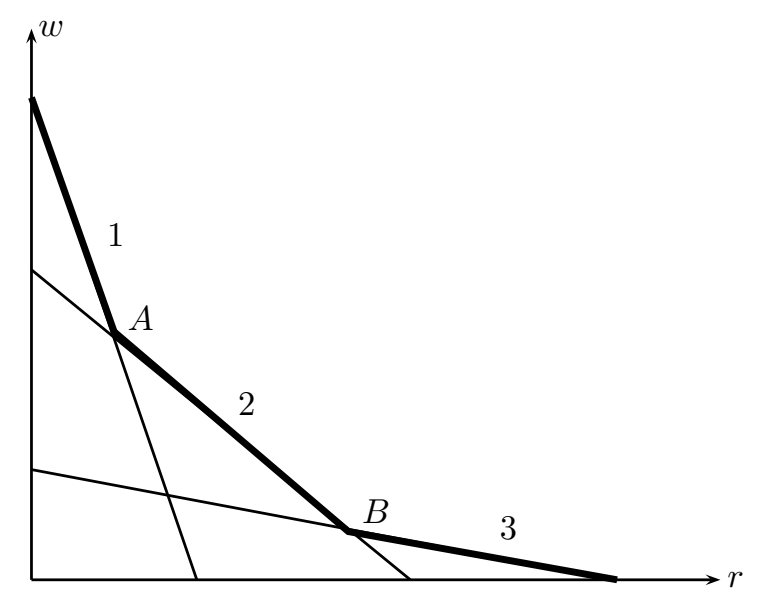

Figure 4.28: The wage-profit frontier for equation (4.24) and $p_{1}=1$

Therefore, despite the nonlinearities shown in Figure 4.27, in the simple case considered here the so-called "1reswitching of techniques" cannot occur: it is not possible for a technique to be profitable at a high level of $w$ and then again at a low level of $w$. This is no longer true if more than one capital good is allowed for, thus increasing the number of commodities. ${ }^{21}$

\subsubsection{One-physical-input simplifications}

Consider the special case of a continuously differentiable neoclassical production function analyzed in Section 2.7. Its unit isoquant $1=$ $F(K, L)$ can also be represented (under certain assumptions) by a function $a_{1}=\psi(l)$ as shown in Figure 4.29. Let $p=1$, and $\delta=0$, the wage-profit curve for each $l$ is given by $w l+r \psi(l)=1$,

$$
w=r \psi(l) / l=r k .
$$

By the envelope theorem, the slope of the resulting wage-profit frontiers must satisfy

$$
w^{\prime}(r)=-\psi(l) / l=-k,
$$

${ }^{21}$ See, for example, Jaeger (1979) for details. 
with respect to the technique actually adopted. This is shown in Figure 4.30 .

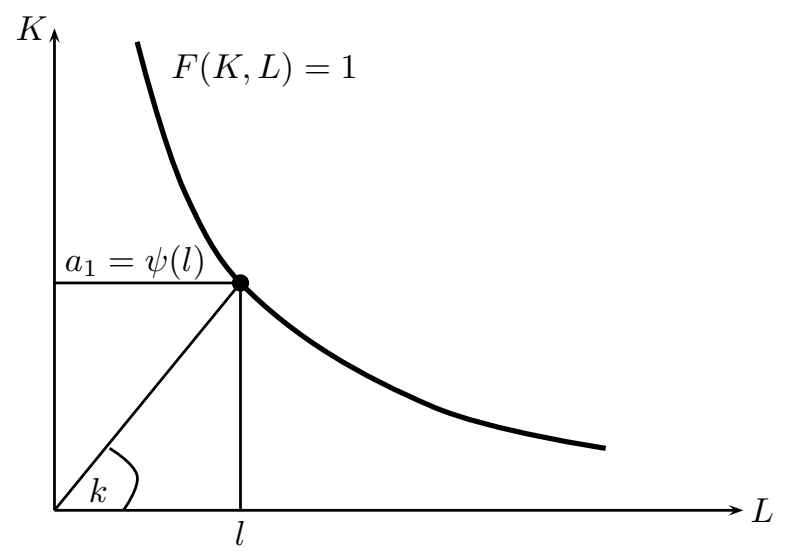

Figure 4.29: Smooth factor substitution in the one-good case $\left(\delta=0, k=a_{1} / l\right)$

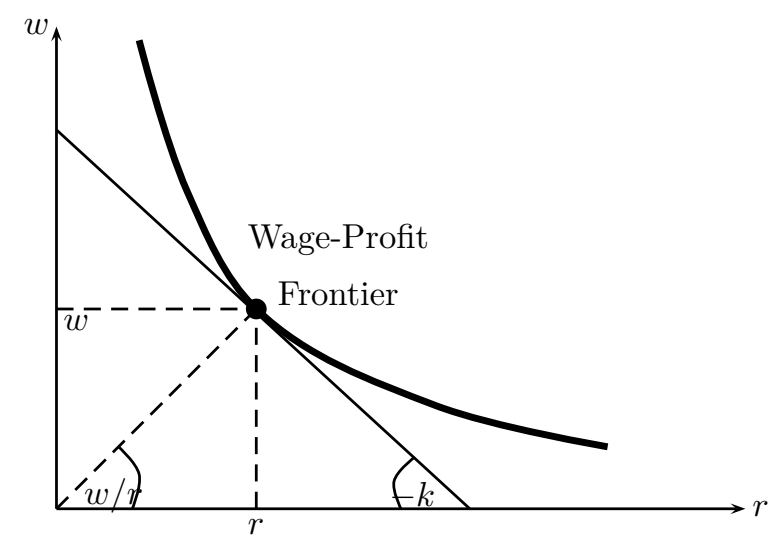

Figure 4.30: Each single technique is in operation at exactly one point of the enveloping strictly convex wage-profit frontier $\left(w^{\max }=1 / l, r^{\max }=1 / a_{1}\right)$

With respect to $p y=w+r k$, along the envelope we have

$$
d(p y)=d w+k d r+r d k
$$


i.e. $d(p y) / d k=r$ due to $d w=-k d r$. Therefore, in the one-good case along the wage-profit frontier the change of the net product relative to the change in the capital stock (both per worker) is always equal to the rate of profit. This is an alternative representation of the Solow growth model of Section 2.7, in which $w=F_{L}(k, 1)=F_{L}(K, L) \Longleftrightarrow$ $F_{K}(K, L)=F_{K}(k, 1)=r$ (assuming constant returns to scale). For any given wage rate, choosing the maximum rate of profit from the existing wage-profit curves (and thus a point on the wage-profit frontier) implies that the profit rate must equal the marginal product of capital in this case. Thus the characterization of the process of income distribution of the Solow growth model, shown in Figure 4.30, seems to apply to a Ricardian world as well, i.e. the neoclassical wisdom that rising wages (profit rates) will be associated with rising (falling) capital intensities seem valid also in a one-good Ricardian world. The relationship

$$
\frac{K}{L} \uparrow \Longleftrightarrow \frac{w}{r} \uparrow
$$

between relative factor usage and relative factor reward, so plausible from the perspective of a single firm, thus also seems to apply to the whole economy in a Ricardian (and not only a neoclassical) environment. ${ }^{22}$

In the earlier example with two goods, with one (basic) investment and one (nonbasic) consumption good, this result also seems to hold, because the wage-profit curves are linear if $p_{1}=1$ is used to normalize prices. Assuming for the sector of basics $a_{11}=\psi\left(l_{1}\right)$ as in the one-good case thus appears to lead to the same conclusion. Yet, we have to use $p_{1} k$ now instead of $k(r)$ and also obtain the functional relationships $p_{1}(r), k(r, g)$, since $p_{1}(r), k(g)$ holds for any given technique in the technology set. In particular

$$
d w=-p_{1} k d(p y)
$$

does not hold in general - not even for a single wage-profit curve - so that

$$
d(p y)=d w+p_{1} k d r+r d\left(p_{1} k\right)
$$

(for $k=k(g))$ does not in general reduce to

$$
d(p y)=p_{1} k d r .
$$

Hence, the marginal productivity rule is not generally valid in

${ }^{22}$ See Weizsäcker (1971, II.1) for further details. 
a Ricardian framework with more than one homogeneous good. Moreover, the possibility of reswitching of techniques in the case of two, or more, basic commodities raises further doubts on the existence of a strictly positive relation between $K / L$ and $w / r$, despite the fact that wage-profit frontiers are determined solely by the basic sectors.

With this result in mind we turn to a classical representation of full long-period positions in economies where substitution is allowed for, without any restrictions imposed on the value of marginal productivities in equilibrium. We consider again the case of one basic and one nonbasic commodity. Analogous to Figure 4.5 above we now obtain Figure 4.31 (where $\kappa=1$ for simplicity at the point of the envelope considered).

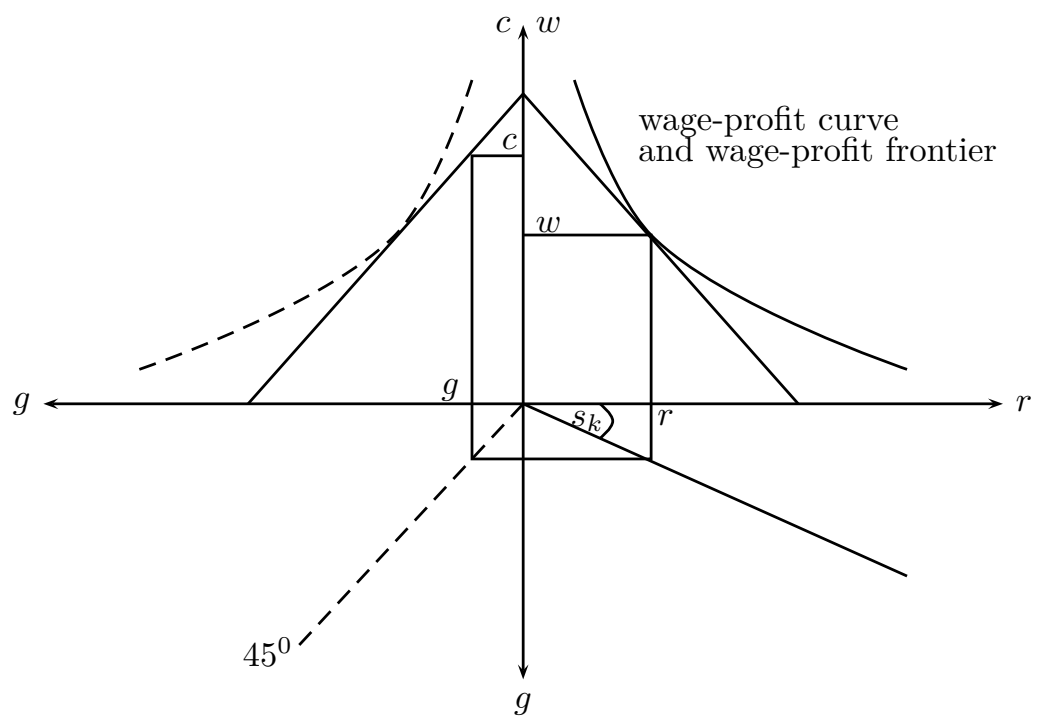

Figure 4.31: The classical closure and the choice of technique

The consumption-growth frontier (the outer envelope of the consumption-growth curves) provides no information concerning optimal techniques for a given real wage $w$ and savings propensity $s_{k}$. The choice of technique is decided in the upper right-hand part of Figure 4.31, through profit rate maximization, and this need not correspond to a point on the consumption-growth frontier. A social planner could realize a higher $c$ for any given $g$ (if $g \neq r$ ) by adjusting the rate of profit to the $(g, c)$-combination selected. But in a free market economy this is not feasible, and the outcome is determined by the 
conflict on the level of $w$, profit maximization and the capitalists' propensity to save. For example, variations in $s_{k}$ will change $g$ and thus $p_{1}(r) k(g)$ without any change in $r$. Both of the observations just made, though, will lose their power if extreme classical saving habits prevail $\left(s_{w}=0, s_{k}=1\right)$.

The next steps of the analysis would be to consider matrices with two basic commodities

$$
A=\left(\begin{array}{ll}
a_{11} & a_{12} \\
a_{21} & a_{22}
\end{array}\right), \quad l=\left(l_{1}, l_{2}\right),
$$

where at most $a_{22}$ can be assumed as zero. The linearity associated with the normalization rule $p_{1}=1$ will no longer obtain, and reswitching of techniques may occur even in the simple two-good case. Further, in the case with $n$ goods (with $p b=1, l x=1$ ) we have

$$
w=\frac{1}{l(I-(\delta+r) A)^{-1} b}, \quad c=\frac{1}{l(I-(\delta+g) A)^{-1} b},
$$

implying that fairly complicated wage-profit frontiers may arise from the existence of multiple techniques.

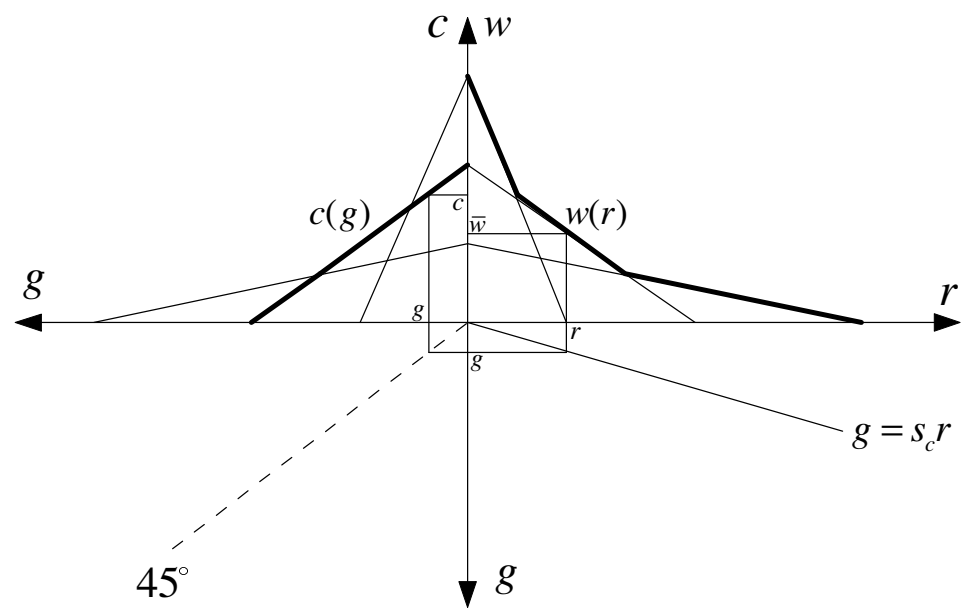

Figure 4.32: The classical closure of the two degrees of freedom in the case of substitution for $\kappa>1, \kappa=1, \kappa<1$

Nevertheless, the following accounting identities hold

$$
p y=w+r p A x=c+g p A x,
$$


if $l x=1$ and $p b=1$ hold, since

$$
w l+(\delta+r) p A=p \Rightarrow w+r p A x=p(I-\delta A) x=p y,
$$

and

$$
c b+(\delta+g) A x=x \Rightarrow c+g p A x=p(I-\delta A) x_{2}=p y .
$$

Figure 4.32 shows the classical closure in an economy with three alternative techniques and the numéraire $p_{1}=1$ in the two-good economy analyzed thus far. In capitalist market economies, the choice of technique is determined by the $w(r)$ curve and the technique chosen has to be mapped onto the $c$ - $g$ diagram in order to determine consumption $c$ per head, which is larger than workers' consumption $\bar{w}$ per head (given that $\left.s_{w}=0, s_{c}=s_{k} \in(0,1)\right)$.

As a final remark, we note that such a system may also be closed assuming a subsistence wage (or a given wage rate) as in Chapter 1 and the question of determinacy of a long-period equilibrium price and quantity position may also be approached from a Walrasian or a Keynesian perspective (see Marglin (1984) for a thorough discussion).

\subsection{Conclusions}

In this chapter we have considered a Classical manufacturing economy producing one intermediate (investment) good and one consumption good. We have discussed its production price system and its balanced growth path solutions and have shown how they can be combined into a complete description of the economy, given certain assumptions on savings and a certain growth trend (determined by the natural growth rate of the labor force). Then, we have analyzed the issue of an invariable measure of value, and the possibility of factor substitution and choice of technique. We have argued that Ricardo's hope of discovering an objective measuring stick to explain movements in relative prices cannot be satisfied by Sraffa's Standard Commodity. We have shown that choice of techniques casts serious doubt on the neoclassical theory of income distribution.

It is worth stressing that the wage-profit frontier is the result of profit rate maximization and should thus underlie all competitive theories of capital accumulation, regardless of the particular closure of the system-Marxian, neoclassical, or Keynesian. The existence, or lack thereof, of substitution in production does not provide a means for discriminating between alternative approaches. The ex post equality between the marginal product of capital and the rate of profit is simply a consequence of profit rate maximization (in a one-dimensional setup) 
and thus neither an advantage nor a disadvantage of a specific approach to balanced growth and uniform profitability (Marglin 1984).

More broadly, this chapter shows how progress from the simple economies considered in Chapters 2 and 3 to a more detailed investigation of price and quantity magnitudes sheds light on many key topics in Classical economics, and in particular on distributive conflict. However, we have not yet investigated such economies from the perspective of a System of National Accounts (and its 'real' magnitudes) as was sketched in Chapters 1-3. The question of what occurs behind the nominal magnitudes that drive the actions of economic agents remains open for now. The Ricardian foundations laid in this chapter represent a starting point for the more thorough analysis of the distributional conflict between labor and capital developed in Part II. 



\section{The von Neumann-Sraffa Model}

In the previous chapters, we have examined classical production prices in linear models in which each industrial sector produces a single output - also known as the basic Leontief model. We have proved that, under some general assumptions on technology, production prices are well-defined, unique and strictly positive. We have interpreted these production prices as a long-period equilibrium: prices of production emerge when capitalist profit-maximizing behavior, and workers' competition for jobs, ensure that a uniform profit rate and a uniform wage rate emerge in all sectors. Furthermore, under some additional mild assumptions, all sectors are operated in a classical long-period equilibrium.

How robust are these results? Are they confined to the basicand arguably rather special - Leontief model, with single outputs, no fixed capital, a single type of homogeneous labor? Or can we extend the classical theory of production prices to more general economies? Furthermore, if production prices are conceived of as the equilibrium, resting position of the classical economy, is this equilibrium stable? In other words, do production prices actually emerge as the outcome of dynamic competitive processes that characterize capitalist economies?

This chapter extends the analysis of Chapter 4 to explore the von Neumann model with joint production and multiple activities and to examine the dynamics of price and quantity magnitudes in the economy. We first define the balanced growth path-or von Neumann/Sraffa equilibrium - of these economies, in which a uniform rate of profit, a uniform wage rate and a uniform growth rate emerge in all sectors. We show that under rather general assumptions on technology, a von Neumann/Sraffa equilibrium exists and the main insights of the basic Leontief economy can be generalized.

We then analyze a model of the gravitation of market prices and quantities around, respectively, classical prices of production and

the balanced growth path. The dynamics of the classical model can 
be formulated in a very general way, in a rectangular system with joint production that allows for both process extinction and product extinction in general $n$-good economies. Process extinction occurs when some production processes are not operated in the equilibrium of the classical economy. Product extinction occurs when the long-period equilibrium price of a certain commodity is zero.

We show that, unlike in the basic Leontief model, both process and product extinction can emerge in the von Neumann growth equilibrium. Furthermore, the so-called "cross-dual" dynamics of prices and quantities need not be convergent and even when the system does converge, and Sraffa/von Neumann production prices can be interpreted as the centers of gravity for market prices, convergence may take a long time.

\subsection{The von Neumann growth model}

In Chapters 1-4 we considered only square unit output matrices $B=I$ : each sector produces a unique commodity with a single production method. We now consider a general system of (pure) joint production, allowing for a multiplicity of production techniques even if there is no joint production. As, for example, in a sector which produces a single commodity $j$ by means of $k(j)$ production techniques, i.e. activity vectors $A_{* j_{1}}, \ldots, A_{* j_{k(j)}}$. This special case of multiple production techniques would then be represented by the partial IO structure

$$
\left(A_{* j_{1}}, \ldots, A_{* j_{k(j)}}\right) \mapsto\left(e_{j}, \ldots, e_{j}\right),
$$

where $e_{j}^{\prime}=(0, \ldots, 0,1,0, \ldots, 0)$ is the $j$-th vector in the canonical basis of $\mathbb{R}^{n}$. In general we consider nonnegative input and output matrices $A, B$ with $m$ columns (representing $m$ different activities) and $n$ rows (representing $n$ different commodities). In principle we allow for the possibility that every process produces every commodity $(B>0)$, with the only restriction that the number of such processes be finite. In IO analysis, the input matrix $A$ and the output matrix $B$ are called absorption and make matrices, respectively.

Empirically, in the firms' accounting practices and in the IO tables of the SNA, the natural accounting unit is one year. Hence we do not follow the practice of von Neumann models to assume a fictitious period of production (of which all real periods of production are a multiple or of which it is a common divisor). This modeling technique would imply the introduction of semifinished products after each unit period and their treatment as marketed commodities, which they are not. We consider the period of production as a non-technological-indeed an 
accounting - concept which provides information on costs and proceeds (inputs and outputs) on a calendar basis. There is no real alternative to this conventional timing of inputs and outputs, for there is no common unit for the production period of the $n$ commodities in real life. ${ }^{1}$

Finally, it is important to stress that we do not consider fixed capital, although the Sraffa-von Neumann model does provide a theoretical framework to incorporate fixed capital. In the Sraffa-von Neumann model machines used in production and not completely depleted in the current production period are considered as jointly produced output of the production process. Yet, as shown below, using $(B, A, l)$ in the production price equations implies treating capital goods as if they were outputs that can be traded on perfectly competitive markets at given prices-empirically, a rather unrealistic assumption. Instead, in Part II of the book we follow established accounting practices of firms and in the SNA, and will introduce an appropriate capital stock matrix.

Having defined its range of applicability, we can now outline the Sraffa-von Neumann model. Let the input and output matrices $A=$ $\left(a_{i j}\right), B=\left(b_{i j}\right), i=1, \ldots, n, j=1, \ldots, m$, represent the unit intensities of a linear technology where $a_{i j}$ is the quantity of good $i$ required per unit intensity of technique $j$ and $b_{i j}$ the quantity of good $i$ produced per unit intensity of technique $j$. Let $l_{j}$ be the labor inputs of these techniques and $b_{i}$ the quantity of good $i$ required as subsistence per worker. The matrix $S=\left(b_{i} l_{j}\right), i=1, \ldots, n, j=1, \ldots, m$ is of the same dimension as $A$. Define the augmented input matrix $A^{b} \equiv A+S$ : the consumption of the workforce is included in $A^{b}$ in physical terms, as necessities consumed by workers in each activity. We assume that each technique uses at least one input $\left(A_{\star j} \geq 0\right)$ and that each good can be produced by at least one technique $\left(B_{i \star} \geq 0\right)$ and that labor supply is unlimited and thus does not represent a bottleneck for the solution of the model.

Let $p=\left(p_{1}, \ldots, p_{n}\right)$ be the vector of prices of the $n$ commodities and $x^{\prime}=\left(x_{1}, \ldots, x_{m}\right)$ the vector of intensities at which the given techniques are operated. We are interested in vectors $x$ and $p$ that represent, respectively, a balanced growth path at the common rate $g$ for those goods that are in fact commodities, and a balanced profitability situation with a common profit rate $r$ for those techniques that are operated. This amounts to assuming

${ }^{1}$ Even if such unit existed, it would imply an enormous increase in the dimension of the matrices $A, B$ and in computational intensity. 


$$
\sum_{j=1}^{m} b_{i j} x_{j} \geqq(1+g) \sum_{j=1}^{m} a_{i j}^{b} x_{j}, \quad i=1, \ldots, n,
$$

with $p_{i}=0$ for all $i$ where the inequality is strict, and

$$
(1+r) \sum_{i=1}^{n} p_{i} a_{i j}^{b} \geqq \sum_{i=1}^{n} p_{i} b_{i j}, \quad j=1, \ldots, n,
$$

with $x_{j}=0$ for all $j$ where the inequality is strict. In other words, in a balanced growth path, commodities in excess supply become free goods, and processes with inferior profitability become extinct.

The model can be rewritten in matrix notation as follows:

$$
\begin{aligned}
\left(B-(1+g) A^{b}\right) x & \geqq 0, \quad x \geq 0, \\
p\left(B-(1+g) A^{b}\right) x & =0, \\
p\left(B-(1+r) A^{b}\right) & \leqq 0, \quad p \geq 0, \\
p\left(B-(1+r) A^{b}\right) x & =0 .
\end{aligned}
$$

If $x, p$ solve equations (5.3)-(5.6), then $\alpha x, \beta p$ are also solutions for any $\alpha, \beta>0$. Equations (5.3)-(5.6) therefore involve the determination of $n+m$ unknowns, including the common rate of growth $g$ and the common rate of profit $r$.

5.1.1 von Neumann equilibria: Existence and properties

Let the technology $\left(B, A^{b}\right)$ be productive if there is $x \geq 0$, such that $\left(B-A^{b}\right) x>0$. The next Proposition proves that if technology is productive, the von Neumann model has a well-defined and economically meaningful solution. ${ }^{2}$

\section{Proposition 5.1.}

(1) There is a solution $(x, g, p, r)$ of equations (5.3)-(5.6) which satisfies $p B x>0$ and:

$$
\begin{array}{lll}
p_{i}=0 & \text { if and only if } & (1+g) \sum_{j=1}^{m} a_{i j}^{b} x_{j}<\sum_{j=1}^{m} b_{i j} x_{j}, \\
x_{j}=0 & \text { if and only if } & (1+r) \sum_{i=1}^{n} p_{i} a_{i j}^{b}>\sum_{i=1}^{n} p_{i} b_{i j} .
\end{array}
$$

(2) There holds $g=r>-1$ for any $(x, g, p, r)$ which satisfies equations (5.4) and (5.6), and $p B x>0$.

\footnotetext{
${ }^{2}$ The proof of Proposition 5.1 can be found in Nikaido (1968, 145-147).
} 
(3) If the technology $\left(B, A^{b}\right)$ is productive, then $g=r>0$.

Even if technology is productive, it is not necessarily the case that the common equilibrium rate of growth and profit, $g=r$, is unique. Nikaido $(1968,147)$ provides the following simple example of nonuniqueness:

$$
A^{b}=\left(\begin{array}{ll}
1 & 0 \\
0 & 1
\end{array}\right), \quad B=\left(\begin{array}{ll}
2 & 0 \\
0 & 3
\end{array}\right),
$$

where one has the solutions

$$
\begin{array}{ll}
x^{\prime}=(1,1), & p=(1,0), \quad g=r=1, \\
x^{\prime}=(0,1), \quad p=(1,1), & g=r=2 .
\end{array}
$$

This is a decomposable system of the kind considered in Chapter 1 and thus no sophisticated output matrix $B$ is necessary in order to get non-uniqueness. von Neumann (1937) assumed $A^{b}+B>0$ in order to get the uniqueness of $g=r$ and justified this assumption by means of arbitrarily small additions to the original matrices, for example:

$$
A^{b}=\left(\begin{array}{cc}
1 & 3 \phi \\
2 \phi & 1
\end{array}\right), \quad B=\left(\begin{array}{cc}
2 & 0 \\
0 & 3
\end{array}\right), \quad \phi \approx 0 .
$$

In our view, however, this does not solve the problem, since the solutions will vary dramatically with $\phi$. Therefore, in the next section, we simply assume uniqueness (for square-systems) in order to investigate the stability of the activity vector $x$ and the price system $p$ if the economy is not at a von Neumann equilibrium. Woods (1978, 282 ) briefly states that decomposability (appropriately applied to joint production systems ${ }^{3}$ ) is necessary, but not sufficient for multiple growth rates to occur.

\subsubsection{The case of multiple activities and no joint production}

We now turn to the important special case with multiple activities, but no joint production. In this case we have a rectangular output matrix of the following type

\footnotetext{
3 The IO structure $A, B$ may be called decomposable if there is a proper subset of goods that can be produced by using only inputs from this proper subset.
} 


$$
B=\left(\begin{array}{ccc}
1 \ldots 1 & 0 \ldots 0 \ldots \ldots \ldots 0 & 0 \ldots 0 \\
0 \ldots 0 & 1 \ldots 1 \ldots \ldots \ldots 0 & 0 \ldots 0 \\
\ldots & \ldots \ldots \ldots \ldots \ldots & \ldots \\
\ldots & \ldots \ldots \ldots \ldots & \ldots \\
\ldots & \ldots \ldots \ldots \ldots & \ldots \\
0 \ldots 0 & \ldots \ldots \ldots \ldots & \ldots \ldots
\end{array}\right)
$$

with $k_{1}$ activities for the production of commodity $1, k_{2}$ for commodity 2 and so forth, up to $k_{n}$, each case represented by the 1 's in the corresponding row of $B$ : the matrices $B$ and $A^{b}$ are therefore of dimension $n \times\left(k_{1}+\ldots+k_{n}\right)$. By selecting one process per commodity it is possible to form $\prod_{i=1}^{n} k_{i}$ square subsystems $A_{n}^{b}, B_{n}=I$ of the given IO structure (of the type in Chapter 1).

From these subsystems consider the matrix $A_{n}^{b *}$ with the smallest Frobenius root $\lambda\left(A_{n}^{b *}\right) \leq \lambda\left(A_{n}^{b}\right)$ and thus the fastest expansion path. For simplicity, we assume that $A_{n}^{b *}$ is indecomposable and so that if $A_{n}^{b} \neq A_{n}^{b *}$, then $\lambda\left(A_{n}^{b *}\right)<\lambda\left(A_{n}^{b}\right)$ holds (up to flukes). We also assume that $\lambda\left(A_{n}^{b *}\right)<1$ and thus have a unique solution:

$$
A_{n}^{b *} x_{n}^{*}=\lambda\left(A_{n}^{b *}\right) x_{n}^{*}, \quad p^{*} A_{n}^{b *}=\lambda\left(A_{n}^{b *}\right) p^{*},
$$

with $x_{n}^{*}, p^{*}>0$ and $r_{n}^{*}=1 / \lambda\left(A_{n}^{b *}\right)-1>0$. Then, the following proposition can be proved: ${ }^{4}$

\section{Proposition 5.2.}

(1) The equations (5.3)-(5.6), with $p B x>0$ added, have the solution

$$
x^{*}, \quad p^{*}, \quad g^{*}=r^{*}=1 / \lambda\left(A_{n}^{b *}\right)-1,
$$

where $x^{*}$ denotes the activity vector in $\mathbb{R}_{+}^{m}$ where all the components corresponding to activities in $A^{b}$ not present in $A_{n}^{b *}$ are zero and all others are given by the vector $x_{n}^{*}$.

(2) If $x, p$, and $g=r$ solve equations (5.3)-(5.6), with $p B x>0$, then $x=\alpha_{1} x^{*}, p=\alpha_{2} p^{*}$ for positive constants $\alpha_{1}, \alpha_{2}$ and $g=r=$ $1 / \lambda\left(A_{n}^{b *}\right)-1>0$.

Selecting the square subsystem with the smallest Frobenius root therefore gives us the unique von Neumann solution of a system with multiple activities and no joint production.

\footnotetext{
${ }^{4}$ See Woods $(1978,274)$ for a proof of Proposition 5.2.
} 


\subsubsection{Is von Neumann square?}

Returning to the case of joint production, we close this section with a result from Bidard $(1986,412)$, which builds a bridge between the standard square model of production prices and the von Neumann model. Consider an indecomposable von Neumann model of production, $A^{b}, B$. Under some rather general assumptions, ${ }^{5}$ it is possible to extract from $A^{b}, B$ one square subsystem $A^{b *}, B^{*}$ consisting of operated methods and commodities (goods with positive prices) at the rate $g^{*}=r^{*}$, such that

1. production methods, (respectively goods) inside the truncation are efficient, (respectively not overproduced);

2. the row and column vectors $p^{*}, x^{*}$ of $A^{b *}, B^{*}$ associated with $\frac{1}{1+r^{*}}$ are positive and (up to a factor) unique and when completed by zeros represent equilibrium prices and equilibrium activity levels of the whole von Neumann model of production; and

3. there exists an open interval $\left(\bar{r}, r^{*}\right)$ on which $B^{*}-(1+r) A^{b *}$ is nonnegatively invertible.

In other words, excluding a very small set of production structures, we can generically assume that the number of efficient activities (with respect to the rate of profit they allow for) equals that of commodities (goods not produced in excess) and, moreover, that the square active part $A^{b *}, B^{*}$ of $A^{b}, B$ satisfies $\left(B^{*}-(1+r) A^{b *}\right)^{-1}>0$ for all rates of profit $r$ sufficiently close, but strictly below $r^{*}$. The latter property becomes important if (part of the) labor vector $l$ is not included by means of a subsistence basket into the input matrix, which then allows us to calculate the prices of production, in terms of labor commanded, by means of an expression of the type $p^{*}=l^{*}\left(B^{*}-(1+r) A^{*}\right)^{-1}$.

In the next section, ${ }^{6}$ in the case of pure joint production, we shall start from such a truncation of a general von Neumann model in order to investigate the stability of the unique (up to scale) equilibrium.

\subsection{The gravitation of market prices in a square von Neumann-Sraffa system}

Suppose now that $A^{b}, B$ are square $n \times n$ (augmented) input and output matrices. According to the result in Section 5.1.3, this IO system, in general, can be regarded as the outcome of the von Neumann (1937)

\footnotetext{
${ }^{5}$ In particular, the system must be reducible and satisfy some additional conditions that make it generic, see Bidard $(1986,410)$.

${ }^{6}$ Section 5.2 is a reformulated version of Flaschel and Semmler (1987).
} 
equilibrium concept and as a particular type of Sraffa (1960) model, where labor inputs and workers' consumption are included in the physical input matrix, and constant returns are assumed. We assume that this linear model of production is productive and there exists an equilibrium $\left(R^{*}, x^{*}, p^{*}\right)$ :

$$
\left(B-R^{*} A^{b}\right) x^{*}=M x^{*}=0, \quad p^{*}\left(B-R^{*} A^{b}\right)=p^{*} M=0,
$$

such that both $x^{*}$ and $p^{*}$ are strictly positive, uniquely determined (up to a scale factor), and $R^{*}=1+r^{*}>1$ by the productiveness of $A^{b}, B$.

We study the stability of $\left(R^{*}, x^{*}, p^{*}\right)$. We stipulate the following price and supply dynamics for the above IO system and given activities $x$ and prices $p$ in $\mathbb{R}_{++}^{n}$ :

$$
\begin{aligned}
\dot{x} & =+\widehat{d}_{1} \widehat{x}\left(B-R^{*} A^{b}\right)^{\prime} p^{\prime}=+\widehat{d}_{1} \widehat{x} M^{\prime} p^{\prime} \\
\dot{p}^{\prime} & =-\widehat{d}_{2} \widehat{p}\left(B-R^{*} A^{b}\right) x=-\widehat{d}_{2} \widehat{p} M x
\end{aligned}
$$

where $d_{1}, d_{2} \in \mathbb{R}_{++}^{n}$ are given vectors of adjustment coefficients.

Equation (5.10) states that the rate of change of activity levels $x_{j}$ has the same sign as the term $p\left(B-R^{*} A^{b}\right)_{\star j} x_{j}=p M_{\star j} x_{j}$, i.e. it is proportional to the extra profits of sector $j$ measured by reference to the equilibrium value $R^{*} \cdot{ }^{7}$ Equation (5.11) says that market prices increase (decrease) if supply $B_{i \star} x$ of commodity $i$ falls short of (exceeds) demand $R^{*} A_{i \star}^{b} x$, which is defined by the current input requirements $A_{i \star}^{b} x$ multiplied by the growth factor $R^{*}$. Equations (5.10)-(5.11) describe the dynamics of quantities and prices in terms of percentage changes if one postmultiplies both sides by the diagonal matrices $\widehat{x}, \widehat{p}$, respectively.

Equations (5.10)-(5.11) consider price and quantity dynamics as if in a vacuum - supply bottlenecks, inventory changes, and other features of actual economic adjustment processes are ignored. Yet, they already represent very advanced dynamics in a general model of production, which incorporates Marx's views that capital moves into sectors with excess profits and out of less profitable sectors. This increases supply in surplus sectors and reduces supply in deficit sectors, eventually leading

\footnotetext{
7 Though in this section the proofs of the stability properties of the dynamical systems are provided by referring to the equilibrium profit and growth rate $R^{*}$, computer simulations show that the results are not invalidated if the average rate $R(x, p)=p B x / p A^{b} x$ is used as the benchmark in our dynamical system (see Flaschel and Semmler (1986b,a)). Indeed, one may argue that the average rate is the empirically relevant benchmark and should be used for stability analysis (see also Steedman (1984, 135)).
} 
to pressure on prices and profitability in the former, and to rising prices and profitability in the latter. Surplus sectors may become deficit sectors, and vice versa during this cross-over or cross-dual adjustment process. The interesting question is whether this dynamical process converges to the von Neumann (1937) equilibrium price and quantity ray or cycle around it or even move away from it, leading to explosive trajectories and thus a breakdown of this dynamic process.

The dynamics of quantities and prices also depend on the initial conditions $x=x(0), p=p(0)$. Our analysis is based on these given initial conditions and is thus formulated relative to them. We first rewrite equations (5.10) and (5.11) as follows: ${ }^{8}$

$$
\begin{aligned}
d \ln x / d t & =\tilde{x}=+\widehat{d}_{1} M^{\prime} p^{\prime} \\
d \ln p^{\prime} / d t & =\tilde{p}^{\prime}=-\widehat{d}_{2} M x .
\end{aligned}
$$

Next, we define two row vectors $\psi_{1}, \psi_{2} \in \mathbb{R}_{++}^{n}$ as follows:

$$
\begin{aligned}
& \psi_{1}=x^{*^{\prime}} \widehat{d}_{1}^{-1}, \quad \psi_{1} e=1, e^{\prime}=(1, \ldots, 1), \\
& \psi_{2}=p^{*} \widehat{d}_{2}^{-1}, \quad \psi_{2} e=1, e^{\prime}=(1, \ldots, 1) .
\end{aligned}
$$

Pre-multiplying equations (5.12)-(5.13) by $\psi_{1}, \psi_{2}$, respectively gives:

$\psi_{1} d \ln x / d t=0$, i.e. $\psi_{1} \ln x=\rho_{1} ; \quad \psi_{2} d \ln p^{\prime} / d t=0$, i.e. $\psi_{2} \ln p^{\prime}=\rho_{2}$,

where $\rho_{1}, \rho_{2}$ are constants. Therefore each trajectory $\left(x^{\prime}(t), p(t)\right)$ of the above dynamics must satisfy the side-conditions

$$
\psi_{1} \ln x(t)=\psi_{1} \ln x(0) ; \quad \psi_{2} \ln p^{\prime}(t)=\psi_{2} \ln p^{\prime}(0) .
$$

Furthermore, we choose the unique von Neumann equilibrium quantities and prices from the ray of initially given equilibrium quantities and prices by setting:

$$
\psi_{1} \ln x^{*}=\psi_{1} \ln x(0) ; \quad \psi_{2} \ln p^{*^{\prime}}=\psi_{2} \ln p^{\prime}(0)
$$

Our analysis will thus be restricted to the given initial conditions, the unique equilibrium quantities and prices under consideration, and the trajectories that may or may not converge in the considered submanifold of $\mathbb{R}_{++}^{n}$ to the unique equilibrium within this manifold.

The system of equations (5.10)-(5.11) is defined for all $x, p^{\prime} \in \mathbb{R}_{+}^{n}$.

\footnotetext{
${ }^{8}$ Recall that $\tilde{x}, \ln (x), \ldots$ are applied component-wise with respect to the vectors considered.
} 
However, situations where $x_{j}$ and $p_{i}$ are equal to zero imply $\dot{x}_{j}=0$ and $\dot{p}_{i}=0$, respectively, the hyperplanes which are tangent to the positive orthant $\mathbb{R}_{++}^{2 n}$ of $\mathbb{R}_{+}^{2 n}$ are all invariant sets of the vector field of equations (5.10)-(5.11), i.e. none of its solution curves which start in such a hyperplane can leave it. This implies that the positive orthant is an invariant set, too. The discussion of the stability of the equilibrium $x^{*}, p^{*}>0$ can therefore be restricted to the positive orthant $\mathbb{R}_{++}^{2 n}$ where equations (5.10)-(5.11) are reformulated as follows:

$$
\begin{aligned}
\tilde{x} & =+\widehat{d}_{1}\left(B-R^{*} A^{b}\right)^{\prime} p^{\prime}=+\widehat{d}_{1} M^{\prime} p^{\prime}, \\
\tilde{p}^{\prime} & =-\widehat{d}_{2}\left(B-R^{*} A^{b}\right) x=-\widehat{d}_{2} M x .
\end{aligned}
$$

Definition 5.1. An equilibrium $z^{*}$ of a differential equation system $\dot{z}=$ $f(z)$ is stable if, for every neighborhood $U$ of $z^{*}$, there is a neighborhood $U_{1}$ of $z^{*}$ in $U$ such that every solution $z(t)$ which starts in $U_{1}$ is in $U$ for all $t>0$.

A sufficient condition for the type of stability in Definition 5.1 is the existence of a so-called Lyapunov function $V$ around $z^{*}$, i.e. a continuous function on $U$ (differentiable on $U-\left\{z^{*}\right\}$ ) such that

$$
\begin{aligned}
& V\left(z^{*}\right)=0, \quad V(z)>0 \quad \text { if } z \neq z^{*} \text { and } \\
& \dot{V}=D V(z) \cdot \dot{z}(t) \leqq 0 \quad \text { in } \quad U-\left\{z^{*}\right\},
\end{aligned}
$$

(see Hirsch, Smale, and Devaney (2012)). Such a function will be used to prove the following:

Proposition 5.3. Any equilibrium of the system of equations (5.14)(5.15) described by equation (5.9) is stable.

Proof. Let $z=\left(x, p^{\prime}\right)^{\prime} \in \mathbb{R}_{++}^{2 n}$. Then, equations (5.14)-(5.15) become

$$
\dot{z}=\widehat{d} \widehat{z} Q z \quad \text { or } \quad \tilde{z}=\widehat{d} Q z
$$

where $d \equiv\left(d_{1}, d_{2}\right)^{\prime}$ and

$$
Q \equiv\left(\begin{array}{cc}
0 & M^{\prime} \\
-M & 0
\end{array}\right)=\left(\begin{array}{cc}
0 & \left(B-R^{*} A^{b}\right)^{\prime} \\
-\left(B-R^{*} A^{b}\right) & 0
\end{array}\right) .
$$

Observe that $Q$ is skew-symmetric and does not depend on the vectors $x, p^{\prime}$ (unlike in the case where the average rate of profit is used instead of the equilibrium rate $R^{*}$ ). 
As a Lyapunov function around the equilibrium $z^{*}=\left(x^{*}, p^{*^{\prime}}\right)^{\prime}$, we propose the function $V: \mathbb{R}_{++}^{2 n} \rightarrow \mathbb{R}_{+}$defined by ${ }^{9}$

$$
V(z)=q^{\prime}\left[\left(z-\widehat{z}^{*} \ln z\right)-\left(z^{*}-\widehat{z}^{*} \ln z^{*}\right)\right],
$$

where $q \in \mathbb{R}_{++}^{2 n}$ is given by $q_{k}=d_{k}^{-1}, k=1, \ldots, 2 n$, and $(\ln z)_{i}$ is given by $\ln \left(z_{i}\right)$. The function $V$ is twice differentiable. Its first derivative is given by:

$$
V^{\prime}(z)=q^{\prime}\left(I-\widehat{z}^{-1} \widehat{z}^{*}\right),
$$

which is zero if and only if $z=z^{*}$. The second derivative is given by:

$$
V^{\prime \prime}(z)=\left(\begin{array}{cccc}
q_{1} z_{1}^{*} / z_{1}^{2} & 0 & \ldots \ldots \ldots \ldots & 0 \\
0 & q_{2} z_{2}^{*} / z_{2}^{2} & \ldots \ldots \ldots \ldots & 0 \\
0 & 0 & \ldots \ldots \ldots \ldots & 0 \\
\ldots & \ldots & \ldots \ldots \ldots \ldots & 0 \\
0 & 0 & \ldots \ldots \ldots \ldots \ldots & q_{2 n} z_{2 n}^{*} / z_{2 n}^{2}
\end{array}\right)
$$

and it is positive definite. Therefore the equilibrium $z^{*}$ of equation (5.16) is a strict local minimum of $V$.

The derivative $\dot{V}$ of $V$ along the trajectories of equation (5.16) is

$$
\begin{aligned}
\dot{V} & =V^{\prime}(z)^{\prime} \dot{z}=q^{\prime}\left(I-\widehat{z}^{-1} \widehat{z}^{*}\right) \dot{z} \\
& =q^{\prime}\left(I-\widehat{z}^{-1} \widehat{z}^{*}\right) \widehat{z} \widehat{d} Q z \\
& =q^{\prime} \widehat{d}\left(\widehat{z}-\widehat{z}^{*}\right) Q z \\
& =\left(z-z^{*}\right)^{\prime} Q z \\
& =z^{\prime} Q z=0,
\end{aligned}
$$

since $z^{*^{\prime}} Q=0$ (see equation (5.16)) and $Q$ is skew-symmetric. The function $V$ is therefore constant along all trajectories of equation (5.16) in $\mathbb{R}_{++}^{2 n}$, and so it is a Lyapunov function for $z^{*}$.

Hence cross-duality of price and quantity adjustment in its simplest form gives rise to stability, but not asymptotic stability of the equilibrium. In order to get asymptotic stability, instead of modifying the law of demand and supply that drives prices, we reformulate the law of profitability, i.e. the part of the cross-dual adjustment process driving quantities. To be precise, we assume that capitalist firms also take account of the sign of changes in extra profits (or losses) when moving

\footnotetext{
${ }^{9}$ Due to its construction, the function $V$ should actually be written $V\left(z, z^{*}(z(0))\right)=\ldots$
} 
their capital between sectors. We suggest that rising extra profits speed up the growth rate of the supply in a sector, while falling extra profits tend to reduce the growth effect of supernormal profits (and similarly for negative extra profits). Therefore, in general, the growth rates of activity levels should also be influenced by the distribution of signs in the vector

$$
\xi=\frac{d}{d t}\left(B-R^{*} A^{b}\right)^{\prime} p^{\prime}=\left(B-R^{*} A^{b}\right)^{\prime} \dot{p}^{\prime}, \quad \xi \in \mathbb{R}_{+}^{n} .
$$

The vector $\xi$ shows the direction of change of extra profits (or losses) at a point in time. Integrating equation (5.18) into equations (5.10)-(5.11) we get a new dynamical system:

$$
\begin{aligned}
\dot{x} & =+\widehat{d}_{1} \widehat{x}\left[M^{\prime} p^{\prime}+\beta \xi\right], \\
\dot{p}^{\prime} & =-\widehat{d}_{2} \widehat{p} M x,
\end{aligned}
$$

where $\beta>0$ is an adjustment parameter.

Inserting equation (5.20) into (5.18) gives

$$
\xi=-M^{\prime} \widehat{p} \widehat{d}_{2} M x=E x=-T^{\prime} T x,
$$

where $M=B-R^{*} A^{b}$ and $T=\sqrt{\widehat{p}} \sqrt{\widehat{d}_{2}} M$. With the above notation, the dynamics (5.19)-(5.20) can be represented in compact form by equation (5.22):

$$
\widetilde{z}=\widehat{d}\left(\begin{array}{cc}
\beta E & M \\
-M & 0
\end{array}\right) z=\widehat{d} Q(\beta) z,
$$

where $z=\left(x, p^{\prime}\right)^{\prime}, d=\left(d_{1}, d_{2}\right)^{\prime}$, and $Q=Q(0)$ represent the case analyzed in Proposition 5.3. The matrix $E$ is negative semi-definite, i.e. from equation (5.21), $x^{\prime} E x=-(T x)^{\prime}(T x) \leq 0$ for all $x \in \mathbb{R}_{+}^{n}$; and $x^{\prime} E x=0$ if, and only if, $M x=0$, i.e. $x=x^{*}$, as long as $p>0$ and $x>0$. For the more general system (5.22), the following property holds

$$
\bar{Q}(\beta) \equiv \frac{1}{2}\left(Q(\beta)+Q(\beta)^{\prime}\right)=\left(\begin{array}{cc}
\beta E & 0 \\
0 & 0
\end{array}\right),
$$

where $\bar{Q}(\beta)$ is negative semi-definite.

Using the Lyapunov function (5.17) with regard to equation (5.22) we get 


$$
\begin{aligned}
\dot{V} & =q^{\prime}\left(E-\widehat{z}^{-1} \widehat{z}^{*}\right) \dot{z} \\
& =\left(z-z^{*^{\prime}}\right) Q(\beta) z=z^{\prime} Q(\beta) z-z^{*^{\prime}} Q(\beta) z \\
& =z^{\prime} Q(\beta) z=z^{\prime} \bar{Q}(\beta) z \\
& =\beta x^{\prime} E x \leqq 0 \quad\left(=0 \Longleftrightarrow x=x^{*}\right),
\end{aligned}
$$

since $z^{*^{\prime}} Q(\beta)=0$ and $E=-M^{\prime} \widehat{p}_{2} M$. This inequality implies that the sets $V^{-1}([0, \phi]), \phi>0$ are positively invariant with regard to the dynamics of equation (5.22), i.e. no trajectory which enters such a set can leave it later on. Further, each set $V^{-1}([0, \phi])$ is a compact subset of $\mathbb{R}_{++}^{2 n}$, since equation (5.17) is an additive combination of the strictly convex functions:

$$
V^{i}\left(z_{i}\right)=q_{i}\left(z_{i}-z_{i}^{*} \cdot \ln z_{i}-\left(z_{i}^{*}-z_{i}^{*} \cdot \ln z_{i}^{*}\right)\right) .
$$

Theorem 2 in Hirsch and Smale $(1974,196)$ states the conditions under which the neighborhood around an equilibrium of a dynamical system is asymptotically stable, even when this neighborhood contains no orbit on which the considered Lyapunov function is constant, i.e. the neighborhood lies within the basin of attraction of the equilibrium. Hirsch and Smale's Theorem 2 is not directly applicable to our model, but a modified version of it will allow us to study the asymptotic stability of our dynamical system.

In order to investigate the asymptotic stability of the modified crossdual adjustment process we use the following stability concept:

Definition 5.2. The equilibrium $z^{*}$ of the system (5.19)-(5.20) is globally asymptotically stable if and only if for any $z(0) \in \mathbb{R}_{++}^{2 n}$ and the trajectory $z(t, z(0))$ of equations (5.19)-(5.20), which starts at $z(0)$, there exist scalars $\alpha_{1}, \alpha_{2}>0$ such that

$$
\lim _{t \rightarrow 0} z(t, z(0))=\left(\alpha_{1} x^{*}, \alpha_{2} p^{*^{\prime}}\right) .
$$

Definition 5.2 is a special case of what Hahn $(1982,750)$ and Fisher $(1983,220)$ call "quasi-global stability", appropriately applied to our equilibrium $z^{*}$ which, from a global perspective, is uniquely determined only up to scale factors by the rate $R^{*}$.

According to Hahn $(1982,750)$, a dynamical system or process is quasi-globally stable, if for any $z(0) \in \mathbb{R}_{++}^{2 n}$ all limit points of $z(t, z(0))$ are points of rest (equilibria) of the system. Based on this definition we can prove the following result:

Proposition 5.4. The dynamic process of equations (5.19)-(5.20) is globally asymptotically stable in the sense of Definition 5.2. 
Proof. First we prove that equation (5.22) is quasi-globally stable. To this end we have to show that $V(z(t))$ is convergent for the trajectories $z$ of equation (5.22) and constant if and only if such a trajectory describes a point of rest. The first of these conditions has already been shown, since $V$ is monotonically decreasing along all solution curves $z$ of equation (5.22) and bounded from below. In order to show the second condition, let us assume that $V(z(t))$ is constant for an entire orbit $z(t)=\left(x(t), p(t)^{\prime}\right)^{\prime}, t \geq 0$ in $\mathbb{R}_{++}^{2 n}$. The condition $\dot{V}=0$ implies $x^{\prime} E x=0$ for this orbit, which implies $M x=0$ and thus $x=\alpha_{1} x^{*}$ for a positive scalar $\alpha_{1}$ because of the properties of the sets $V^{-1}([0, \phi])$ (i.e. because of $x, p>0$ throughout). Inserting this result into equation (5.20) gives $\dot{p} \equiv 0$, i.e.

$$
\tilde{z}=\widehat{d}_{1}\left(B-R^{*} A^{b}\right)^{\prime} p^{\prime}=\rho,
$$

where $\rho$ is constant. Pre-multiplying this equation by $\psi_{1}=x^{*^{\prime}} \widehat{d}_{1}^{-1}$ gives $\psi_{1} \tilde{x}=0$. However, since a constant growth rate $\tilde{x}_{j}>0$ is incompatible with our result that the compact sets $V^{-1}([0, \phi])$ are invariant, we get $\tilde{x}_{j}=0$ for all $j$. Hence, $\dot{x} \equiv 0$, since $\psi_{i j}>0$ for $i=1,2$ and all $j$. By Theorem T.1.4 in Hahn $(1982,751)$ the process $(5.22)$ is therefore quasi-globally stable. This completes the proof since as remarked above, global asymptotic stability is a special case of this concept.

Therefore, if capitalists also care about the time rate of change of extra profits when moving their capital from one sector to another, the stipulated price reaction of the 'market' will lead to uniform profitability and growth in the limit, and to the equilibrium prices and activity levels $x^{*}, p^{*}>0$. Our cross-dual adjustment process is thus convergent given $\beta>0$.

The dynamics described by equations (5.19)-(5.20) determine only relative activity levels and relative prices consistently with an approach which includes neither a macro theory of growth nor of inflation. Because of this it might even be claimed that the explicit or implicit existence of a numéraire is unnecessary for an investigation of the stability properties of adjustment processes of relative prices and activity levels. Following Fisher (1983, 25), a proof of quasiglobal stability, a compactness argument, and a demonstration of (local) uniqueness of rest points can be regarded as sufficient to analyze the asymptotic properties of adjustment processes. The stability properties of adjustment processes may change if an a priori condition on invariance is added and if the adjustment process is modified accordingly to satisfy this invariance condition. Yet, such a procedure is arbitrary if the true conditions which restrict prices and activities in 
such an economy are not thereby revealed. The adoption of a numéraire commodity or of another condition of invariance (e.g. of the above type) therefore only contributes to an analysis of the robustness of the analyzed stability with regard to such additional (and arbitrary) restrictions and is therefore ignored here. ${ }^{10}$

\subsection{Process and product extinction in the classical gravitation process}

The results of the previous section can be applied to both Sraffa's and von Neumann's models (if wages are paid ex ante in both cases and represented in physical units). However, the first part of this Section shows that they also hold, with certain qualifications, in the following more general equilibrium model based on a rectangular $n \times m(n<m)$ linear IO model $A^{b}, B,{ }^{11}$ exhibiting more activities than commodities (goods with positive prices). The second part then dispenses with the assumption that all goods are commodities in equilibrium, thus allowing for a treatment of von Neumann models where the number of goodsnot commodities - is larger than the number of activities.

Assumption 5.1. The linear model of production $A^{b}, B$ has an equilibrium $R^{*}>1, x^{*}, p^{*} \geq 0$ which fulfills $p^{*}>0$, i.e. which is characterized by

$$
p^{*} M \leqq 0, M x^{*}=0, \quad p^{*} B x^{*}>0 .
$$

The number of commodities (rows with positive prices) $n$ may now differ from the number of activities $m$, which is only meaningful if $n<m$. Further, according to Bidard (1986) there should be a single truncation of the IO system providing a square subsystem with uniquely determined and strictly positive balanced activity levels and prices except for flukes. However, in this section, we do not exclude flukes and consider general von Neumann models.

\subsubsection{Process extinction}

An interesting special case consists of economies with multiple, but single product activities. As in the more general case, an important question is whether the adjustment processes in Section 5.2 will not only tell something about the process of equalizing profit rates but also

${ }^{10}$ See Franke (1988) for an alternative approach to price and quantity normalization.

11 Again, with $A_{\star j}^{b}, B_{\star j} \geq 0$ for all sectors $j=1, \ldots, m$. 
about the extinction of inferior processes $p^{*} M_{\star j}<0$ (where $x_{j}^{*}=0$ ). Note that we do not allow for product extinction (free goods) in this section, and that the equilibrium is no longer assumed to be unique.

One analytical difficulty in treating this case along the lines of the preceding section is that the domain of the Lyapunov function (5.17) must now be extended in a relatively complex fashion. To examine asymptotic stability it should at least include our reference equilibrium (5.25), i.e. boundary values of $\mathbb{R}_{+}^{n+m}$ for those components where process extinction may occur. It is easily seen, however, that the function (5.17) - which depends on the choice of $z^{*}$ - has a well-defined continuous extension with regard to this new situation, since $z_{j}^{*} \cdot \ln z_{j}$ is zero for these components (and $z_{j}>0$ ). The functions (5.24) must therefore be supplemented and extended by the following functions to provide a full picture of the component-by-component forms of the function $V$ :

$$
V\left(z_{i}\right)=q_{i} z_{i} \text { for } z_{i}^{*}=0 .
$$

On the other hand, function (5.17) cannot be extended to situations $z_{i}=0$ with $z_{i}^{*} \neq 0$. Therefore, the domain of (5.17) is

$$
\mathbb{R}_{++, 0}^{n+m}=\left\{z \in \mathbb{R}_{+}^{n+m}: z_{j}>0 \text { if } z_{j}^{*}>0, z_{j} \geqq 0 \text { if } z_{j}^{*}=0\right\},
$$

(see Rouche, Habets, and Laloy $(1977,263)$ for a related approach). ${ }^{12}$

Proposition 5.5. The equilibrium (5.25) is stable with regard to the adjustment process (5.10)-(5.11) and the domain of definition $\mathbb{R}_{++, 0}^{n+m}$. The stability is asymptotic for all components $j$ where $p^{*} M_{\star j}<0$ holds true.

Proof. Recall first that there is a well-defined continuous extension of the Lyapunov function (5.17) to the domain $\mathbb{R}_{++, 0}^{n+m}$ which contains the equilibrium $z^{*}=\left(x^{*}, p^{*^{\prime}}\right)^{\prime}$. This function allows the same calculations as in the proof of Proposition 5.4 except that we now get:

$$
z^{*^{\prime}} Q(\beta)=z^{*^{\prime}}\left(\begin{array}{cc}
\beta E & M^{\prime} \\
-M & 0
\end{array}\right)=\left(-p^{*} M, 0\right) \geq 0 .
$$

This implies $z^{*^{\prime}} Q(\beta) z=-p^{*} M x$ which gives

$$
\dot{V}=\left(z-z^{*}\right)^{\prime} Q(\beta) z \leqq z^{\prime} Q(\beta) z=\beta x^{\prime} E x=0,
$$

$\overline{12}$ As in the previous section, Proposition 5.4 continues to hold, i.e. the adjustment process of equations (5.19)-(5.20) is quasi-globally stable, and all trajectories that start in $\mathbb{R}_{++, 0}^{n+m}$ have only equilibria as limit points. 
i.e. an additional inequality in terms of the estimation of the behavior of $V$ along $z(t)$. The proof of Proposition 5.4 is then completed by observing that the case $\beta=0$ now gives rise to $\dot{V}<0$ as long as $p^{*} M x<0$, i.e. $x_{j}>0$ for $p^{*} M_{\star j}<0$.

In the case $\beta>0$, we first note that $x^{\prime} E x=0$ is again equivalent to $M x=0$. This remains true since $V^{-1}([0, \phi])$ is still compact and invariant and it has a positive distance from the boundary of $\mathbb{R}_{++}^{n+m}$ for all those components $z_{j}$ where $z_{j}^{*}>0$. The limit points of trajectories which start in $V^{-1}([0, \phi])$ therefore must have positive components for all $j$ where $z_{j}^{*}>0$, i.e. in particular all prices must be positive even in the limit. Hence, $x^{\prime} E x=-x^{\prime} M^{\prime} \widehat{p} \widehat{d}_{2} M x=0$ if and only if $M x=0$. Note, however, that $M x=0$ no longer implies that $x$ is proportional to $x^{*}$, given the possibility of switching of techniques.

In order to show the quasi-global stability of equations (5.19)-(5.20) in this general case we need to show that $V$ is constant if and only if $z$ is a point of rest of equations (5.19)-(5.20), (see Hahn (1982, 751)). Note that Hahn's proof of quasi-global stability also applies to the special type of 'orthant' $\mathbb{R}_{++, 0}^{n+m}$ on which the above Lyapunov function is defined, since all limit points of solution curves which start in $V^{-1}([0, \phi])$ must be contained in this set.

Assume now that $\dot{V}=0$ for an entire orbit $z(t)=\left(x(t), p(t)^{\prime}\right)^{\prime}, t \geqq 0$. By equation (5.27) we get

$$
z^{*^{\prime}} Q(\beta) z=0 \text { and } x^{\prime} E x=0 .
$$

This implies $p^{*} M x=0$, i.e. $x_{j}=0$ for all $j$ with $p^{*} M_{\star j}<0$. Because of $M x=0$ we get from equation (5.20) the result $p \equiv 0$ or $p \equiv \phi$, for some $\phi>0$, and $\xi=0$, see equation (5.19). There remains the dynamics

$$
\dot{x}=\widehat{x} \widehat{d}_{1} M^{\prime} p^{\prime} \geqq 0 \quad \text { for } \quad p M \geqq 0,
$$

if $x_{j}>0$ (the cases $x_{j}=0$ imply a situation of no change with regard to these components). The case $p M_{\star j}>0$ can, however, be excluded by observing that a constant vector of prices $p$ would imply a constant and positive rate of growth for $x_{j}$ in contradiction with the fact that $z(t), t \geq 0$ cannot leave $V^{-1}([0, \phi])$. The remaining possibility $p M_{\star j}<$ $0, x_{j}>0$ is also incompatible with our assumptions, since it would imply

$$
\dot{V}=\sum_{x_{j}>0} q_{j}\left(x_{j}-x_{j}^{*}\right) \cdot \tilde{x}_{j} \neq 0,
$$

because of $\tilde{x}_{j}=\phi<0$ for these components $j$, i.e. $d \dot{V} / d t=\sum q_{j} \cdot \dot{x}_{j}$. 
$\tilde{x}_{j}<0$. In sum, we get $\dot{z}=0$ for the above orbit $z(t)$ : this orbit describes a point of rest $z^{* *}$, which fulfills $p^{* *} M \leq 0, M x^{* *}=0, p^{* *}>0, x^{* *} \geq 0$ and $p^{* *} B x^{* *}>0$.

The limit set of each trajectory - the set of points the trajectory converges to - thus consists of rest points only, but it may now contain different economic equilibria.

\section{Corollary 5.1.}

(1) The equilibria $x^{* *}, p^{* *}$ of the system $A^{b}, B$ which are limit points of the solution curves of process (5.19)-(5.20) are of the same type as the equilibrium $x^{*}, p^{*}$ (see equation (5.25)).

(2) The combinations $\left(x^{*}, p^{* *}\right)$ and $\left(x^{* *}, p^{*}\right)$ are also equilibria with regard to the rate $R^{*}$. They form a convex subset $Z^{*}$ of the set of all equilibria of the natural rate $R^{*} \cdot{ }^{13}$

(3) An activity which is inferior at an equilibrium $z \in Z^{*}$ will not be operated in any equilibrium $z \in Z^{*}$.

(4) Denote by $J^{*}$ the (uniquely determined) maximum set of activities which are operated in the set of equilibria $Z^{*}$. Then: $j \in J^{*} \rightarrow x_{j}^{* *}>$ 0 for the equilibria which are limit points of equations (5.19)-(5.20).

Proof. The above assertions follow from the following facts: (1) the functions (5.24) are strictly convex at the global minimum $z_{i}^{*} ;(2)$ they approach infinity if $z$ approaches zero (for $z_{i}^{*}>0$ ); and (3) the invariant sets $V^{-1}([0, c])$ around the equilibrium (5.25) are all compact. This implies that positive components $z_{i}^{*}$ must lead to positive components $z_{i}^{* *}$ by our adjustment process (5.19)-(5.20), which is independent of the particular choice of equilibrium $z^{*}$.

Therefore, this adjustment process does not (and cannot) discriminate between the optimum activities of the different equilibria in the set $J^{*}$, but leads always to an equilibrium where these activities are all jointly operated. Note here that the function $V$ depends on this choice, yet in a manner which is irrelevant for the truth of Proposition 5.5 .

\subsubsection{Product extinction}

We have shown stability, and even global asymptotic stability, for general joint production economies without free goods. Below, we show that these assertions cannot be extended to the case of free goods $\left(p_{j}^{*}=0\right)$. The law of excess demand (instead of the law of profitability)

$\overline{13}$ According to Fujimoto (1975), the natural rate $R^{*}$ is uniquely determined, because of our assumption $p^{*}>0$, see also Bidard (1986). 
must be modified so as to lead to their extinction by disequilibrium dynamics.

Because of $p^{*}>0$, i.e. $M x^{*}=0$, in the previous section the following simple equation proves the monotonicity of $V$ along the orbits of equations (5.19)-(5.20), by equation (5.26):

$$
z^{*^{\prime}} Q(\beta) z=-p^{*} M x \geq 0 .
$$

However, in the general case $x^{*} \geq 0, p^{*} \geq 0$, the expression for $z^{*^{\prime}} Q(\beta) z$ reads

$$
-p^{*} M x+p M x^{*}-\beta x^{*} M^{\prime} \widehat{p} \widehat{d}_{2} M x .
$$

Here only the first two terms are unambiguously nonnegative, while it can be shown that the last vector must be non-positive in a small neighborhood of $\left(x^{*}, p^{*^{\prime}}\right)^{\prime}$, since $M_{i \star} x$ and $M_{i \star} x^{*}$ have the same sign for all $i$ where $M_{i \star} x^{*}<0$ in such a neighborhood.

In order to obtain asymptotic stability in the presence of free goods, the following modification of this process is appropriate:

$$
\begin{aligned}
\dot{x} & =+\widehat{d}_{1} \widehat{x} M^{\prime} p^{\prime}, \\
\dot{p}^{\prime} & =-\widehat{d}_{2} \widehat{p}[M x+\beta \xi],
\end{aligned}
$$

where $\xi=\frac{d}{d t} M x=M \dot{x}$ and $\beta>0$. The rate of change of excess supplies is assumed to exercise an extra influence on our original dynamics in equations (5.10)-(5.11) - now with regard to price, instead of quantity, adjustments. Instead of equation (5.22) we get

$$
\dot{z}=\widehat{z} \widehat{d}\left(\begin{array}{cc}
0 & M^{\prime} \\
-M & \beta E
\end{array}\right) z,
$$

where now $E=-M \widehat{x} \widehat{d}_{1} M^{\prime}$. In the case of no inferior activities $\left(x^{*}>0\right)$, these dynamics may be treated as in the case $p^{*}>0$ : this adjustment is quasi-globally stable, and exhibits product-extinction instead of process-extinction. Modifications of our simple version of the law of demand may therefore be exploitable for the treatment of free goods. Such modifications are, however, not of central importance in this and the previous section, which focuses on capital movements and their stabilizing properties. Furthermore, the analysis of the simultaneous operation of the two additional influences, equations (5.19) and (5.30), is not straightforward and is left for future research. 


\subsection{Conclusions}

We have shown that it is possible to extend the framework of Chapter 4 to include joint production and multiple activities in the von Neumann-Sraffa growth model. We have derived a set of quasi-globally stable dynamics around the balanced growth equilibrium of this von Neumann-Sraffa model. These price-quantity dynamics are illustrative of the so-called "cross-dual" dynamics envisaged by the Classicals, in which long-period natural prices act as centers of gravity for the movements in short-term market prices.

This chapter concludes the presentation of Quesnay and the Classics, bringing Part I of the book to a close. It extends the classical concept of production prices to include joint production and multiple activities in a balanced growth model, and in so doing it explores the boundaries of the classical (Sraffian) model. These boundaries are quite wide, but nonetheless they do not include some key features of modern capitalist economies, such as fixed capital, capital depreciation, and imperfect competition. Furthermore, the analysis in this chapter focuses on production prices and does not tackle the definition of labor values in general economies with joint production, multiple activities, fixed capital, and so on.

These topics, concerning prices and values, will be examined in Part II. We will draw, on the one hand, on Marx's innovations on the Classics to inform a deeper understanding of the role labor values can play in the analysis of advanced capitalist economies; and on the other hand, on modern input-output theory in order to incorporate fixed capital, imperfect competition, and depreciation in the definition of production prices. 


\section{PART II}

Value and Exploitation: Marx's Legacy 

Part I of the book has introduced the basic definitions and tools of input-output theory, and the theoretical tenets of classical economics. We have discussed the concept of classical competition, and the notions of market prices, production prices, and labor values, interpreted as the total labor costs, or requirements, of commodities. Focusing on simple $n$-good Leontief economies with circulating capital and no joint production, we have derived two broad sets of results. On the one hand, we have shown that the classical-Marxian price theory provides a valid alternative to the standard neoclassical general equilibrium theory. We have derived a number of properties of classical production prices, including the proof of the existence of a general distributive conflict between the main social classes in capitalist economies. On the other hand, however, we have proved that, apart from very special cases, production prices are not proportional to labor values. Therefore, contrary to the standard reading of Marxian value theory, labor values do not determine equilibrium prices.

Two main questions immediately arise in the light of these results. First, can the classical-Marxian theory of production prices be extended to more general economies? In particular, can the theory be extended to incorporate key features of capitalist economies and the actual practices of competitive firms? Second, if they are not good predictors of equilibrium prices, what are labor values good for? The received view is that they are at best a useless, and possibly a logically incoherent construct.

Part II engages with Karl Marx's contribution and in particular with his labor theory of value and analyzes both questions. As concerns prices, we generalize the standard classical concept of production prices by incorporating fixed capital and by allowing for imperfect competition. To be precise, on the one hand, we discuss Leontief's notion of a capital matrix and define prices - and profitability -in relation to the capital advanced, or tied up in production, by firms. On the other hand, we relax the assumption of uniform wage rates and profit rates across sectors, to incorporate an empirically-determined 
hierarchy of intersectoral differentials. We argue that this approach to production prices - which has been pioneered by Leontief and Bródyis theoretically rigorous and provides a more realistic description of capitalist economies than the standard Sraffa/von Neumann model. Then we prove that all of the key insights of classical price theory can be extended to our general model.

As concerns labor values, we start from first principles and discuss their theoretical role and the properties that a general definition of labor values should have. Then, based on this characterization, we propose a general definition of labor values as measuring the real labor costs of producing commodities, based on Marx's concepts of individual and (average) labor values. We show that labor values provide the only theoretically sound measures of (sectoral and aggregate) labor productivity and allow us to examine the structural features of capitalist economies beneath the surface of monetary magnitudes. We prove that our definition preserves all of the properties of Marxian value theory in general economies with multiple activities, joint products, fixed capital and so on.

In other words, contrary to the received view, labor values can be defined in a way that is faithful to Marx's original conception, logically coherent, and fully general. Furthermore, far from being metaphysical, the notion of labor value is based on empirically observable magnitudes, and on the actual practices of competitive firms. Labor values thus conceived should be considered as part of the standard System of National Accounts, and can be used to analyze the dynamics of capitalist economies. As such, our approach to Marxian value theory specifies a progressive research agenda in theoretical as well as empirical economics. 


\section{Labor Values: An Axiomatic Approach}

\subsection{Introduction}

In Part I of the book, we have introduced the classical concepts of market prices, production prices, and labor values. Focusing on simple $n$-good Leontief economies with circulating capital and no joint production, we have shown that production prices can be interpreted as the centers of gravitation of market prices and have derived a number of important insights on capitalist economies, including the proof of the existence of a general distributive conflict between the main social classes. We have also proved, however, that, apart from very special cases, production prices are not proportional to labor values. Therefore, labor values do not, and cannot, determine equilibrium prices.

Two questions immediately arise in the light of these results. First, if they are not good predictors of equilibrium prices, what are labor values good for? The received view is that they are at best a useless, and possibly a logically incoherent construct. But, second, even assuming that a logically coherent and meaningful interpretation of the concept of labor values can be provided in the standard linear setting, is it possible to extend it to more general economies? Again, the received view is that outside of the simplest $n$-good Leontief model with homogeneous labor, circulating capital, and a single production technique in every sector, and without joint production, the concept of labor value is meaningless. In his celebrated book, Marx after Sraffa, for example, Steedman (1977) proved by means of examples that in economies with joint production or fixed capital, the standard definition of labor values as IO employment multipliers yields paradoxical results: the labor values of certain commodities, and even aggregate surplus value, can be negative and concludes that Marx's LTV is irremediably flawed - at least as a general theory. ${ }^{1}$

Steedman's negative conclusion - which both reflects and underpins

\footnotetext{
${ }^{1}$ For a thorough discussion see Chapter 10.
} 
a widespread view - on Marxian value theory is based on the premise that his approach to labor values is the only, or at least the natural extension of the standard definition. This is far from obvious. In the standard, simple Leontief setting labor values can be interpreted either as the standard IO employment multipliers or, equivalently, as measures of the total labor costs, or requirements, of commodities. In more general settings, however, this equivalence breaks down, which immediately suggests that there is no single, natural or uncontroversial definition of labor values. Should labor values be interpreted as employment multipliers, as Steedman (1977) suggests, or is it more appropriate to consider them as indices of the real cost (in units of labor time) of producing commodities?

Or should they be something else altogether? In a seminal article Morishima (1974) argued that, in general economies, the definition of labor values and their relationship to prices of production should be approached in a specific (optimizing) way. To be precise, in the case of joint production (and multiple activities), Morishima (1974) suggested that the values of any nonnegative bundle of goods is the mimimum amount of direct labor necessary to produce it choosing among all possible alternative techniques. The concept of labor values underlying this extension of Marx's LTV-true labor values in Morishima's own words - has been widely considered as an appropriate generalization of Marx's theory, ${ }^{2}$ or at least as an appropriate starting point for alternative optimizing definitions (Roemer 1981, 2002; Matsuo 2008). In the context of our discussion, Morishima's optimizing definition has also been considered as a valid solution to the problems highlighted by Steedman (1977).

Yet, the adequacy of Morishima's definition, and its ability to capture the core features of Marx's LTV, have been questioned. Steedman (1976), for example, has raised various doubts by pointing out that a Marxian definition of labor values should be additive in its construction and based on actual data, not hypothetical techniques, and it should lead to strictly positive labor values for all marketed commodities. Further, Roemer (1981) has argued that in more general settings, Morishima's definition does not really solve the issues posed by Steedman's counterexamples - in particular concerning the relation between aggregate surplus value and aggregate profits - and he has also shown (Roemer 1982) that if Morishima's definition is adopted,

\footnotetext{
${ }^{2}$ See Nutzinger (1976), Wolfstetter (1976), Murata (1977), Takeda (1978), Morishima and Catephores (1978), Cogoy (1979), Roemer (1980a), and Fujimoto and Opocher (2010).
} 
then no clear relation between agents' class and exploitation status can be derived: contrary to the standard Marxian intuition, for example, it may happen that relatively rich agents emerge as exploiters in equilibrium. Therefore he has proposed a refinement of Morishima's definition which is meant to avoid Steedman's counterexample and these problems, too. ${ }^{3}$

In summary, as soon as one abandons the simple, standard Leontief context there is no obvious definition of labor values. A number of alternative approaches exist which are based on different interpretations of Marx's LTV and have different properties. How does one adjudicate between them? In this chapter, rather than immediately presenting our definition, we start from first principles and explicitly discuss the theoretical role of labor values ('what labor values are good for') and, consequently, the properties that a general definition of labor values should have. From this perspective, our approach bears a broad conceptual similarity with the axiomatic method typical of social choice theory. For it is possible to conceive of any approach to value theory as (implicitly) defining a set of problems (including definitions of the main variables: prices, values, technology, competition, and so on); formulating a list of desirable properties (axioms) of the LTV, including the specification of the role of value analysis; and then exploring the set of 'solutions' to those problems - i.e. the set of admissible definitions of labor values. ${ }^{4}$

In our view, an axiomatic approach has two major advantages: conceptually, it focuses the discussion on the key tenets of the Marxian LTV, forcing one to make its fundamental properties explicit. But it also clarifies the conceptual underpinnings and logical structure of different definitions, which allows for constructive criticism of existing definitions and the development of new ones.

\subsection{The labor content of commodities: The real side of social interdependence}

The starting point of our analysis is the description of capitalist economies as highly complex systems characterized by a high degree of social interdependence and by the interconnected role of production and circulation of commodities. In a Marxian perspective, a theoretical

\footnotetext{
${ }^{3}$ For a discussion see Yoshihara and Veneziani (2010) and Veneziani and Yoshihara (2012, 2015a).

${ }^{4}$ For a novel axiomatic approach to Marxian theory see Veneziani and Yoshihara (2012, 2015a), Yoshihara and Veneziani (2010), and Mohun and Veneziani (2017).
} 
analysis of capitalism requires the development of a conceptual apparatus that allows one to capture real phenomena, and especially production relations and production processes, beneath the surface of market interactions. Classical IO theory is, in our view, uniquely placed to provide the tools for this theoretical endeavor.

The United Nations' System of National Accounts (SNA) developed by Richard Stone and his research group is a rigorous classification system of production and exchange activities. It considers many complexities of capitalist economies - including joint production and fixed capital - and attempts to construct both stock and flow matrices to capture the dynamics of an economy, and real magnitudes like real GDP and labor productivity. The real magnitudes of the SNA are theoretical concepts which capture relevant economic phenomena that underlie nominal magnitudes. Thus, they are akin to the centers of gravity developed by the classicals and Marx, which identify a theoretical reference point for the analysis rather than an actual, attainable state.

Accordingly, in our interpretation, the UN's SNA is more classical than neoclassical in nature, in the sense that its concepts focus on average magnitudes rather than on marginal conditions in a perfectly competitive environment. Classical-Marxian prices of production exemplify this type of average magnitude, because competitive forces can yield a uniform profit rate only over a long period of time. They are centers of gravity around which market prices fluctuate in the presence of ruthless competition. They identify an abstract reference point for analysis, rather than the actual position of an economy.

We interpret Marx's LTV as the other building block of the analysis of real phenomena. It aims to identify the real magnitudes behind nominal, market phenomena by focusing on the qualitative notion of 'abstract labor' and on its quantitative expression 'labor content'. From this perspective, labor content is the (average) labor time 'embodied' in a good, in the sense of full-cost accounting in terms of labor time spent (on average) in the production of commodities. Labor values are thus an accounting construct simultaneously determined alongside prices of production. The question then is, what are the rigorous relationships between labor value accounting and prices of production (or actual market prices) accounting? Or, more generally, what are the relations between labor and monetary magnitudes? The aim is not to prove some sort of transformation theorem, but to identify the relationships between theoretical accounting definitions used for economic reasoning and the centers of gravity of the actual price-quantity developments.

Based on this interpretation of Marx's LTV as a precursor, and part, 
of the modern SNAs, a number of-both qualitative and quantitativeproperties can be identified that a definition of labor values should satisfy. These properties concern the relationships between 'real' value magnitudes and observed nominal variables like profit, wages, value added, and so on. The properties discussed in this chapter are widelyalbeit often implicitly - shared in the literature and many debates in value theory arise from the failure of the main received definitions to satisfy one, or more of the axioms in general economies. Below, we discuss these properties and illustrate their importance starting from the standard, simple Leontief production model $(A, l)$. In Leontief economies, labor values are defined as

$$
v=v A+l,
$$

whereas prices of production (or 'natural' prices) are

$$
p=(1+r)(p A+w l) .
$$

\subsection{Basic principles when generalizing labor values}

In this section, we describe some basic properties of labor values that are meant to capture some implications of the notion of labor values as measuring the labor embodied or contained in commodities, where - as Marx emphasized - labor is abstract rather than concrete, simple rather than compound, social rather than private, and necessary rather than wasted, and so labor values should be measured in units of socially necessary labor-time.

First of all, note that, under the usual assumptions on technology (including the productiveness and indecomposability of $A$ ) both labor values in equation (6.1) and production prices in equation (6.2) are strictly positive. In more general models, however, and especially in models with joint production, neither the equilibrium Sraffa/von Neumann price nor the labor value of a certain good will necessarily be positive. Yet, as Steedman (1977) noted, if labor values are meant to capture the amount of labor embodied, or contained in goods, then they cannot be negative. Further, if the labor contained in commodities is necessary rather than wasted, then arguably free goods should have no value. Formally:

(P1) Commodity Correspondence Principle: The price of a good is positive if and only if its labor value is positive. In particular, the labor value of a free good is zero.

For the individual commodity, the LTV is specified as the 
proportionality between production prices and labor values. This is how it appeared in Smith's pre-capitalist "early and rude state", as a "commodity law of exchange"; Ricardo extended this to a capitalist economy with produced means of production and found that in general such a commodity law of exchange did not hold. It was rather superseded by the "capitalist law of exchange", specified as the determination of prices that supported an equalized rate of profit. ${ }^{5}$ Marx then tried to show that the capitalist law of exchange merely modified the commodity law of exchange in the sense that it took value from where it was produced and redistributed it according to total capital advanced. In Marx's particular procedure, the deviations generated by this redistribution summed to zero, and one may argue that, in a certain (ontological) sense, they could not meaningfully do anything else. It is this insight that may be called the conservation principle.

(P2) Conservation principle: Aggregate value added in prices equals total direct labor.

In actual capitalist economies, individual goods can be produced using various production methods, and they can be either the only output of a given sector, or the joint product of an industry producing a range of outputs at the same time (or both). If this is the case, then conceptually one should distinguish between the labor embodied in individual commodities measured at the level of a single firm, or industry, or production method adopted - which may be called individual values - and the amount of social labor embodied in a commodity taking into account all firms, industries and production methods producing the good-which may be called labor values. Formally,

(P3) Individual plus Market-value Principle: Labor values are averages of individual values, which in turn are derived from actual production data by means of labor values.

So far, we have been talking about the labor contained in commodities, and we have analyzed labor values in models with a single type of homogeneous labor. Yet in actual capitalist economies a range of heterogeneous labor inputs are used in production and agents

\footnotetext{
5 The terminology "commodity law of exchange" to describe price/value proportionalities, and "capitalist law of exchange" to describe the determination of prices that support an equalized rate of profit, is used by Foley and Duménil (2008), Foley (2011), and Foley and Mohun (2016).
} 
possess quantitatively and qualitatively different skills. In order to deal with such heterogeneity, Marx emphasizes that different types of labor should be converted, or reduced to simple labor. The question concerns the appropriate conversion rates that allow to reduce a range of labor inputs into a single quantity. Despite some debates on the appropriate concept of "abstract labor", we argue that Marx $(1976,1981)$ shared the views of the other classical economists, whereby "the different kinds of labour are to be aggregated via the (gold) money wage rates" (Kurz and Salvadori 1995, 324). According to Smith, for example,

It is often difficult to ascertain the proportion between two different quantities of labour. The time spent in two different sorts of work will not always alone determine this proportion. The different degrees of hardship endured, and of ingenuity exercised, must likewise be taken into account. There may be more labour in an hour's hard work, than in two hours easy business; or in an hour's application to a trade which it cost ten years labour to learn, than in a month's industry, at an ordinary and obvious employment. But it is not easy to find any accurate measure either of hardship or ingenuity. In exchanging indeed the different productions of different sorts of labour for one another, some allowance is commonly made for both. It is adjusted, however, not by any accurate measure, but by the higgling and bargaining of the market, according to that sort of rough equality which, though not exact, is sufficient for carrying on the business of common life (Smith 2000, 34-35).

One can similarly interpret Ricardo's (1951, Ch. I, sec. II) arguments that "The estimation in which different quantities of labour are held, comes soon to be adjusted in the market with sufficient precision for all practical purposes, and depend much on the comparative skill of the labourer, and intensity of the labour performed". Then: ${ }^{6}$

(P4) Labor-Unit Principle: Labor is homogenized by means of wage differentials.

Two important features of the Labor-unit Principle should be stressed. First, in addition to having solid theoretical foundations in classical economics, the conversion of different types of labor by means of wage rates also coincides with the actual definitions in the UN's SNA and with the practices of IO theorists and statisticians. In fact, in actual IO table data on labor inputs are never in "physical" terms and, at least to some extent, different labor inputs are aggregated using wages. Second, it has an important, and possibly controversial

\footnotetext{
${ }^{6}$ The labor-unit principle allows for the preservation of the price-value theorem discussed below.
} 
implication: in the presence of heterogeneous labor inputs, it is not possible to determine labor values based on purely technological data and prior to, and independently of, price information. In the standard, interpretation of labor values as predictors of equilibrium prices, this is obviously rather problematic. But if labor values are meant to capture the (real, labor) cost of producing commodities in actual capitalist economies, there is no reason why labor values should, definitionally, be independent of price information.

In fact, in economies with multiple activities, joint production and fixed capital, it is generally impossible, or meaningless, to derive fullcost accounting based on technological information only and price information is necessary in order to define the total (labor) costs of commodities. In these cases, if labor values are meant to capture the structure of actual capitalist economies, they should be consistent with the practices of capitalist firms.

(P5) Imputation Principles: If full-cost accounting (of any type) is not possible by means of (physical) IO data alone, the actual practices of firms have to be applied appropriately to close the degrees of freedom in the definition of total costs.

Finally, if labor values are meant to measure the amount of labor embodied, or contained in a given good, then - intuitively-small changes in the amount of direct labor necessary to produce a given good, or small changes in input requirements, should not yield large variations in its labor value. In simple Leontief economies, under the standard assumptions, $v=l(I-A)^{-1}$ and the vector of labor values varies continuously with changes in $(A, l)$. The next axiom generalizes this intuition, and requires that small changes in the production technology do not yield discontinuous variations in labor values.

(P6) Labor-Value Continuity Principle: Labor values change continuously with technology.

Properties (P1)-(P6) are weak and reasonable, and they can all be traced back to Marx's own writings. Yet, they are not trivial, especially in the light of the transformation problem debates in the 1970s and $1980 \mathrm{~s},{ }^{7}$ and most of the definitions of generalized labor values in the literature violate at least one of (P1)-(P6).

\footnotetext{
7 See, in particular, (P1), (P2) and (P4). See Holländer (1982) for a definition of labor values that violates (P4).
} 


\subsection{Simple quantitative features of the Labor Theory of Value}

Principles (P1)-(P6) focus on definitional aspects of the LTV, imposing some exegetical and theoretical constraints on labor values. The next set of properties focus especially on the relation between price and value magnitudes and capture some intuitions that highlight the relevance of Marxian value theory in the analysis of capitalist economies.

A first important principle of Marxian value theory captures the link between social labor time and value creation in capitalist economies. In the standard Leontief setting, it can be formalized as follows.

Marxian Aggregation Theorem (MAT). Consider a production technology $(A, l)$. Let $x$ be the aggregate gross output vector and let $y=(I-A) x$. Then, the value of the net product is equal to total labor time expended:

$$
v y=l x .
$$

Proof. With $v=l(I-A)^{-1}$ and $y=(I-A) x, v y=l(I-A)^{-1}(I-A) x=$ $l x$.

This property is fundamental to Marxian value theory in general, and central - indeed, definitional - in recent approaches by Duménil (1980), Foley (1982), Duménil et al. (2009), and Yoshihara and Veneziani (2009), among others. We therefore state it as a general axiom.

(T1) Marxian Aggregation Theorem: In any given period, the labor value of the net product equals total labor time expended.

The lack of correspondence between values and prices under general conditions is widely accepted, and indeed both Ricardo and Marx themselves knew that in capitalist production economies production prices could not be proportional to labor values. Thus, as Foley (2000) notes, the discourse on Marx's theory of value and the transformation problem shifted to attempts to preserve consistency in the accounting of values and prices along three conditions seen in Marx's own presentation of the transformation:

(1) total price is equal to total value;

(2) total surplus value is equal to total profit;

(3) the rate of profit in value terms is equal to the rate of profit in price terms.

Consider, again, the standard Leontief economy in which labor values are defined by equation (6.1). It is well known that in general, 
conditions (1) and (2) above cannot hold simultaneously: the system (6.2) has $n$ equations in $n+2$ unknowns - the $n$ prices and the two distributive variables $w, r$. The system is closed by specifying a value for one distributive variable and by choosing a numéraire. Recall that $b$ denotes the $n \times 1$ subsistence bundle of workers and suppose that workers do not save and consume all their wages. Then, in the standard approach to Marxian value theory (also known as the "dualist" approach, see Mohun and Veneziani (2017)) the value of the gross product (or total value) is $v x$ and total surplus value is $v(I-A-b l) x$. Total price is then $p x$ and total profit is $p(I-A) x-w l x$, with $w$ denoting the wage. The specification of the subsistence wage leaves one degree of freedom and so, whenever the organic composition of capital differs across sectors - so that $p \neq \phi v$-one can specify the numéraire so that either (1) or (2) holds, but not both.

Let $C$ denote what Marx calls constant capital - the raw materials and non-labor inputs used up in the production process - and let $V$ represent Marx's variable capital - the total living labor used in production. Let $S$ denote the total surplus value produced in an economy. Let the subscripts $v$ and $p$ respectively denote any of $C, V$, $S$, or the rate of profit $r$ measured in units of embodied labor-value terms - or price terms. Marx presents the rate of profit as the ratio of surplus value to the total capital advanced in production, i.e. the sum of constant and variable capital. It is also implicit in Marx's presentation of the transformation of values into prices that the aggregate rate of profit be the same when calculated from value magnitudes as when calculated from price magnitudes. However, just as Marx's first two conditions do not hold, this third condition cannot either.

The value rate of profit can be written as

$$
r_{v}=\frac{S_{v}}{C_{v}+V_{v}}=\frac{v(I-A-b l) x}{v A x+v b l x}
$$

and the price rate of profit is

$$
r_{p}=\frac{S_{p}}{C_{p}+V_{p}}=\frac{p(I-A) x-w l x}{p A x+w l x} .
$$

As for conditions (1) and (2), so long as $p \neq \phi v$ then $r_{v} \neq r_{p}$ and Marx's condition (3) does not hold. The problems of correspondence between value magnitudes and price magnitudes are further demonstrated by the fact that the wage may not necessarily be equal to subsistence, i.e. it could be the case that $w \neq p b$, and because workers do not 
purchase their subsistence bundle in terms of labor values, it is not possible that $w=v b$ could hold.

The lack of correspondence between values and prices-leading to Marx's three conditions not holding simultaneously - has led many authors to reject Marx's LTV and any role at all for labor values in economic analysis, see Samuelson $(1970,1971)$ and Steedman (1977) for example. This conclusion is unwarranted. It is based on a specific, predictive interpretation of the LTV whereby relative labor values are supposed to determine relative production prices. It is also based on a specific, dualist definition of values and value magnitudes in which money is absent, or just a casual afterthought - a veil on real phenomena. Yet these are by no means the only reasonable interpretations of Marxian value theory.

First of all, it is not clear that Marxian value theory can only be interpreted as a predictive exercise. For "there are at least three distinct non-metaphysical interpretations of the [LTV], viz. (i) descriptive, (ii) predictive and (iii) normative" (Sen 1978, 175). As for (i), Sen (1978, 176) notes that "Any description relies on factual statements. But it also involves a selection from the set of factual statements that can be made pertaining to the phenomenon in question: some facts are chosen and others ignored. The selection process is part of the exercise of description, and not a 'metaphysical' exercise." One may argue that in the LTV "it is the activity of production that is being described, and the selection criterion is focused on 'personal participation' " (Sen 1978, 177). It focuses analysis on human effort and refuses "to give the same status to the ownership of [natural resources and capital] in describing participation in production as personal participation through labour" (ibid.). Thus, alternative formulations of the LTV "have to be judged in terms of the motivation of the exercise of description in the particular case in question" (Sen 1978, 178). ${ }^{8}$

In a descriptive interpretation, labor magnitudes are useful to understand the dynamics of capitalist economies. And because the key variable to analyze capitalism is the rate of profit, Marxian value theory should be able to explain profitability. We formulate this idea by requiring that the value rate of profit and the price rate of profit be exactly equal in a balanced growth path and approximately equal in more general settings.

(T2) Profit-Rate Theorem: The average (labor) value- and price-rate of profit, respectively $r_{v}$ and $r_{p}$, are empirically nearly of the same magnitude and equal to each other if a uniform rate of growth is

\footnotetext{
${ }^{8}$ For a comprehensive discussion see Mohun and Veneziani (2017).
} 
given:

$$
\begin{gathered}
r_{v}=\frac{v(I-A-b l) x}{v A x+v b l x}, \quad r_{p}=\frac{p(I-A) x-w l x}{p A x+w l x}, \\
r_{v} \approx r_{p} .
\end{gathered}
$$

As already noted, if $r>0$ and the structure of production $\left(A_{j}, l_{j}\right)$ is different in different sectors - a different organic composition of capital in Marx's terminology - there exists no scalar $\phi>0$ such that $p=\phi v$. Stated differently, for any positive profit rate, prices of production will generally not be proportional or equal to labor values except by pure accident. In simple Leontief economies, the next result describes the conditions under which prices are proportional to labor values.

Marxian Proportionality Theorem (MPT). Prices of production $p$ are proportional to labor values $v$ for some scalar $\phi>0$ if and only if $r=0$ or $A_{i} / l_{i}=A_{j} / l_{j}$ for all $i, j=1, \ldots, n$ but $i \neq j$ :

$p=\phi v \Longleftrightarrow$ either $r=0$ or $\frac{A_{i}}{l_{i}}=\frac{A_{j}}{l_{j}}$ for all $i, j=1, \ldots, n$ but $i \neq j$.

Because the MPT is well known in the literature, no formal proof is given here. The proportionality of production prices and labor values when all sectors have the same organic composition of capital is a counterfactual result that incorporates a methodologically important proposition of Marx's LTV, which underlies the analysis in Capital, Vol.I. Therefore we generalize it as follows.

(T3) Price/Value Theorem: Uniform ratios of profits to wages (in terms of whatever prices) in all sectors of production imply proportionality between labor values and these prices.

In Leontief economies, the system of labor values in equation (6.1) allows for the definition of an aggregate rate of exploitation that captures the relationship between surplus labor and surplus value creation. Marx uses the terms rate of exploitation and rate of surplus value interchangeably, defining either as: the ratio of surplus value to variable capital; the ratio of surplus labor to necessary labor; and the ratio of unpaid to paid labor time. ${ }^{9}$ Formally, in the standard dualist approach, the rate of exploitation $\epsilon$ is

\footnotetext{
${ }^{9}$ While the consistency of these three ratios comes into question when $p \neq$ $\phi v$, in the rest of the book we show that a meaningful relationship between surplus labor and surplus value can be derived.
} 


$$
\epsilon=\frac{S_{v}}{V_{v}}=\frac{v(I-A-b l) x}{v b l x}=\frac{v(I-A) x}{v b l x}-1=\frac{1}{v b}-1
$$

A simpler way to consider $\epsilon$ is that for any given working day measured in hours $h$ and the value of labor-power $v_{w}=v b l x$ scaled to a single working day, we have

$$
\epsilon=\frac{h-v_{w}}{v_{w}}=\frac{h}{v_{w}}-1
$$

Thereby capturing the division of the working day between necessary and surplus labor time.

The purpose of the LTV is to reveal the origin of profits, the key variable in capitalist economies. At its most basic, capitalist society is a class society of workers and capitalists; these classes exist in antagonistic relation to each other, and that antagonism is based on the extraction of surplus labor from the working class by the capitalist class. Extraction of surplus labor is called "exploitation", and it characterizes all types of class society. But while exploitation is obvious in for example slave societies and feudal societies, it is not obvious in capitalist societies where market transactions are voluntary. The purpose of the LTV is to then show how voluntary participation in markets nonetheless generates exploitation.

In the literature on mathematical Marxian economics, this intuition has been captured by the Fundamental Marxian Theorem (FMT), which establishes that there can be no profits without exploitation. Exploitation is a necessary and persistent condition of capitalist economies. Without exploitation there is no explanation for the source of profit, or many of the dynamics of capitalist economies. Formally, the FMT for the $n$-good case with $n$ activities and no joint production is as follows.

Fundamental Marxian Theorem (FMT). The rate of profit $r$ and the rate of surplus value $\epsilon$ are always positive, zero, or negative simultaneously.

Proof. The rate of profit can be given as follows and is a function of the rate of exploitation:

$$
\begin{aligned}
r & =\frac{v(I-A-b l) x}{v(A+b l) x}=\frac{v(I-A-b l) x}{v b l x} \frac{v b l x}{v(A+b l) x} \\
& =\epsilon \cdot \frac{1}{1+v A x / v b l x} .
\end{aligned}
$$


As long as vblx $>0, r=0 \Longleftrightarrow \epsilon=0$, and $r \lessgtr 0 \Longleftrightarrow \epsilon \lessgtr 0$.

The FMT captures the idea that value theory provides the foundations for the Marxian theory of exploitation, showing that profits result from the exploitation of labor, and can be generalized as follows: ${ }^{10}$

(T4) Fundamental Marxian Theorem: The rate of exploitation is positive if and only if the aggregate price rate of profit is positive

$$
\epsilon>0 \Longleftrightarrow r_{p}>0
$$

The FMT is a rather weak way of capturing the idea that, in private ownership economies with unequal distribution of productive assets, profits derive from the exploitation of workers. For it does not impose any quantitative restrictions on the relation between aggregate profits and aggregate surplus value. Yet, one may argue that in Marxian value theory aggregate profits are just the monetary representation of aggregate surplus value, as profits transfer social surplus and social labor from workers to capitalists. One way of capturing this intuition is in the next axiom.

(T5) Redistribution Theorem: Total profit $s_{p}$ is equal to total surplus value $s_{v}$ :

$$
s_{p}=p(I-A) x-w l x=v(I-A-b l) x=s_{v},
$$

and the rate of exploitation is the ratio of total profits to total wages:

$$
\epsilon=\frac{p(I-A) x-w l x}{w l x} .
$$

Finally, although labor values do not, and cannot, in general be taken as predictors of equilibrium prices some robust relations can be established between the two sets of variables. For example, in the standard Leontief setting, it is possible to derive a precise functional relation - more precisely, a correspondence - between prices of production and labor values (see Pasinetti (1977, Ch. 5, Appendix) and Roemer (1981, Ch. 8, sec. 2)). Even in the simplest linear economies, however, this correspondence is not particularly transparent, or informative: for all sectors $i$, price-value differences

$\overline{10}$ Axiom (T4) restricts the appropriate definition of the value of labor power in a way that is consistent, for example, with the 'New Interpretation' (Duménil 1980, 1983; Foley 1982, 1986b). 
depend on how the composition of capital in the production of $i$ differs from that in the production of the commodity used as numéraire, both compositions being evaluated at prices of production. But they also depend upon the "the intricate network of relations between rate of profit and prices in the whole economic system" (Pasinetti 1977, 136). A simpler, and more transparent - albeit significantly weaker - relation between the two magnitudes can be stated in the following axiom, which also generalizes a property of labor values in Leontief economies. ${ }^{11}$

(T6) Labor-Commanded Theorem: Labor values are smaller than (market or production) prices when these prices are normalized by the money wage rate, if all sectors earn positive profits.

(T1)-(T6) are formally weak and reasonable properties that any general definition of labor values should satisfy. But they are by no means trivial. We have shown that they hold in single (nonjoint) production systems with no fixed capital, but they are not easily generalized to general production technologies and all of the received definitions of exploitation violate one or more of (T1)-(T6). For example, the definitions proposed by Morishima (1974), Roemer (1982), and Matsuo (2008) violate (T1) and (T5).

\subsection{Pragmatic uses and applications of the notion of labor values}

In the previous sections, we have discussed several, mostly theoretical properties that the LTV should satisfy. An approach to value theory that satisfies both (P1)-(P6) and (T1)-(T6) preserves some of the key theoretical and methodological insights of Marx's LTV. This is a crucial step in the construction of a logically consistent and theoretically relevant approach to the LTV. Yet, the ultimate judgement on value theory depends on its ability to contribute to causal explanations of economically relevant phenomena. In this section, we discuss some possible applications and developments of the LTV, which attempt to link the theoretical notion of labor values to actual data. We list them without discussing each of them individually because they share a common motivation: contrary to the received view, far from being metaphysical, the Marxian LTV is meant to provide a framework for the empirical analysis of capitalist economies.

${ }^{11}$ (T6) is relevant from an empirical viewpoint in that it identifies market prices as a useful upper bound for values. 
(A1) Leontief Multiplier Theorem: Monetary IO calculations of total labor costs per unit of output value provide the value/price ratios of individual commodities also in general production systems - if IO tables are calculated appropriately (by means of the industry technology hypothesis, see Chapter 10).

(A2) Labor Productivity Measurement: The reciprocal of labor values $1 / v_{i}$ are the appropriate measures of sectoral labor productivity.

(A3) Technical Change Theorem: Profitable capital-using labor-saving technical change raises labor productivity.

(A1)-(A3) are not as central in the literature as some of the other axioms listed in this chapter - for example, the FMT-but they also feature prominently in Marx's LTV. The link between labor content and labor productivity, for example, is central in Marx's notion of labor values:

In general, the greater the productivity of labour, the less the labourtime required to produce an article, the less the mass of labour crystallized in that article, and the less its value. Inversely, the less the productivity of labour, the greater the labour-time necessary to produce an article, and the greater its value. The value of a commodity, therefore, varies directly as the quantity, and inversely as the productivity, of the labour which finds its realization within the commodity (Marx 1976, 131).

According to (A2), this definition of labor content provides a concept of labor values which measures changes in industry-level labor productivity and a conceptual apparatus to understand long-run impacts of technical change, and in particular, as (A3) states, how capital-using labor-saving (Marx-biased) technical change generally reduces the labor content of commodities.

More generally, (A1)-(A3) incorporate the idea that the LTV can provide interesting insights on a wide range of empirical issues. These assertions are investigated in the chapters that follow.

\subsection{Conclusions}

It is well known that, unless the same technology is adopted in all sectors, production prices are not proportional to labor values and thus the former are not-in any meaningful sense - determined by the latter. In the received view, this implies that Marx's LTV is at best redundant. Further, Steedman (1977) has famously argued that, outside of the simplest Leontief economies, the notion of labor value is logically incoherent. In this chapter we have put these standard results 
in the literature into a broader perspective and have developed a novel methodological discussion of Marxian value theory.

Starting from first principles, we have explicitly discussed various possible interpretations of the Marxian LTV ('what labor values are good for') and, consequently, the properties that a proper, general definition of labor values should have. We have argued that, among other things, labor values should be definitionally nonnegative, and the labor value of any commodity should be zero if and only if its price is zero. Further, different types of labor should be homogenized using wages as the relevant conversion rates. We have also formally stated some key results in mathematical Marxian economics, including a relation between exploitation and aggregate profits (the FMT), the proportionality between prices and values when the equilibrium rate of profit is zero, and the aggregate equality between total direct labor spent in production and the labor value of the net product.

The axioms listed in this chapter are formally weak and theoretically robust, and represent the core of Marx's LTV - indeed, they can all be traced back to Marx's own writings. They are generalizations of properties that the standard definition of labor values possesses in simple Leontief models. Yet they are not trivial: the main received definitions do not satisfy (one or more of) them in more general economies, in particular if joint production and fixed capital are considered. Morishima's (1974; 1976b) celebrated definition of labor value as the minimum amount of direct labor necessary to produce a specific bundle of commodities (the so-called 'true' labor value of this bundle) guarantees uniqueness and nonnegativity of labor values and satisfies (P4)-(P5), and-under certain assumptions on technology(T5). ${ }^{12}$ In economies with joint production, however, it does not fulfill (T1) and (P3), for example. Based on the discussion in this chapter, it follows that Morishima's approach cannot be considered as a robust extension of Marx's ideas on value.

Perhaps more importantly, the above properties are also not trivial in the sense that they point to the theoretical and empirical significance of the LTV. Contrary to the received predictive view, labor values can be used for descriptive and normative purposes in order to understand some fundamental, structural characteristics and tendencies of capitalist economies, beneath the surface of monetary magnitudes and market phenomena. As argued above, for example,

${ }_{12}$ See, however, Petri's critique (Petri 1980). For a more general, recent discussion, see Matsuo (2008), Veneziani and Yoshihara (2012, 2015a), Yoshihara (2017). 
(T1), (T2), (T4), and (T5) suggest that value theory identifies a link between profits and exploitation, and more generally between the creation and distribution of value, and the creation and distribution of aggregate income. Similarly, (A2)-(A3) suggest that labor values can be used in the analysis of the real effects of competition driven by technical change, and the dynamics of productivity, technical progress and accumulation.

In closing this chapter, two important points should be made to clarify the nature and scope of our argument. First, it may be objected that the above properties are not axioms: they should be, and indeed usually are, proved as results in a given economic environment, under certain conditions. Yet the central relevance of each such relation in value theory is such that "its epistemological status in our understanding is as a postulate. We seek a model which will make our postulated belief true" (Roemer 1982, 152). The FMT, for example, has been central in debates on Marx's LTV and specifically for the development of new definitions of labor values (and exploitation). Hence, we consider them as axiomatic properties that any appropriate definition should satisfy.

Second, the main contribution of this chapter is methodological. We have identified a number of axioms that generalize some key properties of labor values in the standard Leontief setting and, in our reading, characterize Marx's LTV. This list is not meant to be exhaustive. For example, one may argue that labor values should provide the foundations for Marx's General Law of Accumulation (Marx 1976, Ch. 25), which requires a macroeconomic presentation in real terms that is independent of base periods. Labor values may play an important role in this analysis, because the implied fluctuations in economic growth may be very long-phased.

Although the list may be expanded further, it suffices for our purposes. In the rest of Part II, we present a definition of labor values that satisfies all properties in general linear economies, thanks to a specific reformulation of 'additivity'. This can be done by adopting an accounting procedure within the traditional linear approach to labor values which allows us to deal with multiple production activities, joint products, fixed capital, and so on. Our approach remains therefore close to Marx's general ideas about value, generalizing it to economies with some characteristics - such as joint production-which had not been considered by Marx.

Conceptually, the approach proposed in this book is quite simple. Marx's labor values should be interpreted as an IO accounting construct homologous to the total labor costs defined in Richard Stone's SNA. 
These labor values do not predict prices. But they can be used to construct indices of real labor productivity at the sectoral level.

We shall not prove that our approach provides the unique definition that satisfies the above axioms. It is well known, for example, that the proof of the FMT does not suffice to characterize a unique definition of labor values in general economies and there are several definitions that satisfy (T5) (Veneziani and Yoshihara 2012, 2015a). Indeed, our approach is conceptually close to the 'New Interpretation' (Duménil 1980; Foley 1982, 1986b) in terms of the emphasis on the monetary aspects of capitalist economies and the empirically grounded notion of values. And there likely exist other conceivable definitions that satisfy the above properties. Nonetheless, the axioms identified in this chapter should narrow down the range of admissible definitions significantly. 



\section{Labor Productivity and the Law of Decreasing Labor Content}

\subsection{Introduction}

Heterodox, and more specifically Marxist, economists have long held the belief that the inherent functioning of the capitalist system - and in particular the forces driving technical change, including class struggleleads to a tendential decrease in the amount of labor necessary to produce (or, indeed, embodied in) commodities. One of the clearest and most rigorous formulations of this intuition is due to Farjoun and Machover (1983), who derived the celebrated law of decreasing labor content (henceforth, LDLC). In their probabilistic approach: if $C$ is a commodity produced over a certain period of time, then "there is virtual certainty (probability very near 1 ) that the labor content of one unit of $C$ will be lower at the end of the period than it was at the beginning" (Farjoun and Machover 1983, 97). Further, more explicitly than other authors, Farjoun and Machover put the LDLC at the center of the analysis and considered it as one of the key defining features of capitalist economies: it is "the most basic dynamic law of capitalism, archetype of all capitalist development" (Farjoun and Machover 1983, 139). Therefore, Farjoun and Machover's contribution represents a natural starting point for this chapter, whose main focus is labor productivity and its relation with technical change in capitalist economies.

Granting that the LDLC characterizes capitalist economies, two questions immediately arise. First, why is the LDLC important from a theoretical viewpoint? Second, how can the LDLC be derived, or deduced, from the functioning of capitalist market economies? This chapter analyzes both questions in a general IO model, which is shown to provide a natural framework to formulate and derive the LDLC, and to understand its theoretical relevance.

Section 7.2 addresses the first question and it shows the salience of the notion of labor content for the understanding of labor productivity. 
The law is often seen as self-evidently relevant, because it is considered as equivalent to the law of increasing labor productivity (see, for example, Farjoun and Machover (1983, 11, 139) and passim). And labor productivity plays a key role in economic theories of growth and employment, including issues of innovation, structural change, income distribution, and so on. Yet the relevance of the LDLC for understanding trends in labor productivity is far from obvious: virtually all of the received productivity measures - as developed for instance in the UN's System of National Accounts (henceforth, SNA. See also OECD (2001); BLS (2008)) - focus on real GDP per unit of labor, or on some notion of 'real value added' per unit of labor, in order to measure the performance of (different sectors of) the economy. If the conventional SNA measures properly capture labor productivity, then one may argue that the notion of labor content is either misleading or at best redundant.

A thorough critical analysis of the standard SNA measures of sectoral as well as aggregate labor productivity is provided, from an IO perspective. The analysis of the structural features of the economy allowed by the IO framework forcefully shows that the SNA measures are inappropriate to capture production conditions, and shifts in efficiency and technology, owing to the central role of relative prices and final demand in their construction. Measures of sectoral and total labor productivity should be based on technological data as much as possible (subject to an unavoidable degree of aggregation), and they should not definitionally depend on price variables. The IO employment multipliers - that is, the labor values of Marxian economic theory ${ }^{1}$ - provide (in reciprocal form) theoretically sound measures of sectoral and economy-wide labor productivity, with purely technological foundations - insofar as IO coefficients can be interpreted as pure quantity magnitudes.

Thus, Section 7.2 proves that the law of increasing labor productivity cannot be properly understood unless the LDLC is formulated. Yet the results also have broader implications for productivity analysis, because they show that the shortcomings of the standard indices are more serious than it is acknowledged in the mainstream literature (e.g., Durand (1994); Cassing (1996); Schreyer (2001)) and that a proper understanding of labor productivity requires a focus on labor content. IO tables should always be an integral part

\footnotetext{
${ }^{1}$ Total labor costs and employment multipliers are identical in Leontief models, but can differ in more general economies. For a discussion, see Chapters 8-10.
} 
of the SNA and the point of reference for all productivity measures at the macro- and meso-level of economic activity. ${ }^{2}$

Critiques of standard SNA productivity measures from an IO perspective and the use of employment multipliers to measure productivity are not novel (see, among the others, Gupta and Steedman (1971); Steedman (1983); Wolff (1985, 1994); de Juan and Febrero (2000); Almon (2009)). ${ }^{3}$ This chapter presents a new set of arguments that emphasize the central relevance of relative prices and final demand in the definition of the standard measures, but none of the main criticisms crucially depends on changes in relative prices over time, so that well-known issues relating to index number construction are not focal. Moreover, a unified theoretical framework for the analysis of productivity measures is provided, which is based on a novel axiomatic method. Rather than comparing different measures in terms of their implications in various scenarios, this chapter starts from first principles and formalizes some theoretically desirable properties that any measure of labor productivity should satisfy. ${ }^{4}$ To be precise, the main axiom focuses on changes in productivity and states that labor productivity at $t$ in the production of good $i$ has increased relative to the base period, if a unit increase of the net product of good $i$ demands less labor than in the base period. This is a weak restriction and it incorporates the key intuitions behind the main productivity measures in the literature. Yet it characterizes the IO measures, whereas the conventional SNA indices do not satisfy it in general owing to their inherent dependence on relative prices and final demand.

The second major contribution of this chapter, in Section 7.3, is a rigorous analysis of the conditions under which profitable innovations lower labor values, thereby raising productivity and

${ }^{2}$ The importance of IO tables in productivity analysis is acknowledged in the mainstream literature (see, for example, Schreyer $(2001,50)$ ).

${ }^{3}$ In Richard Stone's original formulation of the UN's SNA, there are definitions of labor productivity that are conceptually analogous to the classical-Marxian measures (e.g., United Nations $(1968,69)$ ). This chapter suggests that it is unfortunate that this approach has been abandoned. It should be noted that productivity measures based on total labor costs are used both in Marxian theory, and in Sraffian, classical, and IO approaches. For this reason, in the rest of the chapter, they are sometimes referred to as classical-Marxian indices.

4 The adoption of an axiomatic approach to analyze Marxian themes is quite novel. Seminal contributions include Yoshihara (2010), Yoshihara and Veneziani (2009), Veneziani and Yoshihara (2011, 2012, 2015a, 2017b), and Mohun and Veneziani (2017). 
increasing consumption and investment opportunities. To be precise, in this chapter the $n$-commodity general equilibrium models analyzed by Roemer (1977, 1980b) are generalized into two main directions. First, following the approach developed by Flaschel (2010), the circulating capital model is extended to the treatment of fixed capital proposed by Bródy (1970) in a seminal contribution. This is important because fixed capital-or, more precisely, capital tied up in productionis a key feature of capitalist economies and it is at the center of innovation processes but, as various authors have argued, the standard von Neumann framework has serious theoretical and empirical limitations ${ }^{5}$ Second, following one of the key insights of Farjoun and Machover (1983), no condition on uniform profit rates is imposed and the conclusions hold for any vector of prices expressed in terms of the wage unit. This extension is both empirically and theoretically relevant, because general equilibrium-type constructions, including uniform profit rate models, may be unsatisfactory as representations of allocation in market economies (for a thorough discussion, see Flaschel et al. (2012b).

In this general framework, different forms of technical change can be considered, and a deterministic theoretical foundation for the LDLC can be derived. In fact, it can be proved that profitable fixed-capitalusing labor-saving innovations lead to productivity increases. Given that capital-using labor-saving technical change has characterized most of the phases in the evolution of capitalism (Marquetti 2003), this result provides theoretical foundations for the conclusion that labor values tend to fall, and labor productivity tends to rise, over time in capitalist economies. These results are consistent with the Marxian analysis of technical change and the historical tendencies of capitalism (see Foley (1986a) and Duménil and Lévy $(1995,2003)$ ), and identify one of the key dynamic laws of capitalism, describing the link between profitable innovations, the tendential rise in the Marxian technical composition of capital, and long-run increases in labor productivity. ${ }^{6}$

The formal analysis also has broader implications concerning the social effects of capitalists' individual decisions. For it can be proved that there is no clear-cut relationship between profitable

\footnotetext{
${ }^{5}$ See Bródy (1970) and, more recently, Flaschel, Franke, and Veneziani (2012b). For an extension of Roemer's (1977) model to von Neumann economies see Roemer (1979) and Dietzenbacher (1989).

${ }^{6}$ Given the focus of the chapter, the LDLC is not analyzed in the context of the broader set of dynamic laws of capitalism. However, some possible links and avenues for further research are briefly discussed below and in the concluding section.
} 
technical change and social welfare in capitalist economies: capitalists' maximizing behavior is neither necessary nor sufficient for the implementation of productivity-enhancing and welfare-improving innovations.

The analysis in Section 7.3 is related to the classical literature on technical change, distribution, and the evolution of capitalism (for recent contributions, see Duménil and Lévy (2003); Foley (2003); Petith (2008)). Yet unlike in the latter contributions, an explicit microeconomic perspective is adopted, which emphasizes capitalists' profit-maximizing behavior in highly disaggregated economies. Moreover, although the chapter sheds some light on the influence of distributive conflict on technical change, the focus is not on the general relation between technical change and distribution, or on the much-debated effect of technical progress on profitability. ${ }^{7}$ Instead the effect of individually optimal capitalist decisions on productivity and social welfare is thoroughly explored. Finally, although the process generating innovations is not explicitly formalized, the analysis can be supplemented with the classical-Marxian evolutionary model of technical change developed by Duménil and Lévy (1995, 2003).

The focus of this chapter is primarily theoretical and methodological: the chapter provides a general analysis of the relationships between prices, technical change, and labor productivity. Yet our analysis has clear empirical implications, and an empirical appraisal of the main theoretical conclusions of this chapter is provided in Chapter 8 .

\subsection{The labor content of commodities and the measurement of labor productivity}

The point of departure of the analysis is the standard IO Table 7.1, which shows economic activity in a particular year in the $n$ sectors of the economy. The notation is standard: $p(t)=\left(p_{1}(t), \ldots, p_{n}(t)\right)$ is the $1 \times n$ vector of prices of the $n$ commodities at time $t ; x_{i j}(t)$ is the amount of good $i$ used as intermediate input in the production of good $j ; x_{i}(t)$ is the gross output of $\operatorname{good} i ; f_{i}(t)$ is the final demand of good $i$.

At the most general level, labor productivity can be defined as a ratio between an index of output and an index of labor input. One possibility is to use gross output as a measure of real product and to

\footnotetext{
7 See, for example, Himmelweit (1974), Michl (1994) and the literature therein. Some implications of the analysis for these classical debates are briefly discussed in Section 7.4 below.
} 


\begin{tabular}{|c|c|c|c|}
\hline $\begin{array}{c}\text { Delivery } \\
\text { from } \downarrow \text { to } \rightarrow\end{array}$ & Sector $1 \ldots$ Sector $n$ & $\begin{array}{c}\text { Final } \\
\text { demand }\end{array}$ & Row sum \\
\hline Sector 1 & $x_{11}(t) p_{1}(t) \ldots x_{1 n}(t) p_{1}(t)$ & $f_{1}(t) p_{1}(t)$ & $x_{1}(t) p_{1}(t)$ \\
\hline . & . & . & . \\
\hline . & . & . & . \\
\hline . & . & . & . \\
\hline Sector $n$ & $x_{n 1}(t) p_{n}(t) \ldots x_{n n}(t) p_{n}(t)$ & $f_{n}(t) p_{n}(t)$ & $x_{n}(t) p_{n}(t)$ \\
\hline Value added & $Y_{1}(t) \quad \ldots$ & - & $Y(t)$ \\
\hline Column sum & $\ldots \quad x_{n}(t) p_{n}(t)$ & $F(t)$ & \\
\hline
\end{tabular}

Table 7.1: The standard form of an IO table

define labor productivity as gross output per unit of direct labor. As is well known, however, this measure is appropriate only in the rather special case of technical progress affecting all factors proportionally. Further, gross output based indices of productivity are sensitive to the degree of vertical integration: ceteris paribus, gross output based productivity rises as a consequence of outsourcing, even if there are no changes in technology and production conditions.

Therefore most of the literature focuses on value added. ${ }^{8}$ Two methods are used to obtain real output measures starting from value added data. The single deflation method requires deflating all entries (both outputs and inputs) in the nominal Table 7.1 by a common price deflator, say $P$. Single-deflated value added in sector $i$ is then $Y_{i}^{s}(t)=$ $Y_{i}(t) / P$, and at the aggregate level $Y^{s}(t)=\sum_{i=1}^{n} Y_{i}^{s}(t)$. In contrast, the method of double deflation attempts to measure everything in constant prices, that is, with regard to Table 7.1 it attempts to replace current prices $p(t)$ with the prices $p(0)$ of a base year $t=0$. This method, however, cannot be directly applied to the Value Added row in Table 7.1, whose entries are pure value magnitudes, and the double deflated sectoral values added $Y_{i}^{d}(t)$ are obtained indirectly by applying the accounting consistency requirement of the nominal Table 7.1 to its analogue in constant prices. This means that $Y_{i}^{d}(t)$ is the value added that would have resulted in sector $i$, had the prices in Table 7.1 remained constant after the base year.

${ }^{8}$ For an approach focusing on gross output, see Hart (1996) and Stiroh (2002). 
Value added in base year prices remains a value magnitude and not a quantity independent of relative prices, and therefore both single- and double-deflated value added are problematic notions in productivity analysis. "Value added is... not an immediately plausible measure of output: contrary to gross output, there is no physical quantity that corresponds to a volume measure of value-added" (Schreyer 2001, 41). Rather than measures of sectoral real output, single deflated values, $Y_{i}^{s}(t)$, should be interpreted as indices of sectoral real incomes, with only a distant relation with technological conditions. Any such measure as $Y_{i}^{s}(t) / L_{i}(t)$ - where $L_{i}(t)$ denotes the work hours employed in sector $i$-represents at best real purchasing power per unit of labor, rather than sectoral labor productivity. In contrast, the economic meaning of sectoral double deflated value added is rather unclear: since $Y_{i}^{d}(t)$ in general differs from $Y_{i}^{s}(t)$, for any $i$, then $Y_{i}^{d}(t)$ does not measure output correctly, and in addition it has nothing to do with real purchasing power. It is a purely fictitious quantity representing the income per worker that would have emerged if prices had remained constant at the level of the base year.

These well-known conceptual problems, though, are usually considered as minor, and in virtually all of the literature on labor productivity, value-added measures of real output, and in particular the double-deflated values, $Y_{i}^{d}(t)$ and $Y^{d}(t)$, are used. Sectoral and macroeconomic labor productivity are defined, respectively, as $\pi_{i}^{c}(t)=$ $Y_{i}^{d}(t) / L_{i}(t)$, and $\pi^{c}(t)=Y^{d}(t) / L(t)$, where $L(t)=\sum_{i=1}^{n} L_{i}(t)$, and

$$
\pi^{c}(t)=\sum_{i}\left(\frac{L_{i}(t)}{L(t)}\right) \cdot\left(\frac{Y_{i}^{d}(t)}{L_{i}(t)}\right)=\sum_{i}\left(\frac{L_{i}(t)}{L(t)}\right) \cdot \pi_{i}^{c}(t) .
$$

Value added based indices are considered theoretically and empirically meaningful. Indices based on single-deflated value added are deemed appropriate to analyze issues relating to economic welfare, whereas "for the purposes of measuring efficiency and productivity [double deflated measures are] to be preferred" (Stoneman and Francis 1994, 425) (see also Cassing (1996)). Several doubts can be raised on both claims, and in general on the standard approach to productivity analysis.

In IO analysis, it is common to choose the units of the $n$ commodities so that, in the base period, $p(0)=e^{\prime} \equiv(1, \ldots, 1)$. The double or rowwise 'price deflated' Table 7.1 can then be expressed in matrix notation as in Table 7.2. Following common practice in IO analysis, the matrix of intermediate inputs $X$ can be transformed into the matrix of input coefficients $A=X \hat{x}^{-1}$, and the $1 \times n$ vector of direct labor inputs $L=\left(L_{1}, \ldots, \ell_{n}\right)$ can be similarly transformed into a vector of labor 


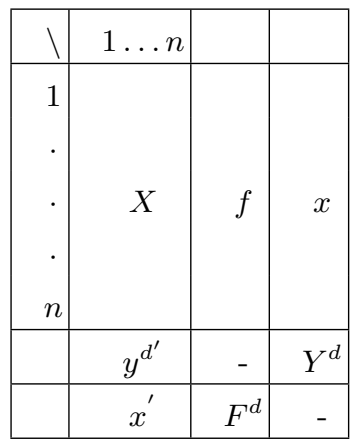

Table 7.2: Elementary IO table in matrix notation

coefficients $l=L \hat{x}^{-1} \cdot{ }^{9}$ Then, the macro-identity $Y^{d}=p(0) f=F^{d}$ behind Table 7.2 can be expressed in matrix notation as follows

$$
Y^{d}=p(0) y^{d}=p(0)(I-A) x=p(0) f=F^{d} .
$$

In contrast, the labor time spent, directly and indirectly, in the production of the $n$ goods is given by $v=\left(v_{1}, \ldots, v_{n}\right)=l(I-A)^{-1}$ and the IO, or classical-Marxian measures of sectoral labor productivity are defined as $\pi_{i}^{m}=1 / v_{i}$. In the rest of this section, a general framework is provided to compare productivity measures. In order to avoid problems of interpretation, the structural coefficients $(A, l)$ are considered as the parameters of a linear technology, as in standard IO practice.

One of the key shortcomings of the SNA measures is their sensitivity to changes in relative prices that do not reflect any shift in production conditions. Consider, for example, a simple economy with one capital good and one pure consumption good, such that in period $t$ the technical coefficients, $a_{i j}$, are $0<a_{11}<1, a_{12}>0$, and $a_{21}=a_{22}=0$. If a single price deflator $P$ is used, which includes prices of all sectors, as in standard index number theory, then quite puzzlingly real value added in sector 1 may be affected by changes occurring in sector 2 even if good 2 does not enter the production of good 1, either directly or indirectly. In general, when output prices change relative to input prices, the single deflation method will detect variations in productivity even if production conditions are unchanged. ${ }^{10}$

\footnotetext{
${ }^{9}$ For the sake of notational simplicity, in the rest of the chapter, the timing of vectors will be omitted, whenever this is clear from the context.

${ }^{10}$ For related analyses of the sensitivity of the SNA measures to changes in relative prices see Durand (1994), Hart (1996), and Almon (2009).
} 
Productivity indices based on double deflated value added fare no better. Consider the IO matrix $\tilde{A}$ in constant prices where the standard normalization $p(0)=e^{\prime}$ is not adopted, so that $\tilde{a}_{i j}=p_{i}(0) a_{i j} / p_{j}(0)$, for all $i, j$. Similarly, $\tilde{l}_{j}=l_{j} / p_{j}(0)$ and thus the same relationship holds for labor values: $\tilde{v}_{j}=v_{j} / p_{j}(0)$. Because the investment good sector is homogeneous with respect to inputs and outputs:

$$
\pi_{1}^{c}=\frac{1-p_{1}(0) a_{11} / p_{1}(0)}{l_{1} / p_{1}(0)}=\frac{1-a_{11}}{l_{1} / p_{1}(0)}=\frac{p_{1}(0)}{v_{1}},
$$

so that relative prices do not distort $\pi_{1}^{c}$, which coincides with the IO measure. For the consumption good sector, however, a different conclusion holds:

$$
\begin{aligned}
\pi_{2}^{c} & =\frac{1-p_{1}(0) a_{12} / p_{2}(0)}{l_{2} / p_{2}(0)}=\frac{p_{2}(0)-p_{1}(0) a_{12}}{l_{2}} \neq \frac{1}{v_{2} / p_{2}(0)} \\
& =\frac{1}{\left(v_{1} / p_{1}(0)\right) p_{1}(0) a_{12} / p_{2}(0)+l_{2} / p_{2}(0)}=\frac{1}{\left(v_{1} a_{12}+l_{2}\right) / p_{2}(0)} .
\end{aligned}
$$

The numerator of $\pi_{2}^{c}$ depends on relative prices, and thus on their structure and on the base period used: different vectors $p(0)$ lead to different values of $\pi_{2}^{c}$ regardless of production conditions. To be sure, labor values are also measured relative to output value, but this only means that each time series of labor values is divided by the constant price of the corresponding good, which does not distort the internal structure of the time series itself: for any given $j, 1 / v_{j}$ is only rescaled and its growth rate is independent of prices. In general, whereas the indices $\pi_{j}^{c}$ depend on the conceptually dubious double deflated values added, the vector $v$ is derived from the meaningful, volume-oriented double deflated entries of the IO table $\tilde{A}$.

The previous conclusions can be generalized and made more rigorous, by analyzing alternative approaches in a unified framework, in which some desirable properties of productivity measures are defined ex ante. Let $e_{i}=(0, \ldots, 1, \ldots, 0)^{\prime}$ be the $i$-th unity base vector. Definition 1 formalizes the notion of increases in labor productivity.

\section{Definition 7.1.}

(1) Labor productivity at $t$ has increased with regard to commodity $i$, relative to the base period, if and only if an increase of the net product $f$ by one unit of commodity $i$ demands less labor than in the base period. Formally, let $x_{i}(t)=(I-A(t))^{-1} e_{i}$ and let $\ell_{i}(t)=$ $l(t) x_{i}(t)$ : labor productivity has increased if and only if $\ell_{i}(t)<\ell_{i}(0)$. 
(2) If $\ell(t) \leq \ell(0)$ then labor productivity at $t$ has increased in the whole economy, with respect to the base period.

Definition 7.1 does not aim to capture all aspects of labor productivity, and it only constrains changes in productivity. From an epistemological viewpoint, it can be seen as an axiom: whatever else a measure of productivity may do, it should satisfy Definition 7.1, which sets some minimal restrictions on productivity measures. From this perspective, Definition 7.1 has a number of attractive features. First, it has a firm technological foundation which captures only shifts in productive conditions and efficiency: purely monetary magnitudes are irrelevant and final demand plays only an auxiliary role. ${ }^{11}$ This is certainly a desirable property of labor productivity measures, as many authors have argued (e.g. OECD (2001)). Second, by focusing on goods, rather than sectors, Definition 7.1(1) incorporates the interdependencies between sectors and it allows one to capture the relation between technical change and social welfare. This may seem more controversial, but a similar concern for the role of intermediate inputs and vertical integration actually motivates the use of valueadded based - as opposed to gross output based - indices in the mainstream literature (e.g. Schreyer (2001, 41ff)): they are preferred because they capture interindustry transactions and "provide an indication of the importance of the productivity measurement for the economy as a whole. They indicate how much extra delivery to final demand per unit of primary inputs an industry generates" (Schreyer 2001, 42). Third, Definition 7.1(2) may be deemed rather stringent, especially if $n$ is large, as it requires (weakly) monotonic increases for all goods. From an axiomatic perspective, however, it sets a very weak and intuitive restriction on any productivity measure. This is even more evident if a (neoclassical) notion of productivity as measuring economic welfare is adopted, for in this case Definition 7.1(2) is analogous to a Paretian condition capturing vector-wise improvements in consumption and investment opportunities.

Definition 7.1 is by no means trivial, however. For example, in Definition 7.1 all labor is implicitly treated as productive. This may be deemed objectionable from a Marxist viewpoint: some labor might be considered unproductive and therefore not count. ${ }^{12}$ Thus, Definition 7.1 is not vacuous and it does incorporate substantive assumptions. It

${ }^{11}$ The original net product $f$ is irrelevant in Definition 7.1 , thanks to the linearity of the technology.

12 This would also force a distinction between labor embodied and Marxian value. 
is not clear theoretically how to incorporate a productive-unproductive labor distinction into a micro-based IO framework, because what is productive from the perspective of an individual capital might be unproductive from the perspective of total social capital, and from this perspective Definition 7.1(2) does not hold. That is, the IO approach requires the maintenance of vertical additivity in an IO table, and the productive-unproductive labor distinction effectively contests it. But it is worth remarking first, that the distinction between productive and unproductive labor is far from being widely accepted among Marxist economists, ${ }^{13}$ and second, that the issue is irrelevant in a comparison with standard SNA productivity measures, because the latter treat all labor as productive anyway.

The next result states that Definition 7.1 characterizes the classicalMarxian measures of labor productivity.

Proposition 7.1. For a given commodity $i, \ell_{i}(t)<\ell_{i}(0)$ if and only if $\pi_{i}^{m}(t)>\pi_{i}^{m}(0)$. Furthermore, if the whole economy is considered $\ell(t) \leq \ell(0)$ if and only if $\pi_{i}^{m}(t) \geq \pi_{i}^{m}(0)$, for all $i=1, \ldots, n$, with strict inequality for some $i$.

Proof. By the definition of $v$, for any final demand $f, L=\ell e=l x=$ $l(I-A)^{-1} f=v f$. The latter expression implies $\ell_{i}(t)=v(t) e_{i}=v_{i}(t)$ and the desired result follows.

In other words, labor productivity with regard to good $i$ increases if and only if the amount of labor directly and indirectly embodied in good $i$ decreases. Further, any index of aggregate labor productivity satisfies Definition 7.1(2) if and only if it is monotonic in the vector of labor values. Proposition 7.1 provides theoretical foundations to the classical-Marxian indices as the appropriate indicators of labor productivity. One may object that the indices $\pi_{j}^{m}$ have the disadvantage that they cannot be deduced only from data that characterize sector $j$, and it is this property that drives Proposition 7.1. Yet the standard value-added based measures cannot be defined based only on data from sector $j$, either, even though the dependence on the other sectors is less evident than in $\pi_{j}^{m}$. It is in fact impossible to formulate and interpret nominal value added $Y_{j}$ - as well as 'real' value added $Y_{j}^{s}$, or $Y_{j}^{d}-$ without reference to a price system (even if prices may not appear explicitly, owing to the normalization $p(0)=e^{\prime}$ ). SNA measures do depend on the data of the other sectors via the price vector, butunlike for $\pi_{j}^{m}$ - the sectoral influences are unexplained and depend on the contingent institutional and market conditions of the base

\footnotetext{
${ }^{13}$ For a thorough discussion, see Mohun (1996) and the subsequent debate.
} 
year. The rigorous technological foundation which characterizes the classical-Marxian indices is lost. Therefore, it should not be surprising that the standard SNA measures cannot correctly capture either sectoral or aggregate labor productivity. This is proved in the following propositions.

Proposition 7.2 states that the SNA and the classical-Marxian indices of sectoral labor productivity coincide only in a very special case.

Proposition 7.2. Let $p(0)=e^{\prime}$. The equality $\pi_{j}^{c}=\pi_{j}^{m}=1 / v_{j}$, for all $j=1, \ldots, n$ holds if and only if $\pi_{j}^{c}=\pi^{c}$, for all $j=1, \ldots, n$.

Proof. $(\Leftarrow)$ Suppose that $\pi_{j}^{c}=\pi^{c}$, all $j=1, \ldots, n$. Then $e^{\prime}-e^{\prime} A=\pi^{c} l$, or equivalently $\left(1 / \pi^{c}\right) e^{\prime}=l(I-A)^{-1}=v$.

$(\Rightarrow)$ Suppose that $\pi_{j}^{c}=1 / v_{j}$, all $j=1, \ldots, n$. Let $\widehat{\pi}^{c}$ denote the diagonal matrix with $\pi_{j}^{c}, j=1, \ldots, n$, on the main diagonal. Since $\pi_{j}^{c}=$ $1 / v_{j}$, all $j=1, \ldots, n$, then $v \widehat{\pi}^{c}=e^{\prime}$, or equivalently, $v=e^{\prime}\left(\widehat{\pi}^{c}\right)^{-1}$. By definition, $v=l(I-A)^{-1}$ and thus, by the latter equation: (i) $e^{\prime}=l(I-A)^{-1} \widehat{\pi}^{c}$. Further, by definition $e^{\prime}(I-A)=l \widehat{\pi}^{c}$, or: (ii) $e^{\prime}=l \widehat{\pi}^{c}(I-A)^{-1}$. Then it is immediate to show that (i) and (ii) have a meaningful solution only if $\widehat{\pi}^{c}=\pi^{c} I$, for some positive $\pi^{c}$.

By Proposition 7.2, any differences in the two sectoral indices must be examined in relation to sectoral productivity differences. The next result instead shows that the SNA measure of aggregate productivity satisfies Definition 7.1(2), if final demand is constant.

Proposition 7.3. Suppose that $f(t)=f(0)=f>0$. If $v(t) \leq v(0)$ then $\pi^{c}(t)>\pi^{c}(0)$. Furthermore, $\pi^{c}(t)>\pi^{c}(0)$ if and only if $v(t) f<$ $v(0) f$.

Proof. The result follows noting that $\pi^{c}(t)=p(0) f / L(t)$ and that the equality $L(t)=v(t) f$ holds, as shown in Proposition 7.1.

In other words, technical change yielding increases in productivity according to Definition 7.1(2) implies a corresponding change in the SNA macroeconomic measure of labor productivity. Further, the change in technology decreases the expenditure of human labor for the production of a given vector of final demand $f$. Thus, Proposition 7.3 suggests that movements in the SNA aggregate measure map changes in the IO indicators, if final demand is constant. Yet Proposition 7.3 does not necessarily hold if final demand varies, nor does it hold at the sectoral level.

Consider the two-sector economy described in Table 7.3, where process 1 is subject to technical change between $t=0$ and $t=1$. 
Let $p(0)=e^{\prime}$ and assume $w=1$. First, technical change in sector 1 is capital-using and labor-saving, in the sense that it increases the value of intermediate inputs, but it lowers labor costs, at current prices. Second, technical change is profitable, because unit costs in sector 1 decrease from 0.9 to 0.86 . Third, the SNA sectoral productivity measure increases in sector 1 and remains constant in sector 2 :

$$
\pi_{1}^{c}(1) \approx 1.44>\pi_{1}^{c}(0)=1.25, \quad \pi_{2}^{c}(1)=\pi_{2}^{c}(0)=8 .
$$

In contrast, fourth, the classical-Marxian measures, $\pi_{1}^{m}, \pi_{2}^{m}$, decrease:

$$
\begin{aligned}
& \pi_{1}^{m}(1) \approx 1.58<\pi_{1}^{m}(0) \approx 1.70, \quad \text { and } \\
& \pi_{2}^{m}(1) \approx 2.92<\pi_{2}^{m}(0) \approx 3.09 .
\end{aligned}
$$

\begin{tabular}{c|cccc}
\hline \hline structure $\backslash$ period & \multicolumn{3}{|c}{$t=0$} & \multicolumn{2}{c}{$t=1$} \\
\hline matrix of intermediate inputs $A$ & 0.1 & 0.3 & 0.44 & 0.3 \\
& 0.4 & 0.3 & 0.1 & 0.3 \\
\hline labor inputs $l$ & 0.4 & 0.05 & 0.32 & 0.05 \\
\hline \hline
\end{tabular}

Table 7.3: A two-sector economy with profitable capital-using and labor-saving technical change (at constant prices $p(0)=e^{\prime}, w=1$ )

The technical change described in Table 7.3 leads to a sharp divergence in the standard indices, $\pi_{i}^{c}$, and the IO indices, $\pi_{i}^{m}$, which can move in opposite directions. Therefore, by Proposition 7.1, the example in Table 7.3 proves that the SNA sectoral measures, $\pi_{i}^{c}$, do not satisfy Definition 7.1. Noting that these conclusions can be generalized to $n$-good economies, they can be summarized in the next Proposition. ${ }^{14}$

Proposition 7.4. Suppose that $f(t)=f(0)>0$. For any good $i$, if $\ell_{i}(t)<\ell_{i}(0)$ then $\pi_{i}^{c}(t)$ may increase, decrease, or remain constant relative to $\pi_{i}^{c}(0)$. Furthermore, it is possible to have $\ell(t) \leq \ell(0)$, but $\pi_{i}^{c}(t) \leqq \pi_{i}^{c}(0)$, for all $i$, with strict inequality for at least some $i$.

In other words, the standard sectoral productivity indices do

${ }^{14}$ In Table 7.3 the reciprocal of the direct labor time per unit of output, $1 / l_{i}(t)$, also increases in sector 1 and remains constant in sector 2 . Therefore Proposition 7.4 can be extended to the indices $\pi_{i}^{l}(t)=1 / l_{i}(t)$ which are also sometimes used to measure productivity. 
not satisfy the minimal requirements set out in Definition 7.1, even under the restrictive assumption of a constant final demand. The shortcomings of the SNA measures $\pi_{i}^{c}$ derive primarily from the fact that they crucially rely on price information and do not properly reflect changes in technology. As a result, they can show increases in productivity in every sector even if the net production possibilities of the economy are deteriorating. Actually, by Proposition 7.3, the SNA aggregate index $\pi^{c}$ correctly reflects changes in the whole economy whenever final demand is constant, but Table 7.3 shows that $\pi^{c}$ and the sectoral measures $\pi_{j}^{c}$ can move in opposite directions (in the example, $\pi^{c}$ increases), if the sectoral allocation of labor changes appropriately (see equation (7.1)). Hence, the SNA sectoral measures do not provide useful information concerning the sectors leading to movements in aggregate labor productivity.

It is worth stressing that the proof of Proposition 7.4 is completely general. In Table 7.3, only profitable technical change is considered, but this is unnecessary to establish the proposition. It is however theoretically relevant because it shows that the result is not driven by some peculiar, or economically meaningless, combination of parameters. Further, none of the conclusions depends on the assumption of capitalusing, labor-saving technical change, and it is easy to construct similar examples with other types of innovations.

Although the previous analysis has focused on sectoral productivity measures, the standard approach to aggregate productivity is also unsatisfactory, and the SNA measure $\pi^{c}$ does not satisfy Definition 7.1(2) in general. To see this, consider again a two-good economy with technical change between $t=0$ and $t=1$. At any $t$, let $L(t)=l(t) x(t)$, so that, by the definition of labor values, $L(t)=v(t) f(t)=v_{1}(t) f_{1}(t)+$ $v_{2}(t) f_{2}(t)$. Then, dropping time subscripts for the sake of notational simplicity, for a given technology $(A, l)$, the net product transformation line is given by:

$$
f_{2}=\left(L-v_{1} f_{1}\right) / v_{2}=L-\pi_{2}^{m} f_{1} / \pi_{1}^{m}, \quad \text { with } \quad \pi_{1}^{m}=1 / v_{1}, \pi_{2}^{m}=1 / v_{2} .
$$

Figure 7.1 shows that if $\pi_{1}^{m} / \pi_{2}^{m} \neq p_{2}(0) / p_{1}(0)$, there can be a change in final demand from $f^{0}$ to $f^{1}$, and a simultaneous change in technology $(A, l)$, such that $v(t) \leq v(0)$ and the net product transformation line shifts out, but $\pi^{c}(0)=p(0) f^{0}>\pi^{c}(1)=$ $p(0) f^{1}$. Noting that this argument can be easily generalized to $n$-good economies, it can be summarized in the next Proposition.

Proposition 7.5. Suppose that $f(t) \neq f(0)$. If $\ell(t) \leq \ell(0)$, then $\pi^{c}(t)$ may increase, decrease, or remain constant relative to $\pi^{c}(0)$. 


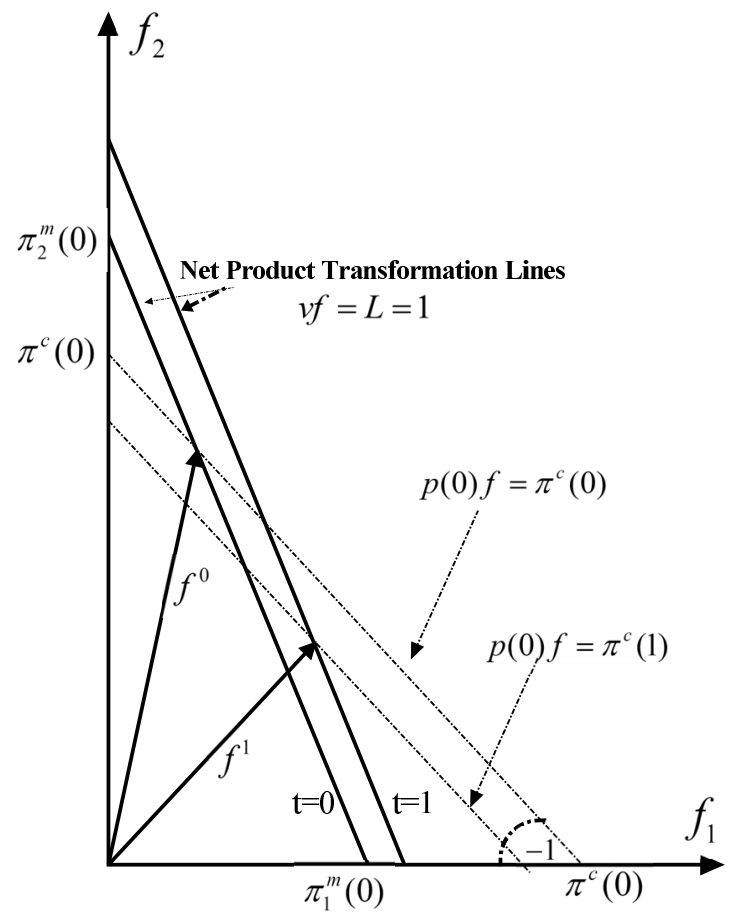

Figure 7.1: An increase in net production possibilities and a decrease in the conventional measure of aggregate labor productivity $\left(p(0)=e^{\prime}\right)$

Proposition 7.5 concludes the theoretical analysis of labor productivity measures. The previous results prove that the SNA sectoral measures do not meet the requirement set out in Definition 7.1(1). By Proposition 7.5, the SNA aggregate measure $\pi^{c}$ does not satisfy the very weak condition in Definition 7.1(2), either: it can detect a decline in productivity in the economy even if the net production possibilities unambiguously increase. Neither the sectoral nor the aggregate SNA productivity measures are adequate to capture shifts in technology and efficiency. Besides, Propositions 7.4 and 7.5 imply that, contrary to the received view, value added based measures are also inadequate to capture economic welfare, for an expansion of the net production possibilities increases social welfare. ${ }^{15}$ Again, the problem with standard measures is that they crucially depend on

$\overline{15}$ Proposition 7.5 also applies to measures based on single deflated aggregate value added. 
relative prices and final demand, in a manner that is independent from technical conditions.

In the mainstream literature, changes in relative prices over time have long been known to cause significant problems in index number construction, and especially in analyses of movements in SNA productivity measures based on fixed-base Paasche or Laspeyres indices. This has motivated a move towards the adoption of the chained Fisher index of real value added, whereby the square root of the product of Paasche and Laspeyres indices is taken for pairs of adjacent years, which are then chained together. It is still debated whether this effectively removes the problems associated with relative prices changing over time, especially given that it comes at the cost of losing additivity of the components and that the Fisher index is undefined when either the Laspeyres or the Paasche index is negative - a not unlikely occurrence (see Schreyer (2004) and Meade (2010)). But the key point here is that although the main shortcoming of standard measures is shown to be their reliance on relative prices (and final demand), nothing in the above analysis hinges upon changes in relative prices over time. The issue of the choice of the appropriate index number is therefore secondary for the key arguments, and the adoption of chained Fisher indices does not solve any of the problems highlighted above.

Firstly, the propositions hold for any two periods, and therefore a fortiori for any two adjacent periods. Hence, per se chaining is not relevant for the present analysis, for chained indices coincide with the standard, fixed-base indices when two adjacent periods are considered. Secondly, and perhaps more importantly, none of the above results depends on relative prices changing between periods 0 and $t$, and therefore they hold even if relative prices remain constant over time, and equal to the base period prices. In this case, however, there is no issue concerning the choice of the appropriate index number capturing changes in the standard SNA productivity measure, as they all coincide. Thus, the adoption of a chained Fisher index makes no difference for our key conclusions. Certainly, if technological conditions change, relative prices are likely to vary and therefore it may be unrealistic to assume them to remain constant over time. Yet, from a theoretical perspective, this is an appropriate assumption which allows us to identify a number of key limitations of standard productivity measures in addition to the well-known problems caused by changes in relative prices. The limitations of the productivity indices based on some notion of real value added are deep and suggest that the notion of labor content is essential to capture labor productivity. They also imply that the law 
of increasing labor productivity cannot be properly understood unless the LDLC is formulated.

\subsection{Technical change and the law of decreasing labor content}

Section 7.2 proves that the classical-Marxian indices $\pi_{j}^{m}=1 / v_{j}$ represent the only theoretically sound measures of labor productivity, which capture both its technological and its welfare aspects, and thus the LDLC is crucial in order to understand the dynamics of a capitalist economy. This section examines the relationship between prices and productivity, by analyzing the conditions under which profitable innovations lower labor values.

Technologies are now more generally described by a 3 -tuple $(K, A, l)$, where $K$ is a stock matrix whose generic entry $K_{i j}$ denotes the amount of good $i$ that is tied up in the production of good $j .{ }^{16}$ Everything is expressed per unit of output. For the sake of simplicity, it is assumed that the output matrix is equal to the identity matrix, $I$, but all results can be extended to technologies with multiple activities as well as joint production, provided the framework outlined in Chapters 9 and 11 to define labor content is adopted. In order to avoid a number of uninteresting technicalities, and with no loss of generality, the following standard assumption is made on technology.

Assumption 7.1. For any technology $(K, A, l), A$ is productive and indecomposable, and $l>0$.

Assumption 7.1 has two main implications. First, in this chapter technical changes in the various sectors of the economy are considered separately. ${ }^{17}$ Yet Assumption 7.1 implies that the effects of sectoral innovations extend throughout the economy. Second, let $p_{w j}=p_{j} / w$ be the price of good $j$ in terms of the wage unit, so that $p_{w}=p / w$ is the vector of wage prices. In what follows, it is not assumed that $p_{w}$ represents long-run production prices: it may well be a vector of (normalized) market prices. By Assumption 7.1, the Leontief-inverse exists and is strictly positive, and so the next Lemma immediately

${ }^{16}$ For a detailed explanation of the treatment of fixed capital see Bródy (1970) and Chapter 8. In this section, it is still assumed that the matrix of depreciation of fixed capital is equal to zero, i.e. $A^{\delta}=0$, but all results can be extended to the matrix $\bar{A}=A+A^{\delta}$, and the corresponding labor values.

17 The reader is referred to Bródy (1970) for a discussion of the prerequisites for an analysis of technical change in a Leontief IO system. 
follows, which extends a well-known property of prices of production with uniform profit rates to any vector of wage prices which allows for positive profits.

Lemma 7.1. Under Assumption 7.1, if $p_{w}>p_{w} A+l$, then $p_{w}>v=$ $l(I-A)^{-1}>0$.

Thus, labor commanded prices are a useful upper bound to estimate embodied labor costs even if no restrictive assumption on uniform profit rates is made.

Let $r_{j}$ be the profit rate on capital advanced in sector $j$. Definition 7.2 distinguishes various forms of technical change, depending on their effect on unit costs and on labor values, and on whether they tend to substitute labor for capital, or vice versa.

\section{Definition 7.2.}

(1) Technical change $\left(K_{\star j}, A_{\star j}, l_{j}\right) \mapsto\left(K_{\star j}^{*}, A_{\star j}^{*}, l_{j}^{*}\right)$ is profitable if and only if, at initially given prices $p_{w}$ such that $p_{w j}=r_{j} p_{w} K_{\star j}+$ $p_{w} A_{\star j}+l_{j}$ and $r_{j}>0$ :

$$
r_{j} p_{w} K_{\star j}+p_{w} A_{\star j}+l_{j}>r_{j} p_{w} K_{\star j}^{*}+p_{w} A_{\star j}^{*}+l_{j}^{*}
$$

(2) Technical change $\left(K_{\star j}, A_{\star j}, l_{j}\right) \mapsto\left(K_{\star j}^{*}, A_{\star j}^{*}, l_{j}^{*}\right)$ is progressive if and only if

$$
v=v A+l>v^{*} A^{*}+l^{*}=v^{*} .
$$

Similarly, technical change is regressive if and only if $v<v^{*}$.

(3) Technical change $\left(K_{\star j}, A_{\star j}, l_{j}\right) \mapsto\left(K_{\star j}^{*}, A_{\star j}^{*}, l_{j}^{*}\right)$ is: capital using $(K U)$ if and only if $p_{w} K_{\star j}<p_{w} K_{\star j}^{*}$; capital saving $(K S)$ if and only if $p_{w} K_{\star j}>p_{w} K_{\star j}^{*}$; labor using $(L U)$ if and only if $l_{j}<l_{j}^{*}$; and labor saving $(L S)$ if and only if $l_{j}>l_{j}^{*}$.

Definition 7.2 generalizes the definitions in Roemer (1977) to economies with capital tied up in production and to any vector of wage prices, $p_{w}$ : profits are treated as a mere residual and no assumptions are made on the uniformity of profit rates or on the determination of $p_{w} \cdot{ }^{18}$ It is important to note that in Definition $7.2(3)$, innovations are defined in monetary terms and thus are significantly more general than in Roemer (1977), in that they allow for non-monotonic changes in capital requirements. Finally, it is worth noting that in Definition 7.2(2) it is not restrictive to focus on technical changes where all labor values

${ }^{18}$ In Roemer (1977), cost-reducing innovations are called viable, but the notion of profitability more explicitly conveys the idea of monetary, rather than physical, magnitudes. 
change in the same direction. If technical change occurs in one sector at a time, this will not produce value changes in opposite directions in different sectors (see Roemer $(1977,410)$ ).

Next, define the following auxiliary intermediate input matrix:

$$
A^{*+}=\max \left\{A^{*}, A\right\} \geqq A^{*} .
$$

The auxiliary matrix $A^{*+}$ is a mathematical construct that will be useful to derive the formal theorems below. In particular, if $j$ is the sector subject to technical change, then $\left(K_{\star j}, A_{\star j}, l_{j}\right) \mapsto\left(K_{\star j}^{*}, A_{\star j}^{*+}, l_{j}^{*}\right)$ might be loosely interpreted as technical change using the most circulating capital intensive technique. Note also that $A_{\star j}^{*+} \geq A_{\star j}$ if and only if $A_{i j}^{*}>A_{i j}$, for at least some $i$. Based on $A^{*+}$, a specific class of innovations is considered below and the following assumption is made:

Assumption 7.2. For any profitable KU-LS technical change $\left(K_{\star j}, A_{\star j}, l_{j}\right) \mapsto\left(K_{\star j}^{*}, A_{\star j}^{*}, l_{j}^{*}\right)$, the following inequality holds: $p_{w} A_{\star j}+$ $l_{j}>p_{w} A_{\star j}^{*+}+l_{j}^{*}$.

Assumption 7.2 states that the main part of the cost-reduction process occurs via changes in the capital that is tied up in production, which allows for significant reductions in labor costs, whereas changes in intermediate inputs are unsystematic and secondary, and therefore profitable even if the auxiliary matrix $A^{*+}$ is considered. Assumption 7.2 rules out only secondary profitable technical changes, and yields no major loss of generality in the analysis of LS innovations. Formally, Assumption 7.2 provides a link between the effect of technical progress on fixed capital and changes in the use of intermediate inputs. Then, the first key result on technical change in general economies with fixed capital can be derived.

Theorem 7.1. Given Assumption 7.1. Let $p_{w}>p_{w} A+l$.

(i) Under Assumption 7.2, all KU-LS profitable technical changes are progressive.

(ii) However, there are KU-LS progressive technical changes which are not profitable.

Proof. Part $(i)$. Suppose first that $A_{\star j}^{*} \leqq A_{\star j}$, and thus $A^{*} \leqq A$. Since $l \geq l^{*}$, then by Assumption 7.1 it immediately follows that $v^{*}<v$. Suppose next that $A_{i j}^{*}>A_{i j}$, for some $i$. Consider the auxiliary matrix $A^{*+}$ and define the vector of auxiliary labor values $v^{*+}=v^{*+} A^{*+}+l^{*}$. By Assumption 7.2, $p_{w} A_{\star j}+l_{j}>p_{w} A_{\star j}^{*+}+l_{j}^{*}$, or, equivalently, $p_{w}\left(A^{*+}{ }_{-}\right.$ $A)-\left(l-l^{*}\right) \leq 0$, with both terms in brackets being semi-positive by 
assumption. By Lemma 7.1, $0<v<p_{w}$, and so the latter inequality implies

$$
v\left(A^{*+}-A\right)-\left(l-l^{*}\right) \leq 0,
$$

and thus

$$
v A^{*+}+l^{*} \leq v A+l=v .
$$

By recursive application of the latter inequality, we get:

$$
v(t+1)=v(t) A^{*+}+l^{*} \leq v(t), t=0,1,2, \ldots, \text { with } v(0)=v .
$$

This sequence is bounded below and monotonically decreasing and thus converges to

$$
v(\infty) A^{*+}+l^{*}=v(\infty)=v^{*+}
$$

Therefore, by Assumption 7.1 it follows that $v^{*+}<v$, so that $\left(A_{\star j}^{*+}, l_{j}^{*}\right)$ is progressive with respect to $\left(A_{\star j}, l_{j}\right)$. Finally, note that by definition $A_{\star j}^{*+} \geqq A_{\star j}^{*}$, and therefore $v^{*}=v^{*} A^{*}+l^{*} \leqq v^{*+}=$ $v^{*+} A^{*+}+l^{*}$, which implies $v>v^{*+} \geqq v^{*}$.

Part (ii). The desired result follows noting that there exist technical changes with $v^{*}<v$, such that $p_{w} A_{\star j}+l_{j} \leqq p_{w} A_{\star j}^{*}+l_{j}^{*}$ at the initial price vector $p_{w}>p_{w} A+l$, because the latter is not proportional to $v$ in general, and noting that for KU-LS technical changes this implies $r_{j} p_{w} K_{\star j}+p_{w} A_{j \star}+l_{j}<r_{j} p_{w} K_{\star j}^{*}+p_{w} A_{\star j}^{*}+l_{j}^{*}$.

Remark 7.3. The recursive argument used in Part $(i)$ can be modified to provide an alternative demonstration of Proposition 8 in Roemer (1977).

Theorem 7.1 is quite general and by no means obvious. For it proves that cost-reducing innovations that substitute fixed capital for labor are progressive, even if no stringent assumption is made concerning the effect of technical change on intermediate inputs. Therefore, in general, LS innovations will reduce the labor content of goods and increase net production possibilities. Yet profitable KU-LS innovations do not fully exploit the potential of technical progress to increase labor productivity. For there exist feasible technologies that will not be adopted by capitalists that would yield social welfare improvements by increasing net production possibilities.

The proof that profitable KU-LS innovations increase consumption and investment opportunities has relevant implications for the LDLC and the understanding of capitalist economies. For it derives a systematic relationship between certain forms of technical change, profit maximizing behavior, and labor values. Empirically, one may conjecture that distributive conflict and increasing wages have 
introduced a bias in the direction of technical change towards KULS changes that may partly explain the secular increase in labor productivity observed in capitalist economies. Theoretically, although class conflict is not analyzed here, one may construct a plausible scenario in which wage increases induce KU-LS technical change, and so a decrease in labor content. This argument may provide microfoundations to the LDLC, which need not be based on-but, of course, can be supplemented by - probabilistic considerations. The price implications of technical changes may indeed be chaotic, as Farjoun and Machover (1983) argued, but the quantity implications investigated in this chapter are independent of such chaotic behavior.

The result in Theorem 7.1, however, cannot be extended to other types of innovations. Theorem 7.2 proves that there may be profitable KS-LU innovations that reduce the economy's net production possibilities, and thus social welfare.

Theorem 7.2. Given Assumptions 7.1 and 7.2. Let $p_{w}>p_{w} A+l$.

(i) All KS-LU progressive technical changes are weakly profitable.

(ii) However, there are KS-LU profitable technical changes which are not progressive. More precisely, technical change is progressive if and only if $v_{j}>v A_{\star j}^{*}+l_{j}^{*}$.

Proof. Part $(i)$. If $p_{w} A_{\star j}+l_{j} \geqq p_{w} A_{\star j}^{*}+l_{j}^{*}$, then the desired result immediately follows from Definition 7.2(3). Therefore suppose $p_{w} A_{\star j}+$ $l_{j}<p_{w} A_{\star j}^{*}+l_{j}^{*}$. Since technical change is progressive, then by Lemma $7.1 p_{w}>v>v^{*}$. The latter inequalities imply that $p_{w}>p_{w} A^{*}+l^{*}$. Suppose, by way of contradiction, that $p_{w j}=r_{j} p_{w} K_{\star j}+p_{w} A_{\star j}+l_{j}<$ $r_{j} p_{w} K_{\star j}^{*}+p_{w} A_{\star j}^{*}+l_{j}^{*}$. Since $p_{w j}>p_{w} A_{\star j}^{*}+l_{j}^{*}$, this implies that there is a $r_{j}^{\prime} \in\left(0, r_{j}\right)$, such that $p_{w j}=r_{j}^{\prime} p_{w} K_{\star j}^{*}+p_{w} A_{\star j}^{*}+l_{j}^{*}$ and since by assumption $p_{w} K_{\star j}>p_{w} K_{\star j}^{*}$, it follows that $r_{j}^{\prime} p_{w} K_{\star j}+p_{w} A_{\star j}+l_{j}<$ $r_{j}^{\prime} p_{w} K_{\star j}^{*}+p_{w} A_{\star j}^{*}+l_{j}^{*}=p_{w j}$. The latter inequality implies that the KU-LS technical change $\left(K_{\star j}^{*}, A_{\star j}^{*}, l_{j}^{*}\right) \rightarrow\left(K_{\star j}, A_{\star j}, l_{j}\right)$ is profitable and therefore, since the premises of Theorem 7.1 are satisfied, it is progressive so that $v^{*}>v$, a contradiction. Therefore, we have $p_{w j}=$ $r_{j} p_{w} K_{\star j}+p_{w} A_{\star j}+l_{j} \geqq r_{j} p_{w} K_{\star j}^{*}+p_{w} A_{\star j}^{*}+l_{j}^{*}$.

Part (ii). First of all, note that if KS-LU technical change $\left(K_{\star j}, A_{\star j}, l_{j}\right) \rightarrow\left(K_{\star j}^{*}, A_{\star j}^{*}, l_{j}^{*}\right)$ is profitable, this has no implication on the inequality $v_{j} \gtreqless v A_{\star j}^{*}+l_{j}^{*}$. Then, we prove that technical change is progressive if and only if $v_{j}>v A_{\star j}^{*}+l_{j}^{*}$.

First, note that $v_{j}>v A_{\star j}^{*}+l_{j}^{*}$ implies $v A^{*}+l^{*} \leq v A+l=v$, and therefore it is possible to construct an infinite sequence

$$
v(t+1)=v(t) A^{*}+l^{*} \leq v(t), t=0,1,2, \ldots, \text { with } v(0)=v,
$$


which is monotonically decreasing, and bounded below, and thus converges to $v(\infty) A^{*}+l^{*}=v(\infty)=v^{*}, v^{*}>0$. By Assumption 7.1 it follows that $v>v^{*}$.

Next, note that if $v_{j}=v A_{\star j}^{*}+l_{j}^{*}$, then $v=v^{*}$. Finally, suppose $v_{j}<v A_{\star j}^{*}+l_{j}^{*}$. Then $v \leq v A^{*}+l^{*}$ and we can consider the following monotonically increasing sequence

$$
v(t) \leq v(t) A^{*}+l^{*}=v(t+1), t=0,1,2,3, \ldots, \text { with } v(0)=v .
$$

By Lemma 7.1, $v<p_{w}$ and by profitability it follows that $p_{w} A^{*}+l^{*} \leq$ $p_{w}$. Therefore:

$$
v(t) \leq v(t) A^{*}+l^{*}=v(t+1)<p_{w} A^{*}+l^{*} \leq p_{w}, t=0,1,2, \ldots
$$

Hence the sequence is bounded above by $p_{w}$, and so it converges to:

$$
v(\infty)=v(\infty) A^{*}+l^{*}=v^{*}, \quad v^{*}>0 .
$$

By Assumption $7.1 v<v^{*}$ must hold

Together with Theorem 7.1, Theorem 7.2 provides a full description of technical change in a capitalist economy with capital tied up in production. Theorem 7.2 characterizes the conditions under which KS-LU progressive technical change occurs: KS-LU innovations are progressive, and thus increase social welfare, if and only if they reduce the labor content of a commodity in terms of the old labor values. Thus, Theorem 7.2 implies that the problematic situation with respect to technological regress is, generally speaking, the labor-using case. To be specific, labor productivity falls if the following inequalities hold simultaneously

$r_{j} p_{w} K_{\star j}+p_{w} A_{\star j}+l_{j}>r_{j} p_{w} K_{\star j}^{*}+p_{w} A_{\star j}^{*}+l_{j}^{*}, \quad l_{j}<l_{j}^{*}, \quad v_{j}<v A_{\star j}^{*}+l_{j}^{*}$.

In Theorem 7.2, labor values move all in the same direction, i.e., if labor productivity falls in some sectors, then it falls in all of them. It is unambiguously clear whether the set of net production possibilities expands or contracts. In the KS-LU case with $v_{j}<v A_{\star j}^{*}+l_{j}^{*}$, it contracts, as the labor contents of all goods rise. Hence capitalist choices leading to KS-LU technical change may have adverse effects on economic development, since they may undermine the LDLC and thus decrease consumption and investment opportunities, and periods characterized by KS-LU technical change may be plagued by productivity slowdowns.

Theorems 7.1 and 7.2 generalize Roemer's (1977) results in 
economies with circulating capital and they identify some systematic connections "between the visible and the invisible - between price and labour-content" (Farjoun and Machover 1983, 84). As noted above, given the KU-LS nature of technical progress in actual capitalist economies, Theorem 7.1 sheds some light on the LDLC, by identifying a link between profit-driven individual actions and the dynamics of labor content. In contrast, Theorem 7.2 can be interpreted as identifying another (potential) failure of the invisible hand. The case $v_{j}=v A_{\star j}^{*}+l_{j}^{*}$ is the dividing line that separates strictly falling from strictly rising labor contents. This dividing line is expressed in terms of labor values, and thus it is not visible to agents in the economy, who take their profit-maximizing decisions based on price magnitudes. As a result, individually rational decisions may lead to socially suboptimal outcomes.

\subsection{Conclusions}

This chapter analyzes the law of decreasing labor content (LDLC) originally formulated by Farjoun and Machover (1983). First, the relevance of the LDLC is shown. It is argued that the IO indices based on the Marxian labor values are theoretically sound measures of labor productivity, whereas conventional indices based on real value added per worker are theoretically questionable and less reliable empirically. The notion of labor content is necessary to analyze labor productivity and the LDLC is central in order to understand the dynamics of capitalist economies.

Second, the dynamics of labor productivity in capitalist economies is analyzed in a general linear model with fixed capital. It is proved that capitalists' maximizing behavior is neither necessary nor sufficient for the implementation of productivity-enhancing and welfare-improving innovations. Further, it is shown that the type of capital-using labor-saving profitable innovations that have characterized capitalist economies tend to lower labor values, which provides a deterministic foundation for the LDLC. In the next chapter, some empirical evidence is also provided, which shows that the LDLC holds in the German economy after the reunification.

The analysis in this chapter can be extended in various directions. From a general theoretical viewpoint, this chapter can be interpreted as showing that profitable innovations that increase the Marxian technical composition of capital, raise labor productivity. This is a strong result in itself, but it immediately raises two questions. First, why does this sort of technical change occur? And, second, what are 
its implications in terms, e.g. of class incomes and distribution? As noted in the Introduction, both questions lie beyond the scope of this chapter but they are important issues in political economy and suggest important avenues for further research. A framework that may be worth considering in order to analyze both questions is developed in Chapter 12, in which a system of prices of production with the usual properties is derived which allows for non-uniform profit rates and wage rates, provided intersectoral wage and profit differentials are assumed to be fixed. In particular, we derive a generalized wage-profit curve, which may allow one to extend Himmelweit's (1974) argument. Further, within that framework, it should be possible to generalize the Okishio theorem, whereby any profitable technical changes lead to an increase in all of the sectoral profit rates, provided the real wage, and the structure of profit and wage rate differentials, remain constant. Yet, a thorough exploration of these issues must be left here for future research.

Further, in this chapter, we have considered some special forms of technical progress, focusing on certain changes in the physical coefficients of a given technology. Yet one may argue that the appropriate definition of technical change should be in monetary, rather than physical terms, and different types of changes affecting intermediate inputs should be defined according to whether $p_{w} A^{*} \leq$ $p_{w} A, p_{w} A^{*} \geq p_{w} A$, or $p_{w} A^{*}=p_{w} A$, and likewise for other coefficients. Although such changes are not considered in this chapter, the analysis in Section 7.3 does suggest some interesting lines for further research, which are consistent with the probabilistic approach suggested by Farjoun and Machover (1983).

As proved in Section 7.3, under different assumptions concerning technology, technical change in a given sector is progressive-and thus the LDLC holds - if and only if $v A^{*}+l^{*} \leq v$. Therefore, the next question is: under what general assumptions concerning technical change does the latter inequality hold? In chapter 7 of their book, Farjoun and Machover (1983) prove that the LDLC obtains in a probabilistic framework as the cumulative result of a sequence of capital-saving labor-preserving technical changes, where the cost of physical inputs decreases while labor requirements remain constant. To be specific, we interpret their argument as proving, in probabilistic terms, that if technical change $\left(A_{j}, l_{j}\right) \rightarrow\left(A_{j}^{*}, l_{j}^{*}\right)$ is such that $l^{*}=l$, then the following relation holds:

$$
p_{w} A^{*} \leq p_{w} A \Rightarrow v A^{*} \leq v A
$$


Interestingly, they do not extend their conclusions to the new vector of labor values $v^{*}$, which solves the matrix equation $v^{*}=v^{*} A^{*}+l$, but following the formal reasoning developed in Section 7.3, it is sufficient to iterate the sequence of decreasing vectors $v(k)$

$$
v(k+1)=v(k) A^{*}+l, v(0)=v=v A+l \rightarrow v^{*}=v^{*} A^{*}+l,
$$

to prove that $v^{*} \leq v$ must hold true, with strict inequalities under Assumption 7.1 and somewhat weaker formulations if nonbasic commodities are included.

Hence, in the light of our analysis, Farjoun and Machover's (1983) result on the LDLC is fairly strong, as it significantly generalizes the analysis of capital-using technical progress, and it represents a first important step from physical inequalities to sectoral price aggregates. The necessary condition for the above argument to hold is simply that $p_{w}\left(A^{*}-A\right) \leq 0$ implies $v\left(A^{*}-A\right) \leq 0$, which is true whenever $p_{w}=$ $\alpha v$, for some scalar $\alpha .{ }^{19}$ In their framework, the LDLC is based on assumptions concerning probability distributions of $p_{w}$ (relative to $v$ ) and a sequence of changes of the type described above, from which they can deduce the probability that the statement $\left[p_{w}\left(A^{*}-A\right) \leq 0\right.$ implies $\left.v\left(A^{*}-A\right) \leq 0\right]$ be true.

The latter argument, however, can be generalized to other types of technical change. Consider first labor-saving capital-preserving technical change, where the matrix $A$ remains constant. This immediately implies falling labor content, since $l^{*}-l \leq 0$ implies $v A+l^{*} \leq v$. But then it should also be possible to extend Farjoun and Machover's (1983) conclusions and the LDLC to the case of capitalsaving (in terms of prices) labor-saving technical change.

The cases of capital-saving labor-using, or capital-using laborsaving, technical progress are less clear-cut, if changes in technology are defined in terms of sectoral price aggregates, rather than in physical terms. Focusing on cost-reducing innovations, we now need to prove that the following condition holds:

$$
p_{w} A^{*}+l^{*} \leq p_{w} A+l \Rightarrow v A^{*}+l^{*} \leq v=v A+l .
$$

One may object that capital-using technical change in terms of prices $p_{w}$ is unrealistic with profit-maximizing firms, since it is unlikely that the use of intermediate inputs is systematically increasing per unit

${ }^{19}$ As we have seen at various times throughout the book, the latter equality holds, for example, if $p_{w}$ is a vector of prices of production and the composition of capital is uniform across sectors. 
of output produced. Yet, the input matrix $A$ also contains items related to fixed capital consumption (depreciation of buildings and machinery) and it is indeed conceivable that the amount of fixed capital per worker, and thereby also the consumption of these items, is increasing in terms of labor commanded prices. ${ }^{20}$ In this case, the IO matrix $A$ should be augmented to include durable investment goods and a depreciation matrix $A^{\delta}$ for these commodities should also be included in the computation of labor values. Then, labor values are defined as usual, namely: $v=v\left(A+A^{\delta}\right)+l$.

It is much more difficult to properly formulate investment criteria for the choice of technique when items with a longer life span than one year are used in capitalist production. For the sake of simplicity, here we still assume that they imply cost-reduction with respect to the matrix $\left(A+A^{\delta}\right)$ so that we may still focus on the condition

$p_{w}\left(A^{*}+A^{\delta *}\right)+l^{*} \leq p_{w}\left(A+A^{\delta}\right)+l \Rightarrow v\left(A^{*}+A^{\delta *}\right)+l^{*} \leq v\left(A+A^{\delta}\right)+l=v$.

Therefore Farjoun and Machover's probabilistic reasoning must now be applied to this theoretical implication in order to investigate the effect of sequences of technical change and the LDLC. This reconsideration of their argument goes beyond the scope of the book and is left for future research.

$\overline{{ }^{20} \text { See Bródy }}$ (1970) for its detailed discussion in terms of capital advanced, capital consumed and turnover times of capital advancements. 


\section{The Sources of Aggregate Profitability: Marx's Theory of Surplus Value Revisited}

\subsection{Introduction}

One of the key contributions of recent debates in value theory and in exploitation theory is the idea that there is no 'transformation problem' to be solved in Marx's labor theory of value (LTV). Labor values are not meant to provide an explanation of classical production prices. In line with much of the recent literature, we have argued, however, that a coherent account of values and the labor content of goods can be provided that is able to inform the empirical and theoretical analysis of the basic laws of capitalism, of the exploitative nature of capitalist relations of production, and of the determinants of the key magnitudes of capitalist economies, especially from a macroeconomic perspective. ${ }^{1}$

To be specific, in Chapter 7 , we have shown that the standard measures of labor content used in the IO literature, the classic Marxian labor values, represent the only sound labor productivity indices. Further, by using the standard Marxian labor values, we have provided a general proof of the law of decreasing labor content (LDLC) - arguably one of the key laws of capitalist accumulation - by demonstrating that profitable technical change that tends to replace living labor with capital (that is, capital-using labor-saving technical change) tends to decrease the labor content of commodities.

This chapter aims to show that a system of labor accounts can be developed, which is independent from money accounts and which provides theoretically and empirically relevant insights on observed phenomena in capitalist economies. To be precise, we analyze a dual interpretation of Marx's theory of profitability whose structure is

\footnotetext{
${ }^{1}$ See the classic contributions by Duménil (1980), Foley (1982), Flaschel (1983a), and, more recently, Mohun (2004), Yoshihara (2010), and Veneziani and Yoshihara (2012, 2015a, 2017b).
} 
conceptually analogous to that of a System of National Accounts (SNA), in which real magnitudes form a dual structure with respect to prices and quantities. A real SNA is designed to promote an understanding of the key processes driving the motion of nominal magnitudes. Analogously, Marx's labor value aggregates were designed as the essential elements of an understanding of what happens underneath the surface of the process of capital accumulation.

In particular, in this chapter we argue that labor values - or total labor costs, in our interpretation-provide important insights on one of the most important issues in Marxian economics, namely the determinants of the general, or economy-wide, rate of profit, and the LTV explains the key variables determining profitability. And Marx had a clear interest in demonstrating that his LTV explained the key variables determining profitability. Concerning the value and price of intermediate inputs, however, he wrote:

It is necessary to bear in mind this modified significance of the cost price, and therefore to bear in mind too that if the cost price of a commodity is equated with the value of the means of production used up in producing it, it is always possible to go wrong (Marx 1981, 265).

This chapter shows that this logical error in the comparison of value and price magnitudes may be empirically irrelevant and that the key insights of the Marxian theory of exploitation and of the profitability of a capitalist economy are valid.

More generally, far from being metaphysical constructs, labor values can be used to investigate empirically some of the fundamental dynamic laws and tendencies of capitalist economies. To this aim, we adopt a standard IO theoretic approach in the tradition of Leontief and Bródy (1970). Thus, we introduce the notions of capital consumed and capital advanced in production and Leontief's concept of a capital stock matrix, and discuss how to compute it based on the available input output data. This is important because profitability should be measured in relation to the stock of capital advanced, and tied up in production both at the sectoral and at the aggregate level. Our model is rather general in that it includes matrices of capital depreciation and capital advanced for production in every sector, and it allows for imported inputs and heterogeneous labor. We define labor values on the basis of the United Nations' SNA: as in standard IO theory, labor values correspond to the total labor costs in the production of goods.

Based on this IO framework, we define the economy-wide price and value rates of profit and analyze them at the theoretical level. The price rate of profit is standard and needs no further comment. 
In this chapter we define the value rate of profit by focusing on the standard notion of the value of labor power as the labor embodied in the workers' consumption bundle. However, our theoretical focus is on class relations and profitability, rather than individual consumption, and so we abstract from savings and focus on a notional consumption bundle that captures workers' capacity to consume. ${ }^{2}$ We identify three main determinants of the value rate of profit: the creation of absolute and relative surplus value, and technical change and accumulation (via their effects on the value of the total capital stock). We then show that differences between the value rate of profit and the price rate of profit depend on the differences between the wage share and the (standard) value of labor power, and between the price and value measures of the total capital stock.

Finally, an empirical appraisal of the main theoretical conclusions of this chapter and the previous one is provided in Section 8.4, based on the IO dataset from the German economy (1991-2000) constructed by Kalmbach et al. (2005). The empirical evidence confirms the main conclusions: first, SNA measures of labor productivity can be rather misleading and quite different from the theoretically sound IO indices. Second, the LDLC holds for the German economy, and it affects the evolution of aggregate profitability - a fact that is not easily visible by just looking at the IO tables. Third, we show that although prices and labor values of individual commodities may deviate significantly, at the aggregate level any such differences are irrelevant and the economywide value and price profit rates coincide up to negligible deviations. To be sure, this does not allow us to draw any robust inferences about causality, yet the correlation between the two rates is so strong and robust as to suggest that a common set of mechanisms is at play. Therefore the key insights of the Marxian theory of exploitation and of the profitability of a capitalist economy seem to be valid, for the creation of absolute and relative surplus value are the essential sources of profitability in a capitalist economy, together with changes in the value of the total capital stock.

\subsection{The SNA approach to Marxian labor values}

Consider an economy with $n$ goods produced in $n$ sectors. In any given period, technology and demand in the economy are described as follows: $A$ is the $n \times n$ matrix of intermediate inputs, $l$ is the $1 \times n$ vector of direct labor inputs, $x$ is the $n \times 1$ vector of gross output, $y$ is the $n \times 1$

\footnotetext{
${ }^{2}$ For a discussion, see Veneziani and Yoshihara (2015a, 2017b).
} 
vector of final demand. ${ }^{3}$ In addition, we follow Bródy's (1970) seminal approach to fixed capital and identify the $n \times n$ matrices of capital advanced $K$ and of capital depreciation $A^{\delta}$. The standard definition of Marxian labor values in this context is then given by:

$$
v=v\left(A+A^{\delta}\right)+l, \quad \text { i.e. } \quad v=l\left(I-\left(A+A^{\delta}\right)\right)^{-1} .
$$

Hence, for each sector $j=1, \cdots, n$, the labor value $v_{j}$ of the good produced in $j$ depends on direct labor and on the consumption of labor imputed to both intermediate inputs and depreciated capital equipment. As discussed in Chapter 1, some weak conditions on the matrix $A+A^{\delta}$ are sufficient to guarantee that the previous equation has a well defined solution and the vector $v$ is strictly positive whenever the vector $l$ is.

Labor values play a prominent role in Marxian economics and have been at the center of vast debates on their role and significance. However, the vector $v$ is also important in standard IO analysis, and we believe that the relationship between Marxian economics and the IO approach is deeper than a mere formal similarity. It is indeed of central importance, and it suggests a theoretical and empirical reconsideration of labor values.

The SNA approach to the measurement of total labor costs in production is most clearly presented in Richard Stone's formulation of the UN's SNA (United Nations 1968), which contains various theoretically rigorous definitions of labor productivity. Consider, for example, technical change in a simple economy with circulating capital only. Let $(A, l),\left(A^{*}, l^{*}\right)$ denote, respectively, technology in the base and in the current period, and let $v, v^{*}$ be the associated vectors of total labor costs. Let $x^{*}$ denote a vector of gross output producible with the new technology. In the UN's SNA, the change in labor productivity between the two periods, $\Lambda^{*}$, is defined as follows (United Nations 1968, $69)$ :

$$
\Lambda^{*}=\frac{l(I-A)^{-1}\left(I-A^{*}\right) x^{*}}{l^{*} x^{*}}=\frac{v\left(I-A^{*}\right) x^{*}}{v^{*}\left(I-A^{*}\right) x^{*}} .
$$

Note that the previous definition holds for any $x^{*}$ and that by definition the following relation holds between gross and net output $y: A^{*} x^{*}+$ $y=x^{*}$. Therefore, setting $y=(1,0, \cdots, 0)^{\prime}, x^{*}$ represents the activity vector that produces one unit of commodity 1 as net product and the

\footnotetext{
${ }^{3}$ Because the data refer to a specific time period, all variables should be dated, however we omit time subscripts for the sake of notational simplicity.
} 
SNA equation of the change in labor productivity becomes:

$$
\Lambda^{*}=\frac{v y}{v^{*} y}=\frac{v_{1}}{v_{1}^{*}} .
$$

This is just the relative change in Marx's index of labor productivity, $\frac{1 / v_{1}^{*}}{1 / v_{1}}$, as considered in the quoted passage from volume one of Capital (Marx 1976, 131) in Chapter 6. The pragmatism of national accounting, when based on a sound IO methodology, thus leads to the Marxian measure of labor productivity, although this was not in the intentions of Richard Stone and his research group in the 1950s and 1960s.

In this chapter, we use the SNA concept of total labor costs in production, i.e. the standard Marxian labor values of commodities, to reconsider his theory of surplus value as the explanation of the essential sources of profitability in a capitalist economy. We discuss the theory of surplus value from both a theoretical and an empirical viewpoint, focusing on the German economy after the reunification.

Our empirical investigation is based on the dataset developed by Kalmbach et al. (2005) describing the German economy for the years 1991-2000. A full description of the dataset and of the production structure of the seven macro sectors is in Chapter 1. The technological coefficients of the 7-sectoral aggregation are reported in Table 8.1, which shows the matrix of intermediate inputs $A$ and the vector of labor coefficients $l$ for the year 1995. The corresponding depreciation matrix, $A^{\delta}$, is in Table 8.2. The entries of $A, l, K, A^{\delta}$, and $v$ (as well as $x$, $y$, and $c$ below) are all measured per million Euro of output value. ${ }^{4}$ Let $p_{n}$ be the vector of average nominal market prices. We have derived $p_{n}$ from data on nominal output levels $x_{n}$ and real output levels $x$ (based on constant prices of the year 1995).

Three characteristics of our empirical framework should be noted here, which are important in the context of value theory. First, the definition of labor values includes the depreciation matrix $A^{\delta}$, and therefore physical joint production can be ignored. Second, Kalmbach et al. (2005) define the average nominal wage level by dividing the sum of all wage incomes by total employment. Formally, suppose that there is a set $\{1, \cdots, \mathcal{T}\}$ of types of workers in the economy, and let $\tau \in\{1, \cdots, \mathcal{T}\}$ denote a generic type. Let $w_{n_{\tau j}}$ denote the nominal wage rate paid to a worker of type $\tau$ employed in sector $j$ and let $l_{\tau j}$

\footnotetext{
${ }^{4}$ Owing to data limitations, employment is measured in terms of workers and not in terms of hours worked. For more details, see Kalmbach et al. (2005) and Chapter 1 above. Note that the double-deflated coefficients $\tilde{a}_{i j}$ are used to characterize the entries of $A$.
} 


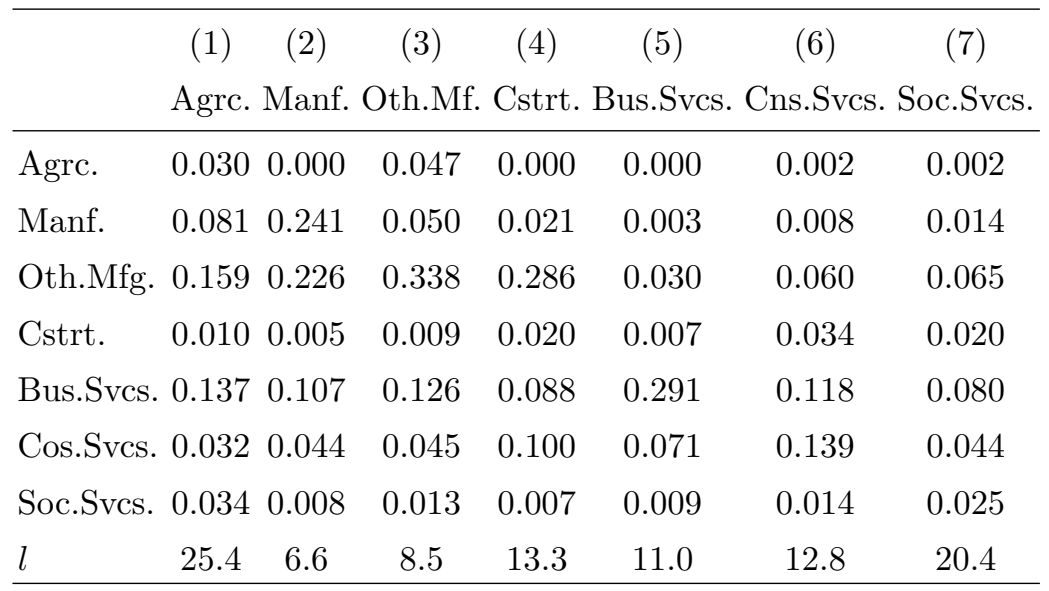

Table 8.1: Technological coefficients of the 7-sectoral aggregation IO matrix (Germany, 1995, sectors 1-7 as columns, per million Euro of output value)

\begin{tabular}{lccccccc}
\hline & $(1)$ & $(2)$ & $(3)$ & $(4)$ & $(5)$ & $(6)$ & $(7)$ \\
& Agrc. & Manf. & Oth.Mf. & Cstrt. & Bus.Svcs. & Cns.Svcs. Soc.Svcs. \\
\hline Agrc. & 0.0008 & 0.0002 & 0.0003 & 0.0001 & 0.0004 & 0.0006 & 0.0004 \\
Manf. & 0.0455 & 0.0126 & 0.0161 & 0.0061 & 0.0233 & 0.0322 & 0.0251 \\
Oth.Mfg. & 0.0562 & 0.0156 & 0.0199 & 0.0075 & 0.0288 & 0.0398 & 0.0310 \\
Cstrt. & 0.0452 & 0.0125 & 0.0160 & 0.0060 & 0.0231 & 0.0320 & 0.0249 \\
Bus.Svcs. & 0.0223 & 0.0062 & 0.0079 & 0.0030 & 0.0114 & 0.0158 & 0.0123 \\
Cns.Svcs. & 0.0090 & 0.0025 & 0.0032 & 0.0012 & 0.0046 & 0.0063 & 0.0049 \\
Soc.Svcs. & 0.0004 & 0.0001 & 0.0001 & 0.0000 & 0.0002 & 0.0003 & 0.0002 \\
\hline$\sum$ & 0.1793 & 0.0498 & 0.0635 & 0.0239 & 0.0918 & 0.1270 & 0.0989 \\
\hline
\end{tabular}

Table 8.2: Depreciation matrix $A^{\delta}$ (Germany, 1995)

denote the amount of time worked by her. Then, the average nominal wage is obtained dividing $\sum_{\tau} \sum_{j} w_{n_{\tau j}} l_{\tau j}$ by total employment $l x$. This implies that wage differentials are used to homogenize labor inputs when defining labor values, ${ }^{5}$ consistently with our theoretical focus on the aggregate wage level $w_{n}$ and on the wage share $w$.

\footnotetext{
${ }^{5}$ We believe that this is in line with what Marx $(1976,134-135)$ states when
} 
Third, Kalmbach et al. (2005) construct the matrix $A$ using data on the industrial consumption of domestic and imported commodities. Thus $A$ provides the technological matrix of intermediate inputs irrespective of their origin. This implies that labor values are measured on the basis of the domestic technology and independently of the conditions under which the imported commodities were actually produced. This treatment is very similar to the approach pioneered by Gupta and Steedman (1971) and in our view it is a more appropriate way of dealing with international labor values than Steedman's (2008) model, in which terms of trade directly enter the definition of labor values. Our approach has two advantages: first, to measure labor values as if foreign commodities were domestically produced reduces significantly data intensity and the likelihood of measurement errors. Second, our approach is adequate if one wants to study the economy not only from the viewpoint of labor productivity, but also from a reproduction perspective where the flow relationships of actual inputs to outputs are the focus of interest, independently of whether the inputs are produced domestically or internationally. ${ }^{6}$

Our notion of labor values is therefore specific in that we adopt a particular treatment of international trade (domestic techniques matter, but not the origin of inputs), heterogeneous labor (wage differentials are used to compare different skills), and fixed capital. But it is also general, in the sense that it holds in an open economy with a complex technology using fixed capital. As shown in Chapters 9, 10, and 11 , our definition of labor values is indeed completely general; it displays no joint production/fixed capital anomalies of the type constructed by Steedman (1977); and - unlike in Steedman's (1977) approach - it is firmly based on the actual behavior of firms (see Bródy (1970)).

Finally, following standard practice in advanced IO analysis (see Bródy (1970)), we use a capital stock matrix $K$ (also measured per million Euro of output value) in order to calculate rates of profit with respect to the capital advanced in production. The capital stock matrix for Germany in 1995 is shown in Table 8.3. ${ }^{7}$

he refers to a social process, fixed by custom. See Veneziani and Yoshihara (2013b) and Chapter 6 above.

6 The role of terms of trade for calculating and comparing value and price rates of profit might be more relevant to analyze international trade and unequal exchange between countries. However, the issue of international exploitation is contentious and a proper treatment lies beyond the scope of this book. For different views see Negishi (1989, 210-213), Veneziani (2009a,c), and Veneziani and Yoshihara (2017a).

7 This matrix represents 'capital advanced', which is not only so-called 'fixed 


\begin{tabular}{|c|c|c|c|c|c|c|c|}
\hline & (1) & (2) & (3) & (4) & (5) & (6) & (7) \\
\hline & Agrc. & Man & $\mathrm{O}$ & Cstrt. & Bus.Svcs & Cns.Sves & Soc.Sves \\
\hline Agrc. & 0.0156 & 0.0043 & 0.0055 & 0.0021 & 0.0080 & 0.0111 & 0.0086 \\
\hline Manf. & 0.3705 & 0.1028 & 0.1312 & 0.0493 & 0.1897 & 0.2625 & 0.2044 \\
\hline Oth.Mfg. & 0.4959 & 0.1377 & 0.1756 & 0.0660 & 0.2539 & 0.3513 & 0.2736 \\
\hline Cstrt. & 0.9031 & 0.2507 & 0.3199 & 0.1202 & 0.4624 & 0.6398 & 0.4982 \\
\hline Bus.Svcs. & 0.22 & 0.0615 & 0.0785 & 0.0295 & 0.1135 & 0.1570 & 0.1223 \\
\hline Cns.Sves. & 0.08 & 0.0232 & 0.0296 & 0.0111 & 0.0427 & 0.0591 & 0.0460 \\
\hline Soc.Svcs. & 0.0074 & 0.0020 & 0.0026 & 0.0010 & 0.0038 & 0.0052 & 0.0041 \\
\hline$\sum:$ & 2.0 & 3 & 0.7429 & 0.2792 & 1.0739 & 1.4861 & 1.1572 \\
\hline
\end{tabular}

Table 8.3: Capital stock matrix K (Germany, 1995)

\subsection{Average value and price gross rates of profit}

By definition, in every period, the labor value of aggregate net output is equal to total direct labor performed; formally, $v y=l x$. For a given arbitrary vector of market prices, $p_{n}$, instead, no such relation holds. However, we can normalize market prices $p_{n}$ by a factor $\gamma$ such that the normalized value of net output is equal to the total labor time employed by firms. Formally, we let $p=\gamma p_{n}$, where $\gamma$ is such that $p y=l x$. Hence, if the average nominal wage is $w_{n}$, the wage share is given by $w=\frac{w_{n} l x}{p_{n} y}=\gamma w_{n}$, and, supposing labor inputs to be paid ex post, the economy-wide gross price rate of profit is:

$$
r_{p}=\frac{(1-w) l x}{p K x}=\frac{1-w}{w} \frac{w l x}{p K x},
$$

where $\epsilon_{p}=\frac{1-w}{w}$ represents Marx's rate of exploitation according to the 'New Interpretation' (Duménil 1980, 1984; Foley 1982, 1986b; Foley

capital', but also for example the average inventory of wheat in bread production (see Bródy (1970, 35-37) for the discussion of the turnover time of such goods). Sector 7 includes all government services (including the military) as well as private social services (provided, for example, by religious organizations). At this high aggregation level, Sector 2 may hold inventories from agriculture and may have capital advances concerning social services, like public administration costs and more. 
and Duménil 2008; Duménil et al. 2009) and $\frac{l x}{p K x}$ is the aggregate labor/capital ratio in terms of market prices.

In our empirical analysis, we assume that the structure of workers' consumption is constant throughout the period considered. Formally, we take as given the aggregate consumption vector of workers $\tilde{c}$ and define $c=\delta \tilde{c}$, where $\delta$ is a positive number such that $p c=w$ (or $\left.p_{n} c=w_{n}\right)$. In other words, for a given consumption structure $\tilde{c}, c$ is the consumption vector that workers could afford at the given wage rate (setting aside savings, taxes, and so on). It provides the potential (not the actual) consumption basket of workers (per unit of labor), given the relative structure of their consumption basket as contained in $\tilde{c}$. Given this normalization, the price rate of profit can be written as $r_{p}=\frac{(1-p c) l x}{p K x}$. Table 8.4 shows the aggregate consumption basket of German workers in the year 2000 and it compares it with aggregate net output in the seven sectors.

\begin{tabular}{lccc}
\hline Sector & $\tilde{c}$ & $y$ & $\tilde{c} / y(\%)$ \\
\hline Agrc. & 5470.0 & 6054.8 & 90.342 \\
Manf. & 36997.1 & 177601.8 & 20.832 \\
Oth.Mfg. & 95319.9 & 178301.5 & 53.460 \\
Cstrt. & 2327.7 & 103454.7 & 2.250 \\
Bus.Svcs. & 60821.5 & 119990.0 & 50.689 \\
Cns. Svcs. 481019.8 & 525536.2 & 91.529 \\
Soc. Svcs. & 411098.7 & 413658.3 & 99.381 \\
\hline
\end{tabular}

Table 8.4: Consumption vs. final demand (million Euro)

The vector $\tilde{c}$ in table 8.4 is based on data containing all household consumption (including pure capitalists). Unsurprisingly, the percentage of consumption in net output is very high in agriculture and in consumer services, and it is lowest in construction which by definition produces investment goods. Although the percentage of net output consumed by workers in the business-related services sector may seem too high, this is due to the fact that the sector also contains the services of lawyers, the production of software, and other products which can also be purchased by the household sector. Note also that intermediate consumption of these products is significant as shown in Table 8.1 .

It is worth briefly commenting on the high percentage of consumption in net output in the social services sector. This derives 
from our choice to include in the vector $\tilde{c}$ all of the social services consumed by the household sector, including those - such as education, health care, elderly care, administration, police, military, and even 'Christian' services - that are mainly financed through 'government' activities based on tax revenues. If we only focused on the social services directly paid for by the household sector, the percentage of consumption in net output would fall from above $90 \%$ to slightly above $10 \%$. To be sure, the inclusion of government-driven consumption into the workers' consumption bundle is an open and controversial issue, from a general theoretical viewpoint. However, it turns out to be of secondary importance in the context of our analysis of aggregate profitability. For the choice of the bundle $\tilde{c}$ is relevant for the composition of the workers' reference consumption bundle, but not for consumption levels, which are instead determined by the wage. Indeed, it can be shown that our empirical results remain essentially identical if only social services directly paid for by the households are included.

As we have already argued various times throughout the book, we view labor values as a theoretical skeleton behind the surface of price-quantity dynamics, i.e. as part of a SNA, which is designed to understand the capitalist process of economic and social reproduction. Labor values are important to detect the fundamental laws of motion of capitalism. From a Marxian perspective, the first and most important step is to understand the essential determinants of the average (not necessarily uniform) price rate of profit or - put differently - to what extent the generation of absolute and relative surplus value, and technical progress drive profitability. Our next step, then, is to analyze profitability from the perspective of labor accounting.

Given the consumption bundle $c$, the standard interpretation of Marx's theory is that the value of labor power coincides with the labor embodied in $c$, namely $v c$ (here measured in terms of workers' potential consumption). The average value rate of profit can then be defined as follows:

$$
r_{v}=\frac{v\left(I-A-A^{\delta}\right) x-v c l x}{v K x}=(1-v c) \frac{l x}{v K x}=\frac{1-v c}{v c} \frac{v c l x}{v K x},
$$

where $\epsilon=\frac{1-v c}{v c}$ is the standard definition of the rate of exploitation and $\frac{l x}{v K x}$ represents the labor/capital ratio in value terms. The average value rate of profit thus depends on three fundamental Marxian magnitudes: the value of labor power, $v c$, the total amount of hours worked, $l x$, and the labor value of the total capital stock, $v K x$. The value of labor power is related to what Marx called the generation of relative surplus value: a 
decrease in workers' wages and consumption, or technological progress lowering the value of consumption goods lowers the value of labor power and thus increases the value rate of profit. The hours actually worked by the workforce have to do with the generation of absolute surplus value, and can be increased if monthly hours worked are increased, if absenteeism is forced down, if holidays are reduced and if (not covered by the model) work intensity is increased, thus driving an increase in the value rate of profit.

The labor value of the total capital stock is affected by accumulation and technical change. Accumulation tends to increase the labor value of the capital stock and thus to lower the value rate of profit. It is difficult, instead, to make clear theoretical predictions on the effect of technical change. In Chapter 7, we have proved that under mild assumptions, any cost reducing innovation that substitutes fixed capital for living labor (i.e. the Marxian fixed-capital-using labor-saving technical change) in a given sector decreases labor values in all sectors. Noting that capital-using labor-saving technical change has characterized most phases of capitalist development (Marquetti 2003), this result provides theoretical foundations to the LDLC and may explain the tendential decrease in labor costs observed in empirical studies, and shown in Table 8.5 below.

If this law is sufficiently strong, then the reduction in labor values may outweigh the increase in the capital stock $K x$ (and the decrease in $l x$ ) thus increasing the value rate of profit. The tension between increases in the capital stock and decreases in labor values can be considered as a fundamental contradiction in the capitalist process of technical change, which is related to Marx's discussion of the role of changes in capital intensity-the so-called technical composition of capital. Whether the LDLC is sufficiently strong to outweigh the countervailing effects is an empirical issue: in a Schumpeterian perspective, the strength of the law may depend on the phase of the long-wave under consideration. In the phase of radical process and product innovations, relatively small increases in capital intensity may yield major increases in productivity, thus lowering the labor value of the total capital stock. As the wave of innovations phases out, larger investments are required to obtain relatively smaller increases in productivity, leading the value of the total capital stock to rise.

To summarize: the basic forces driving an increase in the value rate of profit are a decrease in the value of labor power, an increase in working hours (or working intensity), and technical change leading to a significant decrease in labor values that outweighs any increase in fixed capital. 
Having identified the determinants of the value rate of profit $r_{v}$, the question is whether this can help us to understand movements in the actual price rate of profit $r_{p}$. For it is price, not value magnitudes that are relevant for economic agents and their decisions. By equations (8.2) and (8.3), the following relation between the price and the value rates of profit can be derived:

$$
\frac{r_{v}}{r_{p}}=\frac{(1-v c)}{(1-p c)} \frac{p K x}{v K x}, w=p c .
$$

If total labor costs explain the bulk of costs mirrored by actual prices, then $r_{v}$ and $r_{p}$ will be close. However, the two rates of profit will also be similar if sectoral differences cancel out in the aggregate such that both the labor value of the workers' consumption bundle is close to its monetary value, and the price and value measures of the total capital stock are similar. In this case, the theoretical value rate of profit and the actual price rate of profit would differ only by unsystematic, historically determined price-value deviations that cannot be exploited by the capitalist class as a whole, and any factors influencing the value rate will also affect the price rate of profit in the same direction.

The theoretical and empirical relation between the price rate of profit $r_{p}$ and the value rate $r_{v}$ is arguably the fundamental topic in Marx's labor theory of value. In the next sections, we empirically investigate the relation between price and value magnitudes and whether the production-based rate $r_{v}$ provides a proxy for the average price rate of profit $r_{p}$. The aim is to understand whether the forces underlying the evolution of $r_{v}$ also drive the changes in $r_{p}$. In this case, the Marxian theory of exploitation could be used as foundation for an analysis of overall profitability, with the key determinants of the value rate also driving the dynamics of the observed rate of profit $r_{p}$, which is the key variable of interest for the capitalist class as a whole.

\subsection{Labor values and the LDLC: Empirical results}

This section provides an empirical illustration of the main concepts and propositions concerning technical change, productivity, and the LDLC discussed in this chapter and in the previous one. In order to calculate the labor values of the seven sectors, the formula $v=l\left(I-A-A^{\delta}\right)^{-1}$ is used in each of the ten years. Table 8.5 shows the time series of labor values for the Germany economy (1991-2000). ${ }^{8}$ The classical-Marxian

\footnotetext{
8 The empirical analysis in this section actually focuses on production sectors, rather than produced commodities, in contrast with Definition
} 
measures of labor productivity, $\pi_{j}^{m}$, are then derived as the reciprocal of the entries of $v$. These measures can then be compared to conventional measures of labor productivity $\pi_{j}^{c}$, which are derived by dividing each of the 70 real value added items (per $10^{6}$ Euro output value) by the corresponding labor coefficient (per $10^{6}$ Euro output value). The time series of the two productivity measures for six of the seven sectors are shown in Figure 8.1.9

\begin{tabular}{|c|c|c|c|c|c|c|c|}
\hline & (1) & (2) & (3) & (4) & (5) & (6) & (7) \\
\hline & Agrc. & Man & Oth.Mf & Cstrt. & Bus.Svcs & Cns.Sve & Soc.Sves \\
\hline 1991 & 54.68 & 26.53 & 30.23 & 28.53 & 24.45 & 27.22 & 30.69 \\
\hline 1992 & 47.44 & 25.82 & 28.78 & 27.40 & 23.82 & 26.67 & 29.88 \\
\hline 1993 & 45.24 & 26.55 & 28.55 & 27.77 & 23.62 & 26.95 & 30.04 \\
\hline 1994 & 44.65 & 25.09 & 27.48 & 27.12 & 23.40 & 26.32 & 29.63 \\
\hline 1995 & 42.95 & 23.83 & 27.30 & 27.60 & 23.32 & 25.64 & 29.45 \\
\hline 1996 & 38.76 & 23.87 & 26.46 & 27.65 & 23.06 & 25.29 & 29.45 \\
\hline 1997 & 37.81 & 22.98 & 25.62 & 27.02 & 22.83 & 25.02 & 29.11 \\
\hline 1998 & 37.42 & 22.94 & 25.63 & 26.92 & 22.39 & 25.02 & 28.92 \\
\hline 1999 & 36.46 & 23.46 & 24.89 & 26.65 & 21.82 & 24.93 & 28.86 \\
\hline 2000 & 35.90 & 22.39 & 23.79 & 26.14 & 22.03 & 24.99 & 28.63 \\
\hline
\end{tabular}

Table 8.5: Labor values or total labor costs (persons per million Euro of output value)

The empirical evidence confirms our main conclusions. Concerning the measurement of labor productivity, the data show that the two series $\pi_{j}^{m}, \pi_{j}^{c}$ are very different, as expected from the analysis in Section 7.2. Firstly, apart from the remarkable exception of sector 3, the levels of the two measures are sharply different in all sectors and in virtually every year of the sample, with no recognizable overall pattern (in some sectors $\pi_{j}^{c}$ is higher than $\pi_{j}^{m}$, but the opposite happens in other sectors) and with differences even in the relative ranking of sectors in terms of

7.1 above. Albeit theoretically relevant, this distinction is practically secondary given the high level of aggregation, and the specific choice of the seven macro sectors by Kalmbach et al. (2005).

${ }^{9}$ Social services are omitted because they are subject to processes that in general are not determined by profit-maximizing firms. Overall, labor productivity in this sector has a similar pattern as in sector 6 . 
their labor productivity. By Proposition 7.2, this is to be expected, given the wide sectoral differences in productivity. Secondly, even the qualitative behavior of the two indices over time is very different, as expected from Proposition 7.4. In sector 4, both the trend and the year-on-year behavior of the two variables are markedly different. The Marxian measure of productivity has risen over time, while the conventional SNA measure shows a sharp increase immediately after the German reunification but a significant decline thereafter. Even setting aside the construction sector (where measurement problems may play a role), in various instances the two indices provide opposite verdicts concerning the direction of change of labor productivity over time. Particularly striking examples are sector 2: 1995-96 (and to a lesser extent 1997-98); sector 3: 1994-95 (and to a lesser extent 199798); sector 5: 1993-1995; and last but not least sector 6: 1997-2000, which is characterized by a similar, if less pronounced, overall pattern as sector 4 .

In sum, the theoretical differences between the two measures do give rise to significant empirical discrepancies. The standard SNA indices $\pi_{j}^{c}$ lack theoretical foundations, as argued in Section 7.2 above, and they can also be very misleading in empirical analysis, as the evidence in Figure 8.1 forcefully shows.

Concerning the relation between prices, profits, and labor values, all the tables in Figure 8.1 show that the LDLC holds for the German economy (1991-2000). The classical-Marxian indices of labor productivity show a clear upward trend in all sectors. This result seems robust and it is consistent with the findings of previous studies (e.g., Gupta and Steedman (1971), Wolff (1985), de Juan and Febrero (2000)), although only few contributions explicitly focus on sectoral productivities.

\subsection{Three main sources of aggregate profitability}

This section provides an empirical illustration of the relation between $r_{p}$ and $r_{v}$. Based on a sectoral analysis, this relation is far from obvious. For as shown in Flaschel, Fröhlich, and Veneziani (2013b), the variance of price-value ratios are not insignificant. For example, in 1991 the vector of price-value ratios $\mu_{j}=\frac{p_{j}}{v_{j}}(j=1, \ldots, 7)$ is:

$$
\begin{gathered}
\left(\mu_{1}=0.56, \mu_{2}=1.13, \mu_{3}=1.02, \mu_{4}=0.97\right. \\
\left.\mu_{5}=1.18, \mu_{6}=1.03, \mu_{7}=0.89\right)
\end{gathered}
$$



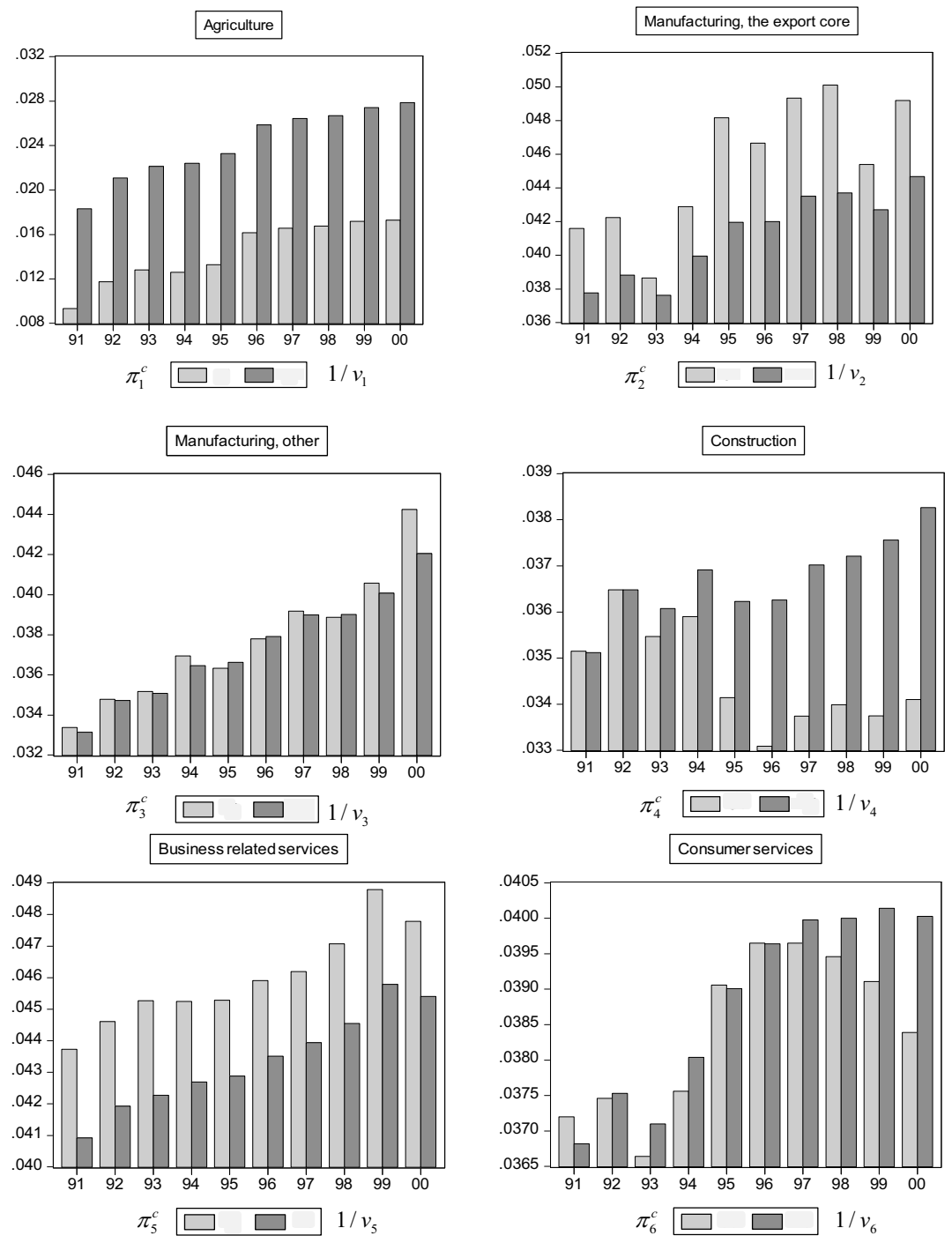

Figure 8.1: Comparing conventional and Marxian labor productivity indices: $\pi_{j}^{c}, 1 / v_{j}$ 
In other words, the price-value ratio is highest in the business-related services sector and lowest in agriculture, with noticeable differences between values and prices also in other sectors. This pattern is persistent throughout the sample. However, as noted above, what really matters is whether sectoral deviations cancel out at the aggregate level. Table 8.6 shows the value and the price rate of profit, and their deviation and it proves that indeed sectoral price-value deviations do not really matter in the aggregate. The price rate of profit clearly mirrors the value rate of profit up to negligible deviations.

\begin{tabular}{ccccccc}
\hline Year & $w=p c$ & $v c$ & $v c / p c$ & $r_{p}(\%)$ & $r_{v}(\%)$ & $r_{v} / r_{p}$ \\
\hline 1991 & 0.698 & 0.714 & 1.02 & 14.7 & 14.3 & 0.969 \\
1992 & 0.717 & 0.730 & 1.02 & 13.3 & 13.1 & 0.979 \\
1993 & 0.728 & 0.736 & 1.01 & 12.1 & 12.0 & 0.989 \\
1994 & 0.715 & 0.724 & 1.01 & 12.8 & 12.7 & 0.989 \\
1995 & 0.714 & 0.723 & 1.01 & 12.8 & 12.5 & 0.981 \\
1996 & 0.713 & 0.720 & 1.01 & 12.8 & 12.5 & 0.981 \\
1997 & 0.699 & 0.708 & 1.01 & 13.5 & 13.2 & 0.981 \\
1998 & 0.700 & 0.710 & 1.01 & 13.4 & 13.0 & 0.970 \\
1999 & 0.706 & 0.715 & 1.01 & 13.0 & 12.7 & 0.973 \\
2000 & 0.710 & 0.724 & 1.02 & 12.8 & 12.4 & 0.965 \\
\hline
\end{tabular}

Table 8.6: Main results

These results provide an empirical illustration of what Flaschel et al. (2013b) call the 'fuzzy connection' between the average price and value rates of profit. Any sectoral deviations between market prices and labor values are in general such that they wash out at the aggregate level. ${ }^{10}$ Table 8.6 also shows that the average price rate of profit has fallen in 1991-1993 and in 1997-2000, possibly due to cyclical fluctuations in economic activity.

An economy can be analyzed from three different perspectives, focusing either on the physical flow of commodities, or on nominal (price) flow magnitudes, or on abstract labor flows. Relating the labor-time oriented SNA to the flow of actually observed (quantity

10 Actually, a number of empirical studies have identified a very strong correlation between prices and labor values even at the sectoral level. See, for example, Shaikh (1984, 1998), Cockshott and Cottrell (1997), Tsoulfidis and Maniatis (2002), and Fröhlich (2013). 
and price) magnitudes is not a 'transformation problem'. Rather, it provides an alternative representation of the economy, as Stahmer (2000) has convincingly argued. From this perspective, our empirical analysis shows that there exists a clear relationship, or connection, between the average price rate of profit and the average value rate of profit, which is so strong as to suggest that the Marxian concepts of absolute and relative surplus value, and his analysis of technical change and accumulation may provide the basis for the explanation of the determinants of the average price rate of profit.

The non-negligible sectoral price-value deviations and the strong correlation between aggregate price and value profit rates suggest - in Marxian terms - that distinct processes may regulate the generation and the distribution of profits. Aggregate profitability can only be improved by Marxian mechanisms: an increase in absolute surplus value $(l x \uparrow)$ or in relative surplus value $(v c \downarrow)$, and a decrease in the labor value of the capital stock - for example, due to technical change which raises labor productivity (lowers $v$ ) so much that it outweighs any countervailing effects on the value of the capital stock. Examples of innovations that may lead to a decrease in the value of aggregate capital are Harrod-neutral technical change or, as mentioned above, Schumpeterian innovation waves leading to radical process and product innovations. Table 8.7, however, suggests that there has been no systematic decrease in the labor value of the capital stock, $v K x$, in the German economy during 1991-2000, although labor productivity $\frac{1}{v_{j}}(j=1, \ldots, 7)$ increased.

Two last points are worth making concerning our results, and in particular the ratios shown in Tables 8.6 and 8.7. First, the labor value of the workers' consumption basket has always been greater than its price equivalent, the labor value of the capital stock has always been smaller than its price equivalent, and the value rate of profit has always been smaller than the price rate of profit. From our point of view, there is no obvious explanation for these phenomena. Second, however, it is worth stressing again the remarkably strong relations between aggregate value and price magnitudes: the differences between value and price expressions of profitability, aggregate capital and workers' purchasing power are negligible. Thus, among other things, Tables 8.6 and 8.7 suggest that it may be immaterial, from an empirical viewpoint, whether one defines the value of labor power as the labor embodied in the workers' normalized (potential) consumption bundle, $v c$, or by equating it to the wage share, $w$, as in the so-called New Interpretation (Duménil 1980; Foley 1982). Indeed, Flaschel et al. (2013a) have shown that the price rate of profit and the value rate of profit coincide up to 


\begin{tabular}{ccccc}
\hline Year & $l x$ & $v K x$ & $p K x$ & $v K x / p K x$ \\
\hline 1991 & 38.45 & 77.13 & 78.89 & 0.9777 \\
1992 & 37.88 & 78.35 & 80.51 & 0.9731 \\
1993 & 37.37 & 82.18 & 83.81 & 0.9805 \\
1994 & 37.30 & 81.2183 .14 & 0.9768 \\
1995 & 37.38 & 82.48 & 83.52 & 0.9876 \\
1996 & 37.27 & 83.3783 .87 & 0.9941 \\
1997 & 37.21 & 82.3583 .16 & 0.9902 \\
1998 & 37.61 & 84.2784 .51 & 0.9971 \\
1999 & 38.08 & 85.61 & 85.80 & 0.9978 \\
2000 & 38.76 & 86.45 & 87.82 & 0.9844
\end{tabular}

Table 8.7: Employment, labor value of capital stock, price value of capital stock, and relative value of capital stock (measured in million persons)

negligible deviations, even if one adopts the latter approach. ${ }^{11}$ These remarkable regularities suggest that theoretical distinctions between alternative approaches to Marxian value theory may turn out to be irrelevant at the empirical level.

\subsection{Conclusions}

This chapter provides an empirical analysis of two key issues in value theory focusing on the German economy (1991-2000) based on the IO theoretic interpretation of labor values as part of a SNA designed to understand the laws of development of capitalist economies behind the surface of nominal magnitudes.

First, we analyze the dynamics of labor productivity in the German economy 1991-2000. We show that the LDLC (or law of increasing labor productivity, in our approach) holds in all sectors as predicted in Marxian theory. Second, we show that, empirically, there exists a strong relation between (aggregate) price and value magnitudes - especially between the economy-wide value and price profit rates. Although this does not allow us to draw any robust inferences about causality, the

${ }^{11}$ Adopting the New Interpretation, however, the value rate of profit is always greater than the price rate. 
correlation between the two rates is so strong and robust as to suggest that a common set of mechanisms is at play.

Thus Marx's theory of exploitation may provide the foundations for an understanding of profitability in a capitalist economy, and systematic changes in profitability can be analyzed by focusing on labor value magnitudes which are more informative than the corresponding price expressions, due to the 'chaotic' nature of the processes of commodity exchange. The three key mechanisms highlighted in Marx's theory of exploitation seem central in determining profitability for the capitalist class as a whole: the production of absolute and relative surplus value, and the capitalist laws of technical change and accumulation. In a capitalist economy, aggregate profitability can be increased by increasing total labor hours worked (or the intensity of work); by reducing the value of labor power; or by introducing innovations that significantly increase labor productivity.

\subsection{Appendix}

\subsubsection{Construction of depreciation and capital stock matrices}

In this subsection we examine how capital stock matrices can be constructed based on the rather limited, available statistics. There are two kinds of data in the IO tables somewhat related to what we need. First, the data on the sectors' total depreciation. We can use them to get an indication of the different levels of capital installed in the single sectors. Second, the investment vector as a component of final demand may give us an idea of the composition of the sectoral capital stocks. Combining this information we can construct coefficients that, distinguished by goods and sectors, proxy replacement investment. Clearly, a number of heroic assumptions are necessary. They are explained in the following five steps.

Step 1: The first assumption postulates that in all sectors the capital stock has the same composition of capital goods. Formally, let the composition be represented by proportions $\kappa_{1}, \ldots, \kappa_{n}$ (which sum up to unity, though this is not essential). If $k_{i j}$ designates the capital good $i$ installed in sector $j$ per unit of its output, the assumption says that in all sectors the capital good vector $\left(k_{1 j}, \ldots, k_{n j}\right)$ is proportional to the composition vector $\left(\kappa_{1}, \ldots, \kappa_{n}\right)$. Denoting the proportionality factor in sector $j$ by $\alpha_{j}$, the relationship reads

$$
k_{i j}=\alpha_{j} \kappa_{i}, \quad i, j=1, \ldots n .
$$

Step 2: The vector $\left(\kappa_{1}, \ldots, \kappa_{n}\right)$ is obtained from the composition 
of the economy's investment vector (gross investment, inclusive of imports). Regarding good $i$, the IO tables distinguish between its investment as plant, $I_{i}^{p l t}$, and its investment as equipment, $I_{i}^{e q t}$. I being overall investment, $I=\sum_{k}\left(I_{k}^{p l t}+I_{k}^{e q t}\right)$, the proportions $\kappa_{i}$ are specified as

$$
\kappa_{i}=\left(I_{i}^{p l t}+I_{i}^{e q t}\right) / I \quad i=1, \ldots n .
$$

Step 3: We suppose that a pure plant capital good deteriorates at a rate $\overline{\delta^{p l t}}$ per year, and a pure equipment capital good at rate $\delta^{e q t}$. The depreciation rate $\delta_{i}$ of good $i$ is then supposed to be a weighted average of the two polar rates, where the weight derives from the proportions of the two types of investment $I_{i}^{\text {plt }}$ and $I_{i}^{e q t}$. Concretely,

$\delta_{i}=\eta_{i} \delta^{p l t}+\left(1-\eta_{i}\right) \delta^{e q t}, \quad$ where $\eta_{i} \equiv I_{i}^{p l t} /\left(I_{i}^{p l t}+I_{i}^{e q t}\right)$.

Owing to data limitations, the rates $\delta^{p l t}$ and $\delta^{e q t}$ are chosen by considering the level of aggregate capital to which they eventually give rise.

Step 4: Although the actual accounting notion and theoretical concept of the physical depreciation of the capital stock are quite different, we put them on an equal footing. We determine the level of the capital goods in sector $j$ by the condition that the total depreciation resulting from the $\delta_{i}$ equals sector $j$ 's empirical depreciation per unit of output, denoted as $d_{j}$. The coefficients $k_{i j}$ must therefore solve: $\sum_{i} \delta_{i} k_{i j}=d_{j} .{ }^{12}$ The proportionality factors $\alpha_{j}$ in equation (8.5) are then obtained by substituting $\alpha_{j} \kappa_{i}$ for $k_{i j}$ and solving for $\alpha_{j}$, which yields

$$
\alpha_{j}=d_{j} / \sum_{i} \delta_{i} \kappa_{i}, \quad j=1, \ldots n
$$

Step 5: Given steps 1-4, it is natural to suppose that replacement investment is identical to the physical deterioration of the capital stock. Denoting replacement investment of $\operatorname{good} i$ per unit of output $j$ by $a_{i j}^{\delta}$, we have

$$
a_{i j}^{\delta}=\delta_{i} k_{i j}
$$

On the basis of empirical data on $d_{i}, I_{i}^{p l t}$ and $I_{i}^{e q t}$, our algorithm

$\overline{12}$ The coefficients $k_{i j}$ can be added up in a column $j$ if we recall that empirically they all have the unit 'worth 1 mill. Euro in prices of 1995'. Naturally, the same applies to the $\kappa_{i}$. 
for constructing the capital stock matrix $K=k_{i j}$ and the replacement investment, or depreciation matrix $A^{\delta}=a_{i j}^{\delta}$ is as follows: obtain $\left(\kappa_{1}, \ldots, \kappa_{n}\right)$ from equation (8.6); compute the $\delta_{i}$ 's by means of equation (8.7); use equation (8.8) to determine the $\alpha_{j}$ 's; get $k_{i j}$ from equation (8.5); lastly, get $a_{i j}^{\delta}$ from equation (8.9).

Consider again the German IO tables. For $d_{i}$ we take the 1995 data because this is the last year for which real data on depreciation (and wage payments) are available. ${ }^{13}$ The investment data, $I_{i}^{p l t}$ and $I_{i}^{\text {eqt }}$, are taken from 2000 (this is without loss of generality since only the composition of the investment matters, which does not vary much). With respect to our 7-sectoral aggregation, these data are reported in the first three rows of Table 8.8.
(1)
(2)
(3)
(4)
(5)
(6)
(7)

Agrc. Manf. Oth.Mf. Cstrt. Bus.Svcs. Cns.Svcs. Soc.Svcs. $\sum$

\begin{tabular}{lcccccccc}
\hline$d_{i}:$ & 17.93 & 4.98 & 6.35 & 2.39 & 9.18 & 12.70 & 9.89 & \\
$\frac{I_{i}^{\text {plt }}}{I}:$ & 0.73 & 0.52 & 3.68 & 43.06 & 3.46 & 0.94 & 0.35 & 52.72 \\
$\frac{I_{i}^{\text {eqt }}}{I}:$ & 0.02 & 17.14 & 19.96 & 0.00 & 7.11 & 3.04 & 0.00 & 47.28 \\
$\kappa_{i}:$ & 0.74 & 17.66 & 23.64 & 43.06 & 10.57 & 3.98 & 0.35 & \\
$\delta_{i}:$ & 5.16 & 12.28 & 11.33 & 5.00 & 10.05 & 10.73 & 5.00 & \\
\hline
\end{tabular}

Table 8.8: Data underlying the construction of matrices $K$ and $A^{\delta}$

Note: All ratios in percent. $d_{i}$ is depreciation per unit of output (Germany 1995); $I_{i}^{\text {plt }}$ and $I_{i}^{\text {eqt }}$ are investment in plant and equipment, respectively, $I$ is total investment (in 2000); $\kappa_{i}$ and $\delta_{i}$ result from equations (8.6)-(8.7), given $\delta^{p l t}$ and $\delta^{e q t}$ from Table (8.9).

The vector of weights $\kappa_{i}$ in the fourth row is the sum of the two preceding rows (one rounding error apart). It may seem peculiar that agricultural products, consumer services and even social services (sectors 1,6 and 7) can statistically become plant or equipment, but this is not relevant since the percentages are fairly low. On the other hand, it certainly accords better with common economic sense that the output of the construction sector 4 is exclusively used as plant and makes up $43.06 \%$ of total investment. The same holds for most of the

${ }^{13}$ It is also convenient that for this year real and nominal data are identical. 
investment goods produced by the two industrial sectors (sectors 2 and $3)$.

Investment data are also used to deduce the annual depreciation rates $\delta_{i}$ of the capital goods from equation (8.7). The $\delta_{i}$ reported in the fifth row of Table 8.8 are based on $\delta^{p l t}=1 / 20=5.0 \%$ and $\delta^{e q t}=$ $1 / 8=12.5 \%$ (values which are justified below). Observe that $\delta_{4}=\delta_{7}=$ $\delta^{p l t}=5.0 \%$ because all investment in construction and social services is in plants, while almost all of the capital goods bought by the industrial sector 2 (the export core) are equipment, so that $\delta_{2} \approx 12.5 \%$.

As noted above the depreciation rates $\delta^{p l t}$ and $\delta^{e q t}$ are chosen based on the total capital stock that they imply. Empirically, we have the following information. In 1994, the economy-wide ratio of gross capital to (gross) value added was 2.9, while in 1995 the net capital stock was $63.1 \%$ of the gross capital stock. The notion more appropriate for us is the net capital stock, since over the lifetime of capital goods depreciation is deducted from their initial value. This gives us a desired capital / value added ratio (CVAR) of 0.631.2.9 =1.83. Once the matrix $K$ is determined from $\delta^{p l t}, \delta^{e q t}$ and equations (8.5)-(8.8), we have to compute the ratio CVAR $=p K x / p\left(I-A_{T}\right) x$, where $x$ and $p=e$ are the empirical vectors of 1995 . Table 8.9 reports these ratios for several combinations of $\delta^{p l t}$ and $\delta^{e q t}$.

\begin{tabular}{ccc}
\hline$\delta^{p l t}$ & $\delta^{\text {eqt }}$ & CVAR \\
\hline $1 / 25$ & $1 / 10$ & 2.34 \\
$1 / 25$ & $1 / 8$ & 2.00 \\
$1 / 25$ & $1 / 7$ & 1.81 \\
$1 / 25$ & $1 / 6$ & 1.60 \\
$1 / 20$ & $1 / 10$ & 2.18 \\
$1 / 20$ & $1 / 8$ & 1.88 \\
\hline
\end{tabular}

Table 8.9: CVAR resulting from different values of $\delta^{\text {plt }}$ and $\delta^{\text {eqt }}$

Our prior is that plant deteriorates at a rate between $1 / 20$ and $1 / 30$ per year, while deterioration of equipment is $1 / 10$ per year or faster. Beginning with a pair $\delta^{p l t}=1 / 25, \delta^{\text {eqt }}=1 / 10$, the table gives us a ratio CVAR $=2.34$, which is much too high. Therefore by equations (8.7)(8.8), the rest of the table explores combinations yielding lower ratios. The small grid of pairs $\delta^{p l t}, \delta^{e q t}$ and the results are self-explanatory. 
On this basis the two pairs with CVAR $=1.81$ and CVAR $=1.88$ are equally good. ${ }^{14}$ Since a depreciation rate of $1 / 8$ appears slightly less arbitrary than $1 / 7$, and since a depreciation rate of $1 / 25$ for plant is perhaps too low, we set $\delta^{p l t}=1 / 20=5.0 \%$ and $\delta^{e q t}=1 / 8=$ $12.5 \%$. Table 8.3 describes the capital stock matrix $K$ that derives from equation (8.5) based on the values of $\kappa_{i}$ and $\delta_{i}$ in Table 8.8, and the $\alpha_{j}$ computed in equation (8.8), for the German economy in 1995.

\begin{tabular}{ccccccccc}
\hline & $(1)$ & $(2)$ & $(3)$ & $(4)$ & $(5)$ & $(6)$ & $(7)$ \\
\multicolumn{7}{c}{ Agrc. } & Manf. Oth.Mf. Cstrt. Bus.Svcs. & Cns.Svcs. Soc.Svcs. Mean \\
\hline$I I_{j}:$ & 48.19 & 63.04 & 62.69 & 52.29 & 41.16 & 37.44 & 25.09 & 46.55 \\
$W_{j}:$ & 21.37 & 28.72 & 26.87 & 32.09 & 31.57 & 24.12 & 58.97 & 32.05 \\
$A_{j}^{\delta}:$ & 17.93 & 4.98 & 6.35 & 2.39 & 9.18 & 12.70 & 9.89 & 8.57 \\
$P_{j}:$ & 12.51 & 3.26 & 4.09 & 13.23 & 18.10 & 25.74 & 6.05 & 12.84 \\
$K_{j}:$ & 209.75 & 58.23 & 74.29 & 27.92 & 107.39 & 148.61 & 115.72 & 105.99 \\
\hline$r_{j}:$ & 5.97 & 5.60 & 5.51 & 47.39 & 16.85 & 17.32 & 5.23 & 12.81 \\
\hline
\end{tabular}

Table 8.10: Empirical sectoral rates of profit (Germany, 1995)

Note: $I I_{j}, W_{j}, A_{j}^{\delta}, P_{j}, K_{j}$ are intermediate inputs, wage payments, depreciation, profits, and capital of sector $j$, all expressed in percent of gross output; $r_{j}$ is the rate of profit, $r_{j}=100 \cdot P_{j} / K_{j}$. Profits are output minus $\left(I I_{j}+W_{j}+A_{j}^{\delta}\right)$.

The last row in Table 8.3 computes the column sums per 1 mill. Euro output in prices of 1995. The number in column 2, for example, indicates that, the sector has capital goods installed that at the prices of 1995 are worth 0.5823 mill. Euro. Thus, the column sums can be said to represent the sectoral ratios of capital to gross output. At first glance it is perhaps surprising that the three services sectors 5, 6 and 7 have significantly higher ratios than the two industrial sectors 2 and 3. If a high capital-output ratio were really an indicator of a high 'degree of industrialization', then agriculture (sector 1) would be the most industrialized and construction would be the least industrialized.

Our main motive for constructing the capital stock matrix $K$ is

${ }^{14}$ We have also checked that the differences in the sectoral rates of profit are rather small. 


\begin{tabular}{|c|c|c|c|c|c|c|c|c|}
\hline & (1) & (2) & (3) & (4) & (5) & (6) & (7) & \\
\hline & Agrc. & Manf. & Oth.M & Cstrt. & Bus.Svcs & Cns.Sves & oc.Svcs & Mean \\
\hline 1991: & 3.75 & 7.79 & 11.51 & 51.56 & 19.49 & 16.84 & 4.68 & 13.82 \\
\hline 1992 & 5.26 & 4.04 & 9.06 & 57.49 & 17.78 & 16.27 & 5.19 & 12.93 \\
\hline 1993: & 4.70 & -0.21 & 7.14 & 51.13 & 17.37 & 15.62 & 4.71 & 11.91 \\
\hline 1994: & 5.62 & 1.87 & 7.17 & 52.52 & 17.84 & 16.24 & 5.67 & 12.72 \\
\hline 1995 & 5.97 & 5.60 & 5.51 & 47.39 & 16.85 & 17.32 & 5.23 & 12.81 \\
\hline 1996 : & 7.05 & 4.28 & 6.56 & 42.56 & 16.46 & 17.97 & 5.20 & 13.05 \\
\hline 1997: & 7.44 & 6.87 & 7.10 & 40.54 & 16.70 & 18.37 & 4.99 & 13.50 \\
\hline 1998: & 6.77 & 7.86 & 7.71 & 39.88 & 17.76 & 18.17 & 5.05 & 13.84 \\
\hline 1999: & 5.83 & 5.73 & 8.19 & 40.17 & 18.01 & 17.74 & 4.85 & 13.67 \\
\hline
\end{tabular}

Table 8.11: German sectoral profit rates (profits per unit of capital goods advanced) over the $1990 \mathrm{~s}$

that it enables us to obtain the appropriate sectoral rates of profit, where in the denominator the material inputs $\left(p A_{T}\right)_{j} x_{j}$ are replaced with the capital stocks $(p K)_{j} x_{j}$. The relevant data to compute sectoral profit rates in Germany, 1995, are collected in Table 8.10. The sectoral capital stocks per unit of output are in the fifth row of Table 8.10. In 1995 nominal and real magnitudes coincide, so that $p=(1, \ldots, 1)$, and the numbers in the fifth row are just the column sums of Table 8.3 (multiplied by 100). It should be clear from the above discussion that they are strictly proportional to the sectoral depreciation statistics $A_{\star j}^{\delta} \cdot{ }^{15}$

The resulting profit rates are shown in the last row of Table 8.10 and seem in line with intuition. The only exception is the unrealistically high profit rate in the construction sector 4 . Table 8.10 shows that the sector's share of profit in gross output is not very noticeable and so such a high profit rate must derive from a comparatively low capital in use. Although measurement errors may play a role, the large gap with the other sectors remains remarkable, even puzzling. In contrast, the profit rates in the two industrial sectors 2 and 3 are remarkably, albeit not implausibly, low.

Table 8.11 employs the procedure of equations (8.5)-(8.8) to derive

${ }_{15}$ The figures in rows 1-4 are measured as percentages of the sectoral outputs. 
the sectoral rates of profit over the $1990 \mathrm{~s}^{16}$ It demonstrates that the profit rates in Table 8.10 for the year 1995 are largely representative of the whole period. One exception is sectors 2 and 3, where 1995 is the only year in which sector 2 scores a higher profit rate. On the whole, the profit rates are relatively stable over the 1990s with the exception of the construction sector, whose profit rate has steadily declined after an extraordinary peak in 1992. The profit rates show that after the short boom following the German unification the first half of the 1990s was for most sectors a rather poor period, with a substantially better output performance over the rest of the decade. For the construction sector, however, it was just the other way round.

\footnotetext{
${ }^{16}$ The last year for which all the data categories we need are available is 1999 . After 1995 wages and depreciation are only available as nominal data, and so we used nominal data for the whole decade. This means that the capital stock coefficients are nominal magnitudes, which does not matter for the computation of the profit rates.
} 



\section{Actual Labor Values with Multiple Activities}

\subsection{Introduction}

In Chapters 7 and 8 , we have considered linear economies in which each sector produces a single good using only one method of production. In this setting, labor values can be defined as the standard IO employment multipliers: they are unique and nonnegative, and they are strictly positive if and only if the corresponding equilibrium prices are positive. They vary continuously with changes in technology and they are proportional to prices if and only if either profits are zero, or the same technology is used in all sectors. At the aggregate level, they capture the relation between total net output and aggregate labor spent in production, and between total profits and the exploitation of the working class. They also provide theoretically robust and empirically meaningful measures of labor productivity.

This chapter extends our analysis to economies in which each industry produces a single good but multiple activities are used in each sector $j$. Formally, in each industry $j$ good $j$ is produced using $k(j)$ production techniques $\left(A_{\star j_{h}}, l_{j_{h}}\right) h=1, \ldots, k(j)$ where $k(j) \geqq 1$ with strict inequality for at least some $j$. How should labor values be defined in this context? A straightforward application of the Leontief formula (treating goods produced with different techniques as if they were different goods) would be highly misleading: labor values thus defined might capture firm- (or activity-) specific employment multipliers - the changes in employment induced by changes in demand for certain goods produced using a specific production method. But they do not measure the average, social real cost of producing a given good in terms of human labor - the labor content of individual commodities.

The definition of labor values in linear economies with single outputs and multiple activities has not been extensively studied in the literature. In a relatively less known, old contribution, Murata (1977) has introduced a definition for labor values of commodities based on 
minimal labor contents, in the spirit of Morishima's (1974) famous optimizing approach. He has proved that if this definition is adopted, then the "Fundamental Marxian Theorem" (FMT) holds in economies with multiple activities. Yet this definition has several disadvantages: like Morishima's definition, it takes no account of Marx's own concept of labor values in the presence of intrasectoral competition; it leads to several technical difficulties; and it separates the concept of labor contents from the measurement of labor contents of commodities that is conventionally used based on monetary Leontief tables.

In this chapter we provide an alternative definition that extends the standard approach to economies with multiple activities. The key step for the appropriate generalization of the concept of labor values as measuring the real (labor) costs of producing goods is to note that the existence of alternative methods of production requires to distinguish the concept of (average) labor values and the notion of individual values. Conceptually, this approach very closely follows Marx's own discussion in Marx (1976) on the analysis of labor values in the presence of significant firm heterogeneity within sectors. Marx's general attitude in these cases was to ground theoretical concepts in firms' actual practices rather than looking at the dominant conventions among economic theorists.

The distinction between individual and average requirements is standard in IO theory. Starting from the original structure $(A, l)$, a standard square IO table is derived by aggregating the activities of each sector - using the activity levels that characterize the individual activities - into some suitably defined 'average' technology $(\bar{A}, \bar{l})$. Formally, the $A_{\star j_{h}}, h=1, \ldots, k(j)$ of each sector are combined into a single column vector as follows:

$$
\bar{A}_{\star j}=\left(x_{j_{1}} A_{\star j_{1}}+\ldots+x_{j_{k(j)}} A_{\star j_{k(j)}}\right) /\left(x_{j_{1}}+\ldots+x_{j_{k(j)}}\right),
$$

and likewise for the corresponding labor inputs. In other words, different production methods are summed at their activity level and then are divided by the total output generated in the sector. Labor values are then defined as the solution of $v \bar{A}+\bar{l}=v$, and represent the average total labor costs of producing the various commodities with respect to the multiple activities that are operated in each sector.

An important feature of our approach is that the properties of the single activities $\left(A_{\star j_{h}}, l_{j_{h}}\right)$ carry over to the average technology $(\bar{A}, \bar{l})$ - as long as the market for single activities remains the same. For example, if all single activities are viable then so is the average technology. Further, if CU-LS technical change takes place with respect 
to some activities, and activity levels remain invariant, then this type of technical change will also characterize the average inputs $(\bar{A}, \bar{l})$. This allows us to show that our extension of the standard concept of labor values has all of the desired properties of Marxian value theory discussed in Chapter 6 and to generalize the standard propositions of Marx's economics, including the FMT.

\subsection{Average and individual labor values in single product systems}

In this section, we analyze labor values in economies with multiple activities in the context of Steedman's (1977, 140-141) model of a twosector simple reproduction economy with a given real wage $b$ (corn) paid in advance and two (circulating) capital goods $M$ and $C$, where $M$ stands for 'material' (later: machines) and $C$ (corn) is consumed by both workers and capitalists. Direct labor inputs are denoted by $l_{i}$. The period of production (a year) is uniform between and within both sectors. This will allow us to introduce our concept of labor values and also to compare it with Steedman's (1977) definition. ${ }^{1}$

Given the rather specific nature of the examples analyzed in this section, and to facilitate comparison with Steedman (1977), we use a slightly different notation. Following Steedman (1977, 141), we take the following IO table as given. We assume simple reproduction so that $M_{2}+M_{2}^{\prime}=M_{1}$, and $Q-C$ is the net output of the system. Let $v_{m}, v_{c}$ denote the labor values of 'material' and 'corn', respectively. According to Marx $(1976,434,530)$, when more than one technique - here in the production of corn - is used, individual values must also be considered. Hence, let $\widetilde{v}_{c}, \widetilde{v}_{c}^{\prime}$ be the two individual values of corn with respect to the two processes (no such distinction is necessary for the first sector). The relation between individual values and labor values can be described as follows.

$$
\frac{\widetilde{v}_{c} Q_{2}+\widetilde{v}_{c}^{\prime} Q_{2}^{\prime}}{Q}=v_{c}
$$

i.e. the labor value of corn is the weighted average of individual values, where the weights are given by the output shares of the processes of the corn-producing sector. Then, by Marx's (1976, 434, 530-531) definition of individual values, we have:

\footnotetext{
${ }^{1}$ See also the discussion in Chapter 11.
} 


$$
\begin{aligned}
v_{c} C_{2}+v_{m} M_{2}+l_{2} & =\widetilde{v}_{c} Q_{2}, \\
v_{c} C_{2}^{\prime}+v_{m} M_{2}^{\prime}+l_{2}^{\prime} & =\widetilde{v}_{c}^{\prime} Q_{2}^{\prime}, \\
v_{c} C_{1}+l_{1} & =v_{m} M_{1},
\end{aligned}
$$

i.e. the process-dependent individual value of a commodity-here of

\begin{tabular}{|c|c|c|c|c|c|c|}
\hline Material & Corn & Labor & & Material & Corn & \\
\hline 0 & $C_{1}$ & $l_{1}$ & $\rightarrow$ & $M_{1}$ & 0 & M-sector \\
\hline$M_{2}$ & $C_{2}$ & $l_{2}$ & $\rightarrow$ & 0 & $Q_{2}$ & \\
\hline$M_{2}^{\prime}$ & $C_{2}^{\prime}$ & $l_{2}^{\prime}$ & $\rightarrow$ & 0 & $Q_{2}^{\prime}$ & \\
\hline \multirow[t]{2}{*}{$M_{2}+M_{2}^{\prime}$} & $C=$ & $L=$ & $\rightarrow$ & $M_{1}$ & $Q=$ & Totals \\
\hline & \multicolumn{3}{|c|}{$C_{1}+C_{2}+C_{2}^{\prime} l_{1}+l_{2}+l_{2}^{\prime}$} & \multicolumn{2}{|r|}{$Q_{2}+Q_{2}^{\prime}$} & \\
\hline
\end{tabular}
one unit of corn - is the sum of the labor values of physical inputs plus the direct labor time individually needed.

Table 9.1: A version of Steedman's two-sector corn economy

Equations (9.1)-(9.4) form a system of 4 equations in 4 unknowns $\widetilde{v}_{c}, \widetilde{v}_{c}^{\prime}, v_{m}, v_{c}$. Substituting equation (9.1) into equations (9.2)-(9.4), we obtain:

$$
\begin{aligned}
v_{m} M_{1} & =\frac{\widetilde{v}_{c} Q_{2}+\widetilde{v}_{c}^{\prime} Q_{2}^{\prime}}{Q} C_{1}+l_{1}, \\
\widetilde{v}_{c} Q_{2} & =\frac{\widetilde{v}_{c} Q_{2}+\widetilde{v}_{c}^{\prime} Q_{2}^{\prime}}{Q} C_{2}+v_{m} M_{2}+l_{2}, \\
\widetilde{v}_{c}^{\prime} Q_{2}^{\prime} & =\frac{\widetilde{v}_{c} Q_{2}+\widetilde{v}_{c}^{\prime} Q_{2}^{\prime}}{Q} C_{2}^{\prime}+v_{m} M_{2}^{\prime}+l_{2}^{\prime} .
\end{aligned}
$$

Equations (9.1)-(9.4) confirm that additive calculations are indeed the basis for the determination of individual and (average) labor values. However, it is immediate to show that in general labor values cannot be derived from the original rectangular IO system in the standard purely additive way, and do not satisfy $v B=v A+l$.

In order to analyze the properties of individual and labor values, in the rest of this section we assume that revenues exceed the costs of physical inputs in every process. Formally: 
Assumption 9.1. There exist prices $p_{m}, p_{c}>0$ for 'material' and 'corn' such that:

$$
\left(p_{m}, p_{c}\right)\left(\begin{array}{ccc}
0 & M_{2} & M_{2}^{\prime} \\
C_{1} & C_{2} & C_{2}^{\prime}
\end{array}\right)<\left(p_{m}, p_{c}\right)\left(\begin{array}{ccc}
M_{1} & 0 & 0 \\
0 & Q_{2} & Q_{2}^{\prime}
\end{array}\right) .
$$

The next result proves that individual labor values exist and are unique and positive.

Proposition 9.1. Under assumption 9.1, the system of equations (9.5)-(9.7) has exactly one, strictly positive solution $\left(v_{m}, \widetilde{v}_{c}, \widetilde{v}_{c}^{\prime}\right)$.

Proof. In matrix notation, equations (9.5)-(9.7) can be written as

$$
\begin{aligned}
& \left(v_{m}, \widetilde{v}_{c}, \widetilde{v}_{c}^{\prime}\right)\left(\begin{array}{ccc}
0 & M_{2} & M_{2}^{\prime} \\
x_{2} C_{1} & x_{2} C_{2} & x_{2} C_{2}^{\prime} \\
x_{2}^{\prime} C_{1} & x_{2}^{\prime} C_{2} & x_{2}^{\prime} C_{2}^{\prime}
\end{array}\right)+\left(l_{1}, l_{2}, l_{2}^{\prime}\right) \\
& =\left(v_{m}, \widetilde{v}_{c}, \widetilde{v}_{c}^{\prime}\right)\left(\begin{array}{lll}
M_{1} & 0 & 0 \\
0 & Q_{2} & 0 \\
0 & 0 & Q_{2}^{\prime}
\end{array}\right)
\end{aligned}
$$

with $x_{2}=Q_{2} / Q$ and $x_{2}^{\prime}=Q_{2}^{\prime} / Q$. Let $\widetilde{A}$ denote the (transformed) matrix of inputs, $\widetilde{B}$ that of outputs, and $l$ the vector of direct labor inputs. The system can be written as

$$
\begin{aligned}
\left(v_{m}, \widetilde{v}_{c}, \widetilde{v}_{c}^{\prime}\right) \widetilde{A}+l & =\left(v_{m}, \widetilde{v}_{c}, \widetilde{v}_{c}^{\prime}\right) \widetilde{B}, \quad \text { or } \\
\left(v_{m}, \widetilde{v}_{c}, \widetilde{v}_{c}^{\prime}\right) \widetilde{A} \widetilde{B}^{-1}+l \widetilde{B}^{-1} & =\left(v_{m}, \widetilde{v}_{c}, \widetilde{v}_{c}^{\prime}\right) .
\end{aligned}
$$

Further, by assumption 9.1 , and noting that $x_{2}+x_{2}^{\prime}=1$, we obtain:

$$
\begin{array}{ll}
\left(p_{m}, p_{c}, p_{c}\right) \widetilde{A} & <\left(p_{m}, p_{c}, p_{c}\right) \widetilde{B}, \quad \text { or } \\
\left(p_{m}, p_{c}, p_{c}\right) \widetilde{A} \widetilde{B}^{-1} & <\left(p_{m}, p_{c}, p_{c}\right),
\end{array}
$$

which yields the desired result by known theorems on nonnegative matrices (see Nikaido (1968, Ch. II)).

Then, by summing equations (9.6) and (9.7) one immediately gets: 


$$
\begin{aligned}
& \left(v_{m}, v_{c}\right)\left(\begin{array}{cc}
0 & M_{2}+M_{2}^{\prime} \\
C_{1} & C_{2}+C_{2}^{\prime}
\end{array}\right)+\left(l_{1}, l_{2}+l_{2}^{\prime}\right) \\
& =\left(v_{m}, v_{c}\right)\left(\begin{array}{cc}
M_{1} & 0 \\
0 & Q
\end{array}\right)
\end{aligned}
$$

Equation (9.8) says that (average) labor values can be directly computed from specifically aggregated data, without using individual values. $^{2}$ By summing the two equations in (9.8), it follows that total employment is equal to the labor value of the net product $Q-C$ of Table 9.1. Formally:

$$
L=v_{c}(Q-C)
$$

By equation (9.8), it also follows that the ratio of surplus value to the total value of labor power is equal to $\epsilon$, the rate of exploitation per labor hour bought by the capitalists.

$$
\epsilon=\frac{1-v_{c} b}{v_{c} b}=\frac{v_{c}(Q-C)-v_{c} b L}{v_{c} b L} .
$$

The previous analysis confirms some of the key results of the book: our labor values are well-defined, positive, unique, they satisfy the value added identity, and allow for an unambiguous definition of the rate of exploitation. The next proposition establishes the link between exploitation and aggregate profits.

Proposition 9.2 (FMT). $\epsilon>0$ if and only if there exist positive prices $\left(p_{m}, p_{c}\right)$ such that $p_{c} Q+p_{m} M_{1}-p_{c} C-p_{m}\left(M_{2}+M_{2}^{\prime}\right)-p_{c} b L>0$.

Proof. Because $M_{1}=M_{2}+M_{2}^{\prime}$, aggregate profits are equivalent to $p_{c}(Q-C-b L)>0$, which in turn is equivalent to $\epsilon>0$.

In other words, the rate of exploitation is positive if and only if there exist positive prices $\left(p_{m}, p_{c}\right)$ which yield positive (aggregate) profits. This concludes the proof that our definition of labor values satisfies the main axioms of Marx's LTV discussed in Chapter 6 in single output economies with multiple activities. It is also possible to extend the results on technical change and labor productivity derived in Chapter 7. Consider the following classification of different types of technical progress, which closely follows analogous definitions in Chapter 7.

\footnotetext{
${ }^{2}$ Assumption 9.1 can be weakened to hold for the aggregate system in equation (9.8).
} 


\section{Definition 9.1.}

(1) Technical change in a given sector is capital-using labor-saving $(C U-L S)$ if and only if either material or corn inputs increase and labor input decreases (CS-LU or CS-LS technical change is defined in a similar way).

(2) Let $\left(v_{m}, v_{c}\right)$ and $\left(v_{m}^{*}, v_{c}^{*}\right)$ denote labor values before and after technical change, respectively. Technical change in a given sector is progressive (resp. regressive) if and only if

$$
\left(v_{m}^{*}, v_{c}^{*}\right) \leq\left(v_{m}, v_{c}\right) \quad\left(\operatorname{resp} .\left(v_{m}^{*}, v_{c}^{*}\right) \geq\left(v_{m}, v_{c}\right)\right) .
$$

(3) Technical change in a given sector is profitable if, at the given initial prices $p_{m}, p_{c}$, it reduces average costs.

Proposition 9.3 extends the analogous results by Roemer. In order to prove it, it suffices to note that the same reasoning as in Roemer (1977) can be applied to the aggregate technology

$$
\left(\begin{array}{cc}
0 & M_{2}+M_{2}^{\prime} \\
C_{1} & C_{2}+C_{2}^{\prime}
\end{array}\right)\left(\begin{array}{cc}
M_{1} & 0 \\
0 & Q
\end{array}\right)^{-1},\left(l_{1}, l_{2}+l_{2}^{\prime}\right)\left(\begin{array}{cc}
M_{1} & 0 \\
0 & Q
\end{array}\right)^{-1} .
$$

First, if technical change affecting one of the three original activities is CU-LS, then technical change is also CU-LS if the aggregate technology is considered. Similarly, technical change that is profitable according to the original disaggregated technology is also profitable for the aggregated one. Third, if profits are positive in each activity, then they are positive if the aggregated technology is considered, which in turn implies $\left(v_{m}, v_{c}\right)<\left(p_{m}, p_{c}\right)$.

\section{Proposition 9.3.}

(1) All CU-LS profitable technical changes are progressive, but there are progressive $C U$-LS changes that are not profitable.

(2) All CS-LU progressive technical changes are profitable, but there are profitable $C S-L U$ changes that are not progressive.

Proposition 9.3 is conceptually similar to the results derived in Chapter 7: given our interpretation of Marxian labor values as indicators of labor productivity, it confirms that profitable CU-LS changes (which Marx considered typical of capitalism) always raise labor productivity, but not all of the CU-LS changes which raise labor productivity are adopted by capitalists as they do not reduce costs. On the other hand, CS-LU changes which raise labor productivity are 
always profitable for capitalists, but there may be cost reducing CS-LU changes which lower labor productivity in at least one sector.

The above analysis shows that the existence of multiple production techniques in use naturally leads to the distinction between individual and average labor values: the latter are defined as weighted averages of the former, and the former are given by the column sums of the given IO table made homogeneous by help of the latter. Although individual values are not necessary to compute the corresponding labor values, they are necessary to explain them. This should be particularly obvious for an interpretation of labor values as productivity indices. For individual values can be used not only to calculate average (labor) values, but also to consider the variance of labor content within each sector. Labor values as defined above are well defined and positive. They satisfy all of the key properties of Marx's LTV discussed in Chapter 6 and do not display any of the anomalies identified by Steedman (1977). ${ }^{3}$

The results derived in this section and the definitions of individual and average labor values are not restricted to two-sector models, constant returns to scale, stationary economies, and the like. All that is needed is an ex-post Leontief table with multiple activities together with a price vector $p$, such that value added is positive in every sector. We extend our analysis in the next section.

\subsection{Individual and average labor values}

Consider a non-joint production structure of the Leontief type which includes the simultaneous operation of more than one activity for at least one commodity. ${ }^{4}$ Let $n$ be the number of commodities and $m$ the number of processes where $m>n$. Then the spectrum of production activities, each of which is characterized by a set of fixed input coefficients per unit of output, is given by the following rectangular net unit-output matrix $\tilde{M}=I-A$, see Murata $(1977,138)$ :

$\tilde{M}=$

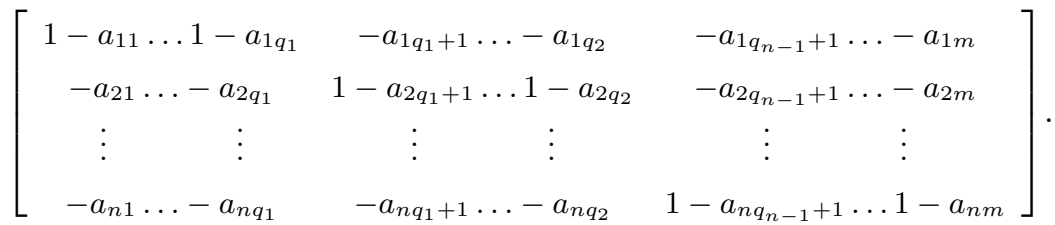

${ }^{3}$ We shall return to this issue in the next chapters.

${ }^{4}$ As in the seminal contribution by Murata (1977). 
Here, $a_{i j} \geq 0$ stands for the input of commodity $i$ required per unit of output of process $j$, which is commodity $k$ if and only if

$$
q_{k-1}+1 \leq j \leq q_{k}, \quad\left(q_{0}=0, \quad q_{n}=m\right),
$$

i.e. the above subdivision $q_{1}, \ldots, q_{n}$ indicates the type of commodity and thus the sector of the economy process $j$ belongs to.

The associated row vector $L$ of labor requirements per unit of output is:

$$
L=\left(l_{1}, \ldots, l_{q_{1}}, l_{q_{1}+1}, \ldots, l_{q_{2}}, \ldots, l_{q_{n-1}+1}, \ldots, l_{m}\right) \in \mathbb{R}_{+}^{m} .
$$

Finally, the intensities $d_{j}$ of processes $j$ with respect to the total output of the corresponding sectors $k$ is:

$$
d=\left(d_{1}, \ldots, d_{q_{1}}, \ldots, d_{q_{n-1}+1}, \ldots, d_{m}\right) \in \mathbb{R}_{+}^{m} .
$$

For all sectors $k=1, \ldots, n, q_{k-1}+1 \leq j \leq q_{k}$ and $\sum_{j=k_{q-1}+1}^{q_{k}} d_{j}=1$. Therefore, $M=\tilde{M} \widehat{d}$ and $L=\tilde{L} \widehat{d}$ represent matrices of the same type as $\tilde{M}$ and $\tilde{L}$, the only difference being that each column $j$ is multiplied by the weight $d_{j}$.

In general there is no vector $\bar{v}=\left(\bar{v}_{1}, \ldots, \bar{v}_{n}\right) \in \mathbb{R}_{+}^{n}$ that solves:

$$
\bar{v} M=L .
$$

Therefore, Murata $(1977,138)$ introduces a new definition of labor values $v^{*}$ based on certain best minimizers. Formally:

$$
v^{*}=L M^{\prime}\left(M M^{\prime}\right)^{-1},
$$

which represents a complex multiplication and summation of the original IO coefficients of the rectangular matrices $M=\tilde{M} \widehat{d}, L=\tilde{L} \widehat{d}$.

But the problem stated with respect to (9.12) did not escape Marx's attention. In Capital, Vol.1, for example, he writes:

Now let some capitalist contrive to double the productivity of labour, and to produce twenty-four instead of twelve articles in the course of a working of 12 hours. The value of the means of production remaining the same, the value of each article will fall. . The individual value of these articles is now below their social value; in other words, they have cost less labour-time than the great bulk of the same article produced under the average social conditions... The real value of a commodity, however, is not its individual, but its social value; that is to say, its value is not measured by the labour-time that the article costs the producer in 
each individual case, but by the labour-time socially required for its production (Marx 1976, 434).

Let $v_{k}^{j}$ be the individual labor value of commodity $k$ produced in process $j$, let $v=\left(v_{k}^{j}\right)$, and let $\bar{v}_{i}$ be the labor value of commodity $i$. We interpret Marx as suggesting:

$$
\begin{aligned}
v_{k}^{j} & =\sum_{i=1}^{n} \bar{v}_{i} a_{i j}+l_{j}, \quad q_{k-1}+1 \leq j \leq q_{k}, \\
\bar{v}_{k} & =\sum_{j=q_{k-1}+1} d_{j} v_{k}^{j} .
\end{aligned}
$$

Inserting equation (9.14) into equation (9.15) gives:

$$
\bar{v}_{k}=\sum_{j=q_{k-1}+1}^{q_{k}} d_{j}\left(\sum_{i=1}^{n} \bar{v}_{i} a_{i j}+l_{j}\right)=\sum_{i} \bar{v}_{i} \sum_{j} d_{j} a_{i j}+\sum_{j} d_{j} l_{j} .
$$

Let $\bar{M}$ and $\bar{L}$ denote, respectively, the $n \times n$ matrix and the $i \times n$ vector obtained from $M, L$ by pure summation of the columns of each sector. ${ }^{5}$ Equation (9.16) can then be equivalently expressed in the following standard form:

$$
\bar{v} \bar{M}=\bar{L}, \quad \bar{v}=\left(\bar{v}_{1}, \ldots, \bar{v}_{n}\right) .
$$

We henceforth assume $\bar{L}>0$ and that there exist prices $\bar{p}=$ $\left(\bar{p}_{1}, \ldots, \bar{p}_{n}\right) \geq 0$ such that $\bar{p} \bar{M}>0$, i.e. value added is positive with respect to each sector.

\section{Proposition 9.4.}

(i) Equation (9.17) has exactly one solution $\bar{v}$ given by

$$
\bar{v}=\bar{L} \bar{M}^{-1} \text {, i.e., } \bar{v}_{k}=\bar{L} \bar{M}_{1 k}^{-1}+\ldots+\bar{L}_{n} \bar{M}_{n k}^{-1} \text {. }
$$

The vector $\bar{v}$ is strictly positive. The vector $v$ therefore must be positive, too.

(ii) Given this vector of labor values $\bar{v}>0$, we have for all column vectors $\bar{x} \in \mathbb{R}_{+}^{n}$ :

$$
\bar{v} \bar{M} \bar{x}=\bar{L} \bar{x} .
$$

${ }^{5}$ Compare this with the symbolic partition in the presentation of $\tilde{M}$ and recall that weights $d_{j}$ have been attached to the respective columns of $\tilde{M}, \tilde{L}$ to obtain the two matrices $M, L$ and that these weights sum to one with respect to each sector. 
Proof. It is well known that $\bar{p} \bar{M}>0$ implies that $\bar{M}$ is nonnegatively invertible (Nikaido 1968, 102-103, 107). Note, that $I-\bar{M}$ can be made indecomposable by adding suitably small numbers $\varepsilon>0$ without changing the assumption on values added. $\bar{M}^{-1} \geq 0$ together with $\bar{L}>0$ then immediately prove the first part of the statement. Then the second part of the statement follows from $\bar{v} \bar{M} \bar{x}=\bar{L} \bar{M}^{-1} \bar{M} \bar{x}=\bar{L} \bar{x}$.

By Proposition 9.4 we have provided a definition of positive labor values on the basis of simple calculations. We have performed this task by taking seriously the Marxian notion of individual value, an indispensible - yet commonly neglected - ingredient of Marx's average or social labor values, whenever the number of activities exceeds the number of produced commodities. ${ }^{6}$ Further, the labor value of the net product $\bar{M} \bar{x}$ resulting from sectoral activity levels $\bar{x}$ is equal to the sum of direct labor performed at these activity levels.

Another feature of Marx's conception of labor values is worth stressing. In Marx, value analysis "analyzes the relationships post factum and, therefore, applies after techniques have been chosen and after wages have been spent ..." (Wolfstetter 1973, 804). This feature of Marxian value analysis is taken into account by Murata (1977) with the help of the relative intensity multipliers $d_{j}$. Activities that are not operated (and exactly these) are represented by zero columns in the matrices $M, L$ and could have been equally well eliminated from the original matrices $\bar{M}, \bar{L}$. The determination of activity levelsi.e. the choice of technique - is not considered, which makes the model particularly handy for our purposes.

\subsection{The measurement of labor contents}

We now compare our measure of labor values $\bar{v}$ with the conventional measurement of labor contents by means of observed transaction tables. Let prices $\bar{p}>0$ be given as in Proposition 9.4 and let $p$ denote the extended price vector

$$
p=\left(\bar{p}_{1}, \ldots, \bar{p}_{1}, \bar{p}_{2}, \ldots, \bar{p}_{2}, \ldots, \bar{p}_{n}\right) \in \mathbb{R}^{m} .
$$

The monetary IO tables can be derived from $\bar{M}$ :

$$
\tilde{G}=\widehat{\bar{p}} \tilde{M} \widehat{p}^{-1}, \quad G=\widehat{\bar{p}} M \widehat{p}^{-1} \quad \text { and } \bar{G}=\widehat{\bar{p}} \bar{M} \widehat{\bar{p}}^{-1} .
$$

${ }^{6}$ We note in passing that equation (9.19) does not hold if Murata's (1977) definition is adopted. 
In each of these tables, physical coefficients $a_{i j}$ have been multiplied by $\bar{p}_{i}$ and divided by $p_{j}$ so that they represent value input of commodity $i$ per $\$$ of value output of process $j$ (or sector $k$ ). ${ }^{7}$ Then:

Proposition 9.5. Let $L, \bar{L}$ be defined as in the previous subsection.

(1) The horizontal summations defined for matrix $M$ imply

$$
\overline{\overline{\bar{p}} M \widehat{p}^{-1}}=\widehat{\bar{p}} \bar{M}^{-1} \text {. }
$$

(2) $e \bar{G}>0$, i.e. $\bar{G}$ is nonnegatively invertible.

(3) The equation

$$
\bar{X} \bar{G}=\bar{m} \quad\left[\bar{m}=\bar{L} \widehat{\bar{p}}^{-1}\right]
$$

has a unique semi-positive solution $\bar{X}=\bar{v} \widehat{\bar{p}}^{-1}$ where

$$
\bar{v}=\bar{L} \bar{M}^{-1}
$$

and $\bar{v}>0$. This solution is strictly positive if $\bar{L}_{k}$ is positive for at least one basic sector $k$ of $\bar{M}$.

(4) The vector $X \in \mathbb{R}^{m}$ defined by

$$
X=\bar{X} G-m, \quad m=L \widehat{p}^{-1}
$$

represents the vector of deviations of individual from average labor values per \$ of output value:

$$
X_{j}=\left(v_{k}^{j}-\bar{v}_{k}\right) / \bar{p}_{k}, \quad q_{k-1}+1 \leq j \leq q_{k} .
$$

(5) Let $b \geq 0$ be a bundle of commodities. The total labor content of bundle $b$ with respect to labor inputs $L$ is determined by

$$
\bar{v} b=\bar{X} \widehat{\bar{p}} b .
$$

Proof. 1. The proof is straightforward, since the employed summation is of the same kind on both sides of the equality sign and since all summed columns $j, q_{k-1}+1 \leq j \leq q_{k}$, have been pre-multiplied by the same diagonal matrix $\widehat{\bar{p}}$ and have been divided by the same price $\bar{p}_{k}$.

2. $e \bar{G}=\bar{p} \bar{M} \widehat{\bar{p}}^{-1}>0$, because $\bar{p} \bar{M}>0$.

3. The result follows from Proposition 9.4, noting that $\bar{x} \bar{G}=$ $\bar{v} \bar{M} \hat{\bar{p}}^{-1}=\bar{L} \hat{\bar{p}}^{-1}$. Futhermore, if $\bar{L}_{k}$ is positive for at least one basic sector $k$ of $\bar{M}$, i.e. $\bar{M}_{k \star}^{-1}>0$ (the $k$-th row of $\bar{M}^{-1}$ is positive), then $\bar{v}=\bar{L} \bar{M}^{-1} \geq \bar{L}_{k} \bar{M}_{k \star}^{-1}>0$ and $\bar{p}>0$.

\footnotetext{
$\overline{{ }^{7} \text { Note that }} p_{j}=\bar{p}_{k}$ for the processes $j: q_{k-1}+1 \leq j \leq q_{k}$.
} 
4. $\bar{X} G-m=\bar{v} \widehat{\bar{p}}^{-1} \widehat{\bar{p}} M \widehat{p}^{-1}-L \widehat{p}^{-1}=(\bar{v} M-L) \widehat{p}^{-1}$. Yet, $(\bar{v} M-L)_{k j}$ is equal to $v_{k}^{j}-\bar{v}_{k}$, by equation (9.14), which yields the desired result.

5 . The result is obvious.

Proposition 9.5 has various implications. It shows that the conventional measurement of labor content by help of square monetary tables such as $\bar{G}$ and of equation $(9.23)^{8}$ leads us back to our definition of labor values, equations (9.14)-(9.15), based on a rectangular matrix $M$ and the corresponding weights $d$. Thus, this result confirms our method of definition. However, more importantly, it justifies those methods of measurement of labor contents, based on monetary data of kind $G$ without looking into the derivation procedure of $G$-provided these matrices have been established as proposed above. ${ }^{9}$ Furthermore, by Proposition $9.5(1)$ the matrix $\bar{G}$ can also be obtained by means of such horizontal summations, based on matrix $G$. Lastly, by Proposition $9.5(5)$, the labor content of any given set of commodities $b \geq 0$ is, in principle, measurable. ${ }^{10}$

\subsection{Prices, profits, and rate(s) of exploitation}

This section provides some basic propositions of Marx's LTV, and analyzes some relations between labor values $\bar{v}$ and prices. Let $\bar{C} \in \mathbb{R}_{+}^{n}$ denote the average consumption of the working-class population per unit of labor performed (in a given time interval). Let $\bar{p}>0$ denote actual commodity prices. Gross profits per unit of sectoral product are defined by the following accounting relationship:

$$
\bar{p} \bar{M}=\bar{p} \bar{C} \bar{L}+\bar{F} \text {, i.e., } \quad \bar{F}=\bar{p}(\bar{M}-\bar{C} \bar{L}) .
$$

${ }^{8}$ This equation can also be expressed as: $\bar{X} \bar{G}=\bar{X}-\bar{A} \widehat{\bar{p}} \bar{A} \widehat{\bar{p}}^{-1}$. Note that the inverse coefficients $\bar{G}_{k l}^{-1}$ used to solve this system are also directly connected to the physical data: $\bar{G}_{k h}^{-1}=\bar{p}_{k} \bar{M}_{k h}^{-1} \bar{p}_{h}^{-1}$, which justifies their interpretation as the monetary form of, average, intermediate product contents.

${ }^{9}$ We have not discussed here the necessity (arising from statistics) of obtaining matrices of a manageable size. The procedures and problems of aggregating sectors to "departments" in the framework of the labor theory of value are considered in Morishima and Seton (1961).

${ }^{10}$ Examples of the measurement of such labor contents by help of monetary Leontief tables can be found in the literature in a variety of investigations. For example, with respect to a measurement of labor productivity in Roman (1974) and with respect to labor values and the rate of surplus value in Wolff (1979). 
Here $\bar{p} \bar{M}$ denotes the vector of values added (per sector and unit of commodity produced) and it is assumed to be positive, we also define

$$
\begin{aligned}
& s=\bar{v}(\tilde{M}-\bar{C} \tilde{L})=\bar{v} \tilde{M}-\bar{v}_{w} \tilde{L}, \quad \bar{v}_{w}=\bar{v} \bar{C} \\
& \bar{s}=\bar{v}(\bar{M}-\bar{C} \bar{L})=\bar{v} \bar{M}-\bar{v}_{w} \bar{L} \\
& \epsilon=s\left(\widehat{\overline{v_{w} \tilde{L}}}\right)^{-1}, \bar{\epsilon}=\bar{s}\left(\widehat{\bar{v}_{w} \tilde{L}}\right)^{-1}
\end{aligned}
$$

where the scalar $\bar{v}_{w}$ describes the value of labor power per unit of labor performed; $s, \bar{s}$ are the vectors of surplus values per process and per sector (per unit of commodity output); and $\epsilon, \bar{\epsilon}$ represent the vectors of the corresponding rates of surplus value (which are well defined only when $\tilde{L}$ and $\bar{L}$ are positive).

Proposition 9.6. There is a uniform sectoral rate of surplus value and this rate is equal to the rate of surplus labor.

$$
\bar{\epsilon}_{1}=\ldots=\bar{\epsilon}_{n}=\left(1-\bar{v}_{w}\right) / \bar{v}_{w},
$$

Proof. Using equation (9.17)

$$
\begin{aligned}
\bar{\epsilon}_{k} & =\left(\sum_{i=1}^{n} \bar{v}_{i} \bar{M}_{i k}-\bar{v}_{w} \bar{L}_{k}\right) /\left(\bar{v}_{w} \bar{L}_{k}\right) \\
& =\left(\bar{L}_{k}-\bar{v}_{w} \bar{L}_{k}\right) /\left(\bar{v}_{w} \bar{L}_{k}\right)=\left(1-\bar{v}_{w}\right) / \bar{v}_{w}
\end{aligned}
$$

Remark 9.2. Proposition 9.6 corresponds to statements made by Marx in volumes one $(1976,304-306)$ and three $(1981,241-242,275)$ of Capital, a detailed examination of which can be found in Cogliano (2011, 2013).

The overall rate of surplus value (or exploitation) is independent of activity levels as long as intrasectoral market shares remain unchanged. Note that rates of surplus value are not uniform at the level of single activities. Further, there is no need here for a process of equalization of such rates of surplus value, nor is there any obvious rationale for such a process, since activities are chosen depending on prices $\bar{p}$, where $\bar{p} \neq \bar{v}$ in general - if the composition of capital is not uniform across sectors.

Next we prove a version of the FMT. Assume for the sake of simplicity that the augmented matrix of inputs $(\bar{C} \bar{L})^{+}=I-\bar{M}+\bar{C} \bar{L}=$ $\bar{A}+\bar{C} \bar{L}$ is indecomposable (Nikaido 1968, 105), i.e. we exclude luxury 
goods from the following considerations. ${ }^{11}$ Let $\bar{H}$ be an arbitrary $n \times n$ matrix with semi-positive columns $\bar{H}_{\star j} \geq 0$ for all $j=1, \ldots, n$.

Proposition 9.7. Any semi-positive solution $v \geq 0, \alpha \geqq 0$ of

$$
v(\bar{M}-\bar{C} \bar{L})=\alpha v \bar{H}, \quad v=\left(v_{1}, \ldots, v_{n}\right) \in \mathbb{R}^{n}, \alpha \in \mathbb{R}
$$

will be strictly positive, provided that there exists a nonnegative row vector $u \in \mathbb{R}^{n}$ such that $u(\bar{M}-\bar{C} \bar{L}) \geq 0$.

Proof. Since $(\bar{C} \bar{L})^{+}$is indecomposable, Lemma 7.4 in Nikaido (1968) says that $v>0$. And the assumption $u(\bar{M}-\bar{C} \bar{L}) \geq 0$ implies that $\bar{M}-$ $\bar{C} \bar{L}=I-(\bar{C} \bar{L})^{+}$is nonnegatively invertible (Nikaido 1968, 107); indeed, $(\bar{M}-\bar{C} \bar{L})^{-1}$ is strictly positive, because of the indecomposability of $(\bar{C} \bar{L})^{+}$. Multiplying $(9.30)$ by $(\bar{M}-\bar{C} \bar{L})^{-1}$ then implies:

$$
v=\alpha v \bar{H}(\bar{M}-\bar{C} \bar{L})^{-1},
$$

which implies that $\alpha>0$. Finally, since all columns of $\bar{H}$ and therefore of $\bar{H}(\bar{M}-\bar{C} \bar{L})^{-1}$ are semi-positive, it follows that a vector $v \geq 0$ and a number $\alpha \geq 0$, such that (9.30) is fulfilled, will exist with respect to each such $\bar{H}$, i.e. that the above assertion and the calculations have not been made with respect to an empty set of objects.

Remark 9.3. Equation (9.30) will always have solutions under the assumptions made.

The formulations of the FMT in the literature have focused on two special rates $\alpha$, namely the rate of surplus value and the rate of profit. ${ }^{12}$ The above version of the FMT then states that each of the following assumptions

(a) The vector of sectoral surplus values is semi-positive;

(b) The rate of surplus value is positive;

(c) The vector of sectoral profits is semi-positive;

(d) The rate of profit is positive;

${ }^{11}$ This assumption can be weakened, provided the structure of basic vs. nonbasic industries is suitably taken into account.

12 Note that a thorough treatment of fixed capital is excluded from the present model. See Murata $(1977,144)$ for an ex post inclusion of capital stocks which differs from the usual treatment by Sraffa and others and which is included in the above presentation of the FMT (at least formally). Similarly, the current presentation avoids the technicalities arising from Murata's complicated definition with regard to rectangular matrices $M$, instead of our average square matrices $\bar{M}$. 
implies the existence of the above vector $u$ and the positiveness of all $\bar{H}$-rates $\alpha$, including the rates of profit and of surplus value. ${ }^{13}$ Note that $H_{\star j} \geq 0$ for all $j=1, \ldots, m$ is not necessary to prove Proposition 9.7 and that $\epsilon_{j} \geq 0$ is not included in any of its implications.

\subsection{Conclusions}

This chapter demonstrates that if our definition of labor values is adopted, the key propositions and theorems of the basic Marxian model can be extended to the case of multiple activities. The rate of surplus value is larger than or equal to the rate of profit; values are close to production prices if the profit rate is not too large or the sectoral compositions of capital are not too different; and the price and the value rates of profit are similar - apart from the two reasons just mentionedwhen sectoral growth rates do not deviate very much from the average rate of growth. In summary, even in the case of multiple activities, the Marxian theory of profits as the product of exploitation holds and can be analyzed empirically as in Chapter 8 .

It may be objected, however, that the conclusions of this chapter do not hold only for labor and similar results can be obtained if one considers any other primary factor, thus suggesting that there is nothing specific about the labor value rate of profit (and therefore the Marxian theory of exploitation) as compared to that of other primary factors (as suggested, for example, in the literature on the socalled Commodity Exploitation Theorem, see Roemer (1982), Bowles and Gintis (1981), Yoshihara and Veneziani (2010), and Veneziani and Yoshihara (2010)). We do not find this objection entirely compelling.

First of all, there are many primary factors, (e.g. natural sources of energy) for which the assumption $\bar{L}>0$, and therefore the existence of a complete set of sectoral rates of surplus value (or compositions of capital), is unrealistic. Actually, normalizing units of measurement, such factors would normally have

$$
\bar{L}=e_{i}^{t}=(0, \ldots, 0,1,0, \ldots, 0) \in \mathbb{R}^{n},
$$

where $i$ denotes the industry which extracts the factor in question. As a result, the vector of these factor contents $\bar{v}$ will be equal to $\bar{M}^{-1}$,

\footnotetext{
${ }^{13}$ Provided that labor values have been defined in such a way that a uniform sectoral rate of surplus value results from a uniform consumption bundle $\bar{C}$, which is the case if our definition is applied: $v(\bar{M}-\bar{C} \bar{L})=\alpha v \bar{C} \bar{L} \rightarrow$ $v \bar{M} \sim \bar{L}$, cf. Propositions 9.4 and 9.6.
} 
which means that this factor does not behave very differently from an intermediate one as far as factor content is concerned.

Nevertheless, one might wish to establish an 'FMT', say, with respect to coal contents, too. In this case, $\bar{C}$ would have to be interpreted as the average consumption of coal mine owners per unit of coal supplied. And from (9.32) we would get

$$
\bar{s}_{i}=\bar{M}_{i \star}^{-1}(\bar{M}-\bar{C} \bar{L})=e_{i}-\left(\bar{M}_{i \star}^{-1} \bar{C}\right) e_{i}=\left(I-\bar{M}_{i \star}^{-1} \bar{C}\right) e_{i}
$$

In this case, only one sectoral rate of 'surplus coal-value' can be meaningfully considered. Despite this peculiarity, some kind of equivalence between 'surplus coal-value', or 'coal exploitation', and profits can be established:

$$
\bar{F}>0 \rightarrow \bar{M}_{i \star}^{-1} \bar{C}<1, \quad \text { i.e., } \quad \bar{s}_{i}>0 .{ }^{14}
$$

Therefore gross profits are positive only if coal mine owners supply more coal than they receive in their consumption basket $\bar{C}$. From a purely formal viewpoint, a 'Coal Exploitation Theorem' can be established, e.g. with regard to mine owners, too.

However, and this is a second, more important point in favor of using labor as the appropriate exploitation numéraire, gross profits from equation (9.34) are now defined with respect to mine owners, not workers' consumption. Hence, the equivalence between 'coal exploitation', and profits has quite a different economic content, since this notion of profit is not what Marx was - and capital theory is interested in. Therefore, we shall not consider the questions that the Commodity Exploitation Theorem may raise any further, and return to the original concept of the price rate of profit and the FMT.

\footnotetext{
$\overline{{ }^{14} \bar{F}>0 \rightarrow}(\bar{M}-\bar{C} \bar{L})^{-1} \geq 0 \rightarrow 0<\operatorname{det}\left(\bar{M}^{-1}(\bar{M}-\bar{C} \bar{L})\right)=\operatorname{det}(I-$ $\left.\left(\bar{M}^{-1} \bar{C}\right) \bar{L}\right)=\operatorname{det}\left(I-\left(\bar{M}^{-1} \bar{C}\right) e_{i}^{\prime}\right)=1-\bar{M}_{i \star}^{-1} \bar{C}$. See Murata $(1977,140)$ and the usual Hawkins-Simon conditions.
} 



\section{Joint Production in a (Marxian) System of National Accounts}

\subsection{Introduction}

As discussed in Chapter 5, in economies with joint production there is no one-to-one correspondence between sectors and commodities, and each industry may produce more than one good. Consequently, the non-diagonal entries of the output matrix are not zero and both the input and the output matrices are typically rectangular. This has some relevant conceptual and formal implications for price and value theory. For one, in models with joint production the standard employment multipliers of IO theory are well defined and meaningful, and they measure the changes in (sectoral and aggregate) employment resulting from variations in final demand, but unlike in simple Leontief economies, they do not necessarily measure the real total labor costs, or requirements, of producing commodities. As shown by Steedman (1977) in his celebrated book, in economies with joint production the standard employment multipliers may be negative (see Chapter 11 for a discussion), while real labor costs, or requirements, should arguably be definitionally nonnegative.

But given that certain commodities may be produced by more than one industry using different technologies, how can the joint outputs of each sector, or activity, be disentangled so that the real (labor) costs of producing a good in the economy can be computed? The (labor and non-labor) inputs used in a given sector produce a bundle of outputs: how much of each input, and especially labor, is used to produce one of the commodities in such bundle? There is no obvious, or natural way of disentangling inputs and outputs. In the previous chapter we have suggested a way of allocating labor costs when each industry produces a single output but multiple production techniques are used, based on the introduction of the distinction between individual and (average) labor values. This procedure allows us to reduce a rectangular production technology to a square Leontief system and Marxian value theory can 
still be analyzed based on merely physical, production-based quantities by applying the standard IO techniques to the 'average' technology $(\bar{A}, \bar{l})$. In this chapter, and the next, we aim to extend this intuition and provide a general definition of labor values - conceived of as the real labor costs of producing goods - based on IO theory and the actual accounting practices of capitalist firms.

In this chapter, in particular, we critically discuss the alternative techniques used in IO theory to disentangle outputs and reduce rectangular, joint production systems to standard square Leontief technologies.

The use of input-output tables in analysis depends on two basic assumptions. The homogeneity assumption requires that each sector produces a single output with a single input structure and that there is no automatic substitution between the outputs of different sectors. The proportionality assumption states that the inputs into each sector are a linear function only of the level of output of that sector, i.e. that the amount of each kind of input absorbed by any particular sector varies in direct proportion to that sector's total output (United Nations 1973, 20).

This quotation suggests that IO analysis should select and use the statistical data available to derive a final IO table in order to apply the standard Leontief model, despite the more general technological structures (multiple activities, multiple outputs) behind such tables. In this chapter, we raise some doubts on the interpretation of this procedure focusing on a specific, yet widely used application of IO tables, i.e. the determination of total labor costs, or requirements, both in money value and in physical terms. This task, however, requires some care:

In the pure theory of input-output the coefficients are regarded as relating to the physical quantities of commodities used in producing a given physical quantity of another commodity. In practice, however, almost all tables are prepared in money values. This is necessary because commodities are usually too heterogeneous to permit a purely physical measure. . It is important, however, that the coefficients in value terms should be interpreted in physical terms, and treated as if they were technical coefficients (United Nations 1973, 20).

This chapter shows that this interpretation cannot be justified, not only because of aggregation problems - which are not considered herebut also because of some deep mathematical problems which may be involved in the IO value-relationships. Indeed, although we focus on the issues raised by joint production, the key point holds in general: it is 
conceptually impossible to disentangle and conceptually separate price and quantity magnitudes, and it makes no sense to treat IO coefficients 'as if they were technological coefficients'.

In our approach, however, the application of the well-known formula for total labor costs based on monetary IO tables leads to magnitudes that can be sensibly interpreted in physical terms. To be specific, we argue that the appropriate way to disentangle the joint outputs of each sector or activity is by using the so-called "industry technology hypothesis" which splits up all inputs in proportion to the relative value of output in the output basket of the joint production activity. In the full-cost accounting techniques of business administration, this is called the "sales value method", since the relative proceeds of the items in a joint bundle determine the amount of joint inputs these single items have to bear.

The simple Leontief model is extended following Sraffa (1960), whose corresponding price equations are also used to derive the IO value-coefficients. There is one important conceptual difference to keep in mind, however. In the Sraffa-von Neumann tradition, joint production systems provide the theoretical framework to incorporate fixed capital: all machines whose life extends over more than one production period are treated as if they were a joint output of the production process. Loosely speaking, at the end of the production period industries are conceived of as producing a certain amount of output(s) and old (used) machines.

In this chapter, and in the next, we consider economies with 'pure' joint production, namely economies with circulating capital only and where more than one output is produced in at least one sector. In the Sraffa-von Neumann approach, natural (or production) prices require both the existence of perfect second-hand markets for fixed capital goods (conceived of as joint products of production activities) and the equalization of profit rates across sectors on circulating capital. This approach is theoretically rigorous and formally elegant but several of its assumptions have been questioned. First, the treatment of fixed capital as a joint product does not properly reflect the actual behavior of firms. As argued by Bródy (1970) in his stock-flow analysis, fixed capital is arguably not a jointly marketable product. Further, and related, the notion of the sectoral rates of profit implied by the Sraffian approach neglects replacement investment and, perhaps more importantly, it relates profits to the flow of material inputs rather than to the sectors' capital stock that ties up the money invested. In the light of these difficulties, below we focus on pure joint production; fixed capital is discussed in Chapters 8 and 13 and Section 11.8.1. 


\subsection{Joint production: The litmus test}

In this section we analyze the general joint production system considered in Sraffa (1960, Ch. VII). We consider a system of $m$ distinct processes, each of which turns out, in various proportions, the same $n$ products (some of which may have a zero coefficient): "An industry or production-process is consequently characterized no longer by the commodity which it produces, but by the proportions in which it uses and the proportions in which it produces, the various commodities" (Sraffa 1960, 45). For the sake of simplicity, and with no loss of generality, we first consider the case of a square system with $n=m$.

These proportions, and their absolute levels, are considered as given by Sraffa, "so that no question arises as to the variation or constancy of returns" (Sraffa 1960, v). Let $a_{i j}\left(b_{i j}\right)$ denote the total input (output) of commodity $i$ with respect to process $j$ and $l_{j}$ the corresponding input of direct labor. Let $r$ denote the uniform rate of profit on capital advanced, $w$ the uniform wage rate (paid ex post), and $p_{1}, \ldots, p_{n}$ the prices of the $n$ goods. These prices are related to $r$ and $w$ via the following equations:

$$
\begin{array}{ccc}
\left(a_{11} p_{1}+\ldots+a_{n 1} p_{n}\right)(1+r)+l_{1} w & = & 6_{11} p_{1}+\ldots+6_{n 1} p_{n} \\
\vdots & \vdots \\
\left(a_{1 n} p_{1}+\ldots+a_{n n} p_{n}\right)(1+r)+l_{n} w & = & 6_{1 n} p_{1}+\ldots+6_{n n} p_{n}
\end{array}
$$

or, in matrix notation

$$
p B=(1+r) p A+w l, \quad A=\left(a_{i j}\right), B=\left(b_{i j}\right), l=\left(l_{j}\right) .
$$

The assumption of square matrices $A, B$ allows Sraffa to avoid the use of inequalities in system (10.1). And with respect to prices $p$ he simply states that "only those methods of production are practicable which, in the conditions actually prevailing (i.e. at the given wage ...) do not involve other than positive prices" (Sraffa 1960, 44).

Because we aim to examine the definition of total labor costs with respect to a given IO table, the latter assumption need not concern us. We may simply assume such positive prices as given and use them as part of the data necessary for the construction of an IO table using the methodology of the UN's System of National Accounts (Stone 1968). The simple structure of Sraffa's price equations (10.1), the focus on joint production and the neglect of many other features of the economy (taxation, foreign trade, etc.) considerably simplify the presentation of the table to be derived. 
It is worth stressing again that we apply the price equations (10.1) only to the case with pure joint production and do not consider them appropriate to deal with fixed capital. The application of equation (10.1) to the case of fixed capital is a purely academic exercise because it contradicts firms' actual practices and assumes the existence of perfectly competitive resale markets for used machinery. There are indeed some markets where used machinery (for example, the heavy machinery used in construction) is leased, but it is not legitimate to assume this happens in all sectors. In most activities, bookkeeping and the valuation of used machinery is of an altogether different type (see also Chapter 7).

Moreover, the real distinction is not one between fixed and circulating capital based on a uniform period of production (which does not exist in reality), but - as discussed in detail in Bródy (1970) between capital advanced and capital consumed. This distinction applies to all commodities, because, for example, some wheat must be advanced in the production of bread. Physical inputs must be distinguished by their turnover times, which approximately separates intermediate inputs from durable inputs by being smaller or bigger than one.

Finally, an issue that has received insufficient attention is the level of disaggregation at which equations (10.1) should be applied, especially in the case with multiple activities of very old and very modern type. Should all used machineries within factories be evaluated by applying a uniform rate of profit, and the related prices of production, to the very large set of items that exist in reality? The pragmatic IO approach of Leontief (1941, 1953, 1986), Stone (1968), and Bródy (1970) based on industries - and not on millions of physical IO structures - is clearly preferable in that it maintains a firm empirical grounding. But this approach, too, can face some problems as shown below.

\subsection{Input-output methods: Which one to choose?}

One important motivation for the laborious construction of IO tables with a considerable degree of sectoral disaggregation was the calculation of the indirect employment effects associated with the expected change in activity levels for the U.S. economy at the end of World War II. The associated "total employment multipliers" were considered by Leontief $(1941,1944)$ in various editions of his book: The Structure of American Economy 1919-1939.

Later writers have adopted Leontief's procedure to construct indices of total labor productivity in a seemingly identical manner (see 
e.g. Evans (1953)). This new interpretation of the same formal measure strengthened the (still prevailing) view that employment multipliers are positive numbers under all economically sensible circumstances. Yet, as shown in Chapter 11, this conclusion is incorrect under joint production, and so the additional characterization of employment multipliers as indices of labor productivity is premature, because unlike the former, the latter must be positive by definition.

A notion which captures atemporal total changes in employment associated with definite changes in final demand need not also properly capture inter-temporal changes in labor productivity outside of the simple Leontief model. It is astonishing that IO analysts have long considered technologies with joint outputs as their methodological starting point, but have significantly underestimated the possibility that employment multipliers be negative.

Likely, this is because they have accepted the idea that IO data should be rearranged so as to allow for the application of the simple Leontief model - and the single interpretation of system labor requirements it offers. As a consequence, negative IO coefficients were declared to be "manifestly absurd" whenever they were observed (see e.g. United Nations $(1968,39)$ ), and no attempt was made to interpret the product by product labor-content calculations in the light of the product by sector accounts they were actually derived from.

This section offers such an interpretation for two of the four basic rules used to rearrange product by sector accounts into product by product IO accounts, i.e. for methods which transfer inputs and outputs between sectors. It is also shown that methods based on the transfer of outputs alone are not meaningful in general and thus should be discarded, even if the numerical difference with the theoretically sound methods is negligible, unless they are used explicitly as a numerical simplification. This may help to avoid surprises if the repeated application of atheoretical approximations suddenly leads to absurd results.

\subsubsection{A physical input-output example}

Consider the following physical data on input and output in Table 10.1. Certain standard row and column sums of the basic IO tabulation in Table 10.1 are missing because of the heterogeneity of products. In order to fill the gaps, the entries of Table 10.1 have to be recalculated in monetary terms. Using the (equilibrium) prices $p_{1}=1 / 3, p_{2}=1$ for products 1,2 and $w=1$ for labor, one obtains the system of monetary accounts in Table 10.2, where the input matrix $A$ is replaced by the absorption matrix $U$ and the (transpose of the) output matrix 
$B$ is replaced by the make matrix $V .{ }^{1}$ This is the basic schematic arrangement of monetary IO data of the UN's (1968) SNA, from which IO tables are derived. Four methods can be considered to derive a single IO, or Leontief, table from the above data.

\begin{tabular}{|c|c|c|c|c|}
\hline Products & Sectors & \multirow{2}{*}{$\frac{\text { Final Demand }}{8}$} & Totals & \multirow[b]{2}{*}{ Product 1} \\
\hline & 250 & & 33 & \\
\hline & $0 \quad 10$ & 7 & 17 & Product 2 \\
\hline $30 \quad 5$ & & & & Sector 1 \\
\hline $3 \quad 12$ & & & & Sector 2 \\
\hline & $\begin{array}{ll}5 & 1 \\
& \\
- & - \\
\end{array}$ & & & Primary Inputs \\
\hline $33 \quad 17$ & & & & Totals \\
\hline
\end{tabular}

Table 10.1: A joint production example economy

\subsubsection{Method 1: Industry coefficients}

In this case, neither outputs nor inputs are transferred between different sectoral accounts in order to obtain an ordinary Leontief table $Q .{ }^{2}$ Instead, in each sector products are regarded as if they were homogeneous to the characteristic product (i.e. the main product produced) of that sector. Therefore Table 10.2 becomes Table 10.3, which is now of the classical Leontief type. ${ }^{3}$ Input coefficients $Q_{i j}, u_{j}$ of intermediate products and labor can then be obtained in the usual way, by normalizing outputs to 'one', which gives

$$
Q=\left(\begin{array}{cc}
5 / 9 & 0 \\
0 & 10 / 13
\end{array}\right), \quad u=(1 / 3,1 / 13)
$$

${ }^{1}$ For a more formal definition of the matrices $U, V$ see Section 10.4 below.

${ }^{2}$ We use a different notation to denote the technology here in order to stress the differences with the standard Leontief matrix of single-output systems.

${ }^{3}$ In Table 10.3 final demand must be adjusted to reflect the hypothetical change in homogeneity assumed and note the same holds in other tables. 


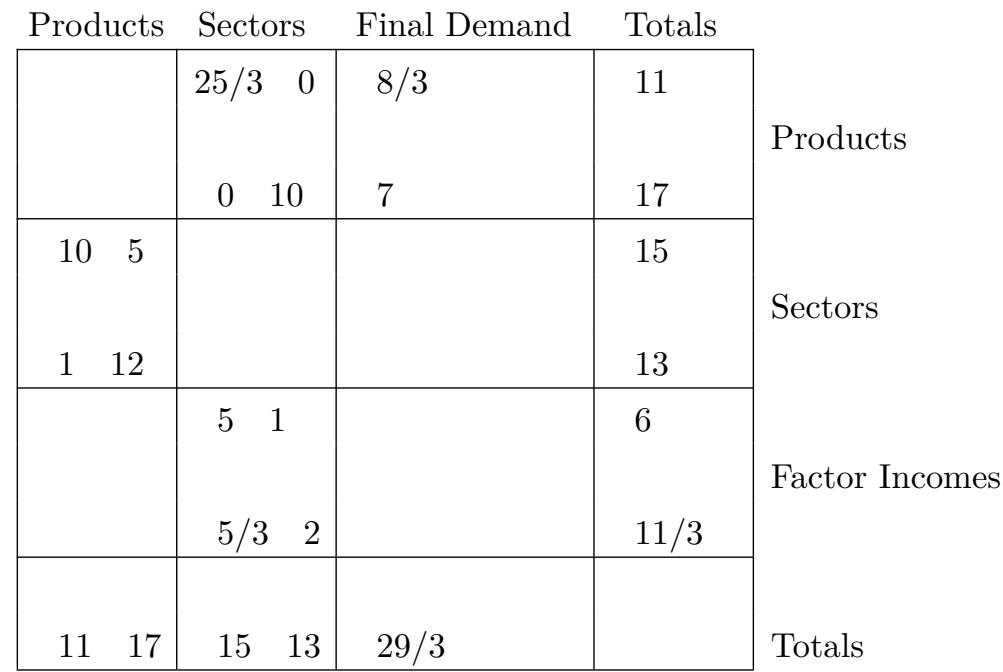

Table 10.2: The monetary equivalent of Table 10.1

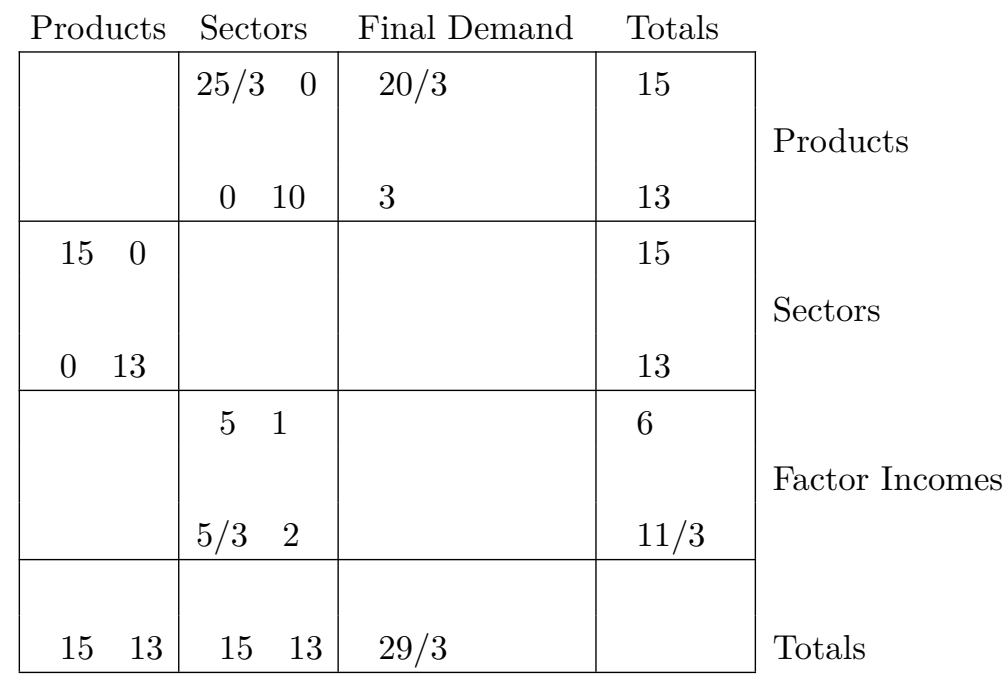

Table 10.3: An arbitrary homogeneity assumption

Note that $Q$ is identical with the matrix $S$ of industry coefficients considered in Sections 10.4-10.5 below. The Leontief structure $Q, u$ thus gives the unit-costs (per $\$$ of output value) structure of the various sectors $j$ regardless of the sector considered, and can be used 


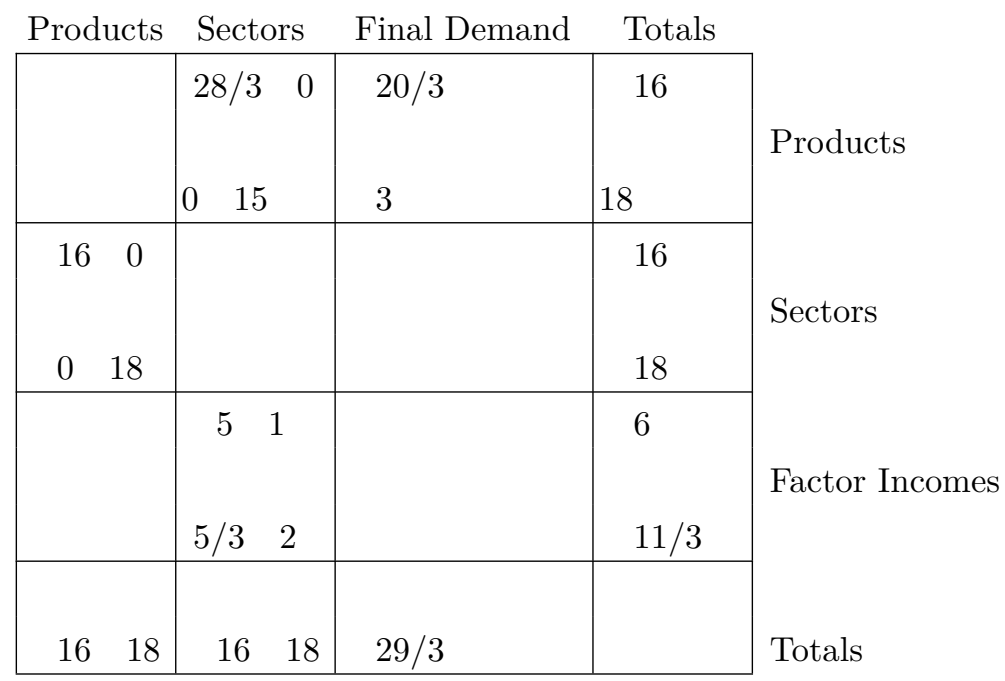

Table 10.4: An arbitrary sales transfer assumption

to calculate sectoral employment multipliers by means of the classical multiplier formula of single-product systems. In our example, this gives

$$
\begin{aligned}
z & =u(I-Q)^{-1} \\
& =(1 / 3,1 / 13)\left(\begin{array}{cc}
1-5 / 9 & 0 \\
0 & 1-10 / 13
\end{array}\right)^{-1} \\
& =(3 / 4,1 / 3) .
\end{aligned}
$$

The resulting total labor inputs $z_{i}$ per $\$$ of commodity sector $i$ thus seem perfectly normal. They imply that the index ' 1 ' is associated with the larger total employment effect.

One problem with this simple approach, however, is that it is not clear how to actually increase total employment in the economy. Should we stimulate the production of commodity 1 (irrespective of where it is produced) or should we stimulate sector 1 (irrespective of what this sector produces in the end)? That the above rearrangement of outputs is completely arbitrary can also be noted by considering an isolated (hypothetical) change of the price of good 1 from $p_{1}=1 / 3$ to $p_{1}^{\prime}=1$ : this leads to a change in the employment multipliers from $(3 / 4,1 / 3)$ to $(1 / 2,1 / 5)$.

We conclude that this method of calculating employment multipliers 
is not theoretically sound and cannot be used to deal with the product by sector difficulties of joint production.

\subsubsection{Method 2: The output method}

The output method (cf. United Nations $(1968,39)$ ) differs from Method 1 because it tries to remove the arbitrary homogeneity assumption underlying the use of industry coefficients $S_{i j}$ as entries of the Leontief matrix $Q_{i j}$. The output method can be described as follows: transfer all off-diagonal outputs to the diagonal by horizontal as well as by vertical displacement. Eliminate the double-counting of outputs by adding all off-diagonal elements of the make matrix $V$ to the corresponding elements of the absorption matrix $U$. This method does not change the value added in each sector, but, unlike method 1, it involves the transfer of outputs between different production accounts, and it depends on the assumption that the principal product of each sector can be unambiguously identified.

In the context of our example, in addition to Table 10.3, it is assumed that co-products are sold and thus transferred to the production accounts of the industry in which they are characteristic products (or where they are principally produced). This implies that such co-products now appear both as an input and as an output (of equal amount) of their characteristic industry. Thus, one may hope to overcome the lack of homogeneity involved in Table 10.3, since this method acknowledges that the output matrix of Table 10.3 is based on heterogeneous entities, which in a second step are then transferred (sold) to that sector which characteristically produces them.

An inspection of Table 10.3 shows that by this second step we have to add one (respectively, five) units of value to the input and output accounts of sector 1 (respectively, sector 2 ). The vector of total employment effects is now given by

$$
\begin{aligned}
z & =(5 / 16,1 / 18)\left(\begin{array}{cc}
(1-28 / 48) & 0 \\
0 & (1-5 / 6)
\end{array}\right)^{-1} \\
& =(5 / 6,1 / 3)
\end{aligned}
$$

Again this vector is subject to considerable changes if isolated changes in commodity prices occur. Further, since sector 1 now appears to be related to the production of commodity 1 only, ${ }^{4}$ it seems even more plausible that the production of commodity 1 be subsidized, which - as argued below-would lead to a reduction in total employment. The

\footnotetext{
${ }^{4}$ See, however, United Nations $(1968,39)$ for some interpretational doubts.
} 
output method thus only superficially corrects the arbitrariness of method 1 to calculate the employment multipliers associated with the joint production system of Table 10.1.

This analysis suggests that some of the inputs $U_{i j}$ must be transferred, along with outputs, in order to achieve better results, even though the simultaneous transfer of inputs is much more difficult than transferring joint outputs that appear as the off-diagonal elements in the make matrix $V$. This task is necessary to derive the theoretically appropriate measures from the monetary IO data of Table 10.2.

10.3.1.3 Method 3: The commodity-technology hypothesis

Neither method 1, where jointly produced goods are simply considered as homogeneous, nor its 'improvement' (method 2) which hypothetically assumes that co-products are 'sold' to their characteristic sector (which then sells them to final demanders), are suitable to analyze the total employment effects in joint production systems. Outputs cannot be simply separated from inputs in the way proposed by the output method if one aims to examine the labor requirements of commodities.

A possible alternative is given by the so-called commodity technology hypothesis $(\mathrm{CTH})$. This hypothesis assumes that there exists a uniquely determined unit-cost structure of commodities from which Table 10.2 is derived by means of (institutionally determined) product mixes based on constant returns to scale economies, see Section 10.4 below. Of course, this hypothesis cannot be true in the example considered in Table 10.1.

Let us nevertheless calculate the IO rearrangements and the employment multipliers according to the CTH. First, let $\alpha_{i k j}$ denote the input value of the $i$-th commodity per unit of output value of the $k$-th commodity when the latter is produced in the $j$-th industry. By definition, the total input $p_{i} A_{i j}$ of the $i$-th commodity in industry $j$ can then be expressed by:

$$
\sum_{k} p_{k} B_{k j} \alpha_{i k j}=p_{i} A_{i j}, \quad \text { all } \quad i, j .
$$

Formally, the CTH can be translated as assuming that for any $(i, k)$, $\alpha_{i k j}=\alpha_{i k}$ for all $j$, and thus $\alpha_{i k}$ is the uniquely determined cost which results from commodity $i$ as employed in the production of commodity $k$.

For the example in Table 10.2, we obtain: 


$$
\begin{aligned}
10 \alpha_{11}+5 \alpha_{12} & =25 / 3 \\
10 \alpha_{21}+5 \alpha_{22} & =0 \\
\alpha_{11}+12 \alpha_{12} & =0 \\
\alpha_{21}+12 \alpha_{22} & =10 .
\end{aligned}
$$

In matrix notation equations (10.3)-(10.6) read:

$$
\left(\begin{array}{ll}
\alpha_{11} & \alpha_{12} \\
\alpha_{21} & \alpha_{22}
\end{array}\right)\left(\begin{array}{cc}
10 & 1 \\
5 & 12
\end{array}\right)=\left(\begin{array}{cc}
25 / 3 & 0 \\
0 & 10
\end{array}\right)
$$

The IO table, or cost-structure, of commodity $\times$ commodity type is given by

$$
Q=\left(\begin{array}{ll}
\alpha_{11} & \alpha_{12} \\
\alpha_{21} & \alpha_{22}
\end{array}\right)=\left(\begin{array}{cc}
20 / 23 & -5 / 69 \\
-10 / 23 & 20 / 23
\end{array}\right)
$$

Wage costs to produce commodities 1 and 2 must be calculated in the same way:

$$
\begin{aligned}
10 \alpha_{01}+5 \alpha_{02} & =5 \\
\alpha_{01}+12 \alpha_{02} & =1 .
\end{aligned}
$$

Equations (10.7)-(10.8) imply $u=\left(\alpha_{01}, \alpha_{02}\right)=(11 / 23,1 / 23)$.

The IO table $Q$ is absurd due to its negative entries. The application of the classical Leontief-multiplier formula here gives

$$
\begin{aligned}
z & =u(I-Q)^{-1} \\
& =(11 / 23,1 / 23)\left(\begin{array}{cc}
9 & 5 \\
-30 & 9
\end{array}\right)=(-3,2) .
\end{aligned}
$$

This result emerges because equation (10.9) is just a mathematical transformation of the standard employment multiplier equations. To see this, re-write (10.9) in the form $z(E-Q)=u$ and post-multiply this equation by the transpose of the make matrix $V^{\prime}$. This leads to:

$$
(5,1)=z(I-Q)\left(\begin{array}{cc}
10 & 1 \\
5 & 12
\end{array}\right)=z\left[\left(\begin{array}{cc}
10 & 1 \\
5 & 12
\end{array}\right)-\left(\begin{array}{cc}
25 / 3 & 0 \\
0 & 10
\end{array}\right)\right]
$$

which is the original system of multiplier equations expressed in monetary terms. 
We conclude that the above procedure of deriving IO data $(Q, u)$ from which employment multipliers are then calculated in the classical Leontief way represents but a complicated detour in the application of the multiplier formula based on the inverse of $V^{\prime}-U$ (instead of $I-Q$ ). In order to apply this methodology, a virtual 'technology' assumption is necessary to derive matrix $Q$, which may have economically meaningless (from the viewpoint of IO theory) negative entries. Nevertheless, it is appropriate to interpret results in terms of employment multipliers (see the next section), which do not require any intermediate steps of doubtful technological content. Thus, Table 10.2 should not be replaced by the following "absurd" Table 10.5 of product $\times$ product type in the hopeless attempt to show the inputs of commodities into commodities as in the Leontief model. ${ }^{5}$

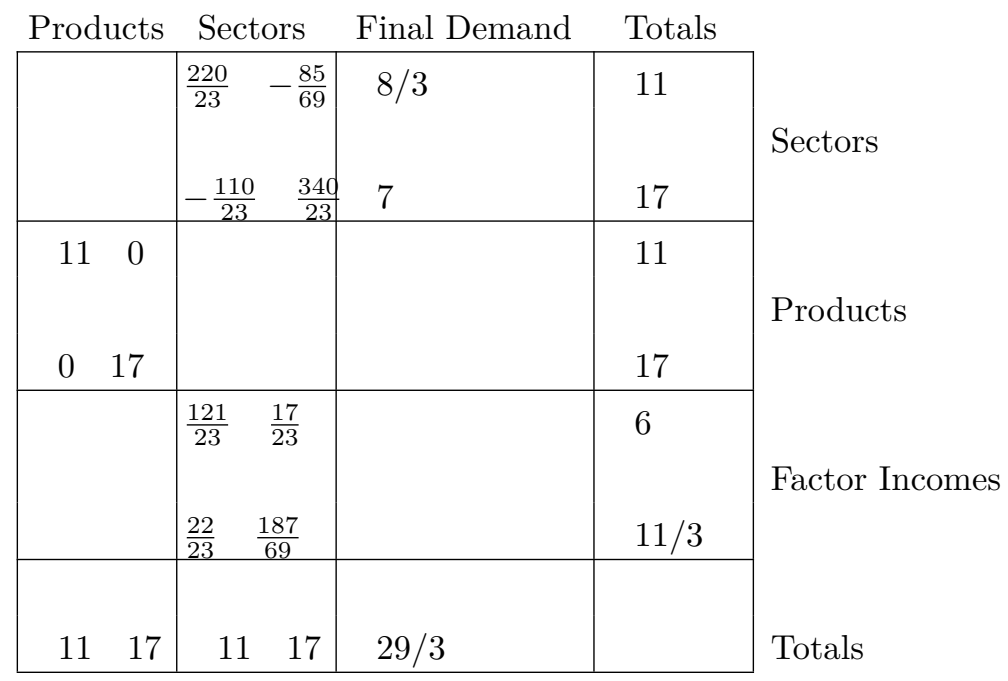

Table 10.5: Negative coefficients in single IO tables

10.3.1.4 Method 4: The industry-technology hypothesis

Another procedure designed to overcome the problems of the output method is based on the so-called industry-technology hypothesis (ITH), which assumes that the cost-structure of commodities is determined by their industry of origin, i.e. by the industry coefficients considered in method 1. Formally, this implies that for any $(i, j), \alpha_{i k j}=\alpha_{i j}$, for all $k$,

\footnotetext{
${ }^{5}$ Flaschel (1980) provides a thorough examination of the misconceptions
} underlying this treatment of the original IO data. 
and therefore the cost-structure of commodities is no longer uniquely determined if joint production is involved. Average cost-structures of commodities must be derived in order to obtain a standard IO table, and this is usually done using market shares as weights. This procedure leads to IO data $(Q, u)$, which by the conventional Leontief-multiplier formula just measures the indices of labor productivity $z \approx(0.72,0.59)$. This is the vector of labor values per unit of output value and not per unit of product (with $p=(1 / 3,1)$ ).

We conclude our analysis of the IO procedures to overcome the problems of joint production by applying the ITH to Table 10.2 in order to transfer co-products to their characteristic industry, as shown in Table 10.6.

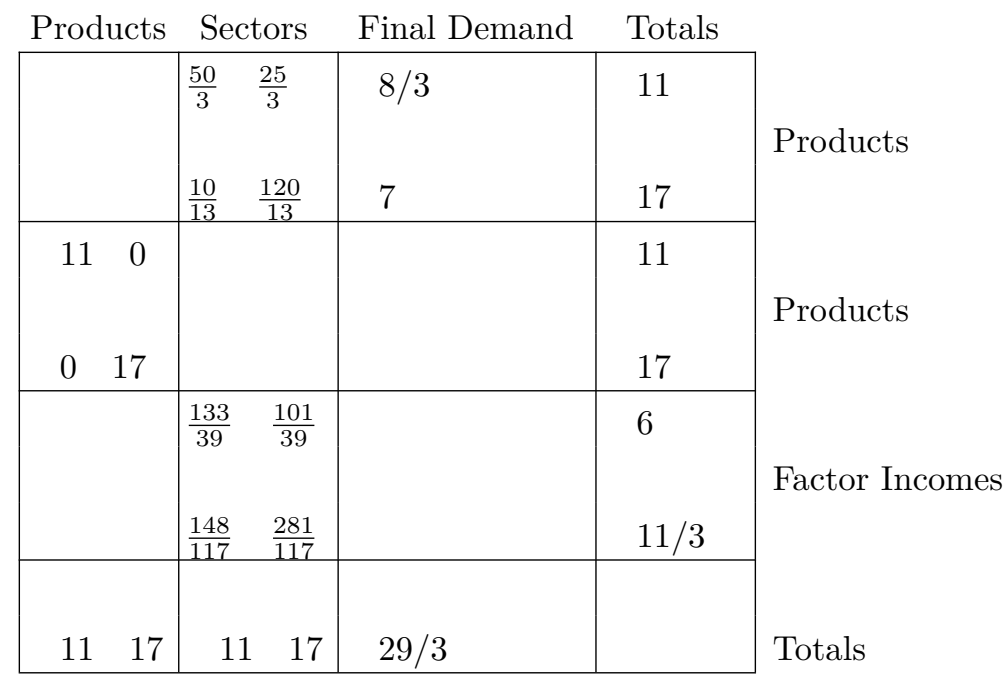

Table 10.6: A method that rules out negative coefficients in single IO tables

Table 10.6 confirms that no "absurd" IO coefficients are possible if the ITH is used, even though this is not due to any property of the given technology as the name 'industry technology hypothesis' might misleadingly suggest. In other words, the matrix of industry coefficients $S$ should not be considered as a final IO table because of the arbitrary homogenization of jointly produced goods. The matrix $S=\left(p_{i} A_{i j} / p B_{\star j}\right)$ is but an intermediate step in the derivation of the IO table of method 4 (where $Q=S T$, see the next section). The strategy of manipulating only outputs (to establish some sort of homogeneity) does not lead to meaningful measures of labor-requirements in the presence 
of joint products. Instead, the two methods of transferring outputs and inputs lead to sensible measures: the method based on the CTH correctly measures the employment effects associated with final demand changes and constant technical conditions. The method based on the ITH captures productivity effects associated with technical changes. The analysis in this section shows that once joint production is taken into consideration the two sets of indices differ and different methods for the derivation of the IO table $Q$ should be employed depending on the purpose of the analysis.

Further, only after one specifies whether the aim is to measure employment multipliers, or indices of labor productivity, can one analyze whether, e.g. the output method-albeit theoretically doubtful - may serve as a numerical approximation to the type of labor requirements to be measured. Finally, it should be noted that methods 1,2 , and 4 cannot be used to analyze supply bottlenecks deriving from joint production, since these methods remove all rigidities due to joint production accounts.

In sum, there are only two theoretically sound methods: the CTH approach and the ITH approach. They are analyzed in the next section in a general model with $n$ commodities and $m$ sectors. However, as argued above, method 3 is in fact redundant, since whatever insight it provides - in the calculation of employment multipliers - can also be obtained by using the make and absorption matrices directly. It is therefore redundant to transform these two matrices into a single IO table, which has no meaningful interpretation, because the assumption of a single cost structure behind the rectangular input and output matrices is in general wrong. Only the ITH approach is economically meaningful in the case of pure joint production, because it mirrors what firms (should) do at the micro-level, namely use their relative benefit, or sales value method for disentangling joint costs in their cost accounting. If the given situation is one of pure joint productionand not just a mixture of single activities into an hypothetical bundle of activities - the ITH is the appropriate choice in that it avoids an empiricist, atheoretical approach.

\subsection{The 'Commodity Technology' and 'Industry Technology' Hypotheses}

Consider a general model with $n$ commodities and $m$ sectors, with $n \leq m$, characterized by pure joint production expressed in price $\times$ quantity terms: 


$$
\begin{aligned}
V^{\prime}= & \left(p_{i} B_{i j}\right), \text { the }(\text { transposed }) \text { make matrix based on } \\
& \text { the physical outputs } B_{i j} \text { of commodities } i \text { by process } j \\
& (i=1, \ldots, n, \quad j=1, \ldots, m), \\
U= & \left(p_{i} A_{i j}\right), \text { the absorption matrix based on the physical inputs } \\
& A_{i j} \text { of commodities } i \text { into process } j .
\end{aligned}
$$

In this system the distribution of zeros and positive entries may be quite arbitrary. ${ }^{6}$

Let $\alpha_{i k j}$ be the input value of the $i$-th commodity per unit of output value of the $k$-th commodity when the latter is produced in the $j$-th industry. By definition the total input $p_{i} A_{i j}$ of the $i$-th commodity in industry $j$ can then be expressed as:

$$
\sum_{k} p_{k} B_{k j} \alpha_{i k j}=p_{i} A_{i j}, \quad \text { all } \quad i, j .
$$

Note that the coefficients $\alpha_{i k j}$ are not among the initial data $U, V$, but have to be derived by means of further assumptions or conventions on these matrices.

Let us consider the CTH first. This hypothesis assumes that there is a unique input vector per $\$$ of output value for each good $i$ (independent of the producing industry $j$ ). The value $\alpha_{i k j}$ can then be reduced to $\alpha_{i k}$ and the set of values $\alpha_{i k}, i, k=1, \ldots, n$, describes the input structure of commodities, as desired. Equation (10.11) in this case implies:

$$
\sum_{k}\left(p_{k} B_{k j} / p B_{\star j}\right) \alpha_{i k}=p_{i} A_{i j} / p B_{\star j},
$$

where $p B_{\star j}=\sum_{i} p_{i} B_{i j}$. The right-hand side of (10.12) describes (normalized) 'industry coefficients' $S_{i j}=\left(p_{i} A_{i j} / p B_{\star j}\right)$ and the bracket on the left-hand side just gives the coefficients of 'commodity mixes' $M_{k j}=\left(p_{k} B_{k j} / p B_{\star j}\right)$. Since both of these coefficient structures represent mere derivatives of the given matrices $U, V$, equation (10.12) - given in matrix notation by $\left(\alpha_{i k}\right) M=S$-can be used to determine the assumed, but still unknown input structure $Q=\left(\alpha_{i k}\right)$ of commodities $i$ into commodities $k$, in the case of square matrices $U, V$ and an invertible matrix $M$ :

$$
Q=\left(\alpha_{i k}\right)=S M^{-1} .
$$

\footnotetext{
${ }^{6}$ See Sraffa (1960) of this structure and Cressy (1976) on the uses of matrices $U, V$.
} 
Under these common (Sraffa 1960, s.VII) assumptions, a unique set of coefficients $\alpha_{i k}$ emerge from the CTH.

But it is unclear that the CTH produces a sensible IO structure in this economy. For there is no technologically (or otherwise) determined input structure of commodities different and independent from the input structures of industries in a system of pure joint production. In general, post-multiplication of $S$ by $M^{-1}$-which is closely related to the inverse of the output matrix $B$ - does not lead to any sensible input structures of commodities, and yields a number of negative, and thus economically meaningless entries. As argued in the previous section, these (meaningless) negative entries are associated with (meaningful) negative employment multipliers (see also Steedman (1977, Ch. 11) and Chapter 11). In these cases, the CTH is an unnecessary detour in the derivation of the employment multipliers of a given input and output structure, which can be directly obtained by investigating the matrix $(B-A)^{-1}$ and applying it to the vector of direct labor inputs.

Things are different if the ITH is employed. In the ITH, it is assumed that the input vector per $\$$ of output value of industry $j$ is independent of the specific product from which this dollar has been obtained. Hence, input amounts of commodities are always determined solely by the unit cost structure of their industry of origin. Formally:

$$
\alpha_{i k j}=\alpha_{i j}=p_{i} A_{i j} / p B_{\star j}=S_{i j},
$$

which is only a reformulation of the definitional equation (10.11). In contrast to the $\mathrm{CTH}$, therefore, no additional information can be drawn from equation (10.11) in connection with the ITH. This is the reason why critics argue that the ITH "in the context of the input-output model leaves the form of the industry-into-commodity transformation matrix entirely undetermined" (Cressy 1976, 117).

This claim is not convincing, though: neither the CTH nor the ITH should be analyzed abstracting from the overall IO methodology, according to which "the input coefficients of an aggregated IO sector are weighted sums of the constituent coefficients of the original sectors, the weights being the relative size of each sector's output" (United Nations 1968, III). In the economies considered here, these weights are given by

$$
T_{j k}=B_{k j} / \sum_{j} B_{k j}, \quad T=\left(T_{j k}\right) .
$$

Both the CTH and the ITH are only aimed at disentangling subsidiary production, i.e. at transferring outputs and inputs so that only one commodity output is attached to each input vector. It is an 
advantage of the CTH that, by assuming a unique input structure per commodity, it makes equation (10.15) redundant. In contrast, if the ITH is adopted, a situation of multiple 'activities' per commodity sector necessarily follows from the existence of subsidiary products, i.e. equation (10.15) is meaningful and must be used. In this case, equations (10.14)-(10.15) can be used to derive the desired matrix $Q$ :

$$
\alpha_{i k}=\sum_{j} T_{j k} \alpha_{i j} \text {, all } i, k \text {, i.e. } Q=\left(\alpha_{i k}\right)=\left(\alpha_{i j}\right) T=S T \text {. }
$$

The transformation matrix (now denoted as $T$ instead of $M^{-1}$ ) is thus uniquely determined, which dispels criticisms (e.g. Cressy (1976, 115)) and confirms the result obtained in United Nations (1968, 3.86), although admittedly not by means of the ITH alone.

It is important to emphasize, however, that disentangling joint products by help of the ITH is only an intermediate step, leading to the rectangular IO structure $S$. The ITH cannot therefore be used alone, for example, to determine prices. ${ }^{7}$

Finally, it is worth noting that the ITH can be applied for any $n$ and $m$ with $n \leq m$. In any case, the matrix $S$ of industry coefficients post-multiplied by the matrix $T$ of market shares leads to a nonnegative square IO table of commodity $\times$ commodity type, which is thus not based on facts of 'technology' alone. Indeed, there exist no technological facts that alone can be used to disentangle subsidiary production under pure joint production.

The next sections show that the ITH is indispensable in any calculation of the total labor costs of commodities and the related labor productivity indices. In fact, it is necessary to disentangle joint products by an appropriate accounting method and to deal with the resulting multiple activities, instead of using inverted net output matrices as in the calculation of employment multipliers. ${ }^{8}$

\footnotetext{
7 As is done, for example, in Cressy (1976). In contrast, using equation (10.15) and focusing on the resulting IO table $Q$ of type $n \times m$, one obtains perfectly reasonable price equations.

8 Following Sraffa (1960), we have started this chapter analyzing square input and output matrices (or square solutions of rectangular von Neumann models, see Chapter 5 above). In the case of general rectangular make and absorption matrices the Moore/Penrose inverse must be used instead to calculate employment multipliers, see e.g. Shinozaki, Sibuya, and Tanabe (1972).
} 
10.5 The determination of labor contents via U.N. IO tables

Table 10.7 presents the IO data in the customary form (see United Nations (1968, 48ff); United Nations (1973, 24)). The notation in Table 10.7 is standard. The price equations (10.2) can equivalently be expressed by

$$
(1+r) e^{\prime} U+y^{\prime}=e^{\prime} V^{\prime}=(V e)^{\prime} \quad \text { or } \quad e^{\prime} U+y^{\prime}+z^{\prime}=g^{\prime},
$$

which gives the central accounting identity of the above SNA interpreted in terms of Sraffa's (1960) two-class economy, as shown in equation (10.1).

\begin{tabular}{|c|c|c|c|c|}
\hline & Goods & Industries & Final demand & Totals \\
\hline Goods & & $\begin{array}{l}U=\hat{p} A \\
U_{i j}=p_{i} a_{i j}\end{array}$ & $\begin{aligned} f & =V^{\prime} e-U e \\
& =\hat{p}(B-A) e\end{aligned}$ & $q=V^{\prime} e$ \\
\hline Industries & $\begin{array}{l}V=(\hat{p} B)^{\prime} \\
V_{j i}=p_{i} b_{i j}\end{array}$ & & & $\begin{aligned} g & =V e \\
& =(p B)^{\prime}\end{aligned}$ \\
\hline $\begin{array}{l}\text { Factor } \\
\text { incomes }\end{array}$ & & $\begin{array}{l}y^{\prime}=w l \\
z^{\prime}=\operatorname{rp} A\end{array}$ & & $\eta=\ldots$ \\
\hline Totals & $\begin{aligned} q^{\prime} & =e^{\prime} V \\
& =(\hat{p} B e)^{\prime}\end{aligned}$ & $g^{\prime}$ & $\eta=p(B-A) e$ & \\
\hline
\end{tabular}

Table 10.7: The theoretical IO framework of the SNA

This identity contains the basic structural data of IO tabulations, namely the absorption matrix $U$ of commodity $\times$ industry type, the make matrix $V$ of industry $\times$ commodity type and the vector of sectoral wage incomes $y^{\prime}$. Horizontal and vertical summations with respect to $V$ define output values of industries and commodities, respectively, from which capital incomes and final demands can be calculated as residuals.

To determine the effects of an increase in final demand on total labor requirements, it is usually considered necessary to calculate a square system of IO coefficients of commodity $\times$ commodity type. Otherwise it is not possible to obtain a meaningful chain of indirect effects. The result of such a calculation has been presented above in the form of the IO matrix $Q$ and the vector of direct labor requirements $u^{\prime}$. There are several ways of arriving at such a table $Q$, each of which starts from certain 'technology' assumptions about the input structure of the $n$ commodities. Yet, for the technology considered, only one method satisfies the following reasonable criteria: 
(a) The IO structure derived should be nonnegative under all economically reasonable circumstances.

(b) The method adopted should allow for the determination of IO tables and of total labor requirements of commodities with a rectangular make matrix $V$.

While the first criterion is indispensable if an input structure of commodities is assumed to exist, the second can be justified with regard to actual commodity classification problems (cf. United Nations $(1973,37))$. Further, in a general joint production model such as the one underlying equations (10.1), only one piece of information on the input structure of commodities - namely their industry of origin - can be reasonably assumed to be available in general.

It is easy to derive an IO table which connects the final demand for commodities $f$ with the levels of output necessary to meet it. For this purpose define the following matrices:

(a) The matrix of industry coefficients: $S=U \hat{g}^{-1}=\hat{p} A \widehat{p B}^{-1}$, which represents the value of inputs of industries per $\$$ of output value.

(b) The matrix of market shares: $T=V \hat{q}^{-1}=B^{\prime} \hat{p}(\hat{p} \widehat{B e})^{-1}=B^{\prime} \widehat{B e}^{-1}$, which consists of purely physical coefficients $T_{j i}=b_{i j} / \sum_{j} b_{i j}$.

Using the expressions in the first two rows of Table 10.7 and the definitions of $S$ and $T$ :

$$
\begin{aligned}
q & =U e+f=S \hat{g} e+f=S g+f=S V e+f=S T \hat{q} e+f \\
& =S T q+f=Q q+f \text { or } \\
q & =(I-Q)^{-1} f=(I-S T)^{-1} f .
\end{aligned}
$$

This is the IO model of commodity $\times$ commodity type based on the ITH (United Nations 1968, 1973; Armstrong 1975) that establishes the desired link between gross and net output of commodities.

In order to clarify the transformations involved, we examine the ITH in more detail. The ITH asserts that each $\$$ of output value of, say, industry $j$ has the same input structure, irrespective of the type of commodity for which this $\$$ stands. Hence, the average input structure for commodity $i: Q_{\star i}$, per $\$$ of output value, is given by the sum of industry coefficients $S_{\star j}, j=1, \ldots, m$, each weighted by the market share of industry $j$. Formally, for all goods $i$,

$$
Q_{\star i}=T_{1 i} S_{\star 1}+\ldots+T_{m i} S_{\star m}, T_{1 i}+\ldots+T_{m i}=1 .
$$

The resulting (average) input structure $Q$ (per $\$$ of output value) of 
commodities, and the associated vector of wage costs $u^{\prime}$, are therefore given by

$$
Q=S T=U \hat{g}^{-1} V \hat{q}^{-1}, u^{\prime}=y^{\prime} \hat{g}^{-1} V \hat{q}^{-1} .
$$

This is exactly the IO structure used above, which exploits the available information on commodity inputs, and represents the final form of IO data for various types of ex post calculations and for planning procedures based on projections.

Having made the necessary transfers of outputs and inputs to arrive at $\left(Q, u^{\prime}\right)$, total wage costs $z$ per $\$$ of commodity output can be measured by the standard formula (United Nations 1973, 103f):

$$
z^{\prime}=z^{\prime} Q+u^{\prime}=u^{\prime}(I-Q)^{-1} .
$$

Dividing direct and total wage costs: $(u, z)$ by wages $w$ leads to an analogous system:

$$
Z^{\prime}=z^{\prime} / w=\left(z^{\prime} / w\right) Q+u^{\prime} / w=\left(u^{\prime} / w\right)(I-Q)^{-1} .
$$

The solution $Z$ of equation (10.20) is often interpreted as describing (physical) labor, or manpower, requirements per unit of output value, i.e. the desired measure.

Expressing equation (10.20) in terms of the original data leads to

$$
Z^{\prime}=l^{\prime} \widehat{p B}^{-1} B^{\prime} \widehat{B e}^{-1}\left(I-\hat{p} A \widehat{p B}^{-1} B^{\prime} \widehat{B e}\right)^{-1} .
$$

But it is not obvious from this expression that the coefficients $Z_{j}$ have the content usually ascribed to them. If $B=I$, then $Z=l \hat{p}^{-1}(I-$ $\left.\left.\hat{p} A \hat{p}^{-1}\right)^{-1}\right)=l(I-A)^{-1} \hat{p}^{-1}$, which indeed gives a physical expression of labor requirements per $\$$ of output value. In the literature, the same interpretation is extended to the formally identical equations (10.20)(10.21) for joint production systems, based on the assumptions quoted in the introduction to this chapter. The question arises, then, whether these assumptions are indeed reasonable. Let us examine further the IO table

$$
Q=\hat{p} A \widehat{p B}^{-1} B^{\prime} \widehat{B e}^{-1}=S T .
$$

Given price equations (10.1) and the effects of a changing wage rate $w$ on such prices $p$ (see Sraffa (1960, Ch. III)), it should be obvious that $Q$ is not constant with respect to changes in the distribution of income, even if the technology is constant, and so it cannot be meaningfully treated as a purely physical magnitude. Moreover, a change in final demand leads both to changes in the input structures $Q_{\star i}$ and to a 
different set of input vectors $q_{i}$ per $\$$ of output value of commodities $i$, even under the favorable conditions of a fixed coefficient/constant returns technology $A, B, l$ (and no changes in prices $p$ ), which raises further doubts on the interpretation of equations (10.20)-(10.21) as providing the system labor requirements in physical terms per unit of output value.

To see this, note that the impact of changes in the vector of activity levels $x=\left(x_{1}, \ldots, x_{k}\right)^{\prime}$ on $A, B$ can be expressed by $A \hat{x}, B \hat{x}$, respectively. Applied to the definition of $Q$ this gives

$$
\begin{aligned}
Q & =\hat{p} A \hat{x} \widehat{p B} \hat{x}^{-1}(B \hat{x})^{\prime}(\widehat{B \hat{x} e})^{-1} \\
& =\hat{p} A \hat{x} \hat{x}^{-1} \widehat{p B}^{-1} \hat{x} B^{\prime} \widehat{B x}^{-1}=\hat{p} A \widehat{p B}^{-1} \hat{x} B^{\prime} \widehat{B x}^{-1}
\end{aligned}
$$

which is clearly dependent on the activity levels $x$.

As a result, both the idea of treating "coefficients $Q_{i j}$ as if they were technical coefficients" and the proportionality assumption (see the opening quote of this chapter) seem deceptive. The homogeneity assumption also seems dubious, for equation (10.17) implies that inputs into commodities have in fact been homogenized by use of average considerations, and so they, too, depend on (changing) market shares.

Although an assumption of constant technical conditions is not very convincing with respect to the IO table $Q$, there is no reason to believe that "failure to meet these requirements can lead to inaccurate results" in the analysis of physically interpretable measures of total labor costs in general joint production systems. To show this, a new definition of total labor requirements based on the complete set of data given by equation (10.1) is introduced in the next section, which should clarify what is actually measured by equations (10.19)-(10.20).

\subsection{A 'physical' approach to the determination of the labor contents of commodities}

In order to determine physically-based labor costs in pure joint production systems, both technological and economic considerations must be taken into account. Indeed, there is a long history of attempts to deal with joint production by means of economic imputations. These attempts have analyzed the problem of managerial cost accounting, and have established a widely accepted and general principle: the allocation of joint costs by means of the relative benefit received from the unit of costing. This is the so-called sales value method of cost accounting applied by firms. In the presence of joint products, this rule reverses the 
relationship between input costs and output prices in that sales ratios in the outputs of firms are used to determine costs, and not vice versa.

In the previous section, we employed a related - in fact, in principle, analogous - principle in the form of the ITH, interpreted as an assumption about technology, to transform the cost structures of the given micro-units by means of macro-coefficients: the market shares (instead of the above mentioned product mix coefficients of firms) to desired IO coefficients $Q_{i j}$. This section shows that the above principle of cost allocation, i.e. the sales value method, combined with the technological relationships that determine total labor requirements in the customary way, suffices to introduce a unique definition of such labor requirements. The issue of the measurement of physically-based labor costs is solved by means of a single new allocation rule rooted in actual managerial cost accounting.

To apply this rule of cost allocation to our economies, note that the relative revenue associated with the unit of costing, e.g. from the $i$-th commodity, is given by

$$
M_{i j}=p_{i} \sigma_{i j} / p B_{\star j}=p_{i} \sigma_{i j} / \sum_{i} p_{i} \sigma_{i j},
$$

with respect to industry $j$. The rule of the sales value method states that $M_{i j}$ of the inputs $A_{\star j}, l_{j}$ in the production of total output $B_{\star j}$ must be allocated to the output $b_{i j}$ of commodity $i$ as part of the joint output basket $B_{\star j}$ :

$$
M_{i j} A_{\star j}, M_{i j} l_{j} \longrightarrow b_{i j}
$$

There is another peculiarity that must be considered in order to derive the equations that determine total labor costs. If some activities display joint products, equation (10.23) results in multiple activities for at least one commodity sector $i$. In sector $i$, the (still unknown) average labor costs $v_{i}$ of commodities $i$ and the labor costs can be written as:

$$
M_{i j}\left(v_{1} a_{1 j}+\ldots+v_{k} a_{k j}\right), M_{i j} l_{j},
$$

and must be regarded as specific to the production of commodity $i$ by process $j$. Hence, they give rise to individual total labor costs $v_{i}(j)$ per unit of product only, i.e.:

$$
M_{i j}\left(v_{1} a_{1 j}+\ldots+v_{k} a_{k j}+l_{j}\right)=v_{i}(j) b_{i j} .
$$

Average total labor costs can then be determined by employing the 
matrix of market shares $T$ described above (whose application can be justified with the same arguments as for equation (10.17) in the previous section):

$$
v_{i}=T_{1 i} v_{i}(1)+\ldots+T_{k i} v_{i}(k) .
$$

Proposition 10.1 shows that equations (10.22), (10.24), and (10.25) suffice to determine total labor costs in a unique and economically meaningful way, which is related to the definition of labor costs in the previous section.

Proposition 10.1. Let $\bar{A} \equiv A M^{\prime} \widehat{B e}^{-1}$ and $\bar{l} \equiv l^{\prime} M^{\prime} \widehat{B e}^{-1}$. Suppose $p>0$ and $w>0$.

(1) The vector $v=\left(v_{1}, \ldots, v_{k}\right)$ of average total labor requirements in (10.25) solves the following (square) system of Leontief equations:

$$
v=v \bar{A}+\bar{l} \text {. }
$$

(2) We have $Q=\hat{p} \bar{A} \hat{p}^{-1}$ and $u^{\prime} / w=\bar{l} \hat{p}^{-1}$.

(3) If $e^{\prime} U<g^{\prime}$, there exists a unique and strictly positive solution to (10.26).

Proof. 1. Summing equations (10.24) with respect to $j$ gives

$$
\sum_{j} M_{i j}\left(v_{1} a_{1 j}+\ldots+v_{k} a_{k j}+l_{j}\right)=\sum_{j} v_{i}(j) b_{i j} .
$$

Equations (10.25) and $T_{j i}=6_{i j} / \sum_{j} b_{i j}$ imply $\sum_{j} v_{i}(j) b_{i j}=v_{i} \sum_{j} b_{i j}=$ $v_{i}(\widehat{B e})_{i i}$, while the left-hand side of the above equation can be expressed as

$$
\sum_{j} M_{i j}\left(v A_{\star j}+l_{j}\right)=\sum_{j}\left(v\left(M_{i j} A_{\star j}\right)+M_{i j} l_{j}\right)=v A M_{i \star}^{\prime}+l M_{i \star}^{\prime} .
$$

Thus, since $v A M_{i \star}^{\prime}+l M_{i \star}^{\prime}=v_{i}(\widehat{B e})_{i i}$, for all $i=1, \ldots, n$, we obtain the desired result:

$$
v A M^{\prime}+l M^{\prime}=v \widehat{B e} .
$$

2. Note that $M^{\prime}=\hat{g}^{-1} V$. Then

$$
\begin{aligned}
\hat{p} \bar{A} \hat{p}^{-1} & =\hat{p} A M^{\prime} \widehat{B e}^{-1} \hat{p}^{-1}=\hat{p} A M^{\prime}(\hat{p} \widehat{B e})^{-1} \\
& =\hat{p} A M^{\prime} \hat{q}^{-1}=\hat{p} A \hat{g}^{-1} V \hat{q}^{-1} \\
& =\hat{p} A \hat{g}^{-1} T=\hat{p} A \widehat{p B}^{-1} T=S T=Q .
\end{aligned}
$$


Similarly: $\bar{l} \hat{p}^{-1}=l M^{\prime} \widehat{B e}^{-1} \hat{p}^{-1}=l M^{\prime} \hat{q}^{-1}=l \hat{g}^{-1}=u^{\prime} / w$.

3. The matrix $Q=S T=U \hat{g}^{-1} T$ is nonnegative and it satisfies:

$$
e^{\prime} Q=e^{\prime} U \hat{g}^{-1} T<g^{\prime} \hat{g}^{-1} T=e^{\prime} T=e^{\prime} B^{\prime} \widehat{B e}^{-1}=(B e)^{\prime} \widehat{B e}^{-1}=e^{\prime},
$$

because by assumption $e^{\prime} U<g^{\prime}$, and $T=e^{\prime} B^{\prime} \widehat{B e}^{-1}$. Hence, the matrix $I-Q$ is nonnegatively invertible (Lancaster 1968), which proves that $z$ in equation (10.19) and therefore $Z$ in equation (10.20) and $v$ in equation (10.26) are uniquely determined and positive.

In other words, the physical input columns $A_{\star j}$ and the direct labor inputs $l_{j}$ can be rearranged by help of $M_{i j}$ (see equation (10.23)) and by simple weighted summations, to yield a square IO matrix $\bar{A}$ and a vector of labor inputs $\bar{l}$, which define the physical background for the IO table $Q$ and the vector $u^{\prime} / w$, and for total labor requirements $Z$ (see (10.26)). In fact, by part (2) the solution $Z$ of (10.20) is identical to $v^{\prime} \hat{p}^{-1}=\left(v_{1} / p_{1}, \ldots, v_{k} / p_{k}\right)$, which is the vector of physical labor requirements per $\$$ of corresponding outputs. Part (3) proves that equations (10.26), and therefore (10.20), have a unique, economically meaningful solution provided value added is positive in each industry. In sum, the seemingly complicated expression (10.21) for $Z$ has a clear and uniquely determined physical content.

However, Proposition 10.1 shows that it makes no sense to claim 'constant technical conditions' for the derived IO table $Q$. For price $\times$ quantity aggregates such as $M_{i j}$ are involved in its construction, a fact which is not obvious when looking at the original transformation of industry coefficients $S$ by means of physical ratios $T_{i j}$ on the basis of the ITH. The lack of suitable constant technical conditions behind $Q$ and the inclusion of an economic imputation in the derivation of total labor requirements $v$ raises serious doubts on the use of these coefficients for the determination of employment effects. It is not really constant technology, but indeed the analysis of a changing technology to which the above physically based cost coefficients should be best suited, if the relationship to managerial cost-accounting is taken into account.

\subsection{Conclusions}

Economies with joint production raise some difficult formal and conceptual issues for IO theory. The basic conceptual apparatus of IO analysis is based on the reduction of production technologies to the standard square Leontief system, with one input produced by each 
sector, or activity. If, however, certain commodities may be produced by more than one industry using different technologies, it is not clear how to disentangle the joint outputs of each sector, or activity, in order to obtain a square single-output system. The (labor and non-labor) inputs used in a given sector produce a bundle of outputs: how much of each input, and especially labor, is used to produce one of the commodities in such bundle? There is no obvious, or natural way of disentangling inputs and outputs.

In this chapter, we have critically discussed the alternative techniques used in IO theory to disentangle outputs and reduce rectangular, joint production systems to standard square Leontief technologies. To be specific, we have argued that, the appropriate way to disentangle the joint outputs of each sector or activity is by using the so-called "industry technology hypothesis" which splits up all inputs in proportion to the relative value of output in the output basket of the joint production activity. In the full-cost accounting techniques of business administration, this is called the "sales value method", since the relative proceeds of the items in a joint bundle then determine the amount of joint inputs these single items have to bear.

Our discussion has focused in general on IO theory and thus it may seem somewhat removed from the classical-Marxian themes of this book. Nonetheless, the issues raised in this chapter have important implications for Marxian theory and the definition of labor values. As we have shown in this chapter, for example, if inputs and outputs are disentangled using the "industry technology hypothesis", the application of the well-known formula for total labor costs based on monetary IO tables leads to magnitudes that can be sensibly interpreted in physical terms.

In the next chapter, we build on this intuition, and on the distinction between individual and (average) labor values, in order to provide a general definition of labor values conceived of as the real total labor costs, or requirements, of producing commodities. This definition is logically consistent, theoretically sound and empirically relevant, and it solves some of the issues raised in the literature in economies with joint production. 


\section{Actual Labor Values with Joint Production}

\subsection{Introduction}

In Chapters 7 and 8, we have shown that, in simple Leontief economies with circulating capital and no joint production, labor values are logically coherent and empirically relevant. Albeit meaningless as predictors of relative equilibrium prices, Marxian values provide the only theoretically sound indices of labor productivity, and they yield fundamental insights on the dynamics of capitalist economies. It is not clear, however, whether these conclusions continue to hold outside of the simple Leontief model.

In Chapter 9 we have extended the key insights of Marx's LTV to linear production economies in which each sector produces a single good but multiple activities are used to produce the various goods. The key step for the generalization of the concept of labor values as measuring the real (labor) costs of producing goods is to note that the existence of alternative methods of production requires, following Marx, to distinguish the concept of (average) labor values and the notion individual values. We have provided an extension of the concept of labor values that captures the key insights of Marx's LTV.

In this chapter, we build on the distinction between (average) labor values and individual values and provide a general definition of labor values - actual labor values - that is theoretically robust and empirically meaningful, and that preserves all of the key propositions of Marxian value theory identified in Chapter 6 in economies with joint production. The key to this breakthrough is the distinction between the concept of labor embodied in, or real cost of production (in terms of labor) of commodities and the notion of IO employment multipliers. Outside of the simplest Leontief economies, the two concepts do not necessarily coincide. The former is definitionally nonnegative, unlike the latter. In fact, Steedman's (1977) famous demonstration that in economies with joint production, the labor values of certain commodities, and even aggregate surplus value, can be negative follows 
from his definition of labor values as IO employment multipliers. Rather than proving Marxian value theory conceptually flawed, this argument shows that Steedman's definition is inappropriate in the case of joint production (or multiple activities). Marxian labor values should measure the labor embodied in, or real (labor) cost of producing certain commodities.

In a seminal article Morishima (1974) proposed that, in general economies, the labor embodied in any bundle of goods-its labor value - be defined as the mimimum amount of direct labor necessary to produce it choosing among all possible alternative techniques. The concept of labor values underlying this definition - 'true labor values' to paraphrase Morishima - has been widely considered as an appropriate generalization of Marx's LTV ${ }^{1}$ or at least as an appropriate starting point for alternative optimizing definitions (Roemer 1981, 2002; Matsuo 2008). ${ }^{2}$ Furthermore, Morishima's optimizing approach has been considered as a valid solution to the problems highlighted by Steedman (1977).

Yet, the adequacy of Morishima's definition, and its ability to capture the core features of Marx's LTV, have been questioned. Morishima's definition satisfies only a subset of the axiomatic properties that capture the key tenets of Marxian value theory discussed in Chapter 6: Morishima's 'true' values are unique and nonnegative. As Steedman (1976) has noted, however, Morishima's labor values are not additive. Roemer (1981) has shown that although the FMT holds in the standard von Neumann equilibria, Morishima's definition does not really solve the issues posed by Steedman's counterexamplesin particular concerning the relation between aggregate surplus value and aggregate profits - in more general settings. Further, Morishima's 'true' values are not necessarily proportional to production prices if all industries have the same organic composition of capital. And so on.

In this chapter we propose an alternative definition of Marxian labor values conceived of as measuring the labor embodied in, or the real (labor) costs of producing certain commodities based on the analysis of joint production in Chapter 10. To be specific, we show that if jointly produced outputs and their inputs are disentangled by means of economic imputations which reflect the benefit received from each unit of costing - i.e. using the "sales value method" - then individual

${ }^{1}$ See Nutzinger (1976), Wolfstetter (1976), Murata (1977), Takeda (1978), Morishima and Catephores (1978), Cogoy (1979), Roemer (1980a), and Fujimoto and Opocher (2010).

${ }^{2}$ For a discussion see Yoshihara and Veneziani (2010) and Veneziani and Yoshihara (2011, 2012, 2015a). 
and average labor values can be defined as in the case with multiple activities based on the square input matrix (and its corresponding labor input vector) obtained from IO methodology in the case of the "industry technology hypothesis".

To be sure, our approach is not purely technological, unlike Morishima's: information on price and market shares is indispensable in order to identify the labor values of individual commodities. In our view, this is not a shortcoming. For, first, as argued in Chapter 10, if joint production is considered, a purely technological definition of labor values is not necessarily appropriate theoretically and it seems quite distant from the actual accounting practices of profit maximizing firms. Indeed, with joint production it is conceptually impossible to disentangle and separate price and quantity magnitudes. Second, any definition of labor values based on the technology actually used by firms is inevitably - albeit implicitly — based on price information, for the production technique used by profit-maximizing capitalists depends on the vector of commodity prices. Morishima's definition is priceindependent because it defines labor values based on labor-minimizing, and therefore possibly counterfactual production activities. As a result, unlike in our approach, Morishima's 'true values' bear a very tenuous relation with actual empirical data.

Third, and perhaps more important, we show that actual labor values are well defined, positive and unique, and preserve the main properties of Marx's LTV discussed in Chapter 6, including the key propositions on price-value relationships, such as the FMT, in linear economies with joint production. Thus, actual labor values display none of the paradoxical features shown in Steedman's (1977) famous examples while having solid empirical foundations and providing important insights on the dynamics of actual capitalist economies.

A final caveat is worth making at this point. This chapter focuses primarily on value theory in economies with pure joint production, namely in economies with circulating capital only and where more than one output is produced in at least one sector. Fixed capital raises different and complex issues, such as the appropriate treatment of depreciation, and - as argued in Chapter 10 - the standard treatment of fixed capital as a joint product is not entirely compelling. The definition of labor values in economies with fixed capital is therefore briefly considered in the Appendix. 


\subsection{An alternative definition of labor values}

For a better comparison, we develop our ideas on value within the von Neumann model, as in Morishima (1974). Our definitional procedure, however, can be applied to any other equilibrium or disequilibrium situation for prices and quantities (provided that there exist prices such that value added is positive in all spheres of actual production).

The symbols $A=\left(a_{i j}\right), B=\left(b_{i j}\right)$ denote the $n \times m$ matrices of inputs and outputs, respectively; $l=\left(l_{j}\right)$ is the strictly positive $1 \times m$ vector of direct labor inputs and $c=\left(c_{i}\right)$ is the $n \times 1$ consumption basket, i.e. the real wage per labor hour. The semi-positive vectors $B_{\star j}, A_{\star j}$ denote the $j$-th columns of $B$ and $A$ and $B_{i \star}, A_{i \star}$ their $i$-th rows. ${ }^{3}$

Consider a given solution $(x, g, p, r)$ - a von Neumann equilibriumto the following system of inequalities:

$$
\begin{aligned}
B x & \geqq(1+g)(A+c l) x, \quad x \geq 0, \\
p B & \geqq(1+r) p(A+c l), \quad p \geq 0, \\
(1+g) p(A+c l) x & =p B x=(1+r) p(A+c l) x, \\
p B x & >0, \quad p c l x>0 .
\end{aligned}
$$

Such a solution will be interpreted as the actual allocation in the economy: $r, p$ are, respectively, Marx's equilibrium rate of profit and the corresponding prices of production, and $x, g$ are a solution to his scheme of reproduction. ${ }^{4}$ Because of $p B x>0$ we have $r=g>-1$.

In our view, labor values are ex post defined magnitudes (after production decisions and wage contracts have been made). Therefore only actual data-here based on $A, B, l$ and $c$-should be used in the definition of labor values.

In order to deal with the complexities inherent in such a general model, it is important to note that joint production implicitly entails the existence of multiple activities. Hence, Marx's concept of individual value additively connected to the labor value of physical inputsshould be used, and our definition results in a relationship of the following kind

$$
\tilde{v}_{j} B_{\star j}=v A_{\star j}+l_{j}, \quad j=1, \ldots, m,
$$

where $\tilde{v}_{j}$ is the $1 \times n$ vector of individual values of the products of

\footnotetext{
${ }^{3}$ For further details on the von Neumann model, see Morishima (1976a).

${ }^{4}$ For a discussion of Marx's schemes of reproduction see Desai and Veneziani (2009).
} 
process $j$, i.e. $\tilde{v}_{j} B_{\star j}$ is the individual value of the whole output basket; and $v$ is the $1 \times n$ vector of labor values, i.e. $v A_{\star j}$ is the total labor value of physical inputs employed in the production of $B_{\star j}$.

To arrive at this result from the above data, we proceed as follows:

(1) We split joint production $B_{\star j}$ into single-product 'activities' by imputing joint inputs $A_{\star j}$ to their single outputs in proportion to the relative share of commodity $i$ in the output value of sector $j$, $p_{i} b_{i j} / p B_{\star j}$. This is the so-called sales value method, a well-known principle in managerial accounting for the allocation of joint costs (cf. Moore and Jaedicke $(1972,498)$ ).

(2) This procedure yields single-product systems with multiple activities, in general. It is at this stage that the notion of individual value has to be taken into account. These individual values are equal to the sum of individual costs reckoned in (average) labor values plus the direct labor individually expended. In turn, individual values provide the basis for the (average) labor values of commodities, when they are weighted according to output proportions, or market shares, $b_{i j} x_{j} / B_{i \star} x$, and summed over all processes which produce the commodity in question.

(3) Steps (1)-(2) lead to a linear and quadratic equation system based on a disaggregated 'technology' $\tilde{A}, \tilde{l}, \tilde{B}$, where $\tilde{B}$ is a regular diagonal matrix that can be reduced to the identity matrix $I$ by the renormalization of the activity levels associated with it. ${ }^{5}$ Formally, the resulting system is as follows:

$$
\tilde{v}=\tilde{v} \tilde{A} \tilde{B}^{-1}+\tilde{l} \tilde{B}^{-1}
$$

This is the standard system for determining labor values, but it is now applied to individual values.

(4) Average labor values are derived from individual values by the averaging process at point (2) and they relate to individual values by help of individual activities as described at point (2).

Having disentangled joint production through economic imputation, the rest of our procedure consists of an application of Marx's concept of 'individual value' from the initial assumptions on $A, B, l$. This construction generalizes the definition which is applied when joint production and multiple activities are not present. It refers to the activities actually used, additively, and it reflects productivity differences with respect to the production alternatives employed by contrasting individual and average labor expenditures.

${ }^{5}$ The notation $\widetilde{A}, \widetilde{l}, \widetilde{B}$ is explained in detail below. 
To be specific, let us assume $B_{\star j} \geq 0$ and $B_{i \star} \geq 0$ : each process produces at least one good and each good - except labor-is produced by some process. Let $(x, g, p, r)$ be the equilibrium solution of equations (11.1)-(11.4), which is assumed to represent the 'actual data' in what follows. In order to define labor values, we restrict our attention to those equilibria that satisfy the following condition:

$$
p B_{\star j}>p A_{\star j} \text { for each } x_{j}>0,
$$

thus value added is positive for the processes activated in equilibrium. By equation (11.2), this assumption is satisfied whenever $r \geq 0$, but the converse is not true. Operated activities therefore exhibit positive wage costs, and since

$p B_{\star j} x_{j}=(1+r) p(A+c l)_{j} x_{j}$ for all $j \quad$ [see equations $\left.(11.2)-(11.3)\right]$, activities with $x_{j}>0$ cannot produce free goods only, i.e. $p B^{j}$ must be positive. Only these types of activities factor into the derivations below.

To start with, consider the $j$-th sector of our reference economy and define:

$$
\beta_{i j}=p_{i} \sigma_{i j} / p B_{\star j} \Longleftrightarrow p B_{\star j}>0, x_{j}>0 \quad\left(\beta_{i j}=0 \text { otherwise }\right) .
$$

The number $\beta_{i j}$ is the relative share of commodity $i$ in the output value of sector $j$.

Next, consider the $i$-th product and define:

$$
\alpha_{i j}=6_{i j} x_{j} / B_{i \star} x \Longleftrightarrow B_{i \star} x>0, p_{i}>0 \quad\left(\alpha_{i j}=0 \text { otherwise }\right) .
$$

The number $\alpha_{i j}$ represents the market share of process $j$ with respect to product $i$.

By definition, for all $i, j$, we have $\sum_{j} \alpha_{i j}=1, \sum_{i} \beta_{i j}=1$, and:

$$
\alpha_{i j}>0 \Longleftrightarrow \beta_{i j}>0 \Longleftrightarrow\left(p_{i}, b_{i j}, x_{j}\right)>0 \text {. }
$$

Let $\beta$ denote the following matrix of dimension $m \times(n \cdot m)$ :

$$
\left(\begin{array}{cccc}
\beta_{11} 0 \ldots 0 & \beta_{21} 0 \ldots 0 & \ldots & \beta_{n 1} 0 \ldots 0 \\
0 \beta_{12} \ldots 0 & 0 \beta_{22} \ldots 0 & \ldots & 0 \beta_{n 2} \ldots 0 \\
\vdots & \vdots & \vdots & \vdots \\
0 \ldots 0 \beta_{1 m} & 0 \ldots 0 \beta_{2 m} & \ldots & 0 \ldots 0 \beta_{n m}
\end{array}\right)
$$


The product $A \beta$ contains the columns $A_{\star j}$ of $A$ duplicated by help of weights $\beta_{i j}$ :

$$
\left(\beta_{11} A_{\star 1}, \ldots, \beta_{1 m} A_{\star m}, \beta_{21} A_{\star 1}, \ldots, \beta_{2 m} A_{\star m}, \ldots, \ldots\right) .
$$

Similarly, let $\alpha$ be the following $(n \cdot m) \times n$ matrix:

$$
\left(\begin{array}{cccc}
\alpha_{11} & 0 & \ldots & 0 \\
\vdots & \vdots & \ldots & 0 \\
\alpha_{1 m} & 0 & \ldots & 0 \\
0 & \alpha_{21} & \ldots & 0 \\
0 & \vdots & \ldots & 0 \\
0 & \alpha_{2 m} & \ldots & 0 \\
\vdots & \vdots & \vdots & \vdots \\
0 & 0 & \ldots & \alpha_{n 1} \\
\vdots & \vdots & \ldots & \vdots \\
0 & 0 & \ldots & \alpha_{n m}
\end{array}\right)
$$

The product $\alpha A$ then contains the rows $A_{i \star}$ of $A$, duplicated by help of weights $\alpha_{i j}$ in the following order:

$$
\alpha A=\left(\alpha_{11} A_{1 \star}^{\prime}, \ldots, \alpha_{1 m} A_{1 \star}^{\prime}, \alpha_{21} A_{2 \star}^{\prime}, \ldots, \alpha_{2 m} A_{2 \star}^{\prime}, \ldots, \ldots\right)^{\prime} .
$$

Let $\bar{A}=\alpha A \beta: \bar{A}$ is a $(n \cdot m) \times(n \cdot m)$ matrix resulting from the simultaneous application of both duplicating procedures. Let $\bar{B}$ be the $(n \cdot m) \times(n \cdot m)$ diagonal matrix:

$$
\bar{B}=\operatorname{diag}\left\{6_{11}, \ldots, 6_{1 m}, 6_{21}, \ldots, 6_{2 m}, \ldots, b_{n 1}, \ldots, 6_{n m}\right\} .
$$

Note that (duplicated) outputs are listed in their respective order here. Similarly, let the $1 \times n \cdot m$ vectors $\bar{p}, \bar{l}$ be defined by

$$
\begin{aligned}
\bar{p} & =\left(p_{1}, \ldots, p_{1}, p_{2}, \ldots, p_{2}, \ldots, p_{n}, \ldots, p_{n}\right) \\
\bar{l} & =\left(\beta_{11} l_{1}, \ldots, \beta_{1 m} l_{m}, \ldots, \beta_{n 1} l_{1}, \ldots, \beta_{n m} l_{m}\right)=l \beta
\end{aligned}
$$

We are constructing a derived (or disentangled) technology $\bar{A}, \bar{l}, \bar{B}$, where $\bar{A}_{\star j}, \bar{l}_{j} \rightarrow \bar{b}_{j j}$ and $\bar{p}$ is an expanded price vector that gives the prices of all outputs produced in every sector. Finally, let $\tilde{\alpha}$ (respectively $\tilde{\beta}, \tilde{p}, \tilde{l})$ be defined by eliminating all rows (respectively all columns) from 
the matrix $\alpha$ (respectively $\beta, \bar{p}, \bar{l}$ ) whenever the corresponding row (and column) in $\bar{B}$ has zero coefficients only. Let $\tilde{A}, \tilde{B}$ be defined by eliminating all columns and rows from the matrices $\bar{A}, \bar{B}$, whenever the corresponding row (and column) in $\bar{B}$ has zero coefficients, i.e. whenever a product $i$ is not produced by process $j .{ }^{6}$

Thus, $\tilde{B}$ is a regular diagonal matrix by definition. The following Lemma can be proved: ${ }^{7}$

Lemma 11.1.

$$
\tilde{\alpha} A \tilde{\beta}=\tilde{A}, \quad l \tilde{\beta}=\tilde{l} .
$$

Proof. To prove that $\tilde{\alpha} A \tilde{\beta}=\tilde{A}$, note that by the definition of $\alpha$ and $\beta$, to strike out a row (column) of $\alpha(\beta)$ corresponds to eliminating the same row (column) of $\bar{A}$ by help of the product $\tilde{\alpha} A(A \tilde{\beta})$. A similar $\operatorname{argument}$ proves $l \tilde{\beta}=\tilde{l}$.

The next Lemma proves another property of the key variables of the model.

Lemma 11.2.

$$
(\tilde{p} \tilde{\alpha})_{i}=(\bar{p} \alpha)_{i}=p_{i} \geq 0 \quad \text { if } \quad B_{i \star} x>0 .
$$

Proof. With respect to $\alpha$ we have

$$
(\tilde{p} \tilde{\alpha})_{i}=\bar{p} \alpha_{i \star}=\sum_{j=1}^{m} p_{i} \alpha_{i j}=p_{i} \sum_{j=1}^{m} \alpha_{i j}=p_{i},
$$

for each $i$ with $B_{i \star} x>0$, since

$$
\sum_{j=1}^{m} \alpha_{i j}=\left\{\begin{array}{lll}
1 & \text { if } & p_{i}>0 \\
0 & \text { if } & p_{i}=0
\end{array} .\right.
$$

And reducing $\alpha$ to $\tilde{\alpha}$ makes no difference, because no positive elements of $\alpha$ are involved in this reduction.

Note that the dual relationship $\tilde{\beta} \tilde{x}=\beta \bar{x}=x$ holds without any qualification, since $x_{j}>0 \Rightarrow p B_{\star j}>0$. Finally, we derive a property of production prices that is useful in order to prove our main result.

\footnotetext{
${ }^{6}$ In a simple Leontief system, where $B=I$, this leads us back to the $n \times n$ matrix $\tilde{B}=I$. In the case of a strictly positive matrix $B$, instead, the dimension would be $(n \cdot m) \times(n \cdot m)$.

7 Note, however, that the vector $\tilde{p}$ may still have zero components. By equations (11.6)-(11.7), we have eliminated only zero columns and rows.
} 
Lemma 11.3. Let $\tilde{c}=\tilde{\alpha} c$. Then

$$
\tilde{p} \tilde{B} \geq(1+r) \tilde{p}(\tilde{A}+\tilde{c} \tilde{l}) .
$$

Proof. For any $i, j$, by definition the right-hand side of equation (11.17) is $p_{i} \sigma_{i j}$, with $b_{i j}>0$. As for the left-hand side, note that by definition, $(1+r) \tilde{p}(\tilde{A}+\tilde{c} \tilde{l})=(1+r) \tilde{p} \tilde{\alpha}(A+c l) \tilde{\beta}$. Further, by Lemma 11.2, $\tilde{p} \tilde{\alpha}=p$ (up to $B_{i \star} x=0$ ), and thus for every $i, j$ :

$$
(1+r) \beta_{i j} p(A+c l)_{\star j}=(1+r) \frac{p_{i} 6_{i j}}{p B_{\star j}} p(A+c l)_{\star j}
$$

if $x_{j}>0$ and $p B_{\star j}>0$ hold; see equations (11.5) and (11.9)-(11.10) and note that $B_{i \star} x=0 \Rightarrow(A+c l)_{i \star} x=0 \Rightarrow 6_{i j}=0$ for $x_{j}>0$. As in this case we also have

$$
p B_{\star j}=(1+r) p(A+c l)_{\star j}
$$

by equations (11.2)-(11.3), then $p_{i} \sigma_{i j}=(1+r) \beta_{i j} p(A+c l)_{\star j}$. On the other hand, if $x_{j}=0$, it follows from equation (11.8) that:

$$
(1+r) \beta_{i j} p(A+c l)_{\star j}=\beta_{i j}=0,
$$

which yields the inequality sign in equation (11.17), if $p_{i} \sigma_{i j}>0$.

Proposition 11.1 establishes some properties of the derived system.

\section{Proposition 11.1.}

(1)

$$
\tilde{p} \tilde{A} \leq \tilde{p} \tilde{B}, \quad \text { i.e. } \quad \tilde{p} \tilde{A} \tilde{B}^{-1} \leq \tilde{p},
$$

with $(\tilde{p} \tilde{A})_{i \star}=(\tilde{p} \tilde{B})_{i \star}$ if and only if the coefficient $b_{i j}$ in the diagonal of $\tilde{B}$ is associated with $p_{i}=0$ (in this case the corresponding column in $\tilde{A}$ vanishes, too).

(2) There exists $\tilde{p}_{\varepsilon} \in \mathbb{R}_{+}^{h}$, with $h \leq n \cdot m$, such that

$$
\tilde{p}_{\varepsilon} \geq \tilde{p} \quad \text { with } \quad \tilde{p}_{\varepsilon} \tilde{A}<\tilde{p}_{\varepsilon} \tilde{B} .
$$

(3) $I-\tilde{A} \tilde{B}^{-1}$ is nonnegatively invertible.

Proof. 1. First of all, note that for any given $j$, the inequality (11.21) can be written as $\beta_{i j} p A_{\star j}<p_{i} b_{i j}$, where $\sigma_{i j}>0$ by the definition of $\tilde{B}$. If $x_{j}=0$ then by definition $\beta_{i j}=0$ and the statement immediately follows. Hence, suppose that $x_{j}>0$. If $p_{i}=0$, then by definition $\beta_{i j}=0$ 
and inequality (11.21) holds as an equality. If $p_{i}>0$, then the result follows as in Lemma 11.3, noting that by equation (11.5), $p A_{\star j}<p B_{\star j}$.

2. Define $p_{\varepsilon}$ by

$$
p_{\varepsilon i}= \begin{cases}p_{i} & \text { if } p_{i}>0 \\ \varepsilon>0 & \text { if } p_{i}=0\end{cases}
$$

and $\bar{p}_{\varepsilon}, \tilde{p}_{\varepsilon}$ in the same way as $\bar{p}, \tilde{p}$. As in Lemma 11.2, it is immediate to prove that $\tilde{p}_{\varepsilon} \tilde{\alpha}=p$ (up to $B_{i \star} x>0$ ), and therefore $\tilde{p}_{\varepsilon} \tilde{\alpha}=\tilde{p} \tilde{\alpha}$. Therefore $\tilde{p}_{\varepsilon} \tilde{A}=\tilde{p}_{\varepsilon} \tilde{\alpha} A \tilde{\beta}=p A \widetilde{\beta}=\tilde{p} \tilde{\alpha} A \tilde{\beta}=\tilde{p} \tilde{A}<\tilde{p}_{\varepsilon} \tilde{B}$, because we have $\tilde{p}_{\varepsilon} \tilde{B}>0$ by construction and noting that the equality sign in $\tilde{p} \tilde{A} \leq \tilde{p} \tilde{B}$ holds only for the vanishing components of $\tilde{p} \tilde{A}$.

3. By part (2), it is easy to find a positive matrix $A_{\varepsilon} \geq A$ which fulfills $\tilde{p}_{\varepsilon} \tilde{A}_{\varepsilon}<\tilde{p}_{\varepsilon} \tilde{B}$, or equivalently $\tilde{p}_{\varepsilon} \tilde{A}_{\varepsilon} \tilde{B}^{-1}<\tilde{p}_{\varepsilon}$. From Nikaido (1968), Theorems 7.1(ii), 7.2(iv), and 7.4(i) we then get for the dominant root $\lambda$ of $\tilde{A} \tilde{B}^{-1}$, denoted as $\lambda\left(\tilde{A} \tilde{B}^{-1}\right)$ :

$$
\lambda\left(\tilde{A} \tilde{B}^{-1}\right) \leq \lambda\left(\tilde{A}_{\varepsilon} \tilde{B}^{-1}\right)<1 .
$$

It follows that $I-\tilde{A} \tilde{B}^{-1}$ is nonnegatively invertible.

Remark 11.1. This disentangled production structure is profitable in the same way as the original structure $A, B$.

Given Proposition 11.1, we can define the labor values of goods produced in specific sectors of the economy:

Definition 11.2. The vector of individual labor values $\tilde{v} \in \mathbb{R}^{h}, n \leq$ $h \leq n \times m$ is defined by:

$$
\tilde{v}=\tilde{l} \tilde{B}^{-1}\left(I-\tilde{A} \tilde{B}^{-1}\right)^{-1}=\tilde{l}(\tilde{B}-\tilde{A})^{-1} \geq 0 .
$$

Define in addition an expanded vector of 'individual values': $\tilde{v}_{(j)}=$ $\left(\tilde{v}_{1(j)}, \ldots, \tilde{v}_{n(j)}\right) \in \mathbb{R}^{n}$ concerning process $j$ only, built on the positive individual values $v_{i(j)}$ of this process as they are defined by the vector $\tilde{v}$, where the added components $\tilde{v}_{i(j)}$-in correspondence to $b_{i j}$-are equal to zero if $b_{i j}=0$ holds, i.e. if good $i$ is not produced by process $j$. The order in the vector $\tilde{v}$ runs from product 1 to product $n$ and lists for each product the positive individual values (corresponding to the positive entries in process 1 up to process $m$ ).

Definition 11.3. The vector of (average) labor values is

$$
v=\tilde{v} \tilde{\alpha} .
$$


The vector $v$ is of the same dimension as $p$; its components $v_{i}$ represent the weighted averages of the individual labor values with the weights $\alpha_{i j}$, the market shares of processes $j$ with respect to product $i$. In other words, labor values are averages of individual values in the case of intrasectoral competition (as discussed by Marx $(1976,315)$ ). The next Proposition derives three additional properties of our definition of labor values.

Proposition 11.2. For all $i=1, \ldots, n$ and $j=1, \ldots, m$ :

(1) $\tilde{v}_{i(j)}=0$ if and only if $b_{i j}>0$ is associated with $p_{i}=0$ or $x_{j}=0$.

(2) $v_{i}=0$ if and only if $p_{i}=0$ or $B_{i \star} x=0$.

(3) $v A \tilde{\beta}+l \tilde{\beta}=\tilde{v} \tilde{B}$, i.e. $v A_{\star j}+l_{j}=\tilde{v}_{(j)} B_{\star j}$ for all processes $j$ that are operated.

Proof. 1. Recall that by Definition $11.2 \tilde{v}_{i(j)}$ corresponds to $\sigma_{i j}$ and has been set equal to zero if $b_{i j}=0$, coefficients which are not contained in the matrix $\tilde{B}$, however. By Definition 11.2 , we have $\tilde{v}=\tilde{l} \tilde{B}^{-1}+$ $\tilde{v} \tilde{A} \tilde{B}^{-1}$. Because of equations (11.8) and (11.15) we know that $\tilde{l}$ has zero components if and only if $p_{i}=0$ or $x_{j}=0$. It thus suffices to show that the corresponding columns in $\tilde{A} \tilde{B}^{-1}$ or $\tilde{A}$ are zero, too. Since $\beta_{i j}=0$ we do know this already with respect to $\tilde{\beta}$. But multiplication by $A$ and then by $\alpha$ from the left preserves this property, thereby implying its validity for $\tilde{A}=\tilde{\alpha} A \tilde{\beta}$, too (see equation (11.15)).

2. From Definition 11.3, it follows that we have

$$
v_{i}=\sum_{j=1} \alpha_{i j} \tilde{v}_{i(j)} .
$$

From equation (11.7) we therefore have $v_{i}=0$ if $p_{i}=0$ or $B_{i \star} x=0$. If, on the other hand, $p_{i}>0$ and $B_{i \star} x>0$ there exists a $j \in\{1, \ldots, m\}$ such that $b_{i j}, x_{j}>0$. By equation (11.8), we then get $\alpha_{i j}>0$ and by part (1) we get $\tilde{v}_{i(j)}>0$ which implies $v_{i}>0$.

3. $v A \tilde{\beta}+l \tilde{\beta}=\tilde{v}(\tilde{\alpha} A \tilde{\beta})+l \tilde{\beta}=\tilde{v} \tilde{A}+\tilde{l}=\tilde{v} \tilde{B}$, where the first equality follows from Definition 11.3, the second from the definition of $\tilde{A}$, and the last from Definition 11.2.

In other words, by part (3) there is an additive relationship between individual values of (the bundle of) outputs of process $j$ and the sum of (average) labor values of their corresponding inputs - plus the direct labor time individually performed (Marx 1976, 434). By parts (1) and (2) both types of values are positive if and only if the corresponding product is a produced good at the individual or at the social level, respectively. Then: 


$$
p_{i} B_{i \star} x=(1+g) p_{i}(A+c l)_{i \star} x \quad \text { implies } \quad(A+c l)_{i \star} x>0 \quad \text { for all } i .
$$

However, the converse is not true in this model. Goods may have use value, but if they are overproduced their exchange value is zero. Since such goods should not be considered as commodities, it is consistent with Marx's theory that their labor value be zero (Marx 1976, 129-131).

Propositions 11.1 and 11.2 conclude the definitional part of this chapter. Note that in addition to positivity, uniqueness of labor values also follows from Propositions 11.1 and 11.2, and Definitions 11.2 and 11.3 .

In closing this section, it is worth discussing our method in relation to steps (1)-(4) above: the post-multiplication of $A$ by $\tilde{\beta}$ disentangles joint production into coexisting multiple activities as described in step (1) and the pre-multiplication of $A$ by $\tilde{\alpha}$ is mathematically equivalent to the averaging procedure in step (2), since by Definition 11.3 we have

$$
\tilde{v} \tilde{\alpha} A=v A \text {, }
$$

for example, $\tilde{\alpha}$ should be seen as the necessary link between $\tilde{v}$ and $v$.

\subsection{Properties of value and price relationships}

This section extends the basic set of relations between labor value aggregates, monetary aggregates, and total direct labor time $l x$ that hold in the simple Marxian production model to von Neumann economies with joint production and multiple activities. Let $\bar{x}=$ $\left(x_{1}, \ldots, x_{m}, \ldots, x_{1}, \ldots, x_{m}\right)^{\prime} \in \mathbb{R}^{m \cdot n}$ and, consistently with the notation in the previous section, define $\tilde{x}$ by eliminating the elements of $\bar{x}$ which correspond to zeros in equation (11.13). Let $y=(B-A) x$ denote the actual net product. The next proposition generalizes a result originally derived by Sato (1979), and then defined by Wolfstetter (1980) as the Marxian Aggregation Theorem (MAT), according to which the value of net product is equal to total labor time expended. This is a fundamental theorem in Marxian value theory, and it is central in various modern approaches (see Duménil (1980), Foley (1982, 1986b), Foley and Duménil (2008), Duménil et al. (2009), Yoshihara and Veneziani (2009)).

Proposition 11.3.

$$
v y=v(B-A) x=\tilde{v}(\tilde{B}-\tilde{A}) \tilde{x}=\tilde{l} \tilde{x}=l x .
$$

Proof. First, using an argument dual to Lemma 11.2, it is immediate to prove that $\tilde{\beta} \tilde{x}=x$. Then, the first equality in the statement follows from 
the definition of $y$. As for the second equality, note that by Definition 11.3 and $\tilde{\beta} \tilde{x}=x$, it follows that $v(B-A) x=\tilde{v}(\tilde{\alpha} B \tilde{\beta}-\tilde{\alpha} A \tilde{\beta}) \tilde{x}=$ $\tilde{v}(\tilde{\alpha} B \tilde{\beta}-\tilde{A}) \tilde{x}$. Furthermore, although $\tilde{\alpha} B \tilde{\beta} \neq \tilde{B}$, we have $\tilde{v} \tilde{\alpha} B x=\tilde{v} \tilde{B} \tilde{x}$, because $\tilde{v} \tilde{\alpha}=\sum_{j=1}^{m}\left(\tilde{v}_{i(j)} \alpha_{i j}\right)_{i=1, \ldots, n}$, and $B x=\left(B_{i \star} x\right)_{i=1, \ldots, n}$ imply that

$$
\begin{aligned}
\tilde{v} \tilde{\alpha} B x & =(\tilde{v} \tilde{\alpha})(B x)=\sum_{i=1}^{n}\left(\sum_{j=1}^{m} \tilde{v}_{i(j)} \alpha_{i j}\right) B_{i} x= \\
& =\sum_{i=1}^{n} \sum_{j=1}^{m} \tilde{v}_{i(j)} \alpha_{i j} B_{i} x=\sum_{i=1}^{n} \sum_{j=1}^{m} \tilde{v}_{i(j)} b_{i j} x_{j}
\end{aligned}
$$

where the latter equality follows from the definition of $\alpha_{i j}$ equation (11.7) - and Proposition 11.2(1). Then, the third equality in the statement follows from Definition 11.2, and the last equality follows from Lemma 11.1 and $\tilde{\beta} \tilde{x}=x$.

By Proposition 11.3, there is no waste of labor in the aggregate, and although relative prices are relevant in the definition of labor values (at a specific point solely) they do not enter the determination of $v y$, the labor value of net national product. Proposition 11.3 does not hold for Morishima's (1974) 'true' labor values, nor for any of the optimizing definitions stemming from Morishima's, such as Roemer's (1981) and Matsuo's (2008). ${ }^{8}$

In order to derive our next proposition, recall that $\widetilde{c}=\widetilde{\alpha} c$ and define

$$
\tilde{z}=(\tilde{B}-\tilde{A})^{-1} \tilde{c} \text { and } z=\tilde{\beta} \tilde{z} .
$$

These definitions are dual to $\tilde{l}, \tilde{v}, v$, and therefore it is immediate to prove that they satisfy a relationship dual to Proposition $11.2(3): \tilde{B} \tilde{z}=$ $\tilde{A} \tilde{z}+\tilde{c}=\tilde{\alpha} A z+\tilde{\alpha} c$. Note also that $\tilde{z} \neq \tilde{x}$ and $z \neq x$ by definition and that $v c$ is positive given $p c>0$ from equation (11.4). The next result proves that the rate of surplus value and the rate of exploitation are equal.

\section{Proposition 11.4.}

$$
\frac{v y-v(c l x)}{v(c l x)}=\frac{1-v c}{v c}=\frac{1-l z}{l z} .
$$

Proof. The first equality, concerning the rate of surplus value and the rate of surplus labor, follows by noting that $v y=l x$ by Proposition

\footnotetext{
${ }^{8}$ For a discussion, see Veneziani and Yoshihara (2012, 2015a).
} 
11.3. The second equality follows from

$$
v c=\tilde{v} \tilde{\alpha} c=\tilde{v} \tilde{c}=\tilde{v}(\tilde{B}-\tilde{A}) \tilde{z}=\tilde{l} \tilde{z}=l \tilde{\beta} \tilde{z}=l z,
$$

where the first four equalities follow, respectively, from the definitions of $v, \tilde{c}, \tilde{z}$, and $\tilde{v}$; the fifth follows from Lemma 11.1; and the last equality follows from the definition of $z$.

Note that individual rates of exploitation, or surplus value

$$
\epsilon_{j}=\frac{v B_{\star j}-v A_{\star j}-v c \cdot l_{j}}{v c \cdot l_{j}}
$$

are not uniform in general. In contrast, since we have assumed a uniform wage basket per hour worked (which fulfills $\tilde{v} \tilde{c}=\tilde{v} \tilde{\alpha} c=v c$ ) and since our method of defining individual values is the standard one, it can easily be seen that rates of surplus value are uniform at the level of individual values with respect to the operated part of the disentangled data $\tilde{A}, \tilde{l}, \tilde{B}, \tilde{c}$. This uniform rate must be equal to the aggregate rate of surplus value $\epsilon:(1-\tilde{v} \tilde{c}) / \tilde{v} \tilde{c}$. Further, since $(1+r) \tilde{p}(\tilde{A}+\tilde{c} \tilde{l})=\tilde{p} \tilde{B}$ (inactive processes are ignored by Lemma 11.3), the well-known relationship $\epsilon \geq$ $r$ of the basic Marxian model (with $B=I$ ) holds also in our general framework. In summary, our definition allows us to generalize many of the standard insights proved in the basic Leontief model. ${ }^{9}$

Finally, it is worth noting that we have meaningful solutions on both the value and the quantity side: $\tilde{l}, \tilde{v}, v \geq 0$ and $\tilde{c}, \tilde{z}, z \geq 0$, respectively. Instead, as Wolfstetter (1976) noted, in Steedman's (1977) purely additive approach to labor values where

$$
v(B-A)=l,
$$

the dual to $(11.25)$

$$
(B-A) z=c,
$$

may have meaningless solutions. As Wolfstetter (1976) argued, the purely additive approach given by equations (11.25)-(11.26) is too narrow to imply economically meaningful solutions beyond the standard single-product model. The next result proves that a robust

\footnotetext{
${ }^{9}$ A uniform rate of surplus value (or rate of exploitation) is assumed by Marx in his own presentation of the transformation of labor values into prices of production. Marx bases this on the mobility of labor and competition among workers, in much the same way as his assumption of a uniform profit rate. For further discussion see Cogliano $(2011,2013)$ and Foley (2011, 2016).
} 
relation between profit and exploitation exists if our definition is adopted.

Proposition 11.5. The rate of profit $r$ and the rate of surplus value $\epsilon$ are always positive, zero or negative simultaneously.

Proof. By Proposition 11.2(2), $v B x=(1+g) v(A+c l) x$ and because $g=r$ :

$$
\begin{aligned}
r & =\frac{v(B-A-c l) x}{v(A+c l) x}=\frac{v(B-A) x-v c l x}{v c l x} \frac{v c l x}{v(A+c l) x} \\
& =\epsilon \cdot \frac{1}{1+v A x / v c l x} .
\end{aligned}
$$

The desired result then follows noting that $v c l x>0$.

The proof of Proposition 11.5 is relatively simple because (except for the uninteresting case $B_{\star j} x=0$ ) goods are free in value terms if and only if they are free in price terms, which is not true for Morishima's 'true' labor values. This allows us to use Marx's equality between the price and the value rate of profit (thanks to the assumption of a von Neumann equilibrium). However, the proof does not depend essentially on this identity, as our discussion after Proposition 11.4 suggests.

Proposition 11.6 derives the conditions for the validity of Marx's aggregate equalities.

Proposition 11.6. The following conditions are equivalent:

$$
\begin{array}{ll}
\text { (1) } & v(B-A-c l) x=p(B-A-c l) x \text {; } \\
\text { (2) } & v(A+c l) x=p(A+c l) x \\
\text { (3) } & v B x=p B x .
\end{array}
$$

Proof. The desired result follows noting that by Proposition 11.2(2) and $g=r, v B x=(1+r) v(A+c l) x$, whereas by equation (11.3) $v B x=$ $(1+r) v(A+c l) x$.

In other words, (1) total surplus value equals total profits exactly when (3) 'the sum of values is equal to the sum of prices' with regard to gross national product (or (2) with regard to total inputs advanced).

Recall that $\tilde{v}_{i(j)}=\tilde{v}_{f(i, j)}$ if $b_{i j}>0$ and $\tilde{v}_{i(j)}=0$ if $b_{i j}=0 .{ }^{10}$ The next Proposition proves that prices and individual values are proportional.

${ }^{10}$ The function $f(i, j)$ is a mapping from $i, j$ to the relevant entry of the vector $\tilde{v}$ in Definition 11.2. 
Proposition 11.7. For all $i, j$, if $b_{i j}>0$, there exists $\phi_{j} \in \mathbb{R}_{+}$, independent of $i$, such that

$$
\tilde{v}_{i(j)}=\phi_{j} p_{i}
$$

Proof. If $x_{j}=0$, then by Proposition 11.2(1) $\tilde{v}_{i(j)}=0$ for all $i$, and the result immediately follows by setting $\phi_{j}=0$. Hence, suppose $x_{j}>0$, so that $p B_{\star j}>0$, as proved in Section 11.2. Then by Proposition 11.2(3) we have

$$
v A_{\star j} \frac{p_{i} \sigma_{i j}}{p B_{\star j}}+l_{j} \frac{p_{i} b_{i j}}{p B_{\star j}}=\tilde{v}_{i(j)} b_{i j},
$$

or equivalently,

$$
\tilde{v}_{i(j)}=p_{i}\left(\frac{v A_{\star j}}{p B_{\star j}}+\frac{l_{j}}{p B_{\star j}}\right)=p_{i} \phi_{j} \quad \text { when } \quad 6_{i j}>0 .
$$

In other words, individual values are proportional to prices of production for all those outputs that are produced by one and the same process $j$ (for $j=1, \ldots, m$ ). This proportionality holds for $m$ disjoint sections of the whole array $\tilde{v}$ of individual values, but in general it does not not hold across sectors, and it does not carry over to labor values $v$.

Next, let the vectors $p^{*}$ and $\tilde{p}^{*}$ be formed from $p$ and $\tilde{p}$ as follows: replace $p_{i}$ in $p$ and $\tilde{p}$ by ' 0 ', whenever $v_{i}$ and $\tilde{v}_{i(j)}=0$, respectively, i.e. in the uninteresting cases: $B_{i \star} x=0, x_{j}=0$. Otherwise, $p_{i}^{*}=p_{i}$ and $\tilde{p}_{i}^{*}=\tilde{p}_{i}$. The next result generalizes the well-known proportionality result in the case of equal organic composition of capital across sectors.

Proposition 11.8. (Marxian Proportionality Theorem). Normalize $p^{*}$ such that $p^{*}(B-A) x=l x$. Suppose that $r>0$. Then, the following statements are equivalent:

$$
\begin{aligned}
& \text { (1) } \tilde{p}^{*}=\tilde{v} ; \\
& \text { (2) } p^{*} B=p^{*} A+l ; \\
& \text { (3) } \frac{p^{*} A_{\star j}}{l_{j}} \text { is independent of } j \text {; }
\end{aligned}
$$

when attention is restricted to processes actually activated $x_{j}>0$.

Proof. (1) $\Longleftrightarrow$ (2). Suppose (1) holds, by Proposition 11.7, $\tilde{p}^{*}=\tilde{v}$ implies $\phi_{j}=1$ for all $j$ such that $x_{j}>0$. Furthermore, by Lemma 11.2 and Definition 11.3, $p^{*}=\tilde{p}^{*} \tilde{\alpha}=\tilde{v} \tilde{\alpha}=v$. Therefore by Proposition 11.7 and noting that $p_{i}^{*} b_{i j}>0$ for at least some $i$ 


$$
\frac{p^{*} A_{\star j}}{p^{*} B_{\star j}}+\frac{l_{j}}{p^{*} B_{\star j}}=1 .
$$

We therefore have $p^{*} A_{\star j}+l_{j}=p^{*} B_{\star j}$ for all $j$ such that $x_{j}>0$. The proof that (2) $\Rightarrow(1)$ is straightforward.

(3) $\Rightarrow$ (2). First note that the change in $p^{*}, \tilde{p}^{*}$ has no effect on the active part of the economy. Therefore, let $w=p c$ and note that since we are considering sectors $j$ such that $x_{j}>0$, at a von Neumann equilibrium we have

$$
p^{*} B=(1+r) p^{*}(A+c l)=(1+r)\left(p^{*} A+w l\right) .
$$

(2) $\Rightarrow(3)$. Subtracting $p^{*} B=p^{*} A+l$ from equation (11.27), we obtain:

$$
r p^{*} A+(1+r)(w-1) l=0,
$$

or $p^{*} A \sim l$, since $r>0$ implies $w \neq 1$, because of the normalization adopted. The assumption of a uniform composition of capital $\left(p^{*} A \sim\right.$ $l \sim w l \sim p^{*} B$ ) then implies that $r$ can be reduced to zero by a suitable increase in $w$ without changing the structure of $p^{*}$ and the above equality sign. And because of the chosen normalization this will be at $w=1$ which leads to

$$
p^{*} B=p^{*} A+l .
$$

By Propositions 11.7 and 11.8, our approach also preserves a key feature of the classical understanding of labor values: both individual and (average) labor values are proportional to prices of production in the case of a uniform organic composition of capital. The equation for labor values $v$ in this case reduces to

$$
v B=v A+l \quad \text { because of } v=p,
$$

i.e. to Steedman's (1977) celebrated purely additive approach. In other words, Steedman (1977) adopts in the general model a definition that is only appropriate in the special case of equal organic composition of capital. We shall return to these important insights below.

\subsection{Joint production and the main products of industries}

In this section, we analyze some properties of individual and labor values that may be useful in empirical applications. We derive a method for computing labor values without calculating individual values first. Then, we discuss some empirical approximations to the correct labor 
values under special assumptions concerning market shares and the structure of sectoral outputs.

To begin with, recall that by Definition $11.3 v=\tilde{v} \tilde{\alpha}$ and by Proposition 11.2(3)

$$
\tilde{v} \tilde{\alpha} A \tilde{\beta}+l \tilde{\beta}=v A \tilde{\beta}+l \tilde{\beta}=\tilde{v} \tilde{B} .
$$

Post-multiplying the latter equation by $\tilde{B}^{-1} \tilde{\alpha}$ gives

$$
v A \tilde{\beta} \tilde{B}^{-1} \tilde{\alpha}+l \tilde{\beta} \tilde{B}^{-1} \tilde{\alpha}=\tilde{v} \tilde{\alpha}=v,
$$

which allows one to determine labor values without using individual labor values $\tilde{v}$, while individual values $\tilde{v}$ can then be obtained by equation (11.28).

In order to operationalize equation (11.29), it is worth noting that $\tilde{\beta} \tilde{B}^{-1} \tilde{\alpha}=\tilde{\beta} \breve{\alpha}$, where $\breve{\alpha}$ is obtained from $\tilde{\alpha}$ by replacing each $\alpha_{i j}=$ $b_{i j} x_{j} / B_{i \star} x$ by $\breve{\alpha}_{i j}=x_{j} / B_{i \star} x$. Using the definitions of the matrices $\alpha$ and $\beta$, this product can be calculated as:

$$
\begin{aligned}
& \tilde{\beta} \breve{\alpha}=\left(\begin{array}{ccc}
\beta_{11} \breve{\alpha}_{11} & \ldots & \beta_{n 1} \breve{\alpha}_{n 1} \\
\vdots & & \vdots \\
\beta_{1 m} \breve{\alpha}_{1 m} & \ldots & \beta_{n m} \breve{\alpha}_{n m}
\end{array}\right)=\left(\frac{p_{i} b_{i j} x_{j}}{p B_{\star j} \cdot B_{i \star} x}\right) \\
& =\left(\begin{array}{ccc}
\frac{x_{1}}{p B_{\star 1}} & \ldots & 0 \\
\vdots & & \vdots \\
0 & \ldots & \frac{x_{m}}{p B_{\star m}}
\end{array}\right)\left(\begin{array}{ccc}
b_{11} & \ldots & b_{n 1} \\
\vdots & & \vdots \\
b_{1 m} & \ldots & b_{n m}
\end{array}\right)\left(\begin{array}{ccc}
\frac{p_{1}}{B_{1 \star} x} & \ldots & 0 \\
\vdots & & \vdots \\
0 & \ldots & \frac{p_{n}}{B_{n \star} x}
\end{array}\right),
\end{aligned}
$$

where $j=1, \ldots, m$ represent the $m$ rows and $i=1, \ldots, n$ represent the $n$ columns of the corresponding matrices. The interpretation of these seemingly complicated expressions is simple: the original equation (11.28) expressing the relation between labor values and individual values is derived by means of an economic decomposition of joint production by help of the matrix of revenue shares $\tilde{\beta}$, for each joint output basket. The 'single-product system' obtained is then aggregated again by forming the 'average' of the 'activities' which 'produce' the same product by help of weights which reflect market shares: $\tilde{\alpha}$. To perform this procedure, 'processes' have to be standardized to the same activity level which is equivalent to summing the columns in $A \tilde{\beta}$ that belong to the same output type weighted with activity levels $x_{1}, \ldots, x_{m}$ and divided by $B_{i \star} x$, the total output of that industry.

The next Proposition provides some approximate relations that can 
be used to compute labor values in various special cases. The proof is straightforward and therefore omitted.

Proposition 11.9. Assume that $p>0, x>0$ holds true. Then:

(1) Let the number of processes $m$ be equal to the number of goods $n$. Assume the approximate equalities:

$$
p_{j} 6_{j j} \approx p B_{\star j} \quad \text { and } \quad b_{j j} x_{j} \approx B_{\star j} x \quad(j=1, \ldots, n) .
$$

Then $\tilde{\beta} \tilde{B}^{-1} \tilde{\alpha} \approx I$, and labor values may approximately be calculated by

$$
v A+l=v B .
$$

(2) Suppose that for all $j \in\{1, \ldots, m\}$ there exists (exactly one) $i_{j} \in$ $\{1, \ldots, n\}$ such that $p_{i_{j} j} b_{i_{j} j} \approx p B_{\star j}$. Then the relation between labor values and individual values can be described approximately by

$$
v A+l=v \hat{B}, \quad \hat{B}=\operatorname{diag}\left\{6_{i_{1} 1}, \ldots, b_{i_{m} m}\right\} .
$$

(3) Suppose that for all $i \in\{1, \ldots, n\}$ there exists (exactly one) $j_{i} \in$ $\{1, \ldots, m\}$ such that $b_{i j_{i}} x_{j_{i}} \approx B_{i_{\star}} x$. Then we can determine labor values approximately by solving:

$$
v A \tilde{\beta}+l \tilde{\beta}=v \hat{B}, \quad \hat{B}=\operatorname{diag}\left\{b_{i j_{1}}, \ldots, b_{n j_{n}}\right\},
$$

where $\tilde{\beta}$ has been reduced by eliminating all columns where $\beta_{i j}$ fulfills $j \neq j_{i}$.

In other words, in general economies with joint production, the standard formula for the computation of labor values is a good approximation provided good $j$ dominates in the proceeds obtained from the $j$-th activity, and the output of good $j$ in the economy as a whole is obtained mainly by employing the $j$-th activity. Furthermore, if the proceeds of each process mainly stem from one of its products, labor values are determined approximately as in pure substitution systems. Finally, if each of the $n$ commodities is mostly produced by one process, then the formula in part (3) provides an alternative approximation for the derivation of labor values.

\subsection{Employment multipliers and labor values in joint production systems: Steedman after Stone}

In this section, we compare the notion of "employment multipliers" used in Steedman (1977, Ch. 11) with our definition of labor values 
in the context of a two-sector model. and show how the jointproduct convention underlying our concept of labor values allows us to generalize Marx's ideas on the additivity of the value creation process without running into the anomalies described in Steedman (1977). In contrast to Steedman's employment multipliers, which reflect the system's labor requirements to produce additional quantities of net output, our concept of labor values incorporates the idea that average values of jointly produced goods vary continuously with prices (salesratios) so that the labor value of a good whose price approaches 0 , must also approach zero.

As shown above (see also Flaschel (1983b)), in the von Neumann model both concepts of total labor requirements allow one to preserve Marx's methodological device of explaining the general nature of profits first under the assumption that commodities exchange at their labor values (in volumes 1 and 2 of Capital) by assuming a uniform composition of capital. Hence, any differences must arise in the analysis of additivity of value creation in joint production systems with different compositions of capital. In the latter case, Steedman (1977) famously demonstrated that a purely technologically determined notion of "labor costs" is deeply unsatisfactory in relation to Marx's theoretical objectives. ${ }^{11}$ Our extension of Marx's labor values is based on the principles of full-cost accounting for jointly produced outputs and is consistent with Marx's view that actual production plays a dominant part in the process of profit creation (Sen 1978, 179).

\subsubsection{Employment multipliers}

Steedman (1977) considers the following two-sector examples of a von Neumann model (assuming wages are paid ex post). The technology is:

$$
B=\left(\begin{array}{cc}
6 & 3 \\
1 & 12
\end{array}\right), \quad A=\left(\begin{array}{cc}
5 & 0 \\
0 & 10
\end{array}\right), \quad l=(1,1)
$$

Steedman considers three different wage baskets per unit of labor input: $c^{1}=(3 / 6,5 / 6), c^{2}=(6 / 5,3 / 5), c^{3}=(3 / 7,6 / 7)$. The relevant von Neumann equilibrium prices (measured in wage-units) are given in each of these cases by: $p_{1}=1 / 3, p_{2}=1, w=1=\left(p_{1}, p_{2}\right) c^{\prime}$. On the quantity side, the three equilibrium employment levels are $L^{1}=$ $6, L^{2}=5$, and $L^{3}=7$, respectively, and the activity vectors are:

$$
x_{1}^{1}=5, x_{2}^{1}=1 \text { or } x_{1}^{2}=3, x_{2}^{2}=2 \text { or } x_{1}^{3}=6, x_{2}^{3}=1 .
$$

${ }_{11}$ This does not mean, however, that Steedman's labor costs cannot be useful for other purposes. 
In each case, the growth rate is equal to the rate of profit with $g^{i}=$ $r^{i}=0.2, i=1,2,3$. Equilibrium net output $y^{i}=(B-A) x^{i}, i=1,2,3$ is: $y^{1}=(8,7)^{\prime}, y^{2}=(9,7)^{\prime}, y^{3}=(9,8)^{\prime}$.

In comparing equilibria 1 and 2 , Steedman finds that a change in employment $\Delta L=L^{2}-L^{1}=-1$ corresponds to $(\stackrel{\wedge}{=})$ a change in net output $\Delta y=y^{2}-y^{1}=(1,0)^{t}$ of one unit of commodity 1 . And when comparing equilibria 2 and 3 he finds:

$$
\Delta L=+2 \stackrel{\wedge}{=} y=(0,1)^{\prime} .
$$

These calculations show that a unit increase in the net output of commodity 1 (respectively 2) is accompanied by a change in total employment of ' -1 ' (respectively ' +2 '), numbers which describe the "employment multipliers" of this technique (Steedman 1977, 158).

It is easy to prove that the well-defined employment multipliers $\left(z_{1}, z_{2}\right)=(-1,2)$ solve

$$
\begin{aligned}
6 z_{1}+z_{2} & =5 z_{1}+1, \\
3 z_{1}+12 z_{2} & =10 z_{2}+1,
\end{aligned}
$$

i.e. they can be calculated from the technological data in the usual purely additive way.

Do these employment multipliers properly extend Marx's concept of labor value? And, in particular, do they allow one to pursue Marx's theoretical aims? Steedman's well-known answer is that employment multipliers are the proper extension of the Marxian definition of labor value, but they are useless for the main aim of value theory, namely for the quantitative determination of the uniform rate of profit. We would argue, instead, that Steedman's strictly additive definition of value in equations (11.30)-(11.31) does not properly capture some of Marx's key principles of value determination. A careful reading of the passages on labor value found in Marx's Capital suggests the following problems:

1. The view that labor gets 'incorporated' in the commodities produced with it (actually or by means of a theoretical imputation) is meaningless in the presence of joint products, if it is understood in terms of 'technology' alone. For there no longer exists a sensible chain of commodity inputs purely determined by technological data which can be characterized as having gone into the production of single units of net output. Subsystems $(B-A)^{-1} y$ of gross output needed to produce a certain basket of net output $y$ no longer represent a sensible way of determining the amount of labor 'embodied' in it. This is demonstrated in the above example by 
the fact that ' -1 ' hours of labor cannot be characterized as having been 'incorporated' into commodity 1 .

2. In Marx's Capital additivity in labor value determination is restricted to average conditions of production. It is unclear that the above example describes such average conditions, since one of the two processes is absolutely inferior to the other.

3. The change from $y^{1}$ to $y^{2}$ which results in an extra production $(1,0)^{\prime}$ goes hand in hand with an increase in labor productivity, as more net output is produced with less labor: $(8,7)^{\prime} / 6 \rightarrow(9,7)^{\prime} / 5$. Marx did connect changes in labor productivity with changes in labor value. Steedman's values, however, show no change at all: $z_{1}, z_{2}$ remain unchanged.

We conclude that Steedman's purely technological interpretation of labor values - determined jointly and additively as the sum of values of the means of production and the new value added by "living labor"- - is unsatisfactory. It destroys their dual characterization as presented in Morishima (1973, Ch. 1) for single-product activities, and by applying Marx's treatment of constant and variable capital to the case of joint inputs without further qualifications, it lays the ground for the refutation of the FMT.

\subsection{Labor values}

We now apply our definition of labor values to Steedman's (1977) example and show that it better captures Marx's LTV. Because our method of labor value calculation is a strict ex post calculation, technological data are not sufficient, and in general any actual quantity and price configurations of the period under consideration could be used, including disequilibrium allocations. For the sake of simplicity, however, we focus on von Neumann equilibria and start from prices $p_{1}=1 / 3, p_{2}=1$ and the following IO configurations (realized in case 1):

\begin{tabular}{c|cccccc} 
& \multicolumn{3}{|c}{ Good 1 Good 2 Labor } & \multicolumn{4}{c}{ Good 1 Good 2 } \\
\hline Process 1 & 25 & 0 & 5 & $\rightarrow$ & 30 & 5 \\
\hline Process 2 & 0 & 10 & 1 & $\rightarrow$ & 3 & 12 \\
\hline
\end{tabular}

Our approach starts from the separation of joint activities based on the proportions of the proceeds of each good within each joint output basket. These ratios are given by: 


$$
\begin{aligned}
& \beta_{11}=\frac{p_{1} b_{11}}{p B_{\star 1}}=\frac{(1 / 3) \cdot 6}{(1 / 3) \cdot 6+1 \cdot 1}=\frac{2}{3}, \beta_{21}=\frac{p_{2} b_{21}}{p B_{\star 1}}=\frac{1}{3}(\text { process } 1), \\
& \beta_{12}=\frac{p_{1} b_{12}}{p B_{\star 2}}=\frac{(1 / 3) \cdot 3}{(1 / 3) \cdot 3+1 \cdot 12}=\frac{1}{13}, \beta_{22}=\frac{p_{2} b_{22}}{p B_{\star 2}}=\frac{12}{13}(\text { process } 2),
\end{aligned}
$$

for each of the three equilibria. Based on these coefficients, we can derive four single-product 'processes'. The transformation matrix is

$$
\widetilde{\beta}=\left(\begin{array}{cccc}
\frac{2}{3} & 0 & \frac{1}{3} & 0 \\
0 & \frac{1}{13} & 0 & \frac{12}{13}
\end{array}\right) .
$$

Then, we have to aggregate the processes 'producing' the same good according to market shares, thereby arriving at a two-equation system for the two unknown labor values, $v_{1}, v_{2}$, as described in equation (11.29):

$$
\left(v_{1}, v_{2}\right)=\left(v_{1}, v_{2}\right) A \widetilde{\beta} \breve{\alpha}+l \widetilde{\beta} \breve{\alpha}
$$

In order to apply equation (11.32), first of all, note that we need not worry about the difference between, for example, $\bar{\beta}$ and $\widetilde{\beta}$, since all of the entries of $B$ are positive. Next, note that in all three equilibria the following data are unchanged:

$$
\begin{aligned}
& \widetilde{B}=\left(\begin{array}{cccc}
6 & 0 & 0 & 0 \\
0 & 3 & 0 & 0 \\
0 & 0 & 1 & 0 \\
0 & 0 & 0 & 12
\end{array}\right), A \widetilde{\beta}=\left(\begin{array}{cccc}
\left(\frac{2}{3}\right) 5 & 0 & \left(\frac{1}{3}\right) 5 & 0 \\
0 & \left(\frac{1}{13}\right) 10 & 0 & \left(\frac{12}{13}\right) 10
\end{array}\right), \\
& l \widetilde{\beta}=\left(\frac{2}{3}, \frac{1}{13}, \frac{1}{3}, \frac{12}{13}\right) .
\end{aligned}
$$

In contrast, the matrix $\breve{\alpha}$ depends on the equilibrium considered. Recalling the definition in the previous section, we derive: 


$$
\begin{aligned}
\text { Case } 1: \breve{\alpha}=\left(\begin{array}{cc}
\frac{5}{33} & 0 \\
\frac{1}{33} & 0 \\
0 & \frac{5}{17} \\
0 & \frac{1}{17}
\end{array}\right), \quad \text { Case } 2: \breve{\alpha}=\left(\begin{array}{cc}
\frac{3}{24} & 0 \\
\frac{2}{24} & 0 \\
0 & \frac{3}{27} \\
0 & \frac{2}{27}
\end{array}\right), \\
\text { Case } 3: \breve{\alpha}=\left(\begin{array}{cc}
\frac{6}{39} & 0 \\
\frac{1}{39} & 0 \\
0 & \frac{6}{18} \\
0 & \frac{1}{18}
\end{array}\right) .
\end{aligned}
$$

Therefore, in Case 1 we obtain

$$
A \widetilde{\beta} \breve{\alpha}=\left(\begin{array}{cc}
\frac{5}{33} \frac{10}{3} & \frac{5}{17} \frac{5}{3} \\
\frac{10}{13} \frac{1}{33} & \frac{1}{17} \frac{120}{13}
\end{array}\right) \quad \text { and } \quad l \widetilde{\beta} \breve{\alpha}=\left(\frac{133}{1287}, \frac{101}{663}\right),
$$

and from equation $(11.29)$ labor values are: $\left(v_{1}, v_{2}\right) \approx(0.237,0.587)$. In Case 2,

$$
A \widetilde{\beta} \breve{\alpha}=\left(\begin{array}{cc}
\frac{10}{3} \frac{3}{24} & \frac{3}{27} \frac{5}{3} \\
\frac{10}{13} \frac{2}{24} & \frac{2}{27} \frac{120}{13}
\end{array}\right) \quad \text { and } \quad l \widetilde{\beta} \breve{\alpha}=\left(\frac{7}{78}, \frac{37}{351}\right)
$$

and labor values are $\left(v_{1}, v_{2}\right) \approx(0.204,0.452)$. In Case 3 :

$$
A \widetilde{\beta} \breve{\alpha}=\left(\begin{array}{cc}
\frac{10}{3} \frac{6}{39} & \frac{5}{3} \frac{6}{18} \\
\frac{10}{13} \frac{1}{39} & \frac{120}{13} \frac{1}{18}
\end{array}\right) \quad \text { and } \quad l \widetilde{\beta} \breve{\alpha}=\left(\frac{53}{507}, \frac{19}{117}\right)
$$

and labor values are $\left(v_{1}, v_{2}\right) \approx(0.239,0.606)$. In all three cases labor values are smaller than prices of production measured in wage-units: $p_{1}=1 / 3, p_{2}=1$. More importantly, in contrast to Steedman's approach, our definition allows us to identify a rise in labor productivity, when comparing Cases 2 or 3 with 1 , which clearly reflects the changes in the ratios $y^{i} / l^{i}$. The change in productivity between Cases 2 and 3, instead, is less clear cut, because labor values move in opposite directions.

As concerns individual values, by Proposition 11.2(3), we apply the formula $v A \tilde{\beta}+l \tilde{\beta}=\tilde{v} \tilde{B}$, to obtain the following system of equations 


$$
\begin{aligned}
6 \widetilde{v}_{1} & =v_{1} \frac{10}{3}+\frac{2}{3}, \\
3 \widetilde{v}_{2} & =v_{2} \frac{10}{13}+\frac{1}{13}, \\
\widetilde{v}_{3} & =v_{1} \frac{5}{3}+\frac{1}{3}, \\
12 \widetilde{v}_{4} & =v_{2} \frac{120}{13}+\frac{12}{13} .
\end{aligned}
$$

It immediately follows that $3 \widetilde{v}_{1}=\widetilde{v}_{3}$ and $3 \widetilde{v}_{2}=\widetilde{v}_{4}$, which confirms the proportionality result established in Proposition 11.7. For the three equilibria analyzed, individual values are (index $1,2=$ Good 1 , index $3,4=$ Good 2):

$$
\begin{array}{rlr}
\left(\tilde{v}_{1}, \tilde{v}_{2}, \tilde{v}_{3}, \tilde{v}_{4}\right) & \approx(0.24,0.18,0.72,0.54) & \text { Case } 1 \\
& \approx(0.22,0.14,0.66,0.42) & \text { Case } 2 \\
& \approx(0.25,0.18,0.75,0.54) & \text { Case } 3
\end{array}
$$

These values show that both commodities are produced by less labor in the second process, which is absolutely superior to the first: one unit of labor is converted into more than twice as much net output by the second process. But as the inputs of each of our two commodity sectors indeed stem from both activities - the distribution of inputs is governed by the ratios in output-value - both commodity sectors employ inputs produced under both favorable and unfavorable conditions. Productivity differences therefore decrease to some extent, thereby narrowing the gap in individual values actually observed. ${ }^{12}$

Another way to analyze productivity differences between the two sectors is to calculate

$$
l^{0}=v(B-A)=v\left(\begin{array}{l}
13 \\
12
\end{array}\right) \approx \begin{cases}(0.83,1.90) & (\text { Case } 1) \\
(0.65,1.50) & (\text { Case 2) } \\
(0.84,1.92) & (\text { Case 3) }\end{cases}
$$

${ }^{12}$ The change in employment $L^{2}-L^{1}=-1$ associated-according to Steedman (1977) - with the change in net output $y^{2}-y^{1}=(1,0)^{t}$ is now represented by $v^{2} y^{2}-v^{1} y^{1}=v^{2}\left(y^{2}-y^{1}\right)+\left(v^{2}-v^{1}\right) y^{1}=L^{2}-L^{1}=-1$. This captures the double nature of the change in the labor value of net output, which is equal to the change in total employment. This change is not driven simply by a change in net output (as captured by the term $v^{2}\left(y^{2}-y^{1}\right)$ ), for given labor values, as in Steedman's approach: the overall change is also driven by the change in labor values themselves (as in the term $\left.\left(v^{2}-v^{1}\right) y^{1}\right)$. 
and to regard these numbers as 'socially necessary direct labor inputs'. These values show the hypothetical technological changes in labor inputs that have to take place in order that all processes look 'normal'13 with respect to the average labor values determined above. ${ }^{14}$ To achieve this kind of normality labor productivity measured in the usual narrow sense (output per labor input) has to rise in the first and to fall in the second process, thus confirming the productivity differences between the two activities.

In the first two volumes of Capital, Marx's analysis is based on the assumption of a uniform composition of capital. ${ }^{15}$ In the rest of this section, we compare Steedman's definition of labor values and ours in this theoretically important case. Consider the following modification of the above IO configurations.

\begin{tabular}{l|ccccccc} 
& \multicolumn{4}{|c}{ Good 1} & Good 2 & Labor & \multicolumn{3}{r}{ Good 1 } & Good 2 \\
\hline Process 1 & 25 & 0 & 5 & $\rightarrow$ & 30 & 5 \\
\hline Process 2 & 0 & 10 & 6 & $\rightarrow$ & 3 & 17 \\
\hline
\end{tabular}

Note that $p_{1}=1 / 3, p_{2}=1, w=1$ again represent prices of production, which now imply a uniform composition in the two processes: $(25 / 3) / 5=5 / 3$. This is not changed by the reallocation $\bar{A}, \bar{l}$ and $\tilde{A}, \tilde{l}$ of the inputs by means of the method employed to disentangle joint products, and the reallocated production data $\tilde{A} \tilde{B}^{-1}, \tilde{l} \tilde{B}^{-1}$ also exhibits a uniform composition of capital at the above prices. This implies that our labor values - equation (11.32) -and prices $p=$ $(1 / 3,1)$ are proportional, with a proportionality factor that can be determined by: $\left(v_{1}, v_{2}\right)(8,12)^{t}=11$, implying $\left(v_{1}, v_{2}\right)=(1 / 4,3 / 4)$. By Proposition 11.8, Steedman's (1977) employment multipliers, $z$, must then be identical to labor values $v$ :

${ }^{13}$ It is perhaps in this sense that Sen's $(1978,178)$ characterization of "socially necessary labor time" as "involving counterfactuals" has to be understood.

14 The hypothetical technology $A, B, l^{0}$ will not necessarily generate the same $v$ or even $\tilde{v}$ as before.

15 According to Marx (1981, 252), "in different branches of industry unequal profit rates prevail, corresponding to the different organic composition of capitals, and, within the indicated limits, corresponding also to their different turnover times; so that at a given rate of surplus-value it is only for capitals of the same organic composition-assuming equal turnover times - that the law holds good, as a general tendency, that profits stand in direct proportion to the amount of capital, and that capitals of equal size yield equal profits in the same period of time." 


$$
z=(5,6)\left(\left(\begin{array}{ll}
30 & 3 \\
5 & 17
\end{array}\right)-\left(\begin{array}{ll}
25 & 0 \\
0 & 10
\end{array}\right)\right)^{-1}=(5,6)\left(\begin{array}{ll}
7 & -3 \\
-5 & 5
\end{array}\right) / 20=(1 / 4,3 / 4) .
$$

This confirms that the two definitions are different only when joint production occurs and the composition of capital is not uniform. In the latter case, a simple application of Marx's additive process of value creation (as outlined in Capital, Vol.I) - with no averaging process involved - is arguably misplaced and no firm conclusion on the LTV can be drawn based on the anomalies thereby obtained. ${ }^{16}$

The above results show that our definition of labor values solves the problems of the standard additive approach, and satisfies the key properties of Marx's LTV (discussed in Chapter 6). It may be objected that our approach implies the dependence of labor values on prices. There are at least four arguments to defend our definition:

1. Labor values depend on prices even before introducing joint production, because, for example, the choice of technique depends on prices. Thus their basic methodological status is not changed by our extension.

2. The rule to allocate joint costs to the different units of costing is important to the extent that it is the only general rule of cost accounting that allows for a uniform rate of profit to result from price-setting behavior and competition. Yet, this rule makes costs and profits accruing to a particular item of joint outputs depend on the price of this product.

3. The use of price magnitudes to define labor costs is not alien to Marx's own theory. In Capital, labor values are deduced from the notion of exchange value: things may be useful and the product of human labor, yet their labor values are zero because they have no exchange value (Marx 1976, 131). Besides, relative wages are sometimes used by Marx to make labor homogeneous. ${ }^{17}$

4. If full-cost accounting for jointly produced commodities by means of the sales value method is accepted for price magnitudes as the general method of allocating joint costs to the various outputs produced, then the same method should be applied to full labor-cost accounting (value magnitudes), too. This, in our view, is the only meaningful way to examine Marx's theory in which labor values

${ }^{16}$ It is worth noting, however, that the problems of Steedman's technological value-accounting method arise at the same level of abstraction at which the 'transformation problem' itself arises (Marx 1981, Ch. 9).

17 See also the discussion in Section 11.2 and Marx (1976, Chs. 1, 3), (1981, Ch. 10) on further possible influences of markets and prices on labor value determination. 
provide a sort of real-cost accounting framework, which relates monetary magnitudes to the functioning of the production process and reveals the coercive nature of the latter. Labor cost accounting should apply the same principles as in ordinary cost accounting.

5. When the composition of capital is uniform, prices that differ from labor values can be eliminated from the data used to determine our labor values $v$ (see Propositions 11.1, 11.2, and 11.8). This means that the theoretical priority associated with the Marxian use of labor values (Marx 1981, Ch. 10) remains intact despite the technological complexities of joint production. This is a considerable advantage of our definition: it is unnecessary to completely revise the theoretical structure of Marx's analysis of labor values.

\subsection{Conclusions}

Steedman (1977) has shown that in the presence of joint production, labor values cannot be meaningfully defined on purely technological grounds. Economic conventions are necessary to overcome the incompleteness of technological data in general models, as Sen (1978, 178) also suggested. One such convention is used in Morishima (1974), in the form of a second choice function. An alternative convention has been applied here to the economic configuration $(x, g, p, r)$ by focusing on a popular accounting principle, the sales value method, employed by firms to impute joint costs.

We have complemented Marx's characterization of labor values by introducing a single device (or 'convention') that allows us to deal with a feature of actual technologies that he did not consider. We have thus extended the traditional definition of labor values to the case of joint production and multiple activities in such a way that all of the properties discussed in Chapter 6 are satisfied (provided additivity is interpreted as holding for the determination of individual labor values).

Our definition embodies an empirically oriented approach to Marx's LTV, whereby " "value' in the Marxian sense is not metaphysical as is often claimed but an observable and operational magnitude" (Okishio 1963, 291) (see also Sen $(1978,176)$ ). As discussed in this chapter, and the previous one, given the data collected in the standard IO tables, one can derive a square monetary Leontief matrix $M$ of commodity $\times$ commodity kind (together with suitably calculated direct labor inputs $m$ ), with respect to which our labor values $v$ fulfill the customary formula for the calculation of labor values per $\$$ of output value 


$$
z M+m=z \quad ; \quad z=\left(v_{1} / p_{1}, \ldots, v_{n} / p_{n}\right)
$$

Therefore, even in the presence of joint production, labor values remain observable, correspond to the standard measure of labor content, and can be derived from standard monetary Leontief tables.

Based on our definition of labor values, the following problems can therefore be empirically analyzed that are central in Marx's theory:

1. The degree of non-equivalence between values and prices (Okishio 1963, 291), and the importance of proportionality theorems such as Proposition 11.8.

2. Marx's analysis of the price rate of profit - via the value rate of profit - in terms of his main labor value aggregates.

As we have argued in Chapter 8, the relation between the price and value rates of profit - and its consequences - lies at the heart of Marx's investigation and it remains a key question even though the two rates are not equal, contrary to Marx's original intuition. Once the possibility for such deviations has been recognized, artificial examples such as Steedman's (1977) are not particularly insightful. Instead, empirical investigations are necessary to evaluate the possible links between the two rates, and our definition of labor values provides a firm basis for these analyses, including in economies with joint production.

Further, as Sen $(1978,182)$ has noted: “... one may be interested in the relationship between values and prices even if this is not a convenient way of calculating prices with given physical data, or a good way of predicting future prices. Value is then treated not as an 'intermediate product' in some calculational or predictive exercise but as a concept of interest in its own right." In our view, this is the right way to conceive of labor values, and in this book we have derived several theorems relating such labor values to issues of allocation and technical change. This line of research has to be pursued in order to discover the true content of Marx's LTV. This chapter demonstrates that the existence of joint products in our view is no hindrance to this research program.

\subsection{Appendix}

In this chapter, we have focused on economies with pure joint production and have ignored fixed capital. Here we briefly address some of the issues that fixed capital raises for the LTV and compare different approaches, starting from Steedman's (1977) seminal analysis. 
11.8.1 Fixed capital: Steedman's example of falling efficiency reconsidered

Consider the following technological description of a process which employs a durable capital good $M$ ('machines') to produce the consumption- and seed-commodity $C$ :

$$
\begin{aligned}
& \begin{array}{lllllllll}
C & M & L & C & M & L & C & M & L
\end{array}
\end{aligned}
$$

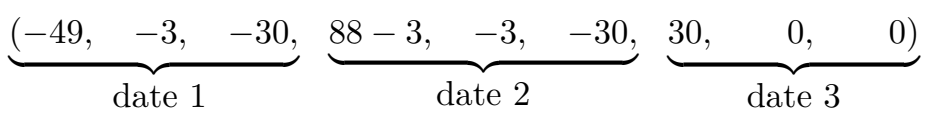

$$
\begin{aligned}
& \text { production period } 1 \quad \text { production period } 2
\end{aligned}
$$

In this example, 3 machines together with 49 units of corn and 30 units of labor have to be advanced to produce 88 units of corn at the end of the first period and can be employed again (but with falling efficiency) to produce 30 units of corn at the end of the second period by using up 3 units of corn and 30 units of labor at its beginning. At the end of the second period the machine is physically worn out with no use value left.

Following Sraffa's (1960) methodology, Steedman (1977, 145, Table III) gives the following alternative description of this economy with fixed capital:

$$
\begin{aligned}
49 C+3 M_{\text {new }}+30 L & \rightarrow 88 C+3 M_{\text {old }}, \\
3 C+3 M_{\text {old }}+30 L & \rightarrow 30 C .
\end{aligned}
$$

The two phases of the process are shown separately by introducing one additional good: the one-year-old machine: $M_{\text {old }}$. This reformulation aims to make the price equations of Sraffa's square joint production systems applicable to the case of fixed capital, too. In this approach, prices of production are calculated in such a way that a uniform rate of profit emerges at each stage of the machine-using process. In order to do this, the characterization of corn production in equations (11.33)-(11.34) must be completed by adding a machineproducing process. This is done by Steedman as follows:

$$
3 C+3 L \rightarrow 3 M_{\text {new }} .
$$

Taking corn as the numéraire, and assuming the real wage to be $b=(2 / 3) C$, he then obtains the following prices for the two types of machines ( $m$ : new machine; $o$ : old machine):

$$
p_{m}=2, p_{o}=2 / 3,
$$


corresponding to a uniform rate of profit of $20 \%$. Based on these prices - which are taken as the actual market prices in the followingtwo thirds of the value of the new machine is written down in the first period and the remaining value in the second.

In order to calculate 'correct labor values', Steedman (1977) applies the principle of uniform profitability to a zero rate of profit, i.e. to a hypothetical real wage $b$, and characterizes Marx's labor value calculations as being purely additive as far as new commodities are concerned and strictly linear with regard to fixed capital and depreciation. As a result, he obtains a negative labor value for the old machine by his first method and incoherent results, i.e. an overdetermined equation system, by his second one.

Yet it is not compelling to conduct value depreciation - which, like any depreciation process, is not a matter of technology alone-by adopting the counterfactual assumption of zero profits, while ignoring actual data, and in particular the actual depreciation rates $(2 / 3,1 / 3)$. Arguably, economic data should be used to calculate 'embodied labor time', in case technological data do not suffice. It is a well-established fact in managerial cost-accounting that in general one cannot arrive at a determination of full costs on purely technological grounds without using economic imputations. Why should things be different with respect to the calculation of 'real costs'-here with regard to labor?

As shown in this chapter, a consistent definition of labor values can be provided in the presence of 'pure joint production': joint commodity outputs and their inputs should be disentangled by means of economic imputations which reflect the sales benefit received from each unit of costing, which reflects the share of each unit of costing in total revenue. The prices of production in equation (11.36) and the implied depreciation rates $(2 / 3,1 / 3)$ obey the same 'benefit principle', which imputes joint effort in such a way that uniform profitability (here over stages of production) emerges. If this principle is employed for the case of fixed capital, it is possible to avoid Steedman's anomalies of labor value depreciation as we now show.

Equations (11.33)-(11.34) indicate that fixed capital implies the existence of production alternatives, so that the method developed in this chapter can be applied. Unlike in the case of pure joint production, only the fixed capital good has to be imputed to the different stages of production, and not the whole input basket. This method replaces the artificial output: $M_{\text {old }}$ by the actual deduction from the (value of the) input: $3 M_{\text {new }}$, and thus it reduces this model to the case of multiple activities.

Treating Table 11.1 as Table 9.1, we obtain the following labor 


\begin{tabular}{|c|c|c|c|c|c|}
\hline & $M C$ & $L$ & $M$ & $C$ & \\
\hline & $\begin{array}{ll}0 & 3\end{array}$ & $3 \rightarrow$ & 3 & 0 & M-sector \\
\hline \multirow{2}{*}{\multicolumn{3}{|c|}{$(2 / 3) \cdot 34930 \rightarrow$}} & 0 & 88 & \\
\hline & & & & & C-sector \\
\hline \multirow[t]{2}{*}{$(1 / 3) \cdot$} & $\begin{array}{ll}3 & 3 \\
\end{array}$ & $30 \rightarrow$ & 0 & 30 & \\
\hline & 355 & $63 \rightarrow$ & & 118 & Totals \\
\hline
\end{tabular}

Table 11.1: The two-sector corn economy again

values of 'corn' and '(new) machines' (see equation (9.8)):

$$
\begin{aligned}
& \left(v_{m}, v_{c}\right)\left(\begin{array}{rr}
3 & 0 \\
52 & 3
\end{array}\right)+(3,60)=\left(v_{m}, v_{c}\right)\left(\begin{array}{rr}
3 & 0 \\
0 & 118
\end{array}\right), \\
& \text { i.e., }\left(v_{m}, v_{c}\right)=(2,1)
\end{aligned}
$$

which are the same as those obtained by Steedman (1977, 145), as they stem from the same aggregated system. But instead of $v_{o}=-1$, the book value of the old machine (in terms of labor content) is $v_{o}=$ $(1 / 3) v_{m}=2 / 3$. Moreover, instead of extending this additive approach to a three equations system, there are two further equations (9.2)-(9.3) to consider, which determine the two individual values of corn for the two stages of production:

$$
\widetilde{v}_{c} \approx 0.943, \widetilde{v}_{c}^{\prime} \approx 1.167,
$$

which are connected to the (average) labor value of corn by equation (9.1):

$$
v_{c}=\frac{88 \widetilde{v}_{c}+30 \widetilde{v}_{c}^{\prime}}{118} .
$$

Hence, no anomaly in labor values can be observed, if the notion of individual value is adopted to take into account productivity differences at the two stages of the corn-producing process. In contrast, Steedman's (1977) approach yields anomalies because it starts from the assumption that: $v_{c}=\widetilde{v}_{c}=\widetilde{v}_{c}^{\prime}$ (leading to three, rather than five equations), an assumption that is justified only in very special cases.

What about Marx's original value calculations? As Steedman (1977, 140) noted, "... Marx worked in terms of linear value depreciation but was aware that it was an over-simplification." Therefore, let us 
adopt the depreciation rates $\delta_{1}=\delta_{2}=1 / 2$ instead of Sraffa's $(2 / 3,1 / 3)$. In addition to $v_{m}, v_{c}$-which are unchanged-we obtain the individual values: $\widetilde{v}_{c} \approx 0.932, \widetilde{v}_{c}^{\prime} \approx 1.200$, and the book value (in terms of labor content) $v_{o}=(1 / 2)$ for the old machine. Thus Marx's "oversimplification" makes little difference (and produces no "internal incoherence"), but has the advantage of getting rid of price-determined nonlinear depreciation procedures. It may, therefore, be regarded as an approximation, which works better the lower the amount of fixed capital per unit of output.

The above analysis shows that negative labor values cannot emerge from the application of our methodology to such examples with falling efficiency: as long as $\delta_{1}, \delta_{2} \geq 0, \delta_{1}+\delta_{2}=1$, values are well-defined, regardless of the actual definition and size of depreciation rates. It is not the specific choice of depreciation rates that prevents the occurrence of anomalies, but rather the structure of our approach. The treatment of depreciation may change, but as long as fixed capital is understood as implying the existence of production alternatives, thus leading to the analysis of 'individual values', no anomaly can occur in the determination of 'real labor costs'. It is the neglect of the latter concept that makes Steedman's (1977) 'correct value calculations' an unsatisfactory generalization of Marx's labor values.

Two provisos are worth making at this point concerning our definition. First, the range of admissible depreciation rates $\delta_{1}, \delta_{2}$ above is not sufficient to establish proportionality between prices of production and labor values. Indeed, labor values $v_{m}, v_{c}$ do not even depend on $\delta_{1}, \delta_{2}$. Second, the independence of $v_{m}, v_{c}$ from $\delta_{1}, \delta_{2}$ and their equality with zero-profit prices is due to the assumption that simple reproduction prevails, in which case the aggregated system in equation (9.8) does not depend on $\delta_{1}, \delta_{2}$.

Summarizing, uniform profitability implies equal individual values - i.e. uniform productivity at all stages of production of a given technique - only in very special cases. In general, different stages of production are characterized by different real costs with respect to their homogeneous output. Thus, averages have to be formed to arrive at unique labor values for each commodity.

\subsubsection{Rising efficiency and rising book values of machinery}

So far, we have excluded negative depreciation rates, but Steedman (1977) also considers an example with $\delta_{1}<0, \delta_{2}>0$. The technological description of this example of rising efficiency (in integrated form) is in Table 11.2, whose interpretation is analogous to Table 11.1.

The output of corn in the first period does not exceed the input 


\begin{tabular}{|c|c|c|c|c|c|c|c|c|c|}
\hline $\mathrm{C}$ & $\mathrm{M}$ & $\mathrm{L}$ & $\mathrm{C}$ & $\mathrm{M}$ & $\mathrm{L}$ & $\mathrm{C}$ & $\mathrm{M}$ & $\mathrm{L}$ & \\
\hline-1 & 0 & -5 & 0 & 5 & 0 & 0 & 0 & 0 & M \\
\hline-9 & -5 & -10 & $10-15$ & 0 & -25 & 25 & 0 & 0 & C \\
\hline \multicolumn{3}{|c|}{ date 1} & \multicolumn{3}{|c|}{ date 2} & \multicolumn{3}{|c|}{ date 2} & \\
\hline
\end{tabular}

Table 11.2: The two-sector corn economy with rising efficiency

necessary for the second period, but there is a net output of $25+10-$ $(15+9)$ units of corn in the whole process.

To apply Sraffa's book values to the determination of labor values, we have to determine Sraffa's prices first (which are omitted by Steedman (1977) in this example). Let $b=0.2 C$ and set $p_{c}=1$. We need to solve:

$$
\begin{aligned}
(1+r)(1+0.2 \cdot 5) & =5 p_{m} \\
(1+r)\left(9+5 p_{m}+0.2 \cdot 10\right) & =10+5 p_{o} \\
(1+r)\left(15+5 p_{0}+0.2 \cdot 25\right) & =25
\end{aligned}
$$

The solution of this system is: $p_{m} \approx 0.4204$ (the price of the new machine), $p_{o} \approx 0.7540$ (the book value of the old machine), and $r \approx 5.1$ (the uniform rate of profit).

The rising (physical) efficiency of capital is reflected in rising vintage prices and "might be the result of the 'running-in' of the new machine" (Steedman 1977, 142). One would imagine, however, that the "runningin" of the new machine resulting in increased efficiency during its second year must be accompanied by running-in costs - a special kind of investment expenditure that appreciates capital and that may not be easily identifiable. In order to illustrate this point, for the sake of simplicity, in the rest of the section we assume that 3 of the 9 units of corn used at the beginning of the first period are indeed running-in costs. For example, they may be necessary either:

(a) to have a sufficient output in period one, or

(b) to make the one-year-old machine functional again in date $2,{ }^{18}$ or

${ }^{18}$ In this case, there exists an alternative activity in the corn sector that may be succinctly described as: $(-6,-5,-10,10,0,0)$. In other words, the three units of corn are not invested in date 1 and therefore the machine 
(c) to raise the output of corn in each period by two units (from 8 to 10 and from 23 to 25 respectively). ${ }^{19}$

Let us set aside these possible interpretations of the 3 units of corncosts for the moment and compute labor values. Steedman $(1977,144)$ sets $v_{c}=\widetilde{v}_{c}=\widetilde{v}_{c}^{*}$ by his "correct value accounting" method and solves:

$$
\begin{aligned}
v_{c}+5 & =5 v_{m} \\
9 v_{c}+5 v_{m}+10 & =10 v_{c}+5 v_{o} \\
15 v_{c}+5 v_{o}+25 & =25 v_{c}
\end{aligned}
$$

which gives $v_{c}=4, v_{m}=1.8, v_{o}=3$.

He concludes "that with machines of rising efficiency, value depreciation can be negative" (Steedman 1977, 144). Yet there is nothing paradoxical in the fact that additional investments $(3 C$ in our example) may lead to rising book values of machinery - both at a rate of profit of $5.1 \%$, leading to $p_{o}>p_{m}$, and at a zero profit rate, leading to $v_{o}>v_{m}$. The only thing to explain here is how the running-in cost is reflected in the above system of price equations, where no distinction is made between those corn-costs which circulate with their product (6C in our example) and those which remain fixed with the machinery during its useful life $(3 C)$. This topic, however, is beyond the scope of this book.

Unlike in Steedman's definition, we distinguish between $v_{c}$ and $\widetilde{v}_{c}, \widetilde{v}_{c}^{\prime}$. Using Sraffa's vintage prices, we get the depreciation rates:

$$
\delta_{1}=\frac{p_{m}-p_{o}}{p_{m}} \approx-0.794, \delta_{2} \approx 1.794 .
$$

Therefore, by adopting an economically determined allocation of the 5 machines to the two stages of corn production, as in the previous section we obtain Table 11.3.

Using equations (9.2)-(9.3), we get:

cannot be used in date 2, which in turn implies that no output of corn is obtained in date 3 . This activity can be neglected, though, because it is strictly inferior at equilibrium prices.

19 Again, this implies that there exists an alternative activity in the corn sector that may be succinctly described as: $(-6,-5,-10,8-15,0,-25,23)$. In other words, the three units of corn are not invested in date 1 and therefore output in dates 2 and 3 is lower by two units. This activity can be neglected, though, because it is strictly inferior at equilibrium prices. 


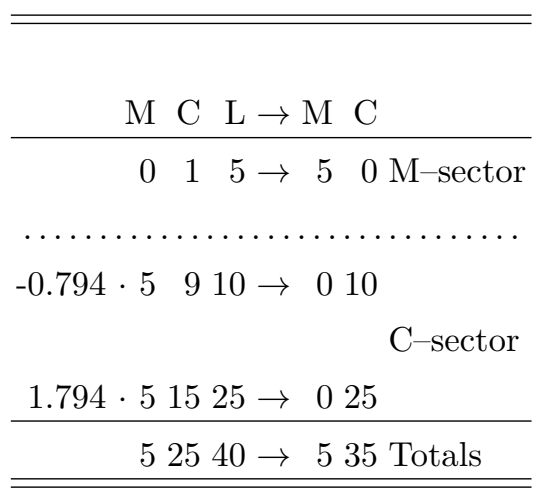

Table 11.3: The two-sector corn economy with Sraffa's vintage prices

$$
\begin{aligned}
\widetilde{v}_{c} & \approx 3.886, \widetilde{v}_{c}^{\prime} \approx 4.046, \quad v_{c}=\frac{10 \widetilde{v}_{c}+25 \widetilde{v}_{c}^{\prime}}{35}=4, \\
v_{m} & =1.8 \quad v_{o}=(1.794) \cdot v_{m} \approx 3.229 .
\end{aligned}
$$

In the example with falling efficiency, our distinction between individual and average labor values ruled out negative labor values, unlike in Steedman's approach. In the example with rising efficiency, instead, our definition of $v_{o}$ (and $\widetilde{v}_{c}, \widetilde{v}_{c}^{\prime}$ ) displays the same qualitative features as Steedman's. This is because there is no anomaly in rising book values, as noted above; on the contrary, it is quite natural for the labor value of machine vintages to rise if investment expenditures (implying 'appreciation') exceed depreciation.

We can now consider the three kinds of investment expenditures (a)-(c) listed above. As truncations can be excluded here, there is no difference between them with regard to Sraffa's method of price determination. ${ }^{20}$ But at least when measuring labor value and productivity there might be a difference between the three types of investment. Consider our example of 3 units of corn as "running-in" costs. Setting these costs aside, the two stages of the corn-producing sector can be depicted as follows:

$$
\begin{aligned}
& 6 C+5 M_{\text {new }}+10 L \rightarrow 10 C \\
& 15 C+5 M_{\text {old }}+25 L \rightarrow 25 C .
\end{aligned}
$$

$\overline{20}$ This method only discriminates between these types of investment when they imply different truncations, by representing the combined effect of depreciation allowances and 'appreciation' efforts at each stage by a single number: the (possibly positive) change in book-value. 
Consequently, the depreciation rates for period 1 and 2 are

$$
\delta_{1}=2 / 7 ; \quad \delta_{2}=5 / 7
$$

if we assume proportionality between activity level and depreciation, and regard constant efficiency to be in line with the application of linear depreciation. As concerns the three types of "running-in" costs, we suggest to interpret case (a) as implying appreciation rates $\gamma_{1}=1, \gamma_{2}=$ $0^{21}$ and case (c) as implying (approximately) $\gamma_{1}=2 / 7, \gamma_{2}=5 / 7$.

As shown in subsection 11.8.1, in the case of simple reproduction (average) labor values do not depend upon depreciation rates. But with regard to individual values and equations (9.2)-(9.3) we suggest to define these values in the presence of appreciation by:

$$
\begin{array}{r}
6 v_{c}+\gamma_{1} 3 v_{c}+\delta_{1} 5 v_{m}+10=10 \widetilde{v}_{c} \\
15 v_{c}+\gamma_{2} 3 v_{c}+\delta_{2} 5 v_{m}+25=25 \widetilde{v}_{c}^{\prime} .
\end{array}
$$

In case (a) we get: $\widetilde{v}_{c} \approx 4.857, \widetilde{v}_{c}^{\prime} \approx 3.657$ and $v_{o}=\left[\gamma_{2}\left(3 v_{c}\right)+\right.$ $\left.\delta_{2}\left(5 v_{m}\right)\right] / 5 \approx 1.286$, i.e. rising labor productivity within the process considered and a falling book value.

In case (b) we get: $\widetilde{v}_{c} \approx 3.657, \widetilde{v}_{c}^{\prime}=4=v_{c}$ and $v_{o} \approx 3.686$, i.e. the opposite result as compared to (a).

In case (c) we get: $\widetilde{v}_{c}=\widetilde{v}_{c}^{\prime}=4=v_{c}$ and $v_{o}=3$, which is what Steedman (1977) describes as "correct value accounting" (a special case, where appreciation exceeds depreciation).

But regardless of how $\delta_{i}, \gamma_{i}$ are determined, it is always possible to associate with given depreciation and appreciation coefficients a positive vector of individual values describing productivity changes within the fixed-capital-using process(es) relative to their (positive) averages: the Marxian labor values. It is unnecessary to identify the best depreciation procedures in order to show that labor values and the transfer of labor value from durable means of production to commodity output can be defined without anomalies.

${ }^{21}$ This is probably the only case covered by Sraffa's method of price computation. 



\section{Production Prices and Imperfect Competition, Part I}

\subsection{Introduction}

In Part I of the book, we have shown that in Smith's "early and rude state of society" - in which labor is the only means of productionthe "commodity law of exchange" (Foley 2011; Foley and Mohun 2016) holds and there exist well-defined relationships between labor values and prices, such that one can conclude that labor values determine relative (natural or labor commanded) prices. But this is not true in a capitalist economy with produced means of production, in which natural prices and labor costs diverge in general. The commodity law of exchange is superseded by the "capitalist law of exchange" (Foley 2011; Foley and Mohun 2016), specified as the determination of prices that support an equalized rate of profit. This raises doubts on the standard, predictive interpretation of the LTV as a theory of the determination of relative prices but, as we have argued throughout the book, it does not necessarily mean that the classical-Marxian theory of prices and values is irremediably flawed.

In particular, in the previous chapters, we have argued that Marxian labor values - conceived of as the real labor costs of producing commodities - are formally well-defined, theoretically sound and empirically meaningful magnitudes. And this conclusion is not limited to simple Leontief economies: we have extended the definition of labor values to general economies with multiple activities, joint production, fixed capital, and so on. Contrary to the received view, although labor values do not explain prices, they are theoretically important to understand the laws and dynamics of capitalist economies, and in this role they are an integral part of the United Nations' SNA. We have not offered a solution to the transformation problem because, in our view, no transformation from value to price magnitudes is either meaningful or necessary, and therefore no "problem" arises.

In this chapter, and the next, we return to the classical-Marxian 
theory of production prices. In Chapters 4 and 5, we proved that production prices are well-defined and can be generalized to $n$-sector linear economies - including pure joint production-by means of the Perron-Frobenius theory of nonnegative matrices. Therefore, as far as the valuation of different commodities is concerned, the Classical theory of restless competition analyzed in Part I provides a theory of longperiod natural prices as centers of gravity for market prices, and the foundations for an analysis of income distribution as the product of class conflict, rather than merely technological factors (unlike in the neoclassical approach).

In the classical long-period approach analyzed in Chapters 4 and 5, production prices entail the equalization of profit rates and wage rates across sectors. Conceptually, this is meant to reflect the idea that prices of production are centers of gravity of market prices and emerge due to competitive pressures that tend to remove any sectoral differences in wage and profit rates. Empirically, however, the evidence does not clearly support the existence of a strong tendency towards profit rate or wage rate equalization. To the contrary, a number of empirical studies, and our own analysis of the German economy 1991-2000 suggest that intersectoral differences are persistent and indeed show a remarkable stability over time.

In this chapter, we extend the classical production price equations to incorporate the existence of persistent, and stable, wage and profit rate differentials. We show that, under the usual assumptions on technology, the generalized production prices are unique, well-defined and strictly positive. Moreover, all of the standard results of Classical price theory - including the existence of a negative relation between wages and profits-continue to hold.

\subsection{Differentiated sectoral wage and profit rates}

The standard von Neumann/Sraffa concept of Classical production prices is based on two fundamental assumptions. First, competitive forces dominate, at least in the long period, and capital and labor mobility tend to remove all sectoral differences in wage and profit rates. Second, fixed capital should be conceived of as a perfectly marketable joint output of the various production processes and the (uniform) profit rate should be computed on circulating capital only. These assumptions make the von Neumann/Sraffa model extremely elegant but they are both theoretically and empirically rather questionable. For one thing, "Secondhand capital goods are notoriously hard to value as they are often single items for which markets are quite thin" (Desai 
2015, 226). But then it seems doubtful to treat them as perfectly marketable jointly produced outputs. ${ }^{1}$

For another, even setting aside the treatment of fixed capital, and granting that the profit rate should be computed only based on circulating capital, the assumption of uniform wage and profit rates across sectors is empirically doubtful. On the one hand, there is the wellknown problem of different sectoral turnover times of the intermediate inputs, that is, the time it takes until capital outlays are recovered. On the other hand, sectoral profitabilities will differ for various systematic reasons, including differential risk, and perhaps more importantly a number of persistent distortions of competition related to the degree of concentration, the extent of entry barriers, and the degree of collusion between firms.

Several empirical studies have shown persistent differences in sectoral profit rates, including among many others, Semmler (1984, 106) and Duménil and Lévy (2002). In the latter study, for example, the authors find that in the U.S., industries with very large capital-labor ratios are totally different from other industries in that they persistently earn an extremely low rate of profit. Similarly, as shown in Chapter 8 we have shown that a strikingly persistent structure of significantly different sectoral profits characterizes the German economy (19912000). Although we compute profitability focusing on fixed capital, it is not difficult to show that a persistent heterogeneity in sectoral profit rates remains even if one focuses on circulating capital.

Sectoral wage rates do not seem to show any clear tendency towards uniformity, either. This is true even if they are aggregated across larger sectors, as in our 7-sectoral standard aggregation. To get a first impression of the sectoral differentiation of wage rates, we compile the basic data for Germany in Table 12.1, for the year 1995 and with respect to our standard aggregation.

The differences in the sectors' (average) wage rates and the ranking of sectors should not come as a great surprise. Nonetheless, it is worth stressing that all wage rates are somewhat biased downward. The $L_{i^{-}}$ statistics in Table 12.1 include independent businessmen, whereas $W_{i}$ are the wage payments to employees only. The ratios $w_{i}=\mathrm{W}_{i} / \mathrm{L}_{i}$ will therefore be subject to a certain degree of measurement error especially in sectors $i=1$ and, to a lesser degree, $i=6$, the agricultural and consumer services sectors, respectively. This should not affect our main

\footnotetext{
${ }^{1}$ Further, as argued in Chapter 8 , the theoretically and empirically relevant distinction in computing production prices is not between fixed and circulating capital but rather between capital advanced and capital consumed.
} 

(1)
(2)
(3)
(4)
(5)
(6)
(7)

Agrc. Manf. Oth.Mf. Cstrt. Bus.Svcs. Cns.Svcs. Soc.Svcs. Sum

\begin{tabular}{llllllll}
\hline$W_{i}: 9382$ & 99663 & 196801 & 78819 & 180355 & 178708 & 253172 & 996900 \\
$L_{i}: 1117$ & 2301 & 6216 & 3266 & 6272 & 9449 & 8761 & 37382 \\
$w_{i}: 8399$ & 43313 & 31660 & 24133 & 28756 & 18913 & 28898 & 26668
\end{tabular}

Table 12.1: Sectoral price components (Germany, 1995)

Note: $W_{i}, L_{i}, w_{i}$ are, respectively: wage payments, the number of persons (in 1000s) working in the sector, and the sector's average wage rate per year (in Euro), $w_{i}=\mathrm{W}_{i} / \mathrm{L}_{i}$.

conclusions below, however, especially concerning the effect of wage variations, since the ratios of the wage rates of different sectors are assumed to be constant.

To be sure, the empirical evidence on sectoral convergence is not unequivocal and other studies, including in the classicalMarxian tradition, have identified empirically some tendencies towards equalization (see, for example, the recent study by Scharfenaker and Semieniuk (2017)). Yet the analysis of the German economytogether with other empirical work-strongly suggests that it is worth considering departures from the standard long period model of production prices with uniform wage and profit rates.

\subsection{Production prices with persistent wage and profit rate differentials}

The incorporation of differentiated wage and profit rates into the standard production price equations with circulating capital is straightforward. Considering both versions with wages paid ex-post and ex-ante, it reads

$$
\begin{aligned}
& p_{i}=\left(1+r_{i}\right)(p A)_{i}+w_{i} l_{i}, \quad i=1, \ldots, n, \\
& p_{i}=\left(1+r_{i}\right)\left((p A)_{i}+w_{i} l_{i}\right), \quad i=1, \ldots, n .
\end{aligned}
$$

We study this set of relationships from three different angles. First, the profit rates $r_{i} \geq 0$ and the nominal wage rates $w_{i}>0$ are taken as exogenously given and we search for the conditions on the profit rates that permit an economically meaningful solution for the price vector $p$. Second, the real sectoral wage rates are taken as given and we 
characterize the set of profit rates $r_{1}, \ldots, r_{n}$ for which a meaningful price vector $p$ exists. Third, we postulate a fixed structure of profit and wage rates, in the form of constant ratios, and derive a wage-profit frontier in this setting.

Consider the first question. Clearly, the real wage rates are determined as residuals, and should be rather low in the presence of high profit rates $r_{i}$. In the special case of equal profit rates, we know that the profit rate must be smaller than the maximum rate $R$. Dropping the equalization assumption, some (but not all) sectors may have a rate above this boundary. But certainly, there should be other upper-bounds (for the sectors producing basic commodities). The following proposition, originally proved by Grillo (1976), derives a precise condition and provides a nice connection to the quantity side.

Proposition 12.1. Let $r_{i} \geq 0$ and $w_{i}>0$ be given in equations (12.1) and (12.2). Then each of the two price systems has a meaningful solution $p \geq 0$ if and only if the sectoral rates of profit are sufficiently low in the following sense: there exists a gross output vector $x \geq 0$, together with a corresponding net output vector $y=x-A x \geq 0$, such that

$$
r_{i} \leq \frac{y_{i}}{x_{i}-y_{i}}=\frac{y_{i}}{(A x)_{i}}, \quad \text { for all } i,
$$

and with strict inequality in at least one $i$.

Proof. First let $q_{i}=1+r_{i}$ and observe that $r_{i} \leq y_{i} /\left(x_{i}-y_{i}\right)$ is equivalent to $q_{i} \leq x_{i} /\left(x_{i}-y_{i}\right)$. Furthermore, let $Q$ be the diagonal matrix with entries $q_{i}$ and let $\tilde{l}_{i}=w_{i} l_{i}$. Then equation (12.1) can in compact form be written as $p=p A Q+\tilde{l}$. As for equation (12.2), simply specify $\tilde{l}_{i}=\left(1+r_{i}\right) w_{i} l_{i}$. We use the fact that the solution $p=\tilde{l}(I-A Q)^{-1}$ exists and is semi-positive if and only if the dominant eigenvalue of $A Q$ is less than unity.

To demonstrate that the proposition's condition is necessary, suppose $\lambda^{\star}(A Q)<1$. Then there exists $d \in \mathbb{R}^{n}, d \geq 0$, such that $(I-A Q) d \geq 0$. Next, let $x=Q d$ and $y=(I-A) x$. We thus get $0 \leq(I-A Q) d=(I-Q) d+(I-A) Q d=(I-Q) d+(I-A) x=$ $d-Q d+y=Q^{-1} x-x+y$ and, by premultiplication with $Q$, $x-Q(x-y) \geq 0$. Considering this vector inequality component-wise and dividing the components by $x_{i}-y_{i}$ if the expression is positive it yields the condition $\left(x_{i}-y_{i}\right.$ is nonnegative anyway, so that the inequality sign is maintained; if $x_{i}-y_{i}=0, q_{i}$ and thus $r_{i}$ may be arbitrarily large).

To show the reverse, suppose the condition is satisfied. Accordingly, let $x, y$ be two semi-positive vectors related by $x=A x+y$ that entail 
$q_{i} \leq x_{i} /\left(x_{i}-y_{i}\right)$ for all $i$, where at least one inequality is strict (and $x_{i}-y_{i}=0$ is admitted). Since $x_{i}-y_{i}=(A x)_{i} \geq 0$, it is easily seen that the inequalities are equivalent to $x-Q^{-1} x \leq y$. Setting $d=$ $Q^{-1} x$, this in turn is equivalent to $y-(Q-I) d \geq 0$. Thus we obtain $y=x-A x=Q d-A Q d=(Q-I) d+(I-A Q) d$, and furthermore $(I-A Q) d=y-(Q-I) d \geq 0$. Since $d \geq 0, \lambda^{\star}(A Q)<1$ follows.

Therefore, in each sector $i$ the profit rate must not exceed the "surplus ratio", which is given by the ratio of the final demand $y_{i}$ for good $i$ over the good's material inputs that are required by this and the other sectors to produce the economy's net product $y \in \mathbb{R}_{+}^{n}$. Note, however, that $x$ and $y$ need not be the quantities actually produced and demanded: any appropriately chosen vectors may do. In particular, with $x=(I-A)^{-1} y$ the condition for equations (12.1) and (12.2) to have a meaningful solution $p \geq 0$ may be rewritten as

$$
r_{i} \leq R_{i}=R_{i}(y) \equiv \frac{y_{i}}{\left[A(I-A)^{-1} y\right]_{i}} \quad \text { for all } i,
$$

with strict inequality for at least some $i$.

Observe that in the presence of nonbasics, condition 12.3 allows for $(A x)_{i}=0$, in which case $r_{i}$ may become arbitrarily large. If equation (12.3) holds with equality for all $i$, then $w_{1}=w_{2}=\ldots=w_{n}=0$ in equation (12.1) or (12.2). ${ }^{2}$ In this case, with respect to an underlying (hypothetical) net output vector $y$, the sectoral rates of profit $r_{i}=$ $R_{i}(y)$ are maximal in the sense that no sector can possibly achieve a higher profit rate, unless another sector lowers its rate (this intuition is verified below). In other words, a sector's maximum rate of profit depends on the other sectors' profit rates, and generally also on their distribution; it will make a difference whether two profit rates $r^{a}$ and $r^{b}$ are, in that order, prevailing in sector $j$ and $k$, or in sector $k$ and $j$.

The set $\mathcal{R}$ of all maximum sectoral rates of profit can be described by letting the vector $y$ vary over a suitable (by normalization) subset of the nonnegative orthant in $\mathbb{R}^{n}$, say a simplex. We then have the characterization

$$
\mathcal{R}=\left\{\left(r_{1}, \ldots, r_{n}\right): \exists y \in \mathbb{R}_{+}^{n} \text { with } \sum_{j} y_{j}=1 \text { and } r_{i}=R_{i}(y) \forall i\right\} .
$$

A sector $i$ 's maximum of all its (conditional) maximum rates of profit is obtained if all other sectors earn zero profits. It can be computed by setting $y=e_{i}$, i.e.

\footnotetext{
${ }^{2}$ Formally, it suffices to replace the inequality signs with an equality sign in the proof of Proposition 12.1.
} 


$$
\max _{y} R_{i}(y)=R_{i}\left(e_{i}\right)
$$

which entails $r_{j}=R_{j}\left(e_{i}\right)=0$ for all other sectors $j \neq i$. In general the $R_{i}\left(e_{i}\right)$ will not be equal across sectors.

In a two-sector world, for example, the set $\mathcal{R}$ traces out a downwardsloping curve in the $\left(r_{1}, r_{2}\right)$-plane with intercepts $\left(0, R_{2}\left(e_{2}\right)\right)$ and $\left(R_{1}\left(e_{1}\right), 0\right)$. The inner points of the curve can be computed by letting $y_{1}$ vary from 0 to 1 and setting $y_{2}=1-y_{1}, r_{1}=R_{1}(y), r_{2}=R_{2}(y)$. Since the $R_{i}(y)$ are fractions of the $y$-components, the connection between the two end-points will not be a straight line. Applying this procedure to the empirical two-sectoral matrix $A_{T}$ in equation (1.8)-which we treat as the relevant matrix $A$-yields the outer curve in Figure 12.1. This geometric locus of the sectoral maximum rates of profit is clearly concave. The area below this boundary represents the set of all sectoral profit rates $\left(r_{1}, r_{2}\right)$ that are a priori admissible in equations (12.1) and (12.2).

\subsection{Production prices assuming real wage rates to be given}

We now turn to the second point in the analysis of equations (12.1) and (12.2), and assume real wages to be exogenously given. Measuring them again in terms of a wage basket $c \in \mathbb{R}_{+}^{n}$, let $\omega_{i}$ be the corresponding real wage rates, so that

$$
w_{i}=\omega_{i} p c, \text { all } i
$$

Equations (12.1) and (12.2) can be more compactly written if we define a matrix $C=\left(c_{i j}\right) \equiv \omega_{j} c_{i} l_{j}$, with $i, j=1, \ldots n$, which allows us to write the row vector $\left(w_{1} l_{1}, \ldots, w_{n} l_{n}\right)$ conveniently as $p C$. Entry $c_{i j}$ is the quantity of good $i$ consumed by workers that are employed in producing one unit of good $j$, for which they spend a fraction of their nominal wage $w_{i} l_{i}$.

Let $\mathbf{r}=\left(r_{1}, \ldots, r_{n}\right)$ and let $Q(\mathbf{r}) \equiv \operatorname{diag}\left\{1+r_{1}, 1+r_{2}, \ldots, 1+r_{n}\right\}$ be the diagonal matrix with entries $1+r_{i}$. Equations (12.1) and (12.2) can be reformulated as

$$
\begin{aligned}
& p=p(A Q(\mathbf{r})+C) \\
& p=p((A+C) Q(\mathbf{r})) .
\end{aligned}
$$

It is clear that for equations (12.7) and (12.8) to admit a meaningful solution, wages must not be too high. This means that a suitable production vector $x$ should exceed the material inputs and the 


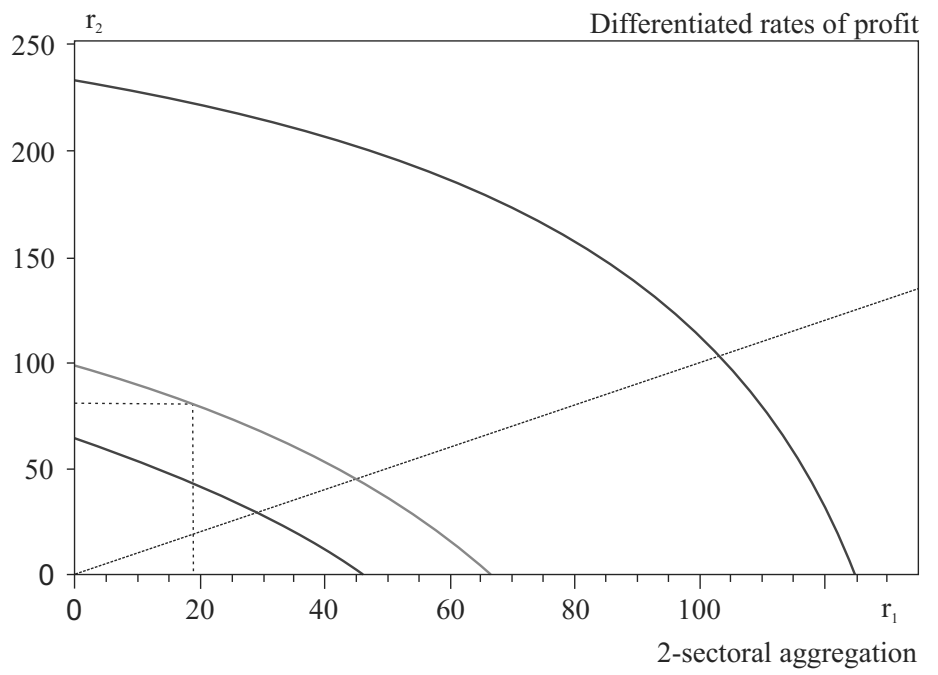

Figure 12.1: Differentiated sectoral rates of profit under exogenous real wage rates

Note: With respect to the two-sectoral aggregation for $A=A_{T}$ in Table 1.8, the outer curve is the set of sectoral maximum rates of profit. The middle curve represents the set $\mathcal{R}(\boldsymbol{\omega})$ in proposition 12.2 , where $\boldsymbol{\omega}=\left(\omega_{1}, \omega_{2}\right)$ are the empirical real wage rates expressed in the consumption basket $c$ (see Table 12.2), while the lower curve depicts the same set for a $25 \%$ increase in real wages. The dotted lines perpendicular to one another indicate the empirical profit rates in $1995, r_{1}=r_{2}$ on the dash-dotted line starting at the origin, and $r_{1}=r_{2}=45.99 \%$ at the intersection of the middle curve with the diagonal straight line identifies the empirical average rate of profit.

workers' consumption associated with it. The matrix augmented by the consumption coefficients, $A+C$, must be productive. Proposition 12.2 establishes the existence of solutions and the trade-off of the sectoral profit rates discussed above in the case with zero wages: one rate can only rise if some other rate decreases. This relationship is strict if $A+C$ is assumed to be indecomposable, which seems plausible at least with positive wages.

Proposition 12.2. Let $\boldsymbol{\omega}=\left(\omega_{1}, \ldots, \omega_{n}\right)$, and suppose that $A+C$ is productive and indecomposable. Then the set $\mathcal{R}(\boldsymbol{\omega})$ of all sectoral profit rates $\mathbf{r}=\left(r_{1}, \ldots, r_{n}\right)$ fulfilling equations (12.7) or (12.8), respectively, is a non-empty one-dimensional manifold in $\mathbb{R}_{+}^{n}$ where for every $\mathbf{r} \in$ 
$\mathcal{R}(\boldsymbol{\omega})$, the corresponding price vector $p$ is strictly positive. Choosing any sector $k$, its rate of profit $r_{k}$ is represented by a differentiable function $f_{k}$ of the other profit rates,

$\mathbf{r} \in \mathcal{R}(\boldsymbol{\omega}) \quad$ if and only if $\quad r_{k}=f_{k}\left(r_{1}, \ldots, r_{k-1}, r_{k+1}, \ldots, r_{k}\right)$.

Furthermore, all partial derivatives of $f_{k}$ are negative (given that $r_{k}>$ $0)$,

$$
\partial r_{k} / \partial r_{j}=\partial f_{k} / \partial r_{j}<0 \quad \text { for } j \neq k
$$

Proof. We formulate the proof with respect to equation (12.7). The treatment of equation (12.8) is analogous.

The important thing to note is that, by virtue of the indecomposability assumption, an increase (decrease) of any profit rate $r_{i}$ causes the dominant eigenvalue of $A Q(\mathbf{r})+C$ to increase (decrease) strictly. Moreover, a solution of equation (12.7) exists if $\mathbf{r}$ induces $\lambda^{\star}[A Q(\mathbf{r})+C]=1 ; p$ is then the left-hand eigenvector, which is strictly positive. Thus, one may begin with a sufficiently small vector $\mathbf{r}$ such that $\lambda^{\star}[A Q(\mathbf{r})+C]<1$, which is possible since the productivity assumption implies $\lambda^{\star}[A Q(0)+C]<1$. Then any sector $i$ may be chosen and its profit $r_{i}$ increased until the dominant eigenvalue equals unity.

Increasing a rate $r_{i}$ in a situation where $\mathbf{r} \in \mathcal{R}(\boldsymbol{\omega})$ raises the eigenvalue above 1 . It can be brought back to this level by sufficiently lowering $r_{k}$. Existence of a function $f_{k}$ and their partial derivatives follows from applying the Implicit Function Theorem to the equation in $\mathbf{r}, F(\mathbf{r}) \equiv \operatorname{det}[\lambda I-A Q(\mathbf{r})-C]=0$, where the eigenvalue $\lambda$ is fixed at $\lambda^{\star}=1$ (and since the determinant is differentiable to any desired order).

Proposition 12.2 says that a function $f_{k}$ with the stated property exists, but not that $f_{k}$ can be expressed in closed form. In fact, the computation of $r_{k}$ involves (iteratively) solving an eigenvalue equation, so that in practice one has to resort to numerical methods.

The proof of Proposition 12.1 goes equally through if one replaces $\tilde{l}$ with the zero vector, the inequality signs with the equality sign, and the matrix $A$ with the augmented matrix $A+C$. Then one obtains the situation of equation (12.8). The profit rates satisfying equation (12.8) can thus be readily characterized by the next proposition, where the concept of 'net output' now includes workers' consumption.

Proposition 12.3. Suppose that the matrix $A+C$ is productive. Then for the sectoral profit rates $\mathbf{r}=\left(r_{1}, \ldots, r_{n}\right)$ a price vector $p \geq 0$ 
satisfying equation (12.8) exists if and only if there exists a gross output vector $x \geq 0$, together with a corresponding 'net output' vector $y=x-A x-C x \geq 0$, such that

$$
r_{i}=\frac{y_{i}}{x_{i}-y_{i}}=\frac{y_{i}}{(A x)_{i}+(C x)_{i}}, \quad \text { for all } i .
$$

In a two-sector world, the frontier of the maximum sectoral rates of profit of Proposition 12.1 is illustrated by the outer curve in Figure 12.1. We want to do the same for Proposition 12.2, focusing on wages paid ex-post. To this end, we have to determine empirical values for the two-dimensional vectors $l, c$ and $\boldsymbol{\omega}$. This is done in Table 12.2 which focuses on Germany in 1995 and aggregates all industries into Manufacturing and Services. We also add the two-sectoral rates of profit (which correspond to the $r_{i}^{(1)}$ for the 7-sectoral aggregation in Table 12.1, and yield the same average rate of profit). We normalize the consumption basket $c$ such that $p c=p_{1} c_{1}+p_{2} c_{2}=1\left(p_{1}=p_{2}=1\right.$ for 1995), and so the wage rates $w_{i}$ reflect directly the real wages, $\omega_{i}=0.001 \cdot w_{i}$ (given that the quantities are measured in 1 mill. Euro in 1995-prices). Hence $r_{i}=r_{i}^{(1)}$ are the resulting profit rates for wages paid ex-post.

\begin{tabular}{lcc}
\hline & \multicolumn{2}{c}{ Sector } \\
& $(1)$ & $(2)$ \\
& Manufacturing & Services \\
\hline$c_{i}:$ & 32.950 & 67.050 \\
$l_{i}:$ & 9.420 & 14.060 \\
$w_{i}:$ & 29.819 & 25.008 \\
$r_{i}:$ & 18.950 & 82.060 \\
\hline
\end{tabular}

Table 12.2: Empirical two-sectoral data for equation (12.7) (Germany, 1995)

Note: The components of the consumption vector are in percent; $l_{i}$ are persons per 1 mill. Euro output; $w_{i}$ are given in 1000 Euro per job; the profit rates $r_{i}$ are defined by $r_{i}=r_{i}^{(1)}=\left[p_{i}-(p A)_{i}-\right.$ $\left.w l_{i}\right] /(p A)_{i}$, where $p=(1,1)$ for 1995 , and $A=A_{T}$ from equation (1.8).

Equipped with these data, we can determine the consumption matrix $C$ and the set of sectoral profit rates $\mathcal{R}(\boldsymbol{\omega})$ for this special case 
of equation (12.7). More specifically, we fix $r_{1}$ successively at different values and solve equation (12.7) for the corresponding value of $r_{2}$, which is the value that causes the dominant eigenvalue of $A Q(\mathbf{r})+C$ to be unity. In the notation of Proposition 12.2, this procedure yields the function $r_{2}=f_{2}\left(r_{1}\right)$.

It may, however, be noted that in a broader perspective another approach is more useful, which applies an iterative method. To begin with, it - correctly - presupposes that an algorithm is available to compute the dominant eigenvalue of semi-positive matrices. Given $r_{1}$, we exploit the fact that the function $\phi=\phi\left(r_{2}\right) \equiv \lambda^{\star}\left[A Q\left(r_{1}, r_{2}\right)+C\right]-1$ is strictly increasing in $r_{2}$ and choose two, possibly extreme, values $r_{2}^{a}$ and $r_{2}^{b}$ that entail $\phi\left(r_{2}^{a}\right)<0$ and $\phi\left(r_{2}^{b}\right)>0$. Then a straight line is drawn from $\left(r_{2}^{a}, \phi\left(r_{2}^{a}\right)\right)$ to $\left(r_{2}^{b}, \phi\left(r_{2}^{b}\right)\right)$ and the value $r_{2}^{c}$ is determined where it intersects the zero line. If $\phi\left(r_{2}^{c}\right)<0, r_{2}^{a}$ is replaced with $r_{2}^{c}$, if $\phi\left(r_{2}^{c}\right)>0$, $r_{2}^{b}$ is replaced with $r_{2}^{c}$. After that, a new round is started. In this way the points of intersection approximate, step by step, the (unique) value $r_{2}$ at which the function $\phi\left(r_{2}\right)$ vanishes up to any desired degree of precision. The procedure just described is the regula falsi, which for well-behaved functions such as our $\phi$ is well known to converge quite rapidly, and certainly. ${ }^{3}$

The adoption of regula falsi allowed us to derive the middle curve in Figure 12.1, which is the geometric locus $\mathcal{R}(\boldsymbol{\omega})$ of all pairs of profit rates $r_{1}$ and $r_{2}$ that are compatible with the empirical wages and consumption structure in equation (12.7). The curve is situated considerably below the frontier of the sectoral maximum rates of profit. The dotted lines indicate the two profit rates from Table 12.2 that actually prevailed in 1995. The dash-dotted line depicts equal rates of profit $r_{1}=r_{2}$, and it shows how much the empirical configuration deviates from the stylized uniform rate of profit. The cross identifies the point where $r_{1}=r_{2}=45.99 \%$, which is just the average rate of profit in 1995. This point is very close to the middle curve. Hence the error made by hypothesizing a uniform rate of profit in equation (12.7) is very small, if we compare the resulting profit rate with the empirical average rate of profit. In contrast, the associated prices will be quite different from the empirical prices $p_{1}=p_{2}=1$. Finally, the lowest curve in Figure 12.1, below the middle line, is the set $\mathcal{R}(\boldsymbol{\omega})$ that results from a uniform $25 \%$ increase in the empirical real wage rates. It shows that the sectoral profitabilities would be severely affected by such a (hypothetical) event, unless it is compensated by falling labor coefficients.

\footnotetext{
${ }^{3}$ See, for example, Press, Flannery, Teukolsky, and Vetterling $(1986,248)$.
} 


\subsection{Distributive conflict and the wage-profit frontier}

In closing this chapter, consider the third point, namely a study of the wage-profit relationship. If for that purpose profit and wages are each to be represented by a single variable, we need to make some changes. The most natural assumption in this respect is to postulate constant ratios of the sectoral wage and profit rates. ${ }^{4}$

To formalize this idea, choose an arbitrary reference sector $k$. We hypothesize constant ratios $q_{i}^{r}=r_{i} / r_{k}$ for the sectoral rates of profit, and $q_{i}^{w}=w_{i} / w_{k}$ for the sectoral wage rates. Equation (12.1) can thus be rewritten as ${ }^{5}$

$$
p_{i}=\left(1+r_{k} q_{i}^{r}\right)(p A)_{i}+w_{k} q_{i}^{w} l_{i} .
$$

Next, define the diagonal matrices

$$
Q_{k}^{r}=\operatorname{diag}\left[q_{1}^{r}, q_{2}^{r}, \ldots, q_{n}^{r}\right], \quad Q_{k}^{w}=\operatorname{diag}\left[q_{1}^{w}, q_{2}^{w}, \ldots, q_{n}^{w}\right] .
$$

Hence equation (12.9) becomes

$$
p=p A\left(I+r_{k} Q_{k}^{r}\right)+w_{k} l Q_{k}^{w} .
$$

Before inquiring into the effects of variations of the profit rate, we must determine the maximum value $\tilde{R}_{k}$ that $r_{k}$ can attain. ${ }^{6}$ The formal determination of $\tilde{R}_{k}$ is slightly different from that of the maximum uniform rate of profit in Chapter 3 . To derive $\tilde{R}_{k}$, set $w_{k}=0$ in equation (12.11) and let $r_{k}=\tilde{R}_{k}$. This gives us $p(I-A)=\tilde{R}_{k} p A Q_{k}^{r}$. Postmultiplying both sides by $(I-A)^{-1}, \tilde{R}_{k}$ is the reciprocal of the dominant eigenvalue of the (semi-positive) matrix, $A Q_{k}^{r}(I-A)^{-1}$, i.e.:

$$
\tilde{R}_{k}=1 / \lambda^{\star}\left[A Q_{k}^{r}(I-A)^{-1}\right] .
$$

The special case $Q_{k}^{r}=I$ of uniform profit rates, in which $R=$ $\tilde{R}_{k}=1 / \lambda^{\star}\left[A(I-A)^{-1}\right]$, is equivalent to equation (3.15) in Chapter 3 , $R=\left[1-\lambda^{\star}(A)\right] / \lambda^{\star}(A)$. To verify this, abbreviate $\tilde{\lambda}=\lambda^{\star}\left[A(I-A)^{-1}\right]$ and note that the eigenvalue equation $\tilde{\lambda} p=p A(I-A)^{-1}$ is equivalent

${ }^{4}$ Giannini (1976) is a precursor of our approach but he still assumes wages to be uniform.

${ }^{5}$ The case with ex ante wage payments is treated analogously and therefore omitted.

${ }^{6}$ We use a tilde to underline the difference with $R_{i}$ in equation (12.3). In fact, $\tilde{R}_{k}$ depend on the distribution of the other sectors' profit rates, so that $\tilde{R}_{k}=\tilde{R}_{k}\left(Q_{k}^{r}\right)$ in general. 
to $\tilde{\lambda} p(I-A)=p A \Longleftrightarrow \tilde{\lambda} p=(1+\tilde{\lambda}) p A \Longleftrightarrow[\tilde{\lambda} /(1+\tilde{\lambda})] p=p A=$ $\lambda^{\star}(A) p$, from which it follows that $\tilde{\lambda}=\lambda^{\star}(A) /\left[1-\lambda^{\star}(A)\right]=1 / R$.

Letting $w_{k}=1$ and treating $r_{k}$ as the exogenous distribution variable, equation (12.11) can be solved for the prices in terms of sector $k$ 's labor commanded,

$$
p^{w}=p^{w}\left(r_{k}\right)=l Q_{k}^{w}\left[I-A\left(I+r_{k} Q_{k}^{r}\right)\right]^{-1}, \quad 0 \leq r_{k}<\tilde{R}_{k} .
$$

The real wage rate of sector $k$, measured in the consumption basket $c \in \mathbb{R}_{+}^{n}$, is given by

$$
\omega_{k}=\omega_{k}\left(r_{k}\right)=1 / p^{w}\left(r_{k}\right) c .
$$

The effects of an increase of $r_{k}$ are the same as for an increase in the uniform profit rate in equation (3.13) if $A$ is indecomposable: it increases all entries of the inverse matrix in equation (12.13), which entails that the real wage rate $\omega_{k}$ is a strictly decreasing function of $r_{k}$, showing the class conflict over distribution.

\subsection{Conclusions}

In this chapter, we have extended the classical-Marxian theory of production prices to incorporate the existence of persistent, and stable, wage and profit rate differentials. We have shown that, under the usual assumptions on technology, the generalized production prices are unique, well-defined and strictly positive. Moreover, all of the standard results of production price theory - including the existence of a negative relation between wages and profits-continue to hold. In other words, the key insights of classical-Marxian approach hold even if the empirically unrealistic assumption of uniform profit and wage rates is relaxed. In line with the general approach of this book, this chapter develops what may be called an empirically-informed theoretical concept of production prices.

Although our analysis in this chapter is significantly more general than the standard von Neumann/Sraffa approach (and reduces to the latter in the special case where $w_{i}=w$ and $r_{i}=w$ for all $i$ ), it still suffers from two important limitations: we have neglected capital depreciation, and we have defined the profit rate as a mark-up on intermediate inputs, rather than fixed capital. We extend our formal and conceptual framework in order to incorporate these topics in the next chapter. 



\section{Production Prices and Imperfect Competition, Part II}

\subsection{Introduction}

The choice of techniques and the introduction of technological innovations by profit maximizing firms are central features of advanced economies. They play a fundamental role in theories of value and distribution, and, given their relevance for capital theory, they have been at the center of substantial controversy between alternative approaches. Although most of the contributions have focused on theoretical issues, the empirical analysis of actual economies is arguably crucial, and indeed empirically-oriented claims have played an important role in the debate. For example, the theoretical possibility of paradoxes in capital theory, such as 're-switching' and 'reverse capital deepening', highlighted by the neo-Ricardian school has long been acknowledged, but critics have often argued that such phenomena are unlikely to be found in actual economies. ${ }^{1}$

In Classical theory, the so-called "wage-profit curves" play a prominent role in the analysis of choice of techniques and technical innovations, from both the theoretical and the empirical viewpoint. The empirical wage-profit curves of individual countries have been analyzed, among the others, by Krelle (1976), Ochoa (1987), Cekota (1988), and Silva (1991), who have adopted a circulating capital approach, under the standard assumption of uniform wage and profit rates in all sectors, in order to inquire into the existence of paradoxes in capital theory. ${ }^{2}$ More recently, the circulating capital approach has been significantly generalized by Han and Schefold (2006) who have analyzed the empirical wage-profit curves of nine OECD countries in the period 1986-1990. The main methodological innovation of Han and Schefold (2006) is not to consider data from one country at a certain

\footnotetext{
${ }^{1}$ See Kurz and Salvadori (1995, Ch. 14) for a review of the debate.

${ }^{2}$ For a more detailed discussion of the empirical literature, see Han and Schefold (2006).
} 
point in time, as a given set of data describing an organic economic system. According to them, in a circulating capital approach it is natural to assume that a given process used in a country at a certain date - say, $\left(A_{\star j}, l_{j}\right)$, where $A_{\star j}$ is the $j$-th column of the IO matrix $A$ and $l_{j}$ is the $j$-th entry of the vector of labor coefficients $l$-is also available in principle in a different country at a different point in time. Therefore they implement pairwise comparisons of the IO datasets - for example, they compare Germany 1990 and Canada 1990 - which gives two possible processes for each of the $n$ industries and thus $2^{n}$ possible economy-wide techniques, or mixed IO matrices. On the basis of this type of mixed IO matrices, they calculate the envelopes of wage-profit curves and find the first (limited) evidence supporting the empirical relevance of paradoxes in capital theory.

This chapter focuses on the core theoretical and methodological issues raised by the empirical analysis of wage-profit curves and by the measurement of prices of production. Three main contributions emerge. First, some general shortcomings of empirical studies based on the circulating capital approach with uniform wage and profit rates are shown. It is argued that the appropriate description of alternative techniques should include the capital that must be advanced, or tied up, in a production process (as well as the associated replacement investment). An analysis based on the circulating capital approach arguably does not capture the complexity of the production structure of the economy, with different techniques coexisting in every given period and capital tied up in old production processes, and the determinants of investment decisions. Further, the concept of profit rate that is relevant for capitalists' decisions should arguably focus on fixed, rather than circulating capital, and capital stock matrices should be an essential ingredient in the analysis of prices of production and the associated wage-profit curves.

The shortcomings of an approach neglecting capital stocks are particularly evident in Han and Schefold's comprehensive and original study. The composition of processes from various countries and various times into an artificial intermediate IO structure is of a very hypothetical nature, and it is actually illegitimate if the capital stock matrices underlying the single processes are taken into account. Their analysis is arguably misleading from an empirical perspective, because their results are based on wage-profit curves that are irrelevant for the analysis of the actual choice of technique. Yet, the neglect of capital stock matrices is a common shortcoming of empirical analyses of classical price theory.

Further, as argued in Chapters 8 and 12 the empirical evidence 
provides little support to the standard assumption of uniform sectoral profit rates. This suggests that conventional analyses based on standard production price equations may be misleading, and the assumption of uniform profit rates may not be innocuous in empirical work, especially in economies characterized by persistent and significant noncompetitive features, and imperfections in capital markets. This point was forcefully made by Farjoun and Machover (1983), who argued that the restriction of classical price theory to the consideration of a uniform rate of profit is utterly unrealistic. This is particularly evident if sectoral disaggregation is very high, because the structure of IO tables - which are based on averaging procedures - then starts to show the degree of heterogeneity that characterizes the production of the various commodities.

Second, following the original insight of Farjoun and Machover (1983), an alternative theoretical framework to analyze production prices and the distributive conflict between capital and labor is provided, which can be used to derive empirical wage-profit curves and some testable theorems concerning the relation between prices, distribution, and technical change. To be precise, we argue that the standard production prices should be generalized to allow for the introduction of sectoral profit and wage rate differentials, as well as capital stocks. ${ }^{3}$ A general system of prices of production is set up, under the assumption of fixed intersectoral wage and profit differentials, and a negative relation between profits and wages is derived, thus showing the existence of a generalized wage-profit curve. It is also proved that costreducing technical change can lead to Pareto-improvements (a shift in the generalized wage-profit curve) at the modern margin of the economy, but the empirical IO analysis is complicated by the presence of old techniques alongside new, more efficient ones.

Third, an empirical application of the theoretical framework to the German economy (1991-1999) is presented. We show that the empirical wage-profit curves are fairly close to straight lines - a result stressed by Shaikh in various contributions (e.g. Shaikh and Tonak (1994) and Shaikh (1998)) which confirms the conclusions in section 4.8.2 and extends them to the analysis of fixed capital. Further, the order of techniques is such that a sufficiently big decline in wages would lead the economy 'back in time' to older techniques; only in some of the years considered is the economy positioned on the envelope of the wage-profit curves; and the realized income distribution tuples of subsequent years

\footnotetext{
3 The introduction of capital stocks in the definition of prices of production is not novel. It figures prominently, for example, in the approach proposed by Shaikh (1998). In the latter contribution, however, wage and profit rate differentials are not considered.
} 
have always been outside the wage-profit curves of previous years. In our context, this result is not based just on factor substitution, but also (more importantly) on the occurrence of technical change.

Two important caveats are worth making at this point. Firstly, the main aim of this chapter is not to adjudicate the debate on the empirical relevance of 're-switching' and 'reverse capital deepening'. The focus of the chapter is primarily methodological: it suggests an alternative, general approach to classical price theory, which takes into account capital stocks and allows for persistent profit and wage rate differentials. From this viewpoint, the discussion of the German economy (1991-1999) does not aim to be exhaustive, or fully rigorous from an econometric viewpoint. The dataset constructed by Kalmbach et al. (2005) is used to illustrate the main theoretical points and the implications of the approach proposed, and the empirical results should be taken as a first step towards a more detailed analysis. Secondly, as argued in Chapter 8, the treatment of capital stocks is notoriously difficult and a number of strong assumptions must be made in order to construct appropriate data. Yet, we believe that neither the assumptions nor the findings are unreasonable, and in any case the theoretical and methodological point stands: the presence of capital stocks tied up, or advanced, in the various production processes raises important issues - which are not easily accommodated in the standard framework, e.g. by allowing for joint production-and it is preferable to try to deal with the construction of a capital stock matrix, however imperfect, rather than to assume the issue away.

\subsection{Neo-Ricardian theory and empirical analysis}

In this section, two main shortcomings of the standard analysis of prices of production and wage-profit curves are highlighted. In order to illustrate the core methodological points, consider the extensive empirical study by Han and Schefold (2006). In the latter paper, thirtytwo 36-sectoral IO tables and the corresponding vectors of labor inputs, from the OECD database (for nine countries from the period 1986-1990) serve as data in order to investigate paradoxes of capital theory from the empirical point of view. Let the standard notation hold, where $A$ is the $n \times n$ nonnegative IO matrix, $l$ is the $1 \times n$ positive vector of direct labor inputs, $p$ is the $1 \times n$ price vector, $r$ is the profit rate, and $w$ is the wage rate. To each IO table Han and Schefold apply the conventional definition of prices of production

$$
p=(1+r) p A+w l
$$


in order to calculate the associated wage-profit curve $r(w)$. Han and Schefold compare the thirty-two 36-sectoral datasets in pairs: for example, in their Table 1 they compare Germany 1990 and Canada 1990. When mixed together, this yields two processes for each industry and thus $2^{36}$ possible economy-wide techniques, or mixed IO matrices, on the basis of which the envelopes of the wage-profit curves can be calculated.

This approach has some desirable features. The interpretation of the IO structures $(A, l)$ as sets of interchangeable techniques is arguably a natural extension of the standard circulating-capital approach and it yields a very large amount of data, because there are $\left(\begin{array}{l}32 \\ 2\end{array}\right)$ pairs of mixed IO matrices. The adoption of the standard production price equations greatly simplifies the computation of wage-profit curves, and the empirical analysis in general, thanks to their relatively simple mathematical structure. Yet, two main problems raise doubts on the theoretical robustness and empirical relevance of Han and Schefold's analysis and more generally on the standard approach.

First, the neglect of capital stocks is arguably a major shortcoming in the analysis of choice of techniques, technological innovation, and investment decisions of profit maximizing firms. It may be objected that the absence of fixed capital is only due to the lack of appropriate data and that fixed capital can be incorporated within the standard Sraffa-von Neumann approach, by treating it as a joint product. Yet, this assumption has long been argued to be empirically questionable, especially from the viewpoint of the actual behavior of firms. In fact, the treatment of fixed capital as a joint product in production price equations presupposes the existence of perfectly competitive markets for used machines. This is rather doubtful as fixed capital is arguably not a jointly marketable product. ${ }^{4}$ Further, the notion of the sectoral rates of profit implied by the standard approach has two major shortcomings from the economic point of view, which go beyond the unreasonably high values reported by Han and Schefold (2006). The specification of $r$ in the production price equations above neglects replacement investment and, perhaps more importantly, it relates profits to the flow of material inputs rather than to the sectors' capital stock that ties up the money invested. Instead, Leontief's (1953) almost forgotten concept of a capital stock matrix seems to provide a more appropriate starting point for the analysis of choice of techniques

\footnotetext{
${ }^{4}$ For a more general, and detailed critique, see the stock-flow analysis by
} Bródy (1970, Ch. 1.2) and the related IO literature. 
and technical innovations. For it emphasizes the notions of capital advanced, capital consumed, and the related turnover times as well as the importance of stock-flow accounting in general.

Consider again the dataset constructed by Kalmbach et al. (2005) for the German economy from 1991 to 1999 described in detail in Chapter 1. As argued in the Appendix to Chapter 8, there is no direct information on the coefficients of the capital stock matrix, but data are available in the IO tables that do bear at least some relation with the information needed. In fact, the tables contain data on each sector's total depreciation, which can be used to get an indication of the different levels of capital in place in individual sectors. Moreover, the investment vector that is reported as a component of final demand gives us an approximate idea of the composition of the sectoral capital stocks. Combining this information, we can construct coefficients that, distinguished by goods and sectors, proxy replacement investment. It goes without saying that a number of auxiliary assumptions have to be made in this construction. We believe, however, that they are not unreasonable and in any case it is preferable to tackle the issue of capital stocks, rather than to assume it away. ${ }^{5}$

The capital stock matrix for Germany in 1995 was already shown in Table 8.3; it corresponds to the matrix of intermediate inputs in Table 1.11. Entry $k_{i j}$ designates capital good $i$ in place per unit of output of sector $j$, where $i$ represents plant, equipment, and inventory at different stages of production. That is, $k_{i j}$ is the capital in millions of Euro that is tied up (or advanced) for one year in the production of one million Euro of sector $j$ 's output (in prices of the base year 1995). The last row computes the column sums, which can be viewed as the sectoral capital-output ratios. ${ }^{6}$

If one acknowledges the importance of the capital tied up, or advanced, in a production process, one has to conclude that the compositions of production processes considered in Han and Schefold (2006) have little, if any, economic content. What kind of economies do the mixed intermediate input techniques used in Han and Schefold (2006) (see, for example, Table 1, p.755) represent if the capital that is tied up in the background of their application is in Canada in the case of a German process, and vice versa? Even in the case of our nine years of data for the German economy, what is the meaning of

${ }^{5}$ For a detailed explanation of the methodology used, see the Appendix to Chapter 8 and Flaschel (2009, Ch. 8).

${ }^{6}$ These are the ratios of capital to gross output, and do not include the stock of housing (of consumers). These two features explain the perhaps unfamiliarly low values. 
combining the intermediate inputs of the seven sectors from different years into one single technique, and its hypothetical wage-profit curve, without recognizing that these inputs may be specific to the capital that is tied up in their production process? In general, in empirical and theoretical analyses of the choice of techniques and technical innovation, the appropriate description of techniques should be given by the tuple $\left(A, A^{\delta}, l, K\right)$, where $A^{\delta}$ is the depreciation matrix corresponding to the capital stock matrix $K$, rather than by the simpler structure $(A, l)$ considered in the standard approach, and the actual structure of these matrices in terms of the presence of modern, medium-vintage, and outdated techniques should be explicitly taken into account.

The second major shortcoming of the standard approach concerns the assumption of a uniform rate of profit, for which the empirical evidence for Germany, 1991-1999, provides no justification at all. From Table 8.11 it emerges not only that the assumption of a uniform rate of profit does not hold in Germany in the 1990s, but-even granting the limited length of the time series - also that there is no tendency for profit rates to converge. We admit that capital stock calculations may be fairly inaccurate. There may be a tendency towards the leasing of capital equipments which are therefore tied up in another sector. The construction sector may also exhibit a large amount of semifinished products which should be carefully examined. Such details are however secondary here for the conclusion that the assumption of a uniform rate of profit across these fundamentally different sectors is not of much help in the investigation of the evolution of the German economy. The profit rate differentials emerging from Table 8.11 are too large to be the product of measurement errors. Further, it is worth noting that the above results are in line with other empirical studies. It is particularly striking, for example, that the significant differentials between manufacturing and services rates of return are of the same order of magnitude as those reported by Hopps (2007) for the UK. In general, similar findings are obtained by several authors and for various other countries (see, e.g. Shaikh (2008) and the references therein). ${ }^{7}$ Besides, the assumption of uniform profit rates is likely to become less and less tenable the higher the degree of disaggregation, in particular when it comes to the separation of coexisting modern, average, and outdated processes of production.

In the next section, an alternative theoretical framework is proposed

\footnotetext{
${ }^{7}$ If the standard approach is adopted and profit rates are defined as the ratio of profits over the cost of intermediate inputs, it can be shown that in the German economy profit rate differentials remain significant over the period considered (1991-1999). See Chapter 12 for further discussion.
} 
for the analysis of prices of production and the associated wageprofit curves, which incorporates capital stock matrices and allows for persistent profit (and wage) rate differentials.

\subsection{Theoretical aspects of wage-profit curve measurements}

Let $w_{i}$ denote the nominal wage paid in sector $i, i=1, \ldots, 7$, and let $w_{m}$ denote the nominal wage in an arbitrarily chosen reference sector, $m \in\{1, \ldots, 7\}$; and let a similar notation hold for $r_{i}$ and $r_{m}$, respectively. In this section, we consider prices of production on the basis of given wage and profit rate differentials - respectively, $w_{i} / w_{m}$ and $r_{i} / r_{m}$, fixed in a given base year (say, 1995), between the industries that make up our IO world. The assumption that wage and profit rate differentials do not change over time may seem overly strong, but it can be defended in a number of ways. ${ }^{8}$ The assumption of rigid wage differentials may be justified either along the lines suggested first by Keynes (1936), or just as a first approximation to a fuller analysis, which would endogenize wage differentials and consider their variations over time. The persistence of profit rate differentials, instead, may be due to the existence of lasting noncompetitive factors and imperfections in capital markets. Besides, the assumption of a given hierarchy of profit rates (relative to the rate $r_{m}$ ) can be seen as an empiricallyoriented substitute for the uniform rate of profit assumed in standard neo-Ricardian analyses. ${ }^{9}$

With respect to Table 8.11, we choose a base year (1995) and a reference sector $m$, and fix the then prevailing empirical ratios $q_{i}^{r}=$ $r_{i} / r_{m}$ and $q_{i}^{w}=w_{i} / w_{m}$. This is by no means innocent, since some of the proportions do vary considerably from one year to another, but it seems reasonable as a first approximation. On this basis, we define the following time-independent diagonal matrices:

$$
Q_{m}^{r}=\operatorname{diag}\left\{q_{1}^{r}, q_{2}^{r}, \ldots, q_{n}^{r}\right\}, \quad Q_{m}^{w}=\operatorname{diag}\left\{q_{1}^{w}, q_{2}^{w}, \ldots, q_{n}^{w}\right\} .
$$

These matrices can be used to define to a system of augmented supply

\footnotetext{
${ }^{8}$ For a more detailed defense, we refer to the classic work of Semmler (1984), Mueller (1986, 1990), and Duménil and Lévy (1993).

9 The assumption of fixed wage and profit differentials is - mathematically speaking - a generalization of the Sraffian model which also assumes fixed 'differentials', with all ratios being equal to one. Actually, at this point, we might invoke Han and Schefold's $(2006,749)$ "argument of sufficient rigor in comparison", according to which, although empirical analysis must be as rigorous as possible, it need not be more rigorous than the alternative approach that is criticized, the standard Sraffian approach in our case.
} 
side price equations that take into account the possibility of profit and wage differentials, and explicitly incorporate capital stock and capital stock depreciation matrices: ${ }^{10}$

$$
p=p\left(A+A^{\delta}\right)+w_{m} l Q_{m}^{w}+r_{m} p K Q_{m}^{r}
$$

Equation (13.1) assumes that intersectoral differentials remain constant when the reference wage and profit rates change: in other words, things like the degree of monopoly or market power, the risk structure, the markup structure, etc., are assumed to remain constant in each sector. Thus, for example, if $w_{m}$ rises, all sectoral wage rates (and the general wage rate) increase in constant proportions. And it can be shown that equation (13.1) gives such wage changes more influence on prices in sectors with relatively higher profitability compared to the standard production prices (and vice versa): the same amount of capital advanced has a relatively higher impact on the output price of a sector, the higher the profit rate in this sector. This can be interpreted as reflecting the fact that a bigger market power may give an industry more 'elbowroom' with respect to their output price. Variations in relative wage and profit rates, however, need to be considered in a second step.

Equation (13.1) can be treated similarly to the standard prices of production. ${ }^{11}$ In the rest of this chapter, we assume that the total input matrix $A+A^{\delta}$ is profitable. Formally: ${ }^{12}$

Assumption 13.1. There is a price vector $p>0$ such that $p>p(A+$ $\left.A^{\delta}\right) \geq 0$.

Theorem 13.1 proves that under Assumption 13.1, equation (13.1) has a well-defined solution.

${ }^{10}$ The ratio $K_{i j} / A_{i j}^{\delta}$ can be interpreted as the turnover time $\tau_{i j}$ of the capital stock item $i$ in the industry $j$, where again $i$ may represent machinery, buildings, and the inventories that are related to so-called circulating capital goods (raw materials, etc.). See Bródy (1970) for a detailed discussion of such turnover times. The matrix $A^{\delta}$ is analyzed in more detail in Flaschel (2010).

${ }^{11}$ In the base year 1995, where the nominal and real magnitudes of the IO tables coincide, equation $(13.1)$ is indeed satisfied with $p=(1, \ldots, 1)$ for the given wage rate $w_{m}$ of this year.

12 Since this chapter is methodological in orientation, we do not deal with issues of semi-positivity here, but assume that everything is expressible with strict vector inequalities. For a discussion of the properties of nonnegative input matrices, see Chapter 1 and the references therein. 
Theorem 13.1. Under Assumption 13.1, there is a strictly positive number $r^{\max }$, such that for all $r_{m} \in\left[0, r^{\max }\right)$ prices in sector-m labor commanded are positive and given by

$$
p^{w}=p^{w}\left(r_{m}\right)=l Q_{m}^{w}\left(I-A-A^{\delta}-r_{m} K Q_{m}^{r}\right)^{-1} .
$$

Proof.

1. By Assumption 13.1, it follows that $A+A^{\delta}$ is nonnegatively invertible and its inverse can be expressed as in the standard Leontief multiplier formula

$$
\left(I-\left(A+A^{\delta}\right)\right)^{-1}=\sum_{\tau=0}^{\tau=\infty}\left(A+A^{\delta}\right)^{\tau} .
$$

Therefore, the matrix $K Q_{m}^{r}\left(I-A-A^{\delta}\right)^{-1}$ is also nonnegative.

2. Setting $w_{m}=0$, an eigenvalue equation is obtained which allows us to compute sector $m$ 's maximum rate of profit $r^{\max }$ as the reciprocal of the dominant eigenvalue $\bar{\lambda}$ of the nonnegative matrix $K Q_{m}^{r}\left(I-A-A^{\delta}\right)^{-1}$, i.e.: $r^{\max }=1 / \bar{\lambda}\left[K Q_{m}^{r}\left(I-A-A^{\delta}\right)^{-1}\right]$.

3. Therefore, for all $r_{m} \in\left[0, r^{\max }\right)$ the matrix $K Q_{m}^{r}\left(I-A-A^{\delta}\right)^{-1}$ is characterized by the following vector inequality

$$
\bar{p}\left(A+A^{\delta}+r_{m} K Q_{m}^{r}\right)<\bar{p},
$$

where $\bar{p}>0$ is the dominant eigenvector associated with the eigenvalue $\bar{\lambda}$. This implies that the matrix $B=A+A^{\delta}+r_{m} K Q_{m}^{r}$ is also nonnegatively invertible and its inverse can be expressed as in the standard Leontief multiplier formula (13.3).

Theorem 13.1 generalizes the standard framework, but it does not trivially replicate standard results. For Assumption 13.1 focuses only on $A$ and $A^{\delta}$, but no restriction is imposed, for example, on $K$. Based on Theorem 13.1, the real wage rate $\omega_{m}$ of sector $m$, measured in terms of a given consumption basket $c$, can be defined as follows:

$$
\omega_{m}=\omega_{m}\left(r_{m}\right)=1 / p_{w}\left(r_{m}\right) c
$$

with $\sum_{i} c_{i}=1$, so that the unit is 1000 Euro per job per year.

Equations (13.2) and (13.4) provide a general framework for the analysis of 'normal' prices with frozen relative positions between the workers and between the capitalists of the different sectors and the corresponding wage-profit curve. We suggest that this approach (based on the notions of capital consumed and capital advanced) can be 
empirically relevant when, for example, the consequences of a change in the base rate $r_{m}$ are investigated. The next two theorems derive some properties of the generalized price system concerning both variations in income distribution in a given technological environment, and the distributional effects of technical change at the 'modern margin' of the economy, which may not be easily detected through the use of IO tables (including labor inputs, depreciation matrices, and capital stock matrices) as they are currently measured.

Theorem 13.2 analyzes price changes and income distribution tradeoffs primarily around the actual position of the economy, and for the actual set of production processes activated in a given year (with the different age structure of the capital advancements that represent fixed capital items and that underlie the averages reported in IO data).

\section{Theorem 13.2.}

(1) The price vector $p_{w}\left(r_{m}\right)$ is strictly increasing in the reference profit rate $r_{m}$.

(2) The real wage rate $\omega_{m}\left(r_{m}\right)$ is strictly decreasing in the reference profit rate $r_{m}$.

Proof. Part 1. The result directly follows from Theorem 13.1, noting that by applying the Leontief multiplier formula to the matrix $A+A^{\delta}+$ $r_{m} K Q^{r}$, one obtains:

$$
p_{w}=l Q_{m}^{w} \sum_{\tau=0}^{\tau=\infty}\left(A+A^{\delta}+r_{m} K Q_{m}^{r}\right)^{\tau} .
$$

Part 2. The result follows from part 1 and equation (13.4).

Theorem 13.2 shows that the standard distributive trade-off characterizing class conflict in capitalist economies holds also in our generalized framework, for a given technological and market structure. Our next task is to investigate the effect of technical change on the wage-profit curve and on the structure of distributive conflict. Let us assume that new techniques (indexed by an asterisk *) are entering one, some, or even all of the sectors of the economy such that the following inequality holds ${ }^{13}$

$$
p_{j}>p\left(A_{\star j}^{*}+A_{\star j}^{\delta *}\right)+w_{m} l_{j}^{*}\left(w_{j} / w_{m}\right)+r_{m} p K_{\star j}^{*}\left(r_{j} / r_{m}\right),
$$

for all sectors $j$ where innovation occurs. The profit rate of a new entrant is therefore larger than the average profit rate of incumbents

$\overline{13}$ This type of technical change is analyzed in more detail in Chapter 7. 
whose capital advancements are not yet fully depreciated. Theorem 13.3 characterizes the effect of technical change on prices and distribution.

Theorem 13.3. Let $\omega^{*}$ and $p_{w}^{*}$ denote, respectively, the real wage rate and the price vector defined by equations (13.2) and (13.4), where the averaging techniques $\left(A, A^{\delta}, l, K\right)$ are replaced by the new production processes $\left(A^{*}, A^{\delta *}, l^{*}, K^{*}\right)$. For any given $r_{m}$ :

$$
p_{w}^{*}\left(r_{m}\right)<p_{w}\left(r_{m}\right) \quad \text { and } \quad \omega_{m}^{*}\left(r_{m}\right)>\omega_{m}\left(r_{m}\right) .
$$

Proof. The inequalities

$$
p_{j}>p\left(A_{\star j}^{*}+A_{\star j}^{\delta *}\right)+w_{m} l_{j}^{*}\left(w_{j} / w_{m}\right)+r_{m} p K_{\star j}^{*}\left(r_{j} / r_{m}\right),
$$

can be transformed into equalities again (with the given prices $p$ )

$$
p_{j}=p\left(A_{\star j}^{*}+A_{\star j}^{\delta *}\right)+w_{m} l_{j}^{*}\left(w_{j} / w_{m}\right)+r_{m} p \tilde{K}_{\star j}^{*}\left(r_{j} / r_{m}\right),
$$

by appropriately increasing one or more entries of $K_{\star j}^{*}$ to form a new vector $\tilde{K}_{\star j}^{*}$. This gives rise to the vector equation

$$
p=p\left(A^{*}+A^{\delta *}\right)+w_{m} l^{*} Q_{m}^{w}+r_{m} p \tilde{K}^{*} Q_{m}^{r},
$$

where the innovating sectors use the new production processes only. Using the reference wage as the numéraire to find prices in sector- $m$ labor commanded and rearranging terms gives us an equation similar to $(13.2)$

$$
p_{w}=p_{w}\left(r_{m}\right)=l Q_{m}^{w}\left(I-A^{*}-A^{\delta *}-r_{m} \tilde{K}^{*} Q_{m}^{r}\right)^{-1},
$$

which in turn implies

$$
\begin{aligned}
p_{w} & =l Q_{m}^{w} \sum_{\tau=0}^{\tau=\infty}\left(A^{*}+A^{\delta *}+r_{m} \tilde{K}^{*} Q_{m}^{r}\right)^{\tau} \\
& >l Q_{m}^{w} \sum_{\tau=0}^{\tau=\infty}\left(A^{*}+A^{\delta *}+r_{m} K^{*} Q_{m}^{r}\right)^{\tau} \\
& =p_{w}^{*} .
\end{aligned}
$$

Inserting this into equation (13.4) completes the proof.

Theorem 13.3 states that the immediate replacement of all old processes by innovative techniques allows for Pareto improvements in the distribution of income between capital and labor, and for falling 
prices of production. Note that, in the actual calculation of averaging IO tables, the new techniques are added to the ones that are not yet fully depreciated, i.e. the wage-profit curve of the actual IO table is different from $\omega_{m}^{*}\left(r_{m}\right)$, because in the computation of the latter curve old techniques (of the innovating sectors) are not included: $\omega_{m}^{*}\left(r_{m}\right)$ can be obtained from standard IO tables only when the old techniques of the innovating sectors have become extinct. Therefore the wage-profit curves of actually observed IO systems are hybrid, in the sense that they mix old and new technologies in some averaging way. As a result, although empirical wage-profit curves do provide interesting insights on the choice of techniques and technical innovations in actual economies (as shown, for example, in the next Section), they should be used with caution, keeping in mind that technical change only works at the margin of the IO data, so to speak. It is also not at all obvious whether the outward shift in the curve $\omega_{m}^{*}\left(r_{m}\right)$ proved in Theorem 13.3 is correctly mirrored by the wage-profit curve (13.4) of the actual IO tables, again owing to the hybrid character of the latter.

\subsection{Wage-profit curve measurement: An example}

In this section, the wage-profit curve definition (13.4) is applied to the German economy 1991-1999 as characterized by the IO structures discussed above. There are in principle 9 such curves emerging from the data, but for the sake of visual clarity, Figure 13.1 only shows four of them. The results we obtain in this way are astonishingly straightforward and can be generalized to the remaining five wageprofit curves not shown in Figure 13.1.

Firstly, Figure 13.1 shows that wage-profit curves are fairly close to straight lines, independently of the choice of scaling, a result already stressed by Shaikh in a number of contributions (see, e.g. Shaikh and Tonak (1994) and Shaikh (1998)). Secondly, to a certain degree the actual positions of the economy in the four years considered (marked by a filled square) reflect the business cycle of the period and therefore need not be ordered in a monotonic fashion as far as profit rates are concerned. Nonetheless, they are by and large monotonically increasing in the wage rate as time goes by. But the important result here is that the realized income distribution tuples of subsequent years have been always outside the wage-profit curves of previous years. Only the year 1999 is positioned on the envelope of the wage-profit curves shown, and the envelope itself is made of the wage-profit curves of 1991 and 1999 only. In our context, this result does not depend only on factor substitution, but also (more importantly) on technical change. 
Finally, the order of techniques is such that-in pairwise comparisons, but not always on the envelope - a sufficiently big decline in the wage $\omega_{m}$ would lead us back in time from 1999 to 1996 to 1993 to 1991. This finding might seem difficult to interpret in the generalized framework adopted in this chapter, because unlike in the standard approach, the relation between capital intensity and the slope of the generalized wage-profit curves is not immediately evident, owing to the presence of the markup structures incorporated in the matrices $Q_{m}^{w}, Q_{m}^{r}$ (which, as noted above, reflect a number of factors such as degrees of monopoly, risk structures, relationships to new markets, and so on). Yet, as in the standard approach, in the linear case shown in Figure 13.1, the slopes of the wage-profit curves of different years can be meaningfully compared in terms of capital intensities (see Appendix 13.6). Thus, in Figure 13.1, flatter lines would indicate less capital intensive techniques.

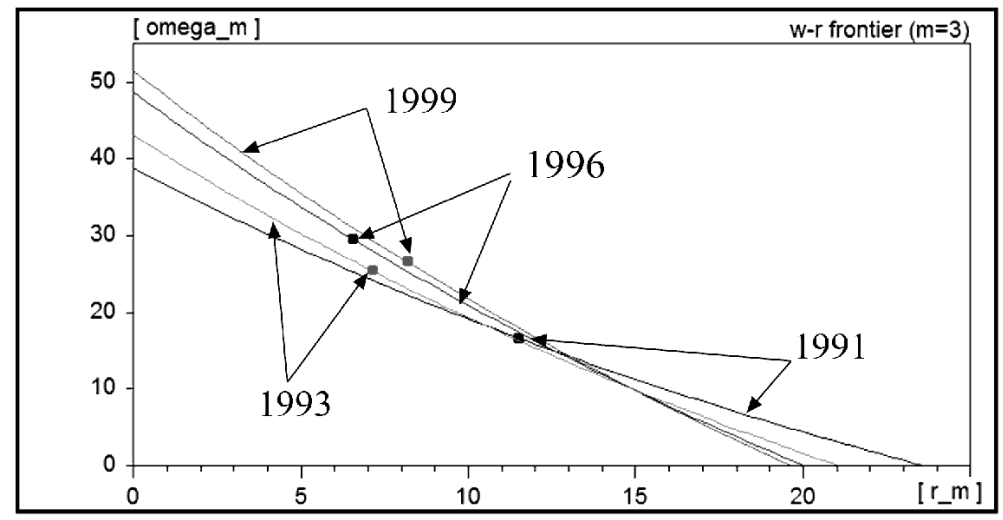

Figure 13.1: Four wage-profit curves and their envelope

Therefore, if a sufficiently big decrease of the wage rate did take place, we would tentatively describe the underlying situation, and its implications, as follows. Each industry has capital tied up in its production process in the form of modern, medium-vintage, and old production processes. In the form of a time-to-rebuild process, we would then expect a return back to older processes so that the profile of processes that characterizes a given industry would start shifting back to older production structures or even techniques that are no longer in use. An example where this happens is the low-wage segment of actual economies where labor-using capital-saving processes can be observed.

Along these lines, empirically-based calculations of wage-profit curves can be meaningful, unlike in a framework where wage and profit 
rates are assumed to be uniform, and certainly unlike in an analysis based on the arbitrary compositions of intermediate input structures from different countries and different times, as in Han and Schefold (2006). We conclude that a Leontief-Bródy approach (augmented by profit rate differentials) may be more appropriate for applied analysis than the purely theoretically-oriented Sraffa-von Neumann approach.

\subsection{Conclusions}

The standard approach to classical price theory is arguably unsatisfactory from an empirical viewpoint. Both the neglect of capital stock matrices - showing the amount of capital tied up, or advanced in production processes - and the assumption of uniform wage and profit rates seem unrealistic. Our alternative proposal for the measurement of empirically relevant wage-profit curves (and the prices of production underlying them) is based not only on the addition of a capital stock matrix $K$ (and a capital stock depreciation matrix $A^{\delta}$ ), but also on the recognition that persistent differences in wage and profit rates may be a characteristic of actual economies, as in the German economy from 1991 to 1999 . The assumption of uniform wage and profit rates greatly simplifies the calculation of prices of production, and the wage-profit curves associated with them, but, as already argued by Farjoun and Machover (1983), it may be rather misleading in applied work.

It may be objected that the IO structures $\left(A, l, K, A^{\delta}\right)$ also provide a set of blueprints from which firms may choose new IO combinations from their sectoral perspective, so that the analytical procedure of Han and Schefold (2006) can still be applied. In our view, such an argument is not entirely compelling.

Firstly, there is the problem of the depreciation of old production activities. Such processes may be slow and hide the role of the most modern techniques in the actual input-output data. Even if all aggregation problems within highly aggregated IO tables were ignored, old and new production activities coexist for some time, so that the reported IO structures are necessarily averages over coexisting vintages of techniques. Secondly, in the presence of fixed capital, investment criteria may be quite complex, ${ }^{14}$ and in any case they are not based on the comparison of economy-wide averaging wage-profit curves. They should have the implication of providing more room for income distribution for capital and labor through technical change, but this implication cannot be used as a criterion for the choice of technique. Finally, empirical wage-profit curves can only be defined in

\footnotetext{
${ }^{14}$ We have formulated an averaging example in Theorem 13.3.
} 
a meaningful way if actual wage and profit rate differentials are taken into account and thus they are by no means pure technologically-based constructs.

The wage-profit curves defined in this chapter are ex-post constructs that show the implications of changes in the baseline profit rate for a certain scheme of production prices (with a given set of wage and profit rate differentials) and for the associated real wage. The analysis holds for a given technology of a given economy in a given year. On this basis it is also possible to analyze (as in Theorem 13.3) how technical change modifies the scope of distributive conflict between capital and labor. Our Figure 13.1 shows in this regard that the realized income distribution tuples of subsequent years have been always outside the wage-profit curves of previous years.

\subsection{Appendix 1: Linear wage-profit curves and capital intensities}

In this Appendix, we generalize the standard equivalence relations holding in economies in which prices are proportional to labor values and the wage-profit curves are linear. These results are interesting per se, in the context of classical price theory, but they also provide formal support to our claims concerning the interpretation of the empirical wage-profit curves in Section 13.4 above.

In order to simplify the notation, let $\bar{l} \equiv l Q_{m}^{w}$ and $\bar{K} \equiv K Q_{m}^{r}$. Firstly, we prove that if prices are proportional to labor values then $\bar{l}$ is the eigenvector associated with the highest eigenvalue of the matrix $D=\left(I-A-A^{\delta}\right)^{-1} \bar{K}$. Formally, we prove that if $p=\gamma \bar{l}\left(I-A-A^{\delta}\right)^{-1}$, some $\gamma>0$, then $\delta \bar{l}=\bar{l} D$, some $\delta>0$. By substituting $p=\gamma \bar{l}(I-$ $\left.A-A^{\delta}\right)^{-1}$ into equation (13.1), one obtains $\gamma \bar{l}=w_{m} \bar{l}+r_{m} \gamma \bar{l}(I-A-$ $\left.A^{\delta}\right)^{-1} K Q_{m}^{r}$, or $\frac{\left(\gamma-w_{m}\right)}{\gamma r_{m}} \bar{l}=\bar{l}\left(I-A-A^{\delta}\right)^{-1} K Q_{m}^{r}$. Letting $\delta=\frac{\left(\gamma-w_{m}\right)}{\gamma r_{m}}$, and noting that $\bar{l}>0$ the desired result follows.

Secondly, and more importantly for the analysis in Section 13.4, it immediately follows from the previous result that if $p=\gamma \bar{l}(I-A-$ $\left.A^{\delta}\right)^{-1}$, some $\gamma>0$, then $(i) \frac{d w_{m}}{d r_{m}}=-k$; and $(i i) p \bar{K}=k \bar{l}$, where $k=\gamma \delta>0 .{ }^{15}$ Thus, if prices are proportional to labor values then the wage-profit curve is linear and in all sectors the following equality holds

${ }^{15}$ The converse implications can be proved with a suitable modification of the argument developed by Kurz and Salvadori (1995, Ch. 4.3) in the standard framework. 


$$
\frac{(p \bar{K})_{i}}{\bar{l}_{i}}=k, \text { for all } i .
$$

The left-hand side of the latter expression can be interpreted as a measure of capital intensity in sector $i$. In fact, the labor vector $\bar{l} \equiv$ $l Q_{m}^{w}$ might be considered as a specific solution of the classical problem related to the reduction of different types of labor by means of the given wage differentials. The vector $\bar{l}$ captures direct labor inputs adjusted for wage differentials and it might be interpreted as labor in 'efficiency units'. Similarly, the capital matrix $\bar{K} \equiv K Q_{m}^{r}$ might be interpreted as providing a measurement of the capital stocks in 'efficiency units' (based on relative profitability), and from this perspective, $k$ might then be considered as a measure of capital intensity in 'efficiency units'.

Although these considerations suggest a promising line for further research, we do not pursue them here and they are unnecessary to establish the claims in Section 13.4. In fact, the comparison of the slopes of the linear wage-profit curves of an economy in different years is now more transparent. Let $k^{j}$ be the slope of the linear wage-profit curves in year $j, j=1,2$. Since $Q_{m}^{r}$ and $Q_{m}^{w}$ are diagonal and time invariant matrices, it follows that

$$
k^{1}<k^{2} \Longleftrightarrow \frac{(p K)_{i}^{1}}{l_{i}^{1}}<\frac{(p K)_{i}^{2}}{l_{i}^{2}} \text {, for all } i .
$$

Noting that prices are proportional to labor values, the ratios in equation (13.7) might be naturally interpreted as a generalization of the standard measure of capital intensities, as argued in the analysis of the empirical wage-profit curves in Section 13.4.

\subsection{Appendix 2: Capital consumed and capital advanced}

In this section we briefly elaborate on the concepts of capital advanced and capital consumed that we have alluded to several times in the book. In the standard approach, capital goods are differentiated into fixed and circulating ones: given a certain base period - say, one monthcirculating capital includes goods that are fully depreciated during the production period (and thus have a depreciation rate of $\delta=1$ ) while fixed capital goods do not fully depreciate $(\delta<1)$. Noting that the choice of production period is largely arbitrary and does not reflect technological relationships, it should be clear that the distinction between fixed and circulating capital is both arbitrary and artificial. Further, rate of return calculations have an institutional nature, as they are conventionally calculated on a yearly basis, a convention which may 
change over time. 'Natural' prices should thus be investigated focusing on their 'natural' time period of calculation, i.e. on a yearly basis. If this empirically motivated choice of 'the period of production' is accepted, then it is not meaningful to assume wages to be paid ex post. Instead, wages, circulating capital, and fixed capital must all be advanced at least to some extent. From the institutional perspective of a yearly evaluation of the activities of firms, concerning their inputs and outputs and the rate of returns, the proper distinction is between capital advanced (on average) and capital consumed for any particular year for any item that is used as an input.

These considerations have relevant implications for production prices. In order to focus on the distinction between capital consumed and capital advanced we shall set aside intersectoral profit and wage differentials and consider a simple two-sector economy with a consumption good and an investment good (machine). Assume that machines are subject to 'radioactive decay': they remain unchanged in quality, but a portion $\delta$ of them disappears without scrapping costs from the sphere of production every year. Capital advanced (per unit of output and continuously reproduced) and capital consumed in the sector of machine production are then represented by $k_{11}$ and $\delta k_{11}$. Setting $p_{2}=1$, in the sector of machinery production the price equation is:

$$
p_{1}=r p_{1} k_{11}+r w k_{01}+\delta p_{1} k_{11}+\tau_{0} w k_{01},
$$

where the parameter $k_{11}$ corresponds to the notation $a_{11}$ used so far, while $k_{01}$ is different from $l_{1}\left(=\tau_{o} k_{01}\right)$, since wages advanced are just $1 / 24$ of wages consumed in the production of one unit of machinery during the year, if wage payments are made on a monthly basis. Ex post payment of wages now at best applies to each month, so that wage funds have to be accumulated monthly to allow wage payments at the beginning or end of each month. Approximately, firms thus keep a stock of funds corresponding to $1 / 24$ of yearly wage payments, i.e. there is some capital tied up to guarantee the timely payment of wages within each 'production' - or, better, 'accounting' - period. Besides $\delta$ we thus have a parameter $\tau_{o}$ that relates wage funds $w k_{01}$ held on average to yearly wage payments $w l_{1}$ per unit of output. A similar relationship applies to the consumption goods sector, where

$$
p_{2}=1=r p_{1} k_{12}+r w k_{02}+\delta p_{1} k_{12}+\tau_{0} w k_{01} .
$$

We assume $\tau_{o}, \delta$ to be uniform throughout the economy for the sake of simplicity. 


$$
K=\left(\begin{array}{cc}
k_{11} & k_{12} \\
0 & 0
\end{array}\right), \quad k_{0}=\left(k_{01}, k_{02}\right) .
$$

The production price equations can be written as

$$
p=\left(p_{1}, p_{2}\right)=(r+\delta) p K+\left(r+\tau_{0}\right) w k_{0} .
$$

Using the former notation $A, l$, equation (13.9) would instead read:

$$
p=(r+\delta) p A+\left(r / \tau_{0}+1\right) w l
$$

This equation confirms that part of wage payments must be considered as advanced capital and should be taken into account accordingly in the calculation of the (uniform) yearly rate of profit $r$. The solution to equation (13.8) is

$$
p=\left(r+\tau_{0}\right) w k_{0}(I-(r+\delta) K)^{-1},
$$

which has a unique solution under standard productiveness assumptions and, for example, given $r$ and $p_{2}=1$.

In a two-commodity economy one cannot distinguish machinery and intermediate inputs and treat them as two separate aspects of the circular flow of capital. We therefore compare the above analysis of machinery and labor inputs into the production of machinery and consumption goods with the situation where intermediate inputs are combined with labor in the two production activities.

In the case of intermediate inputs, say 'corn', one has to distinguish the corn consumed in production in a given year per unit of output from the 'corn' that must be kept as average inventory for an uninterrupted process of production. If corn is ordered for example on a weekly basis in order to allow continuous production, then average inventories of 'corn' are approximately $1 / 104$ of the corn that is consumed in production during a year. More generally, we assume that $k_{1 j}, j=1,2$ represents the amount of corn tied up on average in the production of commodity $j$, while $\tau_{1} k_{1 j}$ (again with uniform $\tau_{1}$ for simplicity) is the amount of corn consumed per unit of output $\left(\tau_{1}=104\right.$ in our example). The price equations for intermediate inputs $j=1$ and consumption goods $j=2$ are:

$$
\begin{aligned}
p_{1} & =r p_{1} k_{11}+\tau_{1} p_{1} k_{11}+r w k_{01}+\tau_{0} w k_{01}, \\
1= & p_{2}=r p_{1} k_{12}+\tau_{1} p_{1} k_{12}+r w k_{02}+\tau_{0} w k_{02},
\end{aligned}
$$

or, using the notation in equation (13.9) 


$$
p=\left(r+\tau_{1}\right) p K+\left(r+\tau_{0}\right) w k_{0} .
$$

Equation (13.11) the same structure as equation (13.10), with the sole distinction that $\delta<1$ holds in the former, while $\tau_{1} \geq 1$ in the latter. The apparently technological distinction between fixed and circulating capital is thus not very relevant and should be replaced by the distinction between capital advanced and capital consumed where the turnover time $1 / \tau$ or $1 / \delta$ may be larger or smaller than the accounting period of one year. ${ }^{16}$

${ }^{16}$ For a more detailed discussion of capital turnover times, see Flaschel (2010). 


\title{
14. Conclusions
}

\begin{abstract}
A scientific theory cannot confine itself to dealing with what is directly observable, to the exclusion of abstract theoretical concepts. The attempt to expunge theoretical concepts, such as labourcontent, from economic theory, leaving only directly observable quantities, such as prices, is a manifestation of instrumentalism, an extreme form of empiricism, which is destructive of all science. Without the concept or labour-content, economic theory would be condemned to scratching the surface of phenomena, and would be unable to consider, let alone explain, certain basic tendencies of the capitalist mode of production (Farjoun and Machover 1983, 97).
\end{abstract}

In the received view, Marxian economics is an object of archaeological interest. The argument goes as follows: the key, distinctive tenet of Marxian economics, the labor theory of value, is at best irrelevant, and likely logically inconsistent. Because the LTV provides the foundations for most of Marx's economic theoriesincluding the theory of exploitation, the theory of accumulation, and the theory of income distribution - and the language in which most of his economic models are expressed-including, for example, the so-called "schemes of reproduction" - then the whole of Marxian economics "is, with a few exceptions, intellectually dead" (Elster 1986, $60)$.

This conclusion is shared by many heterodox economists, most notably Sraffians. According to them, the so-called "transformation problem" arose because Ricardo and Marx saw the LTV as the only practical way to resolve the analytical difficulties posed by the interdependence of prices and distribution. Linear algebra and simultaneous equations now enable us to resolve those same difficulties in a robust fashion, without relying on the LTV. The transformation problem is thereby rendered moot. It no longer is a problem, because there is no longer any need to transform labor values into prices of 
production; indeed, there is no longer any need for labor values at all. The basic class conflict that characterizes capitalism can be rigorously analyzed using the classical theory of production prices, and without appealing to the esoteric notions of exploitation, surplus value, and labor content.

Three main arguments support this widespread negative view on the LTV. First, it is well known that apart from very special cases, (relative) labor values are different from (relative, production) prices. Therefore labor values cannot possibly do what they are meant to do in the standard predictive interpretation of Marx's LTV: they cannot determine, and therefore predict production prices, and the LTV cannot be a theory of equilibrium prices. Besides, and this is the second objection, labor values and prices are determined by the same variables (in particular technology) and information about labor values tells us nothing about what really matters - e.g. for agents' decisions,namely price magnitudes. As Paul Samuelson (1971) famously put it in his 'blackboard theorem', price magnitudes and value magnitudes are independent of each other, with a relation of mutual irrelevance.

The third, fundamental objection is not one of irrelevance but one of logical inconsistency. In his famous book, Marx after Sraffa, for example, Steedman (1977) famously proved that in economies with joint production or fixed capital, both labor values-defined as the standard IO employment multipliers - and aggregate surplus value can be negative even if production prices and aggregate profits are positive. This result confutes the Marxian theory of profits as the product of exploitation, but it also questions the concept of labor values per se. What does it mean to say that the amount of (abstract) labor socially necessary to produce goods is actually negative?

The main aim of this book has been to show that the classical and Marxian approach to economic theory, and specifically the theory of production prices and Marx's LTV are far from dead. Indeed, they can provide interesting insights on the structural features and dynamics of modern capitalist economies. Our conclusions are based both on an alternative - descriptive, rather than predictive -interpretation of the role of value theory, and on a different definition of labor values, which allow us to refute all of the three objections above.

First, we have rejected the standard predictive interpretation of Marxian value theory. One of the key contributions of recent debates in value theory and in exploitation theory, and one of the main arguments of this book, is the idea that there is no 'transformation problem' to be solved in Marx's LTV. Labor values are not meant to provide an explanation of classical production prices. Yet, a coherent account 
of values and the labor content of goods can be provided that is able to inform the empirical and theoretical analysis of the basic laws of capitalism, of the exploitative nature of capitalist relations of production, and of the determinants of the key magnitudes of capitalist economies. Moreover, our approach - and indeed all of the main recent approaches - emphasizes the essential monetary dimension of the economy, and the relation between actual money magnitudes and their labor counterparts. From this perspective, an attempt to 'transform' labor values into production prices is theoretically dubious and empirically meaningless, and the "transformation problem" an irrelevancy.

Second, we have reinterpreted Steedman's (1977) seminal contribution as showing, first, that outside of the simplest Leontief economies the standard employment multipliers do not necessarily coincide with the real costs (in terms of labor) of producing goods, and therefore, second, that there is no obvious definition of labor values in economies with joint production, fixed capital, and so on. Contrary to the received view, Steedman's contribution is therefore not the last nail in the coffin of Marxian value theory. Rather, methodologically, it forces us to reconsider the foundations of value theory and define the properties that a general definition of labor values should possess in the search of a logically consistent and empirically relevant general approach to the LTV.

Third, based on a rigorous reading of Marx's texts, we interpret labor values as indices of the real direct and indirect cost of producing commodities measured in labor units: labor content is the average labor time 'embodied' in a good, in the sense of full-cost accounting in terms of labor time spent on average in the production of commodities. In simple Leontief economies, this is captured by the standard IO employment multipliers. In this book, we propose an extension of this approach to general economies with joint production and fixed capital based on the standard practices of IO theorists and the accounting conventions of profit maximizing firms. In our approach labor values are well defined, logically consistent, nonnegative (and strictly positive for any commodity with positive price) and satisfy several theoretically desirable properties. Labor values are not a logically incoherent construct.

Nor are they irrelevant. For they provide the foundations for a rigorous empirical analysis of capitalist economies. Labor values cannot be used as predictors of production (or market) prices - although, empirically, relative prices are often remarkably close to labor values. In this book, we have shown, however, that a system of labor accounts 
can be developed, which provides theoretically and empirically relevant insights on observed phenomena in capitalist economies. Labor values provide the only theoretically sound measures of sectoral and aggregate labor productivity, and they allow us to theoretically and empirically investigate some deep tendencies of capitalist economies, such as the Law of Decreasing Labor Content. Further, they allow us to develop a dual interpretation of Marx's theory of profits and an explanation of aggregate profitability based on the extraction of relative and absolute surplus value, and the dynamics of labor productivity.

While we have developed a theoretically rigorous and empirically relevant approach to Marx's LTV which holds in general economies with joint production, fixed capital, and so on, we have shown that the standard von Neumann/Sraffa theory of production prices has some important limitations outside the simplest Leontief economies. The von Neumann/Sraffa approach to production prices conceives of fixed capital as a joint product of production processes and assumes that a uniform wage and profit rate emerges in all sectors, where the profit rate is defined as a mark up on circulating capital only. We argue that, first, the appropriate description of alternative techniques should include the capital that must be advanced, or tied up, in a production process (as well as the associated replacement investment). An analysis based on the circulating capital approach arguably does not capture the complexity of the production structure of the economy, with different techniques coexisting in every given period and capital tied up in old production processes, and the determinants of investment decisions. Further, the concept of profit rate that is relevant for capitalists' decisions should focus on fixed, rather than circulating capital, and capital stock matrices should be an essential ingredient in the analysis of prices of production and the associated wage-profit curves. For fixed capital is arguably not a jointly marketable product, and in most cases, there are no markets for used capital goods.

But we have also questioned the empirical relevance of the standard assumption of uniform sectoral wage and profit rates. Several studies, and our own analysis of the German economy (1991-1999) suggest that significant sectoral differences exist and there is little evidence of a tendency towards uniformity. This suggests that conventional analyses based on standard production price equations may be misleading, and the assumption of uniform profit rates may not be innocuous in empirical work, especially in economies characterized by persistent and significant noncompetitive features, and imperfections in capital markets. This point was forcefully made by Farjoun and Machover (1983), who argued that the restriction of Classical price theory to 
the consideration of a uniform rate of profit is utterly unrealistic. This is particularly evident if sectoral disaggregation is very high, because the structure of IO tables - which are based on averaging proceduresthen starts to show the degree of heterogeneity that characterizes the production of the various commodities.

Following the original insight of Farjoun and Machover (1983), and building on the pioneering work of Leontief (1941) on capital stock matrices and Bródy (1970) on the notions of capital advanced and capital consumed, we have developed an alternative theoretical framework to analyze prices of production and the distributive conflict between capital and labor. This approach can be used to derive empirical wage-profit curves and some testable theorems concerning the relation between prices, distribution, and technical change. To be precise, we have argued that conventionally defined prices of production should be generalized to allow for the introduction of sectoral profit and wage rate differentials, as well as capital stocks. We have set up a general system of prices of production, under the assumption of fixed intersectoral wage and profit differentials, and have shown that all of the key insights of classical-Marxian price theory continue to hold, including the existence of a negative relation between profits and wages - a generalized wage-profit curve. Together with our definition of labor values, this provides a general dual frameworkin terms of labor and price magnitudes - to analyze both empirically and theoretically a number of key phenomena occurring in advanced capitalist economies, such as technical change, and its relation with distribution, the dynamics of accumulation and innovation, aggregate profitability, exploitation, and so on.

\subsection{The way forward}

This book is obviously not the last word on Marxian value theory and on the classical-Marxian theory of production prices, and much remains to be done, both empirically and theoretically. For example, we have only briefly touched upon the implications of our definition of values for the Marxian theory of exploitation and class and for the analysis of the class and exploitation status of individual agents. We have analyzed only some aspects of the dynamics of competitive behavior and of the processes of gravitation around production prices. We have completely ignored the Marxian theories of crisis and only mentioned some of the macroeconomic implications of our analysis. The book does provide, however, a general approach to price and value theory which identifies a research programme in the classical-Marxian tradition. In 
closing the book, it is worth summarizing the basic intuition behind our interpretation of Marx's theory.

In a classical-Marxian approach, an economy can be analyzed from three different perspectives - corresponding to Marx's three circuits of capital - focusing either on the physical flow of commodities, or on nominal (price) flow magnitudes, or on abstract labor flows. The structure of our approach to Marx's LTV is conceptually analogous to that of a System of National Accounts, in which real magnitudes form a dual structure with respect to prices and quantities. A real SNA is designed to promote an understanding of the key processes driving the motion of nominal magnitudes. Analogously, Marx's labor value aggregates were designed as the essential elements of an understanding of what happens underneath the surface of the process of capital accumulation. Relating the labor-time oriented SNA to the flow of actually observed (quantity and price) magnitudes is not a 'transformation problem'. Rather, it provides an alternative representation of the economy. And although our interpretation of Marx's LTV is different from the standard predictive view, there is nothing metaphysical about our definition of labor values. Labor accounts provide a framework to understand some of the key magnitudes and dynamic laws of capitalist economies.

In our view, there are close conceptual links between Marxian value theory and the United Nation's (1968) SNA developed by Richard Stone and his collaborators. Stone's formulation of the UN's SNA, for example, contains a general definition of labor productivity of the following type (see United Nations $(1968,69)$ ):

$$
\Lambda^{* * *}=\frac{l(I-A)^{-1}\left(I-A^{*}\right) x^{*}}{l^{*} x^{*}}=\frac{v\left(I-A^{*}\right) x^{*}}{v^{*}\left(I-A^{*}\right) x^{*}} .
$$

If one considers an activity vector $x^{*}$ which produces 1 unit of commodity 1 as net product, such that $A^{*} x^{*}+f=x^{*}, f=$ $(1,0, \ldots, 0)^{\prime}$, then one obtains:

$$
\Lambda^{* * *}=\frac{v f}{v^{*} f}=\frac{v_{1}}{v_{1}^{*}}=\frac{\pi_{1}^{m *}}{\pi_{1}^{m}} .
$$

This is precisely the change in the Marxian concept of labor productivity with respect to commodity 1. In other words, in Stone's work the pragmatism of national accounting has led to the Marxian measure of labor productivity, though nothing of this sort was probably intended when the SNA was established. Our proposal to measure labor productivity by means of the reciprocal of the labor content, or 
total labor costs of producing commodities, is in line both with Marx's discussion in Capital, Vol. I, and with Stone's modern construction of the SNA, even though later revisions of the SNA have returned to an arguably misleading definition of productivity in terms of (real) money value added per work hour.

Perhaps more importantly, Marx's LTV and Stone's formulation of the UN's SNA are similar at a methodological level, and share essentially the same broad vision concerning economic theorizing. Stone's SNA is a rigorous classification system of the activities of an entire economy. It considers many complexities of capitalist economies - including joint production and fixed capital - and attempts to construct both stock and flow matrices that can characterize the evolution of an economy, and real magnitudes like real GDP and labor productivity. The real magnitudes of the SNA are theoretical concepts which capture relevant economic phenomena that underlie nominal magnitudes. Thus, the SNA's real magnitudes are akin to the centers of gravity developed by the classicals and Marx, which serve as reference points for analysis rather than an actual, attainable state of the economy.

Accordingly, our analysis suggests that the UN's SNA is more classical than neoclassical in nature, in the sense that it focuses on average magnitudes rather than on marginal conditions in a perfectly competitive environment. Classical-Maxian production prices exemplify this type of average magnitude, where the uniform profit rate characterizing production prices only comes about through competition over a long period of time. Production prices thus emerge as centers of gravity for the movement in market prices driven by ruthless competition, and serve as an abstract reference point for analysisthey do not depict a concrete state of things. A theory of generalized production prices is thus one of the building blocks for future investigations of the dynamics of competition. The other building block is Marx's LTV, which aims to identify real magnitudes behind nominal ones by focusing on the qualitative concept of 'abstract labor' and on its quantitative expression 'labor content'.

Given this dual representation of the economy, the key issue is not to prove some sort of transformation theorem, but rather to identify the relationships between theoretical accounting definitions used for economic reasoning and the centers of gravity of the actual price-quantity dynamics. Both theoretically and empirically, the aim is to understand the quantitative relationships between defined 'real' value magnitudes and observed nominal variables like profit, wages, value added, and so on. In this book, we have tackled some of these 
relationships, and much remains to be done. Hopefully, we have shown that it is worth doing it. 


\section{References}

Almon, C. (2009). Double trouble: the problem with double deflation of value added and an input-output alternative with an application to Russia. In M. Grassini and R. Bardazzi (Eds.), Energy Policy and Internatinoal Competitiveness. Florence: Firenze University Press.

Armstrong, A. G. (1975). Technology assumptions in the construction of U.K. input-output tables. In W. Gossling (Ed.), Medium Term Dynamic Forecasting. London: Input-Output Publishing Company.

Barna, T. (1975). Quesnay's Tableau in modern guise. The Economic Journal 85, pp. 485-496.

Bidard, C. (1986). Is von Neumann square? Journal of Economics 46 , pp. 407-419.

Blaug, M. (1985). Economic Theory in Retrospect. Cambridge: Cambridge University Press.

BLS (2008). Technical Information about the BLS Major Sector Productivity and Costs Measures. http://www.bls.gov/lpc/lpcmethods.pdf (date last accessed 12 August 2012).

Bortkiewicz, L. v. (1949). On the correction of Marx's fundamental theoretical construction in the third volume of Capital. In P. M. Sweezy (Ed.), Karl Marx and the Close of His System 85 BöhmBawerk's Criticism of Marx. Clifton, NJ: Augustus M. Kelley Publishers.

Bowles, S. and H. Gintis (1977). The Marxian theory of value and heterogeneous labour: A critique and reformulation. Cambridge Journal of Economics 1(2), pp. 173-192.

Bowles, S. and H. Gintis (1981). Structure and practice in the labor theory of value. Review of Radical Political Economics 12(1), pp. $1-26$.

Bródy, A. (1970). Proportions, Prices and Planning: A Mathematical Restatement of the Labor Theory of Value. Amsterdam: NorthHolland.

Cassing, S. (1996). Correctly measuring real value-added. Review of Income and Wealth 42, pp. 195-206. 
Cekota, J. (1988). Technological change in Canada (1961-80): An application of the surrogate wage function. Canadian Journal of Economics 21(2), pp. 348-358.

Cockshott, W. P. and A. F. Cottrell (1997). Labour time versus alternative value bases: a research note. Cambridge Journal of Economics 21(4), pp. 545-549.

Cogliano, J. F. (2011). Smith's 'Perfect Liberty' and Marx's Equalized Rate of Surplus-Value. Department of Economics, The New School for Social Research, WP N. 08/2011.

Cogliano, J. F. (2013). New Directions in Political Economy: Value Theory, Agent-Based Computational Modeling, and the Dynamics of Labor Mobility. Ph. D. thesis, New School for Social Research, New York, NY.

Cogliano, J. F. and X. Jiang (2016). Agent-based computational economics: simulation tools for heterodox research. In F. S. Lee and B. Cronin (Eds.), Handbook of Research Methods and Applications in Heterodox Economics. Cheltenham: Edward Elgar.

Cogliano, J. F., R. Veneziani, and N. Yoshihara (2016). The dynamics of exploitation and class in accumulation economies. Metroeconomica 67(2), pp. 242-290.

Cogoy, M. (1979). Traditionalle und neue Arbeitswerttheorie. Gesellschaft - Beiträge zue Marxschen Theorie 13, pp. 115-139.

Costabile, L. and R. Rowthorn (1985). Malthus's theory of wages and growth. Economic Journal 95, pp. 418-437.

Cressy, R. C. (1976). Commodity and industry technology: symbols and assumptions. The Manchester School 44, pp. 122-131.

de Juan, O. and E. Febrero (2000). Measuring productivity from vertically integrated sectors. Economic Systems Research 12, pp. 65-82.

Desai, M. (1988). The transformation problem. Journal of Economic Surveys 2(4), pp. 295-333.

Desai, M. (2015). Hubris. Why economists failed to predict the crisis and how to avoid the next one. London: Harper Collins.

Desai, M. and R. Veneziani (2009). Rosa Luxemburg's critique of Marx's schemes of reproduction. In R. Bellofiore (Ed.), Rosa Luxemburg and the Critique of Political Economy. Abingdon, Oxon: Routledge.

Dietzenbacher, E. (1989). The implications of technical change in a Marxian framework. Journal of Economics 50, pp. 35-46.

Duménil, G. (1980). De la valuer aux prix de production. Paris: Economica. 
Duménil, G. (1983). Beyond the transformation riddle: A labor theory of value. Science \&S Society 47(4), pp. 427-450.

Duménil, G. (1984). The so-called 'transformation problem' revisited: A brief comment. Journal of Economic Theory 33(2), pp. 340-348.

Duménil, G., D. Foley, and D. Lévy (2009). A note on the formal treatment of exploitation in a model with heterogeneous labor. Metroeconomica 60(3), pp. 560-567.

Duménil, G. and D. Lévy (1993). The Economics of the Profit Rate: Competition, Crises, and Historical Tendencies in Capitalism. Aldershot: Edward Elgar.

Duménil, G. and D. Lévy (1995). A stochastic model of technical change. Metroeconomica 46, pp. 213-245.

Duménil, G. and D. Lévy (2002). The field of capital mobility and the gravitation of profit rates. Review of Radical Political Economics 34(4), pp. 417-436.

Duménil, G. and D. Lévy (2003). Technology and distribution: historical trajectories à la Marx. Journal of Economic Behavior 83 Organization 52, pp. 201-233.

Durand, R. (1994). An alternative to double deflation for measuring real industry value-added. Review of Income and Wealth 40, pp. 303-16.

Dutt, A. K. and R. Veneziani (2010). A Classical-Marxian Model of Education, Growth, and Distribution. DP N. 10, Department of Economics, University of Massachusetts Amherst.

Dutt, A. K. and R. Veneziani (2011). Education, growth and distribution: classical-Marxian economic thought and a simple model. Cahiers d'Economie Politique 61, pp. 157-185.

Elster, J. (1986). An Introduction to Karl Marx. Cambridge: Cambridge University Press.

Evans, D. W. (1953). Indexes of labor productivity as a partial measure of technological change. In Input-Output Relations. Leiden: The Netherlands Economic Institute.

Farjoun, E. and M. Machover (1983). Laws of Chaos. London, UK: Verso.

Felipe, J. and F. M. Fisher (2003). Aggregation in production functions: what applied economists should know. Metroeconomica 54, pp. 208262.

Fine, B. and L. Harris (1979). Rereading Capital. New York, NY: Columbia University Press.

Fisher, F. M. (1983). Disequilibrium Foundations of Equilibrium Economics. Cambridge, UK: Cambridge University Press. 
Flaschel, P. (1980). The derivation and comparison of employment multipliers and labor productivity indexes using monetary and physical input-output tables. Economics of Planning 16, pp. 118129.

Flaschel, P. (1983a). Actual labor values in a general model of production. Econometrica 51(2), pp. 435-454.

Flaschel, P. (1983b). Marx, Sraffa und Leontief. Kritik und Ansätze zu ihrer Synthese. Europäische Hochschulschriften, Frankfurt/M: Peter Lang.

Flaschel, P. (1984). The Standard Commodity and the theory of income distribution. Australian Economic Papers 23, pp. 123-129.

Flaschel, P. (1986). Sraffa's Standard Commodity: No fulfillment of Ricardo's dream of an 'invariable measure of value'. Journal of Institutional and Theoretical Economics 142(3), pp. 588-602.

Flaschel, P. (2009). Macrodynamics: Elements for a Synthesis of Marx, Keynes and Schumpeter. Heidelberg: Springer.

Flaschel, P. (2010). Topics in Classical Mirco- and Macroeconomics. New York, NY: Springer.

Flaschel, P. (2013). 'You have to regulate capitalism, otherwise the criminals will dominate it': Interview with Peter Flaschel. Intervention - European Journal of Economics and Economic Policies 10(1), pp. 2-7.

Flaschel, P., R. Franke, and R. Veneziani (2012a). The measurement of prices of production: An alternative approach. Review of Political Economy 24(3), pp. 417-435.

Flaschel, P., R. Franke, and R. Veneziani (2012b). Reply to Bertram Schefold. Review of Political Economy 24(3), pp. 445-447.

Flaschel, P., R. Franke, and R. Veneziani (2013). Labor productivity and the Law of Decreasing Labor Content. Cambridge Journal of Economics 37(2), pp. 379-402.

Flaschel, P., N. Fröhlich, and R. Veneziani (2013a). The sources of aggregate profitability: Marx's theory of surplus value revisited. Intervention - European Journal of Economics and Economic Policies 10, pp. 299-312.

Flaschel, P., N. Fröhlich, and R. Veneziani (2013b). The sources of profitability. In L. Taylor, A. Rezai, and T. R. Michl (Eds.), Social Fairness and Economics: Economics Essays in the Spirit of Duncan Foley. Abingdon, Oxon: Routledge.

Flaschel, P. and W. Semmler (1986a). Cross-Dual Dynamics for Sraffa/von Neumann Models with Nonsubstitution Properties. Mimeo. University of Bielefeld and the New School for Social Research, New York, NY. 
Flaschel, P. and W. Semmler (1986b). The dynamic equalization of profit rates for input-output models with fixed capital. In W. Semmler (Ed.), Competition, Instability and Nonlinear Cycles. Berlin: Springer Verlag.

Flaschel, P. and W. Semmler (1987). Classical and neoclassical competitive adjustment processes. The Manchester School 55(1), pp. $13-37$.

Fleurbaey, M. (1996). Théories Économiques de la Justice. Paris: Economica.

Foley, D. and G. Duménil (2008). Marxian transformation problem. In S. N. Durlauf and L. E. Blume (Eds.), The New Palgrave Dictionary of Economics. Basingstoke: Palgrave Macmillan.

Foley, D. K. (1982). The value of money the value of labor power and the Marxian transformation problem. Review of Radical Political Economics 14(2), pp. 37-47.

Foley, D. K. (1986a). Money, Accumulation, and Crisis. New York, NY: Harwood.

Foley, D. K. (1986b). Understanding Capital. Cambridge, MA: Harvard University Press.

Foley, D. K. (2000). Recent developments in the labor theory of value. Review of Radical Political Economics 32(1), pp. 1-39.

Foley, D. K. (2003). Endogenous technical change with externalities in a classical growth model. Journal of Economic Behavior \& Organization 52, pp. 167-189.

Foley, D. K. (2011). The long-period method and Marx's theory of value. In V. Caspari (Ed.), The Evolution of Economic Theory: Essays in Honour of Bertram Schefold. Abingdon, Oxon: Routledge.

Foley, D. K. (2016). What is the labor theory of value and what is it good for? In G. Freni, H. D. Kurz, A. M. Lavezzi, and R. Signorino (Eds.), Economic Theory and its History: Essays in Honour of Neri Salvadori. Abingdon, Oxon: Routledge.

Foley, D. K. and S. Mohun (2016). Value and price. In G. Faccarello and H. D. Kurz (Eds.), Handbook of the History of Economic Thought, Volume 3: Developments in Major Fields of Economics. London and Cheltenham: Edward Elgar.

Franke, R. (1988). A note on the Lotka-Volterra gravitation process and its pleasant properties. The Manchester School 56, pp. 147-157.

Freeman, A. and G. Carchedi (1996). Marx and Non-Equilibrium Economics. Brookfield, VT: Edward Elgar.

Fröhlich, N. (2013). Labour values, prices of production and the missing equalisation tendency of profit rates: evidence from the German economy. Cambridge Journal of Economics 37(5), pp. 1107-1126. 
Fujimori, Y. (1982). Wage-profit curves in a von neumann-leontief model: Theory and computation of japan's economy 1970-1980. Journal of Applied Input-Output Analysis 1(1), pp. 43-54.

Fujimoto, T. (1975). Duality and uniqueness of growth equilibrium. International Economic Review 16, pp. 781-791.

Fujimoto, T. and A. Opocher (2010). Commodity content in a general input-output model. Metroeconomica 61 (3), pp. 442-453.

Gehrke, C. and H. D. Kurz (2001). Say and Ricardo on value and distribution. European Journal of the History of Economic Thought 8, pp. 449-486.

Giannini, C. (1976). Saggi di profitto difformi e merce tipo. Giornale degli Economisti e Annali di Economia 35, pp. 349-355.

Grillo, M. (1976). Introduzione di saggi del profitto differenti in uno schema di interdipendenze settoriali. Giornale degli Economisti e Annali di Economia 35, pp. 201-208.

Gupta, S. and I. Steedman (1971). An input-output study of labour productivity in the British economy. Oxford Bulletin of Economics and Statistics 33, pp. 21-34.

Hahn, F. H. (1982). Stability. In K. J. Arrow (Ed.), Handbook of Mathematical Economics. Amsterdam: North-Holland.

Han, Z. and B. Schefold (2006). An empirical investigation of paradoxes: reswitching and reverse capital deepening in capital theory. Cambridge Journal of Economics 30, pp. 737-765.

Harcourt, G. (1972). Some Cambridge Controversies in the Theory of Capital. Cambridge: Cambridge University Press.

Hart, P. E. (1996). Accounting for economic growth of firms in UK manufacturing since 1973. Cambridge Journal of Economics 20, pp. 225-242.

Himmelweit, S. (1974). The continuing saga of the falling rate of profit - a reply to Mario Cogoy. Bulletin of the Conference of Socialist Economists 9, pp. 1-6.

Hirsch, M. W. and S. Smale (1974). Differential Equations, Dynamical Systems, and Linear Algebra. New York, NY: Academic Press, Inc.

Hirsch, M. W., S. Smale, and R. L. Devaney (2012). Differential Equations, Dynamical Systems, and an Introduction to Chaos (3rd ed.). Waltham, MA: Academic Press, Inc.

Holländer, H. (1982). Class antagonism, exploitation and the labor theory of value. Economic Journal 92, pp. 868-885.

Hopps, B. (2007). Profitability of UK companies. London: Office for National Statistics. http://unstats.un.org/unsd/snal1993/handbooks.asp. 
Jaeger, K. (1979). Wachstumstheorie. Eine kapitaltheoretische Einführung. Stuttgart: W. Kohlhammer.

Kaldor, N. (1955). Alternative theories of distribution. Review of Economic Studies 23(2), pp. 83-100.

Kalmbach, P., R. Franke, R. Knottenbauer, and H. Krämer (2005). Die Interdependenz von Industrie und Dienstleistungen: Zur Dynamik eines komplexen Beziehungsgeflechts. Berlin: edition sigma.

Keynes, J. M. (1936). The General Theory of Employment, Interest, and Money. New York, NY: Harcourt.

Kliman, A. J. and T. McGlone (1999). A temporal singlesystem interpretation of Marx's value theory. Review of Political Economy 11(1), pp. 33-59.

Krause, U. (1981). Heterogeneous labour and the fundamental Marxian theorem. Review of Economic Studies 48(1), pp. 173-178.

Krelle, W. (1976). Basic facts in capital theory. Some lessons from the controversy in capital theory. Revue d'Economie Politique 87, pp. 283-329.

Kuczynski, M. and R. L. Meek (1972). Quesnay's Tableau Économique. London: Macmillan.

Kurz, H. D. and N. Salvadori (1992). Morishima on Ricardo. Cambridge Journal of Economics 16, pp. 227-247.

Kurz, H. D. and N. Salvadori (1995). Theory of Production: A longperiod analysis. Cambridge, UK: Cambridge University Press.

Kurz, H. D. and N. Salvadori (2002). One theory or two? Walras's critique of Ricardo. History of Political Economy 34, pp. 365-398.

Lancaster, K. (1968). Mathematical Economics. London: Macmillan.

Leontief, W. (1941). The Stucture of American Economy, 1919-1939: An Empirical Application of Equilibrium Analysis. Oxford University Press.

Leontief, W. (1944). Output, employment, consumption and investment. Quarterly Journal of Economics 58(2), pp. 290-314.

Leontief, W. (1953). Studies in the Structure of the American Economy. New York, NY: Oxford University Press.

Leontief, W. (1986). Input-Output Economics. New York, NY: Oxford University Press.

Marglin, S. (1984). Growth, Distribution, and Prices. Cambridge, MA: Harvard University Press.

Marquetti, A. (2003). Analyzing historical and regional patterns of technical change from a classical-Marxian perspective. Journal of Economic Behavior 65 Organization 52, pp. 191-200.

Marx, K. (1976). Capital: Volume I. New York, NY: Penguin Group. Marx, K. (1978). Capital: Volume II. New York, NY: Penguin Group. 
Marx, K. (1981). Capital: Volume III. New York, NY: Penguin Group.

Matsuo, T. (2008). Profit, surplus product, exploitation and less than maximized utility: A new equivalence proposition on the fundamental Marxian theorem. Metroeconomica 59(2), pp. 249-265.

Meade, D. (2010). Why real value added is not my favorite concept. Studies on Russian Economic Development 21, pp. 249-262.

Michl, T. R. (1994). Three models of the falling rate of profit. Review of Radical Political Economics 24, pp. 55-75.

Mohun, S. (1993). A re(in)statement of the labor theory of value. Cambridge Journal of Economics 18(4), pp. 391-412.

Mohun, S. (1996). Productive and unproductive labor in the labor theory of value. Review of Radical Political Economics 28(4), pp. $30-54$.

Mohun, S. (2003). On the TSSI and the exploitation theory of profit. Capital \&3 Class 27(3), pp. 85-102.

Mohun, S. (2004). The labour theory of value as foundation for empirical investigations. Metroeconomica 55(1), pp. 65-95.

Mohun, S. (2009). Aggregate capital productivity in the US economy, 1964-2001. Cambridge Journal of Economics 33(5), pp. 1023-1046.

Mohun, S. and R. Veneziani (2007). The incoherence of the TSSI: A reply to Kliman and Freeman. Capital \& Class 31(2), pp. 139-145.

Mohun, S. and R. Veneziani (2009). The Temporal Single-System Interpretation: Underdetermination and inconsistency. Marxism21 6, pp. 277-301.

Mohun, S. and R. Veneziani (2012). Reorienting Economics? Philosophy of the Social Sciences 42, pp. 126-145.

Mohun, S. and R. Veneziani (2017). Values, price and exploitation: the logic of the transformation problem. Mimeo. Queen Mary University of London.

Moore, C. and R. Jaedicke (1972). Managerial Accounting. Cincinnati, OH: South-Western Publishing Co.

Morishima, M. (1973). Marx's Economics. New York, NY: Cambridge University Press.

Morishima, M. (1974). Marx in the light of modern economic theory. Econometrica 42(4), pp. 611-632.

Morishima, M. (1976a). Marx from a von Neumann viewpoint. In M. Brown, K. Sato, and P. Zarembka (Eds.), Essays in Modern Capital Theory. Amsterdam: North-Holland.

Morishima, M. (1976b). Positive profits with negative surplus value a comment. Economic Journal 86, pp. 599-603.

Morishima, M. (1989). Ricardo's Economics. Cambridge: Cambridge University Press. 
Morishima, M. and G. Catephores (1978). Value, Exploitation and Growth. London: McGraw Hill.

Morishima, M. and F. Seton (1961). Aggregation in Leontief matrices and the labour theory of value. Econometrica 29(2), pp. 203-220.

Moseley, F. (2000). The "New Solution" to the transformation problem: A sympathetic critique. Review of Radical Political Economics 32(2), pp. 282-316.

Moseley, F. (2016). Money and Totality. Leiden, The Netherlands: Brill.

Mueller, D. (1986). Profits in the Long Run. Cambridge: Cambridge University Press.

Mueller, D. (1990). The Dynamics of Company Profits: An International Comparison. Cambridge: Cambridge University Press.

Murata, Y. (1977). Fundamental Marxian theorem in case of multiple activities. Metroeconomica 29, pp. 137-148.

Negishi, T. (1989). History of Economic Theory. Amsterdam: NorthHolland.

Nikaido, H. (1968). Convex Structures and Economic Theory. New York, NY: Academic Press, Inc.

Nutzinger, H. (1976). Concepts of value in linear economic models. Operations Research Verfahren 26, pp. 717-724.

Ochoa, E. M. (1987). Is reswitching empirically relevant? US wageprofit rate frontiers, 1947-1972. Economic Forum 16, pp. 45-67.

OECD (2001). Measuring Productivity. OECD Economic Studies no. $33,2001 /$ II.

Okishio, N. (1963). A mathematical note on Marxian theorems. Weltwirtschaftliches Archiv 91, pp. 287-298.

Pasinetti, L. L. (1960). A mathematical formulation of the Ricardian system. Review of Economic Studies 27, pp. 78-98.

Pasinetti, L. L. (1962). The rate of profit and income distribution in relation to the rate of economic growth. Review of Economic Studies 29(4), pp. 267-279.

Pasinetti, L. L. (1977). Lectures on the Theory of Production. New York, NY: Columbia University Press.

Petith, H. (2008). Land, technical progress and the falling rate of profit. Journal of Economic Behavior 63 Organization 66, pp. 687-702.

Petri, F. (1980). Positive profits without exploitation: A note on the generalized fundamental Marxian theorem. Econometrica 48(2), pp. $531-533$.

Press, W., B. Flannery, S. Teukolsky, and W. Vetterling (1986). Numerical Recipes - The Art of Scientific Computing. Cambridge: Cambridge University Press. 
Rada, C. and L. Taylor (2006). Empty sources of growth accounting, and empirical replacements à la Kaldor and Goodwin with some beef. Structural Change and Economic Dynamics 17(4), pp. 486-500.

Ricardo, D. (1951). On the Principles of Political Economy and Taxation. Cambridge, UK: Cambridge University Press. Reprinted in 2004 by Liberty Fund, Inc., Indianapolis, IN.

Roemer, J. E. (1977). Technical change and the 'tendency of the rate of profit to fall'. Journal of Economic Theory 16, pp. 403-424.

Roemer, J. E. (1979). Continuing controversy on the falling rate of profit: fixed capital and other issues. Cambridge Journal of Economics 3, pp. 379-398.

Roemer, J. E. (1980a). A general equilibrium approach to Marxian economics. Econometrica 48(2), pp. 505-530.

Roemer, J. E. (1980b). Innovation, rates of profit, and the uniqueness of von Neumann prices. Journal of Economic Theory 22, pp. 451-464.

Roemer, J. E. (1981). Analytical Foundations of Marxian Economic Theory. Cambridge: Cambridge University Press.

Roemer, J. E. (1982). A General Theory of Exploitation and Class. Cambridge, MA: Harvard University Press.

Roemer, J. E. (2002). Equality of opportunity: A progress report. Social Choice and Welfare 19(2), pp. 455-471.

Roman, Z. (1974). Productivity measurement by help of input-output analysis. Jahrbuch Wirtschaft Osteuropas 5, pp. 171-193.

Rouche, N., P. Habets, and M. Laloy (1977). Stability Theory by Lyapunov's Direct Method. New York, NY: Springer Verlag.

Salvadori, N. (2004). Is Ricardian extensive rent a Nash equilibrium? In R. Arena and N. Salvadori (Eds.), Money, Credit and the Role of the State: Essays in Honour of Augusto Graziani. Farnham: Ashgate.

Samuelson, P. A. (1970). The "transformation" from Marxian "value" to competitive "prices": A process of rejection and replacement. Proceedings of the National Academy of Sciences of the United States of America 67(1), pp. 423-425.

Samuelson, P. A. (1971). Understanding the Marxian notion of exploitation: A summary of the so-called transformation problem between Marxian balues and competitive prices. Journal of Economic Literature 9(2), pp. 399-431.

Samuelson, P. A. (1978). The canonical Classical model of political economy. Journal of Economic Literature 16, pp. 1415-1434.

Sargent, T. (1987). Macroeconomic Theory. New York, NY: Academic Press, Inc.

Sato, K. (1979). A note on capital and output aggregation in a general equilibrium model of production. Econometrica 47, pp. 1559-1563. 
Scharfenaker, E. and G. Semieniuk (2017). A statistical equilibrium approach to the distribution of profit rates. Metroeconomica 68(3), pp. 465-499.

Schefold, B. (1986). The Standard Commodity as a tool of economic analysis: A comment on Flaschel. Journal of Institutional and Theoretical Economics 142(3), pp. 603-622.

Schreyer, P. (2001). The OECD productivity manual: a guide to the measurement of industry-level and aggregate productivity. International Productivity Monitor 2, pp. 37-51.

Schreyer, P. (2004). Chain Index Number Formulae in the National Accounts. Eighth OECD-NBS Workshop on National Accounts, 6-10 December.

Semmler, W. (1984). Competition, Monopoly, and Differential Profit Rates. New York, NY: Columbia University Press.

Sen, A. (1978). On the labour theory of value: some methodological issues. Cambridge Journal of Economics 2(2), pp. 175-190.

Seneta, E. (1973). Non-Negative Matrices: An Introduction to Theory and Applications. London: George Allen \& Unwin.

Shaikh, A. (1974). Laws of production and laws of algebra: The humbug production function. Review of Economic Studies 56(1), pp. 115-120.

Shaikh, A. (1984). The transformation from Marx to Sraffa. In E. Mandel and A. Freeman (Eds.), Ricardo, Marx, Sraffa. New York, NY: Verso.

Shaikh, A. (1998). The emprical strength of the labor theory of value. In R. Bellofiore (Ed.), Marxian Economics: A Reappraisal, Vol. 2. New York: St. Martin's Press.

Shaikh, A. (2008). Competition and Industrial Rates of Return. In P. Arestis and J. Eatwell (Eds.), Issues in Finance and Industry. New York, NY: Palgrave Macmillan.

Shaikh, A. (2016). Capitalism: Competition, Conflict, Crises. New York, NY: Oxford University Press.

Shaikh, A. and E. A. Tonak (1994). Measuring the Wealth of Nations. New York, NY: Cambridge University Press.

Shinozaki, N., M. Sibuya, and E. K. Tanabe (1972). Numerical algorithms for the Moore-Penrose inverse of a matrix: Direct methods. Annals of the Institute of Statistical Mathematics 24, pp. 193-203.

Silva, E. A. (1991). The wage-profit curve in Brazil: an input-output model with fixed capital. Review of Radical Political Economics 23, pp. 104-110.

Smith, A. ([1776] 2000). The Wealth of Nations. New York, NY: Random House, Inc. Modern Library Edition. Originally published 
in 1776 as: An Inquiry into the Nature and Causes of the Wealth of Nations.

Solow, R. M. (1956). A contribution to the theory of economic growth. Quarterly Journal of Economics 70(1), pp. 65-94.

Spaventa, L. (1970). Rate of profit, rate of growth and capital intensity in a simple produciton model. Oxford Economic Papers 22(2), pp. $129-147$.

Sraffa, P. (1960). Production of Commodities by Means of Commodities. Cambridge, UK: Cambridge University Press.

Sraffa, P. (Ed.) (1970). The Works and Correspondence of David Ricardo, Volume 1. Cambridge: Cambridge University Press.

Stahmer, C. (2000). The magic triangle of input-output tables. In S. Simon and J. Proops (Eds.), Greening the Accounts. Cheltenham: Edward Elgar.

Steedman, I. (1976). Positive profits with negative surplus value: a reply. Economic Journal 86, pp. 604-608.

Steedman, I. (1977). Marx After Sraffa. London: New Left Books.

Steedman, I. (1979). Trade Among Growing Nations. Cambridge, UK: Cambridge University Press.

Steedman, I. (1983). On the measurement and aggregation of productivity increase. Metroeconomica 35, pp. 223-233.

Steedman, I. (1984). Natural prices, differential profit rates and the classical competitive process. The Manchester School 52(2), pp. 123140.

Steedman, I. (2008). Marx after Sraffa and the open economy. Bulletin of Political Economy 2, pp. 165-174.

Stiroh, K. J. (2002). Information technology and the U.S. productivity revival: what do the industry data say? American Economic Review 92, pp. 1559-1576.

Stone, R. (1968). A system of national accounts. United Nations, Department of Economic and Social Affairs. New York, NY.

Stoneman, P. and N. Francis (1994). Double deflation and the measurement of output and productivity in UK manufacturing 197989. International Journal of the Economics of Business 1, pp. 423437.

Takeda, S. (1978). A note on the fundamental Marxian theorem. Economic Studies Quarterly 49, pp. 67-76.

Tsoulfidis, L. and T. Maniatis (2002). Values, prices of production and market prices: some more evidence from the Greek economy. Cambridge Journal of Economics 26, pp. 359-369.

United Nations (1968). A system of national accounts. New York: Studies in Methods, Series F, No. 2, Rev. 3. 
United Nations (1973). Input-output tables and analysis. New York: Studies in Methods, Series F, No. 14, Rev. 1.

United Nations (1993). System of national accounts. United Nations Statistics Division: http://unstats.un.org/unsd/sna1993/handbooks.asp.

Veneziani, R. (2004). The Temporal Single-System Interpretation of Marx's economics: A critical evaluation. Metroeconomica 55(1), pp. 96-114.

Veneziani, R. (2005). Dynamics, disequilibrium, and Marxian economics. Review of Radical Political Economics 37, pp. 517-529.

Veneziani, R. (2007). Exploitation and time. Journal of Economic Theory 132, pp. 189-207.

Veneziani, R. (2008). A future for (analytical) Marxism? Philosophy of the Social Sciences 38, pp. 388-399.

Veneziani, R. (2009a). Global capitalism and imperialism theory. some issues of substance and method. Review of Political Economy 21, pp. $195-211$.

Veneziani, R. (2009b). The rationality of analytical Marxism. In A. Chitty and M. McIvor (Eds.), Karl Marx and Contemporary Philosophy. Basingstoke: Palgrave Macmillan.

Veneziani, R. (2009c). Rosa Luxemburg on Imperialism: Some issues of substance and method. In R. Bellofiore (Ed.), Rosa Luxemburg and the Critique of Political Economy. Abingdon, Oxon: Routledge.

Veneziani, R. (2012). Analytical Marxism. Journal of Economic Surveys 26, pp. 649-673.

Veneziani, R. (2013). Exploitation, inequality, and power. Journal of Theoretical Politics 25(4), pp. 526-545.

Veneziani, R. and N. Yoshihara (2010). Exploitation and productiveness: The generalised commodity exploitation theorem once again. Bulletin of Political Economy 4, pp. 45-58.

Veneziani, R. and N. Yoshihara (2011). Strong subjectivism in the Marxian theory of exploitation: A critique. Metroeconomica 62, pp. 53-68.

Veneziani, R. and N. Yoshihara (2012). Profits and exploitation: A reappraisal. Advances in Mathematical Economics 16, pp. 85-109.

Veneziani, R. and N. Yoshihara (2013a). Exploitation of labour and exploitation of commodities: a 'New Interpretation'. Review of Radical Political Economics 45, pp. 517-524.

Veneziani, R. and N. Yoshihara (2013b). The Measurement of Labour Content: A General Approach. WP N.704, School of Economics and Finance, Queen Mary University of London. 
Veneziani, R. and N. Yoshihara (2015a). Exploitation in economies with heterogeneous preferences, skills and assets: An axiomatic approach. Journal of Theoretical Politics 27(1), pp. 8-33.

Veneziani, R. and N. Yoshihara (2015b). Unequal exchange, assets, and power: recent developments in exploitation theory. In C. Binder, G. Codognato, M. Teschi, and Y. Xu (Eds.), Individual and Collective Choice and Social Welfare. Berlin: Springer.

Veneziani, R. and N. Yoshihara (2017a). Globalisation and inequality in a dynamic economy: An axiomatic analysis of unequal exchange. Social Choice and Welfare, Forthcoming.

Veneziani, R. and N. Yoshihara (2017b). One million miles to go: taking the axiomatic road to defining exploitation. Cambridge Journal of Economics, Forthcoming.

von Neumann, J. (1937). A model of general economic equilibrium. Review of Economic Studies 13(1), pp. 1-9. Translated version published in 1945-1946.

Weizsäcker, C. C. v. (1971). Steady State Capital Theory. Heidelberg: Springer.

Wolff, E. N. (1979). The rate of surplus value, the organic composition, and the general rate of profit in the U.S. economy, 1947-67. American Economic Review 69(3), pp. 329-341.

Wolff, E. N. (1985). Industrial composition, interindustry effects, and the U.S. productivity slowdown. Review of Economics and Statistics 67, pp. 268-277.

Wolff, E. N. (1994). Productivity measurement within an input-output framework. Regional Science and Urban Economics 24, pp. 75-92.

Wolff, R. D., B. Roberts, and A. Callari (1982). Marx's (not Ricardo's) "transformation problem": a radical reconceptualization. History of Political Economy 14(4), pp. 564-582.

Wolfstetter, E. (1973). Surplus value, synchronized labour costs and Marx's labour theory of value. Economic Journal 83, pp. 787-809.

Wolfstetter, E. (1976). Positive profits with negative surplus value: a comment. Economic Journal 86, pp. 864-872.

Wolfstetter, E. (1980). Value, natural price, duality, and time: a reexamination of some propositions in Marxian economics. Metroeconomica 32, pp. 137-153.

Woods, J. E. (1978). Mathematical Economics. Suffolk: Richard Clay (The Chaucer Press).

Wright, I. (2008). The emergence of the law of value in a dynamic simple commodity economy. Review of Political Economy 20(3), pp. $367-391$. 
Yoshihara, N. (2010). Class and exploitation in general convex cone economies. Journal of Economic Behavior \& Organization 75, pp. 281-296.

Yoshihara, N. (2017). A progress report on Marxian economic theory: On the controversies in exploitation theory since Okishio. Journal of Economic Surveys 31(2), pp. 632-659.

Yoshihara, N. and R. Veneziani (2009). Exploitation as the Unequal Exchange of Labour: An Axiomatic Approach. WP N. 655, Queen Mary, University of London.

Yoshihara, N. and R. Veneziani (2010). Commodity content in a general input-output model: A comment. Metroeconomica 61(4), pp. 740748. 
